\title{
Optical Manipulation and Structured Materials Conference
}

, "Optical Manipulation and Structured Materials Conference," Proc. SPIE 11141, Optical Manipulation and Structured Materials Conference, 1114101 (21 April 2019); doi: 10.1117/12.2535563

SPIE. Event: Optics and Photonics International Congress, 2019, Yokohama, Japan 


\section{PROCEEDINGS OF SPIE}

\section{Optical Manipulation and Structured Materials Conference}

Takashige Omatsu

Editor

22-26 April 2019

Yokohama, Japan

Published by SPIE

Volume 11141 


\section{Contents}

vii Author Index

xi Conference Committee

BISC-OMC JOINT SESSION

JS-3-02 Optical Tweezers in Biology

JS-3-03 Optical trap and laser interferometry in living cells

JS-3-05 Isotropic Quantitative Differential Phase Contrast Microscopy with Vortex Asymmetric Illumination Patterns

JS-3-06 Monitoring mitochondrial dynamics within mitotic apparatus by lightsheet microscopy

\section{ORAL SESSION 1}

OMC-1-01 Ultrafast force-clamp spectroscopy: dissecting the mechanosensitivity of biomolecule interactions on the microsecond time scale

OMC-1-02 Resonance laser effect on optical trapping of cell surface molecules

OMC-1-03 Single-protein and single-nanoparticle trapping using plasmonic nanoaperture array

OMC-1-04 Helical biomaterial breaks spatial symmetry of helical light field

OMC-1-05 Proposed method of single-particle absorption measurement based on optical transport at solid-liquid interface

\section{ORAL SESSION 2}

OMC-2-01 Searches for new physics using optically levitated spheres.

OMC-2-02 Rotation control of nanoparticles by optical force using resonant nonlinear response

OMC-2-03 Optical trapping in extreme conditions

OMC-2-04 Optical trapping of nanoparticles using dimer and trimer plasmonic nanogap antennas

OMC-2-05 Raman Imaging of Plasmonic Ag Nanostructure for Site-Dependent Molecular Trapping Analysis 
ORAL SESSION 3

OMC-3-01 Optomechanics with optically trapped nanoparticles

OMC-3-02 Modulation of orbital torque on nanoparticles by spin angular momentum via inter-particle light-induced force

OMC-3-03 Proposal of an optical-force probe for chirality sensing of metallic nanostructures

OMC-3-04 One-dimensional optical lattices for optical trapping and manipulation along a few-mode silicon waveguide

OMC-3-05 Real-time monitoring of a reagent release from an optically trapped biodegradable microparticle for drug delivery

\section{ORAL SESSION 4}

OMC-4-01 Self-consistent symmetries of structured ultrafast laser fields and plasmonic nanostructures for optimal laser coupling and sensing

OMC-4-02 Investigation of plasmonic lasing by using focused radially polarized beam

OMC-4-03 Vortex mode emission properties of vertical cavity surface emitting laser with external optical feedback

OMC-4-04 Spatial mode generation and detection by means of the sum-frequency upconversion process

OMC-4-05

Q-Switched All-Fiber Laser based on Graphene Oxide in the C- and L-Bands Using Electrical Deposition Method and Pulse Laser Drilling

\section{ORAL SESSION 5}

OMC-5-01 Vector Holographic Trapping and Tweezing

OMC-5-02 AC electrophoretic mobility of an optically trapped colloidal particle

OMC-5-03 Optical vortex ablation creates high viscosity 'ink-jet'

OMC-5-04 Chiral mass-transport of azo-polymers with OAM light field through two photon absorption

OMC-5-05 Optical propulsion of fluorescent diamonds inside a tapered capillary 


\section{ORAL SESSION 6}

OMC-2-05 Assembling and dynamic ejection of polystyrene particles in CW laser trapping at solution surface

OMC-6-01 The History and Future of Optical Manipulation

OMC-6-02 In-plane orbital motion of particles in microchannels induced by optical vortices

\section{ORAL SESSION 7}

OMC-7-01 Numerical demonstration of the alignment of multiple nanoparticles in a wide area beyound single focal laser spot

OMC-7-02 Polarization-Dependence of Optical Trapping on Polystyrene Nanoparticles and Their Assembly Formation

OMC-7-03 Manipulation of molecular ground-state photodissociation on a gold nanoantenna surface Multi-focal holographic SAX microscopy

OMC-7-04 Weak value amplification of skew aberration

OMC-7-05 Cryptanalysis of computational optical ghost imaging cryptosystems via deep learning

OMC-7-06 Metasurface polarizers with ultra-high extinction ratios<br / >in the telecommunication wavelengths

\section{ORAL SESSION 8}

OMC-8-01 Trapping and manipulation of microparticles using optothermal effects

OMC-8-02 Motion of micro-sized colloidal particles induced by optical vortex

OMC-8-03 Mass transfer and composition change during metal sphere migration in glass by continuous laser illumination

OMC-8-04 Optical Manipulation by Photochemistry

OMC-8-05 Two-photon photo-polymerization induced helical microfibers

\section{ORAL SESSION 9}

OMC-9-01 Spectroscopy of Deoxyribonucleic Acid Film: From Ultraviolet to Terahertz

OMC-9-02 Tunable vortex parametric laser with multiple OAM states 
OMC-9-03 The PCF design for more number of OAM modes up to 101 by increasing the number of airholes

OMC-9-04 Visible vortex light source based on a diode pumped Pr $^{3+}$ :YLF laser

OMC-9-05 Efficient generation of intense spatio-temporally controlled light waves

OMC-9-06 Observation and temperature measurement of fast moving metal sphere in a glass with laser illumination

POSTER SESSION

OMC-P-O1 Size- and composition-controlled synthesis of Au-Ag nanorings for plasmonic applications High-Efficient Holographic Photopolymer Based on Fluorinated Epoxy Resin

OMC-P-02 Three-dimensional observations of particle flows in microchannels induced by photothermal effects

OMC-P-03 Optical control of particle oscillation through a rectangular orifice in a microchannel

OMC-P-04 Transition strength of a standing optical vortex beam in monolayer transition metal dichalcogenides

OMC-P-05 Generation of three-dimensional dark hole by hybrid phase plate in super-resolution microscopy

OMC-P-06 Manipulation of DNA using Nano-structured Semiconductor-assisted (NASSCA) Optical Tweezers

OMC-P-07 Laguerre-Gaussian self-trapped beams in optical lattices

OMC-P-08 High Q-factor planar toroidal metamaterial with the ability of strong magnetic field localization

OMC-P-09 Nano-structured Semiconductor-assisted (NASSCA) Optical Tweezers for Size sorting of polystyrene nanospheres

OMC-P-10 Structured nanofiber-based optical cavity for quantum electrodynamics Development of photoplethysmogram sensor with an LED and sampling rate assessment

OMC-P-11 Non-destructive dispersion of quantum dots into buffer gases toward their optical manipulation

OMC-P-12 Wavefront restoration of high-intensity pulsed laser radiation by acousto-optics Dynamic phase imaging of Haematococcus pluvialis cells by transport of intensity equation

OMC-P-13 Two techniques for experimental generation of spiral light beams

OMC-P-14 What will be done with the magnetically trapped superconducting micro particle? 
OMC-P-15 Diagnosis of semiconductor materials such as cadmium chalcogenides by the method of exiton-polariton luminescence

OMC-P-16 Second-harmonic generation in swift $\mathrm{O}^{5+}$ ion irradiated $\mathrm{KTiOPO}_{4}$ ridge waveguide

OMC-P-17 Advanced nanoantenna

OMC-P-18 The resemblance of polarization spectra of polymers between photo- and mechanicallyinduced microstrains

OMC-P-19 Controlling the electrical size of a conducting cylinder by eccentric<br />coating of Matched Impedance Zero Index Metamaterial

OMC-P-20 Spin Momentum Locking in a Tightly Focused Gaussian Beam

OMC-P-21 Nano-post arrays for optical interconnects

OMC-P-22 Multiring pure-phase binary optical elements to tunable axial multi-focus beam intensity

OMC-P-23 Simultaneously achieving a large negative dispersion and a high birefringence over Er and Tm dual gain bands in a square lattice photonic crystal fiber

OMC-P-24 Diffraction-free mapping of arbitrary modes from pump to probe beam via coherent population oscillation in two-level system

OMC-P-25 Optical and Thermal Time-Dependent Analysis<br / >for Simulating Thermal Lens Effect by High Power Lasers

OMC-P-26 Emission lifetime measurement of optically trapped single particles by using stimulated emission

OMC-P-27 Metalens array generated structured light for distance sensing

OMC-P-28 Emergence of optical extreme events from a modified Fresnel zone plate

OMC-P-29 Giant enhancement of cooperative effect in superfluorescence of arranged molecules by nanoscale metallic structures

OMC-P-30 Generation of pure vector field from the interference of two ellipse fields embedded with Cpoints and V-points 


\section{Authors}

Numbers in the index correspond to the numbers in both the table of content.

Akira, Chiba, OMC-9-06

Akiyama, Tomoki, OMC-1-04

Arita, Yoshihiko, OMC-8-05

Ashida, Masaaki, OMC-2-03, OMC-P-14

Ashihara, Satoshi, OMC-7-03

Awaji, Yoshinari, OMC-4-03

Baba, Yuta, OMC-P-11

Bahloul, Faouzi, OMC-P-23

Banerjee, Ayan, OMC-P-20

Barker, Peter, OMC-3-01

Basharin, Alexey, OMC-P-08

Bhebhe, Nkosi, OMC-5-01

Bonatto, Cristian, OMC-P-28

Capitanio, Marco, OMC-1-01

Chaganava, Irakli, OMC-P-18

Chen, Mu Ku, OMC-P-27

Chen, Chin-Yi, JS-3-06

Chen, Bi-Chang, JS-3-06

Chen, Yung-Fu, OMC-9-04

Cheng, Yazhou, OMC-P-16

Cheon, Seunguk, OMC-9-01

Chiba, Akira, OMC-8-03

Chu, Cheng Hung, OMC-P-27

Chuang, Yu-Hsuan, JS-3-05

Cluzel, Benoît, OMC-3-04

Cojocari, Maria, OMC-P-08

Correia, Ricardo, OMC-P-28

Cruz-Gomez, Miguel, OMC-P-07

de Fornel, Frédérique, OMC-3-04

Dey, Koustav, OMC-P-24

Dholakia, Kishan, OMC-6-01, OMC-8-05

Doi, Kentaro, OMC-P-03, OMC-6-02

Du, Jinjin, OMC-P-10

Efimova, Kseniya, OMC-P-13

Favre-Bulle, Itia, JS-3-02

Forbes, Andrew, OMC-4-04, OMC-5-01

Fritsch, Amanda, OMC-P-28

Fujikawa, Seiya, OMC-2-04

Fujiwara, Kayo, OMC-3-05

Fujiwara, Hideki, OMC-5-05

Fukaminato, Tuyoshi, OMC-2-04

Fukushima, Tomohiro, OMC-6-03

Geng, Xi, OMC-2-03

Ghosh, Nirmalya, OMC-P-20

Gu, Huarong, OMC-P-21

Gupta, Subhasish, OMC-P-20

Hadji, Emmanuel, OMC-3-04

Han, Bennian, OMC-7-05

Han, Xue, OMC-1-03

Han, Hai, OMC-7-05

Harvie, Elliot, OMC-1-03
Hashimoto, Sayaka, OMC-P-09

$\mathrm{He}$, Wenqi, OMC-7-05

Hidai, Hirohumi, OMC-8-03

Hirofumi, Hidai, OMC-9-06

Hong, Seongjin, OMC-4-05, OMC-9-03

Hoshina, Masayuki, OMC-2-02, OMC-3-03, OMC-P-29

Hosokawa, Chie, OMC-1-02

Ichijo, Mitsuki, OMC-5-04

lida, Miyako, OMC-3-05

lida, Takuya, OMC-3-02

Iketaki, Yoshinori, OMC-P-05

Iki, Kohei, OMC-5-02

Imai, Takuya, OMC-8-03

Inove, Shin-ichiro, OMC-7-06

Ishihara, Hajime, OMC-1-05, OMC-2-02,

OMC-3-03, OMC-7-01, OMC-7-02, OMC-P-04,

OMC-P-29

Ishii, Shodai, OMC-P-04

Ito, Syoji, OMC-P-26

Iwamoto, Kenta, OMC-8-02

Iwasa, Kohei, OMC-9-05

Iwata, Muneaki, OMC-5-03

Jager, Jean-Baptiste, OMC-3-04

Jeong, Hayoung, OMC-9-01

Juodkazis, Saulius, OMC-P-06, OMC-P-09

Kameda, Shinji, OMC-P-25

Kameyama, Tatsuya, OMC-P-01, OMC-P-1 1

Kaneko, Akihiro, OMC-5-03

Kang, Chul, OMC-9-01

Kawaguchi, Haruki, OMC-8-05

Kawano, Satoyuki, OMC-6-02, OMC-P-02, OMC-

$\mathrm{P}-03$

Kilosanidze, Barbara, OMC-P-18

Kim, Byungjoo, OMC-4-05

Kim, Soeun, OMC-9-01, OMC-9-03, OMC-P-23

Kimura, Yasuyuki, OMC-5-02

Kishi, Tetsuo, OMC-8-03

Kishimoto, Tatsunori, OMC-1-02

Kishkin, Sergey, OMC-P-13

Kitamura, Kyoko, OMC-4-02

Kobulashvili, Irine, OMC-P-18

Kodaira, Akira, OMC-P-05

Kokado, Kensuke, OMC-2-03

Konrad, Thomas, OMC-4-04

Kotova, Svetlana, OMC-P-13

Kotsifaki, Domna, OMC-1-03

Koyama, Ryota, OMC-P-03

Kudo, Tetsuhiro, OMC-2-05

Kudoh, Suguru, OMC-1-02

Kudryashov, Sergey, OMC-4-01 
Kumagai, Hiroshi, OMC-P-05

Kumakura, Mitsutaka, OMC-P-11, OMC-P-14

Kuo, Hsin Yu, OMC-P-27

Kurosawa, Hiroyuki, OMC-7-06

Lee, Junhyung, OMC-8-05

Lee, Yong Soo, OMC-P-23, OMC-9-03

Lee, Chung Ghiv, OMC-P-23

Lee, Yong Soo, OMC-9-01

$\mathrm{Li}$, Yan, OMC-9-03

Li, Wenfang, OMC-P-10

Liao, Meihua, OMC-7-05

Lin, Shulang, OMC-P-21

Lin, Yu-Zi, JS-3-05

Lin, Ren Jie, OMC-P-27

Linklater, Denver, OMC-P-06

Lopez-Aguayo, Servando, OMC-P-07

Lopez-Aguayo, Daniel, OMC-P-07

Losevsky, Nikolai, OMC-P-13

Lu, Jia-Syun, OMC-2-05

Lu, Dajiang, OMC-7-05

Luo, Yuan, JS-3-05

Ma, Yuanyuan, OMC-9-04

Maeda, Maki, OMC-P-10

Maeng, Inhee, OMC-9-01

Malik, Tayyab, OMC-P-19

Mamuti, Roukuya, OMC-9-02

Maruyama, Takashi, OMC-P-05

Masuda, Keigo, OMC-1-04, OMC-5-04

Masuhara, Hiroshi, OMC-2-05

Matsumoto, Mitsuhiro, OMC-3-05

Matsuo, Reimon, OMC-8-05

Matsusaka, Souta, OMC-8-03

Matsuura, Tomoki, OMC-7-01, OMC-7-02

Minowa, Yosuke, OMC-2-03

Miyamoto, Yoko, OMC-7-04

Miyamoto, Katsuhiko, OMC-1-04, OMC-5-03,

OMC-5-04, OMC-8-05, OMC-9-02, OMC-9-04

Miyasaka, Hiroshi, OMC-P-26

Miyauchi, Akira, OMC-6-03

Mizuno, Daisuke, JS-3-03

Molchanov, Vladimir, OMC-P-12

Monteiro, Fernando, OMC-2-01

Morichika, Ikki, OMC-7-03

Morita, Ryuji, OMC-4-03, OMC-9-05

Morita, Noboru, OMC-8-03

Moriwaki, Yoshiki, OMC-P-14

Moriyasu, Takeshi, OMC-P-11

Murakoshi, Kei, OMC-6-03

Murata, Takeshi, OMC-1-04

Nagai, Koumei, OMC-P-05

Nagura, Ryo, OMC-6-02

Nakagawa, Kohki, OMC-4-03

Nakamoto, Takahiro, OMC-P-02

Nakamura, Ryosuke, OMC-5-03

Nakatsuka, Ryoji, OMC-6-02

Nándor, Bokor, OMC-P-05

Naoi, Jun, OMC-P-14

Nic Chormaic, Síle, OMC-1-03, OMC-P-10

Nishida, Shigeki, OMC-9-02

Nishioka, Nobuyasu, OMC-9-06
Nishizawa, Kenji, JS-3-03

Nito, Fumika, OMC-P-03

Noboru, Morita, OMC-9-06

Nudga, Alexandr, OMC-P-17

Obuchi, Hiroya, OMC-4-02

Oh, Kyunghwan, OMC-4-05, OMC-9-01,

OMC-9-03, OMC-P-23

Oikawa, Shunpei, OMC-6-03

Oka, Kazuhiko, OMC-9-05

Okada-shudo, Yoshiko, OMC-1-04

Okamoto, Shunsuke, OMC-P-26

Oku, Satoshi, OMC-P-05

Omatsu, Takashige, OMC-1-04, OMC-3-02,

OMC-5-03, OMC-5-04, OMC-8-05, OMC-9-02,

OMC-9-04, JS-3-01

Ortega-Mendoza, Gabriel, OMC-8-01

Otsuka, Ryohei, OMC-5-05

Oyamada, Nobuaki, OMC-6-03

Pal, Debapriya, OMC-P-20

Pal, Sushanta, OMC-P-30

Pan, Shuixin, OMC-7-05

Peng, Xiang, OMC-7-05

Picard, Emmanuel, OMC-3-04

Pin, Christophe, OMC-2-04, OMC-3-04, OMC-5-05

Poletaev, Dmitrii, OMC-P-17

Polvonov, Bakhtiyor, OMC-P-15

Prisyazhniuk, Andrei, OMC-P-17

Prokopova, Dariya, OMC-P-13

Quan, Chai, OMC-9-03

Ramirez-San-Juan, Julio, OMC-8-01

Ramos-Garcia, Ruben, OMC-8-01

Rodríguez-Fajardo, Valeria, OMC-5-01

Romagnoli, Priscila, OMC-P-10

Rosales-Guzman, Carmelo, OMC-5-01

Roux, Filippus S., OMC-4-04

Roy, Sourabh, OMC-P-24

Rubinsztein-Dunlop, Halina, JS-3-02

Sakurai, Atsunori, OMC-7-03

Samagin, Sergey, OMC-P-13

Samlan, CT, OMC-7-04

Sarabia-Alonso, Julio, OMC-8-01

Sasaki, Rin, OMC-9-05

Sasaki, Shota, OMC-P-14

Sasaki, Keiji, OMC-2-04, OMC-5-05

Sasamoto, Kosuke, OMC-P-01

Saxena, Aditya, OMC-1-03

Sekkat, Zouheir, OMC-8-04

Senthilkumaran, P., OMC-P-30

Septhon, Bereneice, OMC-4-04

Setoura, Kenji, OMC-P-26

Shimomura, Takayuki, OMC-P-1 1

Shinozaki, Ryo, OMC-5-04

Shiraki, Hirofumi, OMC-P-29

Shoji, Tatsuya, OMC-3-05

Shoji, Tatsuya, OMC-P-06, OMC-P-09

Sokolenko, Bogdan, OMC-P-17

Sotome, Hikaru, OMC-P-26

Souta, Matsusaka, OMC-9-06

Spezzia-Mazzocco, Teresita, OMC-8-01 
Starosek, Alexandr, OMC-P-17

Steinlechner, Fabian, OMC-4-04

Stilgoe, Alexander, JS-3-02

Sudo, Kota, OMC-2-04

Sugino, Yujiro, JS-3-03

Taguchi, Takahisa, OMC-1-02

Takahashi, Genta, OMC-2-04

Takamune, Masato, OMC-P-14

Takao, Ryota, OMC-P-06

Takiyama, Takayuki, OMC-P-1 1

Tamura, Mamoru, OMC-3-02

Tan, Qiaofeng, OMC-P-22

Tao, Yukihiro, OMC-7-01, OMC-7-02

Tardif, Manon, OMC-3-04

Toda, Yasunori, OMC-4-03, OMC-9-05

Torimoto, Tsukasa, OMC-P-01, OMC-P-1 1

Torres, Juan P., OMC-4-04

Toyoda, Kohei, OMC-1-04, OMC-5-03

Truong, Viet Giang, OMC-1-03

Truong, Viet, OMC-P-10

Tsai, Ying-Ju, JS-3-05

Tsai, Din Ping, OMC-P-27

Tsuboi, Yasuyuki, OMC-P-06, OMC-3-05,

OMC-P-09

Tsuji, Tetsuro, OMC-P-02, OMC-P-03, OMC-6-02

Tsujimura, Tempei, OMC-6-02

Tung, Jung-Chen, OMC-9-04

Umeda, Katsuhiro, JS-3-03

Valles, Adam, OMC-4-04

Verma, Onkar, OMC-P-24

Wada, Takudo, OMC-1-05

Wang, Wen-Cheng, JS-3-06

Ward, Jonathan, OMC-P-10

$\mathrm{XU}$, Ning, OMC-P-22

Yamane, Keisaku, OMC-4-03, OMC-5-04,

OMC-9-05

Yatagai, Toyohiko, JS-3-01

Yeh, J. Andrew, JS-3-05

Yokoshi, Nobuhiko, OMC-2-02, OMC-3-03,

OMC-P-29, OMC-P-04

Yokoyama, Tomohiro, OMC-7-01, OMC-7-02

Yoshimura, Shuko, OMC-8-03

Yoshizawa, Taiki, OMC-1-04

Yushkov, Konstantin, OMC-P-12

Zhang, Chenggong, OMC-7-05 
Proc. of SPIE Vol. 11141 1114101-10

Downloaded From: https://www.spiedigitallibrary.org/conference-proceedings-of-spie on 26 Apr 2023 Terms of Use: https://www.spiedigitallibrary.org/terms-of-use 


\title{
Conference Committee
}

\author{
Conference Chair
}

Takashige Omatsu, Chiba University (Japan)

Conference Co-chairs

Hajime Ishihara, Osaka Prefecture University (Japan)

Keiji Sasaki, Hokkaido University (Japan)

Conference Program Committee

Masaaki Ashida, Osaka University (Japan)

Satoshi Ashihara, The University of Tokyo (Japan)

Yung-Fu Chen, National Chiao Tung University (Taiwan)

Síle Nic Chormaic, Okinawa Institute of Science and Technology

Graduate University (Japan)

Kyoko Kitamura

Ryuji Morita, Hokkaido University (Japan)

Kei Murakoshi, Hokkaido University (Japan)

Kyunghwan Oh, Yonsei University (Korea, Republic of)

Seigo Ohno, Tohoku University (Japan)

Hiromi Okamoto, Institute for Molecular Science (Japan)

Ichiro Shoji, Chuo University (Japan)

Yasuhiro Sugawara, Osaka University (Japan)

Yasuyuki Tsuboi, Osaka City University (Japan) 
Proc. of SPIE Vol. 11141 1114101-12

Downloaded From: https://www.spiedigitallibrary.org/conference-proceedings-of-spie on 26 Apr 2023 Terms of Use: https://www.spiedigitallibrary.org/terms-of-use 


\title{
Optical Tweezers in Biology
}

\author{
Alexander B. Stilgoe*a, Itia A. Favre-Bulle ${ }^{a}$, and Halina Rubinsztein-Dunlop ${ }^{a, b}$

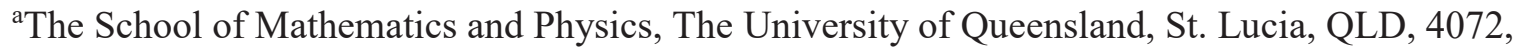 \\ Australia \\ ${ }^{\mathrm{b}} \mathrm{ARC}$ Centre of Excellence for Engineered Quantum Systems, The University of Queensland, St. \\ Lucia, QLD, 4072, Australia
}

\begin{abstract}
Optical tweezers has enabled application and measurement of forces in a highly quantitative way and has been used to discover and study in depth a multitude of biological processes. In this tutorial presentation, we start with overview of the original work by Arthur Ashkins' and the first applications of optical tweezers in biological systems and we describe the principles of optical trapping showing how to measure optical forces and torques. We describe the broad use of optical tweezers with applications ranging from cell manipulation to molecular biology. We conclude the presentation with discussions of the current state of the art of this area of science and discuss further applications of optical tweezers and possible future directions.
\end{abstract}

Keywords: optical tweezers, forces, torques, cells, biophysics, molecular biology, beam shaping, light detection,

\section{Introduction}

Optical tweezers have been widely used in quantitative measurements of biological systems and processes since the first demonstration of the technique in 1986 by Arthur Ashkin 1. They were utilized in biophysics and cell biology to shine light into the understanding of processes taking place in cells and molecules ${ }^{2,3}$. Here we describe optical tweezers, outlining the principal of operation and review their use in biological systems as well as in a number of other micro-systems.

\section{Body \\ 1. Principal of operation and measurement}

Optical tweezers can trap, manipulate and measure forces acting on particles in a range from femto- to nanoNewtons (fN-nN). Optical forces occur due to the conservation of momentum when light scatters off an object. If a particle scatters light such that the average direction of the light is changed, then the particle must experience a force in the opposite direction. We outline various methods of modelling this process, including: Rayleigh scattering, geometrical optics, and an approach based on a full electromagnetic theory. We outline how the effect of the scattering can be measured using various detection systems, such as camera and position sensitive detector, and how these are incorporated into a system to measure forces acting in a broad variety of systems including biological and bio-medically relevant ones.

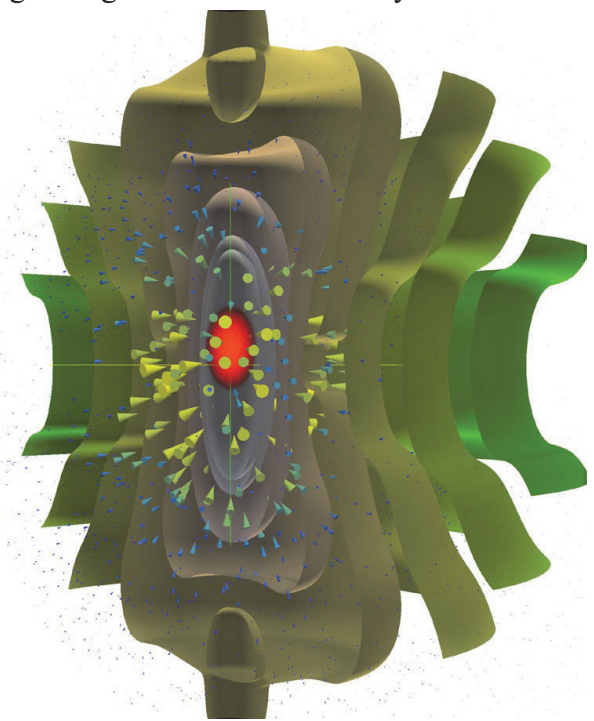

Figure 1: Model and simulation of an optically trapped particle in a highly focused laser beam. Cones depict relative magnitude of optical force. Ellipsoidal patch shows the distribution of particle location under Brownian motion. 3D contours represent the equipotential of intensity of a laser beam.

* stilgoe@physics.uq.edu.au 


\section{Applications to biology}

In the years since their first demonstration optical tweezers have been used to manipulate organelles ${ }^{4}$, and measure molecular motions, such as the compliance of flagella ${ }^{5}$ and kinesin stepping ${ }^{6}$. Optical tweezers have been particularly successful in the realm of biochemistry and have been used to precisely measure DNA, protein and enzyme function ${ }^{3}$. An optical tweezers ability to remotely, and without contact, manipulate has been essential to these experiments and applications. We cover the large range of methods used to illustrate the range of capabilities of the optical tweezers technique.
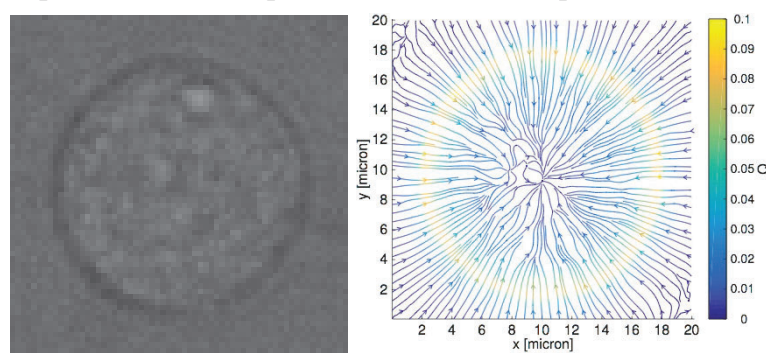

Figure 2: Digital micrograph of Chinese hamster ovary (CHO) cell and optical forces acting on it as a function of position. The overall spherical shape gives a net trapping force, relative changes of individual parts of the cell can be both manipulated and measured with optical tweezers.

\section{Current work, our work and future directions}

We have been using optical tweezers in a number of applications with particular emphases on measurement of optical torque and rotation. This enabled measurement of viscoelastic properties in biologically relevant systems. We also have been concerned with developing methods for using optical tweezers in in-vivo studies of model systems such as zebrafish and combining these with relevant imaging modalities to study in details neuronal systems.

The capabilities of optical tweezers are continually being extended, being now able to trap with greater degree of control over optical forces ${ }^{7,8}$, deeper into the system $^{9,10}$, with precision $^{11}$, and with measurement beyond spherical particles to irregular and deformable particles $^{12,13}$.
Future application of optical tweezers will be considered for measurements inside cells, studies of biological swimmers and their characterization.

\section{References}

1. Ashkin A, Dziedzic JM, Bjorkholm JE, Chu S. Observation of a single-beam gradient force optical trap for dielectric particles. Opt Lett. 1986;11 (5):288-290.

2. Svoboda K, Block SM. Biological Application of Optical Forces. Annual Review of Biophysics and Biomolecular Structures. 1994;23:247-285.

3. Moffitt JR, Chemla YR, Smith SB, Bustamante C. Recent advances in optical tweezers. Annual Review of Biochemistry. 2008;77:205-228.

4. Ashkin A, Schütze K, Dziedzic JM, Euteneuer U, Schliwa M. Force generation of organelle transport measured in vivo by an infrared laser trap. Nature. 1990;348:346.

5. Block SM, Blair DF, Berg HC. Compliance of bacterial flagella measured with optical tweezers. Nature. 1989;338:514.

6. Svoboda K, Block SM. Force and velocity measured for single kinesin molecules. Cell. 1994;77(5):773-784.

7. Taylor MA, Waleed $\mathrm{M}$, Stilgoe AB, Rubinsztein-Dunlop H, Bowen WP. Enhanced optical trapping via structured scattering. Nature Photonics. 2015;9(10):669-673.

8. Phillips DB, Padgett MJ, Hanna S, et al. Shapeinduced force fields in optical trapping. Nature Photonics. 2014;8:400.

9. Favre-Bulle IA, Stilgoe AB, Rubinsztein-Dunlop $\mathrm{H}$, Scott EK. Optical trapping of otoliths drives vestibular behaviours in larval zebrafish. Nature Communications. 2017;8(1):630-630.

10. Zhong M-C, Wei X-B, Zhou J-H, Wang Z-Q, Li Y-M. Trapping red blood cells in living animals using optical tweezers. Nature Communications. 2013;4:1768-1768.

11. Huang R, Chavez I, Taute KM, et al. Direct observation of the full transition from ballistic to diffusive Brownian motion in a liquid. Nature Physics. 2011;7(7):576-580. 
12. Català $\mathrm{F}$, Marsà $\mathrm{F}$, Montes-Usategui $\mathrm{M}$, Farré $\mathrm{A}$, Martín-Badosa E. Extending calibration-free force measurements to optically-trapped rodshaped samples. Scientific Reports. 2017;7:42960.

13. Bui AAM, Kashchuk AV, Balanant MA, Nieminen TA, Rubinsztein-Dunlop H, Stilgoe AB. Calibration of force detection for arbitrarily shaped particles in optical tweezers. Scientific Reports. 2018;8(1):10798.

Proc. of SPIE Vol. 11141 1114101-15 


\title{
Optical trap and laser interferometry in living cells Daisuke Mizuno, Umeda Katsuhiro, Sugino Yujiro, Kenji Nishizawa \\ Department of Physics, Kyushu University, Motooka 744 , Nishiku, Fukuoka 819-0395, Japan
}

\begin{abstract}
Mechanics of living cell interior are governed by cytoskeletons and cytosol. They are extraordinarily heterogeneous and their physical properties are strongly affected by the internally generated forces. In order to understand the out-ofequilibrium mechanics, we have developed a method of microrheology using laser interferometry and optical trapping technology. This method allowed us to probe mechanics and dynamics in living cells with a high spatio-temporal resolution. Microscopic probes in cells are stably trapped in the presence of vigorous cytoplasmic fluctuations, by employing smooth 3D feedback of a piezo-actuated sample stage. To interpret the data, we present a theory that adapts the fluctuation-dissipation theorem (FDT) to out-of-equilibrium systems. We discuss the interplay between material properties and non-thermal force fluctuations in the living cells that we quantify through the violations of the FDT.
\end{abstract}

Keywords: optical trap, laser interferometry, microrheology, cell mechanics

\section{Introduction}

Microrheology (MR) is a method to investigate the mechanical response of soft materials at microscopic $(\sim$ $\mu \mathrm{m})$ range. This technique enables us to measure the viscoelastic properties of the surrounding medium by tracking the motion of imbedded, micron-sized tracer beads [1]. One of the standard implementation of this method is active microrheology (AMR) where tracer beads are actively manipulated with an external force. Then we measure the displacement response of the beads, from which we obtain the viscoelasticity of surrounding media. The other method is passive microrheology (PMR) that passively tracks the imbedded bead's thermal fluctuations. PMR can provides material's rheological properties only at equilibrium since it relies on the fluctuation-dissipation theorem (FDT) to estimate the response function of the probe. Whereas FDT is satisfied only at equilibrium conditions, by comparing the result with PMR to AMR, we can estimate how strongly the system is driven out of equilibrium [2-5].

\section{MR with optical trap and laser interferometry}

Conventional active/passive microrheology has been typically performed using laser interferometry and optical trapping [1]. Laser interferometry (LI) is a technique to detect the position of the probe particle by illuminating it with a probe laser and measuring the interference pattern made between un-scattered and scattered lights [6]. Another laser (drive laser) is tightly focused on the same probe to trap it. Manipulating the focus positions, the arbitrarily controlled optical-trap (OT) force is applied to the probe. Both techniques (LI and OT) require keeping the probe well inside the sub- $\mu \mathrm{m}$-sized tightly-focused laser spot.

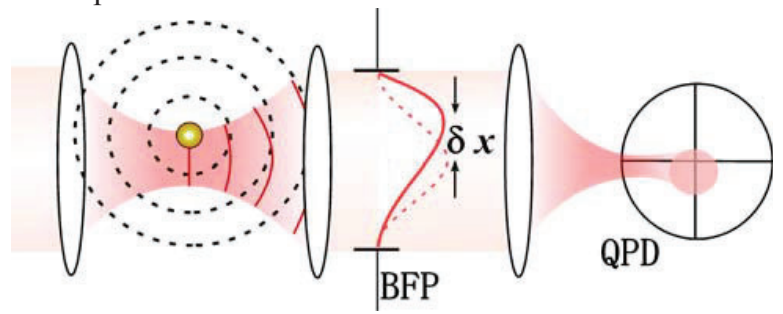

Fig. 1: Schematic illustration of laser interferometry. Image of the interference pattern at the back focal plane (BFP) was projected on to the quadrant photodiode (QPD).

In living cells, mechanoenzymes such as motor proteins create vigorous flows and fluctuations. The probe particle then moves out of the laser focus within the experimental time period. Although strong optical trapping force is able to retain the probe in the focus, a probe trapped in a strong optical potential does not display the mechanical properties of surrounding materials. 


\section{Feedback-tracking active microrheology}

To overcome these issues and perform MR in active environments (typically in living cells), we developed optical-trap-based MR implemented with threedimensional feedback-controlled sample stage [7]. The stage is manipulated by a piezo-mechanical sample stage to cancel large and slow movements of a probe particle so that high-bandwidth, large dynamic-range MR can be performed even in vigorous fluctuations e.g. HeLa cell.

The position of the sample stage $u_{\text {stage }}$ is controlled by the PID feedback as below. Probe position from the laser focus $u_{Q P D}$ measured by LI is used to control the piezo position as

$$
u_{\text {stage }}(t)=1 / \tau \int u_{Q P D} d t,
$$

where $\tau$ (set at $10 \sim 100 \mathrm{~ms}$ ) is the delay time for the feedback response of our experimental setup. Since the total displacement $u$ of the probe in the sample is given as $u=u_{Q P D}+u_{\text {sage }}$ (Fig. 2), eq. (1) yields frequency response relation:

$$
\hat{u}=(1-i \omega \tau) \hat{u}_{\text {stage }}=(1-1 / i \omega \tau) \hat{u}_{Q P D} .
$$

Hereafter, ${ }^{\wedge}$ denotes the amplitude of the sinusoidal signal.

Probe particles are manipulated by a sinusoidal lateral oscillation of the drive laser focus whereas the probe laser is stationary. The optical trapping force applied to the probe particle by drive and probe laser is $k_{d} L \exp (-i \omega t)-\left(k_{d}+k_{p}\right) u_{Q P D}$, where $k_{d}, k_{p}$ refer to the trap stiffness of the drive and probe laser respectively. $L$ is the amplitude of the drive laser oscillation. The Langevin equation for the probe particle under feedback control is then written as

$$
k_{p} u_{Q P D}(t)+\int_{-\infty}^{t} \gamma\left(t-t^{\prime}\right) \dot{u}\left(t^{\prime}\right) d t^{\prime}=k_{d}\left(L e^{-i \omega t}-u_{Q P D}(t)\right)+\zeta(t)+f(t),
$$

where $\dot{u}(t)$ denotes the velocity of the probe in the coordinate system traveling with the feedback-controlled piezo stage. $\zeta(t)$ and $f(t)$ are the thermal and nonthermal forces respectively, and $\gamma(t)$ is the friction function.. The Fourier transform of the ensemble or time average of eq. (3) yields the frequency-dependent response which we rewrite as

$$
\langle\hat{u}(\omega)\rangle^{F B}=\left\langle\hat{u}_{Q P D}(\omega)\right\rangle+\left\langle\hat{u}_{\text {stage }}(\omega)\right\rangle=\alpha(\omega)\left\{\hat{F}(\omega)-\left(k_{d}+k_{p}\right)\left\langle\hat{u}_{Q P D}(\omega)\right\rangle\right\},
$$

where $\hat{F}(\omega) \equiv k_{d} L$. The superscript FB denotes the total displacement when the position of the piezo stage is feedback controlled.
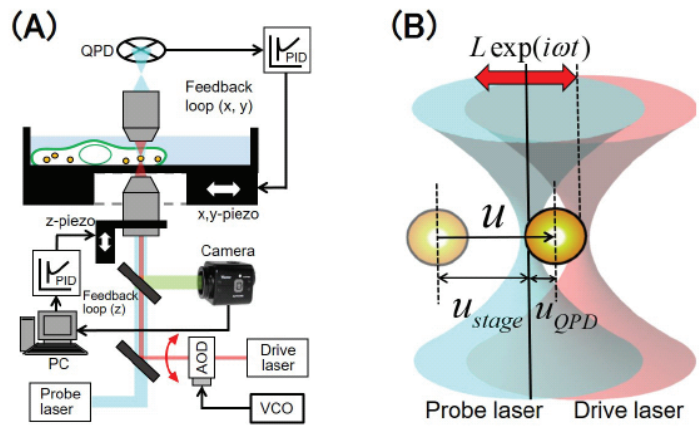

Fig. 2: (A) Schematic of the feedback-tracking microrheology setup. (B) Displacements of a probe and laser positions.

We define the "total" response of the probe movement to the wiggling force applied by the drive laser under the feedback control as $A_{F B} \equiv\langle\hat{u}(\omega)\rangle^{F B} / \hat{F}(\omega)$. By using this definition and substituting eq. (2) into eq. (4), we obtain the relationship between $A_{F B}(\omega)$ and intrinsic response function of a probe in the same media $\alpha(\omega)$ (i.e. response without trap) as

$$
A_{F B}(\omega)=\frac{\langle\hat{u}(\omega)\rangle^{F B}}{\hat{F}(\omega)}=\frac{\alpha(\omega)}{1+\beta \alpha(\omega)},
$$

where $\beta \equiv\left(k_{d}+k_{p}\right) /(1-i \omega \tau)$ is the parameter describing the correction under feedback. $\alpha(\omega)$ is then obtained from the total displacement as

$$
\alpha(\omega)=\frac{A_{F B}}{1-\beta A_{F B}(\omega)}=\frac{\langle\hat{u}(\omega)\rangle^{F B}}{\hat{F}(\omega)-\beta\langle\hat{u}(\omega)\rangle^{F B}} .
$$

The shear viscoelastic modulus $G(\omega)$ of the surrounding material is then obtained via the EinsteinStokes relation extended to frequency response, $G(\omega)=1 / 6 \pi \alpha(\omega) a$ where $a$ is the radius of the probe.

\section{Cell mechanics during cell-cycle progression}

As a typical application of the developed technique, we performed feedback-tracking active microrheology in HeLa cervical cancer cells. HeLa cells were seeded in the glass-bottom dish and incubated until epithelial-like sheets were formed. $2 a=1 \mu \mathrm{m}$ melamine particles (microParticles $\mathrm{GmbH}$ ) coated with polyethelene-glycol 
polymer brush were bombarded into cells with GeneGun (Biorad, 1652257J1).

In Fig. 3, we show shear viscoelasticity of the cytosols in HeLa cells as a function of frequency. We observed that the approximate power-law relation $G(\omega) \propto(i \omega)^{0.5}$ is satisfied in the wide range of frequencies. This response is in contrast to the cytoskeletal networks, for which elastic plateau response $\left[G(\omega) \propto(i \omega)^{0}\right]$ at low frequencies and $G(\omega) \propto(i \omega)^{3 / 4}$ at high frequencies have been typically observed for in vitro [2] and in vivo [7]. Recently, the anomalous viscoelastic response $\left[G(\omega) \propto(i \omega)^{0.5}\right]$ observed in living epithelium was attributed to the dynamics of cytosol rather than cytoskeletons $[7,8]$. In cells, various soft colloids, such as macromolecular assemblies, organelle, etc, are present at their highly condensed state. At the same time, they are forcedly fluidized because of the non-thermal mechanical energies originated from the active metabolism. In this sense, living cytosol is a typical representation of active colloidal glass, which is a current focus of study in the nonequilibrium statistical mechanics

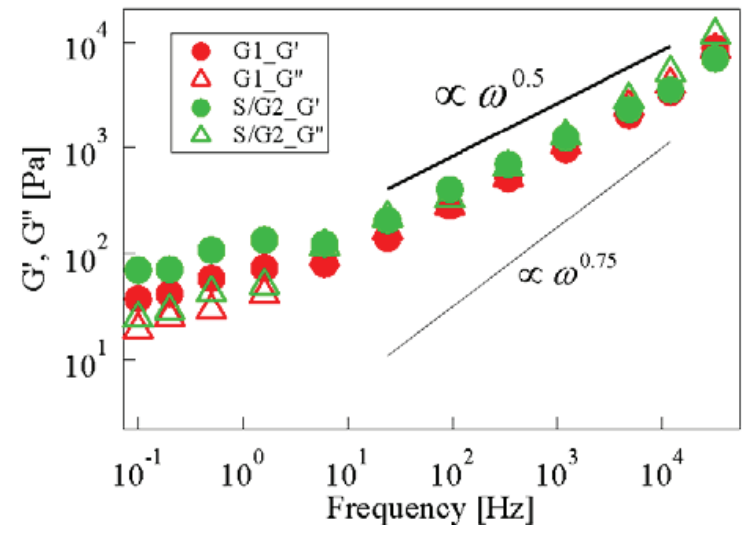

Fig.3: Shear viscoelastic spectrum of HeLa. Fucci2 cells in $\mathrm{G} 1$ and $\mathrm{S} / \mathrm{G} 2$ phases.

field.

The HeLa cells used in this study (HeLa. Fucci2) were labelled with Fucci system that enables to identify G1 and $\mathrm{S} / \mathrm{G} 2$ phases during the cell-cycle progression, by the fluorescence color in the cell nucleus [9]. We found little dependence of rheology during the cell-cycle progression. Although $G(\omega)$ in $\mathrm{S} / \mathrm{G} 2$ phase seems to be slightly increased, we believe this artifact. In this experiment, cells in G1 phase were subjected to microrheology experiments continually until they proceed to $\mathrm{S} / \mathrm{G} 2$ phases. The feedback-tracking microrheology technique allows us to perform experiments with less than $1 \mathrm{~mW}$ laser. For the sake of technical convenience, however, several $\mathrm{mW}$ laser was illuminated to the probe particle. We recently found that such prolonged exposure to several $\mathrm{mW}$ laser affects the probe response (data not shown).

In this study, cells in S, G2 and G1 phases were studied with microrheology. Cells in $M$ Phase could exhibit mechanical properties largely different from other phases. However, $M$ phase cells are rare to find and could be easily eliminated by observing the structure of nucleus under the microscope. Therefore, the finding in this study (little dependence of intracellular rheology on cell-cycle progression from $\mathrm{G} 1$ to $\mathrm{S} / \mathrm{G} 2$ ) allows us to investigate mechanics of cell interiors without strong concern on their cell-cycle dependence.

\section{References}

[1] D. Mizuno, D. A. Head, F. C. MacKintosh, and C. F. Schmidt, Macromolecules 41, 7194 (2008).

[2] D. Mizuno, C. Tardin, C. F. Schmidt, and F. C. MacKintosh, Science 315, 370 (2007).

[3] D. Mizuno, R. Bacabac, C. Tardin, D. Head, and C. F. Schmidt, Phys. Rev. Lett. 102, 168102 (2009).

[4] T. Harada, and S. Sasa, Phys. Rev. Lett. 95, 130602 (2005).

[5] T. Ariga, M. Tomishige, and D. Mizuno, Phys. Rev. Lett. 121 (2018).

[6] F. Gittes, and C. F. Schmidt, Opt Lett 23, 7 (1998).

[7] K. Nishizawa, M. Bremerich, H. Ayade, C. F. Schmidt, T. Ariga, and D. Mizuno, Sci Adv 3, e1700318 (2017).

[8] K. Nishizawa, K. Fujiwara, M. Ikenaga, N. Nakajo, M. Yanagisawa, and D. Mizuno, Sci Rep-Uk 7 (2017). [9] A. Sakaue-Sawano, H. Kurokawa, T. Morimura, A. Hanyu, H. Hama, H. Osawa, S. Kashiwagi, K. Fukami, T. Miyata, H. Miyoshi, T. Imamura, M. Ogawa, H. Masai, and A. Miyawaki, Cell 132, 487 (2008). 


\title{
Isotropic Quantitative Differential Phase Contrast Microscopy
}

$$
\text { with Vortex Asymmetric Illumination Patterns }
$$

\author{
Yu-Hsuan Chuang ${ }^{\mathrm{a}, \mathrm{b}^{*}}$, Ying-Ju Tsai ${ }^{\mathrm{b}, \mathrm{c}}$, Yu-Zi Lin ${ }^{\mathrm{b}}, \mathrm{J}$. Andrew Yeh ${ }^{\mathrm{a}}$ and Yuan Luo ${ }^{\mathrm{b}, \mathrm{d}^{*}}$ \\ ${ }^{a}$ Department of Power Mechanical Engineering, National Tsing Hua University, Hsinchu, 30013, Taiwan \\ ${ }^{\mathrm{b}}$ Institute of Medical Device and Imaging, National Taiwan University, Taipei 10051, Taiwan \\ ${ }^{\mathrm{c}}$ Department of Electrical Engineering, National Taiwan University, Taipei, 10617, Taiwan \\ ${ }^{\mathrm{d}}$ Molecular Imaging Center, National Taiwan University, Taipei, 10672, Taiwan
}

\begin{abstract}
Differential phase contrast (DPC) microscopy is a well-known methodology to recover phase image of transparent specimen under multi-axis intensity measurements. We propose a novel illumination method to achieve isotropic differential phase contrast(iDPC) efficiently and meanwhile improve the accuracy and stability of phase recovery effectively. Our iDPC system is an add-on module setting on the commercial inverted microscope, using a programmable TFT shield to control the illumination pattern. We demonstrate our theoretical approach for iDPC and experimental results by using radial asymmetric pupils to show our accuracy on phase recovery. Besides, we also implemented the new illumination pattern with multiple wavelengths to achieve the same circularly symmetric phase transfer function result in high-speed operation.
\end{abstract}

Keywords: isotropic differential phase contrast; asymmetric pupil; phase recovery; multiple wavelengths

\section{Introduction}

Differential phase contrast(DPC) microscopy[1] based on asymmetric illumination has long been used to retrieve high-resolution qualitative image of a phase specimen. Typically, in DPC techniques, a half-circle pupil with 2axis measurements along vertical and horizontal directions is utilized to reconstruct quantitative images of weak phase samples (i.e. cells). However, some artifacts are produced in the process due to the missing frequencies of phase reconstruction since DPC transfer function is not circularly symmetric with only 2 -axis measurements. Therefore, DPC using 12-axis measurements along twenty-four different angles[2] has been reported recently to obtain missing frequency information such that DPC transfer function becomes circularly symmetric, and thus additional DPC measurements along other axes can significantly improve stability and accuracy for quantitative phase recovery. Here, we present a new approach by using radially asymmetric pupils $[3,4]$ in engineered illumination to acquire pair-wise images for phase recovery and efficiently achieve circularly symmetric transfer function under partially coherent condition.[5] In contrast to conventional DPC using 2 axis measurements, our isotropic DPC does not have missing frequency issue. Besides, compared to the half-circle based DPC method using 12-axis, our approach provides equivalently symmetric transfer function with only 3-axis measurements.

\section{Theory}

By using isotropic differential phase contrast method, we can utilize weak object transfer function to separate the phase from intensity and achieve quantitative phase measurement by pair-wise images[1,2]. Our system can be interpreted by Fourier optics. We use the TFT shield to control our radially asymmetric pupils, so under incoherent illumination condition, the resultant intensity captured on the camera can be written as[2]

$I(r)=\iint|\mathcal{F}\{\mathcal{F}[\mathrm{B}(\mathrm{u}, \mathrm{r})] \cdot[\mathrm{P}(\mathrm{u})]\}|^{2} d^{2} u$,

where $\mathrm{F}$ donates the Fourier transfer function and $\mathrm{u}$ and $\mathrm{r}$ denote spatial coordinates $(x, y)$. I is the intensity on the camera and $\mathrm{P}$ is the pupil function in the objective lens. The transmission function of specimen can be presented as $O(r)=\exp (-\alpha(r)+i \phi(r))$, where $(r)$ denotes the spatial coordinates $(x, y), \alpha(r)$ is attenuation factor, and $\phi(r)$ is the optical phase. Under a weak object approximation, the 
intensity of a weakly scattering specimen in the Fourier space can be written as [6]

$I(u)=\widetilde{H}_{p h a s e} \cdot \widetilde{\phi}(u)+\widetilde{H}_{a m p} \cdot \tilde{\alpha}(u)+\widetilde{H}_{b} \cdot \tilde{\delta}(u)$,

where u, $\tilde{\phi}(u)$, and $\tilde{\alpha}(u)$ represent as spatial frequency coordinates, phase and absorption of the specimen respectively. Therefore, the expression for $\widetilde{H}_{\text {phase }}$ can be written as [7]

$$
\widetilde{H}_{\text {phase }}=j\left[\begin{array}{l}
\iint S\left(\mathrm{u}^{\prime}\right) P\left(\mathrm{u}^{\prime}+\mathrm{u}\right) P^{*}\left(\mathrm{u}^{\prime}\right) d^{2} u^{\prime} \\
-\iint S\left(\mathrm{u}^{\prime}\right) P\left(u^{\prime}\right) P^{*}\left(u^{\prime}-u\right) d^{2} u^{\prime}
\end{array}\right],
$$

\section{Experiment Setup and Results}

In Figure 1, we compare a conventional half-circle pupil with a radially asymmetrical pupil in illumination to see their numerical results of different intensity computed by transfer function $\left(\sum_{\mathrm{i}}\left|\widetilde{\mathrm{H}}_{\text {phase, } x}\right|^{2}\right)$ in multiple axes $(\mathrm{x}=1,2,3)$. Besides, in Figure $1(\mathrm{~g}, \mathrm{~h})$, we can see that by using our radially asymmetric pupils, we can achieve circularly symmetric intensity of transfer function with only 3 -axis measurements, which can be viewed as direct evidence of avoiding artifacts of missing frequencies for phase reconstruction.

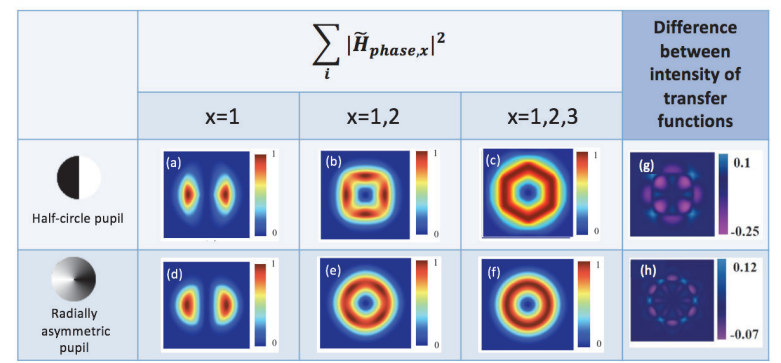

Figure 1. Simulation results of intensity of transfer

function $\left(\sum_{\mathrm{i}}\left|\widetilde{\mathrm{H}}_{\mathrm{phase}, x}\right|^{2}\right)$ along different axes.

Figure 2 is a schematic diagram of the proposed iDPC system setup with the thin-film transistor (TFT) shield located on the Fourier plane of the condenser lens. The TFT shield is controlled by Arduino to show pair-wise radially asymmetric patterns along three different axes. Each pair of images generates a differential phase contrast image, which is utilized to reconstruct quantitative phase information and largely reduce phase reconstruction artifacts of missing frequencies.

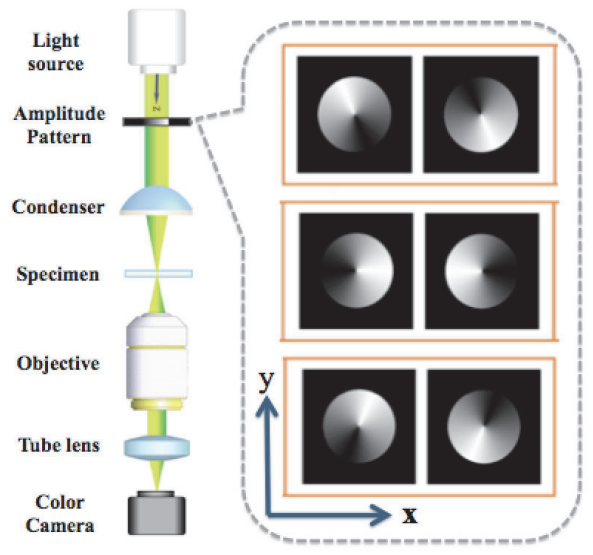

Figure 2. Schematic of our iDPC system setup, using a TFT shield as a programmable aperture.

Figure 3 shows quantitative phase images of live 3T3 mouse fibroblasts cells generated by computationally reconstruct three pairs of DPC images. The color bar in gray scales represents quantitative phase recovery of the cells, and the detailed information of live cells can be clearly observed from the image.
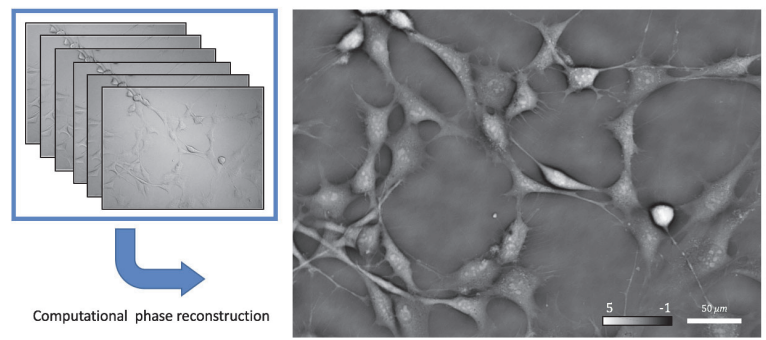

Figure 3. Quantitative phase images of live 3T3 mouse fibroblasts cells obtained using our iDPC system.

\section{Future Work}

Figure 4 We combine our radially asymmetric pattern with multiple wavelengths to shorten image acquisition time. However, different color spectrums may overlap on camera sensor, which is known as "color leakage" problem. This phenomenon will let the information of different color channels mix together and reduce the accuracy of reconstructed images significantly. In order to lower down the influence of color leakage problem, we 
choose the red and blue color in our pattern. The spectrum of red and blue is more separated than the combination with green, so that the visibility of specimen in the unprocessed image can be expected to be higher.

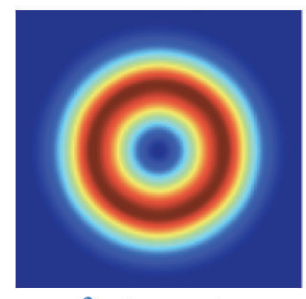

3 -axis approach Radially Asymmetric pupil

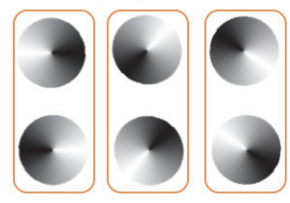

Figure 4. Combine our radially asymmetric pupil with different wavelengths based on the same isotropic circularly symmetric transfer function result.

Figure 5 (a)The quantitative phase image of $10 \mu \mathrm{m}$ microspheres using the multi-wavelength radially asymmetric pupil with 3-axis measurement after color leakage correction. (b)Measured phase distribution of the cross-section along the dashed lines of a zoomed-in microsphere at the solid box region of (a) The scalar bar is $50 \mu \mathrm{m}$.
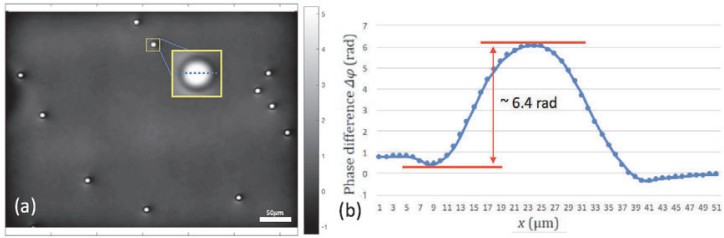

Figure 5. The quantitative phase image of $10 \mu \mathrm{m}$ microspheres and the cross-section phase profile

\section{Conclusions}

We have demonstrated the capability of iDPC to acquire quantitative phase images of label-free cell sample by using radially asymmetric pupils. The proposed iDPC shows isotropic intensity of transfer function, using three-axis measurements with compact system configuration as well as programmable TFT shield. The improvement of accuracy of phase recovery and stability using iDPC has potential to contribute to future study of biophotonics. Furthermore, radially asymmetric illumination can be implemented using multiple wavelengths to obtain quantitative phase images to achieve circularly symmetric phase transfer function, in high-speed operation as well.

\section{References}

[1] S. B. Mehta and C. J. R. Sheppard, "Quantitative phase-gradient imaging at high resolution with asymmetric illumination-based differential phase contrast," Opt. Lett. 34, 1924-1926 (2009).

[2] Tian, L. and Waller, L., "Quantitative differential phase contrast imaging in an LED array microscope." Optics Express, 2015. 23(9): p. 11394. [3] Y.-Z. Lin, K.-Y. Huang and Y. Luo, "Quantitative differential phase contrast imaging at high resolution with radially asymmetric illumination," Opt. Lett. 43, 2973-2976 (2018)

[4] Y.-H. Chuang, Y.-J. Tsai, Y.-Z. Lin and Y. Luo, "Isotropic Quantitative Differential Phase Contrast Microscopy with Radially Asymmetric Illumination," Optics \& Photonics Taiwan, the International Conference (OPTIC 2018)

[5] H.-H. Chen and Y. Luo, "Two-axis isotropic differential phase contrast transfer function using gradient amplitude modulation for phase imaging," in Imaging and Applied Optics 2017 (3D, AIO, COSI, IS, MATH, pcAOP), OSA Technical Digest (online) (Optical Society of America, 2017), CTh4B.5.

[6] Streibl, N., "3-Dimensional Imaging by a Microscope," Journal of the Optical Society of America a-Optics Image Science and Vision, 1985. 2(2): p. 121.

[7] Y.-Z. Lin, H.-H. Chen, K.-Y. Huang, and Y. Luo, "Isotropic Quantitative Differential Phase Contrast Microscopy." Optics \& Photonics Taiwan, the International Conference (OPTIC 2017) 


\title{
Monitoring mitochondrial dynamics within mitotic apparatus by lightsheet microscopy
}

\author{
Wen-Cheng Wang*, Chin-Yi Chen and Bi-Chang Chen \\ Research Center for Applied Sciences, Academia Sinica, Taipei 11529, Taiwan
}

\begin{abstract}
Mitochondria are major energy resources in cell. These highly dynamic organelles are constant fusion and fission inside of cell, especially while cells are at mitotic status. Fusion and fission of mitochondria allowed the inheritances (mitochondrial DNA; mtDNA) and mitochondrial proteins could be exchanged between mitochondria. Sharing the inheritances and mitochondrial proteins is necessary for mitochondria to maintain mitochondrial function. Here, we investigated dynamic of mitochondrial proteins during mitochondrial fusion and fission. We genetically expressed photoswitchable proteins (Dendra2) ${ }^{1}$ into mitochondria and optically monitored the inter-mitochondrial distribution of Dendra2 by lightsheet microscopy ${ }^{2}$. The advantages of lightsheet microscopy, low phototoxicity and fast image speed, make it possible to detect the fusion/fission events of mitochondria during mitosis. We observed that the small portion of photoswitched Dendra2 was transmitted to the whole cell within 15 minutes.
\end{abstract}

Keywords: lightsheet microscopy, mitochondrial protein, mitosis, Dendra2, photoswitch

Frequently mitochondrial fission and fusion (mitochondria dynamics) is one of mechanisms for mitochondria to control the functional quality of mitochondria ${ }^{3,4}$. The protein synthesis at cytoplasm would be imported into mitochondria via a recognition sequence. Targeting signals are usually removed once the protein has reached its correct inter-mitochondrial location. Furthermore, mitochondria also have their own mitochondrial DNA (mtDNA) and could synthesize peptides inside of mitochondria. For both types of proteins, they could be inter-mitochondrially redistributed via fusion and fission.

Mitochondrial matrix, lumen inside of inner mitochondrial membrane, is where metabolic process occurs. There are hundreds of enzyme and proteins required for tricarboxylic acid cycle, urea cycle, and apoptosis cascade and so on. Fusion and fission of mitochondria can result in mitochondrial matrix proteins transferred within mitochondria, ${ }^{5,6}$. In addition, damaged proteins and mutated/damaged mtDNA would be asymmetrically segregated into two daughter cells during cell division $^{7-9}$. Therefore, asymmetry division of mitotic or meiosis cell would generate a mixture of mtDNA ${ }^{10,11}$ within the daughter cells. Mechanisms of asymmetric division and excluding aging/damaged mitochondria regulate stemness preservation of stem cell and multipotency of neural progenitors ${ }^{12-15}$.

Since asymmetry division and fusion/fission play crucial roles in controlling cell function, we are wondering how mitochondria transfer their matrix proteins to mitochondria through fusion and fission. Also, mitotic cells turn into sphere, which become a hindrance for live imaging. To solve this problem, we overcame the obstacles of live imaging requirements by lightsheet microscopy. Increasing spatiotemporal resolutions and reducing phototoxicity to specimen allow us to observe several dynamic subcellular processes without sacrifice of substantial spatiotemporal resolution. By combining with photoswitchable fluorescent proteins, Dendra $2^{1}$, we could monitor the inter-mitochondrial dynamics of mitochondrial proteins during the cell division process of mitosis.

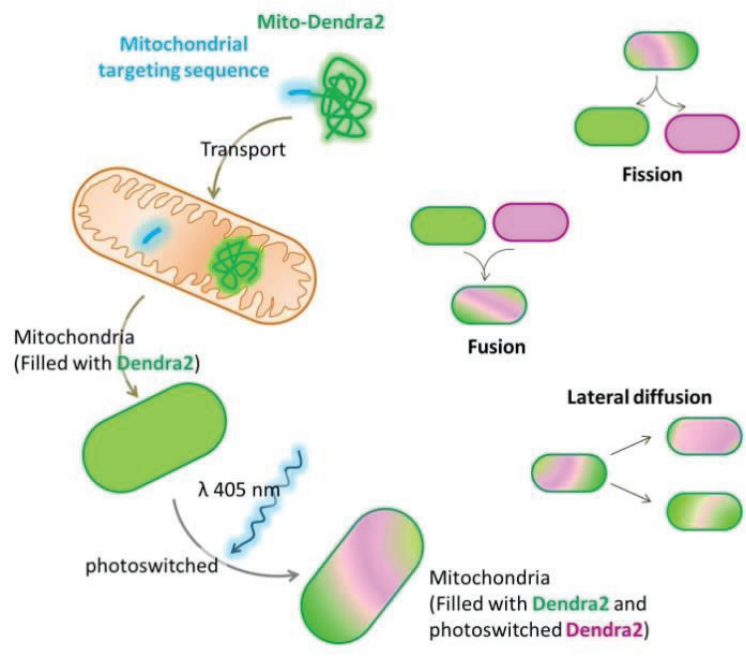

Figure 1. Illustration of mito-Dendra2 targeting into mitochondria and photoswitched by optical approach. 


\section{Body}

In order to monitor how the mitochondrial matrix proteins shuttle between mitochondria, we generated a stable cell line with constant expression of Dendra2 in the mitochondrial matrix (Figure 1). The mitochondrial targeting of Dendra2 are achieved by tagging the mitochondrial matrix targeting sequence on Dendra2 (mito-Dendra2) ${ }^{16}$. The mitochondrial targeting sequence would lead the mito-Dendra2 shuttling between mitochondria and imported into mitochondrial matrix (Figure 1). After the correct transport of mito-Dendra2, the targeting sequence would be removed by the mitochondrial enzyme. The mitochondria filled with mito-Dendra2 could be observed by green fluorescent.

To optically mark a portion of mito-Dendra2, the laser $405 \mathrm{~nm}$ were applied on the localized area of cell (Figure 2A). The exposure to $405 \mathrm{~nm}$ laser would switch the mito-Dendra2 from green to red fluorescent. This results in a mixture population of mito-Dendra2 within mitochondria (Figure 1 and 2). In the figure 2, a redistribution of optically labeled mitochondria with red mito-Dendra2 was observed before the cell entry into metaphase/anaphase. These indicated a rapid exchange and diffusion of mitochondrial material before the cell division. Asymmetry division was observed after the cytokinesis (unpublished data). Taken all together, our data indicated that we could combine the lightsheet microscopy and the optically labelling approach to mark a small population of mitochondria and monitor the dynamics of mitochondrial materials within mitochondria during mitosis.

To further quantify the dynamic of photoswitched mito-Dendra2, we generated a 3D mask to cover all mitochondria (Figure 3A). The 3D mask was used to filter the mito-Dendra2 signals; the photobleaching rate of live image was calculated from the masked green and red signals (Figure 3A). The total intensity of green mito-Dendra2 was gradually decreased (Figure 3B); the photoswitched mito-Dendra2 show slight decay during imaging. We adjusted the intensity decrease by photobleaching correction. The corrected intensity were used for calculating the 560/488 ratio (Figure 3C).

The ratio of $560 / 488$ signals were shown in gray scale (Figure 3D); the ratio signal was gradually diffused from photoswitched area to distal area. To visualize the spatio-temporal change of 560/488 ratio, a color-coded ratio were demonstrated (Figure 3E). In addition, we extract a small region of phtoswitched area to analyze the change of ratio (Figure $3 \mathrm{~F}$ ). We could observe a decreased ratio while the photoswitched mito-Dendra2 diffused and redistributed into distal area (Figure 3C).

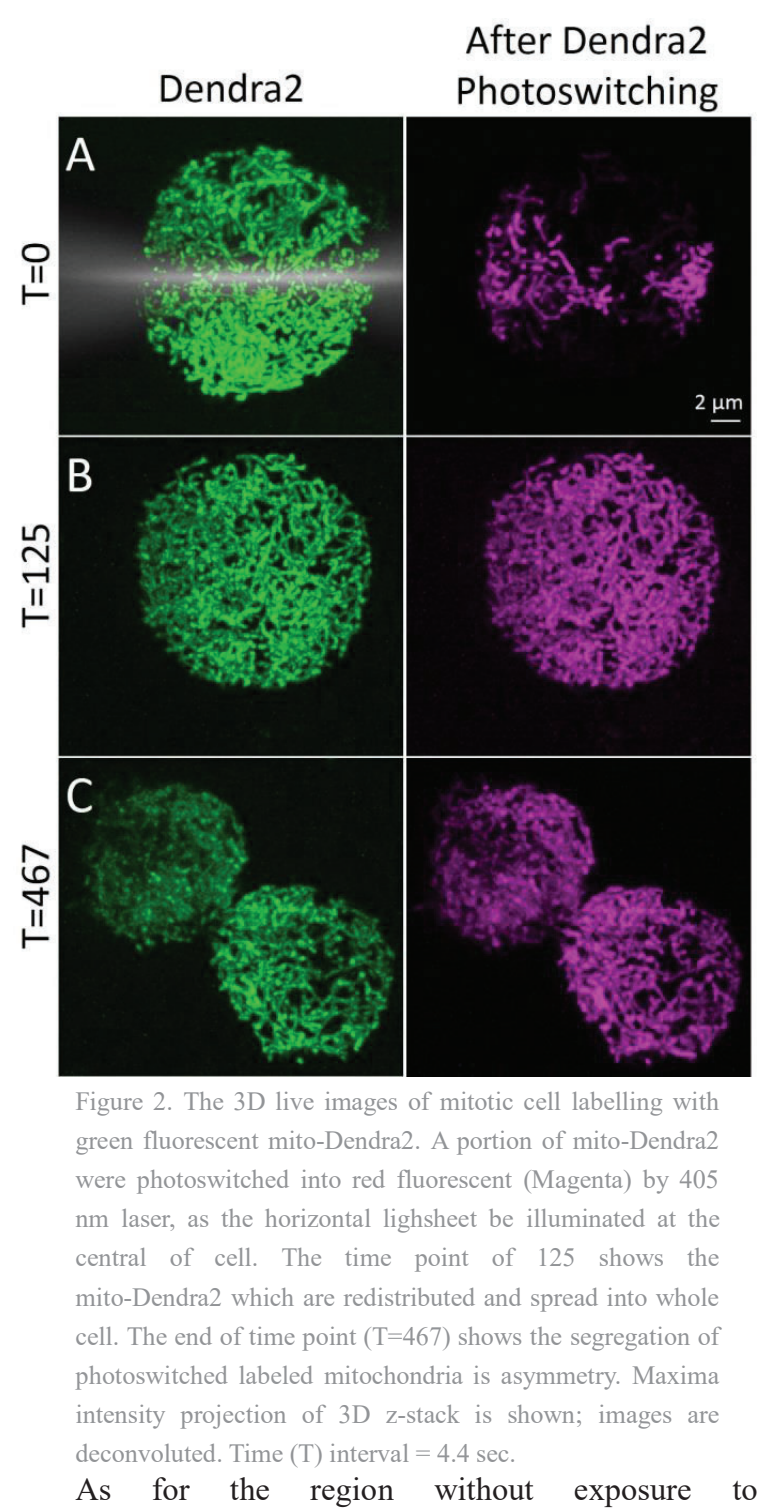

photoswitching (Figure 3G), the redistribution of mito-Dendra2 results in an increase of ratio. According to these results, we could analyze, track and quantify the subcellular dynamics and redistribution of mitochondrial proteins during cell division by lightsheet and optical labelling.

\section{Results and Discussion}

A detailed dynamic of mito-Dendra2 within single mitochondria would be quantified. These would improve our understanding about mito-Dendra2 diffusion and exchange by mitochondrial fusion/fission. Specific mitochondrial proteins with Dendra2 tag would be 

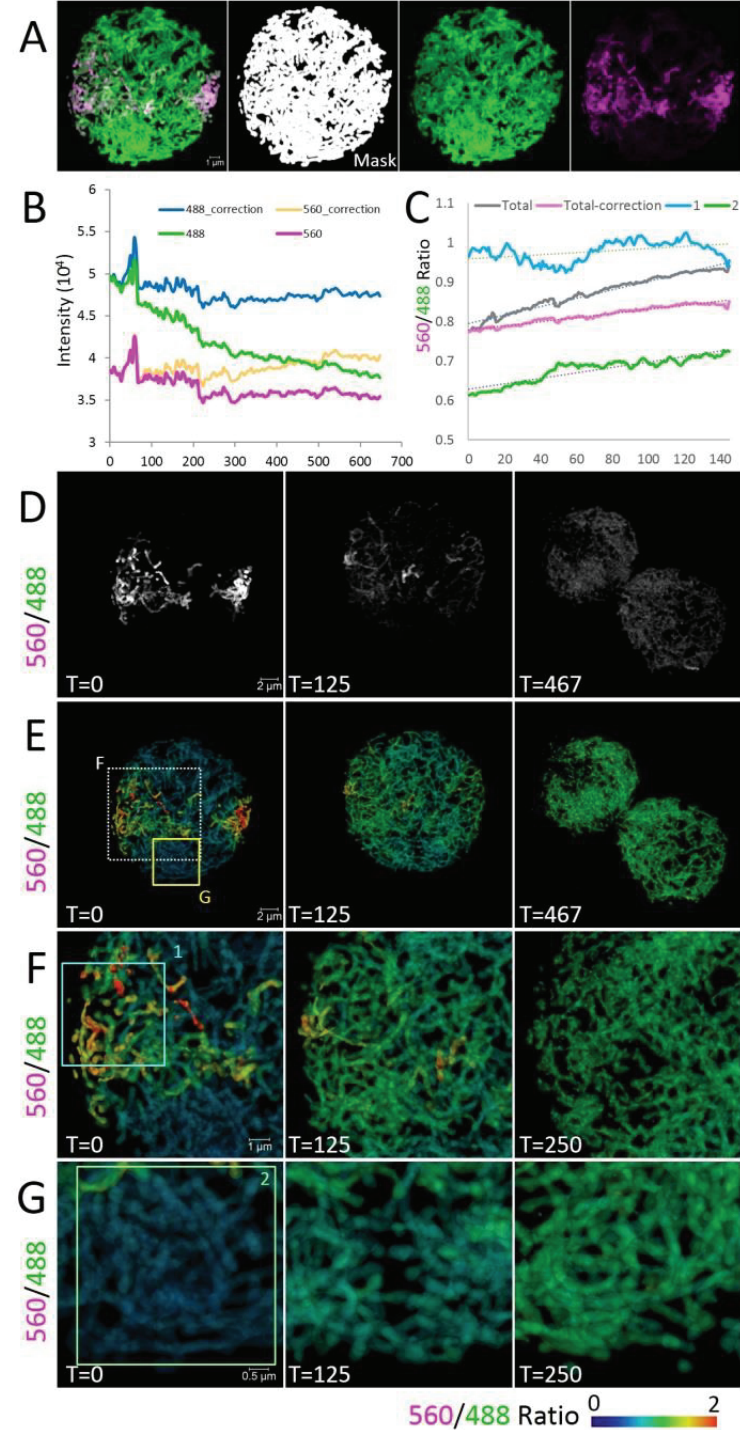

Figure 3. The quantification of mito-Dendra2 dynamic and redistribution. (A) The sum of green and red mito-Dendra2 was used for generated the $3 \mathrm{D}$ mask. The outside signals of both colors were removed by the mask. (B) The total fluorescence intensities were quantified and applied for bleaching correction. (C) The ratio was calculated from the masked signals of each color after bleaching correction. The $560 / 488$ ratio demonstrated in gray scale (D) and color-code (E-G) illustrate the relative population of mito-Dendra2. (F) A selected area form $\mathrm{E}$ (white dashed line box) shows the diffusion and decay of photoswitched mito-Dendra2. (G) A selected area from E (yellow box) shows the gradually increase of photoswitched mito-Dendra2. Quantification of $\mathrm{F}$ and $\mathrm{G}$ was shown in $\mathrm{C}$ studied. These investigations would reveal the correlation of mitochondrial proteins/inheritances and cell division. This research might expectantly increase the understanding of mitochondrial function and pathology of mitochondrial diseases in the future.

\section{References}

1 Chudakov, D. M., Lukyanov, S. \& Lukyanov, K. A. Tracking intracellular protein movements using photoswitchable fluorescent proteins PS-CFP2 and Dendra2. Nat Protoc 2, 2024-2032, doi:10.1038/nprot.2007.291 (2007).

2 Chen, B. C. et al. Lattice light-sheet microscopy: imaging molecules to embryos at high spatiotemporal resolution. Science 346, 1257998, doi:10.1126/science.1257998 (2014).

3 Benard, G. et al. Mitochondrial bioenergetics and structural network organization. J Cell Sci 120, 838-848, doi:10.1242/jcs.03381 (2007).

4 Devine, M. J. \& Kittler, J. T. Mitochondria at the neuronal presynapse in health and disease. Nat Rev Neurosci 19, 63-80, doi:10.1038/nrn.2017.170 (2018)

5 Bockler, S. et al. Fusion, fission, and transport control asymmetric inheritance of mitochondria and protein aggregates. J Cell Biol 216, 2481-2498, doi:10.1083/jcb.201611197 (2017).

6 Zhou, C. et al. Organelle-based aggregation and retention of damaged proteins in asymmetrically dividing cells. Cell 159, 530-542, doi:10.1016/j.cell.2014.09.026 (2014).

7 Aguilaniu, H., Gustafsson, L., Rigoulet, M. \& Nystrom, T. Asymmetric inheritance of oxidatively damaged proteins during cytokinesis. Science 299, 1751-1753, doi:10.1126/science.1080418 (2003).

8 Youle, R. J. \& van der Bliek, A. M. Mitochondrial fission, fusion, and stress. Science 337, 1062-1065, doi:10.1126/science. 1219855 (2012).

9 Lopez-Domenech, G. et al. Miro proteins coordinate microtubule- and actin-dependent mitochondrial transport and distribution. EMBO J 37, 321-336, doi:10.15252/embj.201696380 (2018).

10 Mishra, P. \& Chan, D. C. Mitochondrial dynamics and inheritance during cell division, development and disease. Nat Rev Mol Cell Biol 15, 634-646, doi:10.1038/nrm3877 (2014)

11 Dalton, C. M. \& Carroll, J. Biased inheritance of mitochondria during asymmetric cell division in the mouse oocyte. J Cell Sci 126, 2955-2964, doi:10.1242/jcs.128744 (2013)

12 Katajisto, P. et al. Stem cells. Asymmetric apportioning of aged mitochondria between daughter cells is required for stemness. Science 348, 340-343, doi:10.1126/science.1260384 (2015).

13 Teitell, M. A. Adult stem-like cells exclude "older" mitochondria. Cell Metab 21, 658-659, doi:10.1016/j.cmet.2015.04.023 (2015)

14 Suomalainen, A. Stem cells: Asymmetric rejuvenation. Nature 521, 296-298, doi:10.1038/521296a (2015)

$15 \mathrm{Lu}$, B., Jan, L. \& Jan, Y. N. Control of cell divisions in the nervous system: symmetry and asymmetry. Аnnu Rev Neurosci 23, 531-556, doi:10.1146/annurev.neuro.23.1.531 (2000).

16 Schatz, G. The protein import system of mitochondria. J Biol Chem 271, 31763-31766 (1996) 


\title{
Ultrafast force-clamp spectroscopy: dissecting the mechanosensitivity of biomolecule interactions on the microsecond time scale
}

\author{
Marco Capitanio ${ }^{\mathrm{a}, \mathrm{b}}$ \\ aDepartment of Physics and Astronomy, University of Florence, Italy \\ bLENS (European Laboratory for Non-linear Spectroscopy), Florence, Italy
}

\begin{abstract}
In the last few decades, force has emerged as a fundamental regulatory factor for cell life. The measurement of how force modulates biomolecular interactions is fundamental for the understanding of a large number of different biological processes. We developed a constant-force laser trap that allows us to investigate molecular interactions and subnanometer conformational changes occurring on a time scale of few tens of microseconds. The technique, named ultrafast force-clamp spectroscopy, has been applied to study load-dependent interactions between a biological polymer (DNA, actin, microtubules) and a binding protein. Here, I will review implementation of our technique to the study of the mechanosensitivity of molecular motors and transcription factors during target search along DNA.
\end{abstract}

Keywords: optical tweezers, molecular motors, single molecule biophysics, mechanotransduction, DNA target search

*capitanio@lens.unifi.it; phone +390554572054

\section{Introduction}

Atomic force microscopes, optical tweezers, and magnetic tweezers have enabled the study of a wide range of molecular processes in which force plays a crucial role. However, they are usually inadequate to probe the dynamic interactions that occurs during transduction of mechanical signals on the millisecond and submillisecond time scale ${ }^{1,2,3}$. We developed a constant-force laser trap that allows us to investigate molecular interactions and sub-nanometer conformational changes occurring on a time scale of few tens of microseconds ${ }^{4,5}$. The technique, named ultrafast force-clamp spectroscopy, has been applied to study load-dependent interactions between a biological polymer (DNA, actin, microtubules) and a binding protein.

\section{Molecular Motors}

\section{Body}

Ultrafast force-clamp spectroscopy is particularly useful in deciphering the mechanochemistry of molecular motors. Mechanical transitions in molecular motors often occur on a submillisecond time scale and rapidly follow binding of the motor with its cytoskeletal filament. Interactions of nonprocessive molecular motors with their filament can be brief and last for few milliseconds or fraction of milliseconds. The investigation of such rapid events and their load dependence requires specialized single-molecule tools. Ultrafast force-clamp spectroscopy is a constant-force optical tweezers technique that allows probing such rapid mechanical transitions and submillisecond kinetics of biomolecular interactions, which can be particularly valuable for the study of nonprocessive motors, single heads of processive motors, or stepping dynamics of processive motors ${ }^{5}$. We applied our technique to reveal the mechanosensitivity of the working stroke of non-processive skeletal muscle myosin ${ }^{4}$ and to dissect the mechanosensitivity of the processive motor myosin- $5 \mathrm{~B}^{6}$. 


\section{DNA binding proteins and the DNA target search process}

The maintenance of intact genetic information, as well as the deployment of transcription for specific sets of genes, critically rely on a family of proteins interacting with DNA and recognizing specific sequences or features. The mechanisms by which these proteins search for target DNA are the subject of intense investigations employing a variety of methods in biology. A large interest in these processes stems from the faster-than-diffusion association rates, explained in current models by a combination of $3 \mathrm{D}$ and 1D diffusion ${ }^{7}$. Single molecule fluorescence approaches are particularly amenable for the characterization of protein- DNA interactions aiming at the direct measurement of diffusion constants and the characterization of sequence- dependent processes along a DNA molecule 8,9 . However, fluorescence detection is vulnerable to photobleaching, which limits the observation time, and low signal, which limits the localization precision attainable in single molecule tracking measurements. We studied the mechanism of DNA target search by a single lac repressor protein (LacI) with ultrafast force-clamp spectroscopy, attaining submillisecond and few base-pair resolution ${ }^{10}$.

\section{Acknowledgments}

The research leading to these results has received funding from the European Union Horizon 2020 Programme (H2020-INFRAIA-2014-2015 under grant agree- ment no. 654148 - LASERLAB-EUROPE), by EMPIR under the European Union's Horizon 2020 research and innovation program grant agreement 15HLT01 MetVBadBugs, and by Ente Cassa di Risparmio di Firenze.

\section{References}

[1] Capitanio, M., "Optical Tweezers," [An introduction to Single Molecule Biophysics], Y. L. Lyubchenko, Ed., CRC Press, Taylor \& Francis group (2017).

[2] Capitanio, M. and Pavone, F. S., "Interrogating biology with force: Single molecule highresolution measurements with optical tweezers," Biophys. J. 105(6), 1293-1303 (2013).

[3] Neuman, K. C. and Nagy, A., "Single-molecule
[4] Capitanio, M., Canepari, M., Maffei, M., Beneventi, D., Monico, C., Vanzi, F., Bottinelli, R. and Pavone, F. S., "Ultrafast force-clamp spectroscopy of single molecules reveals load dependence of myosin working stroke," Nat. Methods 9(10), 1013-1019 (2012).

[5] Gardini, L., Tempestini, A., Pavone, F.S., Capitanio, M., "High-speed optical tweezers for the study of single molecular motors," [Molecular Motors: Methods and Protocols, Second Edition], C. Lavelle, Ed., Methods in Molecular Biology, Springer US.

[6] Gardini, L., Heissler, S. M., Arbore, C., Yang, Y., Sellers, J. R., Pavone, F. S. and Capitanio, M., "Dissecting myosin-5B mechanosensitivity and calcium regulation at the single molecule level."

[7] Monico, C., Capitanio, M., Belcastro, G., Vanzi, F. and Pavone, F. S., "Optical Methods to Study Protein-DNA Interactions in Vitro and in Living Cells at the Single-Molecule Level.," Int. J. Mol. Sci. 14(2), 3961-3992 (2013).

[8] Wang, Y. M., Austin, R. H. and Cox, E. C., "Single molecule measurements of repressor protein 1D diffusion on DNA," Phys. Rev. Lett. 97(4), 048302 (2006).

[9] Monico, C., Belcastro, G., Vanzi, F., Pavone, F. S. and Capitanio, M., "Combining singlemolecule manipulation and imaging for the study of protein-DNA interactions," J. Vis. Exp.(90) (2014).

[10] Tempestini, A., Monico, C., Gardini, L., Vanzi, F., Pavone, F. S. and Capitanio, M., "Sliding of a single lac repressor protein along DNA is tuned by DNA sequence and molecular switching," Nucleic Acids Res. 46(10), 5001-5011 (2018). 


\title{
Resonance laser effect on optical trapping of cell surface molecules
}

\author{
Tatsunori Kishimoto $^{\text {a,b }}$, Suguru N. Kudoh ${ }^{\mathrm{b}}$, Takahisa Taguchi ${ }^{\mathrm{c}}$, Chie Hosokawa ${ }^{\mathrm{a}, \mathrm{b}, \mathrm{d}, \mathrm{e}_{*}}$ \\ ${ }^{a}$ Biomedical Research Institute, National Institute of Advanced Industrial Science and Technology \\ (AIST), 1-8-31 Midorigaoka, Ikeda, Osaka 563-8577, Japan \\ ${ }^{\mathrm{b}}$ Graduate School of Science and Technology, Kwansei Gakuin University, 2-1 Gakuen, Sanda, \\ Hyogo 669-1337, Japan \\ ${ }^{\mathrm{c}}$ National Institute of Information and Communications Technology (NICT), 1-4 Yamadaoka, Suita, \\ Osaka 565-0871, Japan \\ dAdvanced Photonics and Biosensing Open Innovation Laboratory, AIST-Osaka University, 2-1 \\ Yamadaoka, Suita, Osaka 565-0871, Japan \\ e Department of Chemistry, Graduate School of Science, Osaka City University, 3-3-138 Sugimoto, \\ Sumiyoshi-ku, Osaka 558-8585, Japan
}

\begin{abstract}
For the purpose of artificial control in synaptic transmission on neuronal cells with optical trapping, we demonstrate optical trapping with resonance effect of nanoparticles and cell surface molecules by simultaneous irradiation with nonresonance and resonance laser beams. The average transit times of quantum-dot (QD) suspensions increased by the simultaneous irradiation with NIR laser and resonance laser, suggesting that single QD was more constrained at the focal spot due to optical trapping potential enhanced with resonance laser irradiation. Moreover, we applied the resonance laser effect to manipulate QD-conjugated AMPA-type glutamate receptors on neuronal cells.
\end{abstract}

Keywords: optical trapping, resonance effect, neuronal cell, quantum-dot

\section{Introduction}

Neuronal cells formed complex networks and communicate via synaptic connections. When neurotransmitters it binds to such as glutamate are released at excitatory synapses, AMPA ( $\alpha$-amino-3hydroxy-5-methyl-4-isoxazolepropionic acid) -type glutamate receptors (AMPARs) located on neurons bind to the glutamate and mediate the post-synaptic depolarization. The molecular dynamics of AMPAR is essential for synaptic plasticity and subsequent modulation in living neuronal network, related with memory and learning [1]. For aiming artificial control of the synaptic transmission, we have applied optical trapping using a focused near-infrared (NIR) laser to manipulate the AMPARs on neurons. We have been reported that optical trapping dynamics of quantum-dot (QD) conjugated AMPARs on neurons depended on initial assembling state of AMPARs [2].

Optical trapping enables non-contact and nondestructive manipulation at the laser focus. Since optical trapping potential depends on particle size and the polarizability, it is generally difficult to trap stably a single nanoparticle in solution. However, highly concentrated nanoparticles or polymers in solution can be trapped and assembled at the focal spot, which has an attractive potential in localized perturbation into molecular dynamics at cell surface. In order to realize optical trapping of single nanoparticles in solutions, the enhancement of optical trapping force with surface plasmon resonance [3] and resonance effect based on nonlinear optical responses [4] have been demonstrated in recent studies. In this study, we propose and demonstrate optical trapping with resonance effect of nanoparticles

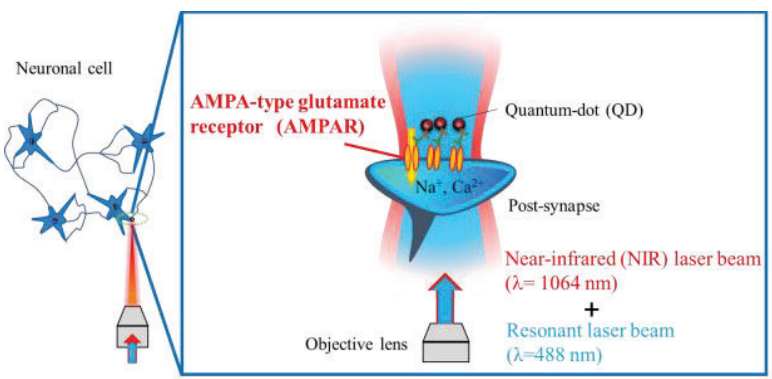

Figure 1. Schematic images of optical trapping of QDAMPARs by simultaneous irradiation with NIR laser $(\lambda$ : 1064-nm) and resonance laser ( $\lambda$ : 488-nm). 
and cell surface molecules on neuronal cells by simultaneous irradiation with NIR laser for conventional optical trapping and resonance laser beams (Fig. 1).

\section{Materials and Methods}

\section{Sample preparations and neuronal cell cultures}

Quantum-dot (QD: CdSe/ZnS nanocrystal) whose diameter was $10-20 \mathrm{~nm}$ and had a peak emission wavelength of $655 \mathrm{~nm}$ was used as samples. QD nanoparticle suspensions diluted in distilled water at the concentration of $1 \mathrm{nM}$ was dropped on a glass substrate and enclosed with a coverglass.

Rat hippocampal neurons were isolated from 18-dayold embryos of a Wistar rat, dissociated by treatment with $0.01 \%$ trypsin, and plated at a density of $1.8 \times 10^{3} \mathrm{cell} / \mathrm{mm}^{2}$ in a glass-bottomed dish. The dissociated neurons were maintained at $37^{\circ} \mathrm{C}$ in a humidified atmosphere containing $5 \% \mathrm{CO}_{2}$ and cultured for 20-24 days in Neurobasal medium. For visualization of AMPARs at cell surface of living neurons, cells were incubated with a mouse monoclonal anti-AMPAR subunit GluR2 antibody, followed by secondary incubation with a QD anti-mouse IgG antibody conjugates.

\section{Experimental setup}

A 1064-nm laser beam from cw-Nd: $\mathrm{YVO}_{4}$ laser for conventional optical trapping and a 488-nm DPSS laser beam for resonance effect were introduced into an inverted microscope and focused onto QD suspensions or QD-AMPARs on neurons using a $100 \times$ oil-immersion objective lens (N.A. 1.3). Light from a mercury lamp transmitted through a band-pass filter (WIG; BP $530-550 \mathrm{~nm}$ ) was used to excite QD-AMPARs on neurons. Fluorescence images were captured by EM-CCD camera. Alternatively, fluorescence emission at the laser focus was detected by an avalanche photodiode and acquired by fluorescence correlation spectroscopy (FCS) analysis.

\section{Results and Discussion}

When a NIR laser for non-resonance with the laser power of $300 \mathrm{~mW}$ was focused on QD nanoparticle suspensions, two-photon excitation fluorescence of QD was measured at the focal spot and autocorrelation function $(\mathrm{ACF})$ curve of the fluorescence intensity was

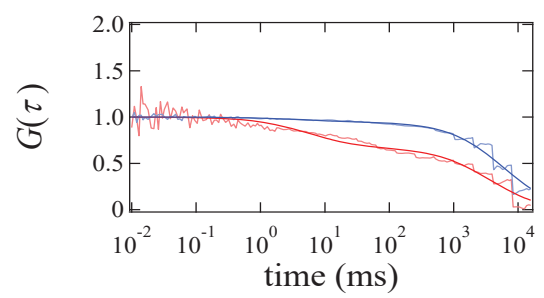

Figure 2. Typical normalized ACF curves of fluorescence intensity of QD-AMPARs at the laser focus on neurons (20DIV) with (blue) and without resonance laser irradiation (red). The NIR laser power and resonance laser power was $300 \mathrm{~mW}$ and $1.5 \mu \mathrm{W}$, respectively.

obtained. The average transit time $\tau_{\mathrm{D}}$ of QD nanoparticle was obtained to be $1.4 \pm 0.16 \mathrm{~ms}(N=10)$, which was in good agreement with the calculation of $\tau_{\mathrm{D}}$ derived from the diffusion coefficient. This suggests that the diffusion property of QD was not affected by optical trapping force based on the NIR laser. In addition to NIR laser, resonance laser with a wavelength of 488-nm under weak laser power of $1.2 \mu \mathrm{W}$ was focused into QDs suspended in water, $\tau_{\mathrm{D}}$ of QD nanoparticle was $11.6 \pm 1.66 \mathrm{~ms}(N=10)$. The $\tau_{\mathrm{D}}$ had tendency to increase with the laser power of 488-nm laser. This suggests that the particle dynamics of QD nanoparticles is more constrained at focal spot due to optical trapping potential enhanced with resonance laser irradiation.

Furthermore, NIR laser at the laser power of $300 \mathrm{~mW}$ and $488-\mathrm{nm}$ laser at the laser power of $0-1.5 \mu \mathrm{W}$ were focused onto QD-AMPARs located on neuronal cell with the culturing days, i.e. 20 days in vitro (20 DIV). When a NIR laser focused into QD-AMPARs on neuronal cell, fluorescence intensity gradually increased at the focal spot, suggesting that QD-AMPARs were optically trapped. For quantitative analysis of QD-AMPARs in an optical trap, the mobility of them at the laser focus was evaluated by FCS. The average transit time $\tau_{\mathrm{D} 2}$ of ACF curves was $3309 \pm 935 \mathrm{~ms}(N=8)$. In addition to the NIR laser, 488$\mathrm{nm}$ laser was simultaneously focused into QD-AMPARs on neuronal cell. The average transit time $\tau_{\mathrm{D} 2}$ increased with the laser power of 488-nm laser (Fig. 2). These results supported that the molecular dynamics of QDAMPARs on neuronal cells became more slowly at focal spot by the simultaneous irradiation with NIR laser and resonance laser. 


\section{Conclusions}

The average transit time of QD nanoparticles suspended in water or QD-AMPARs located on neuronal cells at the focal spot slightly increased by the simultaneous irradiation with NIR laser and resonance laser beam. These results concluded that the nanoparticles dynamics was more constrained at focal spot due to enhancement of optical trapping force based on resonance laser effects.

\section{Acknowledgements}

This work was supported in part by JSPS KAKENHI Grant Numbers JP25700031, JP16H02092, JP16H06504 in Scientific Research on Innovative Areas "NanoMaterial Optical-Manipulation".

\section{References}

[1] Compans, B., Choquet, D., Hosy E., "Review on the role of AMPA receptor nano-organization and dynamic in the properties of synaptic transmission", Neurophotonics 3, 041811 (2016).

[2] Kishimoto, T., Maezawa, Y., Kudoh S. N., Taguchi, T., Hosokawa, C., "Optical trapping of quantum-dot conjugated AMPA-type receptors depend on initial assembling states", Proc. SPIE 10712, 1071204 (2018).

[3] Miyauchi, K., Tawa, K., Kudoh S. N., Hosokawa, C., "Surface plasmon-enhanced optical trapping of quantum-dot conjugated surface molecules on neurons cultured on a plasmonic chip", Jpn. J. Appl. Phys. 55, 06GN04 (2016).

[4] Kudo, T., Ishihara, H., "Resonance optical manipulation of nano-objects based on nonlinear optical response", Phys. Chem. Chem. Phys. 15, 14595 (2013). 


\title{
Single-protein and single-nanoparticle trapping using plasmonic
}

\section{nanoaperture array}

\author{
Domna G. Kotsifaki ${ }^{1}$, Viet Giang Truong ${ }^{1}$, Elliot Harvie ${ }^{1}$, Aditya Saxena ${ }^{1}$, Xue Han ${ }^{1,2}$ and Síle Nic \\ Chormaic $^{1,3, *}$ \\ ${ }^{1}$ Light-Matter Interactions Unit, Okinawa Institute of Science and Technology Graduate University, \\ Onna, Okinawa, 904-0495, Japan \\ ${ }^{2}$ School of Optoelectronic Engineering and Instrumentation Science, Dalian University of \\ Technology, Dalian, China \\ ${ }^{3}$ Université Grenoble Alpes, CNRS, Grenoble INP, Institut Néel, 38000 Grenoble, France
}

\begin{abstract}
We demonstrate optical trapping of single Cytochrome c proteins, using a plasmonic tweezers based on metallic asymmetric nano-aperture arrays. We succeed in immobilizing single proteins with very low in-trap laser intensities. This approach paves the way for selective single-molecule manipulation without labeling or tethering.
\end{abstract}

\section{Introduction}

As one of the building blocks of life, proteins play vital roles in almost all cellular activities. The study of proteins and their function are critical due to their association with diseases such as Alzheimer's, Parkinson's, prions and tumors [1]. There already exist many established methods which can provide valuable information about the mean average state of protein [2]. However, a key benefit of single-molecule techniques is their ability to acquire physical properties of interest from individual protein data and build up a picture of the underlying protein heterogeneity in the system without the need for labeling or tethering [2]. Plasmonic trapping based on metallic nanostructures is an attractive method for monitoring of proteins at the single-molecule level in a label-free, sterile and freesolution environment [3].

Here, we demonstrate three-dimensional optical trapping of a single-protein using plasmonic tweezers by employing low trapping laser intensity. The plasmonic tweezers is based on a unique metallic asymmetric nano-aperture array, with each aperture being separated by a nanoslot [4]. The plasmonic structure provides great tunability of the Fano resonance in the near-infrared region offering singleparticle trapping with high selectivity and specificity. Single Cytochrome c proteins, with a hydrodynamic radius of $1.5 \mathrm{~nm}$, have previously been trapped [5], using a laser wavelength of $980 \mathrm{~nm}$. Our work presented herein, demonstrates the potential of using metallic nano-aperture structures for the controlled optical manipulation of biomolecules and their

*Corresponding author: sile.nicchormaic@oist.jp identification or quantification in heterogeneous environment based on their size and/or refractive index.

\section{Experimental set up}

The optical trapping setup was reported in our earlier works [6,7]. Briefly, the experimental arrangement is based on a modified Thorlabs optical tweezers kit (OTKB), see Fig. 1.

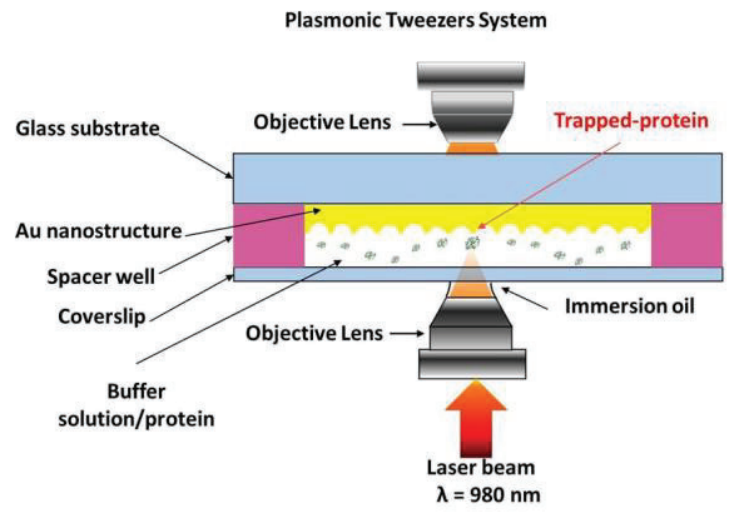

Figure 1. Schematic of the plasmonic tweezers setup.

A Ti:Sapphire laser at $980 \mathrm{~nm}$ was used to generate the optical trap. The beam was focused onto the sample though a 100x microscope objective lens with high numerical aperture (N.A. $=1.25$ ). For the trapping sample, we prepared a solution of Cytochrome c protein with a molecular weight of $12 \mathrm{kDa}$ in phosphatebuffered saline solution $(\mathrm{pH}=7.0)$ [5]. The transmitted light through the plasmonic device was collected using a condenser microscope objective (N.A. $=0.55)$ and measured by a silicon-based avalanche photodiode. The trapping events were recorded as discrete transmission jump $(>10 \%)$ of the laser power through the plasmonic 
nanostructures. The plasmonic structure was fabricated on a $50 \mathrm{~nm}$ thickness gold layer using focusing ion beam, details of which are published in our previous works $[4,6,7]$.

\section{Results and Discussion}

In previous work, we demonstrated trapping of single particles with $30 \mathrm{~nm}$ diameter using a gold plasmonic nanohole array [6]. Figure 2 shows a time trace of the transmission signal of the trapping event, displaying the distinction between the untrapped (black line) and trapped (red line) stage.

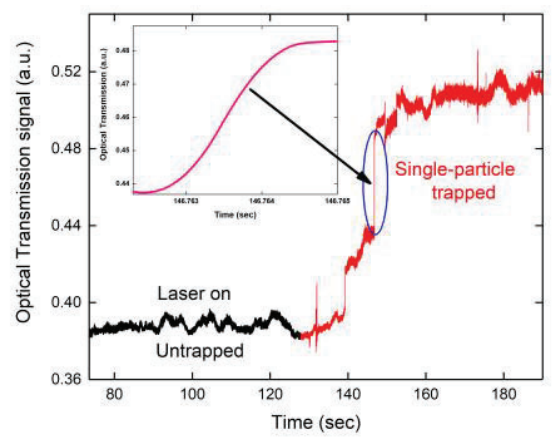

Figure 2. Time trace of the optical transmission signal using the nanoaperture array to trap single polysterene particle [6].

The distinct jump in the transmission signal during the trapping process has an interval time equals to 3 msec as shown in the inset of Fig. 2.

Improving our past successes [7], we recently trapped single proteins using an asymmetric nanoaperture array. Figure 3 shows a trapping event for a single protein using an incident laser intensity of 0.29 $\mathrm{mW} / \mu \mathrm{m}^{2}$. The intensity fluctuations in the trapped state could be due to the differences in size, shape, or orientation changes in the protein within the plasmonic trapping potential. In this work, both the autocorrelation and the trapping transient analyses were used to determine the trap stiffness. The latter is related to the drag force of the fluid as compared to the autocorrelation analysis, which is based on thermal fluctuations. For comparison reasons between our approach and different works, we scale our experimental trap stiffness values to a particle size of 10 $\mathrm{nm}$ for an incident laser intensity of $1 \mathrm{~mW} / \mu \mathrm{m}^{2}$. We obtain a scaled trapped stiffness of $\sim 0.1 \mathrm{fN} / \mathrm{nm}$ for a 10 $\mathrm{nm}$ particle [7] which is comparable to that determined for other plasmonic geometries [8].

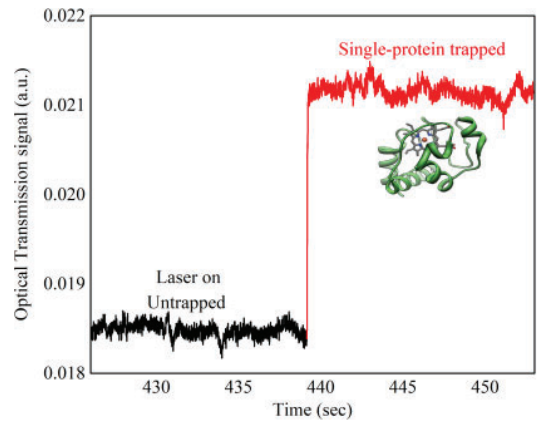

Figure 3. Trapping event of Cytochrome c protein using the asymmetric nanoaperture array.

Our plasmonic tweezers have the benefit of nondestructive application when working with heatsensitive molecules, such as proteins, in their physical environment, i.e. a buffer solution, without destroying them. It is a promising and versatile tool for in vitro optical manipulation at the single-molecule level.

\section{References}

[1] Checler, F., and Alves da Costa, C., "p53 in neurodegenerative diseases and brain cancers," Pharmac. \& Therap. 142, 99-113 (2014).

[2] Miller, H., Zhou, Z., Shepherd, J., Wollman, A.J.M., and Leake, M.C., "Single-molecule techniques in biophysics: a review of the progress in methods and applications," Rep. Prog. Phys. 81, 024601(2018).

[3] Gordon, R. "Biosensing with nanoaperture optical tweezers," Optics and Laser Technology 109, 328-335 (2019).

[4] Sergides, M., Truong, V.G. and Nic Chormaic, S., "Highly tunable plasmonic nanoring arrays for nanoparticle manipulation and detection," Nanotechnology 27, 365301 (2016).

[5] Spinazzi, M., Casarin, A., Pertegato, V., Salviati, L., and Angelini, C., "Assessment of mitochondrial respiratory chain enzymatic activities on tissues and cultured cells," Nature Protocols 7(6), 1235-1246 (2012).

[6] Han, X., Truong, V.G., Thomas, P.S. and Nic Chormaic, S., "Sequential trapping of single nanoparticles using a gold plasmonic nanohole array," Photonics Research 6(10), 981-986 (2018).

[7] Han, X., Truong, V.G. and Nic Chormaic, S., "Efficient microparticle trapping with plasmonic annular aperture arrays," Nano Futures 2, 035007 (2018).

[8] Kontala, A., and Gordon, R., "Quantification of highefficiency trapping of nanoparticles in a double nanohole optical tweezer," Nano Letters 14, 853-856 (2014). 


\title{
Helical biomaterial breaks spatial symmetry of helical light field
}

\author{
Keigo Masuda ${ }^{\text {a }}$, Taiki Yoshizawa ${ }^{\text {a }}$ Tomoki Akiyama ${ }^{a}$, Yoshiko Okada-Shudo ${ }^{\mathrm{b}}$, \\ Takeshi Murata $^{\mathrm{a}, \mathrm{c}}$, Koehi Toyoda ${ }^{\mathrm{a}, \mathrm{c}}$, Katsuhiko Miyamoto ${ }^{\mathrm{a}, \mathrm{c}}$, Takashige Omatsu ${ }^{\mathrm{a}, \mathrm{c}, *}$ \\ ${ }^{a}$ Graduate School of Engineering, Chiba University, Japan \\ ${ }^{b}$ Department of Electronic Engineering, The University of Electro-Communications, Japan \\ ${ }^{\mathrm{c}}$ Molecular Chirality Research Center, Chiba University, Japan \\ Corresponding author: Takashige Omatsu \\ E-mail: omatsu@faculty.chiba-u.jp
}

\begin{abstract}
We discover that optical vortex mode breaks into a rotating twin mode with two bright spots in bacteriorhodopsin suspensions. The rotational direction of the twin mode was determined by the handedness of optical vortex. The rotation speed was proportional to the square of the injected optical vortex intensity.
\end{abstract}

Keywords: bacteriorhodopsin, optical vortices, two photon absorption, singular optics, spatial soliton, modal instability

\section{Introduction}

Optical vortex, possessing an annular intensity profile and carrying an optical orbital angular momentum owing to its helical wavefront, provides new fundamental and applied sciences, including super resolution microscopy, optical telecommunication, and optical manipulation [1]. In particular, it is noteworthy that the OAM of optical vortex enables us to twist the irradiated material, such as, silicon, polymer, and even liquid resin, to form a spiralshaped structures (we call it chiral structures) on a nano/micro-scale [2-4]. The chirality of such structures was also controlled by the handedness of optical vortex, i.e. the twisting direction of the helical wavefront. In recent years, a laser-induced biomaterial-waveguide, in which the optical radiation force collects the cyanobacteria in suspensions inside the optical field to form an optical waveguide, has been reported $[5,6]$. In general, biomaterials, such as bacteriorhodopsin well known as a light-driven proton pump, mostly exhibit a helicity, however, there is no reports concerning the interaction of helical biomaterials and helical optical fields.

In this article, we report on the spatial symmetry breaking of optical vortex propagating through bacteriorhodopsin suspensions, in which optical vortex mode is broken into a twin mode with two bright spots and rotated toward a clockwise or counter-clockwise direction, assigned by the handedness of the optical vortex.

\section{Experiments}

A $1 \mu \mathrm{m}$ picosecond laser with a pulse width of $5 \mathrm{ps,}$ pulse repetition frequency of $40 \mathrm{MHz}$, and an average power of $\sim 50 \mathrm{~mW}$, was used as a laser source. The output was converted onto a right-handed optical vortex with a clockwise helical wavefront by utilizing a spiral phase plate. The generated vortex beam was focused by using a lens with a focal length of $50 \mathrm{~mm}$ onto a front surface of a 1-cm-long glass cuvette including bacteriorhodopsin suspensions (concentration of $\sim 10 \mu \mathrm{M}$ diluted in a $16 \%$ $\mathrm{NaCl}$ solution) without any significant absorption in a near-infrared region.

After the laser was on, the optical vortex mode was broken into a twin mode with two bright spots, and it rotated towards a clockwise direction. When the spiral phase plate was reversed to generate a left-handed optical vortex, the twin mode started to rotate towards counterclockwise direction (Figs. 1(a), (b)).

The rotation speed of the twin mode was approximately proportional to the square of the injected optical vortex intensity mode, as shown in Fig. 2. Also, the rotation of the twin mode accelerated as the vortex pulse was compressed to $3.5 \mathrm{ps}$ (Fig. 2). These results indicated that the symmetry breaking of the optical vortex was originated from the nonlinear interaction between a 
helical optical field and a helical bacteriorhodopsin. In fact, a $\mathrm{NaCl}$ solution without any bacteriorhodopsin molecules never induced such symmetry breaking of the optical vortex mode.

(a)
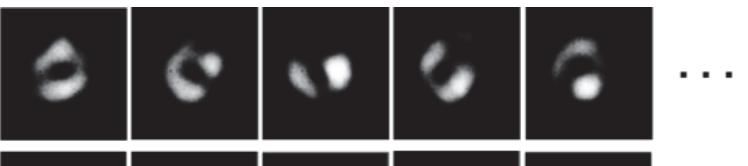

(b)
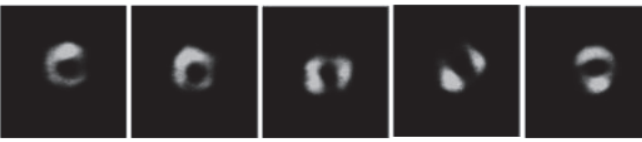

Figure 1. Temporal evolution of a transmitted (a) right- of (b) left-handed optical vortex through bacteriorhodopsin (bR) suspensions.

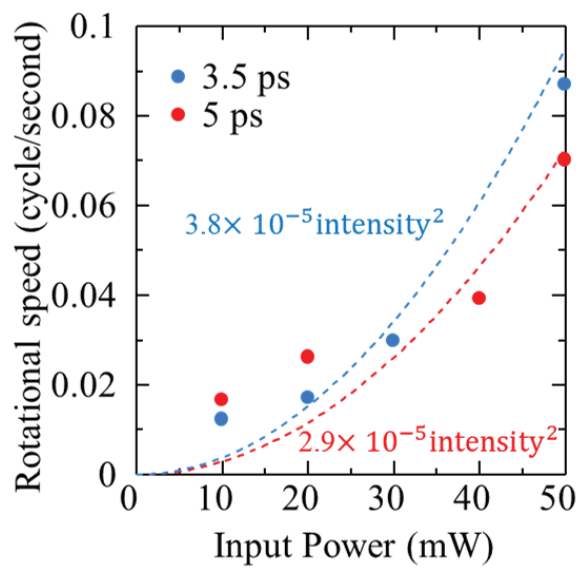

Figure 2. Rotation speed of the twin mode as a function of an injected optical vortex intensity.
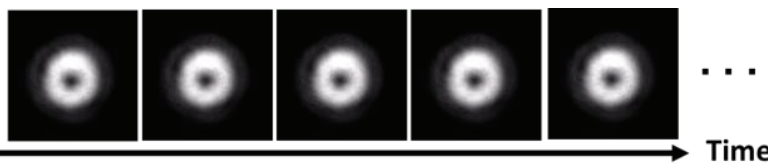

Figure 3. Temporal evolution of a transmitted optical vortex in $\mathrm{NaCl}$ solution without bR molecules.

\section{Conclusion}

We have discovered, for the first time, that helical bacteriorhodopsin molecules break a spatial symmetry of optical vortex mode into a rotating twin mode. The rotational direction of the twin mode was fully determined by the handedness of the optical vortex. The rotation speed was proportional to the square of the intensity of the injected vortex mode.

\section{References}

[1] Allen, L., Beijersbergen, M. W., Spreeuw, R. J. C., Woerdman, J. P., "Orbital angular momentum of light and the transformation of Laguerre-Gaussian laser modes," Phys. Rev. A. 45, 8185 (1992).

[2] Toyoda, K., Takahashi F., Takizawa, S., Tokizane, Y., Miyamoto, K., Morita, R., and Omatsu, T., "Transfer of light helicity to nanostructures," Phys. Rev. Lett. 110, 143603 (2013).

[3] Masuda, K., Shinozaki, R., Kinezuka, Y., Lee, J., Ohno, S., Hashiyada, S., Okamoto, H., Sakai, D., Harada, K., Miyamoto, K., Omatsu, T., "Nanoscale chiral surface relief of azo-polymers with nearfield OAM light," Opt. Express 17, 22197 (2018).

[4] Lee, J., Arita, Y., Toyoshima, S., Miyamoto, K., Panagiotopoulos, P., Wright, E. M., Dholakia, K., Omatus, T., "Photopolymerization with LightFields Possessing Orbital Angular Momenutm: Generation of Helical Microfibers," ACS Photonics, 5, 4156 (2018).

[5] Bezryadina, A., Hansson, T., Gautam, R., Wetzel, B., Siggins, G., Kalmbach, A., Lamstein, J., Gallardo, D., Carpenter, E. J., Ichimura, A., Morandotti, R., Chen, Z., "Nonlinear Self-Action of Light through Biological Suspensions," Phys. Rev. Lett. 119, 058101 (2017).

[5] Barada, D., Juman, G., Yoshida, I., Miyamoto, K., Kawata, S., Ohno, S., Omatsu, T., "Constructive spinorbital angular momentum coupling can twist materials to create spiral structures in optical vortex illumination,” Appl. Phys. Lett. 108, 051108 (2016).

[6] Fardad, S., Salandrino, A., Heinrich, M., Zhang, P., Chen, Z., Christodoulides, D. N., "Plasmonic Resonant Solitons in Metallic Nanosuspensions," Nano Lett. 14, 2498 (2014). 


\title{
Proposed method of single-particle absorption measurement based on optical transport at solid-liquid interface
}

\author{
Takudo Wada*a, Hajime Ishihara, ${ }^{\text {a, }}$ \\ aDepartment of Physics and Electronics, Osaka Prefecture University, 1-1 Gakuen-cho, Naka-ku, \\ Sakai, Osaka 599-8531, Japan \\ bepartment of Materials Engineering Science, Osaka University, 1 Machikaneyama-cho, Toyonaka, \\ Osaka 560-8531, Japan
}

\begin{abstract}
We investigate optical transport of nanoparticles at solid-liquid interface. The evanescent wave transports the particles being bound at the interface, i.e., the degree of freedom of the particle motion is decreased. By motion analysis considering Brownian motion, we find that such a restricted transport substantially improves the transport efficiency. In addition, we show that single-particle absorption characteristics can be estimated from the transport distance spectrum to the incident light energy. This method utilizes the fact that the transport distance of the particle depends on the light absorption of the particle, and it is possible to directly measure the absorption characteristics of a single particle experimentally.
\end{abstract}

Keywords: optical manipulation, transport, counter-propagating waves, evanescent waves, absorption characteristics

\section{Introduction}

Radiation force is caused by the interaction between incident light and target substances, and there are two types; one is a dissipative force that arises from the transfer of light momentum to a substance by the absorption and scattering, and the other is gradient force arising from an electromagnetic interaction between incident light and induced polarization.

The optical manipulation using radiation force for micro-sized particles was theoretically proposed and experimentally demonstrated by Ashkin et al. as optical tweezers $[1,2]$, and that for atomic-sized particles was experimentally demonstrated by Chu et al., as laser cooling [3]. However, the manipulation of nanoparticles has been a challenge because the magnitude of the radiation force is approximately proportional to the particle volume in the Rayleigh scattering regime, which prevents overcoming the problems by the random force from environment. To enhance the exerted force on particles, the manipulation method using electric resonance phenomena has been studied [4], and theoretically demonstrated in simple conditions.

In this contribution, we study the optical manipulation using the electric resonance effect for realistic situations.

*wada-2@pe.osakafu-u.ac.jp; phone 8172 254-9268
For example, we consider the water and glass substrate, in order to suppress Brownian motion by capturing particles with the near field and limiting the degree of freedom of particle motion to 2-dimensional space. With this system, we demonstrate a selective transport of nano-particles, which leads to a scheme to estimate the absorption spectra of single nanoparticles by measuring the transport distance

\section{Theoretical Method}

We use the following expression of the time-averaged radiation force [5] as

$\langle\mathbf{F}(\omega)\rangle=(1 / 2) \operatorname{Re}\left\{\int d \mathbf{r}\left[\nabla \mathbf{E}(\mathbf{r}, \omega)^{*} \cdot \mathbf{P}(\mathbf{r}, \omega)\right]\right\},(1)$

where $\mathbf{E}$ and $\mathbf{P}$ are the time-harmonic response electric field and induced polarization, respectively. The induced polarization consists of the resonant part and the background part. The former one can be written by the following Lorentz model as

$\mathbf{P}(\omega)=\sum_{j}\left|\boldsymbol{\mu}_{j \mathrm{~g}}\right|^{2}\left(\frac{1}{\omega_{j}-\omega-i \gamma_{j}}+\frac{1}{\omega_{j}+\omega+i \gamma_{j}}\right) \mathbf{E}(\omega)$,

where $\boldsymbol{\mu}$ is the dipole moment, $\omega_{j}$ is the electric resonant energy of target substance, $\omega$ is the frequency of the incident light and $\gamma_{j}$ is the dephasing constant. In order to calculate the latter one, background polarization $\mathbf{P}_{\mathrm{b}}$, we use Clausius-Mosotti law including the radiation reaction effect as $[6,7]$, 
$\mathbf{P}_{\mathrm{b}}=a_{\mathrm{CMRR}} \mathbf{E}$,

$a_{\mathrm{CM}}=4 \pi r^{3} \varepsilon_{0} \varepsilon_{2} \frac{m-1}{m+2}$,

$a_{\mathrm{CMRR}}=\frac{a_{C M}}{1-i k^{3} \frac{a_{C M}}{6 \pi \varepsilon_{0} \varepsilon_{2}}}$,

where $\varepsilon_{1}$ and $\varepsilon_{2}$ are the dielectric constants of the target substance and surroundings, respectively and $m=\varepsilon_{1} / \varepsilon_{2}$. On the other hand, the incident evanescent wave at the solid-liquid interface is given by

$$
\begin{aligned}
\mathbf{E}_{\mathrm{t}}=\mathbf{A}_{\mathrm{t}} \exp [- & \frac{\omega}{\mathrm{c}} \sqrt{n_{s}^{2} \sin ^{2} \theta_{i}-n_{1}^{2}} \mathrm{x} \\
& \left.+i\left(\frac{\omega}{c} n_{s} \sin \theta_{i} z-\omega t\right)\right] .
\end{aligned}
$$

Here $\mathbf{A}_{\mathrm{t}}$ is the transmit electric field coefficient, $n_{s}$ and $n_{1}$ are the solid and liquid refractive index, $\theta_{i}$ is the incident angle.

\section{Results and Discussion}

As for the incident lights, we employ two counterpropagating evanescent waves and consider the situation of irradiating them on the glass-water interface (Fig. 1(a)). The incident angle $\theta_{i}$ is set to $\sin \theta_{i}=1.1$ as to exceed the critical angle.

As the targets, we assume the two kinds of particles of nano-diamond; one has $300 \mathrm{NV}$-centers and another has no NV-centers (Fig. 1(b)). Their parameters are as follows: [Nano-diamond] The radius is $20 \mathrm{~nm}$ and the dielectric constant is 5.7. [NV-center] The transition energy $\hbar \omega_{21}=$ $2.32 \mathrm{eV}$. The dipole moments for 1-2 transitions is 4.0 Debye, the dephasing constant is $20 \mathrm{meV}$.

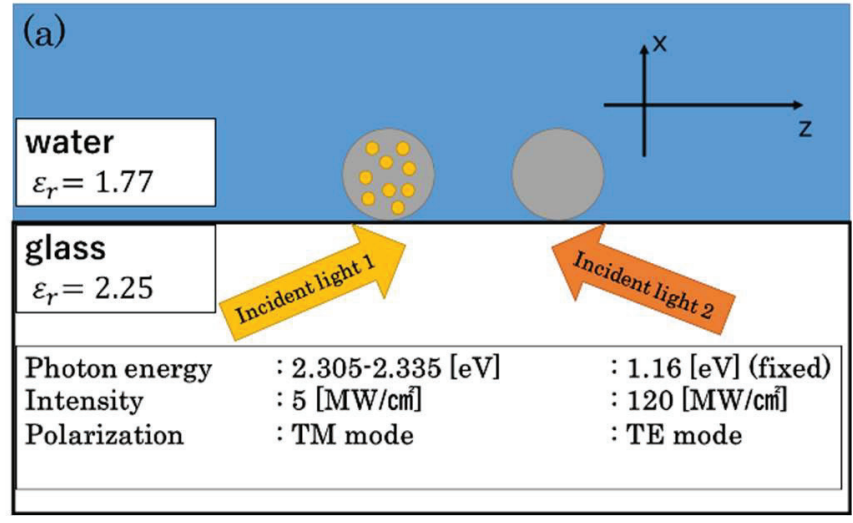

(b)

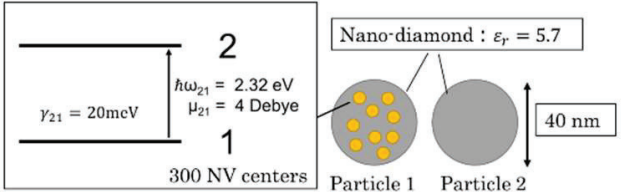

Figure 1. (a) The model of the counter-propagating evanescent waves at the interface between glass and water. (b) The energy diagram of assumed nanoparticles.

In Fig. 2, we show the trajectory of two particles by the motion analysis. It can be seen that resonant particles having NV-centers are swept to the right by resonant light and non-resonant particles are swept to the left. It also shows that the particles are bound to a plane. The arrows indicate the direction of radiation forces; the red arrow is $\mathbf{F}_{1}$, the force applied to the particle 1 , the blue arrow is $\mathbf{F}_{2}$, the force applied to the particle 2, the gray arrows are $\mathbf{F}_{\mathrm{bg}}$, the forces applied to the nano-diamonds and the yellow arrow is $\mathbf{F}_{\text {res }}$, the force applied to the NV-centers. To summarize, it can be written as follows

$\mathbf{F}_{1}=\mathbf{F}_{\text {res }}+\mathbf{F}_{\mathrm{bg}}\left(+\mathbf{F}_{\text {random }}\right)$

$\mathbf{F}_{2}=\mathbf{F}_{\mathrm{bg}}\left(+\mathbf{F}_{\text {random }}\right)$.

Here, $\mathbf{F}_{\text {random }}$ is the random force caused by Brownian motion.

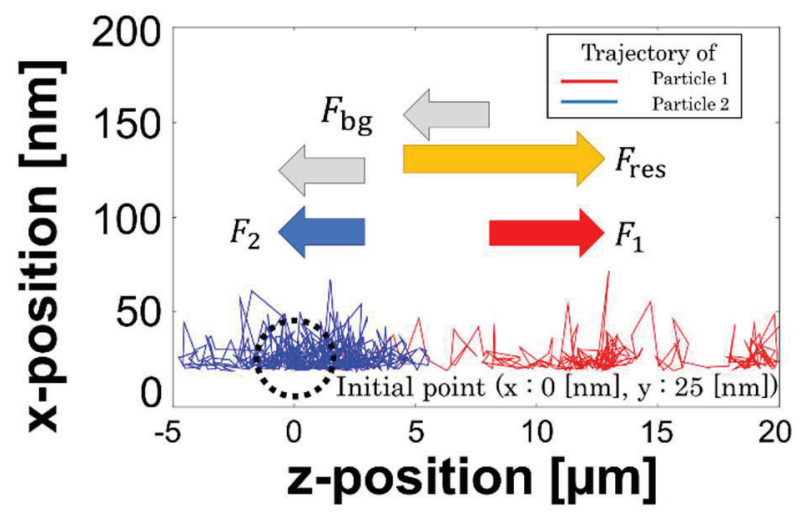

Figure 2. The trajectory of two particles for 5 seconds by using the counter-propagating evanescent waves model; the photon energy 1 is $2.32 \mathrm{eV}$. The red arrow indicates the force applied to the particle 1, which is composed of the background radiation force and the resonant radiation force. The blue arrow indicates the force applied to the particle 2 , and it is equal to the background radiation force. Two particles are bound to the plane by the gradient force and subjected to the random force by Brownian motion. 
In Fig. 3, we show the photon energy spectrum of the transport distance of nano-diamond and the fitting curve by Lorentz function. The distance is the average of the differences between the distances of two particles transported 5 seconds and 100 times for each energy of incident light 1 . As can be seen from the equations (7) and (8), the differences between two radiation forces,

$\Delta \mathbf{F}=\mathbf{F}_{1}-\mathbf{F}_{2}=\mathbf{F}_{\text {res }}$,

depend on the resonant radiation force. From the eqs. (1) and (2), the force depends on the imaginary part of the susceptibility representing the absorption of the particle.

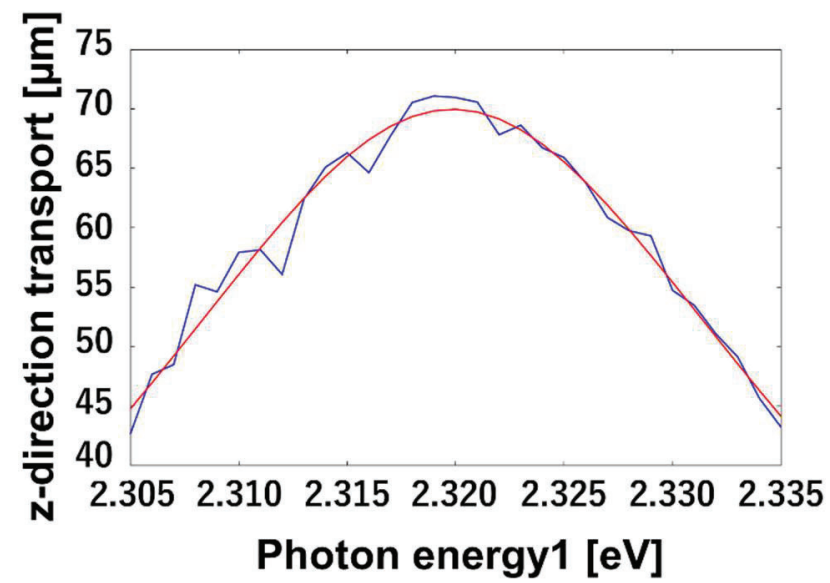

Figure 3. The photon energy spectrum of the transport distance of nano-diamond and the fitting curve by Lorentz function. The resonance center $\omega_{0}=2.320 \mathrm{eV}$ and the dephasing constant of the NV-center $\gamma=19.79 \mathrm{meV}$ are obtained as fitting parameters. The original value we assumed are $\omega_{0}=2.320 \mathrm{eV}$ and $\gamma=20 \mathrm{meV}$.

Therefore, it is considered that the difference between the moving distances of the resonant particles and the nonresonant particles represents the difference in absorption. In fact, when fitting the spectrum of the moving distance with Lorentz function, the resonance center and the dephasing constant of the NV-center are obtained as fitting parameters, which are in good agreement with the original values we assumed.

\section{Conclusion}

We have discussed the particle selective transport at the interface between water and glass and theoretically proposed a method to approach the absorption constant of single nano-particles. Experimentally, it is difficult to evaluate the particle absorption spectra of single particle by measuring the optical signal. However, by using this method, the absorption spectra can be measured directly by observing particle motion. It will be a future subject to propose more realistic model setup for the corresponding experimental study.

\section{Acknowledgement}

This work was supported by JSPS KAKENHI Grant Number JP16H06504 for Scientific Research on Innovative Areas "Nano-Material Optical-Manipulation"

\section{References}

[1] Ashkin, A., "Acceleration and Trapping of Particles by Radiation Pressure," Phys. Rev. Lett. 24, 156 (1970).

[2] Ashkin, A., Dziedzic, J. M., Bjorkholm, J. E. and Chu, S., " Observation of a single-beam gradient force optical trap for dielectric particles," Opt. Lett. 11, Issue 5, 288-290 (1986)

[3] Chu, S. and Wieman, C., "Laser Cooling and Trapping of Atoms: Introduction," J. Opt. Soc. Am. B 6, 20202022 (1989).

[4] Iida, T. and Ishihara, H., "Theoretical Study of the Optical Manipulation of Semiconductor Nanoparticles under an Excitonic Resonance Condition," Phys. Rev. Lett. 90, 057403 (2003).

[5] Iida, T. and Ishihara, H., "Theory of resonant radiation force exerted on nanostructures by optical excitation of their quantum states: From microscopic to macroscopic descriptions," Phys. Rev. B 77, 245319 (2008).

[6] Chaumet, P. C. and Nieto-Vesperinas, M., "Time averaged total force on a dipolar sphere in an electromagnetic field," Opt. Lett. 25, 1065-1067 (2000).

[7] Draine, B. T., "The discrete-dipole approximation and its application to interstellar graphite grains," Astrophys. J. 333, 848-872 (1988). 


\title{
Searches for new physics using optically levitated spheres
}

\author{
Fernando Monteiro ${ }^{\mathrm{a}}$ \\ ${ }^{a}$ Wright Laboratory, Department of Physics, Yale University, New Haven, CT 06520, USA
}

\begin{abstract}
$\mathrm{SiO}_{2}$ spheres with diameter ranging from 5 to $23 \mu m$ are optically levitated in a high vacuum environment. This low pressure environment allows for a center of mass acceleration sensitivity of $0.4 \mu \mathrm{g} / \sqrt{\mathrm{Hz}}$ and the low drag allows for a spinning rates of $5 \mathrm{MHz}$ and rotational damping times of $6 \times 10^{4} \mathrm{~s}$. The electric charge present on the spheres can be controlled on the single electron level until they become charge neutral, further decoupling the sphere from its surroundings. These objects can serve as a tool to probe new physics such as the search for millicharged particles, neutrality of matter and deviation from coulomb's law.
\end{abstract}

Keywords: Optical Levitation, Acceleration Sensitivity, Spin Angular Momentum, Damping Time, New Interactions

\section{INTRODUCTION}

Optically levitated objects were first studied by Ashkin ${ }^{1,2}$ and since then have been used in several applications ranging from biology ${ }^{3-5}$ to fundamental physics. ${ }^{6-8}$ Dielectric nanospheres and microspheres levitated in a high vacuum environment offer an excellent force and acceleration sensor due to the reduced gas molecules collisions and are currently being used for applications in tests of quantum mechanics, ${ }^{9,10}$ precision force and acceleration sensing ${ }^{11-18}$ and fundamental physics. ${ }^{6-8}$ The rotational degree of freedom of these levitated objects is also a subject of study in liquids, ${ }^{19-21}$ air and medium vacuum ${ }^{22-31}$ and high vacuum environments with recent examples in which the measured rotation rates range from $\mathrm{MHz}$ to $\mathrm{GHz}$ for microspheres ${ }^{32}$ and nanospheres ${ }^{33,34}$ respectively.

\section{EXPERIMENTAL SETUP}

$\mathrm{SiO}_{2}$ spheres with diameter ranging from 5 to $23 \mu \mathrm{m}$ are trapped above the focal point of a vertically oriented $1064 \mathrm{~nm}$ laser beam. ${ }^{18,32}$ Two $532 \mathrm{~nm}$ beams are used to image the levitated sphere in the three degrees of freedom and the images are projected into photodiodes that sense the center of mass motion of the sphere. The signal from the photodiodes goes into a field-programmable gate array (FPGA) that is used to control a deflection mirror that steers the levitation beam and controls the position of the sphere in the radial direction, and to an Acousto-Optic Modulator (AOM) that controls the power of the levitating beam, which results in the control of the position of the sphere with respect to the focal point of the trapping beam. Such three dimensional control provides additional center of mass cooling that is necessary for stable levitation in the high vacuum environment. ${ }^{18}$

An Electro-Optic Modulator (EOM) is used to control the polarization of the levitating beam from linear to circular polarization. ${ }^{32}$ Such control enables transfer from the spin angular momentum from this beam to the sphere resulting in controllable rotation rates that can exceed $\mathrm{MHz}$ rates in the high vacuum environment. ${ }^{32}$ Measurement of the angular degree of freedom is performed by a linear polarized imaging beam. After passing through the sphere, this beam is directed to a polarization sensing device that is able to measure modulations of the beam polarization caused by the rotating sphere. ${ }^{32}$ A photograph of the setup in shown in figure 1. 


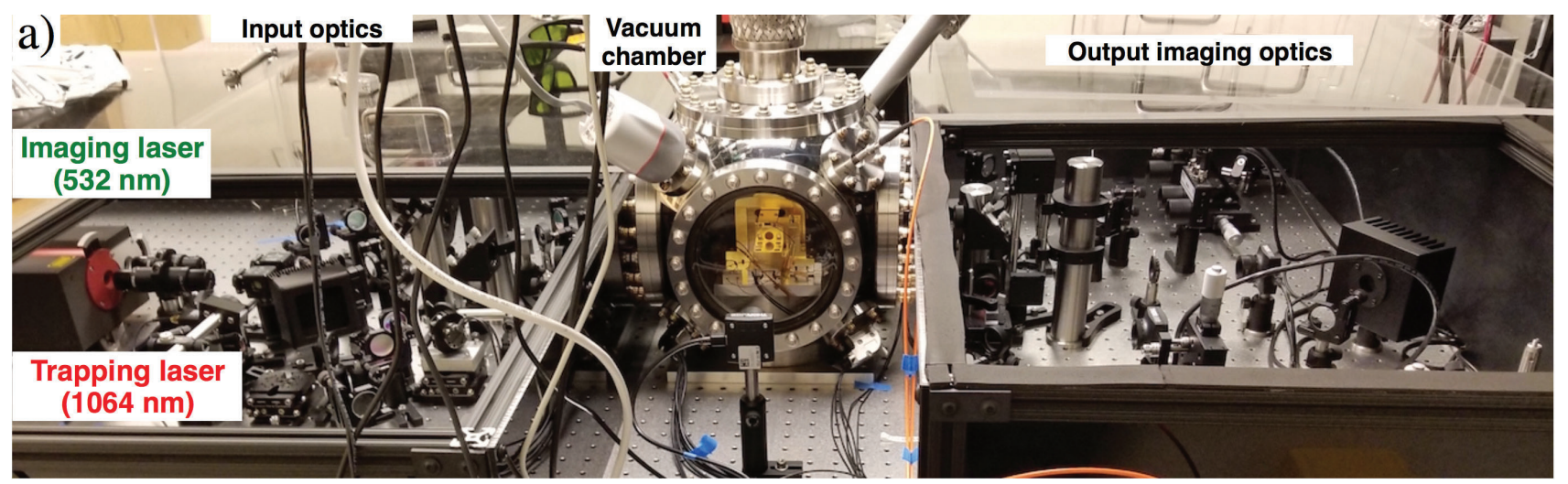

Figure 1. Photograph of the experimental setup with the vacuum chamber in the center, imaging and trapping laser on the left and imaging optics on the right. ${ }^{32,35}$

\section{CURRENT SENSITIVITY}

Two flat and parallel electrodes located in the center of the vacuum chamber and around the levitating sphere are used to created a known oscillating electric field at the sphere's position. ${ }^{18}$ At the same time an UV lamp is used to discharge the sphere. The oscillatory motion of the sphere, due to the oscillatory electric field, is monitored while the sphere is being discharged. The amplitude of this motion decreases in steps that are associated with each electron leaving the sphere. ${ }^{36}$ The amplitude of this motion is used to convert the signal generated by the photodiodes into force when the sphere has one remaining electron. This method is not only used to provide a calibration for the acceleration sensitivity, but can also be used to detect small charges within the sphere. ${ }^{8}$ For the current setup, measured acceleration sensitivities of $0.4 \times 10^{-6} \mathrm{~g} / \sqrt{\mathrm{Hz}}$ are measured for $23 \mu \mathrm{m}$ spheres in diameter. ${ }^{18}$ Such sensitivity corresponds to center of mass temperature on the order of $60 \mathrm{mK}$ and a charge sensitivity of $6 \times 10^{-5} \mathrm{e} / \sqrt{\mathrm{Hz}}$, where $e$ is the electron charge, for electric fields of $5 \mathrm{kV} / \mathrm{mm}$ in between the electrodes.

The spinning rate of the $\mathrm{SiO}_{2}$ spheres with diameter of $10 \mu \mathrm{m}$ and at $10^{-7} \mathrm{mbar}$ can get as high as $5 \mathrm{MHz}$ depending on the trapping beam polarization. ${ }^{32}$ At lower pressures the dissipation of energy of the rotation degree of freedom becomes smaller and results in measured damping times that can be as high as $16 \mathrm{~h}$. A future application of such low damping of the rotational degree of freedom can be the detection of small torques acting on the sphere. ${ }^{34}$

\section{FUTURE SEARCHES OF NEW PHYSICS}

We show in this section how the setup described above can be used for the detection of physics that goes beyond the standard model. ${ }^{8,35}$ We will concentrate on the particular case of the so-called dark or hidden sector which introduces new candidates for dark matter particles and interactions with the standard model. ${ }^{37,38}$ The simplest case of interaction considered by these models is a kinetically mixed dark electromagnetic force corresponding to the gauge group $\mathrm{U}_{D}(1)$ with the standard electromagnetic force corresponding to the gauge group $\mathrm{U}(1) .{ }^{37,38}$ Some of the experimental consequences of this type of model would be a deviation from Coulomb's law ${ }^{37}$ and the existence of dark matter candidates that carry electric charges smaller than the electron charge $|e|$, also called millicharged particles. ${ }^{38,39}$

While our setup can be used to look for deviations from Coulomb's law, ${ }^{35}$ we will focus on how to use the setup described above on the search of millicharged dark matter particles. ${ }^{8}$ This can be accomplished by using the UV light in order to make sure that total number of protons is the same as the total number of electrons on the levitated sphere, applying a large electric field and looking for a response of the center of mass motion of the sphere. ${ }^{8}$ Such center of mass response would be either related to the presence of millicharged particles or backgrounds. Most of the backgrounds are on the form of dipole moments. Such dipoles couple to the gradient of the electric field, which can be minimized by placing large electrodes parallel within $1 \mathrm{mrad}$ and by spinning the spheres in order to average out the dipole moment in the direction of the measurement. Our setup allows 
for an oscillating electric field as high as $5 \mathrm{kV} / \mathrm{mm}$ with charge sensitivity of $6 \times 10^{-5} e / \sqrt{\mathrm{Hz}}$ and our relatively big spheres will enable lower abundances of the millicharged particles to be probed.

\section{CONCLUSION}

Optically levitated spheres in a high vacuum environment can be used as a highly sensitive device to probe new fundamental interactions. We demonstrated that $\mathrm{SiO}_{2}$ spheres with diameter ranging from 5 to $23 \mu \mathrm{m}$ offer high acceleration sensitivity ${ }^{18}$ and low damping associated with the rotational degree of freedom. ${ }^{32}$ Such devices can be used in the search for millicharged particles that have been proposed in some extensions of the standard model.

\section{ACKNOWLEDGMENTS}

This work is supported, in part, by the Heising-Simons Foundation, the National Science Foundation under Grant No. 1653232 and Yale University.

\section{REFERENCES}

[1] Ashkin, A. and Dziedzic, J. M., "Optical levitation by radiation pressure," Appl. Phys. Lett. 19, 283-285 (Oct. 1971).

[2] Ashkin, A., Dziedzic, J. M., Bjorkholm, J. E., and Chu, S., "Observation of a single-beam gradient force optical trap for dielectric particles," Opt. Lett. 11, 288-290 (May 1986).

[3] Ashkin, A. and Dziedzic, J., "Optical trapping and manipulation of viruses and bacteria," Science 235(4795), 1517-1520 (1987).

[4] Ashkin, A., Dziedzic, J. M., and Yamane, T., "Optical trapping and manipulation of single cells using infrared laser beams," Nature 330, 769-771 (Dec. 1987).

[5] Neuman, K. C. and Block, S. M., "Optical trapping," Rev. Sci. Instrum. 75, 2787-2809 (Sept. 2004).

[6] Geraci, A. A., Papp, S. B., and Kitching, J., "Short-range force detection using optically cooled levitated microspheres," Phys. Rev. Lett. 105, 101101 (Aug 2010).

[7] Rider, A. D., Moore, D. C., Blakemore, C. P., Louis, M., Lu, M., and Gratta, G., "Search for screened interactions associated with dark energy below the $100 \mu \mathrm{m}$ length scale," Phys. Rev. Lett. 117, 101101 (Aug 2016).

[8] Moore, D. C., Rider, A. D., and Gratta, G., "Search for millicharged particles using optically levitated microspheres," Phys. Rev. Lett. 113, 251801 (Dec 2014).

[9] Romero-Isart, O., Juan, M. L., Quidant, R., and Cirac, J. I., "Toward quantum superposition of living organisms," New J. of Phys. 12, 033015 (Mar 2010).

[10] Kaltenbaek, R., Hechenblaikner, G., Kiesel, N., Romero-Isart, O., Schwab, K. C., Johann, U., and Aspelmeyer, M., "Macroscopic quantum resonators (maqro)," Exp. Astron. 34, 123-164 (Oct 2012).

[11] Yin, Z.-Q., Geraci, A. A., and Li, T., "Optomechanics of levitated dielectric particles," Int. J. Mod. Phys. B27, 1330018 (2013).

[12] Ether, Jr., D. S., Pires, L. B., Umrath, S., Martinez, D., Ayala, Y., Pontes, B., Araújo, G. R. d. S., Frases, S., Ingold, G.-L., Rosa, F. S. S., Viana, N. B., Nussenzveig, H. M., and Neto, P. A. M., "Probing the Casimir force with optical tweezers," Europhys. Lett. 112, 44001 (Nov. 2015).

[13] Ranjit, G., Atherton, D. P., Stutz, J. H., Cunningham, M., and Geraci, A. A., "Attonewton force detection using microspheres in a dual-beam optical trap in high vacuum," Phys. Rev. A 91, 051805 (May 2015).

[14] Ranjit, G., Cunningham, M., Casey, K., and Geraci, A. A., "Zeptonewton force sensing with nanospheres in an optical lattice," Phys. Rev. A 93, 053801 (May 2016).

[15] Jain, V., Gieseler, J., Moritz, C., Dellago, C., Quidant, R., and Novotny, L., "Direct measurement of photon recoil from a levitated nanoparticle," Phys. Rev. Lett. 116, 243601 (Jun 2016).

[16] Hempston, D., Vovrosh, J., Toro, M., Winstone, G., Rashid, M., and Ulbricht, H., "Force sensing with an optically levitated charged nanoparticle," Appl. Phys. Lett. 111(13), 133111 (2017).

[17] Rider, A. D., Blakemore, C. P., Gratta, G., and Moore, D. C., "Single-beam dielectric-microsphere trapping with optical heterodyne detection," Phys. Rev. A 97, 013842 (Jan 2018). 
[18] Monteiro, F., Ghosh, S., Fine, A. G., and Moore, D. C., "Optical levitation of 10-ng spheres with nano-g acceleration sensitivity," Phys. Rev. A 96, 063841 (Dec 2017).

[19] Friese, M. E. J., Nieminen, T. A., Heckenberg, N. R., and Rubinsztein-Dunlop, H., "Optical alignment and spinning of laser-trapped microscopic particles," Nature 394, 348350 (Jul 1998).

[20] Bishop, A. I., Nieminen, T. A., Heckenberg, N. R., and Rubinsztein-Dunlop, H., "Optical microrheology using rotating laser-trapped particles," Phys. Rev. Lett. 92, 198104 (May 2004).

[21] Bennett, J. S., Gibson, L. J., Kelly, R. M., Brousse, E., Baudisch, B., Preece, D., Nieminen, T. A., Nicholson, T., Heckenberg, N. R., and Rubinsztein-Dunlop, H., "Spatially-resolved rotational microrheology with an optically-trapped sphere," Sci. Rep. 3, 1759 (2013).

[22] He, H., Friese, M. E. J., Heckenberg, N. R., and Rubinsztein-Dunlop, H., "Direct observation of transfer of angular momentum to absorptive particles from a laser beam with a phase singularity," Phys. Rev. Lett. 75, 826-829 (Jul 1995).

[23] La Porta, A. and Wang, M. D., "Optical torque wrench: angular trapping, rotation, and torque detection of quartz microparticles," Phys. Rev. Lett. 92(19), 190801 (2004).

[24] Arita, Y., McKinley, A. W., Mazilu, M., Rubinsztein-Dunlop, H., and Dholakia, K., "Picoliter rheology of gaseous media using a rotating optically trapped birefringent microparticle," Anal. Chem. 83(23), 8855-8858 (2011).

[25] Arita, Y., Mazilu, M., and Dholakia, K., "Laser-induced rotation and cooling of a trapped microgyroscope in vacuum," Nat. commun. 4, 2374 (08 2013).

[26] Mazilu, M., Arita, Y., Vettenburg, T., Auñón, J. M., Wright, E. M., and Dholakia, K., "Orbital-angularmomentum transfer to optically levitated microparticles in vacuum," Phys. Rev. A 94, 053821 (Nov 2016 ).

[27] Hoang, T. M., Ma, Y., Ahn, J., Bang, J., Robicheaux, F., Yin, Z.-Q., and Li, T., "Torsional optomechanics of a levitated nonspherical nanoparticle," Phys. Rev. Lett. 117, 123604 (Sep 2016).

[28] Rahman, A. A. and Barker, P., "Laser refrigeration, alignment and rotation of levitated yb 3+: Ylf nanocrystals," Nat. Photonics 11(10), 634 (2017).

[29] Kuhn, S., Kosloff, A., Stickler, B. A., Patolsky, F., Hornberger, K., Arndt, M., and Millen, J., "Full rotational control of levitated silicon nanorods," Optica 4(3), 356-360 (2017).

[30] Kuhn, S., Stickler, B. A., Kosloff, A., Patolsky, F., Hornberger, K., Arndt, M., and Millen, J., "Optically driven ultra-stable nanomechanical rotor," Nat. Commun. 8, 1670 (2017).

[31] Li, W., Li, N., Shen, Y., Fu, Z., Su, H., and Hu, H., "Dynamic analysis and rotation experiment of an optical-trapped microsphere in air," Appl. Opt. 57, 823-828 (Feb 2018).

[32] Monteiro, F., Ghosh, S., van Assendelft, E. C., and Moore, D. C., "Optical rotation of levitated spheres in high vacuum," Phys. Rev. A 97, 051802 (May 2018).

[33] Reimann, R., Doderer, M., Hebestreit, E., Diehl, R., Frimmer, M., Windey, D., Tebbenjohanns, F., and Novotny, L., "Ghz rotation of an optically trapped nanoparticle in vacuum," Phys. Rev. Lett. 121, 033602 (Jul 2018).

[34] Ahn, J., Xu, Z., Bang, J., Deng, Y.-H., Hoang, T. M., Han, Q., Ma, R.-M., and Li, T., "Optically levitated nanodumbbell torsion balance and ghz nanomechanical rotor," Phys. Rev. Lett. 121, 033603 (Jul 2018 ).

[35] Moore, D. C., "Tests of fundamental physics with optically levitated microspheres in high vacuum," Proc.SPIE 10723, 10723 - 10723 - 10 (2018).

[36] Monteiro, F., Ghosh, S., van Assendelft, E. C., and Moore, D. C., "An apparatus for optical levitation of microspheres in high vacuum with rotational control," Proc.SPIE 10723, 10723 - 10723 - 9 (2018).

[37] Jaeckel, J., "A force beyond the standard model - status of the quest for hidden photons," ArXiv 1303.1821v1 (2013).

[38] Essig, R. et al., "Dark Sectors and New, Light, Weakly-Coupled Particles," ArXiv 1311.0029 (2013).

[39] Brümmer, F. and Jaeckel, J., "Minicharges and magnetic monopoles," Physics Letters B 675(3), 360 - 364 (2009). 


\title{
Rotation control of nanoparticles by optical force using resonant nonlinear response
}

\author{
Masayuki Hoshina ${ }^{a}$, Nobuhiko Yokoshi ${ }^{a}$, Hajime Ishihara*a,b \\ ${ }^{a}$ Graduate School of Engineering, Osaka Prefecture University, 1-1 Gakuen-cho, Naka-ku, Sakai, \\ Osaka, 599-8531, Japan \\ ${ }^{\mathrm{b}}$ Graduate School of Engineering Science, Osaka University, 1-3 Machikaneyama-cho, Toyonaka, \\ Osaka, 560-8531, Japan
}

\begin{abstract}
We theoretically propose a scheme of rotation control of nanoparticles by light using resonant nonlinear optical response. It is known that the Laguerre-Gaussian beam have orbital angular momentum and they can rotate small particles. In the present study, we theoretically demonstrate a selective switching of rotation direction of nanoparticles by optical pumping. The particles have gain by pumping, and the phase of the induced polarization becomes inverted, which leads to the rotation-direction switching. This switching occurs selectively according to the electronic resonance levels of nanoparticles. Further, we study the scheme to rotate nanoparticles and switch its rotation direction in the nanoscale area. Being associated with localized surface plasmons sustained by the nanoscale metallic structures, a circularly polarized light can induce the rotational motion of nanoparticles near the metallic nanogap. This rotation direction can also be inverted by using nonlinear optical response.
\end{abstract}

Keywords: Optical manipulation, Localized surface plasmons, Nonlinear optical response

\section{Introduction}

The techniques to optically manipulate mechanical motions of small particles are promising for pushing nanotechnologies up to the next stage. However, manipulating nanoparticles is not easy as compared with manipulating microparticles because an exerted force becomes extremely weak. In order to overcome this difficulty, the techniques using a steep gradient of the electric field intensity associated with localized surface plasmons has been intensively studied [1], and also, using the electronic resonance effect has been paid attention [2]. In both cases, the nonlinear optical effects easily occur, and it has been clarified that the nonlinear effect played an essential role in the optical trapping experiments using resonant effects [3] and leads to unconventional manipulation schemes [4].

In the present contribution, we propose a new scheme of optical manipulation by using nonlinear optical response. Namely, the switching of the rotation direction of nanoparticles that can be invoked by optical pumping of nanoparticles. This operation can be made in a selective way according to the electronic resonance levels of nanoparticles. Further, we theoretically demonstrate a rotational motion of nanoparticles in a nanoscale area, where the circularly polarized light at nanogap made with tetramer metallic nanostructures show a circular optical current.

\section{Method}

With the use of the discrete dipole approximation method, we numerically calculate the total electric field, $\boldsymbol{E}(\boldsymbol{r}, \omega)=\boldsymbol{E}_{b}(\boldsymbol{r}, \omega)+\int d \boldsymbol{r}^{\prime} \boldsymbol{G}\left(\boldsymbol{r}, \boldsymbol{r}^{\prime}, \omega\right) \boldsymbol{P}_{m}\left(\boldsymbol{r}^{\prime}, \omega\right)$, where $\boldsymbol{P}_{m}(\boldsymbol{r}, \omega)$ is the induced polarization of the nanoparticle, $\boldsymbol{E}_{b}(\boldsymbol{r}, \omega)$ is the background electric field, and $\mathbf{G}\left(\boldsymbol{r}, \boldsymbol{r}^{\prime}, \omega\right)$ is the renormalized Green's function including geometrical information of the system through dielectric function. On the other hand, the polarization of the nanoparticle is calculated by solving the integral equation with the Markovian master equations. Then, we substitute the total electric field and the polarization in the expression of the time-averaged radiation force [5], $\langle\boldsymbol{F}(\omega)\rangle=\operatorname{Re}\left[\int d \boldsymbol{r}[\nabla \boldsymbol{E}(\mathbf{r}, \omega) *] \cdot \boldsymbol{P}_{m}(\mathbf{r}, \omega)\right]$. As a nanoparticle, we assume the particle that is modeled by the typical three-level structure including vibronic levels 
as in Fig. 1(a). (a)

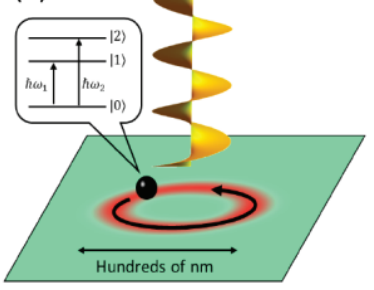

(b)

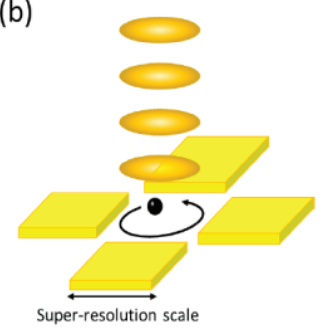

Figure 1: Schematic diagram of the model of incident beam and metallic nano-complex. (a) Image of the conventional type of rotational optical manipulation with Laguerre-Gaussian beam. Its beam waist is 300 $\mathrm{nm}$. (b) Image of super-resolution rotational optical manipulation inside tetramer metallic structures with the circularly polarized plane wave. The nanostructures are assumed to be four gold panels forming nanogaps. The size of each panel is $75 \times 60 \mathrm{x}$ $20 \mathrm{~nm}^{3}$ and the gaps are $14.1 \mathrm{~nm}$. The size of the NP is set to be $5 \times 5 \times 5 \mathrm{~nm}^{3}$. In both cases, incident lights propagate to negative direction of $\mathrm{z}$ axis.

\section{Results}

If the nanoparticle is irradiated with the LaguerreGaussian (LG) beam, the nanoparticle is rotated as illustrated in Fig. 1(a). Here, an interesting effect is the behavior of nanoparticle under the pump beam to create gain of the particle. The direction of rotation, and that along z-axis are inverted. In this case, the direction of dissipative force is inverted for both along $\mathrm{z}$-axis and along orbital direction because of the inversion of excitation probability of the state $|1\rangle$, but the sign of the gradient force is not changed and much stronger than the dissipative force. Thus, the particle is still trapped at the position with a certain negative $\mathrm{z}$ and rotated. In this process, the stimulated emission occurs, where the absorption of one photon induces emissions of two photons. Thus, the observed effect is consistent with the conservation laws of the linear momentum and the orbital angular momentum.

Next, we demonstrate a scheme utilizing the localized optical vortex induced by metallic nano-complex as illustrated in Fig. 1(b). Here, we employ the tetramer structure as the metallic nanostructure for realization of the localized optical vortex, and assume the circularly polarized plane waves injected from the above. We assume that two incident plane waves (manipulation light and pump light) have the spin angular momentum $(\mathrm{s}=+1$ or -1 ) and the energies resonant to $0-1$ and $0-2$ transitions, respectively. The rotation direction of the nanoparticle induced by incident light depends on its spin angular momentum. It should be noted that the spin angular momentum of light can cause the orbital motion of the nanoparticle by utilizing the metallic nano-complex. Figure 2(a) shows the force map inside the tetramer structure in $x-y$ plane, where incident light is only manipulation light and its spin angular momentum and intensity are $\mathrm{s}=+1$ and $100 \mathrm{~kW} / \mathrm{cm}^{2}$, respectively. In this case, the dissipative force saturates and the gradient force is dominant to move the nanoparticle, therefore the rotational force is suppressed and the nanoparticle is attracted toward the gaps where field intensity is strong. On the other hand, under the two-beam excitation, the pump intensity enables to adjust the ratio of the dissipative force to the gradient force. Figure 3(b) shows a force map where we assume the pump light to have the same spin angular momentum $(\mathrm{s}=+1)$ as the manipulation light and both intensities to be $100 \mathrm{~kW} / \mathrm{cm}^{2}$. In this case, the inversion of the excitation rate and stimulated emission between the states 0 and 1 occur, resulting in opposite circulating force induced by the manipulation light. As a result, although both incident lights have the same spin angular momentum, the rotation direction by the manipulation light becomes opposite to that by the pump light, and hence, their rotational forces (dissipative forces) cancel each other. On the other hand, in the case with different spin angular momentum, their rotational forces reinforce each other, hence the orbital motion of the nanoparticle is realized (see, Fig. 3(c)). Also, interestingly, we can avoid pointing the optical force outwards by switching the force direction at nanogaps, where the manipulation light energy is slightly reddetuned to the energy resonant to $0-1$ transition. This rotational force is several tens of times stronger than that in the case of using LG beam without metallic structures. Thus, one can realize rotational manipulation with stronger optical force in super-resolution scale and can control it by the presence/absence of pump light. 


\section{Summary}

In summary, we have theoretically demonstrated the control of rotation direction of the nanoparticles with optical nonlinearity and the way to realize such manipulation at super-resolution scale by utilizing the metallic nano-complex. The results show that the use of nonlinearity and the localized optical vortex will greatly enhance the number of degrees-of-freedom and precision of optical manipulation of nanoparticles.

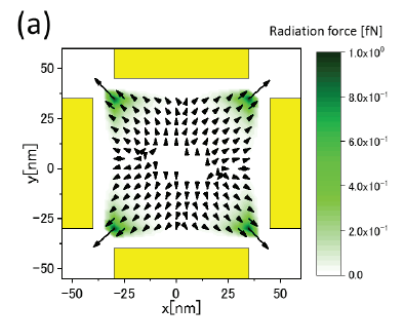

(b)
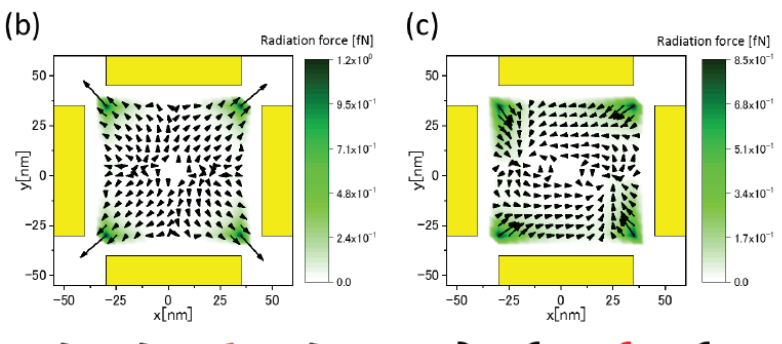

$$
U+\omega \Rightarrow C+0 \quad 0+C \Rightarrow C+C
$$

Figure 2: Radiation force map inside the metallic nanocomplex. Black arrows indicate force vectors in $x-y$ plane. Color bars show the magnitude of the radiation force. Manipulation light and pump light energies are tuned to the resonance with $0-1$ and $0-2$ transitions, respectively. (a) The case with manipulation light alone. The intensity is $100 \mathrm{~kW} / \mathrm{cm}^{2}$ and its spin angular momentum is $s=+1$. (b) The case with both pump and manipulation lights. Both lights have spin angular momentum $\mathrm{s}=+1$ and their intensities are $100 \mathrm{~kW} / \mathrm{cm}^{2}$. (c) The case with both pump and manipulation lights. Pump light and manipulation light have spin angular momentum $\mathrm{s}=-1$ and $\mathrm{s}=+1$ and their intensities are $100 \mathrm{~kW} / \mathrm{cm}^{2}$, respectively. In this case, the energy of manipulation light is slightly red-detuned $(1.798 \mathrm{eV})$. Arrows drawing circle below (b,c) show rotation direction of radiation force caused by the pump and manipulation lights, respectively.

\section{References}

[1] M. L. Juan, M. Righini and R. Quidant, Nat. Photon. 5 (2011) 349

[2] T. Iida and H. Ishihara, Phys. Rev. Lett., 90 (2003) 057403

[3] T. Kudo and H. Ishihara, Phys. Rev. Lett. 109, (2012) 087402

[4] T. Kudo and H. Ishihara, Phys. Chem. Chem. Phys. 15, (2013) 14595

[5] T. Iida and H. Ishihara, Phys. Rev. B77, (2008) 245319 


\title{
Optical trapping in extreme conditions
}

\author{
Yosuke Minowa*, Xi Geng, Kensuke Kokado, Masaaki Ashida \\ Graduate School of Engineering Science, Osaka University, 1-3, Machikaneyama, Toyonaka, Osaka \\ 560-8531 JAPAN
}

\begin{abstract}
Liquid helium is a unique fluid showing many interesting and peculiar properties at low temperature including superfluidity. We demonstrated the optical trapping of single nanoparticles in superfluid helium. The particles were introduced into the optical trapping region via in-situ pulsed laser ablation. Our technique is an important step to realize controllable nanoscale-probing of the superfluidity.
\end{abstract}

Keywords: optical trapping, superfluid $\mathrm{He}$, nanoparticles, laser ablation, optical manipulation

*minowa@mp.es.osaka-u.ac.jp

\section{Introduction}

Optical trapping of micro/nanoparticles is a versatile technique which can be used to study microrheology ${ }^{1}$, mechanical properties of molecules ${ }^{2}$, microcrystalization ${ }^{3}$, and even fundamental physics ${ }^{4}$. In particular, the technique is most suitable to reveal local physical or chemical properties of the surrounding medium and the interaction between the trapped particles and the medium. The optical trapping is, however, often implemented only in room temperature fluids including water, organic solvent, ambient air, and vacuum. In principle, the optical trapping does not require room temperature and cryogenic situation would even more suitable because the small thermal energy should stabilize the optical trapping.

Here we demonstrated that the optical trapping is actually achievable in cryogenic situation. We succeeded in the optical trapping of dielectric nanoparticles in superfluid helium. The superfluid helium is a peculiar low temperature fluid showing very low viscosity, ideally zero viscosity at zero temperature. Although macroscopic viscosity measurement has been reported before, microscopic thermal fluctuation in the superfluid helium is not studied experimentally, which would be a key factor to reveal the details of the elementary excitations, phonon and roton ${ }^{5}$. Moreover, the optical trapping in superfluid helium is an indispensable method to add a local small rotational or linear perturbation onto the superfluid helium.
Our proposed method would be a novel tool to investigate the fundamental quantum physical properties of superfluid helium.

\section{Method}

The whole experiment was executed in the cryostat filled with the superfluid helium whose temperature was maintained around $1.3 \mathrm{~K}$. The continuous wave laser light (wavelength $785 \mathrm{~nm}$, power $150 \mathrm{~mW}$ ) was introduced into the cryostat for the optical trapping. The focusing lens with a high numerical aperture for the optical trapping was mounted within the cryostat. The optical trapping target was dielectric nanoparticles fabricated with in-situ laser ablation. A sintered semiconductor $\mathrm{ZnO}$ was placed in the cryostat and was irradiated with a second harmonic of Nd:YAG laser with a pulse duration of $10 \mathrm{~ns}$, a pulse energy of $1 \mathrm{~mJ}$, and a repetition rate of $10 \mathrm{~Hz}$. The spot size was around $50 \mu \mathrm{m}$. After the laser ablation process, there were many nano and microparticles ejected from the ablated surface ${ }^{6,7}$ and some of them accidentally came around the focusing point of the continuous wave laser light were optically trapped. The trapped particles were visualized through the light scattering. The scattering of the continuous laser light for the optical trapping was enough strong to visualize the particles using a CMOS camera equipped with an imaging lens. 


\section{Results and Discussion}

Figure 1(a) shows a typical image of the optically trapped $\mathrm{ZnO}$ nanoparticles. Single bright dot corresponds to the strong light scattering from the trapped nanoparticles and is highlighted with the arrow in the figure. Figure 1 (b) illustrates a schematic view of the experimental situation. The dotted lines represent the region of the continuous laser light for the optical trapping and the arrow indicates the propagation direction of the light. The nanoparticles was optically trapped exactly at the focusing point of the laser light and stably kept the position more than a several dozen minutes.

(a)

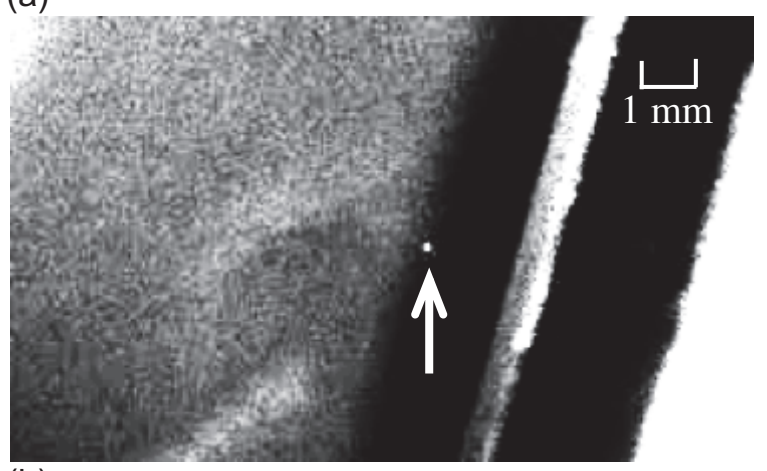

(b)

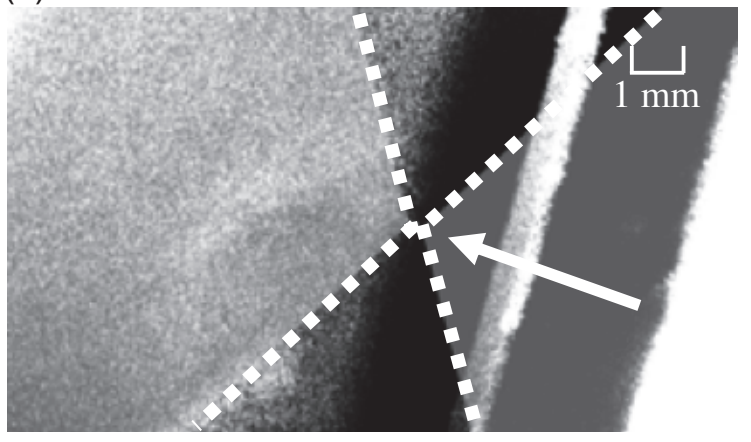

FIG. 1. (a) Optical trapping of $\mathrm{ZnO}$ nanoparticles in superfluid helium. The arrow highlights the trapped particle. (b) The schematics of the continuous laser light path. The dotted lines represent the light focusing region and the arrow indicates the propagation direction.

Our result demonstrated the stable optical trapping of the nanoparticles in superfluid helium and is a unique and yet simple example of the optical trapping in extreme conditions. Our demonstration is a first step toward the nanoscale characterization of the quantum superfluidity in a spatiotemporally resolved manner.

This work was supported by JSPS KAKENHI Grant Numbers JP16H06505, JP17K17841, 16H03884.

\section{References}

[1] Rongxin Huang, Isaac Chavez, Katja M. Taute, Branimir Lukić, Sylvia Jeney, Mark G. Raizen, and ErnstLudwig Florin, "Direct observation of the full transition from ballistic to diffusive Brownian motion in a liquid," Nature Physics, 7, 576-580 (2011).

[2] Karel Svoboda, Christoph F. Schmidt, Bruce J. Schnapp and Steven M. Block, "Direct observation of kinesin stepping by optical trapping interferometry," Nature, 365, 721-727 (1993).

[3] Teruki Sugiyama, Ken-ichi Yuyama, and Hiroshi Masuhara, "Laser Trapping Chemistry: From Polymer Assembly to Amino Acid Crystallization," Acc. Chem. Res., 45 , 1946-1954 (2012).

[4] J. Millen, P. Z. G. Fonseca, T. Mavrogordatos, T. S. Monteiro, and P.F. Barker, "Cavity Cooling a Single Charged Levitated Nanosphere," Phys. Rev. Lett., 114, 123602 (2015).

[5] Xiao Li, Ran Cheng, Tongcang Li, and Qian Niu, "Brownian motion in superfluid 4He,"arXiv:1107.0485 [cond-mat.stat-mech]

[6] Y. Minowa, Y. Oguni, and M. Ashida, "Inner structure of $\mathrm{ZnO}$ microspheres fabricated via laser ablation in superfluid helium," Opt. Express 25, 10449-10455 (2017). [7] Yosuke Minowa, Tatsuro Suzuki, Kenji Setoura, Syoji Ito, Hiroshi Miyasaka, Masaaki Ashida, "Single- particle photoluminescence from cadmium selenide quantum dots fabricated via laser ablation in superfluid helium," J. Nanophoton. 13, 012506 (2018) 


\title{
Optical trapping of nanoparticles using dimer and trimer
}

\section{plasmonic nanogap antennas}

\author{
Christophe Pin*a, Genta Takahashi ${ }^{\mathrm{a}}$, Seiya Fujikawa ${ }^{\mathrm{a}}$, Kota Sudo ${ }^{\mathrm{a}}$, \\ Tuyoshi Fukaminato ${ }^{b}, K_{\text {Keiji Sasaki }}{ }^{\mathrm{a}}$ \\ ${ }^{a}$ Research Institute for Electronic Science, Hokkaido University, Kita 20-jo Nishi 10-chome, Kita-ku, \\ Sapporo, Hokkaido, 001-0020, Japan \\ ${ }^{\mathrm{b}}$ Department of Applied Chemistry and Biochemistry, Kumamoto University, 2-39-1 Kurokami, \\ Chuo-ku, Kumamoto, 860-8555, Japan
}

\begin{abstract}
The excitation of a localized surface plasmon resonance in the nanogap of a plasmonic antenna provides an efficient way to focus some incident light in a nanoscale volume. Enhanced light-matter interactions can then be obtained when a nanoscale object interacts with the confined light. Thanks to enhanced near-field optical forces, plasmonic nanogap antennas can be used as nanotweezers in order to trap nanoparticles. We investigate here the trapping and deposition of nanoparticles such as nanodiamonds and dye-molecule nanoparticles using gold dimer and trimer nanogap antennas.
\end{abstract}

Keywords: optical trapping, nanoparticle, plasmonic antenna, nanoparticle deposition, near-field optical force

\section{Introduction}

Plasmonic nanogap antennas enable focusing light at the nanometer scale by coupling the incident light to localized surface plasmon resonances (LSPRs). This nanoscale confinement of the light can enhance the lightmatter interactions between an incident beam and the nanoscale objects located in the vicinity of the plasmonic nanogap. For instance, the strong field gradient resulting from the excitation of a LSPR generates enhanced nearfield optical forces that make it possible to attract and stably trap nanoparticles. In recent years, nanogap antennas have been used as plasmonic nanotweezers in order to trap various kinds of nanoparticles [1-4].

In this work, we investigate the plasmonic trapping of dielectric nanoparticles using gold dimer and trimer nanoantennas. We first report on the trapping of red fluorescent nanodiamonds. We also investigate the deposition of polystyrene and dye-molecule nanoparticles in the plasmonic nanogaps of dimer and trimer antennas under intense laser irradiation [5].

*sasaki@es.hokudai.ac.jp; phone 011 706-9396

\section{Experimental details}

\section{Plasmonic nanogap antennas}

The plasmonic nano-antennas used in this work were designed using COMSOL Multiphysics in order to optimize the coupling efficiency between the incident light coming from a Nd:YAG laser source $(1064 \mathrm{~nm})$ and the nanogap LSPR of the antenna. We evaluated the influence of the sizes of the antenna and the size of the gap on the optical response of the gold nano-antenna. The photothermal response of the antenna and its environment was also modeled and evaluated by numerical simulation.

The gold nano-antennas were then fabricated by ebeam lithography, gold sputtering, and lift-off techniques. As shown in Figure 1, the optical response of the fabricated nano-antennas was characterized by dark-field spectroscopy in order to check the spectral properties of the antennas' LSPRs. Good agreement was found between the experimental results and the numerical model.

\section{Fluorescent nanoparticles}

In order to investigate the plasmonic trapping of nanoparticles, we used different kinds of fluorescent nanoparticles. In the one hand, we studied the trapping of red fluorescent nanodiamonds for the diamond is a known as a hard dielectric material with a relatively high refractive index (2.39 at $1064 \mathrm{~nm})$. Moreover, the red 
fluorescence of NV- centers is robust (no photobleaching or blinking effects) as it originates from highly stable structural defects. Red fluorescent nanodiamonds with an average size of $50 \mathrm{~nm}$ in water solution were purchased from FND Biotech.

We also investigated the plasmonic trapping of commercial red fluorescent polystyrene nanoparticles with an average size of $40 \mathrm{~nm}$ and self-assembled dyemolecule nanoparticles fabricated by reprecipitation method [5].
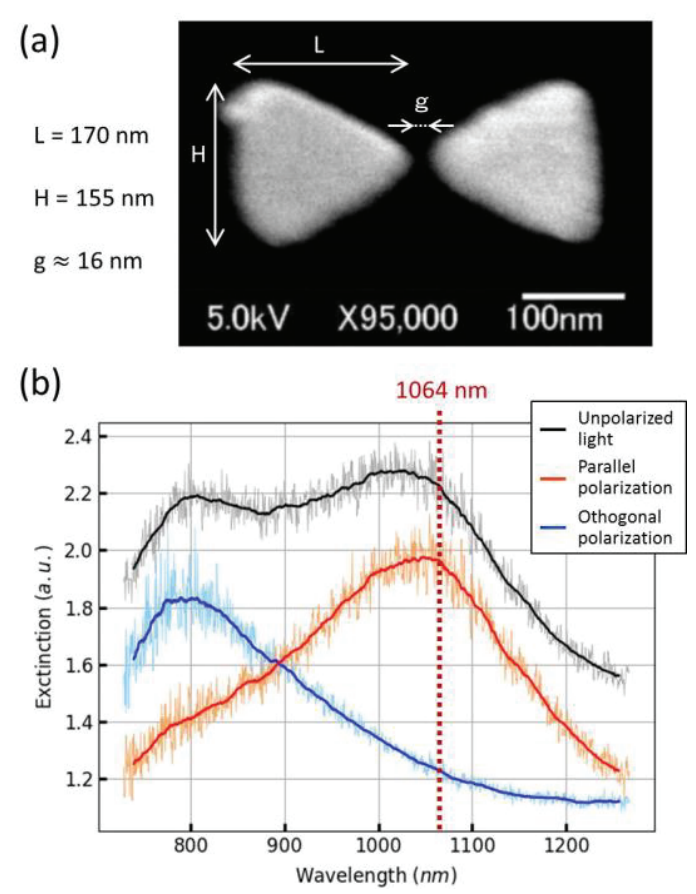

Figure 1. (a) Scanning electron microscope (SEM) image of a gold dimer antenna. (b) Extinction spectra of the previous nano-antenna measured in water environment under different incident light polarization conditions.

\section{Results and Discussion}

\section{Nanodiamond trapping}

By irradiating a gold dimer antenna with an estimated laser intensity of $110 \mathrm{~kW} . \mathrm{cm}^{-2}$, we successfully trapped up to three nanodiamonds simultaneously. As shown in Figure 2, the nanodiamonds were stably trapped, then released after turning off the laser, and finally trapped again after turning on the laser again.

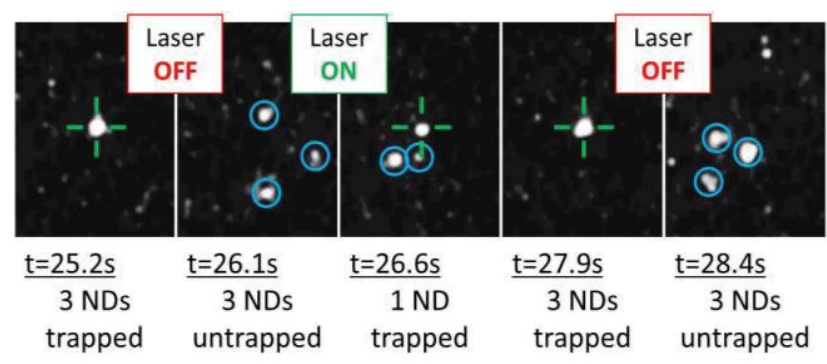

Figure 2. Screenshots taken from the video recording of a nanodiamond trapping experiment. Up to three nanodiamonds were stably trapped in the near-field of a single plasmonic dimer nano-antenna.

\section{Nanoparticle deposition}

We also achieved deposition of nanoparticles in the nanogaps of plasmonic antennas [5]. In the case of offresonant plasmonic nano-antennas, the deposition process requires a focused laser beam with an intensity of about 1 MW. $\mathrm{cm}^{-2}$ to be shined for only few seconds on the target antenna. Nanoparticles located few micrometers away from the antenna are found to be quickly attracted towards the antenna, before being trapped and adsorbed in the vicinity of the nanogap. This process is thought to be due to the combination of a photothermally induced interfacial flow and enhanced near-field optical forces created by the excitation of the nanogap LSPR.

The nanoparticle deposition process described above was successfully applied to polystyrene nanoparticles and self-assembled dye-molecule nanoparticles. This process can be applied for the trapping and deposition of desired nanomaterials. We will present our latest results dealing with plasmonically enhanced fluorescence spectroscopy of nanoparticles. Especially, we aim at probing the chiral response of dye-molecule nanoparticles deposited in the nanogap of trimer antennas.

\section{Acknowledgements}

We acknowledge financial support from JSPS KAKENHI (Grant Numbers JP16H06506, JP18H05205, and JP18H03882). C.P. also acknowledges funding from the Research Institute for Electronic Science, Hokkaido University, and thanks Y. Matsuo, K. Onishi, and W. Nakano from the nanofabrication support team for their technical support. 


\section{References}

[1] Juan, M. L., Righini and M., Quidant, R., "Plasmon nano-optical tweezers," Nat. Photon. 5, 349-356 (2011).

[2] Shoji, T. and Tsuboi, Y., "Plasmonic Optical Tweezers toward Molecular Manipulation: Tailoring Plasmonic Nanostructure, Light Source, and Resonant Trapping," J. Phys. Chem. Lett. 5, 2957-2967 (2014).

[3] Tsuboi, Y., Shoji, T., Kitamura, N., Takase, M., Murakoshi, K., Mizumoto, Y. and Ishihara, H., "Optical Trapping of Quantum Dots Based on GapMode-Excitation of Localized Surface Plasmon," J. Phys. Chem. Lett. 1, 2327-2333 (2010).

[4] Tanaka, Y., Kaneda, S. and Sasaki, K., "Nanostructured Potential of Optical Trapping Using a Plasmonic Nanoblock Pair," Nano Lett. 13, 2146-2150 (2013).

[5] Pin, C., Ishida, S., Takahashi, G., Sudo, K., Fukaminato and T., Sasaki, K., "Nanostructured Potential of Optical Trapping Using a Plasmonic Nanoblock Pair," ACS Omega 3, 4878-4883 (2018). 


\title{
Optomechanics with optically trapped nanoparticles
}

\author{
P. F. Barker ${ }^{1}$ \\ ${ }^{\mathbf{1}}$ Dept. of Physics and Astronomy, University College London, London \\ p.barker@ucl.ac.uk
}

KEY WORDS: nanoparticles, optical tweezers, optomechanics.

Nanoscale oscillators levitated by optical, electric or magnetic fields in high vacuum offer a completely new arena for studies of foundational science and applications [1,2]. The drastic suppression of decoherence potentially allows observation of non-classical states of motion, while the creation of long-lived macroscopic quantum states enable demonstrations of quantum behaviour on very large mass scales. This includes the possibility of creating large macroscopic superpositions, as well as tests of proposed mechanisms of wavefunction collapse at large length scales.

An important requirement for these studies is the development of methods to manipulate and cool the oscillator's centre-of-mass motion as well as controlling the internal temperature. In this talk I will describe our recent work which has demonstrated optical cooling of levitated silica spheres $(200 \mathrm{~nm})$ to milliKelvin temperatures $[3,4]$, as well as internal cooling of optically levitated nanocrystals [5].

1. S. Bose, A. Mazumdar, G. W. Morley, H. Ulbricht, M Toroš, M. Paternostro, A. Geraci, P. F. Barker, M. S. Kim, G. Milburn, Phys. Rev. Lett. 119, 240401 (2017)

2. D. Goldwater, M. Paternostro, P. F. Barker, Phys. Rev. A 94, 010104(R)

3. J. Millen, P. Z. G. Fonseca, T. Mavrogordatos, T. S. Monteiro, P. F. Barker, Phys. Rev. Lett. 114, $123602(2015)$.

4. P. Z. G. Fonseca, E. B. Aranas, J. Millen, T. S. Monteiro, P. F. Barker, Phys. Rev. Lett. 117, 173602 (2016).

5. A. T. M. Anishur Rahman, P. F. Barker, Nature Photonics 11, 634-638 (2017). 


\title{
Modulation of orbital torque on nanoparticles by spin angular momentum via inter-particle light-induced force
}

\author{
Mamoru Tamura ${ }^{\mathrm{a}, \mathrm{b}}$, Takashige Omatsu ${ }^{\mathrm{c}, \mathrm{d}}$, Takuya Iida*a,b \\ ${ }^{a}$ Graduate School of Science, and ${ }^{b}$ Research Institute for Light-induced Acceleration System, \\ in Osaka Prefecture University, 1-2, Gakuen-cho, Naka-ku, Sakai-shi, Osaka, 599-8570, Japan \\ ${ }^{\mathrm{c}}$ Graduate School of Advanced Integration Science, and ${ }^{\mathrm{d}}$ Molecular Chirality Research Center, \\ in Chiba University, 1-33, Yayoi-cho, Inage-ku, Chiba-shi, Chiba, 263-8522, Japan
}

\begin{abstract}
In the optical manipulation, although the spin angular momentum (SAM) makes trapped objects spinning, the orbital angular momentum (OAM) of Laguerre-Gaussian (LG) beam enables to lead the spinning and orbital motion. However, we theoretically discovered that the SAM could modulate the OAM-induced orbital motion of multiple nanoparticles (NPs); the orbital torque exerted on NPs under the LG beam was modulated by the SAM via the inter-particle light-induced force. Our discovery explore a new physical aspect of many-body dynamics of NPs based on the SAM-OAM coupling.
\end{abstract}

Keywords: nanoparticle, optical tweezers, angular momentum, Laguerre-Gaussian beam

\section{Introduction}

Light carries the photon momentum which pushes the irradiated objects for the light propagating direction. In addition, light can have the orbital and spin angular momentum (OAM and SAM) [1,2]. Laguerre-Gaussian (LG) beam so-called optical vortex holds the OAM, and the light with a circular polarization holds the SAM. Such angular momentum also induces a force on objects. The objects trapped off axis under the LG beam show the orbital motion, while the circular polarization leads to the spinning motion. However, these orbital and spinning motions are not independently determined by the OAM and SAM. For example, the OAM can induce the spinning motion for the large objects to be trapped at beam axis [3]. Furthermore, in a helical structure produced by LG beam, it was reported that the helical form was dependent on the total angular momentum as a sum of OAM and SAM [4].

In these examples, the induction of spin motion by the OAM is understandable easily. On the contrary, the SAM should have the same capability with the OAM; also the SAM could lead the orbital motion. Although the tight focusing of LG beam led the orbital motion dependent on the SAM, it was contribution of OAM converted from the SAM [5]. Recently, we theoretically clarified that the SAM could modulate the orbital torque on nanoparticles

*t-iida@p.s.osakafu-u.ac.jp
(NPs) exerted by LG beam, where the inter-particle lightinduced force (LIF) was a key factor. In this study, we demonstrate that the orbital torque exerted on multiple NPs can be modulated by the SAM via the inter-particle LIF.

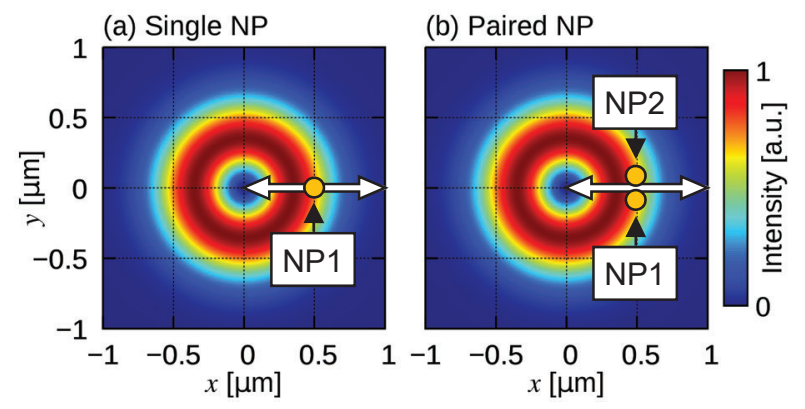

Figure 1. Calculation model. (a) Single NP and (b) paired NPs were scanned from $x=0$ to $1 \mu \mathrm{m}$ as indicated by arrow. Background showed the intensity of LG beam. NPs' size is slightly exaggerated.

\section{Theoretical Method and Model}

As a target of torque evaluation, we assumed the gold NPs (70 $\mathrm{nm}$ in diameter) dispersed in a water. The single NP and paired NPs were located on the focal plane $(z=0)$ of LG beam as shown in Figure 1. The torque when they were scanned from $x=0$ to $1 \mu \mathrm{m}$ were evaluated, where the distance between paired NPs' surface was $10 \mathrm{~nm}$.

To evaluate the torque exerted on NPs, the discrete 
dipole approximation (DDA) was employed. NPs were discretized into the cubic cells with the induced polarization $\mathbf{P}_{i}(\omega)=\chi(\omega) \mathbf{E}_{i}(\omega)$, where $i$ is the index of cells, and $\chi(\omega)=\varepsilon_{\mathrm{p}}(\omega)-\varepsilon_{\mathrm{m}}$ is the electric susceptibility $\left(\varepsilon_{\mathrm{p}}\right.$ is the dielectric function of gold given by the Drude-critical points model [6], and $\varepsilon_{\mathrm{m}}=n_{\mathrm{m}}{ }^{2}=1.33^{2}$ is the dielectric constant of medium). $\mathbf{E}_{i}$ is the response field expressed as,

$$
\begin{aligned}
\mathbf{E}_{i}(\omega)= & \mathbf{E}^{\mathrm{inc}}\left(\mathbf{r}_{i}, \omega\right)+\sum_{j \neq i} \mathbf{G}\left(\mathbf{r}_{i}-\mathbf{r}_{j}, \omega\right) \cdot \mathbf{P}_{j}(\omega) V_{j}, \\
& +\mathbf{S}_{i}(\omega) \cdot \mathbf{P}_{i}(\omega)
\end{aligned}
$$

where $\mathbf{E}^{\text {inc }}$ is the electric field of incident light, $\mathbf{G}$ is the Green's function of electromagnetic field, $V_{i}$ is the volume of $i$-th cell, $\mathbf{S}_{i}(\omega) \cdot \mathbf{P}_{i}(\omega)=\int_{V i} \mathbf{G}(\mathbf{r}, \omega) \cdot \mathbf{P}_{i}(\omega) \mathrm{d} \mathbf{r}$ is the analytical integration of $\mathbf{G}$ for $i=j$. As an incident light, $\mathbf{z}$ propagating circularly polarized LG beam (wavelength $1064 \mathrm{~nm}$ ) was assumed. The electric field is expressed by,

$$
\begin{aligned}
& \mathbf{E}^{\mathrm{inc}}(\mathbf{r}, \omega)=\left[-\sqrt{2} i w_{0} Q(z, \omega) \rho\right]^{|\ell|} \exp (i \ell \varphi) \mathbf{E}^{\mathrm{G}}(\mathbf{r}, \omega), \\
& \mathbf{E}^{\mathrm{G}}(\mathbf{r}, \omega)=-\hat{\boldsymbol{\varepsilon}} E_{0} i w_{0}^{2} Q(z, \omega) \exp \left[i Q(z, \omega) \rho^{2}+i k z\right],
\end{aligned}
$$

where $Q(z, \omega)=k /\left(2 z-i k w_{0}^{2}\right)$. The expression of LG beam is for $p=0$ and arbitrary $\ell$, but $\ell=1$ was employed in this study. $\mathbf{E}^{\mathrm{G}}$ is expression of Gaussian beam, where the spot radius $w_{0}=0.5 \mu \mathrm{m}$, and $E_{0}=\left(2 n_{\mathrm{m}} I_{0} / c \varepsilon_{\mathrm{m}}\right)^{1 / 2}$ gives the amplitude $\left(I_{0}=2 P /|\ell| ! \pi w_{0}^{2}, P=1 \mathrm{~W}\right.$ is power). The polarization is given by $\hat{\boldsymbol{\varepsilon}}=(1, \pm i, 0) / \sqrt{ } 2$, where + and corresponds to the left and right circular polarization (LCP and RCP). $\mathbf{E}_{i}$ and $\mathbf{P}_{i}$ can be obtained by solving the simultaneous equations. From these values and the general expression of LIF [7], LIF exerted on $i$-th cell is given by,

$$
\left\langle\mathbf{F}_{i}^{\text {cell }}(\omega)\right\rangle=\frac{1}{2} \operatorname{Re}\left\{\left[\nabla \mathbf{E}_{i}(\omega)\right]^{*} \cdot \mathbf{P}_{i}(\omega) V_{i}\right\} .
$$

In addition, a sum of it yields the LIF exerted on $k$-th NP,

$$
\begin{aligned}
& \left\langle\mathbf{F}_{k}^{\mathrm{NP}}(\omega)\right\rangle=\sum_{i \in M_{k}}\left\langle\mathbf{F}_{i}^{\mathrm{cell}}(\omega)\right\rangle=\left\langle\mathbf{F}_{k}^{\mathrm{NP}, \text { inc }}(\omega)\right\rangle+\left\langle\mathbf{F}_{k}^{\mathrm{NP}, \mathrm{IP}}(\omega)\right\rangle \\
& =\sum_{i \in M_{k}} \frac{1}{2} \operatorname{Re}\left\{\left[\nabla \mathbf{E}^{\mathrm{inc}}\left(\mathbf{r}_{i}, \omega\right)\right]^{*} \cdot \mathbf{P}_{i}(\omega) V_{i}\right\} \\
& +\sum_{i \in M_{k}} \frac{1}{2} \operatorname{Re}\left\{\left[\sum_{j \neq i} \nabla \mathbf{G}\left(\mathbf{r}_{i}-\mathbf{r}_{j}, \omega\right) \cdot \mathbf{P}_{j}(\omega) V_{j}\right]^{*} \cdot \mathbf{P}_{i}(\omega) V_{i}\right\}
\end{aligned}
$$

where $M_{k}$ is a set of the cell indices composing the $k$-th NP, and LIF can be separated into two terms $\left\langle\mathbf{F}_{k}^{\mathrm{NP} \text {,inc }}\right\rangle$ and $\left\langle\mathbf{F}_{k}^{\mathrm{NP}, \mathrm{IP}}\right\rangle$ by substituting equation (1) for $\mathbf{E}_{i}$, while the differential of third term in equation (1) is negligible. In equation (5), the first term is caused by the incident light
$\mathbf{E}^{\text {inc }}$. On the other hand, the second term is caused by the scattered field from other cells via $\mathbf{G}$, which can be called as inter-cell LIF. However, since the LIF caused by the arbitrary combination $\mathbf{P}_{i}$ and $\mathbf{P}_{j}$ via $\mathbf{G}$ is almost recoil force, the sum for $i, j \in M_{k}$ is cancelled. Thus, the second sum of second term can be approximately replaced by sum for $j \notin$ $M_{k}$, which is namely inter-particle LIF rather than intercell LIF, because $\left\langle\mathbf{F}_{k}^{\mathrm{NP}, \mathrm{IP}}\right\rangle$ is originated from the contribution of cells comprising other NPs.

Based on the expression of LIF, the total orbital torque exerted on the NPs is simply written by,

$$
\left\langle\tau_{z}(\omega)\right\rangle=\sum_{i}\left[\mathbf{r}_{i} \times\left\langle\mathbf{F}_{i}^{\text {cell }}(\omega)\right\rangle\right]_{z} .
$$

However, the mechanical motion of objects are usually considered as a rigid body. Its translational motion is driven by the force exerted on its center-of-mass (CM), i.e. $\left\langle\mathbf{F}_{k}^{\mathrm{NP}}\right\rangle$. Based on this consideration, the another definition of orbital torque should be written by,

$$
\left\langle T_{z}(\omega)\right\rangle=\sum_{k}\left[\mathbf{r}_{k}^{\mathrm{CM}} \times\left\langle\mathbf{F}_{k}^{\mathrm{NP}}(\omega)\right\rangle\right]_{z},
$$

where $\mathbf{r}_{k}^{\mathrm{CM}}$ is the CM position of $k$-th NP.
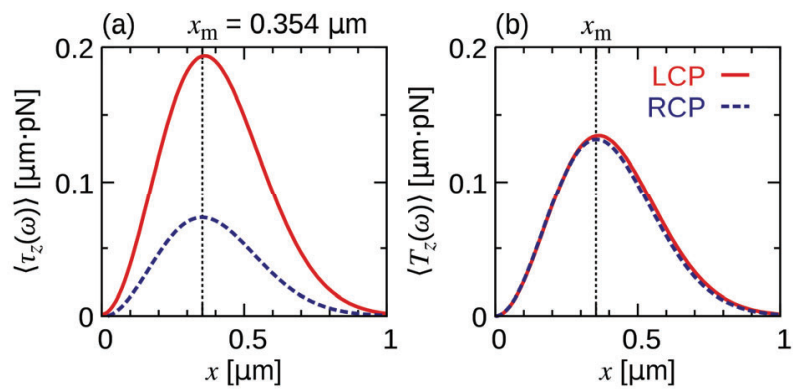

Figure 2. Torque for single NP as a function of $x$ position was evaluated by (a) equation (6) and (b) equation (7). Torque became maximum near $x=x_{\mathrm{m}}$, where the light intensity of LG beam was maximum on $x$-axis.

\section{Results and Discussion}

Figure 2 shows the numerically evaluated orbital torque exerted on the single NP, as a function of $x$-position shown in Figure 1(a). Based on the equation (6) in figure 2(a), the orbital torque for LCP was larger than one for RCP. In contrast, in figure 2(b), the torque evaluated by equation (7) yielded the almost identical values. The origin of this difference was the term of $\mathbf{G}$ in equation (4) after 
substituting equation (1), which was namely intra-particle LIF because it was caused by the interaction between cells in the same particle. This LIF had the distribution in NP and contributed to the spinning of NPs around their CM under the circular polarization. Thus, based on equation (6), which could contribute to modulate the orbital torque. However, this intra-particle LIF was almost canceled in equation (7) and (5) due to the sum for $j \notin M_{k}$. As a result, the difference between LCP and RCP disappeared. Of course, when considering a spinning motion of rigid body, the contribution of SAM should remain as a spin torque.

Next, figure 3 shows the orbital torque exerted on the paired NPs, based on the model of figure 1(b). Needless to say, figure 3(a) by equation (6) showed the different value of torque for LCP and RCP. In addition, we can confirm the clear difference of torque in figure 3(b) employing equation (7). Similar to the discussion for single NP, after the summation, the intra-particle LIF to spin the individual NPs around the CM of each NP was approximately cancelled. However, the inter-particle LIF $\left\langle\mathbf{F}_{k}^{\mathrm{NP}, \mathrm{IP}}\right\rangle$ was not cancelled, which contribute to spin the paired NPs around the CM of whole NPs. Therefore, since NPs were aligned in $y$-direction, the orbital torque was caused from $y$ component of $\mathbf{r}_{k}^{\mathrm{CM}}$ multiplied by $x$-component of $\left\langle\mathbf{F}_{k}^{\mathrm{NP}, \mathrm{IP}}\right\rangle$.
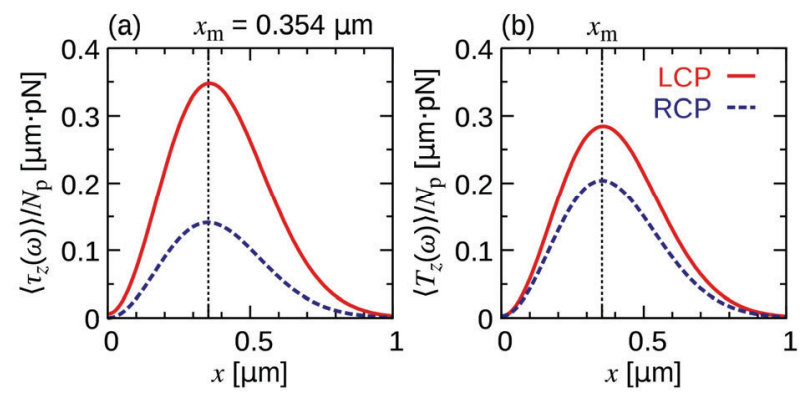

Figure 3. Torque for paired NP as a function of $x$ position was evaluated by (a) equation (6) and (b) equation (7). Torque was normalized by the number of NPs: $N_{\mathrm{p}}=2$.

\section{Conclusion}

In summary, we theoretically demonstrated that the SAM could modulate the OAM-induced orbital motion of multiple NPs via the inter-particle LIF. Based on the DDA, the optical response, LIF, and orbital torque for the single NP and paired NPs were numerically evaluated. For the single NP, the contribution of SAM to the orbital torque disappeared, based on the consideration of translational motion of rigid body. However, for the paired NPs, the SAM modulate the orbital torque on NPs under the LG beam, where the inter-particle LIF to spin the NPs around their CM of whole NPs was an important factor. The obtained results will lead a new physical mechanism of SAM-OAM coupling, and the further development of laser manipulation and processing techniques using LG beams.

\section{Acknowledgements}

This work was supported by Scientific Research on Innovative Areas "Nano-Material Optical-Manipulation" (No. JP16H06507), a Grant-in-Aid for Scientific Research (A) (No. JP18H03884, No. JP17H00856), Grant-in-Aid for Scientific Research (B) (No. JP18H03522, No. JP15H03010), Challenging Research (Exploratory) (No. JP17K19070) from JSPS KAKENHI, JST-Mirai Program (No. JPMJMI18GA), the Key Project Grant Program of Osaka Prefecture University, and the Cooperative Research Program of "Network Joint Research Center for Materials and Devices".

\section{References}

[1] Beth, R. A., "Mechanical Detection and Measurement of the Angular Momentum of Light," Phys. Rev. 50(2), 115-125 (1936).

[2] Allen, L., Beijersbergen, M. W., Spreeuw, R. J. C., Woerdman, J. P., "Orbital angular momentum of light and the transformation of Laguerre-Gaussian laser modes," Phys. Rev. A 45(11), 8185 (1992).

[3] Simpson, N. B., Dholakia, K., Allen, L., Padgett, M. J., "Mechanical equivalence of spin and orbital angular momentum of light: an optical spanner," Opt. Lett. 22(1), 5254 (1997).

[4] Barada, D., Juman, G., Yoshida, I., Miyamoto, K., Kawata, S., Ohno, S., Omatsu, T., "Constructive spin-orbital angular momentum coupling can twist materials to create spiral structures in optical vortex illumination," Appl. Phys. Lett. 108, 051108 (2016).

[5] Zhao, Y., Edgar, J. S., Jeffries, G. D. M., McGloin, D., Chiu, D. T., "Spin-to-Orbital Angular Momentum Conversion in a Strongly Focused Optical Beam,” Phys. Rev. Lett. 99(7), 073901 (2007).

[6] Vial, A., Laroche, T., "Comparison of gold and silver dispersion laws suitable for FDTD simulations," Applied Physics B 93(1), 139-143 (2008)

[7] Iida, T., Ishihara, H., "Theory of resonant radiation force exerted on nanostructures by optical excitation of their quantum states: From microscopic to macroscopic descriptions," Phys. Rev. B 77(24), 245319 (2008). 


\title{
Proposal of an optical-force probe for chirality sensing
}

\section{of metallic nanostructures}

\author{
Nobuhiko Yokoshi $^{\mathrm{a}}$, Masayuki Hoshina ${ }^{\mathrm{a}}$, Hajime Ishihara ${ }^{\mathrm{a}, \mathrm{b}}$ \\ a Department of Physics and Electronics, Osaka Prefecture University, Sakai, Osaka 599-8531, Japan \\ ${ }^{\mathrm{b}}$ Department of Materials Engineering Science, Osaka University, Toyonaka, Osaka 560-8531, Japan
}

\begin{abstract}
We propose a new type of chirality sensing of metallic nanostructures. The essence of our proposal is to evaluate their circular dichroism (CD) from optical force acting on a metallic nano-tip probe. With the use of discrete dipole approximation method, we numerically calculate the optical force on the tip nearby the metallic gammadion array. It is found that the force can three-dimensionally evaluate the $\mathrm{CD}$ that the surface plasmon induces.
\end{abstract}

Keywords: surface plasmon, chirality, optical force, circular dichroism

\section{Introduction}

Surface plasmon on metallic nanostructures enables us to optically access various nanoscale targets through its strong and localized field. One of its various applications is high-sensitive chiral sensing of a single or a few molecules, proteins and so on $[1,2]$. Such a sensing is based on the coupling between chiral molecules and chiral plasmon near designed metallic structures.

The optical responses in the chiral plasmon has been estimated from the circular dichroism (CD) and the optical rotatory dispersion (ORD) in the transmitted farfields [3]. Recently, the profile of the near-field itself is becoming observable by using the aperture-type scanning near-field optical microscope (SNOM) [4]. However, with the above methods only, it is difficult to measure the three-dimensional (3D) structure of the chiral near-field where the chiral molecules should be placed.

Here we propose another approach to figure out the 3D structure of chiral near-field. Our method is to take measure of optical force acting on a metallic nano-probe, which is scanned nearby the metallic structure. We show that the force depends on the circular polarization of the illuminated light, and clearly reflect the $\mathrm{CD}$ of the plasmonic near-field.

\section{Model}

\section{Calculation}

Schematic picture of system setup is shown in Fig. 1 (a). We arrange four of Au gammadion nanostructures on a dielectric substrate (relative permittivity 2.25 , thickness $15 \mathrm{~nm})$. Each gammadion structure is aligned with a spacing of $75 \mathrm{~nm}$.

Here we illuminate the left- or right-handed circularly polarized light from below the substrate. The energy of the light is set to $1.65 \mathrm{eV}$ (wavelength $\lambda \sim 750 \mathrm{~nm}$ ), and the intensity is $1 \mathrm{~kW} / \mathrm{cm}^{2}$. We calculate the optical force on a metallic nanometer-scale probe (Au hemisphere with the radius $50 \mathrm{~nm}$ ), which is scanned in a plane $5 \mathrm{~nm}$ above the nanostructures. In particular, we investigate how the plasmonic chirality affects the vertical component of the optical force.
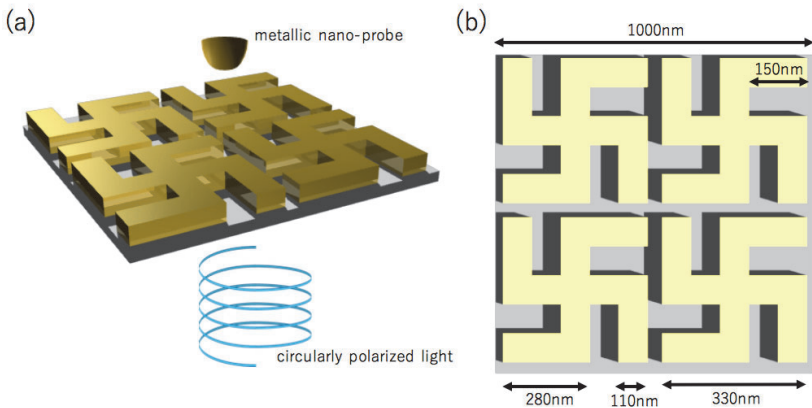

Figure 1. (a) Schematic picture of system setup is shown. The four gammadion structures made of $\mathrm{Au}$ are arranged on a dielectric substrate. The left- or right-handed circularly polarized light is illuminated from below. The Au nano-scale probe tip is scanned to measure the optical force just above the gammadions.

(b) Top view of the system is shown. 
(a)

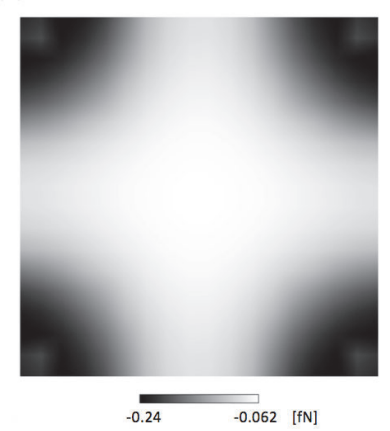

(b)

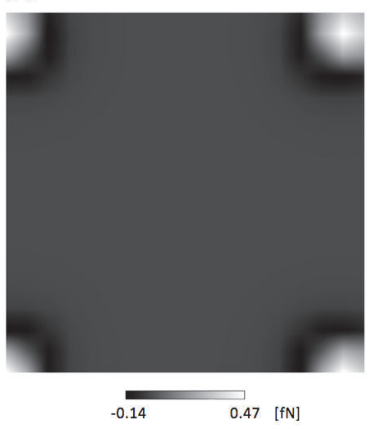

Figure 2. Contour map of the vertical component of the optical force on the Au probe tip. The axes represent the in-plane position of the tip center. The plotted area is $90 \times 90 \mathrm{~nm}^{2}$ including the gap region $\left(75 \times 75 \mathrm{~nm}^{2}\right)$ surrounded by the four gammadions. (a) The case for the illumination of the left-handed circular polarized plane wave light. (b) The case for the right-handed circular polarized light.

\section{Method}

In order to take the geometric information of the metallic nanostructure into account, we solve the Maxwell equation of the whole system (the gammadion and the probe) by using the discrete dipole approximation (DDA) method [5]. In this method, we discretize the whole space by the cubic cells $\left(5 \times 5 \times 5 \mathrm{~nm}^{3}\right)$, and put local susceptibility to each cell. For the cell in Au regions, the susceptibility is given by the Drude model [6]. The total electric field can be calculated through the discretized Maxwell equation by replacing the position integral into the summation with respect to the cells. The 3D optical force acting on the probe tip can be calculated using the total electric field and the induced polarization. It corresponds to the resultant force of the time averaged forces at the cells in the nano-tip region [7].

\section{Results}

We find that the $\mathrm{CD}$, difference between the transmitted light intensity for the left-handed circular polarized illumination and that for the right-handed circular polarized illumination, appears remarkably at the gap region surrounded by the four gammadion. This clearly show that the metallic gammadions induce the strong chiral plasmonic field, which is consistent with the

previous results by the far-field analysis [3]. In addition, our numerical result shows that the vertical component of the total electric field becomes comparable to the in-plane one in the near-field region. This implies that our method can capture the whole figure of the chiral plasmon, which is difficult by SNOM.

In Figs. (a,b), we plot the vertical component of the optical force acting on the Au nano-tip probe. The vertical force, as well as the in-plane component, strongly depends on the circular polarization of the light. In particular, at the edge of the gammadions (the four corners of Figs. $(a, b))$, the vertical force changes its sign depending on the light polarization. These results suggest high possibility of our proposed chiral sensing method.

\section{Conclusion}

We have theoretically proposed a new chiral sensing method of nanoscale metallic nanostructures. In our method, an optical force acting on metallic nano-tip probe is measured. By numerical simulation with DDA method, we showed that our proposal can detect the $3 \mathrm{D}$ profile of the chiral near-field.

Chiral sensing and quantification of a few molecules are getting more important in various fields such as analytical chemistry, biochemistry, pharmacology and basic physics. The $3 \mathrm{D}$ profile of the chiral plasmon, that is localized in nanometer scale, should contribute to these applications. Further optimization of our proposal may lead to higher sensitivity than the present case. Therefore, we believe that this result can bring a new change to the chiral sensing in many fields.

\section{References}

[1] Kuzyk, A., Schreiber, R., Fan, Z., Pardatscher, G., Roller, E.-M., Högele, A., Simmel, F. C., Govorov, A. O. and Lied1, T. 2012. DNA-based self-assembly of chiral plasmonic nanostructures with tailored optical response. Nature 483: 311-314.

[2] Kumar, J., Eraña, H., López-Martínez, E., Claes, N., Martín, V. F., Solís, D. M., Bals, S., Cortajarena, A. L., Castilla, J., and Liz-Marzán, L. M. 2018. Detection of amyloid fibrils in Parkinson's disease using plasmonic chirality. Proc. Nat. Aca. Sci. 115: 3225- 
3230.

[3] Kuwata-Gonokami, M., Saito, N., Ino, Y., Kauranen, M., Jefimovs, K., Vallius, T., Turunen, J. and Svirko, Y. 2005. Giant Optical Activity in Quasi-TwoDimensional Planar Nanostructures. Phys. Rev. Lett. 95: 227401.

[4] Narushima, T. and Okamoto, H. 2013. Strong Nanoscale Optical Activity Localized in TwoDimensional Chiral Metal Nanostructures. J. Phys. Chem. C 117: 23964-23969.

[5] Purcell, E. M. and Pennypacker, C. R. 1973. Scattering and absorption of light by nonspherical dielectric grains. Astro. J. 186: 705-714.

[6] Hoshina, M., Yokoshi, N., and Ishihata, H. 2018. Super-Resolution Trapping: A Nanoparticle Manipulation Using Nonlinear Optical Response. ACS Photon. 5: 318-323.

[7] Iida, T., and Ishihata, H. 2008. Theory of resonant radiation force exerted on nanostructures by optical excitation of their quantum states: From microscopic to macroscopic descriptions. Phys. Rev. B 77: 245319. 


\title{
One-dimensional optical lattices for optical trapping and manipulation along a few-mode silicon waveguide
}

\author{
Christophe Pin*a,b ${ }^{\mathrm{a}}$, Jean-Baptiste Jager ${ }^{\mathrm{b}}$, Manon Tardif ${ }^{\mathrm{b}}$, Emmanuel Picard ${ }^{\mathrm{b}}$, Emmanuel Hadji ${ }^{\mathrm{b}}$, \\ Frédérique de Fornel $^{\mathrm{b}}$, Benoît Cluzel ${ }^{\mathrm{b}}$ \\ ${ }^{a}$ Groupe Optique de Champ Proche - L.R.C. SiNOPTIQ du C.E.A. nDSM-08-36, \\ Laboratoire Interdisciplinaire Carnot de Bourgogne U.M.R. C.N.R.S. 6303, \\ Université de Bourgogne-Franche Comté, 9. Av. A. Savary, 21078 Dijon, France \\ ${ }^{\mathrm{b}}$ Université Grenoble Alpes, C.E.A., I.N.A.C., PHELIQS, SINAPS, F-38000 Grenoble, France
}

\begin{abstract}
Although photonic waveguides have been often used as on-chip optical conveyor belts for transporting trapped particles, we demonstrate here an all-optical approach to create tunable periodic arrays of optical traps along a single silicon waveguide. We show that the simultaneous excitation of two guided modes with different propagation constants leads to a periodic modulation of the near-field optical forces at the surface of the waveguide. Stable trapping of dielectric particles and bacteria is achieved by using this method. We also demonstrate different techniques for manipulating the trapped particles by controlling the light coupling conditions and the laser wavelength. Especially, we show how the period of the optical lattice can be tuned by controlling the laser wavelength, making it possible to finely adjust the trapped particle positions by either pushing or pulling them along the waveguide.
\end{abstract}

Keywords: optical lattice, optical trapping, optical manipulation, optofluidics, silicon photonics, lab-on-a-chip

\section{Introduction}

Since the pioneering work of Kawata and Tani [1], optical forces arising from the evanescent field of the light propagating inside photonic waveguides have been studied for more than two decades. Photonic waveguides are now considered as efficient optical conveyor belts with potential applications for on-chip transportation and sorting of micro- and nanoparticles, as well as living cells and bacteria in liquid solutions [2-7]. Indeed, when a particle interacts with the evanescent tail of a guided mode of a photonic waveguide, the resulting optical force can act both as a trapping force maintaining the particle close to the surface of the waveguide, and as a pushing force propelling the particle in the direction of the light propagation.

In order to achieve stable trapping of a particle, it usually requires the action of an additional photonic microcavity [8-14] or a plasmonic nanostructure [15-17] for creating a hot spot at a fixed position along the waveguide. Yet, recent progress in the design and fabrication of photonic components has enabled the

*benoit.cluzel@u-bourgogne.fr demonstration of periodic arrays of optical traps created by a standing wave formed in a silicon waveguide $[18,19]$. The standing wave was obtained by precisely balancing the power of two counter-propagative waves in a single waveguide.

In this work, we investigate another all-optical approach for periodically modulating the optical forces in the near-field of a few-mode silicon waveguide. We demonstrate that tunable optical lattices can be created by a beating phenomenon between guided modes having different propagation constants [20]. Using such nearfield mode-beating optical lattices, we achieve stable trapping of dielectric particles and e.coli bacteria. We also demonstrate various techniques for manipulating a large number of trapped particles along a single waveguide.

\section{Experimental details}

\section{Photonic silicon waveguides}

The waveguides considered in this work are fabricated on SOI chips using standard microelectronic fabrication techniques. The waveguides have a $510 \times 248 \mathrm{~nm}^{2}$ rectangular cross-section. Numerical simulations were 
performed using COMSOL Multiphysics in order to compute the electric field distributions and the propagation constants of the eigenmodes of such waveguides. As shown in Figure 1, three guided modes can be excited in a silicon waveguide with the considered shape and dimensions. In this work, the different guided modes were selectively excited by controlling the polarization and the fiber-to-waveguide coupling conditions.

\section{Periodic modulation of near-field optical forces}

When two guided modes are simultaneously excited in the same waveguide, they propagate with different propagation constants. This results in a spatially periodic phase difference between the two modes, which impacts the light-matter interactions occurring along the waveguide. This mode-beating phenomenon causes a periodic modulation of the near-field optical forces acting on the particles near the surface of the waveguide. The period $p$ of this modulation is given by:

$$
p_{\mathrm{ij}}=2 \pi /\left|\beta^{(\mathrm{i})}-\beta^{(\mathrm{j})}\right|=\lambda_{0} /\left|n_{\mathrm{eff}}{ }^{(\mathrm{i})}-n_{\mathrm{eff}}{ }^{(\mathrm{j})}\right|
$$

where $\lambda_{0}$ refers to the free-space wavelength and $\beta^{(\mathrm{i})}, \beta^{(\mathrm{j})}$ (respectively $n_{\text {eff }}^{(\mathrm{i})}, n_{\text {eff }}^{(\mathrm{j})}$ ) to the propagation constants (respectively to the effective refractive indices) of the two guided modes.

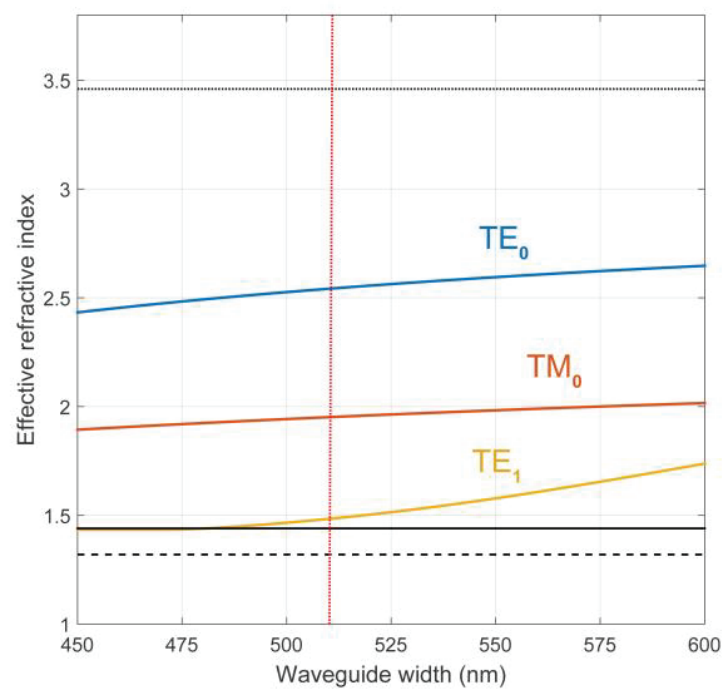

Figure 1. Computed values of the effective refractive indices of the guided modes as a function of the waveguide's width.

\section{Near-field mode-beating optical lattices}

As shown in Figure 2, stable optical trapping of $1 \mu \mathrm{m}$ and $500 \mathrm{~nm}$ fluorescent polystyrene beads was achieved when both transverse-electric (TE) and transversemagnetic (TM) modes were excited in the waveguide. More especially, the beads were trapped in a periodic array above the waveguide with a period of $3.26 \mu \mathrm{m}$. When modifying the coupling conditions, the $500 \mathrm{~nm}$ beads were sometimes trapped in staggered rows on both sides of the waveguide with a period of $1.46 \mu \mathrm{m}$. These experimental results are in good agreement with the results obtained from numerical simulations: while the one-row periodic trap array is formed by the copropagation of both the fundamental TE and TM modes ( $\mathrm{TE}_{0}$ and $\mathrm{TM}_{0}$, respectively), the trapping of particles in staggered rows results from the copropagation of both the fundamental and the first order TE modes $\left(\mathrm{TE}_{0}\right.$ and $\mathrm{TE}_{1}$, respectively). As shown in Figure 2 (c), we also achieved stable trapping of e.coli bacteria in an optical lattice formed by the $\mathrm{TE}_{0}-\mathrm{TM}_{0}$ copropagation.

(a)

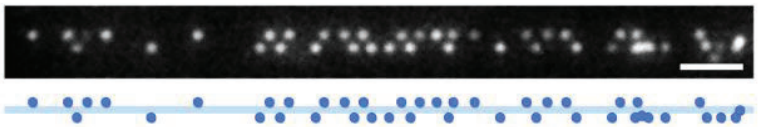

(b)

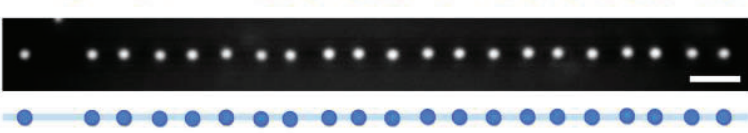

(c)

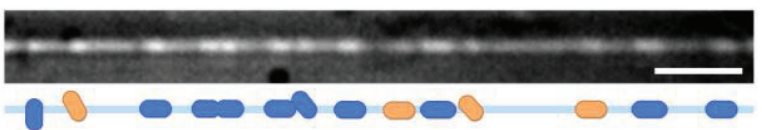

Figure 2. (a) Periodic arrangement of $500 \mathrm{~nm}$ polystyrene beads trapped in staggered rows. (b) Periodic arrangement of $1 \mu \mathrm{m}$ polystyrene beads above the waveguide. (c) Stable trapping of several e.coli bacteria above a single waveguide.

\section{New optical manipulation techniques}

The periodic modulation of the near-field optical forces allows not only for the stable trapping of a large number of particles but also for their manipulation. For instance, modifying the polarization of the incident light and the coupling conditions at the entrance of the waveguide enables switching from one optical lattice configuration to 
another, or to the optical propulsion of the trapped particles. We also show how the period of the optical lattices can be dynamically controlled by tuning the laser wavelength. The particle spacing can therefore be finely tuned and the position of the trapped particles precisely controlled. This makes it possible to move the trapped particles in both directions along the waveguide, even against the direction of the light propagation.

\section{Conclusion}

In this work, we have demonstrated how the simultaneous excitation of pairs of guided modes leads to the periodic modulation of the optical forces in the nearfield of a few-mode waveguide. By exciting the adequate pairs of guided modes, we have been able to trap a large number of particles and bacteria in one-dimensional optical lattices formed along a single waveguide. By controlling the light coupling conditions and the laser wavelength, we have demonstrated different optical manipulation techniques for handling the trapped particles.

\section{Acknowledgements}

This work was performed in cooperation with the Labex ACTION program (contract ANR-11-LABX-000101 ), and with the support of the "Plateforme Technologique Amont" of Grenoble, funded through the CNRS Renatech network.

\section{References}

[1] Kawata, S. and Tani, T., "Optically driven Mie particles in an evanescent field along a channeled waveguide," Opt. Lett. 21, 1768-1770 (1996).

[2] Gaugiran, S., Gétin, S., Fedeli, J.M., Colas, G., Fuchs, A., Chatelain, F. and Dérouard, J., "Optical manipulation of microparticles and cells on silicon nitride waveguides," Opt. Express 13, 6956-6963 (2005).

[3] Yang, A.H., Moore, S.D., Schmidt, B.S., Klug, M., Lipson, M. and Erickson, D., "Optical manipulation of nanoparticles and biomolecules in sub-wavelength slot waveguides," Nature 457, 71-75 (2009).

[4] Ahluwalia, B. S., McCourt, P., Huser, T. and Hellesø, O. G., "Optical trapping and propulsion of red blood cells on waveguide surfaces," Opt. Express 18, 21053 21061 (2010).

[5] Scullion, M.G., Arita, Y., Krauss, T.F. and Dholakia, K., "Enhancement of optical forces using slow light in a photonic crystal waveguide," Optica 2, 816-821 (2015).

[6] Schein, P., O'Dell, D. and Erickson, D., "Orthogonal nanoparticle size, polydispersity, and stability characterization with near-field optical trapping and light scattering," ACS Photon. 4, 106-113 (2017).

[7] Tanaka, T. and Yamamoto, S., "Optically induced meandering Mie particles driven by the beat of coupled guided modes produced in a multimode waveguide," Jpn. J. Appl. Phys. 41, L260 (2002).

[8] Mandal, S., Serey, X. and Erickson, D., "Nanomanipulation using silicon photonic crystal resonators," Nano Lett. 10, 99-104 (2009).

[9] Renaut, C., Dellinger, J., Cluzel, B., Honegger, T., Peyrade, D., Picard, E., de Fornel, F. and Hadji, E., "Assembly of microparticles by optical trapping with a photonic crystal nanocavity," Appl. Phys. Lett. 100, 101103 (2012).

[10] Renaut, C., Cluzel, B., Dellinger, J., Lalouat, L., Picard, E., Peyrade, D., Hadji, E. and de Fornel, F., "On chip shapeable optical tweezers," Sci. Rep. 3, 2290 (2013).

[11] Lin, S. and Crozier, K. B., "Trapping-assisted sensing of particles and proteins using on-chip optical microcavities," ACS Nano 7, 1725-1730 (2013).

[12] Pin, C., Cluzel, B., Renaut, C., Peyrade, D., Picard, E., Hadji, E. and de Fornel, F., "Optofluidic taming of a colloidal dimer with a silicon nanocavity," Appl. Phys. Lett. 105, 171108 (2014).

[13] Pin, C., Cluzel, B., Renaut, C., Picard, E., Peyrade, D., Hadji, E. and de Fornel, F., "Optofluidic near-field optical microscopy: near-field mapping of a silicon nanocavity using trapped microbeads," ACS Photon. 2, 1410-1415 (2015).

[14] Pin, C., Renaut, C., Tardif, M., Jager, J.-B., Delamadeleine, E., Picard, E., Peyrade, D., Hadji, E., de Fornel, F. and Cluzel, B., "On-chip photonic tweezers for photonics, microfluidics, and biology," Proc. SPIE 10252, 1025212 (2017).

[15] Lin, P. T., Chu, H. Y., Lu, T. W. and Lee, P. T., "Trapping particles using waveguide-coupled gold 
bowtie plasmonic tweezers," Lab. Chip 14, 4647-4652 (2014).

[16] Magno, G., Ecarnot, A., Pin, C., Yam, V., Gogol, P., Mégy, R., Cluzel, B. and Dagens, B., "Integrated plasmonic nanotweezers for nanoparticle manipulation," Opt. Lett. 41, 3679-3682 (2016).

[17] Ecarnot, A., Magno, G., Yam, V. and Dagens, B. "Ultra-efficient nanoparticle trapping by integrated plasmonic dimers," Opt. Lett. 43, 455-458 (2018).

[18] Soltani, M., Lin, J., Forties, R. A., Inman, J. T., Saraf, S. N., Fulbright, R. M., Lipson, M. and Wang, M. D., "Nanophotonic trapping for precise manipulation of biomolecular arrays," Nature Nanotech. 9, 448-452 (2014).

[19] Baker, J. E., Badman, R. P. and Wang, M. D., "Nanophotonic trapping: precise manipulation and measurement of biomolecular arrays," WIREs Nanomed. Nanobiotechnol. 10, e1477 (2018).

[20] Pin, C., Jager, J.-B., Tardif, M., Picard, E., Hadji, E., de Fornel, F. and Cluzel, B., "Optical tweezing using tunable optical lattices along a few-mode silicon waveguide," Lab. Chip 18, 1750-1757 (2018). 


\title{
Real-time monitoring of a reagent release from an optically trapped biodegradable micro-particle for drug delivery
}

\author{
Miyako Iida ${ }^{\mathrm{a}}$, Tatsuya Shoji ${ }^{\mathrm{a}}$, Kayo Fujiwara ${ }^{\mathrm{a}}$ Mitsuhiro Matsumoto $^{\mathrm{a}}$, Yasuyuki Tsuboi*a \\ ${ }^{a}$ Graduate School of Science, Osaka City University, 3-3-138 Sugimoto, \\ Sumiyoshi, Osaka 558-8585, Japan
}

\begin{abstract}
Biodegradable poly(lactic-co-glycolic acid) (PLGA) microparticles are representative drug delivery carriers. Although it is important to understand detailed relationships between a drug release mechanism and their properties such as size and shape, analysis for an individual particle has been much difficult due to thermal fluctuations. In the present study, we performed optical trapping of a single PLGA microparticle for real-time monitoring of a reagent release from the particle by means of fluorescence microspectroscopy. We successfully monitored a fluorescence decay of an optically trapped PLGA microparticle. This decay process should reflect a reagent release from the microparticle. Our real-time monitoring method will be a powerful tool for designing an optimized drug delivery system.

Keywords: biodegradable polymer, optical tweezers, fluorescence microspectroscopy, optical force, microparticle, Poly(lactide-co-glycolide) (PLGA)

*twoboys@sci.osaka-cu.ac.jp ; phone+81-6-6605-3693
\end{abstract}

\section{Introduction}

Poly(lactic-co-glycolic acid) (PLGA) has received great interests in medical and pharmaceutical fields because of its biodegradability, biocompatibility, and negligible toxicity. PLGA is useful for drug delivery systems as carriers. Drug molecules are released from PLGA microparticles via gradual decomposition of PLGA in the presence of water. The drug release mechanism is influenced by many factors of PLGA microparticles such as chemical compositions (ratio of copolymerization), molecular weight, surface morphology, shape, and size. In particular, particle size is a crucial important parameter because the decomposition rate strongly depends on the specific surface area of PLGA microparticles. Therefore, it is necessary to understand the detailed relationship between a drug release rate and their particle size. However, due to thermal fluctuation, it has been difficult to access an individual microparticle for chemical analysis.

For addressing the issue, we focused on optical tweezers ${ }^{[1]}$. Optical tweezers are excellent tools for noncontact and nondestructive manipulation of microparticles at the focal point of a laser beam. In the present study, we performed optical trapping of a single PLGA microparticle for real-time monitoring of a release process of fluorescent molecules from the particle by means of fluorescence microspectroscopy.

\section{Experiments}

PLGA microparticles containing rhodamine $6 \mathrm{G}$ as a model drug molecule were prepared using a double emulsion-solvent evaporation technique ${ }^{[2,3]}$. A rhodamine $6 \mathrm{G}$ aqueous solution was emulsified into an acetonitrile solution containing PLGA. The water-in-oil emulsion was added into an aqueous solution of a surfactant with being stirred to form microparticles. After stirring, the microparticles were finally collected by pulification. For a control experiment, we also synthesized poly $(N$-isopropyl acrylamide) (PNIPAM) microparticles containing rhodamine $6 \mathrm{G}$ by radical polymerization ${ }^{[4]}$. By means of dynamic light scattering, we determined diameters of PLGA and PNIPAM microparticles to be $3.0 \pm 2.0 \mu \mathrm{m}$ and $0.30 \pm 0.20 \mu \mathrm{m}$, respectively. For fluorescence microspectroscopy, these microparticles were dispersed in water.

We used a continuous wave (cw) near-infrared (NIR) laser $(\lambda=1064 \mathrm{~nm})$ for optical trapping and a $\mathrm{cw}$ visible laser $(\lambda=532$ $\mathrm{nm})$ for fluorescence excitation. These laser beams were focused in a sample solution with an objective lens $(\times 100$, N.A. $=1.3)$ to obtain fluorescence spectra of an optically trapped single microparticle. 


\section{Results and Discussion}

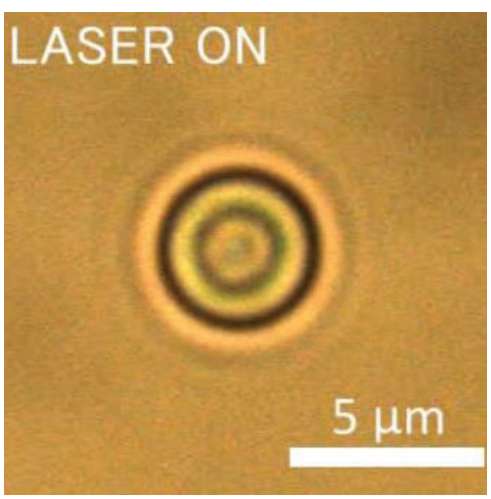

Figure 1. Bright field microscopic image of an optically trapped single PLGA microparticle.

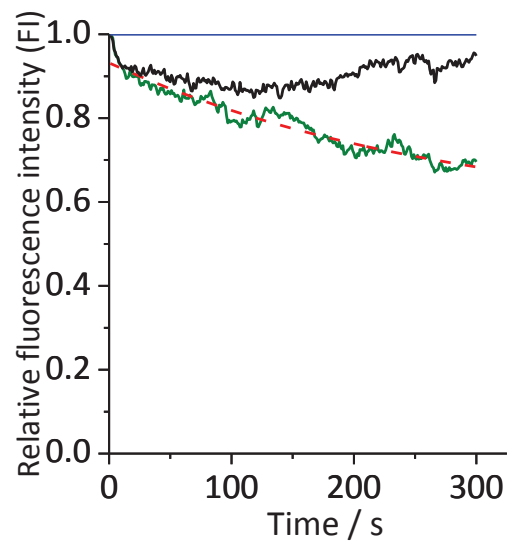

Figure 2. A fluorescence decay profile of an optically trapped single PLGA microparticle (green) together with that of a PNIPAM microparticle (black). Curve fitting with a single exponential function for PLGA microparticle is also shown with a dotted line (red).

Focusing a $1064 \mathrm{~nm}$ laser beam into a sample solution, a single PLGA microparticle with $5.4 \mu \mathrm{m}$ in diameter was trapped due to an optical force at the focal point (Figure 1). We obtained fluorescence spectra of such optically trapped microparticles. Figure 2 shows a temporal profile of fluorescence intensity (FI) from the trapped PLGA microparticle (Figure 1) together with that from a trapped PNIPAM as a control. FI from the PLGA microparticle gradually decayed from 1.0 to 0.7 within 300 seconds. This decay curve was well fitted with a single exponential function. We determined a time constant $\tau$ to be 910 seconds. In the exponential decay, the time constant $\tau$ can be interpreted as the time constant of a release process due to decomposition of PLGA microparticle. Moreover, we found that $\tau$ depended on size of PLGA microparticles. Our technique will be a powerful analytical tool for real-time monitoring of release process from a single PLGA microparticle.

\section{Conclusion}

In this study, we successfully obtained a time constant of reagent release for an optically trapped single PLGA microparticle. The fluorescence decay curve was well fitted single exponential function. The time constant $\tau$ depended on size of a PLGA microparticle. Our optical manipulation technique combined with fluorescence microspectroscopy will be a powerful analytical tool for real-time monitoring of release process from a model particle of drug delivery.

\section{References}

[1]A. Ashkin, J. M. Dziedzic, J. E. Bjorkholm, S. Chu, 'Observation of a single-beam gradient force optical trap for dielectric particles', Opt. Lett., 11, 288-290 (1986)

[2]H. Yan, Y. Hou, P. Niu, K. Zhang, T. Shoji, Y. Tsuboi, L. Zhao, J. Chang, 'Biodegradable PLGA nanoparticles loaded with hydrophobic drugs: confocal Raman Microspectroscopic characterization', J. Mater. Chem. B, 3, 3677-3680 (2015)

[3]J. Yang, R. Zhang, D. Liu, X. Zhou, T. Shoji, Y. Tsuboi, H. Yan, 'Laser trapping/confocal Raman spectroscopic characterization of PLGA-PEG nanoparticles', Soft Matter, 14, 8090-8094 (2018)

[4]Y. Matsumura, K. Iwai, 'Synthesis and thermos-responsive behavior of fluorescent labeled microgel particles based on poly( $N$-isopropylacrylamide) and its related polymers', Polymer, 46, 10027-10034 (2005) 


\title{
Assembling and dynamic ejection of polystyrene particles in CW laser trapping at solution surface
}

\author{
Jia-Syun Lu, ${ }^{1}$ Tetsuhiro Kudo, ${ }^{1}$ Hiroshi Masuhara ${ }^{1,2 *}$ \\ ${ }^{I}$ Department of Applied Chemistry, College of Science, National Chiao Tung Univ. Taiwan, \\ ${ }^{2}$ Center for Emergent Functional Matter Science, National Chiao Tung Univ. Taiwan, \\ Corresponding author:
}

\begin{abstract}
Assembling and ejection dynamics of polystyrene microparticles of 1 micrometer is revealed by applying CW laser trapping at solution surface. Initially a concentric circle-like assembly is rapidly prepared, giving a size much larger than the focus. Assembling evolves, showing some fluctuation in its size, which is accompanied with ejecting of some microparticles. The concentric-like structure is transformed into a hexagonal packing structure, then the ejection is much suppressed. Microparticles are ejected in a linearly aligned manner and its speed is slowed down with time and the distance. Assembling and ejection dynamics is considered in terms of light scattering of the trapping laser.
\end{abstract}

Keywords: optical tweezers, laser trapping, polystyrene particle, solution surface, assembling and ejection

\section{Introduction}

"Optical tweezers" were proposed by Ashkin in 1986 (1) and he was awarded the 2018 Nobel Prize Physics last year. His original idea was presented in the several papers published in early 1970's (2, 3), and "Optical Tweezers" have been widely used in various research areas. We are the first group to demonstrate its high potential in chemistry and extended systematic studies on micro/nano particles, droplets, supramolecules, polymers, micelles, and so on $(4,5)$. All the experiments were carried out in solution, as three-dimensional manipulation of small objects is its characteristic performance as laser-based method. Gradient force is exerted in the focal volume and does not expand so much toward the outside. However, we found novel trapping behavior when we irradiated the trapping laser of $1064 \mathrm{~nm}$ at solution surface. Polystyrene (PS) nanoparticles (NPs) of $200 \mathrm{~nm}$ diameter were trapped not only at the focus but also its outside, forming its single large assembly of a few ten micrometer with a disc-like shape (6). Also we showed that laser trapping of amino acids gives a similar behavior of their clusters and eventually gave a single crystal (7). Here we report a new assembling and ejection dynamics of PS microparticle (MP) of 1 micrometer at solution surface which is obtained by focusing $1064 \mathrm{~nm} \mathrm{CW}$ laser at it solution surface.

\section{Experiment}

Ii is known that high power of $1064 \mathrm{~nm}$ trapping laser is absorbed by $\mathrm{H} 2 \mathrm{O}$, so we used $\mathrm{D} 2 \mathrm{O}$ in order to suppress laser heating effect. The density of PS and $\mathrm{D} 2 \mathrm{O}$ are $1.04 \mathrm{~g} / \mathrm{cm} 3$ and $1.11 \mathrm{~g} / \mathrm{cm} 3$, respectively, thus PS MPs easily float outrambromie Vol. 11141ight are in the same direction. our sample for $15 \mathrm{~min}$ before carrying out the experiment without their aggregation. If the sample solution is kept for longer than $30 \mathrm{~min}$ at room temperature, most of MPs would float near the air/solution surface. To keep the homogeneous condition, we always performed our trapping experiment within $30 \mathrm{~min}$ after taking out the sample from ultrasonication bath. Optical set up for trapping experiment is almost same to that reported previously (6).

\section{Results and Discussion}

MPs are homogeneously dispersed inside the solution before switching on the trapping laser (see Figure $1 \mathrm{~A}$ ). It is worth to notice that some of the MPs look white and dark. The former MPs are located at air/solution interface, namely, on the focal plane, while the dark MPs are inside solution, namely, out of the focal plane. Upon starting the irradiation of the trapping laser, the several MPs were captured rapidly at the focal spot. A few seconds later, the size of the assembly became larger than the focus, but other MPs were still attracted to the assembly. This assembly looked like a concentric circle (Figure $1 \mathrm{C}$ ), but it is not so stable and we often observed the rearrangements of MPs in its assembly.

Sequentially, few MPs are ejected out radially from the assembly in a linear alignment (Figure 1 D), where these ejected MPs moved as a group. When the MPs reached far from the focus, its linear morphology disappeared and MPs were separated as individuals. When we observed the backscattering of $1064 \mathrm{~nm}$ trapping laser from the assembly, the directions of ejection and scattered 


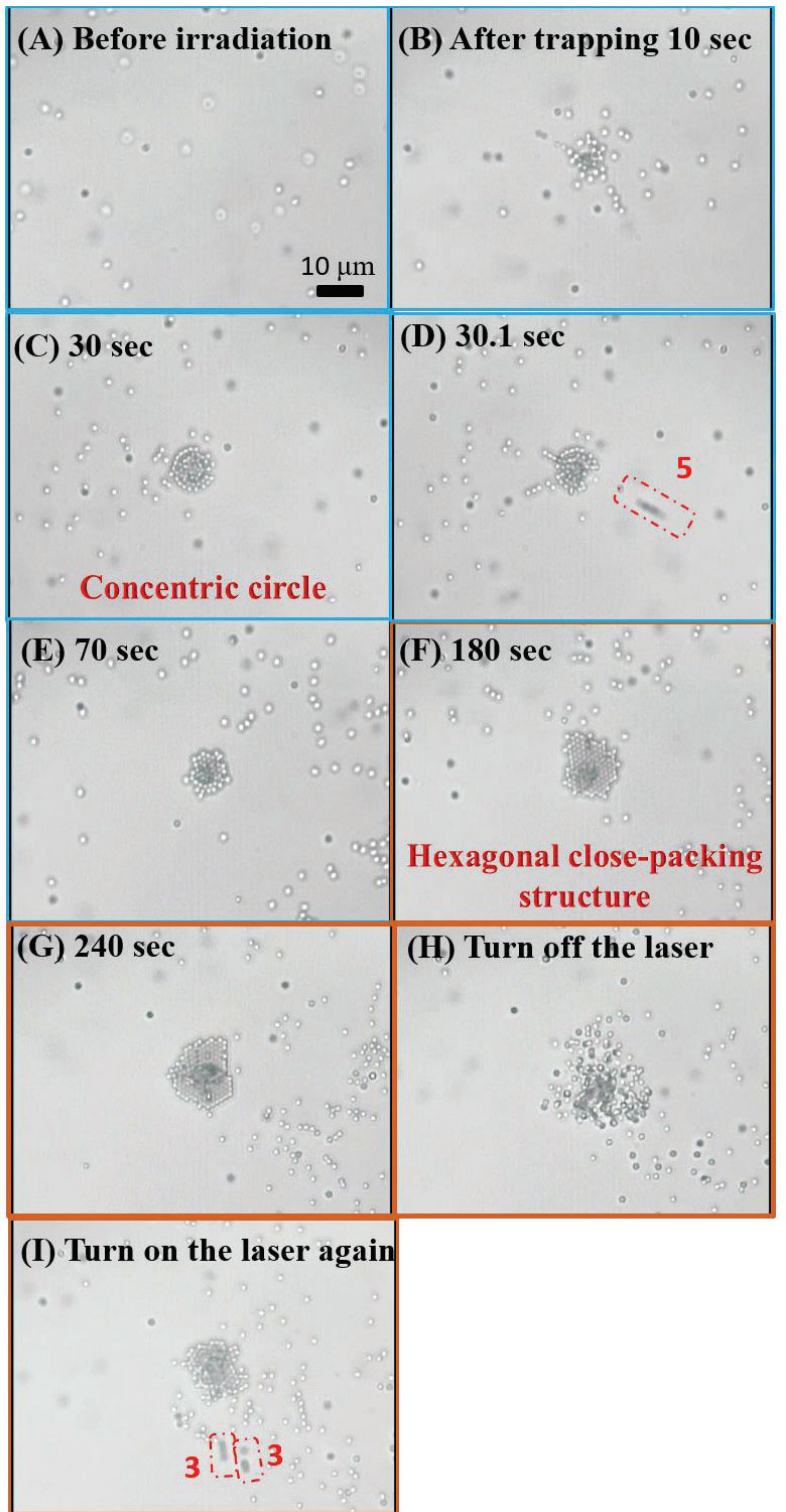

The concentric circle-like assembly sometimes transforms to a hexagonal close-packing structure (HCP) (Figure $1 \mathrm{~F}$ ), then the assembly never returns back to the concentric one again. It is well known that HCP structure is most stable, and

Figure 1. The transmission images of PS MPs at the D2O solution surface. (A) Before irradiation, the MPs are dispersed inside the solution. (B) At $10 \mathrm{sec}$ after turning on the laser, several MPs are trapped at the focus. (C) After $30 \mathrm{sec}$ irradiation, the assembly looks like concentric circle-like one. (D) An ejection of MPs occurs. (E) The concentric circle-like assembly starts to transform to a HCP structure from the edge. (F) Clear HCP structure is prepared. $(\mathrm{G})$ The HCP structure seems still to keep growing. $(\mathrm{H})$ Upon turning off the laser, the HCP assembly dispersed to individual MPs. (I) By turning on laser again, the HCP is recovered.

indeed here the assembly with HCP structure does not undergo rearrangement. It is worth noting that the ejection of MPs is seldom observed from the
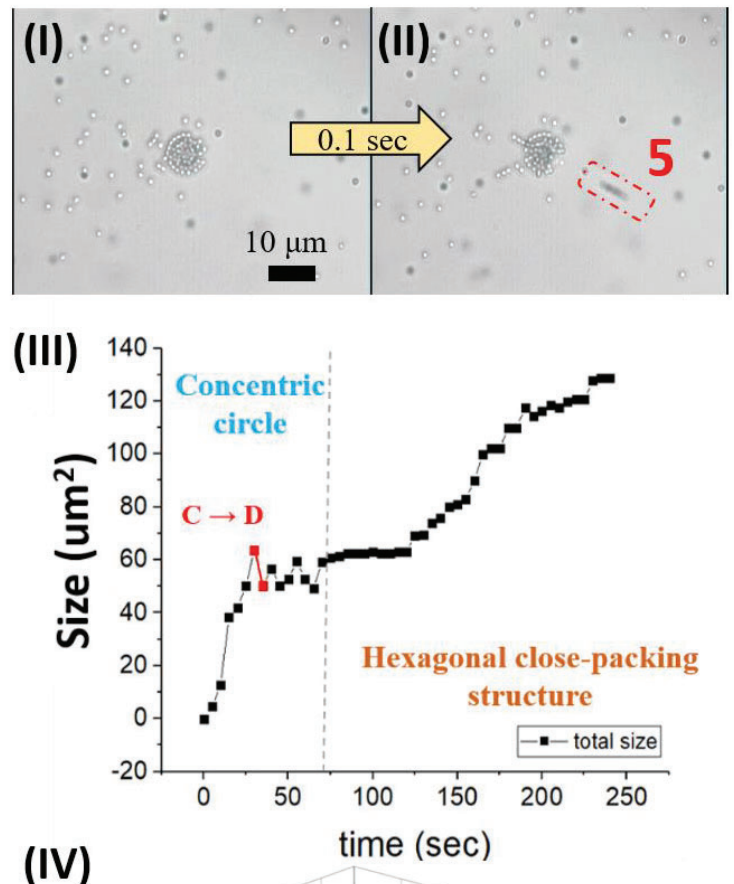

(IV)

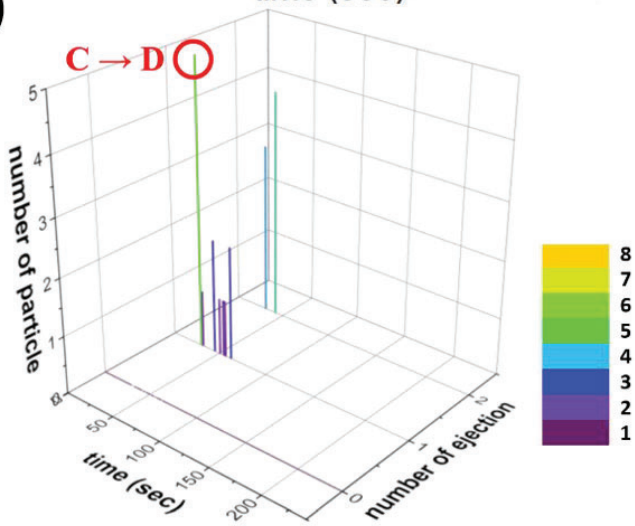

equilibrium state. When we turned off the laser and rapidly turn on it again, the assembly remains with the HCP structure and sometimes shows the ejection.

Figure 2. The transmission images of PS MPs at the D2O solution surface. (I) and (II) show ejectio dynamics of 5 MPs in $0.1 \mathrm{sec}$, corresponding to (C) and (D) in Figure 1. (III) The size of the single assembly of MPs is plotted as a function of the irradiation time. (IV) The X, Y and Z axes represent the irradiation time, the number of ejection taking place during a certain observation period ( 1 and 2 mean individual and simultaneous ejection), how many number of the ejected MP, respectively.

In the assembly size-time diagram of Figure 2 (II), the size of the assembly increases dramatically in the beginning and shows a first saturation with some of the fluctuations. At about $120 \mathrm{sec}$, the size increases again gradually. Here we can correlate (III) and (IV) by "C->D" which corresponds to 5 MPs ejection. The several MPs were ejected out, decreasing the number of MPs in the assembly. Thus, the assembly shrinks after ejections occur, however, some other MPs are still attracted to the 
assembly. The size increase is observed again, giving to the fluctuation in the assembly size.

We measured the velocity of ejection and found the fastest velocity is obtained at the beginning of ejection behavior, then the speed gradually slows down with the time and the distance. We consider that the linear morphology maybe is ascribed to the hydrodynamic force. When the first MP moving at the high speed, several MPs easily follow its direction caused by less resistance. However, the viscosity of solvent reduces the speed of MPs, the force of fluid dynamic getting smaller, then the ejected MPs as a group disperses individually in solution.

\section{Summary}

Until now trapping, assembling, and ejection of PS NPs are reported only for femtosecond laser trapping (8), where a special morphology of ejected NPs like a linear alignment here was not observed. The present work reports for the first time on the ejection behavior in continuous wave $(\mathrm{CW})$ laser trapping experiment. Interestingly they are ejected in a linearly aligned arrangement. These results on expanded assembling and the following ejection can be interpreted in terms of light scattering of the trapping laser of $1064 \mathrm{~nm}$. The important role of solution surface in laser trapping can be discussed by comparing our and previous paper on laser trapping of PS NPs at the glass/solution interface (9).

\section{References}

[1] Ashkin, A., Dziedzic, J. M., Bjorkholm, J. E. and Chu, S., "Observation of a single-beam gradient force optical trap for dielectric particles," Opt. Lett. 11(5) 288-290 (1986).

[2] Ashkin, A., "Acceleration and Trapping of Particles by Radiation Pressure," Phys. Rev. Lett. 24(4), 156-159 (1970).

[3] Ashkin, A., "Atomic-Beam Deflection by Resonance-Radiation Pressure" Phys. Rev. Lett. 25(19), 1321-1324 (1970).

[4] Masuhara, H., De Schryver, F. C., Kitamura, N. and Tamai, N., [Microchemistry: Spectroscopy and Chemistry in Small domain], Elsevier, North-Holland, (1994).

[5] Masuhara, H. and De Schryver, F. C., [Organic Mesoscopic Chemistry], Blackwell Science, New Jersey, (1999).

[6] Wang, S.-F., Yuyama, K., Sugiyama, T. and Masuhara, H., "Reflection Microspectroscopic Study of Laser Trapping Assembling of Polystyrene Nanoparticles at Air/Solution Interface," J. Phys. Chem. C, 120(29) 15578-15585 (2016).

[7] Sugiyama, T., Yuyama, K. and Masuhara, H., "Laser Trapping Chemistry: From Polymer Assembly to Amino Acid Crystallization," Accounts Chem. Res., 45, 1946-1954 (2012).

[8] Usman, A., Chiang, W.-Y. and Masuhara, H., "Optical Trapping and PolarizationControlled Scattering of Dielectric Spherical Nanoparticles by Femtosecond Laser Pulses," J. Photochem. Photobiol. A Chem. 234, 84-90 (2012).

[9] Kudo, T., Wang, S.-F., Yuyama, K. and Masuhara, H., "Optical Trapping-Formed Colloidal Assembly with Horns Extended to the Outside of a Focus through Light Propagation," Nano Lett. 16(5), 3058-3062 (2016). 


\title{
Investigation of plasmonic lasing by using focused radially polarized beam
}

\author{
Kyoko Kitamura*a, Hiroya Obuchi ${ }^{\mathrm{a}}$ \\ ${ }^{a}$ Electronics, Kyoto Institute of Technology, Gosho-Kaido-cho, Sakyo-ku, Kyoto, 606-8585, Japan
}

\begin{abstract}
A focused radially polarized beam effectively induces the plasmon modes of metals which placed near the focus. As part of an investigation into the interactions between focused radially polarized beams and materials, we here numerically study the feasibility of plasmonic lasing by using the focusing properties. By introducing a metal-gain-metal nanostructure, the peculiar electric fields have been obtained that suggests lasing assisted by plasmonic effects.
\end{abstract}

Keywords: radially polarized beam, focused fields, metal nano-structure, FDTD simulation, plasmonic lasing

\section{Introduction}

Radially polarized beams, which possess electric field vectors arranged like the spokes of wheels pointing out from the center in the beam cross-section, have attracted much interest and are expected to be used in many applications in optics, such as improved optical data storage and optical microscopy, because of their peculiar focusing properties. When it is focused with high numerical aperture lens, it generates strong longitudinal fields on the beam axis which can lead the achievements to create a small focal spot size $(<0.4 \lambda)$ with a long depth of focus $(>10 \lambda)$, while it generates zero Poynting energy on the beam axis [1] [2]. We have been reported that such very promising radially polarized beams can be generated by photonic-crystal lasers and we have been demonstrated its focusing properties [3]. However, the practical applications of radially polarized beam have not been shown yet, even though the particular focusing properties on free space had been shown. One of the reasons is surely that interactions between such beams and materials have not been investigated much, because the focusing properties of the beam are very distinct from those of other beams. Previously, we have used a three- dimensional (3D) finite-difference time-domain (FDTD) calculation in order to exploit these focusing properties, in the specific case where a sub-wavelength sized gold metal cubes are arranged on the beam axis through the propagation direction. Then, we showed the electric field enhancement in the tiny spaces between gold cubes arranged on the beam axis along the propagating direction [4]. In present work, we introduced a gain material to the strongly enhanced electric field, and then, investigated of the feasibility of plasmonic lasing by using the field.

\section{Simulation model}

Figure 1 shows the calculation model. We simulated the focusing electro-magnetic fields of a radially polarized beam by exciting the fields under the 3D-FDTD space. The beam is focused with $N A=0.9$ and the excitation plane is at $z=-4 \mu \mathrm{m}$. To introduce gain material and metal nano-structure, we used four-level system and drude model. The center of gain material is located at focal point $(\mathrm{x}=\mathrm{y}=\mathrm{z}=0)$ and the cylindrical metals are sandwiching the material.

*kyoko@kit.ac.jp; phone 8175 724-7405 


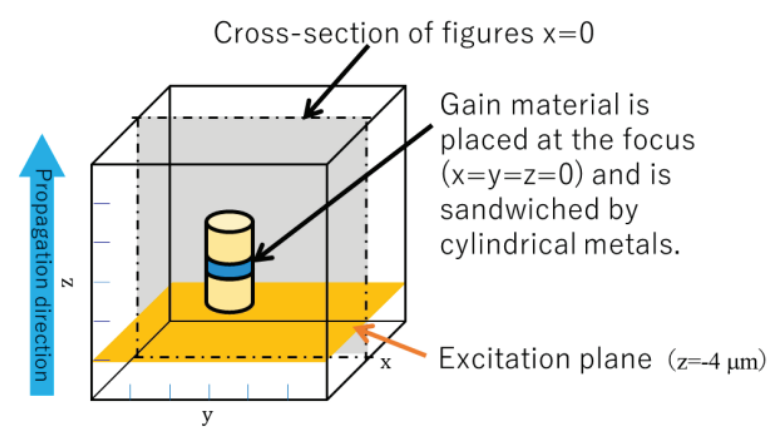

Figure 1. Calculation model of three-dimensional FDTD analysis.

\section{Spectrum and electric field intensity profiles}

Firstly, as part of an investigation, we calculated the case when the gain material has carrier density $N=2 \times 10^{20}$ $\mathrm{cm}^{-3}$, thickness is $65 \mathrm{~nm}$ and when the cylindrical metals are consisted by gold and both of them have $200 \mathrm{~nm}$ diameter and $100 \mathrm{~nm}$ length. Figure 2 show the spectrums when we excited radially polarized beam with the absorption wavelength of the gain material $\left(\lambda_{\mathrm{abs}}=690 \mathrm{~nm}\right)$. The spectrum after pass the materials shows clear red-sift and the spectrum peak wavelength is matched with the emission wavelength of the gain material $\left(\lambda_{\mathrm{emi}}=715 \mathrm{~nm}\right)$.

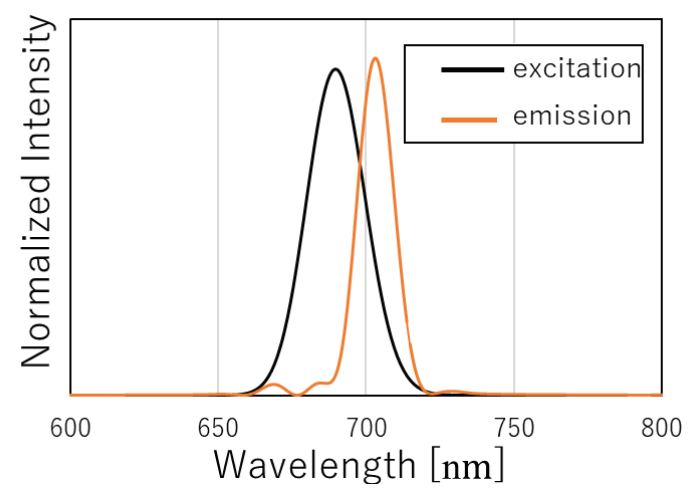

Figure 2. Spectrums when excited the absorption wavelength

Figure 3 show the time-dependent electric field intensity profiles the case when excited with $\lambda_{\mathrm{exc}}=500 \mathrm{~nm}$ (upper) and with $\lambda_{\text {exc }}=690 \mathrm{~nm}$ (lower). The upper cases show the maximum intensities are localized at the edge of gain material, due to the high career density. Whereas, the lower cases show the electric fields flowed into the gain material. When we monitored the time dependent energy, the lower case showed higher Q factor compared with the upper case. From this points, we conclude that the beam coupled with the gain material and emit another wavelength. In addition, compared with when cylindrical metals were perfect conductor or silver, the effects were different. From these calculations, the emission is assisted by the electric enhancement of plasmon mode, which induced by focused radially polarized beam.

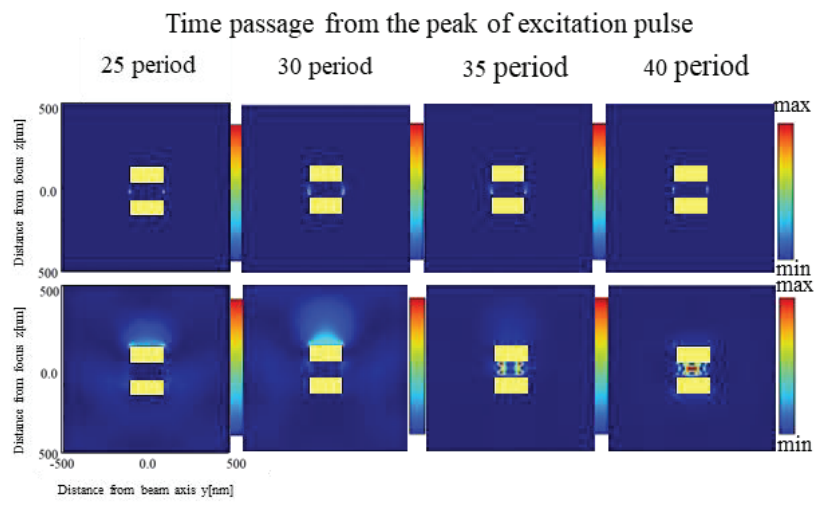

Figure 3. Time-dependent electric field intensity profiles. (upper) $\lambda \mathrm{exc}=500 \mathrm{~nm}$, (lower) $\lambda \mathrm{exc}=690 \mathrm{~nm}$.

\section{Summary}

We have analyzed the feasibility of plasmonic lasing by using focused radially polarized beam and metal-gainmetal nano-structure. The further details will be reported at the conference.

\section{References}

[1] K. Kitamura, K. Sakai, and S. Noda, Opt. Express, vol. 18, pp.4518- 4525 (2010).

[2] K. Kitamura, K. Sakai and S. Noda, Opt. Express, vol.19, pp. 13750- 13756 (2011).

[3] K. Kitamura, M. Nishimoto, K. Sakai and S. Noda, Appl. Phys. Lett., Vol. 101, pp. 221103- 221106 (2012).

[4] K. Kitamura, T. T. Xu and S. Noda, Opt. Express, 21, 32217 (2013). 


\title{
Vortex mode emission properties of vertical cavity surface emitting laser
}

\section{with external optical feedback}

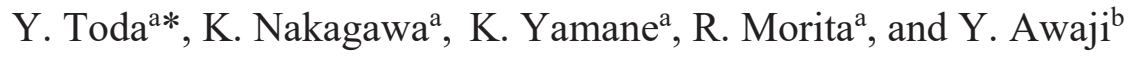 \\ aDepartment of Applied Physics, Hokkaido University, N13W8 Kita-ku, Sapporo, 060-8628 Japan \\ ${ }^{\mathrm{b}}$ National Institute of Information and Communications Technologies, 4-2-1 Nukui-Kitamachi, \\ Koganei, Tokyo, 184-8795 Japan
}

\begin{abstract}
Based on our previous work, in which we demonstrated single orbital angular momentum (OAM) mode emissions from a vertical cavity surface emitting laser (VCSEL) with an external optical feedback, the conditions of the vortex mode emission are investigated in terms of feedback efficiency and polarization. There are two orthogonal polarization configurations for efficient single OAM mode emission, one of which corresponds to the dominant polarization in freerunning VCSEL. Nearly identical emission properties of the two polarizations indicate an independence between the spin and OAM. On the other hand, the stability of the OAM mode emission, which is characterized by the dependencies of the feedback efficiency and injection current, is changed between the two polarizations, suggesting different contributions of the broken rotational symmetry to the spin and OAM.
\end{abstract}

Keywords: Laguerre-Gaussian mode, orbital angular momentum, optical feedback, VCSEL, optical vortex

*toda@eng.hokudai.ac.jp; phone +81 11 706-6627

\section{Introduction}

Vortex beams with phase, polarization and/or other foliation singularities have attracted growing interest in various fields because of their unique physical properties and potential applications. One of the intensively studied applications is the spatial mode division multiplexing (MDM) in optical communications, in which a welldefined series of orbital angular momentum (OAM) modes (or Laguerre-Gaussian (LG) modes with arbitrary radial indices) is expected to be a promising candidate for the multiplexing [1]. In the application, the semiconductor laser for generating controllable OAM mode is a key device, and such OAM mode emissions have been realized using modified vertical cavity surface emitting lasers (VCSELs) [2-5]. There are also more fundamental interests to study the vortex modes in VCSELs as they show collective and spontaneous formation of multiple vortices [6]. In our previous work, single OAM mode emissions were demonstrated from a VCSEL with external optical feedback provided by a spatial light modulator (SLM) [7]. Here the OAM mode was controllable by changing the computer-generated hologram $(\mathrm{CGH})$ on the SLM. In this work, by taking advantage of the controllability of the CGH, we perform a concise OAM mode analysis of the VCSEL in terms of feedback efficiency and polarization. We find that there are two orthogonal polarization configurations responsible for the efficient single OAM mode emission, one of which corresponds to the dominant polarization in free-running VCSEL. The two polarizations show nearly identical OAM emission properties, indicating an independence of the spin and OAM. On the other hand, the stability of the OAM mode emission, which is characterized by the dependencies of the feedback efficiency and injection current, is changed between the two polarizations. The result implies that the rotational symmetry breaking is sensitive to the spin but not to the OAM.

\section{Experiments}

We used a commercially available VCSEL with a large aperture size $(\sim 10 \mu \mathrm{m}$ in diameter) responsible for generating higher-order transverse modes. The active layer consists of $3 \mathrm{Al}_{0.12} \mathrm{Ga}_{0.88} \mathrm{As} / \mathrm{Al}_{0.30} \mathrm{Ga}_{0.70} \mathrm{As}$ quantum wells between distributed Bragg reflectors for $\lambda$-cavity, in which a single longitudinal mode at a wavelength of $\sim 780$ 
$\mathrm{nm}$ is generated. The output from the VCSEL was collimated and introduced onto a SLM. We selected the polarization of the optical feedback by rotating a halfwaveplate with respect to the polarization axis of the SLM. The 1st-order diffraction beam by CGH on the SLM was used as the return light and fed back into the VCSEL while the 0th order diffraction beam was used to evaluate the OAM mode of the output beam. We employed a forked CGH pattern optimized for the single OAM mode emission with $\ell=-1$ (LG-mode). The details of the optimization were described in Ref. [7]. The OAM mode analysis of the output beam was carried out by an OAM-resolved technique [8]. In the free-running VCSEL, a fundamental Gaussian mode ( $\ell=0$; G-mode) with a linear polarization (defined as H-polarization) develops above the threshold current $\left(J_{\mathrm{th}} \sim 1.7 \mathrm{~mA}\right)$. The linear polarization emission indicates the rotational broken symmetry of the VCSEL. By increasing the injection current, additional two OAM modes ( $\ell= \pm 1$; HG-mode) appear at around $J \sim 1.9 \mathrm{~mA}$.

\section{Results and Discussions}

Figure 1 shows typical OAM spectra at different diffraction efficiencies $(\eta)$ and at injection currents $(J)$. Here the H-polarization was selected for the optical feedback. To clarify the OAM mode emission property, the color density plots of the $\ell=-1,0,+1$ modes are displayed as functions of $\eta$ and $J$ in Fig. 2 (a)-(c), respectively. Since each spectrum consists of some of

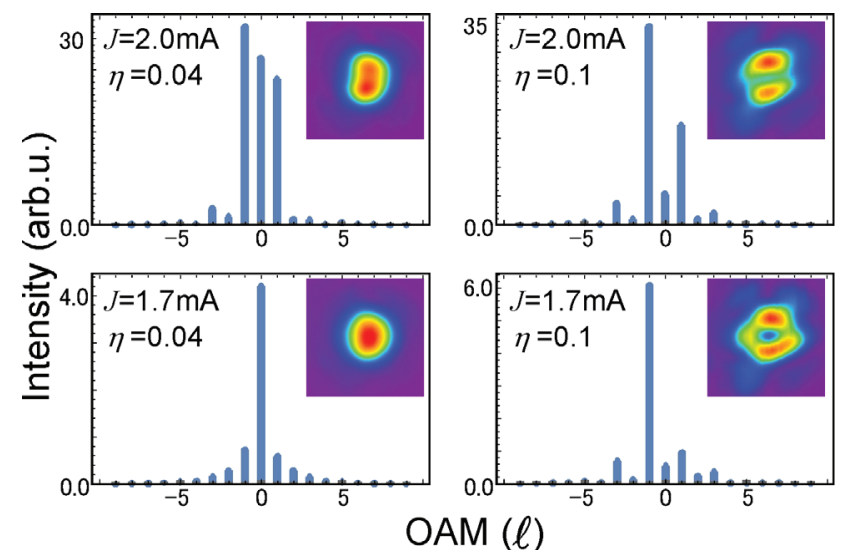

Figure 1. OAM mode spectra of VCSEL with optical feedback at typical injection currents and diffraction efficiencies. Insets show the corresponding output beam profiles.

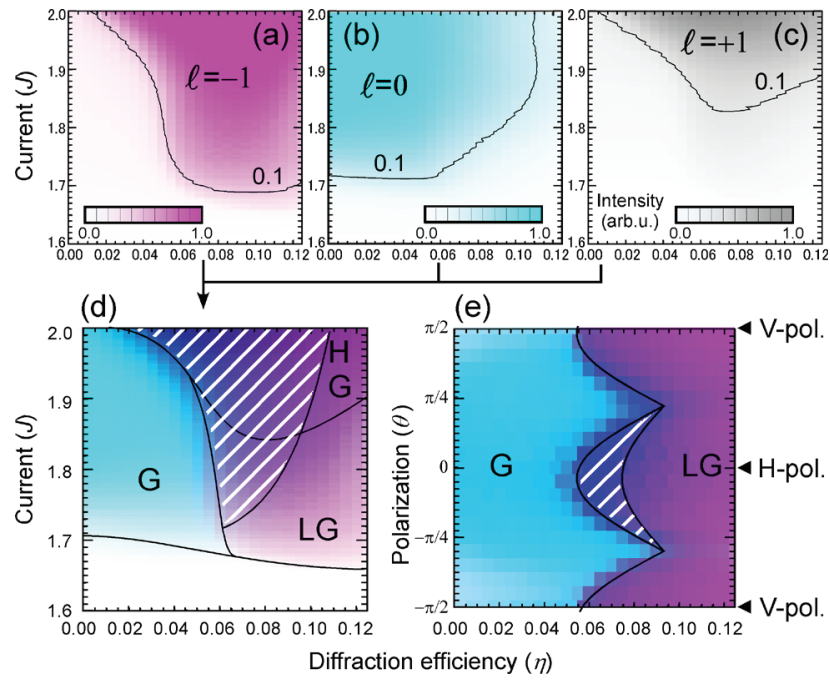

Figure 2. Color density plots of OAM mode intensity for $\ell=$ (a) -1 , (b) $0,(c)+1$ as functions of diffraction efficiency $(\eta)$ and injection current $(J)$. OAM mode properties (d) as functions of $\eta$ and $J$, and (e) as functions of $\eta$ and polarization $(\theta)$.

these 3 modes (Fig. 1), the combination of the plots shows a phase diagram of the spatial mode emission (Fig. 2 (d)). The G-mode is efficient for the weak optical feedback, corresponding to the unlocked region by the optical feedback. The LG mode emission is achieved at above $\eta \sim 6 \%$ and at below $J \sim 1.9 \mathrm{~mA}$. The reduction of the threshold current induced by the optical feedback is observed in the diagram. The efficient optical feedback is also ensured by the frequency shift of the longitudinal mode owing to the resonator Gouy phase shift. The LGmode region shrinks with increasing injection current, and alternatively HG-like degenerate OAM-mode emission becomes dominant. There also exists a crossover between the G- and LG- or HG-mode emissions, in which the output mode is unstable for sharing the modal gain between these two different-order modes with different resonance conditions.

Figure 2 (e) shows a result of the spatial mode property as functions of $\eta$ and polarization $(\theta)$, where $\theta=0$ corresponds to the H-polarization. Here the injection current is fixed at $J=1.72 \mathrm{~mA}$. When $\eta=0$, the G-mode shows a peak at $\theta=0$, which corresponds to the dominant H-polarization in free-running VCSEL. In contrast, LGmode is efficient at $|\theta|=0(\mathrm{H}-)$ and $\pi / 2$ (V-polarizations). The results observed at these two polarizations show 
nearly identical OAM emission properties near the threshold current, indicating the independence of the spin and OAM. On the other hand, the unstable crossover region (hatched area) is changed between the two polarizations. There is no crossover at $|\theta|=\pi / 2$ mainly due to the small gain of the $\mathrm{V}$-polarization for the G-mode. The result implies that the rotational symmetry breaking in the VCSEL affects the spin polarization but is not critical to the OAM.

\section{Conclusion}

We investigated the conditions of the vortex mode emission in terms of feedback efficiency and polarization. There are two orthogonal polarization configurations for the efficient single OAM mode emission, which exhibit nearly identical emission properties. On the other hand, the unstable region arising from the competition between different-order modes is changed between the two polarizations, suggesting different contributions of the broken rotational symmetry to the spin and OAM.

This work is supported by JSPS KAKENHI $16 \mathrm{H} 03876$.

\section{References}

[1] Willner, A. E., Wang, J., and Huang, H., "A different angle on light communications," Science, 337, 655-656 (2012).

[2] Iwahashi, S., Kurosaka, Y., Sakai, K., Kitamura, K., Takayama, N., and Noda, S. "Higher-order vector beams produced by photonic-crystal lasers", Opt. express, 19, 11963-11968 (2011).

[3] Seghilani, MS, Myara, M., Sellahi, M., Legratiet, L., Sagnes, I., Beaudoin, G., Lalanne, P., and Garnache, A., Sci. rep. 6, 38156 (2016).

[4] Huanlu Li, David B. Phillips, Xuyang Wang, YingLung Daniel Ho, Lifeng Chen, Xiaoqi Zhou, Jiangbo Zhu, Siyuan Yu, and Xinlun Cai, "Orbital angular momentum vertical-cavity surface-emitting lasers," Optica 2, 547-552 (2015).

[5] Mochizuki, S., Gu, X., Tanabe, K., Matsutani, A., Ahmed, M., Bakry, A., and Koyama, F., "Generation of vortex beam using Bragg reflector waveguide," Appl. Phys. Express, 7, 022502 (2014).

[6] Jimenez-Garcia, J., Rodriguez, P., Guillet, T., and Ackemann, T., "Spontaneous formation of vector vortex beams in vertical-cavity surface-emitting lasers with feedback”, Phys. Rev. Lett., 119, 113902 (2017).

[7] Toda, Y., Moriya, A., Yamane, K., Morita, R., Shigematsu, K., and Awaji, Y. "Single orbital angular mode emission from externally feed-backed vertical cavity surface emitting laser", Appl. Phys. Lett., 111, 101102 (2017).

[8] Yamane, K., Yang, Z., Toda, Y., and Morita, R. "Frequency-resolved measurement of the orbital angular momentum spectrum of femtosecond ultra-broadband optical-vortex pulses based on field reconstruction," New J. Phys., 16, 053020 (2014). 


\title{
Spatial mode generation and detection by means of the sum-frequency upconversion process
}

\author{
Adam Vallés ${ }^{1,2}$, Bereneice Sephton ${ }^{3}$, Fabian Steinlechner ${ }^{4,5}$, Thomas Konrad $^{6,7}$, \\ Juan P. Torres ${ }^{8,9}$, Filippus S. Roux ${ }^{3,10}$, and Andrew Forbes ${ }^{3}$ \\ ${ }^{1}$ Graduate School of Advanced Integration Science, Chiba University, 1-33 Inage-ku, Chiba, 263-8522, Japan \\ ${ }^{2}$ Molecular Chirality Research Center, Chiba University, 1-33 Inage-ku, Chiba, 263-8522, Japan \\ ${ }^{3}$ School of Physics, University of the Witwatersrand, Private Bag 3, Wits 2050, South Africa \\ ${ }^{4}$ Fraunhofer Institute for Applied Optics and Precision Engineering, Albert-Einstein-Str. 7, 07745 Jena, Germany \\ ${ }^{5}$ Friedrich Schiller University Jena, Abbe Center of Photonics, Albert-Einstein-Str. 6, 07745 Jena, Germany \\ ${ }^{6}$ School of Physics, University of KwaZulu-Natal, Durban, South Africa \\ ${ }^{7}$ National Institute of Theoretical Physics, UKZN Node, Durban, South Africa \\ ${ }^{8}$ ICFO-Institut de Ciencies Fotoniques, Mediterranean Technology Park, 08860 Castelldefels, Barcelona, Spain \\ ${ }^{9}$ Department of Signal Theory and Communications, Universitat Politecnica de Catalunya, Campus Nord D3, 08034 Barcelona, Spain \\ ${ }^{10}$ National Metrology Institute of South Africa, Meiring Naudé Road, Brummeria, Pretoria 0040, South Africa \\ adam.valles@chiba-u.jp
}

The efficient creation and detection of spatial modes of light has become topical of late, driven by the need to increase photon bit-rates in classical and quantum communications. Here we put forward a new spatial mode detection technique based on the nonlinear optical process of sumfrequency generation, also showing that the method can be used to transfer an image from the infrared band to the visible, which implies the efficient conversion of many spatial modes.

There has been a great development in methods to create and detect optical spatial modes, in particular complex structured light fields [1], fuelled for example by the desire for higher bit-rates in classical and quantum communication $[2,3]$. Such mode creation and detection processes are traditionally achieved with tools based on linear optics, i.e., refractive or diffractive field mapping by allowing lossless phase and amplitude modulation of an input beam, or by single step modulation of the phase and/or amplitude. However, mode creation has also been demonstrated with nonlinear optics. With spontaneous parametric down-conversion (SPDC), the method of choice to introduce the concept, one can create photon pairs entangled in their spatial degree of freedom [4]. It is instructive to outline how SPDC generates anticorrelated modes, as schematically depicted in Fig. 1(a) for a Gaussian pump and orbital angular momentum (OAM) modes as an example. When a Gaussian beam pumps the SPDC process, it mediates the generation of paired entangled down-converted photons, signal $(A)$ and idler $(B)$, embedded into spatial modes satisfying the opposite topological charge $\ell_{\mathrm{A}}=-\ell_{\mathrm{B}}$, as shown in the simulated spiral bandwidth of Fig. 1(b).

In this work we make use of the dual process sum-frequency generation (SFG) for the detection and selection of spatial modes, contrasting the similarity with the anticorrelated relation obtained with its analogous nonlinear process, the SPDC.

(a)

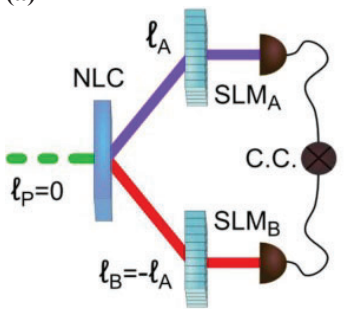

(b)

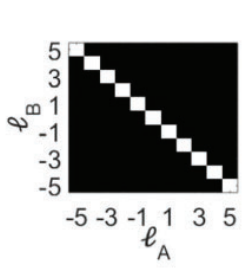

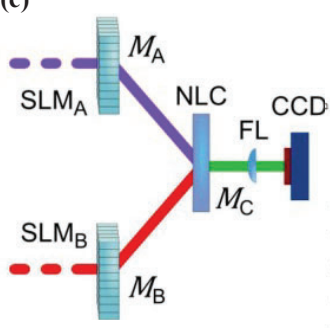

(d)

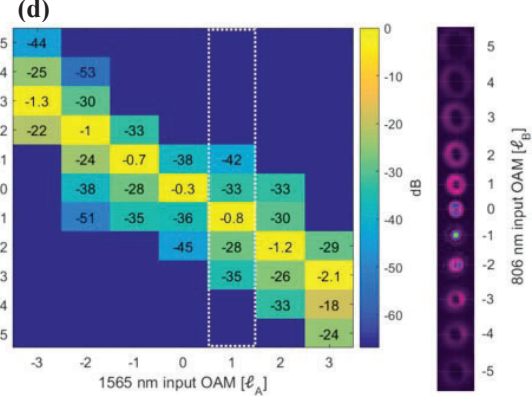

FIG. 1 (a) Traditional quantum experiment where a Gaussian mode pumps a nonlinear crystal (NLC) mediating the generation of two entangled photons with opposite OAM values. (b) Simulated modal spiral spectrum of the down-converted photon pairs. (c) Setup used to demonstrate the spatial mode detection by frequency upconversion. (d) Experimental cross talk results after performing a modal decomposition varying the helical charge of the input OAM mode for $806 \mathrm{~nm}$ ranging within $\ell_{\mathrm{B}}=[3,-3]$, when setting a particular input OAM mode for $1565 \mathrm{~nm}$ within the range $\ell_{\mathrm{A}}=[3,-3]$. The inset of the rightmost is the modal decomposition for the input state $\ell_{\mathrm{A}}=1$, obtaining a maximum power in the centre of coordinates when $\ell_{\mathrm{B}}=-1$.

In the frequency upconversion process, schematically depicted in Fig. 1(c), two incoming signals $\left(M_{\mathrm{A}}\right.$ and $\left.M_{\mathrm{B}}\right)$ are engineered to be in specific states. The upconverted signal is detected in the far field, so that there is a nonzero signal only when the phases are conjugate. In this scheme the nonlinear crystal (NLC) is the detector rather than the generator. Our results shown in Fig. 1(d) confirm the concept of spatial mode detection by upconversion using intense beams carrying orbital angular momentum $\left(\ell_{\mathrm{A}}\right.$ and $\left.\ell_{\mathrm{B}}\right)$ as example. To quantify the quality of this spatial 
mode detection technique, a cross-talk matrix measurement was performed where each column is normalized with all terms adding to unity and finding a $-30 \mathrm{~dB}$ cross-talk on average, thus demonstrating how well we can distinguish between the desired mode and the rest of the orthogonal modes from the same basis [5].
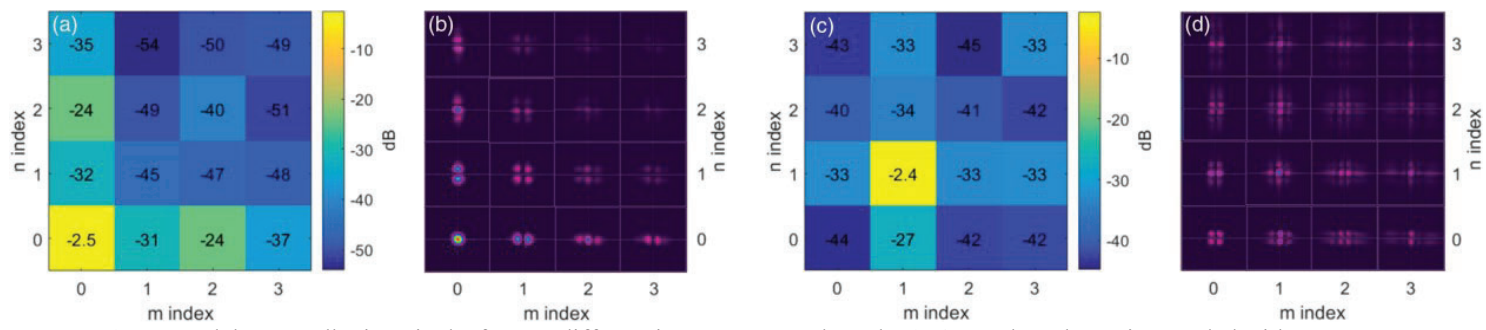

FIG. $2 \mathrm{HG}$ modal cross talk given in $\mathrm{dB}$ for two different input $\mathrm{HG}_{m, n}$ modes. The 1565 -nm laser beam is encoded with (a) $\mathrm{HG}_{0,0}$ and (c) $\mathrm{HG}_{1,1}$, performing a modal decomposition with the 806-nm input laser beam such as $\mathrm{HG}_{n, m}$, with the $m$ and $n$ indexes ranging within $[0,3]$. We show the captures obtained in the right indicating the weightings of the detected modes in the left, by extracting the centre pixel value in the intensity plots for (b) $\mathrm{HG}_{0,0}$ and (d) $\mathrm{HG}_{1,1}$. Dotted crosshairs indicate the central coordinate.

Figures 2(a) and (c) show the measurement outcome when a modal decomposition was performed on a 1565-nm laser beam with a particular encoded $\mathrm{HG}_{m, n}$ input mode, with indexes (a) $m=n=0$ and (c) $m=n=1$. The laser beam centred at $806 \mathrm{~nm}$ was used to perform a tomographic scan by varying the $m$ and $n$ indexes within the range of $[0,3]$, while measuring the resulting signal after the upconversion process. Each cross-talk modal decomposition plot in Figs. 2(a) and (c) has been normalized with all its terms adding to unity, prior to being expressed in dB. A maximum on-axis intensity is obtained only when the $806-\mathrm{nm}$ intense beam is encoded with the modes $\mathrm{HG}_{0,0}$ and $\mathrm{HG}_{1,1}$, as can be seen in Figs. 2(b) and (d), respectively. Here, since $M_{\mathrm{A}}=\mathrm{HG}_{0,0}\left(\right.$ or $\left.\mathrm{HG}_{1,1}\right)$, all on-axis intensities for $M_{\mathrm{B}}$ are zero except for the case when $M_{\mathrm{A}}=M_{\mathrm{B}}^{*}=\mathrm{HG}_{0,0}\left(\right.$ or $\left.\mathrm{HG}_{1,1}\right)$. The average efficiency of the process was $\approx 10^{-4}$, and further study is needed considering whether the efficiency is mode dependent.
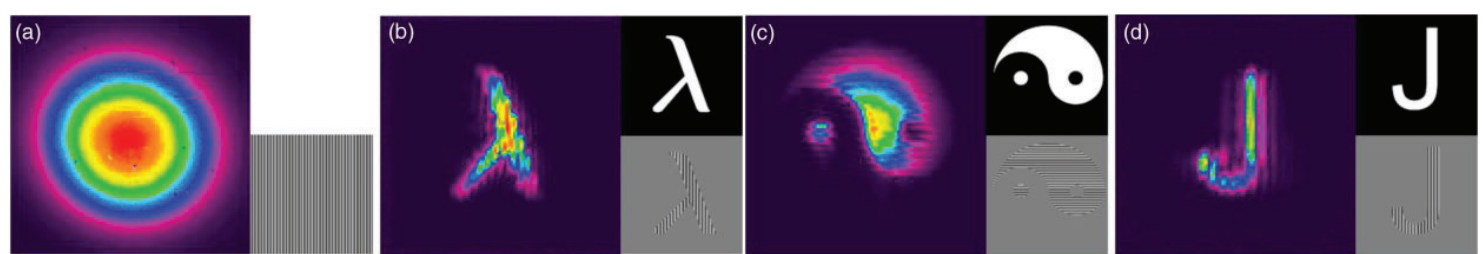

FIG. 3 Upconverted images measured as $M_{\mathrm{C}}$ with $M_{\mathrm{A}}(1565 \mathrm{~nm})$ as a Gaussian, shown in (a), and $M_{\mathrm{B}}(806 \mathrm{~nm})$ set to be (b) lambda, (c) yin yang, and (d) jay symbols. The insets show the applied amplitude modulated mask and the corresponding hologram to imprint the image onto mode $M_{\mathrm{B}}$. The emerging fringes are the Moiré pattern due to the finite resolution of the SLM screen.

The extrapolation to more complex mode structures is limited only by the transverse resolution that can be obtained from the upconversion process. In order to demonstrate the complexity of possible modes, we encoded various complex images in arm $B$, while keeping the other laser beam in arm $A$ as a Gaussian mode. Note that even if we swap the modes in $A$ and $B$, the resulting outcome would be the same. In Fig. 3 we show the resulting images after the upconversion transfer, confirming the dynamic range of the technique, that is, the technique works even with high resolution spatial modes, being able to even transfer the emerging Moiré pattern due to the finite resolution of the spatial light modulator (SLM) screen. To test whether we were measuring only the upconverted $532 \mathrm{~nm}$ signal in all our results and not the residual $806 \mathrm{~nm}$ input (also detectable by the CCD camera), we changed the polarization of the $1565 \mathrm{~nm}$ beam to horizontal, checking that the upconverted signal disappeared completely.

Even though we have demonstrated the technique with high intensity signals, the results are immediately applicable to single photon states too by employing single photon counting detectors and single mode fibres. This experimental configuration paves the way for spatial mode detection of infrared band signals but measuring them in the visible with cheaper and faster silicon-based detector technology.

[1] H. Rubinsztein-Dunlop, et al., "Roadmap on structured light," J. Opt. 19, 013001 (2017).

[2] A. E. Willner, et al., "Optical communications using orbital angular momentum beams," Adv. Opt. Photon. 7, 66 (2015).

[3] B. Ndagano, I. Nape, M. Cox, C. Rosales-Guzman, and A. Forbes, "Creation and detection of vector vortex modes for classical and quantum communications," J. Lightwave Technol. 36, 292 (2018).

[4] S. P. Walborn, C. H. Monken, S. Pádua, and P. H. Souto-Ribeiro, "Spatial correlations in parametric down-conversion," Phys. Rep. 495, 87-139 (2004).

[5] B. Sephton, A. Vallés, F. Steinlechner, T. Konrad, J. P. Torres, F. S. Roux, A. Forbes "Spatial mode detection by frequency upconversion," Opt. Lett. 44, 586 (2019). 


\title{
Q-Switched All-Fiber Laser based on Graphene Oxide in the C- and L-Bands Using Electrical Deposition Method and Pulse Laser Drilling
}

\author{
Byungjoo Kim, Seongjin Hong, and Kyunghwan Oh* \\ Institute of Physics and Applied Physics, Yonsei University, Seoul 03722, South Korea
}

\begin{abstract}
We experimentally deposited a graphene oxide(GO) onto an end face of the optical fiber with the new method by applying electrical arc and pulse laser drilling to GO using commercial fusion splicer and laser ring cavity. We demonstrate a Q-switched Er-doped fiber laser (EDFL) using GO thin film deposited by proposed method as a saturable absorber (SA). We successfully generated stable passively Q-switched EDFL in both C- and L-band using different GO SA without adjusting the optical gain. Detailed GO film deposition method at end face of the optical fiber using electrical arc and pulse laser drilling are discussed. Our proposed deposition method with electrical deposition method and pulse laser drilling might be applied to make a SA with other similar two-dimensional materials.
\end{abstract}

Keywords: Graphene oxide, Q-switching fiber laser, Deposition, Laser drilling, Saturable absorber, C-band, L-band

\section{Introduction}

In recent decades, researchers have devoted great effort into the development of ultrafast pulse lasers based on different materials. Currently, graphene has emerged as promising two-dimensional (2D) material in the fields of material science and photonics. Also graphene oxide has attracted much attention as SA material [1-4] and various fabrication method of SA device using the spray deposition, optical deposition, and chemical vapor deposition on to the end face of optical fiber $[5,6]$. In this work, we demonstrated new deposition method for the Qswitched EDFL with the GO thin films as a SA in C-and L-band regimes. The GO thin film is fabricated by an electrical deposition method by applying the electrical arc and the pulse laser drilling in laser ring cavity. Stable Qswitched pulse are operated at both the center wavelength of $1568.1 \mathrm{~nm}$ in C-band, and $1600.5 \mathrm{~nm}$ in L-band, respectively. The C- and L-band are commonly used in communication systems because the wavelength division multiplexing (WDM) systems that combine the C-band and L-band can increase the transmission capacity $[7,8]$.

\section{Method}

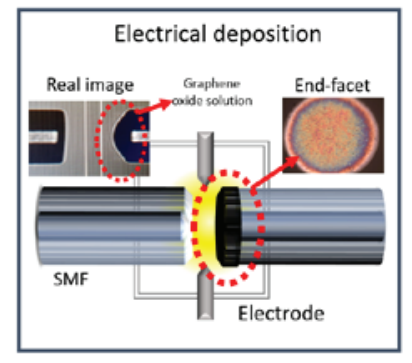

(a)

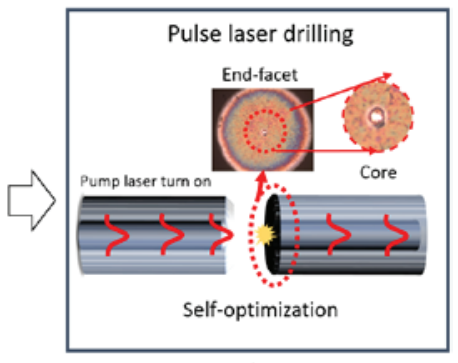

(b)
Figure 1.(a) The process of electrical deposition method (b) The process of pulse laser drilling

The electrical deposition method is shown in Figure 1.(a). The end face of optical fiber was immersed in the prepared GO solution. Using electrical arc in conventional fusion splicer [Fitel S177a], GO thick film was deposited at the end face of optical fiber. By turning on the $980 \mathrm{~nm}$ pump laser, the GO thick film is drilled by the pulse laser as shown in Figure 1.(b). Stable Qswitched EDFL is operated by a thin film. The thickness of GO film was optimized by drilling process automatically in laser cavity [9]. 


\section{Results and Discussion}

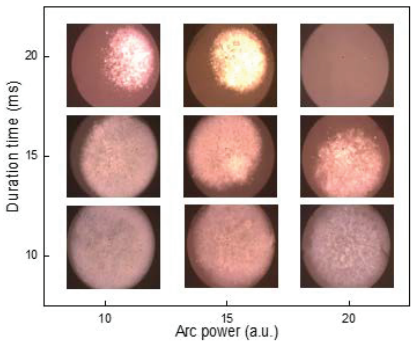

(a)

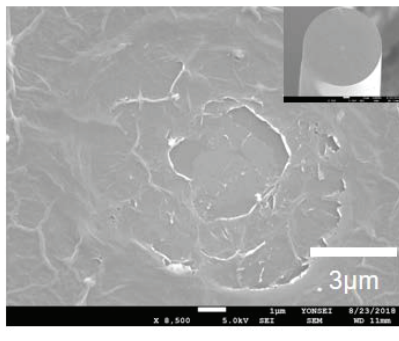

(b)
Figure 2. (a) The cross-section image of end face of optical fiber depending on arc power and duration time. (b) SEM image of the optical fiber core region

Figure 2.(a) shows the GO film at the end face of optical fiber depending on arc power and arc duration of fusion splicer. As shown in the Figure 2.(a), GO film was not deposited at the core with more than $15 \mathrm{~ms}$ duration time, so we chose the adjusted parameters with arc power 10 unit and arc duration time of $10 \mathrm{~ms}$ (Since the absolute arcpower was not supported by the fusion splicer, only the relative values were available in the experiment.). Figure 2.(b) shows a scanning electron microscope (SEM) image of GO film at the end face of optical fiber. As shown in Figure 2.(b), the GO film at the core is thinner than film at the cladding. Note that thickness of GO film was optimized by drilling process automatically for generating a Q-switching laser in our laser cavity.

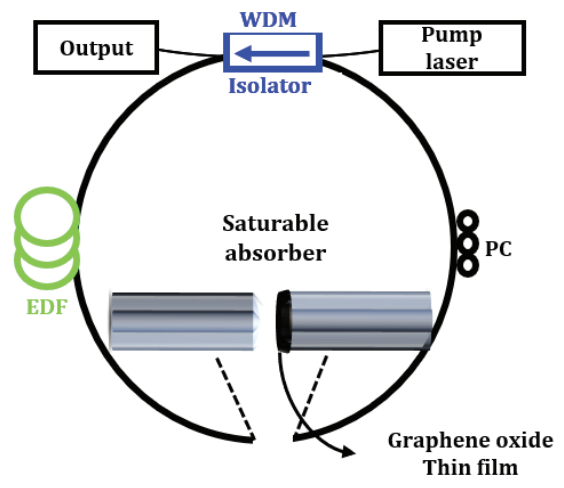

Figure 3. Configuration of the laser ring cavity

Figure 3.(a) shows the ring cavity was pumped to a $980 \mathrm{~nm}$ laser diode through a $980 \mathrm{~nm} / 1550 \mathrm{~nm}$ WDM coupler. An erbium-doped fiber with a length of $1.2 \mathrm{~m}$ provides optical gain in L-band regime. An isolator was used to ensure unidirectional propagation of the laser. A polarization controller adjusted the round-trip polarization state in the fiber ring cavity. An output coupler with the power ratio of 10:90 was used where the $10 \%$ port was used as the laser output port. Prepared GO-deposited optical fiber using our new deposition method was inserted in ring laser cavity as a SA to generate a passive Q-switching.

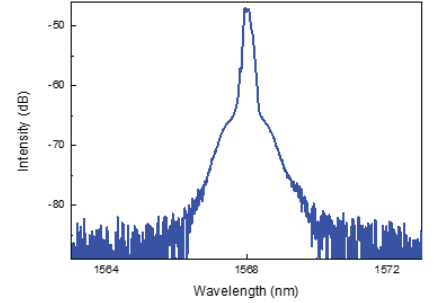

(a)

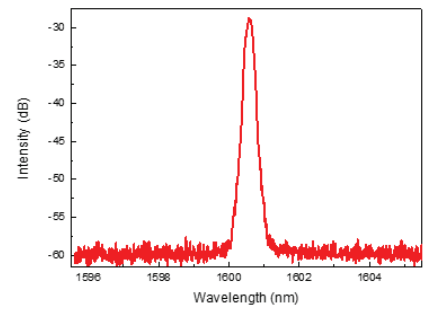

(c)

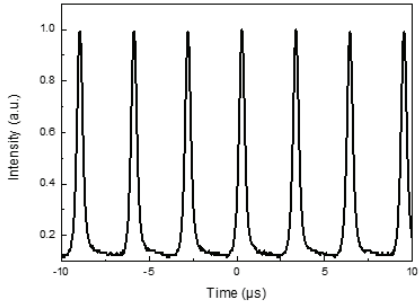

(b)

(d)

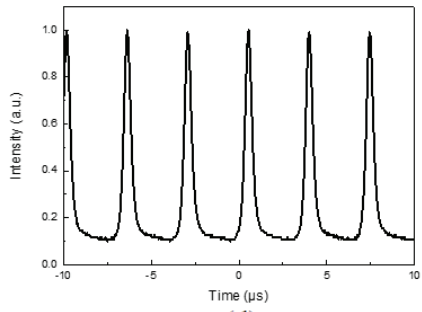

Figure 4. (a) Optical spectrum analyzer of the output data (b) pulse train in the C-band regime (c) Optical spectrum analyzer of the output data (d) pulse train in the L- band regime respectively

In C-band regime, the output spectrum of the Qswitching operation at the pump power of $237.6 \mathrm{~mW}$ is shown in Figure 4.(a) with the central wavelength of $1568.1 \mathrm{~nm}$. The stable Q-switched pulse trains are shown in Figure 4.(b). By changing the pump power increased from $149.3 \mathrm{~mW}$ to $280.8 \mathrm{~mW}$, the repetition rates increased from $22.5 \mathrm{kHz}$ to $52.3 \mathrm{kHz}$, the output power linearly increased from $0.21 \mathrm{~mW}$ to $1.35 \mathrm{~mW}$, and the pulse width decreased from $10.4 \mu \mathrm{s}$ to $4.16 \mu \mathrm{s}$. Furthermore, we fabricated another GO-deposited optical fiber SA and generated a Q-switching laser in L-band. Detailed gain dynamics and wavelength shift need to be further investigated in our proposed method, which is being pursued by the authors. In L-band regime, the output spectrum of the Q-switching operation at the pump power of $193.6 \mathrm{~mW}$ is shown in Figure 4.(c) with the central 
wavelength of $1600.5 \mathrm{~nm}$. The stable Q-switched pulse trains are shown in Figure 4.(d). The pump power increased from $105.2 \mathrm{~mW}$ to $193.6 \mathrm{~mW}$, the repetition rates increased from $6.3 \mathrm{kHz}$ to $17.8 \mathrm{kHz}$, the output power linearly increased from $0.3 \mathrm{~mW}$ to $2.28 \mathrm{~mW}$, and the pulse width decreased from $24.0 \mu$ s to $7.8 \mu$ s respectively.

\section{Conclusion}

In conclusion, we successfully fabricated an all-fiber saturable absorber by depositing the GO film on end face of optical fiber by applying an electrical arc and laser drilling. By using this new method, we successfully generated stable Q-switching all-fiber laser in both C- and L-band with different GO SA without changing the optical gain. This result confirms that our proposed deposition method with electrical arc and laser drilling could generate a Q-switching pulse successfully in different band regimes both C-band and L-band. The proposed method could be further applied to fabricate a SA in optical fiber cavity to generate a Q-switching fiber laser applications.

\section{Acknowledgement}

This work was supported by ICT R\&D Program of MSIP/IITP (2014-3-00524) and supported by Basic Science Research Program through the National Research Foundation of Korea(NRF) funded by the Ministry of Science, ICT \& Future Planning(No. 2016k1A3A1A09918616).

\section{References}

[1] Xu, J., Liu, J., Wu, S., Yang, Q. H., and Wang, P., "Graphene oxide mode-locked femtosecond erbiumdoped fiber lasers," Optics express, 20(14), 1547415480 (2012).

[2] Ahmad, H., Muhammad, F. D., Zulkifli, M. Z., and Harun, S. W., "Graphene-oxide-based saturable absorber for all-fiber Q-switching with a simple optical deposition technique," IEEE Photonics Journal, 4(6), 2205-2213 (2012).

[3] Sobon, G., Sotor, J., Jagiello, J., Kozinski, R., Librant, K., Zdrojek, M., ... and Abramski, K. M., "Linearly polarized, Q-switched Er-doped fiber laser based on reduced graphene oxide saturable absorber," Applied Physics Letters, 101(24), 241106 (2012).

[4] Boguslawski, J., Sotor, J., Sobon, G., Kozinski, R., Librant, K., Aksienionek, M., ... and Abramski, K. M., "Graphene oxide paper as a saturable absorber for Erand Tm-doped fiber lasers," Photonics Research, 3(4), 119-124 (2015).

[5] Woodward, R., and Kelleher, E., "2D saturable absorbers for fibre lasers," Applied Sciences, 5(4), 1440-1456 (2015).s

[6] Martinez, A., and Sun, Z., "Nanotube and graphene saturable absorbers for fibre lasers," Nature Photonics, 7(11), 842 (2013).

[7] Kwon, W. S., Lee, H., Kim, J. H., Choi, J., Kim, K. S., and Kim, S., "Ultrashort stretched-pulse L-band laser using carbon-nanotube saturable absorber," Optics express, 23(6), 7779-7785 (2015).

[8] Hong, S., Lédée, F., Park, J., Song, S., Lee, H., Lee, Y. S., ... and Oh, K., "Mode-Locking of All-Fiber Lasers Operating at Both Anomalous and Normal Dispersion Regimes in the C-and L-Bands Using Thin Film of 2D Perovskite Crystallites," Laser \& Photonics Reviews, 12(11), 1800118 (2018).

[9] Hendow, S. T., Romero, R., Shakir, S. A., and Guerreiro, P. T., "Percussion drilling of metals using bursts of nanosecond pulses," Optics express, 19(11), 10221-10231 (2011). 


\title{
Vector Holographic Trapping and Tweezing*
}

\author{
Carmelo Rosales-Guzman*a, Nkosi Bhebhe ${ }^{\mathrm{b}}$, Valeria Rodriguez-Fajardo ${ }^{\mathrm{b}}$ and Andrew Forbes ${ }^{\mathrm{b}}$ \\ ${ }^{a}$ Wang Da-Heng Collaborative Innovation Center for Quantum manipulation \& Control, Harbin \\ University of Science and Technology, Harbin 150080, China \\ ${ }^{\mathrm{b}}$ School of Physics, University of the Witwatersrand, Private Bag 3, Johannesburg 2050, South Africa
}

\begin{abstract}
Complex vector states of light have found many applications to date. Here we present new approaches for the creation of such optical fields, including multiple scalar and vector states multiplexed from a single hologram. We explore vector combinations that allow shape invariant propagation in free-space, and outline a quantum toolkit for the analysis of such beams. Finally, we combine these advances into an optical trapping and tweezing setup to demonstrate a holographic trap for both vector and scalar modes.
\end{abstract}

Keywords: vector beams, holographic optical tweezers, spatial light modulators, structured light

\section{Introduction}

Structured light is a highly topical field that captures the ability to tailor light in amplitude, phase and polarization [1]. Amongst the many structured light fields are vector beams, characterized by spatially variant polarization patterns. They have found a myriad of applications to date, which have been recently reviewed $[2,3]$. Here we present new approaches for the creation of such optical fields, including the simultaneous generation or multiplexing, from a single hologram, of multiple scalar and vector states. We explore vector combinations that allow shapeinvariant propagation in free-space, and outline a quantum toolkit for the analysis of such beams. Finally, we combine these advances into an optical trapping and tweezing setup to demonstrate a holographic trap for both vector and scalar modes.

\section{Summary of results}

We outline new approaches to the creation of vector beams. We point out that it is possible to create multiplexed vector beams from a single hologram by combining interferometric and holographic approaches, demonstrating all Higher-Order Poincare Sphere beams simultaneously from one device [4]. We show that a common path approach can be used to create propagation invariant flat-top beams by exploiting the efficiency issues of SLMs, combining an undiffracted Gaussian mode with a vortex mode [5]. After the creation step we are able to show accurate analysis of the vector beams by employing quantum tools to form a vector beam analyser. This allows the degree of vectorness to be analysed during propagation. Finally, we deliver these beams into an optical trap and show simultaneous optical trapping and tweezing of scalar and vector beams [6]. We use this to test the trapping efficiency of cylindrical vector vortex beams, including radially and azimuthally polarized beams in the same trap. We believe that this constitutes the first demonstration of a vector holographic optical trap (HOT), extending the functionality of previous scalar HOTs.

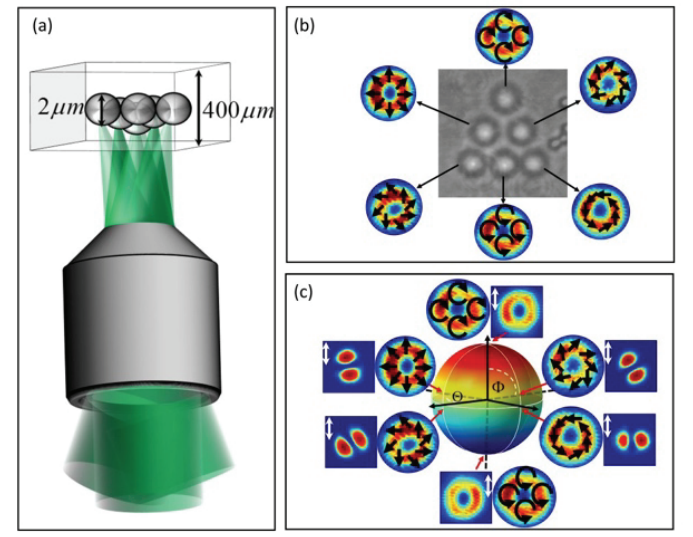

Figure 2. An example of a vector holographic trap for the delivery of scalar and vector beams. 


\section{References}

[1] Rubinsztein-Dunlop, H., et. al., "Roadmap on structured light,” J. Opt. 19, 013001 (2017)

[2] Rosales-Guzman, C., Ndagano, B., and Forbes, A., “A review of complex vector light fields and their applications," J. Opt. 20, 123001 (2018).

[3] Ndagano, B., Nape, I., Cox, M.A., Rosales-Guzman, C., and Forbes, A., "A review of complex vector light fields and their applications,” J. Light. Tech. 36, 292-301 (2018).

[4] Rosales-Guzman, C., Bhebhe, N., and Forbes, A., "Simultaneous generation of multiple vector beams on a single SLM," Opt. Express 25, 25697-25708 (2017).

[5] Bhebhe, N., Rosales-Guzman, C., and Forbes, A., "Classical and quantum analysis of propagation invariant vector flat-top beams," Appl. Opt. 57, 5451-5458 (2018).

[6] Bhebhe, N., Williams, P., Rosales-Guzman, C., Rodriguez-Fajardo, V., and Forbes, A., "A vector holographic optical trap," Scientific Reports 8, 17387 (2018). 


\title{
AC electrophoretic mobility of an optically trapped colloidal particle
}

\author{
Kohei Iki*, Yasuyuki Kimura \\ Department of Physics, Kyushu University, 744 Motooka, Nishi-ku, Fukuoka, 819-0395, Japan
}

\begin{abstract}
It is important to understand the dynamics of ions around charged colloidal particles from the fundamental and application viewpoints. In this study, we develop a new measurement method of AC electrophoretic mobility of a micrometer-sized single colloidal particle in a wide frequency range over four decades. Fast and precise detection of the displacement of the particle is achieved by laser-trapping of the particle and using laser interferometry. The complex mobility of a silica particle of diameter of $1 \mu \mathrm{m}$ dispersed in water is measured from $10 \mathrm{~Hz}$ to $10 \mathrm{kHz}$. The obtained apparent spectrum is found to contain the influence of laser trapping at lower frequencies and the dynamics of electro-osmotic flow induced by the charged cell surfaces. We succeed to observe the dynamics of the electrically induced bulk flow in a $100-\mu \mathrm{m}$-thick cell threedimensionally for the first time.
\end{abstract}

Keywords: electrophoresis, electro-osmosis, optical tweezers, colloid, mobility

\section{Introduction}

AC electrokinetic behavior including electrophoresis, electro-rotation and electro-osmosis have recently been utilized to manipulate mesoscopic objects in micro-scaled devices [1]. However, the dynamics of charged mesoscopic objects dispersed in aqueous solution is complex and their electric response has not been fully understood [2]. The electrophoretic mobility, which is given by the ratio of the electrically induced velocity of a particle to and the applied electric field, is one of the important values to characterize colloidal particles. Although the frequency dependence of the mobility is expected to reflect the dynamics of the surface charge of the particles [2], it has mainly been studied only under a DC electric field.

In this study, a new experimental method to measure the mobility of a single colloidal particle under AC electric field has been developed and the spatial dependence of the mobility spectrum has been studied in three-dimensions. Especially, the spatial dependence of flow field induced by the surface charge of the cell has been measured in a wide frequency range for the first time.

\section{Experiment}

A silica particle with diameter of $1 \mu \mathrm{m}$ dispersed in an ultrapure water is trapped by a strongly focused laser beam (wavelength $830 \mathrm{~nm}$ ). The position of the trapped particle is detected by a quadrant photodiode (QPD) using laser

i.k1024r@gmail.com; phone 81-92-802-4109 interferometry method [3]. This enables us to measure the displacement of the particle with high spatial (nm) and temporal $(10 \mu \mathrm{s})$ resolution. An alternating electric field is applied to the sample cell using a function generator. The thickness of the cell is $100 \mu \mathrm{m}$ and the gap of the $\mathrm{Cu}$ electrodes is $2 \mathrm{~mm}$ (Fig. 1).

The applied electric field $E(t)$ is a sinusoidal one with the amplitude of $E_{0}$ and the angular frequency of $\omega$ as $E(t)$ $=E_{0} \exp (i \omega t)$. The induced displacement of the particle $x(t)$ is represented as $x(t)=x_{0} \exp \{i(\omega t-\delta)\}$ with the amplitude of $x_{0}$ and the phase delay $\delta$. The AC complex mobility $\mu^{*}$ is obtained by $x_{0}, E_{0}$, and $\delta$ as

$$
\mu^{*}(\omega)=\frac{v(\omega)}{E_{0}}=\frac{i \omega x_{0}}{E_{0}} \exp (-i \delta)
$$

The temporal data of $x(t)$ is calculated from the output voltage signal of the QPD.

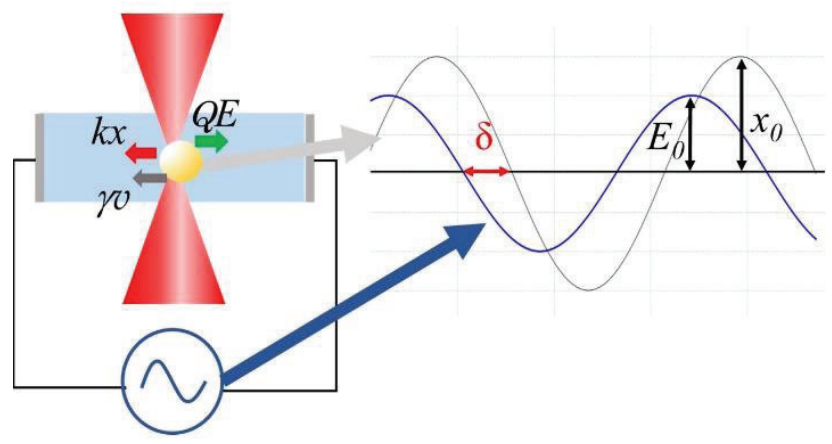

Figure 1. Schematic of the forces applied to an optically trapped particle (left) and the definition of the amplitude $x_{0}, E_{0}$, and the phase delay $\delta$ (right). 


\section{Results and Discussion}

\section{Trapping effect}

The apparent frequency dependence of the measured mobility $\mu^{*}\left(=\mu^{\prime}+i \mu^{\prime}\right)$ from $10 \mathrm{~Hz}$ to $10 \mathrm{kHz}$ is shown in Fig. 2. The observed $\mu$ ' decreases to zero and $\mu$ " exhibits characteristic frequency dependence at the low frequencies. This is an artifact due to the trapping by laser beam. In order to remove this confinement effect, we use the corner frequency in the power spectrum of $|x(\omega)|^{2}$ without electric field. After this correction, $\mu^{\prime}$ is constant and $\mu$ ' is almost zero at the low frequencies as shown in Fig. 3.

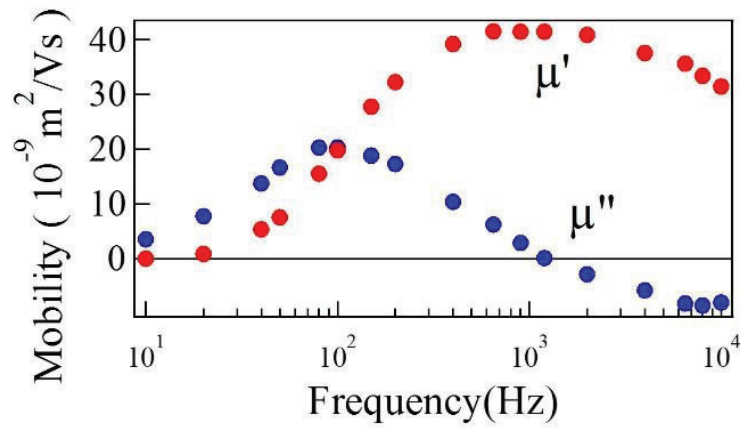

Figure 2. Frequency dependence of apparent complex mobility $\mu^{*}$ of a $1 \mu \mathrm{m}$-silica particle in water.

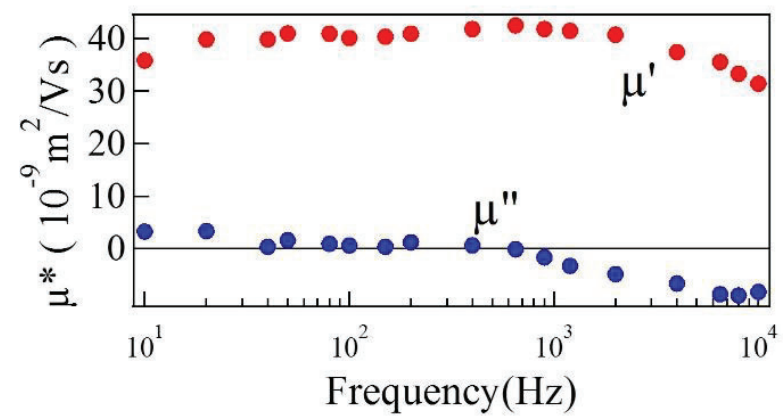

Figure 3. Frequency dependence of complex mobility $\mu^{*}$ of a $1 \mu \mathrm{m}$-silica particle in water after correction.

\section{Electroosmotic flow}

A glass cell wall is known to be charged in an aqueous solution and form an electric double layer near the wall. The counter-ions within the double layer are forced to migrate by the application of electric field and induces a flow inside the cell, which is called an electro-osmotic flow $[1,2]$. The flow field varies with the distance from the cell wall. We measure the mobility $\mu^{*}$ at various height within a cell by changing the height of the trap. In the low frequency region, $\mu^{*}$ greatly depends on the height. In the high frequency region, $\mu^{*}$ becomes zero irrespective of the height. Theoretical hydrodynamic calculation of the flow in a closed parallel plate cell $[4,5]$ is found to make good agreement with the experimental spectrum obtained in this study. It is found that the relaxation behavior appears in the frequency range above $1 \mathrm{kHz}$ in Fig. 3 is due to the relaxation of electro-osmotic flow.

\section{Conclusion}

We developed a new method for AC electrophoretic mobility using optical trap. Detection of the position of the particle by laser interferometry enables us to realize extremely wide frequency measurement. The apparent spectrum contains the influence of laser trapping at low frequencies and the information on the electro-osmotic flow at high frequencies. We succeed in removing the former artifact by using the fluctuation in position without electric field. By measuring the height dependence of the spectrum, the information of spatial dependence of flow field is obtained in wide frequency range. Our method utilizing optical trapping is expected to offer new spatiotemporal information in electro-microfluidics.

\section{References}

[1] Morgan H. and Green N.G., "AC Electrokinetics: colloids and nanoparticles", Research Studies Pr Ltd, Baldock (2002).

[2] Russel, W.B. Saville D.A. and Schowalter W.R., "Colloidal Dispersions", Cambridge University Press, Cambridge, (1992).

[3] Beunis F., Strubbe F., Neyts K., and Petrov D., "Beyond Millikan: The Dynamics of Charging Events on Individual Colloidal Particles." Phys. Rev. Lett. 108, 016101 (2012).

[4] Ramos A., Morgan H., Green N.G.. Castellanos A., "AC Electric-Field-Induced Fluid Flow in Microelectrodes", J. Colloid and Interface Sci., 217, 420, (1999).

[5] Landau L.D. and Lifshitz E.M., "Fluid Mechanics" 2nd ed., Butterworth-Heinemann, Oxford, (1987) 


\title{
Optical vortex ablation creates high viscosity 'ink-jet'
}

\author{
Ryosuke Nakamura ${ }^{1}$, Muneaki Iwata ${ }^{2}$, Akihiro Kaneko ${ }^{3}$, Kohei Toyoda $^{1,4}$, \\ Katsuhiko Miyamoto ${ }^{1,4}$, and Takashige Omatsu ${ }^{1,4}$ \\ ${ }^{1}$ Graduate School of Engineering Chiba University, Chiba 263-8522, Japan \\ ${ }^{2}$ Research and Development Division. Ricoh Institute of Information and Communication Technology. Applied Imaging Development Center, \\ Kanagawa 243-0460, Japan \\ ${ }^{3}$ RICOH CT\&P Division.1st Technology Center, Shizuoka 410-0007, Japan \\ ${ }^{4}$ Molecular Chirality Research Center, Chiba University, Chiba 263-8522, Japan \\ Corresponding Author e-mail address: omatsu@faculty.chiba-u.jp
}

\begin{abstract}
We demonstrate the formation of a high viscosity 'ink-jet' by employing optical vortex laser ablation. The optical vortex induces the radial inward mass transport of the ink towards its dark core through the hydrodynamics and vaporizationinduced recoil pressure, resulting in the creation of the 'ink-jet'. The OAM then forces the melted ink to spin towards clockwise or counter-clockwise direction assigned by the handedness of the vortex pulse, thereby stabilizing the formation of the 'ink-jet'.
\end{abstract}

\section{Introduction}

An ink-jet printing technology, i.e. a non-contact printing process, enables us to shape various patterns, formed of micron-scale liquid droplets, such as color images, flexible organic electric circuits, integrated optical devices. However, it has several drawbacks. For instance, it is difficult to eject high-viscosity ink droplets with a viscosity of $>0.1 \mathrm{~Pa} \cdot \mathrm{s}$, containing functional nanoparticles [1,2]. Also, a smallest diameter of ejected ink droplet is typically limited to $\sim 20 \mu \mathrm{m}$.

Optical vortex possesses a ring-shaped spatial form and an orbital angular momentum (OAM), arising from its helical wavefront with a phase singularity characterized by a topological charge, $\ell$ [3]. Circularly polarized light also carries a spin angular momentum (SAM), associated with its helical electric field [4]. To date, we and our co-workers discovered that the optical vortex laser ablation enables the fabrication of unique structured matters, such as chiral structures, on the irradiated target with the help of SAM [5-9]. In particular, in the silicon needle formation, it was found that the optical vortex forces the silicon droplets to spin, resulting in the efficient accumulation of the melted silicon on the substrate [10].

In this paper, we report on that the optical vortex forces the melted ink to spin, thereby yielding a high viscosity ink-filament, so-called high viscosity 'ink-jet'. This phenomenon will be extended to develop an entirely novel printing technique for high-viscosity functional inks.

\section{Experiments}

An air-dried film with a thickness of $\sim 20 \mu \mathrm{m}$ and an extremely high viscosity of $\sim 4 \mathrm{~Pa} \cdot \mathrm{s}$, formed of a conventional ultraviolet ink, on a silica glass substrate was used as a target sample. A nanosecond green laser was used, and its output was converted to be a circularly polarized first-order optical vortex with $\ell=1$ and $s=1$ by employing a spiral phase plate (SPP) and a quarter-wave plate (QWP). A single optical vortex pulse was loosely focused to be a $\phi 160 \mu \mathrm{m}$ annular spot on the target sample from the backside (the silica glass plate substrate side). The laser pulse energy was then measured to be $\sim 40 \mu \mathrm{J}$.

Temporal evolution of the formation of 'ink-jet' ejected from the film was observed by a high-speed camera (Shimadzu Corp., Hyper Vision HPV-X), for the side view, at a frame rate of $2 \times 10^{6} \mathrm{fps}$. The radial inward mass transport of the ink towards the dark core of the optical vortex occurs, so as to form the 'ink-jet' within approximately $4 \mu \mathrm{s}$ after the irradiation of optical vortex pulse. An additional 2-6 $\mu$ s later, the micron-scale ink droplet (with a dimeter of $\sim 20 \mu \mathrm{m}$ ) was also ejected (Fig. 1(a)), In contrast, the conventional Gaussian pulse 
irradiation scattered mostly the ink, resulting in that the 'ink-jet' has never been created (Fig. 1(b)).

Why can an optical vortex pulse create such 'ink-jet'? Optical vortex collects the melted ink within its dark core through the vaporization-induced recoil pressure to form the 'ink-jet' via an optical vortex-induced capillary-wave. The OAM then should force the melted ink to spin, thereby stabilizing the formation of the 'ink-jet'. In fact, the spinning motion of the droplets was directly observed, and its speed was then measured to be $\sim 10^{5} \mathrm{rpm}$. Also, it is noteworthy that the spinning direction and speed of the droplet were determined by the sign and magnitude of total angular momentum, $J$, i.e. sum of OAM and SAM (Fig.2).
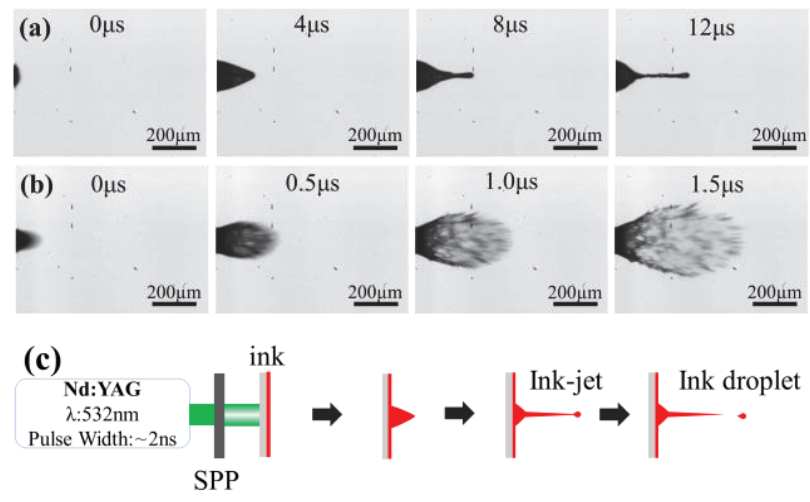

Fig. 1. (a) Time-laps of the formation of 'ink-jet' by optical vortex laser ablation. The optical vortex pulse carried OAM of $\ell=1$ and SAM of $s=1$. (b) Time-laps of the scattered ink droplets by irradiation of a circularly polarized Gaussian beam. The optical vortex and Gaussian pulse energies were then measured to be $40 \mu \mathrm{J}$. (c) Schematic of temporal dynamics of 'ink-jet' formation.

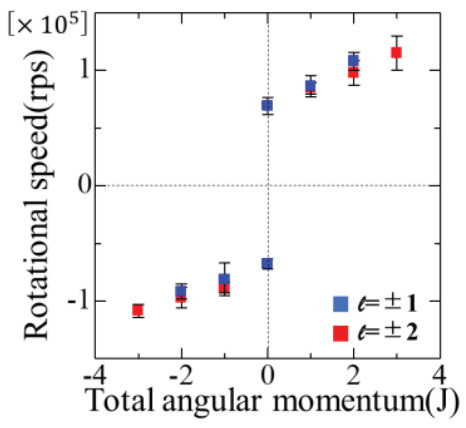

Fig. 2. Rotational speed of the droplets as a function of total angular momentum $(J)$.

\section{Conclusion}

We have demonstrated the formation of the micronscale ink-filament, so called 'ink-jet', by employing optical vortex laser ablation. Such creation of the 'ink-jet' will be potentially extended to develop an entirely novel printing technique, enabling the development of a new generation printed electric circuit formed of a highviscosity ink droplet, containing functional nanoparticles.

\section{References}

[1] Burns, S. E., Cain, P., Mills, J., "Inkjet printing of polymer thin-film transistor circuits," MRS Bulletin. 28, 829-834 (2003).

[2] Cui, X., Boland, T., "Human microvasculature fabrication using thermal inkjet printing technology," Biomaterials. 30, 6221-6227 (2009).

[3] Zhan, Q., "Cylindrical vector beams: from mathematical concepts to applications," Advances in Optics and Photonics, 1, 1-57 (2009).

[4] O’Neil, A. T., MacVicar, I., Allen, L., Padgett, M. J., "Intrinsic and extrinsic nature of the orbital angular momentum of a light beam," Phys. Rev. Lett. 88, 053601 (2002).

[5] Omatsu, T., Chujo, K., Miyamoto, K., Okida, M., Nakamura, K., Aoki, N., Morita, R., "Metal microneedle fabrication using twisted light with spin," Opt. Express, 18, 17967-17973 (2010).

[6] Toyoda, K., Miyamoto, K., Aoki, N., Morita, R., Omatsu, T., "Using optical vortex to control the chirality of twisted metal nanostructures," Nano Lett. 12, 3645-3649 (2012).

[7] Toyoda, K., Takahashi, F., Takizawa, S., Tokizane, Y., Miyamoto, K., Morita, R., Omatsu, T., "Transfer of light helicity to nanostructures," Phys. Rev. Lett., 110, 143603/1-5 (2013).

[8] Takahashi, F., Miyamoto, K., Hidai, H., Yamane, K., Morita, R., Omatsu, T., "Picosecond optical vortex pulse illumination forms a monocrystalline silicon needle," Sci. Rep., 6, 21738/1-10 (2016).

[9] Takahashi, F., Takizawa, S., Hidai, H., Miyamoto, K., Morita, R., Omatsu, T., "Optical vortex pulse illumination to create chiral monocrystalline silicon nanostructures," Physica Status Solidi A, 213, 10631068 (2016). 


\title{
Chiral mass-transport of azo-polymers with OAM light field
}

\section{through two photon absorption}

\author{
Keigo Masuda ${ }^{a}$, Mitsuki Ichijo ${ }^{a}$, Ryo Shinozaki ${ }^{a}$, Keisaku Yamane ${ }^{\text {, }}$ \\ Katsuhiko Miyamoto ${ }^{\mathrm{a}, \mathrm{c}}$, Takashige Omatsu ${ }^{\mathrm{a}, \mathrm{c}, \text {, }}$ \\ ${ }^{a}$ Graduate School of Advanced Integration Science, Chiba University, Japan \\ ${ }^{b}$ Department of Applied Physics, Hokkaido University, Japan \\ ${ }^{c}$ Molecular Chirality Research Center, Chiba University, Japan \\ Corresponding author : Takashige Omatsu \\ E-mail: omatsu@faculty.chiba-u.jp
}

\begin{abstract}
We demonstrate chiral mass-transport of azo-polymers by illumination of tightly focused $1-\mu \mathrm{m}$ picosecond optical vortex pulses with a pulse width of 8-ps through two-photon absorption. The chiral surface relief formation requires picosecond pulses with a relatively long pulse duration. In fact, it is also worth noting that it is difficult to create such chiral surface relief by employing 2-ps optical vortex pulses.
\end{abstract}

Keywords: azo-polymer, optical vortices, two photon absorption, singular optics, laser materials processing

\section{Introduction}

Optical vortex exhibits a helical wavefront to induce an annular intensity profile and carry optical orbital angular momentum (OAM), characterized by topological charge $\ell$ [1]. Such physical properties of optical vortex provide new fundamental and applied sciences in various fields, such as laser scanning microscopy, optical telecommunication, quantum optics, and optical manipulation.

In particular, it is worth noting that optical vortex can twist materials, such as silicon, metal and liquid resin, to form unique structures with a chirality assigned by the twisting direction (i.e. handedness) of the helical wavefront of optical vortex $[2,3]$. In recent years, we and our co-workers have discovered that green continuouswave (cw) optical vortex also twist an azo-polymer film to form a chiral surface relief with the help of spin angular momentum (SAM, $s$ ) associated with a circular polarization [4,5]. Such chiral surface relief will be applied to the chiral plasmonic meta-surface to manipulate the polarization and concentration of chiral molecules and their aggregations.
The two-photon-absorption (TPA) process has always been offering us new fundamental sciences and advanced applications, for instance, as 3-dimentional micro/nanofabrication, etc. [6]. Most studies of surface relief formation in azo-polymers, however, are based on single-photon-absorption (SPA). Surface relief formation via TPA has not yet been experimentally and theoretically established. Surface relief formation via TPA should provide entirely new fundamental phenomena, such as spin and orbital angular momentum coupling in TPA, with nonlinear optics and photochemistry.

As a pioneering work, Ishitobi et.al. have demonstrated two-photon-absorption induced surface relief formation in azo-polymer by irradiation of femtosecond laser pulses [7]. However, the femtosecond laser with a rather shorter time duration in comparison with required time for photoisomerization was ill suitable for efficient mass-transport of azo-polymers. In fact, a height of the generated surface relief was limited to be only $40 \mathrm{~nm}$.

In this paper, we report on chiral surface relief formation of azo-polymers via TPA by the illumination of $1 \mu \mathrm{m}$ picosecond optical vortex pulses. 


\section{Experiments}

Azo-polymer film used in our experiments exhibited no significant absorption in near-infrared region. A $1-\mu \mathrm{m}$ picosecond laser with a pulse width of 8 ps, a pulse repetition frequency of $40 \mathrm{MHz}$, and average power of 40 $\mathrm{mW}$, was conducted, and its output was converted onto a right-handed circularly polarized optical vortex with $\ell=1$ and $s=1$ by utilizing a spiral phase plate (SPP) and a quarter wave plate (QWP). The generated vortex beam was tightly focused to be a $4 \mu \mathrm{m}$ annular spot onto the azo-polymer film surface by a high numerical aperture objective lens $(\mathrm{NA}=0.9)$. The focused laser intensity was then estimated to be $1.1 \mathrm{GW} / \mathrm{cm}^{2}$.
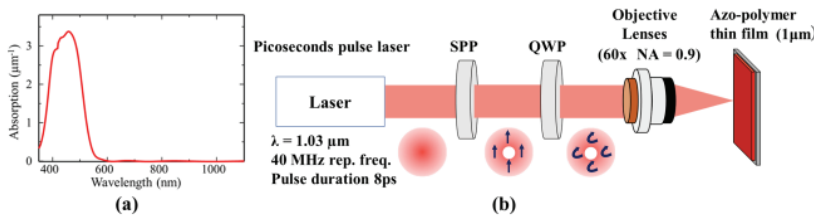

Figure 1. (a) Absorption spectrum of the azo-polymer used in our experiment. (b) Experimental setup.

Figure 2 shows the temporal response of surface relief formation in azo-polymer film. After laser was turned on, mass-transport of azo-polymers occurred along the intensity profile within one minute. Subsequently, azopolymers revolved azimuthally and were directed radially toward the dark core of optical vortex, so as to establish the chiral surface relief within exposure time of 3 minutes. The diameter and height of the relief was measured to be a $3 \mu \mathrm{m}$ and $390 \mathrm{~nm}$, respectively. The twisted direction of the surface relief was then assigned by the handedness of optical vortex in Fig. 3.
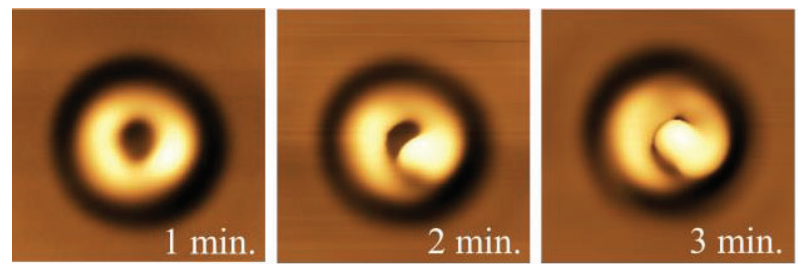

Figure 2. Temporal evolution of right-handed chiral mass-transport of azo-polymer by illumination of 8-ps optical vortex pulses
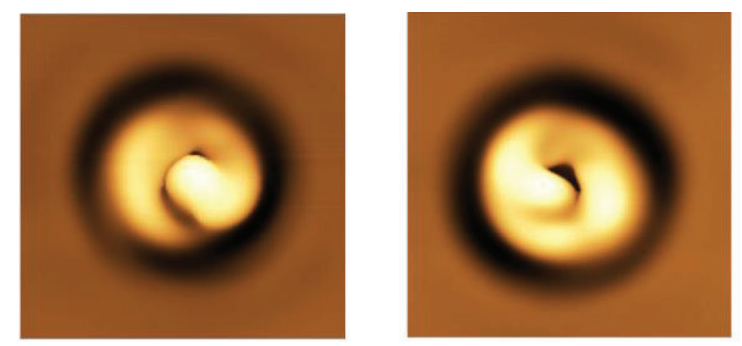

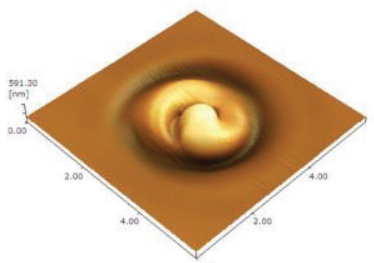

(a)

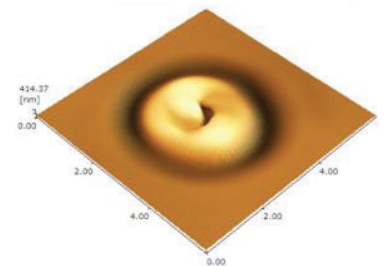

(b)
Figure 3. (a) Right-handed- and (b) left-handed- chiral surface relief in azo-polymer film by illumination of right-handed and left-handed optical vortex pulses.

In contrast, 2-ps right-handed optical vortex pulses, which had a pulse width comparable to the required time for photo-isomerization, impacted the efficient OAM transfer, thereby resulting in the creation of only nonchiral surface relief with a height of $110 \mathrm{~nm}$ even with an exposure time of $\sim 10$ minutes (Fig. 4(a)). Chiral surface relief formation by irradiation of 2-ps vortex pluses required an exposure time of at least 60 minutes.

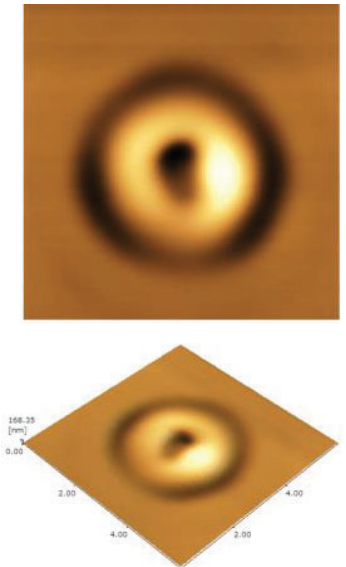

(a)
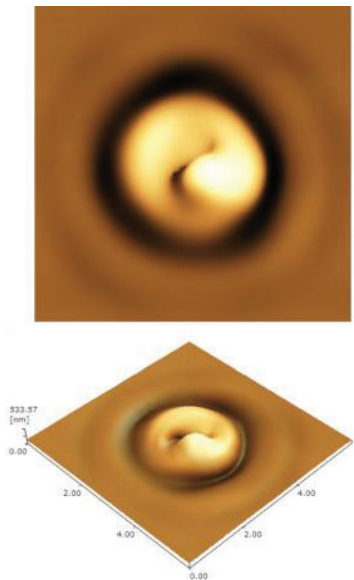

(b)
Figure 4. Surface relief in azo-polymer by illumination of 2-ps optical vortex pulses with exposure time of (a) 10- and (b) 60- minutes. 


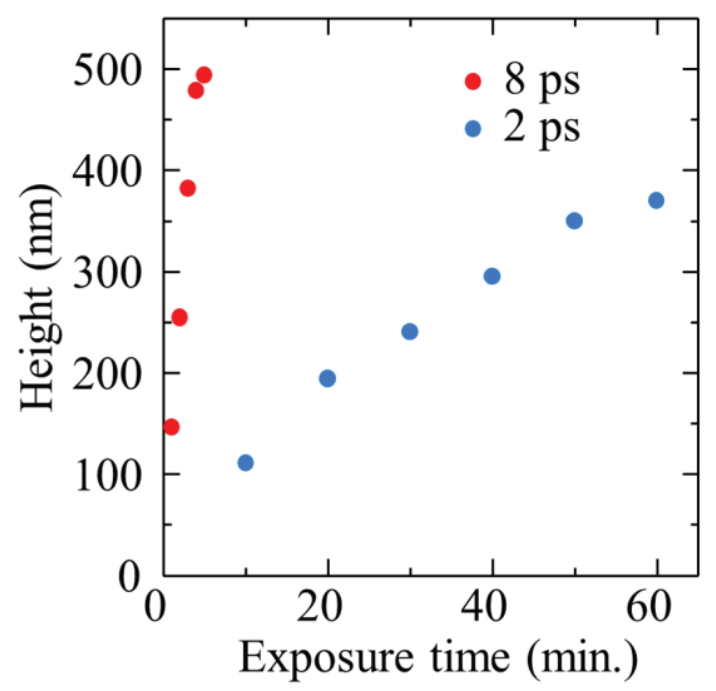

Figure 5. Height of the surface relief, formed by 8 ps and 2 ps optical vortex pulses, at varying the exposure time.

\section{Conclusion}

We have demonstrated, for the first time, TPA-induced chiral mass-transport of an azo-polymer by illumination of $1-\mu \mathrm{m}$ picosecond optical vortex pulses, so as to establish a chiral surface relief with a diameter of 0.7 times the diffraction limit. Efficient chiral surface relief formation requires optical vortex pulses with a longer pulse duration in comparison with the required time for photo-isomerization.

\section{References}

[1] Allen, L., Beijersbergen, M. W., Spreeuw, R. J. C., Woerdman, J. P., "Orbital angular momentum of light and the transformation of Laguerre-Gaussian laser modes," Phys. Rev. A. 45, 8185 (1992).

[2] Toyoda, K., Takahashi, F., Takizawa, S., Tokizane, Y., Miyamoto, K., Morita, R., and Omatsu, T., "Transfer of light helicity to nanostructures," Phys. Rev. Lett. 110, 143603 (2013).

[3] Lee, J., Arita, Y., Toyoshima, S., Miyamoto, K., Panagiotopoulos, P., Wright, E. M., Dholakia, K., Omatus, T., "Photopolymerization with LightFields Possessing Orbital Angular Momenutm: Generation of Helical Microfibers," ACS Photonics, 5, 4156-4163 (2018).

[4] Barada, D., Juman, G., Yoshida, I., Miyamoto, K.,
Kawata, S., Ohno, S., Omatsu, T., "Constructive spinorbital angular momentum coupling can twist materials to create spiral structures in optical vortex illumination,” Appl. Phys. Lett. 108, 051108 (2016).

[4] Masuda, K., Nakano, S., Barada, D., Kurama, M., Miyamoto, K., Omatsu, T., “Azo-polymer film twisted to form a helical surface relief by illumination with a circularly polarized Gaussian beam," Opt. Exp. 25, 12499 (2017).

[6] Kawata, S., Sun, HB., Tanaka, T., Takada, K., "Finer features for functional microdevices," Nature 412, 697 (2001).

[7] Ishitobi, H., Shoji, S., Hiramatsu, T., Sun, HB., Sekkat, Z., Kawata, S., "Two-photon induced polymer nanomovement," Opt. Exp. 16, 14106 (2008). 


\title{
Optical propulsion of fluorescent diamonds inside a tapered capillary
}

\author{
Christophe Pin ${ }^{\mathrm{a}}$, Ryohei Otsuka ${ }^{\mathrm{a}}$, Hideki Fujiwara ${ }^{\mathrm{a}}$, Keiji Sasaki*a \\ ${ }^{a}$ Research Institute for Electronic Science, Hokkaido University, Kita 20-jo Nishi 10-chome, Kita-ku, \\ Sapporo, Hokkaido, 001-0020, Japan
}

\begin{abstract}
In order to achieve efficient optical manipulation and sorting of nanoparticles, both enhanced optical forces and a large interaction volume are required. In this work, we investigate the use of a tapered glass capillary as an optofluidic platform for the optical manipulation of nanoparticles. We perform numerical simulations in order to evaluate the optical properties of glass micro- and nanocappillaries. Then we fabricate tapered capillaries with micrometer and sub-micrometer sizes using a heating and pulling method. After injecting a sample solution containing red fluorescent diamond particles inside a tapered capillary, we couple the light from a $532 \mathrm{~nm}$ laser source into the capillary. Optical propulsion of the brightest particles is achieved using a laser power of few tens of milliwatts. Particle velocities reaching few tens of micrometers per second are measured.
\end{abstract}

Keywords: optofluidics, optical manipulation, optical sorting, nanodiamonds, photonics, partially liquid core waveguide, lab-on-a-chip, lab-in-capillary

\section{Introduction}

Optical forces provide an efficient and contactless way to detect and separate objects in liquid colloidal suspension according to their optical properties. For instance, depending on the wavelength and polarization of the light, object-dependent optical forces can be applied to a large number of particles in solution, allowing for selective optical trapping or selective optical transportation. This effect can be used to achieve optical sorting of nanoparticles with different optical properties [1-7]. Efficient optical manipulation of nanoparticles is yet a challenging task as it usually requires the use of an intense laser light to cover a large interaction volume, or the use of plasmonic and photonic nanostructures that offer only a very limited interaction volume.

In order to address this issue, we investigate here the use of a tapered glass capillary as an optofluidic platform for the optical manipulation of nanoparticles. We first numerically evaluate the optical properties of glass micro- and nanocappillaries. Then we describe the fabrication process that allows us to fabricate micrometer and sub-micrometer size glass capillaries. We finally demonstrate the optical propulsion of fluorescent diamond particles inside a tapered capillary.

\section{Experimental details}

\section{Capillary waveguides}

A large variety of photonic waveguides have already been studied for the purpose of manipulating particles in different integrated optofluidic environments. While single-mode and few-mode waveguides enable efficient near-field optical trapping of particles and living cells [815], hollow-core waveguides provide a better spatial overlap between the light beam and the sample solution [16-18].

We consider here glass micro- and nano-capillaries filled with a water solution. When placed in an air environment, a water-filled capillary acts as an optical waveguide with a partially liquid core, since the refractive indices of the capillary materials $\left(\mathrm{n}_{\text {glass }}=1.5, \mathrm{n}_{\text {water }}=1.33\right.$ ) are higher than the refractive index of air $\left(\mathrm{n}_{\text {air }}=1.0\right)$. Figure 1 shows the electric field distributions of the fundamental modes of capillaries with different sizes at both $1064 \mathrm{~nm}$ and $532 \mathrm{~nm}$ wavelengths. Although the single-mode regime can be reached only in capillaries with sub-micrometer dimensions, larger capillaries may still be used for optofluidic applications as a nonnegligible part of the guide light is located in the evanescent field near the glass-water interface.

*sasaki@es.hokudai.ac.jp; phone 011 706-9396 

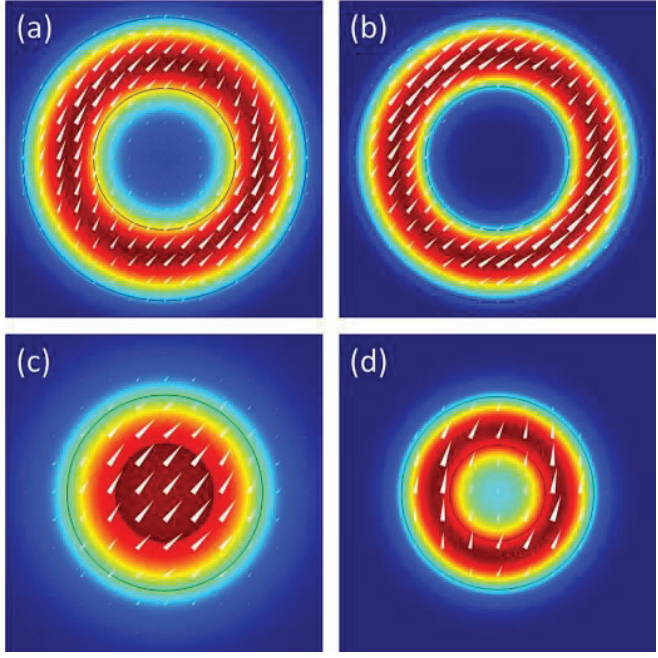

Figure 1. Cross-sections of the electric field distribution of the fundamental guided mode (a),(b) in a $2 \mu \mathrm{m}$ large capillary and (c),(d) in a $700 \mathrm{~nm}$ large capillary at (a),(c) $1064 \mathrm{~nm}$ and (b),(d) $532 \mathrm{~nm}$ wavelengths.

\section{Fabrication process}

Tapered glass capillaries were fabricated using a standard heating and pulling method that was first developed for the fabrication of tapered optical fibers. As shown in Figure 2, capillaries with sub-micrometer sizes were successfully fabricated after optimizing the pulling time and speed.

\section{Fluorescent diamonds}

Red fluorescent diamond particles with an average size of $100 \mathrm{~nm}$ in water solution were purchased from FND Biotech. Diamond is known as a hard dielectric material with a high refractive index value (2.44 at $532 \mathrm{~nm})$. In addition, the NV- centers in the diamond particles show a bright, robust (no photobleaching or blinking effects), red fluorescence.

\section{Optical setup}

In order to perform optofluidic experiments, we developed a home-made system to inject the sample solution inside a tapered capillary. After cleaving the capillary ends, the light from a green laser diode emitting at $532 \mathrm{~nm}$ is coupled to the capillary via a cleaved optical fiber coated with immersion oil. A fluorescence microscope is used to observe and record the motion of the particles inside the capillary.
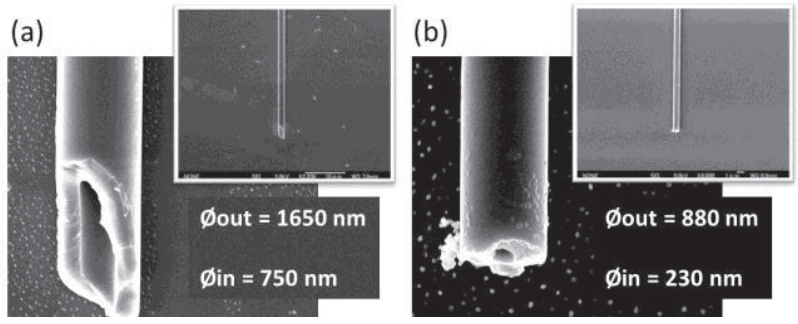

Figure 2. Scanning electron microscopy images of two broken tapered glass capillaries of different sizes.

\section{Results and Discussion}

By monitoring the output power at the end of a solution-filled tapered capillary, we could measure transmission efficiency values reaching $20 \%$. The transmission efficiency takes into account both the fiberto-capillary coupling losses at the input end of the capillary and the radiative losses occurring in the tapered region of the capillary.

Using a tapered capillary of $6.7 \mu \mathrm{m}$ outter diameter and an input laser power of few tens of milliwatts, the propulsion of the brightest diamonds inside the capillary was successfully observed. The experiment was reproduced in a $2.2 \mu \mathrm{m}$ outter diameter capillary. By analyzing the motion of the particles, we could measure particle velocity values reaching few tens of micrometers per second. Varying the input laser power allowed us to control the particle velocity.

\section{Conclusion}

This work aims at developing new optofluidic platforms using tapered glass capillaries. We first investigate the optical properties of water-filled capillaries. Then, we successfully fabricate tapered capillaries with micrometer and sub-micrometer dimensions. After injecting a sample solution containing red fluorescent nanodiamonds inside a tapered capillary, the optical propulsion of bright diamonds is achieved under green laser light illumination.

\section{Acknowledgements}

We acknowledge financial support from JSPS KAKENHI (Grant Numbers JP16H06506, JP18H05205, and JP18H03882). C.P. also acknowledges funding from 
the Research Institute for Electronic Science, Hokkaido University.

\section{References}

[1] Imasaka, T., Kawabata, Y., Kaneta, T. and Ishidzu, Y., "Optical chromatography," Anal. Chem. 67, 17631765 (1995).

[2] Hart, S. J. and Terray, A. V., "Refractive-index-driven separation of colloidal polymer particles using optical chromatography," Appl. Phys. Lett. 83, 5316-5318 (2003).

[3] MacDonald, M. P., Spalding, G. C. and Dholakia, K., "Microfluidic sorting in an optical lattice," Nature 426, 421-424 (2003).

[4] Ploschner, M., Cizmar, T., Mazilu, M., Di Falco, A. and Dholakia, K., "Bidirectional optical sorting of gold nanoparticles," Nano Lett. 12, 1923-1927 (2012).

[5] Tkachenko, G. and Brasselet, E., "Optofluidic sorting of material chirality by chiral light," Nat. Commun. 5 , 3577 (2014).

[6] Nan, F. and Yan, Z., "Creating Multifunctional Optofluidic Potential Wells for Nanoparticle Manipulation," Nano Lett. 18, 7400-7406 (2018).

[7] Shi, Y., Xiong, S., Chin, L. K., Zhang, J., Ser, W., Wu, J., Chen, T., Yang, Z., Hao, Y., Liedberg, B., Yap, P. H., Tsai, D. P., Qiu, C.-W. and Liu, A. Q., "Nanometerprecision linear sorting with synchronized optofluidic dual barriers," Sci. Adv. 4, eaao0773 (2018).

[8] Kawata, S. and Tani, T., "Optically driven Mie particles in an evanescent field along a channeled waveguide," Opt. Lett. 21, 1768-1770 (1996).

[9] Gaugiran, S., Gétin, S., Fedeli, J.M., Colas, G., Fuchs, A., Chatelain, F. and Dérouard, J., "Optical manipulation of microparticles and cells on silicon nitride waveguides," Opt. Express 13, 6956-6963 (2005).

[10] Yang, A.H., Moore, S.D., Schmidt, B.S., Klug, M., Lipson, M. and Erickson, D., "Optical manipulation of nanoparticles and biomolecules in sub-wavelength slot waveguides," Nature 457, 71-75 (2009).

[11] Ahluwalia, B. S., McCourt, P., Huser, T. and Hellesø, O. G., "Optical trapping and propulsion of red blood cells on waveguide surfaces," Opt. Express 18, 2105321061 (2010).
[12] Scullion, M.G., Arita, Y., Krauss, T.F. and Dholakia, K., "Enhancement of optical forces using slow light in a photonic crystal waveguide," Optica 2, 816-821 (2015).

[13] Maimaiti, A., Truong, V. G., Sergides, M., Gusachenko, I. and Chormaic, S. N., "Higher order microfibre modes for dielectric particle trapping and propulsion," Sci. Rep. 5, 9077 (2015).

[14] Schein, P., O'Dell, D. and Erickson, D., "Orthogonal nanoparticle size, polydispersity, and stability characterization with near-field optical trapping and light scattering," ACS Photon. 4, 106-113 (2017).

[15] Pin, C., Jager, J.-B., Tardif, M., Picard, E., Hadji, E., de Fornel, F. and Cluzel, B., "Optical tweezing using tunable optical lattices along a few-mode silicon waveguide," Lab. Chip 18, 1750-1757 (2018).

[16] Garbos, M. K., Euser, T. G. and Russell, P. S. J., "Optofluidic immobility of particles trapped in liquidfilled hollow-core photonic crystal fiber," Opt. Express 19, 19643-19652 (2011).

[17] Unterkofler, S., Garbos, M. K., Euser, T. G. and Russell, P. S. J., "Long-distance laser propulsion and deformation-monitoring of cells in optofluidic photonic crystal fiber," J. Biophotonics 6, 743-752 (2013).

[18] Spesyvtseva, S. E. S., Shoji, S. and Kawata, S., "Chirality-Selective Optical Scattering Force on Single-Walled Carbon Nanotubes," Phys. Rev. Appl. 3, 044003 (2015). 


\title{
The History and Future of Optical Manipulation
}

\author{
Kishan Dholakia, \\ SUPA, School of Physics and Astronomy, University of St Andrews, St Andrews, North Haugh \\ UK KY16 9SS,
}

\section{Tel:0044 1334 463184; Fax 00441334463104 \\ Email:kd1@st-andrews.ac.uk}

The ideas of moving objects using laser beams, evoking concepts such as a "tractor beam" is typically thought of as in the realm of science fiction. In the laboratory science fiction turns into science fact: a powerful technique known as "optical tweezers" (OT) shows that micrometre-sized particles (and even biological material and atoms) can be grabbed, moved and generally manipulated without any physical contact using optical forces. This is a powerful demonstration of the optical dipole or gradient force in action. For such "optical tweezers" Arthur Ashkin was the co-recipient of the Nobel Prize in Physics in 2018. The approach has enabled unprecedented insight into biological molecules such as DNA and molecular motors as well as cell-cell interactions. In the mesoscopic world of optical tweezers, researchers are now harnessing these systems to study a host of fundamental science: this includes advanced colloidal interactions, dynamics of particles in various potentials (with strong analogues to atomic systems), optically bound matter, studies of the optical angular momentum of light. The list is ever growing.

This talk will give a perspective of the history and future of studies in optical manipulation. 


\title{
In-plane orbital motion of particles in microchannels induced by optical vortices
}

\author{
Ryoji Nakatsuka $^{\text {a }}$, Tetsuro Tsuji ${ }^{a}$, Tempei Tsujimura ${ }^{\text {a }}$, Ryo Nagura ${ }^{\text {a }}$, Kentaro Doi ${ }^{\text {, }}$, Satoyuki Kawano*a \\ ${ }^{a}$ Graduate School of Engineering Science, Osaka University, 1-3 Machikaneyama, \\ Toyonaka, Osaka, 560-8531, Japan
}

\begin{abstract}
We experimentally characterize the optical-vortex-induced orbital motion of particles in microchannels for the development of manipulation techniques. The use of channels with small confinement in the beam propagation direction leads to in-plane orbital motion. Thus, simple 2D image analysis enables quantitative evaluations of the diameter and velocity of the microparticle orbital motion. We carry out experiments with different topological charges of the Laguerre-Gaussian beam and compare the orbital diameters with those given by Rayleigh scattering theory. We find that the variation in diameter for different topological charges shows a good agreement with the theory.
\end{abstract}

Keywords: Optical manipulation, Rayleigh scattering, Laguerre-Gaussian beam, Optical vortex

\section{Introduction}

Since Allen's discovery that optical vortices have a polarization-independent orbital angular momentum, various studies have been carried out to characterize optical vortices and explore their applications, such as chirality control and optical trapping. In particular, it has been found that optical vortices can induce orbital motion of microparticles. Detailed analyses of the orbital motion and the extension of the particle diameter range to the nanoscale are important tasks for developing future applications of optical vortices for manipulation. In this paper, we analyze the motion of $1-\mu \mathrm{m}$ particles in microchannels and establish an experimental procedure to characterize the in-plane orbital motion.

\section{Experimental Method}

A liquid crystal on silicon - spatial light modulator (LCOS-SLM) was used to generate an optical vortex. A near-infrared laser $(\lambda=1064 \mathrm{~nm})$ was used as the incident Gaussian beam. In addition, an excitation beam $(\lambda=488$ $\mathrm{nm}$ ) was combined with a Laguerre-Gaussian (LG) beam and these beams were guided to an inverted microscope, as shown in Fig. 1, which was used to observe the fluorescence

*kawano@me.es.osaka-u.ac.jp

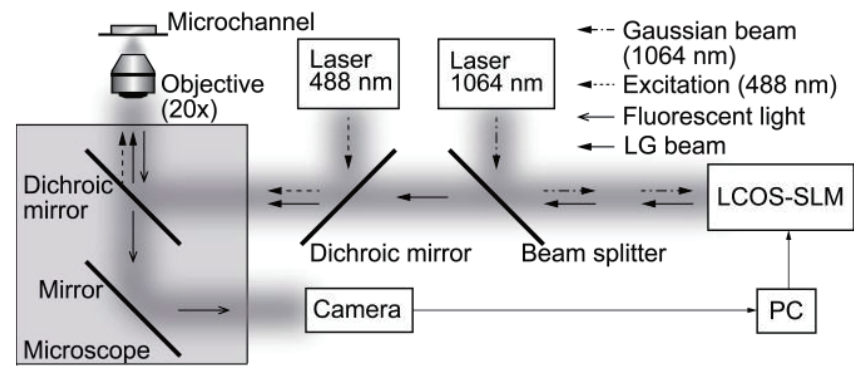

Fig. 1 Overview of optical setup.

of particles in microchannels with a height of $8.1 \mu \mathrm{m}$. Due to the small confinement in the beam propagation direction, we can obtain in-plane 2D video of the particle motion [1]. Since the optical forces strongly decrease with the particle diameter, smaller particles are more difficult to characterize. As a first step toward nanoparticle manipulation, we chose particles with a diameter of $1 \mu \mathrm{m}$ in this study to allow for high-resolution and high-speed observations, and established a method for evaluating the optical-vortexinduced particle motion. The aqueous solution used contained fluorescent polystyrene (PS) particles (radius $a=$ $500 \mathrm{~nm}$ ) and $1 \mathrm{wt} \%$ surfactant Triton X-100 to avoid agglomeration.

\section{Results and Discussion}

The gradient force mainly traps particles on the ringlike laser power intensity distribution with diameter $d_{m}=$ 
$2 w_{0}(m / 2)^{1 / 2}$, where $w_{0}$ is the beam waist at the focal plane [1]. On the other hand, a scattering force, which has an azimuthal component, drives the particle orbital motion. First, we show the orbital motion of two PS particles. When the microchannel was irradiated with an optical vortex with $m=5$, the particles underwent orbital motion, as shown in Fig. 2. Particles 1 and 2 orbited about the optical axis, which is the center of the panels, in the counterclockwise direction with time $t$. To evaluate the orbital diameter $d_{m}$, the images were averaged over entire frames and the resulting fluorescence intensity distributions were analyzed. It was found that $d_{5}=4.6 \mu \mathrm{m}$. An optical vortex with $m=7$ was also employed to investigate the effect of topological charge $m$ on the diameter, and $d_{7}=5.4 \mu \mathrm{m}$ was obtained. The ratio $d_{7} / d_{5}$ predicted by Rayleigh theory [3] is $(7 / 5)^{1 / 2}=$ 1.18 , which is in good agreement with the experimental value of 1.17. Therefore, Rayleigh theory well predicts the effect of topological charge $m$ on the diameter of the orbital motion.

The trajectory of the particle $(x, y)=\left(x_{p}(t), y_{p}(t)\right)$ was analyzed by the particle tracking method. Figure 3(a) shows $x_{p}$ and $y_{p}$ as functions of $t$. It is clear that the particle
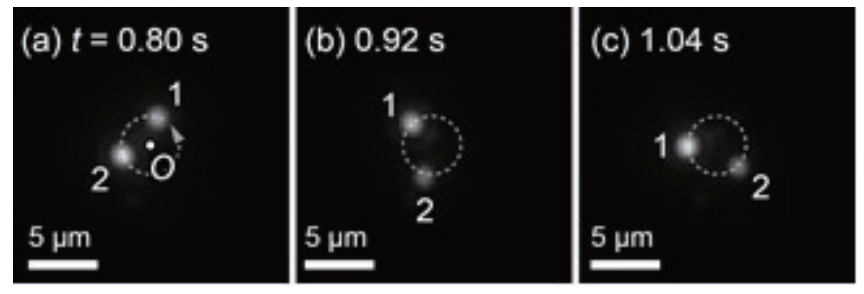

Fig. 2 In-plane orbital motion of particles induced by an optical vortex with a topological charge of $m=5$.
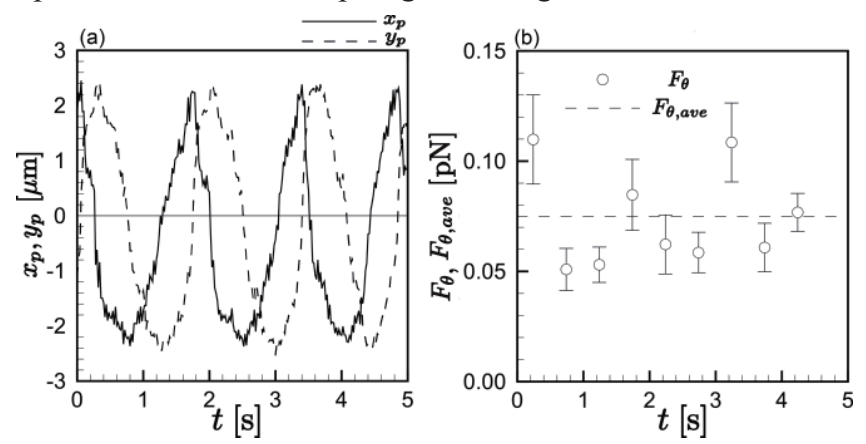

Fig. 3 (a) Trajectory of the particle. (b) Force component in the $\theta$ direction on the particle. underwent orbital motion with radius $r \approx 2.3 \mu \mathrm{m}$. We evaluated the force $F_{\theta}$ in the azimuthal direction $\theta$, which is shown in Fig. 3(b). Since the angular velocity of the particle is constant in time and the Reynolds number $\mathrm{Re}=2 \rho r v_{\theta} \mu^{-1}$ $=5 \times 10^{-5} \ll 1$ is negligibly small-where $\rho=10^{3} \mathrm{~kg} / \mathrm{m}^{3}$ is the mass density of water, $\mu=8.6 \times 10^{-4} \mathrm{~Pa} \cdot \mathrm{s}$ is the viscosity at $300 \mathrm{~K}$, and $v_{\theta}$ is the azimuthal velocity component-we assume that the azimuthal optical force $F_{\theta}$ is balanced with the Stokes drag $F_{\theta}=6 \pi \mu a v_{\theta}$, as in the case of the preceding study [1]. $F_{\theta}$ was $7.5 \times 10^{-2} \mathrm{pN}$ in the present case of the $1-\mu \mathrm{m}$ particles. Note that the radial optical force $F_{r}$ acts as a centripetal force, and was estimated as $2.0 \times 10^{-6} \mathrm{pN}$. In the case of nanoparticles, as the particle diameter decreases, the optical force decreases and Brownian motion becomes prominent; thus, a more careful analysis and a larger data set are necessary.

\section{Concluding Remarks}

In this study, forces acting on particles by an optical vortex were analyzed by the $2 \mathrm{D}$ image analysis of particle orbital motion near the upper wall of a microchannel. Rayleigh scattering theory was found to qualitatively predict the orbital diameter. A similar characterization of nanoparticles is planned for the future, and is expected to extend the range of applications to nanoscale biological objects, such as viruses or allergen particles with diameters of less than several hundreds of nanometers.

In the development of lab-on-a-chip fluidic sensors, combination with electrophoresis [4], thermophoresis [5], electrohydrodynamic flows [6][7][8], and/or the optical control of micro/nanoparticle motion [1][3] has attracted attention, and will be attractive for single-molecule measurements in the research area of bio-nanotechnology.

[1] Nito, F., et al., J. Phys. Chem. C 122, 17963-75 (2018).

[2] Ng, J., et al., Phys. Rev. Lett. 104, 103601 (2010).

[3] Nagura, R., et al., Proc. SPIE 10712, 107121C (2018)

[4] Tanaka, S., et al., Sci. Rep. 6, 31670 (2016).

[5] Tsuji, T., et al., Phys. Rev. Appl. 9, 024035 (2018).

[6] Yano, A., et al., Jpn. J. Appl. Phys. 56, 097201 (2017).

[7] Doi, K., et al., J. Chem. Phys. 148, 204512(2018).

[8] Nagura, R., et al., Micro Nano Lett. 12 526-531 (2017). 


\title{
Raman Imaging of Plasmonic Ag Nanostructure for Site-Dependent Molecular Trapping Analysis*
}

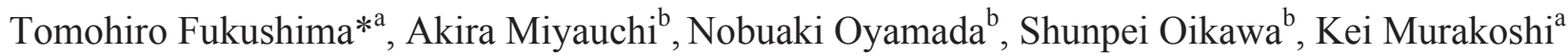 \\ ${ }^{a}$ Department of Chemistry, Faculty of Science, Hokkaido University, \\ Sapporo, Hokkaido 060-0810, Japan \\ ${ }^{\mathrm{b}}$ Graduate School of Chemical Sciences and Engineering, Hokkaido University, \\ Sapporo, Hokkaido 060-0810, Japan
}

\begin{abstract}
Here, we describe Raman imaging of plasmonic Ag nanostructure for site-dependent molecular trapping analysis under electrochemical potential control. Ag nanostructure was prepared by nanosphere lithography (NSL) techniques with the polystyrene templates. Excitation of Ag nanostructure with $633 \mathrm{~nm}$ laser induced the fluorescence from Ag nanocluster and Raman signals from 4,4'-bipyridine molecules. Adsorption states of molecules were defined during optical measurements by controlling the electrochemical potential. Raman imaging was conducted depending on molecular concentration and light intensity to investigate the site-dependent molecular trapping analysis. We found that we can classify the spots showing anomaly in particular to the light intensity from Raman imaging.
\end{abstract}

Keywords: Plasmonic nanostructure, Optical imaging analysis, Surface enhanced Raman spectroscopy, Electrochemistry, Optical trapping

\section{Introduction}

Metal nanostructures can confine incident light to induce localized surface plasmon polariton. Surface plasmon associates steep interfacial electromagnetic fields, which can enhance Raman scattering or luminescent signal from molecules at metal surface $[1,2]$. The enhancement factor heavily depends on the composition, size and shape of metal nanostructures [3]. In addition, Raman spectroscopy is a powerful tool to provide the molecular information such as number of molecules and molecular orientation at metal surface [4]. On the other hand, optical imaging of nanostructure provides statistical information on optical behavior based on the site-dependent time-course analysis. Imaging methods are actually useful because heterogeneity on the nanostructure can be also evaluated.

Here, we describe that our investigation on Raman imaging of plasmonic Ag nanostructure for sitedependent molecular trapping analysis. Ag nanostructure was prepared by beads template methods and optical properties are evaluated under electrochemical potential control. In addition, emission imaging provides us the information on the fluorescence from $\mathrm{Ag}$ nanocluster and surface enhanced Raman scattering (SERS) from surface adsorbing molecules. Interestingly, we found that this emission behavior is heavily dependent on molecular concentration and light intensity. The analysis of blinking behavior provides us the information on the heterogeneity on SERS-active nanostructure.

\section{Experiments}

A periodic array of triangular Ag nanoparticles was prepared on the conductive glass substrate, indium tin oxide (ITO) by the nanosphere lithography (NSL) technique [5]. Polystyrene beads with diameters of 350 $\mathrm{nm}$ were used for the preparation of the template. The Ag was vacuum-evaporated to a thickness of $30 \mathrm{~nm}$. The obtained substrate was denoted Ag NSL. The extinction, scattering and emission spectra of the prepared Ag NSL substrates were recorded using a multichannel spectrometer (MCPD-2000). The extinction and scattering spectra were recorded using as halogen lamp. The emission spectra and images were acquired from the objective-type $(60 \times)$ total internal reflection fluorescence (TIRF) microscopy using a $633 \mathrm{~nm}$ excitation laser $(\sim 0.1$ $\mathrm{mW} \mathrm{cm}^{-2}$ ), in which the emission with the wavelength 
longer than $670 \mathrm{~nm}$ was collected. Electrochemical experiments were conducted by three-electrode electrochemical cell placed on the top of microscope. Ag $\mathrm{NSL}, \mathrm{Ag} / \mathrm{AgCl}$, and $\mathrm{Pt}$ wire were used as working, reference, and counter electrodes respectively (Figure 1a). All the electrochemical potentials were referenced to the $\mathrm{Ag} / \mathrm{AgCl}$.
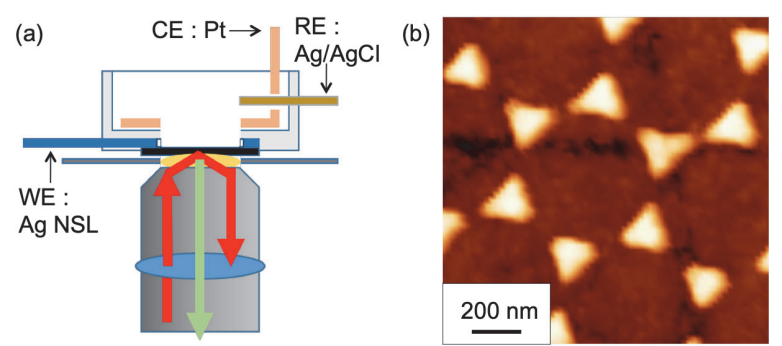

Figure 1. (a) TIRF imaging setup under electrochemical potential control. (b) AFM image of prepared Ag NSL on ITO substrate.

\section{Results and Discussion}

Successful fabrication of the NSL substrate was confirmed from AFM images as shown in Figure 1b. A characteristic array of triangular Ag nanoparticles was observed. Fabricated Ag NSL was used as working electrode and placed on the microscope for the optical measurements and immersed in $0.1 \mathrm{M} \mathrm{NaClO}_{4}$ respectively. Electrochemical potential was kept at $0 \mathrm{~V}$ to avoid the photo-oxidation of Ag NSL under intense laser irradiation during the optical experiments. Extinction spectra of Ag NSL showed broad peak located at $600 \mathrm{~nm}$. Scattering spectra of Ag NSL showed broad peak located at $700 \mathrm{~nm}$.

We studied emission properties of plasmonic Ag nanostructures. Emission spectrum was obtained through excitation of Ag NSL by $633 \mathrm{~nm}$ laser. Fluorescence behavior was observed around $710 \mathrm{~nm}$ which was due to the Ag nanocluster [5]. Addition of 4,4'-bipyridyine (denoted as bpy thereby) molecules into electrolyte did not influenced on neither extinction nor scattering spectrum but influenced on the emission spectrum. Intense sharp peaks are found at $704 \mathrm{~nm}$ and $688 \mathrm{~nm}$, respectively. These peaks are assigned to $1600 \mathrm{~cm}^{-1}$ and $1110 \mathrm{~cm}^{-1}$ in Raman scattering from surface adsorbing bpy molecules, respectively.
We examined the emission imaging of Ag NSL. Figure 2 a showed the $3 \mathrm{D}$ plot of averaged intensity depending on the sites. Averaged intensities of the emission sites were site dependent. This is originated from fluorescence from $\mathrm{Ag}$ nanocluster and SERS signal from molecules as discussed above. Figure $2 \mathrm{~b}$ shows the histogram for the averaged intensity from $\mathrm{Ag} \mathrm{NSL}$ in $0.1 \mathrm{M} \mathrm{NaClO}_{4}$ and $\mathrm{Ag}$ NSL in $0.1 \mathrm{M} \mathrm{NaClO}_{4}$ and $10 \mathrm{mM}$ bpy solution. In the absence of bpy, there was mainly single distribution located at 77 counts in averaged intensity. On the other hand, in the presence of bpy molecules, there were two distinguishable distributions located at 77 counts and 83 in averaged intensity. This can be understood as emission originates from SERS and fluorescence. Therefore SERS signal can be distinguished by considering the averaged emission intensity.

(a)

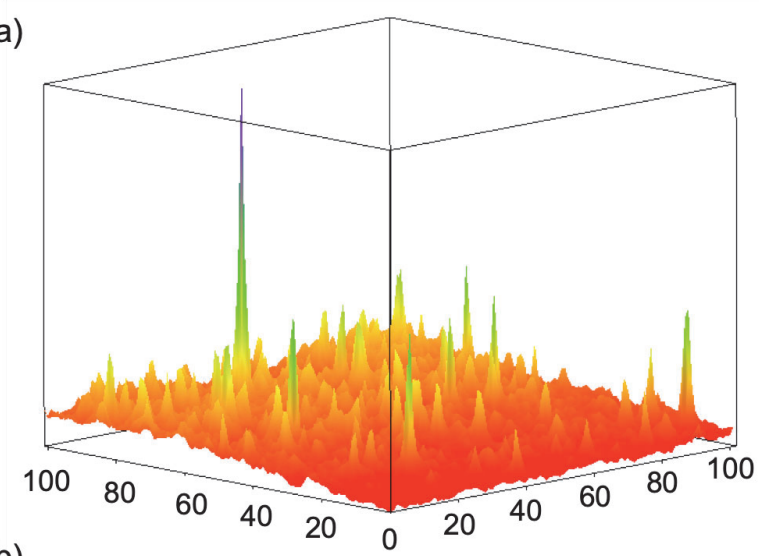

(b)

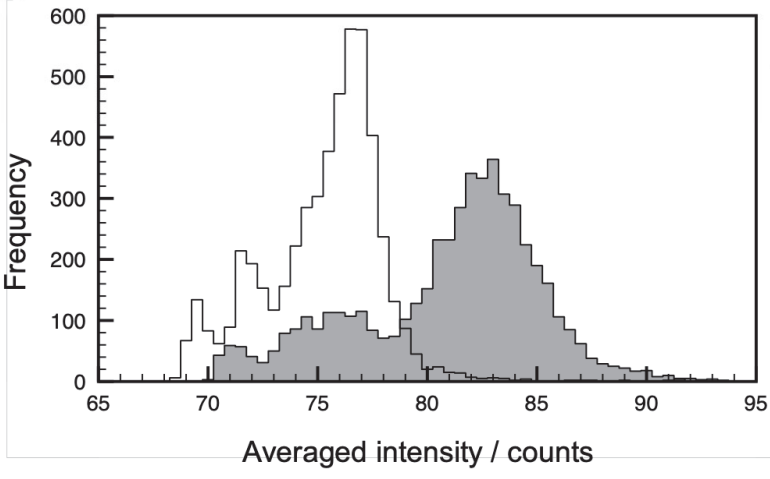

Figure 2. (a) 3D plot for averaged intensity from imaging analysis of Ag NSL in $0.1 \mathrm{M} \mathrm{NaClO}_{4}$ and 10 $\mathrm{mM}$ bpy aqueous solution. (b) Histogram of averaged intensity. White histogram : $\mathrm{Ag} \mathrm{NSL}$ in $0.1 \mathrm{M} \mathrm{NaClO}_{4}$ aqueous solution. Gray histogram : Ag NSL in $0.1 \mathrm{M}$ $\mathrm{NaClO}_{4}$ and $10 \mathrm{mM}$ bpy aqueous solution. 
Blinking behavior was examined through the imaging analysis. As discussed above, averaged intensity of sites are related to the SERS and fluorescent signal. Figure 3 shows the time-course of the intensity of signals at different sites with similar averaged intensity. Multiple "ON" states and intensive blinking were observed in Figure 3a. On the other hand, there were less "ON" state and blinking behavior was seldomly observed in Figure $3 b$.
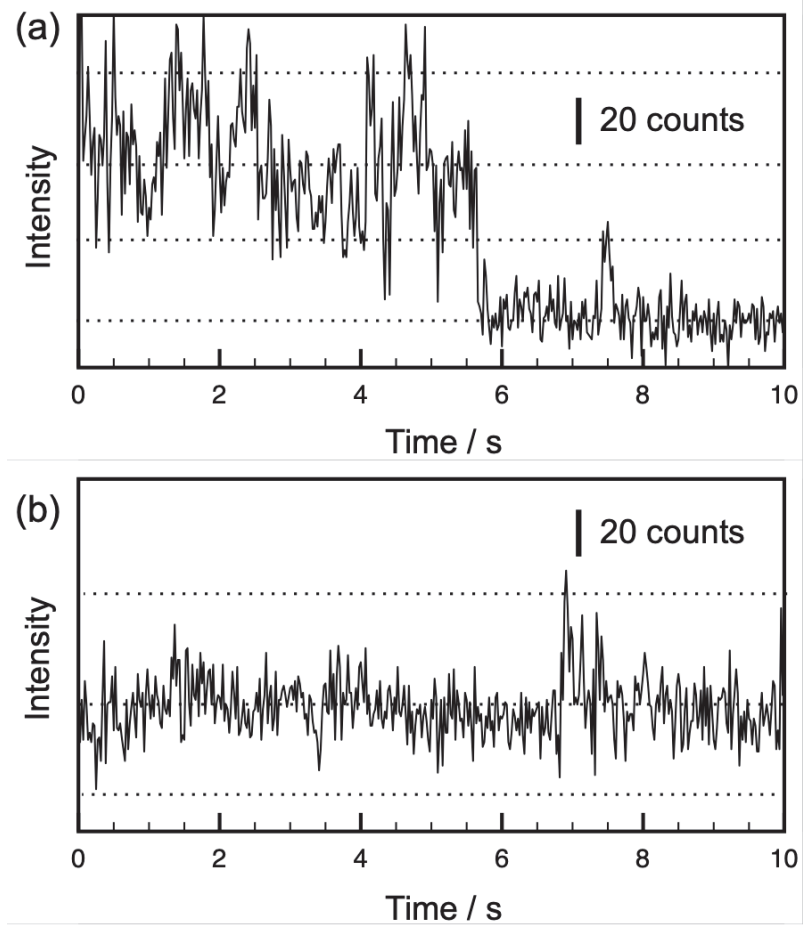

Figure 3. Blinking behavior of Ag NSL in $0.1 \mathrm{M}$

$\mathrm{NaClO}_{4}$ and $10 \mathrm{mM}$ bpy aqueous solution with

different sites with similar averaged intensity.

We found that this blinking behavior was dependent on the bpy concentration and light intensity. By increasing the concentration of bpy molecules in solution, Raman signals were hardly observed. Interestingly, some sites are actually light intensity dependent. As increasing the light intensity, higher intensity in SERS signals was observed. This observation suggests that plasmonic fields might enhance the optical trapping of molecules even at lower intensity.

In conclusion, we successfully demonstrated that Ag nanostructure can be utilized for the Raman imaging of site-dependent molecular trapping analysis. We found that Raman signals can be distinguishable even without spectral information. We also confirmed that SERS signals are dependent on concentration of molecules. Furthermore, we found that there are specific sites, which is actually responsible to the light intensity in terms of SERS signal. We are currently investigating the Raman imaging by using well-defined $\mathrm{Ag}$ nanostructure fabricated by electron beam lithography.

*tfuku@sci.hokudai.ac.jp; +81-11-706-4811

\section{References}

M. Fleischmann, P. J. Hendra, A. J. McQuillan, "Raman Spectra of Pyridine Adsorbed at a Silver Electrode" Chem. Phys. Lett., 26 (2), 163-166 (1974).

[2] M. Inoue, K. Ohtaka, "Surface Enhanced Raman Scattering by Metal Spheres. I. Cluster Effect" J. Phys. Soc. Japan, 52 (11), 3853-3864 (1983).

[3] N. J. Halas, S. Lal, W.-S. Chang, S. Link, P. Nordlander, "Plasmons in Strongly Coupled Metallic Nanostructures" Chem. Rev., 111 (6), 3913-3961 (2011).

F. Nagasawa, M. Takase, H. Nabika, K. Murakoshi, "Polarization Characteristics of Surface-Enhanced Raman Scattering from a Small Number of Molecules at the Gap of a Metal Nano-Dimer" Chem. Commun., 47 (15), 4514-4516 (2011).

[5] B. Takimoto, H. Nabika, K. Murakoshi, "Enhanced Emission from Photoactivated Silver Clusters Coupled with Localized Surface Plasmon Resonance" J. Phys. Chem. C, 113 (27), 11751-11755 (2009).

[6] A. Ashkin, J. Dziedzic, "Optical Levitation by Radiation Pressure" Appl. Phys. Lett. 19, 283-285 (1971).

[7] T. Shoji, Y. Tsuboi, "Plasmonic Optical Tweezers toward Molecular Manipulation: Tailoring Plasmonic Nanostructure, Light Source, and Resonant Trapping” J. Phys. Chem. Lett. 5, 2957-2967, (2014).

M. A. Osborne, S. Balasubramanian, W. S. Furey, D.

Klenerman, "Optically Biased Diffusion of Single Molecules Studied by Confocal Fluorescence Microscopy" J. Phys. Chem. B, 102, 3160-3167, (1998). 


\title{
Numerical demonstration of the alignment of multiple nanoparticles in a wide area beyond single focal laser spot*
}

\author{
Y. Tao ${ }^{a^{*}}$, T. Matsuurab ${ }^{b}$ T. Yokoyama ${ }^{a}$, H. Ishihara ${ }^{a, b}$

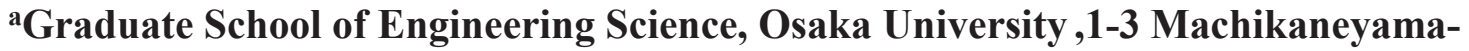 \\ cho, Toyonaka-shi, Osaka, 560-8531, Japan

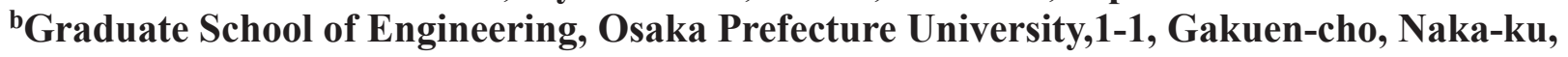 \\ Sakai-shi, Osaka, 599-8531, Japan
}

\begin{abstract}
Nanoscale objects demonstrate various optical response induced by the quantum size effect. The response would be controlled by ordering such small objects on a surface of material or substrate. In this study, we investigate a formation of two-dimensional arrangement by the optical force on multiple gold nanoscale particles. Only a single focused laser is applied to solvent with the particles. A lot of nanoparticles are trapped in the vicinity of focal spot. We focus on the multiple scattering of optical electric fields due to the nanoparticles and calculate the optical force numerically in the self-consistent method. When the linear polarized laser is applied, the nanoparticles show the formation in a linear arrangement. Such formation is not observed for the self-assembled growth, where the hexagonal close-packed structure is formed.
\end{abstract}

Keywords: Optical manipulation, Nano particles, Plasmonic particle, Scattering light

\section{Introduction}

Nanoscale materials show attractive optical properties due to the quantum size effect, where the characteristic lengths of electron, light, and material are comparable. The optical properties, e.g. spectrum of quantum dots [1], can be engineered by a management of their size and structure. Moreover, a regular alignment of nanomaterials would result in an exotic optical response not observed in nature. Such systems are called meta-material [2]. The fabrication of regular structure using nanoobjects is not easy due to difficulties of direct and selective operation.

An optical manipulation is another candidate to form the regular structure of nanomaterials. The optical manipulation is caused by the interaction between light and matter, where the optical force on small objects is induced by a momentum transfer from light to matter and a gradient of electromagnetic fields in space. The former and later forces are called scattering and gradient forces, respectively [3]. Ashkin et al. have demonstrated the optical tweezer by applying a single focused laser on a dielectric particle with microscale diameter [4]. The particles are trapped in the vicinity of focal point since the gradient force is enlarged. The optical manipulation has many advantages, e.g., a noncontact operation. The polarization and frequency degrees of freedom of incident laser lead to rich variety for the optical manipulation.

Recently, a formation of two-dimensional arrangement of polystyrene [5] and gold nanoparticles [6] on the glass substrate by a single focal laser has been reported by Kudo et al. They have observed a time-evolution of assembly of nanoparticles and revealed a dependence of assembly on the linear and circular polarizations. Moreover, they have found the optical trapping beyond the size of focal spot.

In this study, we investigate theoretically a formation of ordered two-dimensional structure of gold nanoparticles particles form scattered electric fields. We simulate the scattered fields numerically. The fields induce the optical force even on the particles located even at the outside of focal area. Each particle moves along the optical force and stops at a stable position determined by an interference between the scattered fields. We show clearly that the ordered structure of trapped nanoparticles by the focal laser is changed by the polarization.

\section{Model and Simulation Method}

In this section, we describe our model and method of numerical simulation. We consider multiple gold spherical nanoparticles in a water solvent. The solvent is sandwiched by two glass substrates. A focal laser is applied to the solvent. The incident direction is perpendicular to the glass substrate and the particles are optically trapped in the vicinity of focal point. We assume that the dynamics of particles is restricted in a two-dimensional space parallel to the substrate.

The optical electric fields are scattered by the multiple particles in the vicinity of focal point. The scattered fields by the particles have to be considered self-consistently. We utilize the T-matrix method [7] to obtain the self-consistent electric fields numerically. The optical force on each particle is evaluated from the Maxwell's stress tensor. This force drives the particle in the 2D plane.

The dynamics of particles follows the Langevin equation with the optical driving forces and the viscosity of solvent. After a short interval in time, all particles change their position. Therefore, the multiple scatterings of fields and the optical forces are also changed. We simulate the dynamics by calculating these processes in iterative.

We assume water and gold as solvent and trapped particles, respectively, whose refractive indices are $n_{\mathrm{w}}=$ 1.33 and $n_{\mathrm{Au}}=0.258+\mathrm{i} 6.97$. The diameter of spherical gold

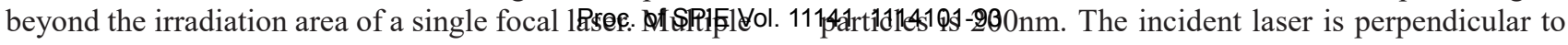




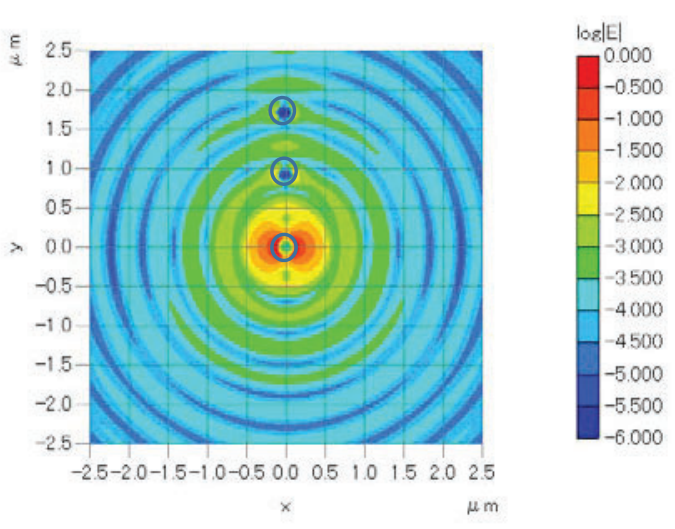

Figure 1. (Color) Electric field with three particles for linear polarization along the $x$-axis. Black circles indicate the position of gold particles.

the $x y$-plane and the center of focal point is located at the origin. The laser shape is described in term of the Gaussian function. The numerical aperture is NA $=0.95$. The wavelength is $1064 \mathrm{~nm}$. The particles can move in the $2 \mathrm{D}$ plane at $z=0$, however, we disregard the presence of glass substrate for simplicity in our numerical calculation. The random force for the Brownian motion is neglected. The viscosity of water is $0.890 \mathrm{mPa} \cdot \mathrm{s}$.

\section{Calculated Results}

\section{Linear polarization}

First, we apply a linear polarized incident field to the nanoparticles. In the case of linear polarization along the $x$-direction, the gold particles are aligned perpendicular to the linear polarization with finite distance between the particles. Figure 1 shows one of simulated results of the position of particles after the time-evolution following the optical driving forces. At the initial condition, one particle is trapped at the center of focal spot, and the others are distributed in the vicinity of the spot (not shown). Figure 1 indicates the final positions, where the particles are stable. The scattering from the central particle gives a main contribution to the scattered fields. If there is only one particle located at the origin, the system and scattered field shows a mirror symmetry with respect $y=0$. The scattered field exhibits an oscillation of field intensity, the period of which oscillation is comparable with halves of the wavelength $\lambda$ of incident light in the medium ( $\lambda \fallingdotseq 800 \mathrm{~nm})$. If one puts the second particle, the particle moves to the high intensity position and surround along the circle. At the end, it arrives on the $x=0$ line, where is the most stable point on the high intensity circle. The third particle is also located finally along or near the $y$-axis.

The presence of second, third, and more particles breaks the mirror symmetry. Moreover, we find an interference of electric fields due to the multiple scattering. The interference results in another stable position on the $y$ axis. If one adds several particles in Fig. 1, one of the particles might be trapped on $x=0$ line with a finite distance from the origin (not shown). It supports an

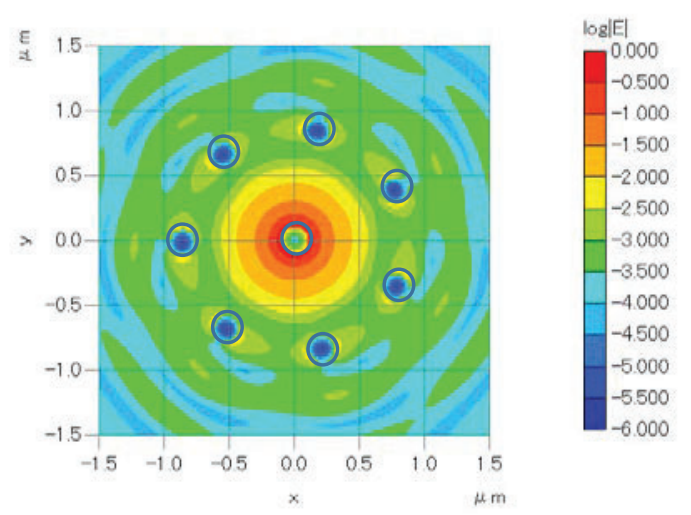

Figure 2. (Color) Electric field with seven particles for clockwise circular polarization. Black circles indicate the position of gold particles.

assembly formation in the $2 \mathrm{D}$ regular arrangement in experiment [7].

\section{Circular polarization}

Next, we consider a circular polarization. In this case, a scattered field is isotropic when only one particle is trapped at the origin. For the second particle, there is no stable position on the high intensity circle to stop the optical driving motion due to the rotational symmetry. Therefore, the (second, third, and more) peripheral particles continue to move along the high intensity circle in the clockwise or counterclockwise direction according to the circular polarization of incident field.

The radius of high intensity circle for the circular polarization is also integer multiple of the wavelength $\lambda$. Thus, the length of circle is $\lambda$. If the distance between the optically trapped particles is roughly $\lambda$, up to six particles are stored on the first circle, which means the hexagonal package. Figure 2 exhibits, however, storage of seven particles. This is note observed in the experiment [7].

\section{Conclusions}

We have demonstrated a numerical simulation of dynamics of multiple gold nanoparticles by a single focused laser. The focused laser induces the optical forces on the particles not only inside the focal spot but also out of the spot. Moreover, the optical forces depend on the polarization of incident laser. The dynamics is accelerated by the optical force. As a consequence, we obtain different $2 \mathrm{D}$ arrangement of gold nanoparticles according to the kinds of polarization.

[1] M. A. Reed et al. J. Vac. Sci. \& Tech., 4, 358 (1986).

[2] D. R. Smith et al. Phys. Rev. Lett., 84, 4184 (2000).

[3] A. Ashkin Phys. Rev. Lett., 24, 156 (1970).

[4] A. Ashkin et al. Optics Lett., 11, 288 (1986).

[5] T. Kudo, et al. Nano Lett., 16, 3058 (2016).

[6] T. Kudo, et al. Nano Lett., 18, 5846 (2018).

[7] D. W. Mackowski and M. I. Mishchenko, J. Opt. Soc.

Am. A 13, 2266 (1996). 


\title{
Polarization-Dependence of Optical Trapping on Polystyrene Nanoparticles and Their Assembly Formation
}

\author{
Tomohiro Yokoyama*a, Tomoki Matsuura ${ }^{\mathrm{b}}$, Yukihiro Tao ${ }^{\mathrm{a}}$, Hajime Ishihara ${ }^{\mathrm{a}, \mathrm{b}}$ \\ ${ }^{a}$ Graduate School of Engineering Science, Osaka University, 1-3 Machikaneyama, Toyonaka, Osaka, \\ 560-8531, Japan \\ ${ }^{\mathrm{b}}$ Graduate School of Engineering, Osaka Prefecture University, 1-1 Gakuen-cho, Naka-ku, Sakai, \\ Osaka, 599-8531, Japan
}

\begin{abstract}
We study theoretically an optical trapping of many nanoparticles by a single focused laser. The optical trapping of nanoparticles at an interface between a water solvent and a glass substrate forms a two-dimensional assembly of particles. Since the trapping force depends on the polarization of incident light, the shape and structure of assembly depends on the polarization. To understand an origin of the assembly formation, we examine numerical simulations of the light scattering and optical force with ordered structures of polystyrene nanoparticles by using the T-matrix method. The electric fields give the optical force on the polystyrene nanoparticles. The simulated results follow a formation of two-dimensional ordered structures beyond the focal size and their polarization-dependence. If the linear polarization is applied, the assembly shows a tetragonal regularly ordered structure. The scattering directions of light from the center of assembly and focal spot are four according to the symmetry of tetragonal structure. The particles swarming around the assembly are attracted to the scattering light by the gradient force, and would form four horns evolved from the assembly. In the case of circular polarization, the assembly of optically trapped particles shows a hexagonal structure. Then, the number of directions of the light scattering becomes six according to the symmetry of assembly. The number of scattering directions corresponds to horns. Our results for both polarizations agree with experiment by Kudo et al. [Nano Lett., 16, 3058 (2016)].
\end{abstract}

Keywords: Optical manipulation, Polystyrene nanoparticles, Assembly formation, T-matrix method

\section{Introduction}

An optical manipulation of small objects is attractive since it is a non-contact technique. The optical manipulation or trapping is proposed first by A. Ashkin in 1970 [1] and developed in physics, chemistry, and biology. The optical trapping utilizes a radiation pressure (scattering force) and a gradient force in a focused laser [2]. These forces are weaker as a volume of trapped material is smaller. Therefore, the optical manipulation for the nanoscale objects is a challenging issue.

Nanoscale materials, e.g. semiconductor quantum dot, are also attractive subject since the quantum and electronic characters depend on their size and shape significantly. Such property is called the quantum size effect. In other words, objects with identical composition but different sizes and shapes work as "different materials" each other. The optical property of such nano-materials also can be engineered by the size, shape, and structure [3]. In addition to the material engineering, the regular structure of such materials may induce and enhance their quantum properties. However, an accurate manipulation of nanoscale objects is not easy due to difficulties of direct and selective operation.
The optical trapping has a potential to form a regular structure of nano-materials. Recently, T. Kudo et al. have reported a formation of two-dimensional arrangement of polystyrene [4] and gold nanoparticles [5] by the optical trapping at an interface of water solvent and glass substrate. This trapping is achieved by a single focal laser. They have observed an assembly of optically trapped nanoparticles around the focal spot of laser. The assembly spreads optical beyond the size of focal spot and shows a regular arrangement of nanoparticles. Moreover, the arrangement depends on a kind of laser polarization.

In this study, we theoretically investigate the optical trapping of many polystyrene nanoparticles at water/glass interfaces. We examine a numerical simulation of light scattering and optical force based on the T-matrix [6]. First, some polystyrene particles are trapped by a focal laser, and the light is scattered by the trapped particles. The scattered electric field of light binds other particles at the outside of focal spot. Then, the bounden particles at the outside extend the scattered field. These iterative processes form the assembly of particles beyond the focal spot. Our numerical calculation supports this scenario. The arrangement of trapped particles depends on the kind of 
linear/circular polarizations. Our results explain the experimental observation in [4] qualitatively.

\section{Model and Method}

In this section, we describe our model and method of numerical calculation. We consider a lot of polystyrene spherical nanoparticles in a water solvent. The solvent is sandwiched by two glass substrates. A focal laser described by the Gaussian function is applied to the solvent. The direction of incident light is perpendicular to the substrate and the particles are optically trapped in the vicinity of focal point. We restrict the dynamics of particles in a twodimensional plane on the substrate.

An incident electric field of light is scattered by the polystyrene particles. In the presence of many scatterers, we have to take multiple scattering processes into account. We utilize the multiple T-matrix method [6] to obtain the electric field self-consistently. The optical force on each particle is evaluated from the electromagnetic fields by using the Maxwell's stress tensor.

In the numerical calculation, we assume polystyrene particles with the refractive index being $n_{\text {poly }}=1.59$ in a water solvent with $n_{\text {water }}=1.33$. The refraction and reflection of light due to the glass substrate are disregarded. The diameter of particles is $D=500 \mathrm{~nm}$. The wavelength of incident laser in vacuum is $\lambda=1064 \mathrm{~nm}$. The focused laser is described by the Gaussian function with a focal waist being $w_{0}=500 \mathrm{~nm}$, which is almost the diffraction limit. In the calculation, we place the particles with a regular structure in the vicinity of focal point. For the regular arrangement, a distance between the particles is $\Delta=550$ $\mathrm{nm}$ corresponding roughly to the experimental observation [4]. We set the $x$ - and $y$-axes parallel to the substrate and the $z$-axis is perpendicular.

\section{Calculated Results}

\section{Linear polarization with tetragonal arrangement}

We apply a focused laser with linear polarization along the $y$-direction when an assembly of polystyrene particles is present at the focal spot. The assembly is formed and kept by the optical force. In our simulation, we assume a regular tetragonal arrangement of nanoparticles [4], shown in Fig. 1. Here, 17 x $17=289$ particles are arranged in the tetragonal distribution.

Figure 1 shows a total electric field which is a sum of incident and scattered fields. Around the focal spot, the total field is relatively stronger due to the incident component. The incident field is scattered by all particles

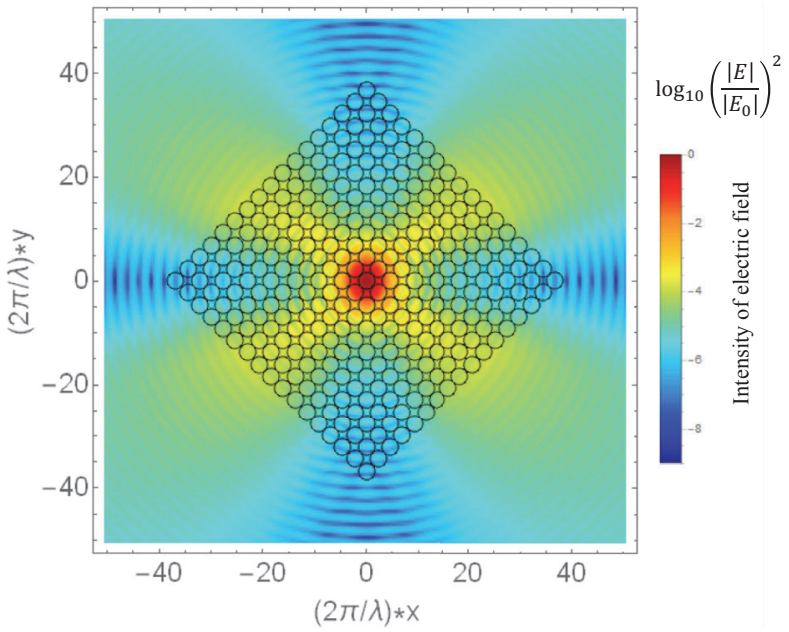

Figure 1. Total electric field with a tetragonal arrangement of 289 polystyrene nanoparticles. An incident light has the linear polarization in the $y$-direction. A normalized field intensity is plotted in the colored- and logarithmic-scale.

They are tilted about 60 degrees from the polarization direction. The trapped and surrounding particles are attracted to the enhanced field by the optical gradient force. Therefore, the spatial-distribution of scattering field supports the experimental observation with four horns developed from the assembly.

To discusses an influence of regular structure of trapped particles on the scattering field, we examine a randomly distributed assembly (not shown). For the random distribution, we find no particular enhancement nor structure of scattering field. Moreover, if the trapped particles form regular structure with multiple layers in the $z$-direction, the enhancement of scattering field is stronger than that by a single layer (not shown). These results mean a selfassembled optical trapping for multiple particles.

\section{2. circular polarization with hexagonal arrangement}

For the linear polarization, the system has a mirror symmetry at $x=0$. The circular polarization brings rotational symmetry if one takes a time average. Therefore, a regular structure of trapped particles shows a hexagonal arrangement [4]. Then, we examine the scattering electric field for the circular polarization by the hexagonal structure in Fig. 2. The number of particles in an assembly is 271 .

The scattering of light by the hexagonal arrangement is directed in six directions in Fig. 2, which follows the 6-fold symmetry of the hexagonal distribution. The surrounding particles are attracted by the gradient force to the scattered field in the six directions. The enhanced field in the six 


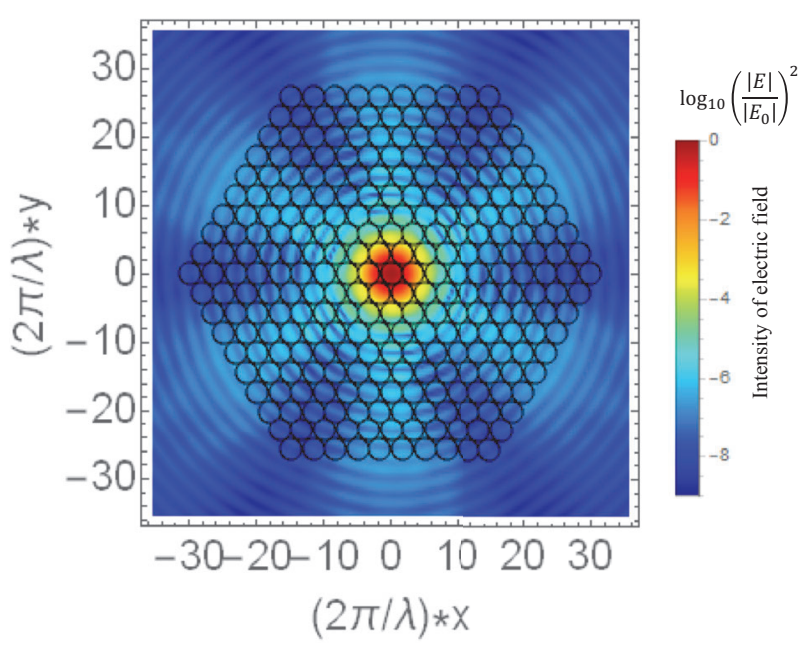

Figure 2. Total electric field with a hexagonal arrangement of 271 polystyrene nanoparticles. An incident light has the circular polarization. A normalized field intensity is plotted in the colored- and logarithmic-scale.

found in the experiment [4]. The scattering directions of light are perpendicular to the edges of particle arrangement, not be directed to its corners. This means that in the assembly, the light is scattered and enhanced by distributed particles, not propagates along the aligned particles. Moreover, the particles in the assembly are pushed by the optical force. The force on each particle is clockwise or counterclockwise direction against the center of laser spot according to the direction of circular polarization. Thus, the optically trapped assembly is rotated. These dynamics may be induced by a transfer of the angular momentum of circular polarization to the particles.

\section{Conclusions}

We have theoretically studied the scattering of light due to the optically trapped polystyrene nanoparticles. When one applies the focused laser to the water solvent with many polystyrene nanoparticles, the particles are optically trapped at the water/glass interface and form an assembly around the focal spot. The assembly of particles depends on the kind of polarization. The incident light is scattered by the trapped assembly, which scattering field depends on the distribution of assembly. Moreover, the scattered field traps surrounding particles. By these processes, we find the polarization-dependent assembly of particles beyond the laser focal spot. Our numerical calculation follows this self-assembled structure and the experimental observation in [4].

\section{References}

[1] Ashkin, A., "Acceleration and Trapping of Particles by Radiation Pressure," Phys. Rev. Lett. 24, 156 (1970).

[2] Iida, T. and Ishihara, H., "Theory of resonant radiation force exerted on nanostructures by optical excitation of their quantum states: From microscopic to macroscopic descriptions," Phys. Rev. B 77, 245319 (2008).

[3] Smith, A. M. and Nie, S., "Semiconductor Nanocrystals: Structure, Properties, and Band Gap Engineering," Acc. Chem. Res. 43, 190 (2001).

[4] Kudo, T., et al., "Optical Trapping-Formed Colloidal Assembly with Horns Extended to the Outside of a Focus through Light Propagation," Nano Lett., 16, 3058 (2016).

[5] Kudo, T., et al., "A Single Large Assembly with Dynamically Fluctuating Swarms of Gold Nanoparticles Formed by Trapping Laser," Nano Lett., 18, 5846 (2018). [6] Mackowski, D. W. and Mishchenko, M. I., "Calculation of the T matrix and the scattering matrix for ensembles of spheres,” J. Opt. Soc. Am. A 13,2266 (1996).

*tomohiro.yokoyama@mp.es.osaka-u.ac.jp;

phone:+81-6-6850-6504 


\title{
Manipulation of molecular ground-state photodissociation
}

\author{
on a gold nanoantenna surface
}

\author{
Ikki Morichika, Atsunori Sakurai, Satoshi Ashihara* \\ Institute of Industrial Science, The University of Tokyo, \\ 4-6-1, Komaba, Meguro-ku, Tokyo, 153-8505, Japan
}

\begin{abstract}
Plasmonic near-field enhancements offer extensive potential for amplifying light-matter interactions. A fascinating yet challenging application of plasmonics is to manipulate chemical reactions via enhanced near-fields. In this contribution, we demonstrate ground-state dissociation of a metal carbonyl compound in a liquid phase by employing plasmonic nearfields of chirped mid-infrared pulses. Both down-chirping and near-field enhancements contribute to enhance multiquantum vibrational excitation and to induce dissociation. Here we use pump-probe spectroscopy to observe cumulative adsorption of the dissociated species on the gold surface. Our results indicate that plasmonics, combined with midinfrared ultrashort pulse technologies, are useful for manipulating vibrationally-mediated chemical reaction control.
\end{abstract}

Keywords: surface plasmon, ultrafast infrared spectroscopy, vibrational ladder climbing, chemical reaction control

\section{Introduction}

Surface plasmon excitation provides electric-field enhancements on a nanometer scale, which offers extensive potential for amplifying light-matter interactions. One of the successful applications in the infrared (IR) region is surface-enhanced infrared absorption spectroscopy (SEIRAS) ${ }^{1}$. Recently, surface plasmons have also been applied to ultrafast IR spectroscopy, where third-order nonlinear signals were substantially amplified ${ }^{2-5}$ by IR-resonant nanoantennas $s^{6,7}$. Although most of the previous studies have focused on spectroscopic applications, plasmonic near-field enhancements may also be applicable to manipulation of chemical reactions via vibrational excitations.

Here we demonstrate vibrationally-mediated molecular ground-state dissociation in a condensed phase, for the first time to the best of our knowledge. Dissociation reaction is achieved through efficient multi-quantum vibrational excitation (or vibrational ladder climbing), driven by plasmonically-enhanced chirped mid-IR pulses. In our pump-probe reflection experiments, the enhanced mid-IR pump pulses efficiently induce multi-quantum vibrational excitation on the $\mathrm{T}_{1 \mathrm{u}} \mathrm{CO}$-stretching mode of $\mathrm{W}(\mathrm{CO})_{6} / n$-hexane solutions, resulting in $\mathrm{CO}$ dissociation. Here we observe cumulative adsorption of the dissociated species on the gold surface upon pump-pulse irradiation.

\section{Methods}

Gold nanoantennas are arranged in 2D arrays of rectangular lattice on a $1-\mathrm{mm}$ thick $\mathrm{CaF}_{2}$ substrate (Fig. 1a). Each nanoantenna is 1300 -nm long, 200 -nm wide, and 100-nm high. The size of the array unit cell is $2.0 \times$ $2.8 \mu \mathrm{m}$. A W(CO) $6 / n$-hexane solution is held between the substrate and another $\mathrm{CaF}_{2}$ window separated by a $25 \mu \mathrm{m}$ Teflon spacer. Our target mode is $\mathrm{T}_{1 \mathrm{u}} \mathrm{CO}$-stretching mode of $\mathrm{W}(\mathrm{CO})_{6}$, which exhibits the center frequency of $1983 \mathrm{~cm}^{-1}$ and the FWHM of $3 \mathrm{~cm}^{-1}$.

The reflectance spectrum for the nanoantenna array with the $\mathrm{W}(\mathrm{CO})_{6} / n$-hexane solution is shown in Fig. 1(b). The broad resonance peak, corresponding to localized surface plasmon resonance of the nanoantennas, is modulated at the absorption band of $\mathrm{W}(\mathrm{CO})_{6}$ with spectral features characteristic to Fano resonance.
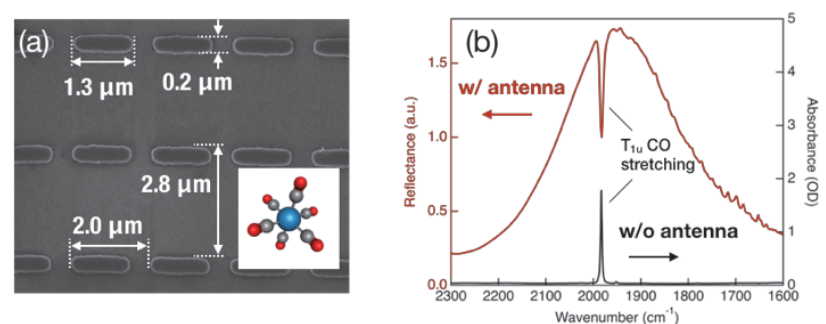

Figure 1. (a) A scanning electron microscope image of the gold nanoantenna arrays. (b) The measured reflectance spectrum for the nanoantenna arrays with a $\mathrm{W}(\mathrm{CO})_{6} / n$-hexane solution.

*ashihara@iis.u-tokyo.ac.jp 


\section{Results and Discussions}

Enhanced pump-probe reflection spectroscopy is performed by using mid-IR femtosecond pulses with the center frequency of $1945 \mathrm{~cm}^{-1}$ and the FWHM bandwidth of $180 \mathrm{~cm}^{-1}$ (Fig. 2a). Pump pulses are down-chirped by linear dispersion in a $40-\mathrm{mm} \mathrm{CaF}_{2}$ crystal, resulting in the group delay dispersion of $-24000 \mathrm{fs}^{2}$.

The transient reflectance change at 2 ps delay time after pump irradiation with fluence of $0.6 \mathrm{~mJ} / \mathrm{cm}^{2}$ is shown in Fig. 2(b). The antenna-coupled system exhibits a positive reflectance change at the fundamental $\mathrm{T}_{1 \mathrm{u}}$ mode frequency $\left(1982 \mathrm{~cm}^{-1}\right)$, attributed to ground-state bleach $(\mathrm{v}=0 \rightarrow 1)$ and stimulated emission ( $\mathrm{v}=1 \rightarrow 0$ ), and negative reflectance changes, attributed to excited-state absorptions: $\mathrm{v}=1 \rightarrow 2$ at $1967 \mathrm{~cm}^{-1}, \mathrm{v}=2 \rightarrow 3$ at $1953 \mathrm{~cm}^{-1}$, $\mathrm{v}=3 \rightarrow 4$ at $1931 \mathrm{~cm}^{-1}, \mathrm{v}=4 \rightarrow 5$ at $1908 \mathrm{~cm}^{-1}, \mathrm{v}=5 \rightarrow 6$ at $1889 \mathrm{~cm}^{-1}$, and $\mathrm{v}=6 \rightarrow 7$ at $1966 \mathrm{~cm}^{-1}$. These resonance frequencies agree with those reported in previous studies ${ }^{8}$. Note that this is the first observation of vibrational excitation up to $\mathrm{v}=6$, whose energy is close to the dissociation energy of one W-C bond.

(a)

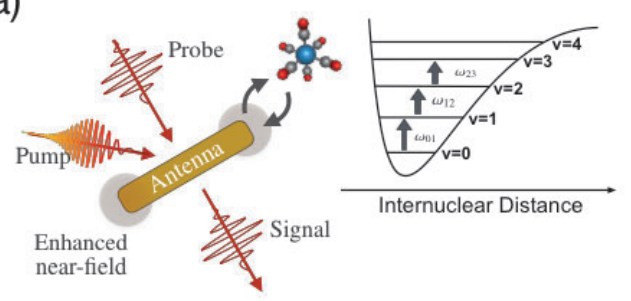

(b)

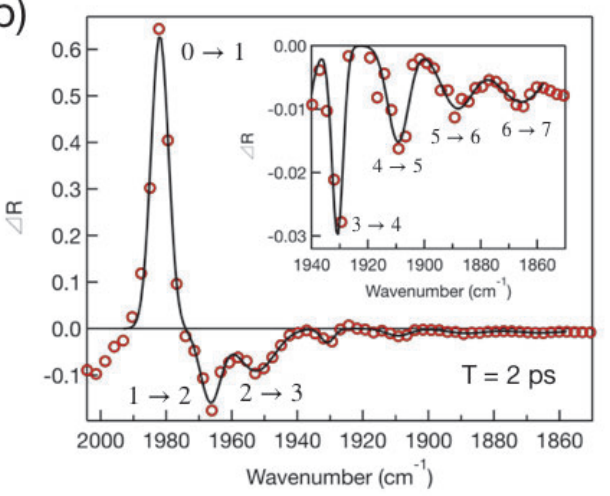

Figure 2. (a) A schematic of the antenna-enhanced vibrational ladder-climbing. (b) The transient reflectance change for the nanoantenna arrays with $\mathrm{W}(\mathrm{CO})_{6} / n$-hexane.
We monitor the transient reflection at 2 ps delay time for an hour, while irradiating the sample with the pump pulse that has group-delay dispersion of $-24000 \mathrm{fs}^{2}$ and fluence of $0.6 \mathrm{~mJ} / \mathrm{cm}^{2}$ at $1 \mathrm{kHz}$ repetition rate. Figure 3 shows the transient reflectance change measured at different irradiation time of 0-60 min. We clearly observe that a new band grows cumulatively with the irradiation. We also observe that the center frequency and the FWHM bandwidth of the band are blue-shifting and narrowing, respectively. After 60-min irradiation, the band exhibits the center frequency of $1930 \mathrm{~cm}^{-1}$ with FWHM of $22 \mathrm{~cm}^{-1}$.

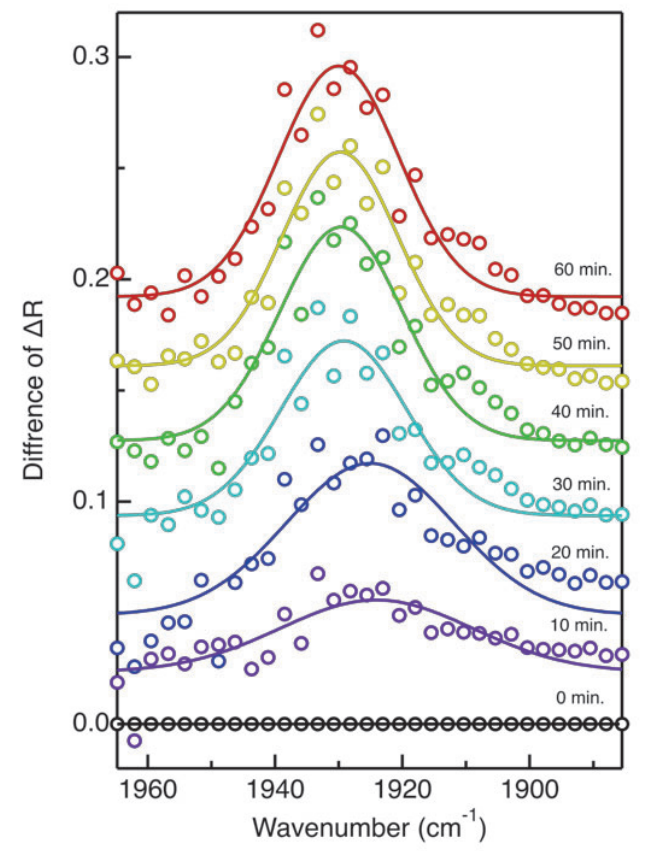

Figure 3. The transient reflectance change measured for different irradiation time of $0-60 \mathrm{~min}$. Solid lines represent Gaussian functions fitted to each spectrum.

The newly appearing band is attributed to ground-state bleach of the $\mathrm{A}_{1} \mathrm{CO}$-stretching mode of the adsorbate, whose resonance frequency coincides with those reported in previous studies. Therefore the presented results indicate that the down-chirped plasmonic near-fields induce $\mathrm{CO}$ dissociation in the carbonyl compounds and the dissociated species are adsorbed on the gold surface. The fact that we observe multi-quantum vibrational excitation up to the $\mathrm{v}=6$ state, whose energy is comparable to the first bond dissociation energy, also suggests the dissociation reaction. 


\section{References}

[1] F. Neubrech, C. Huck, K. Weber, A. Pucci, and H.

Giessen, "Surface-Enhanced Infrared Spectroscopy Using Resonant Nanoantennas," Chem. Rev. 117, 5110 (2017).

[2] P. M. Donaldson and P. Hamm, "Gold Nanoparticle Capping Layers: Structure, Dynamics, and Surface Enhancement Measured Using 2D-IR Spectroscopy," Angew. Chem., Int. Ed. 52, 634 (2013).

[2] O. Selig, R. Siffels, and Y. L. A. Rezus, "Ultrasensitive Ultrafast Spectroscopy Employing the Near Field of Gold Nanoantennas," Phys. Rev. Lett. 114, 233004 (2015).

[3] F. Kusa, I. Morichika, A. Takegami, and S. Ashihara, "Enhanced ultrafast spectroscopy using coupled nanoantenna arrays," Opt. Express 25, 12896 (2017).

[4] I. Morichika, F. Kusa, A. Takegami, A. Sakurai, and S. Ashihara, "Antenna-Enhanced Nonlinear Infrared Spectroscopy in Reflection Geometry," J. Phys. Chem. C 121, 11643 (2017).

[5] A. Gandman, R. Mackin, B. Cohn, I. V. Rubtsov, and L. Chuntonov, "Two-Dimensional Fano Lineshapes in Ultrafast Vibrational Spectroscopy of Thin Molecular Layers on Plasmonic Arrays," J. Phys. Chem. Lett. 8, 3341 (2017).

[6] F. Kusa and S. Ashihara, "Spectral Response of Localized Surface Plasmon in Resonance with MidInfrared Light," J. Appl. Phys. 116, 153103 (2014). [7] F. Kusa, K. E. Echternkamp, G. Herink, C. Ropers, and S. Ashihara, "Optical field emission from resonant gold nanorods driven by femtosecond mid-infrared pulses," AIP Advances 5, 077138 (2015).

[8] T. Witte, J. S. Yeston, M. Motzkus, E. J. Heilweil, and K. L. Kompa, "Femtosecond infrared coherent excitation of liquid phase vibrational population distributions (v > 5)," Chem. Phys. Lett. 392, 156 (2003). 


\title{
Weak value amplification of skew aberration
}

\author{
C T Samlan* and Yoko Miyamoto \\ Department of Engineering science, The University of Electro-communications, Chofu, Tokyo, Japan \\ $-182-8585$
}

\begin{abstract}
Here we propose a weak measurement scheme to experimentally characterize the lowest order skew aberration in a two lens paraxial system. The numerical analysis shows weak value amplification of sub-wavelength skew aberration to the beam waist scale.
\end{abstract}

Keywords: Skew aberration, weak measurement, paraxial optics

*samlanctcmr@gmail.com

\section{Introduction}

Skew aberration is a weak polarization rotation associated with the accumulation of spin-redirection geometric phase by the spin (circular) polarization components due to the parallel transport of the polarization vector along the skew rays [1] of a beam. The lowest order of skew aberration is the linear polarization rotation perpendicular to the meridional plane. Linear polarization aberrations are observed in rotationally symmetric nonpolarizing optical systems such as lenses and curved mirrors even when all Fresnel $s$ and $p$ coefficients are equal [2]. The skew aberration of a two lens paraxial system can be visualized as the rotation of each ray's polarization state between the entrance pupil (I) and the exit pupil (II) as shown in the first two pictures in figure 1 (a). Figure. 1(a)III shows the consequence of linear skew aberration at the image plane, where the right (red) and left (blue) circular polarization components are slightly shifted perpendicular to the meridional plane. Though such shifts due to skew aberrations in paraxial systems are on the order of a few thousandths of the wavelength, it can be observed as a beam width scale effect using an amplification technique called weak measurement.

Although weak measurement was introduced in 1988 in the context of quantum mechanics [3], a strong mathematical analogy with classical wave optics has led the concept of weak measurement to be used as a powerful tool to amplify and measure subwavelength optical effects such as spatial shifts and angular deflections of beams [49] and optical rotations $[10,11]$. In a weak measurement, a property of a system (the observable) is first weakly coupled to a separate degree of freedom (the meter) of the system, then the information about the observable is obtained from the meter. In this work we employ the weak measurement to amplify and characterize the linear weak skew aberration of a paraxial system of two thin lenses.

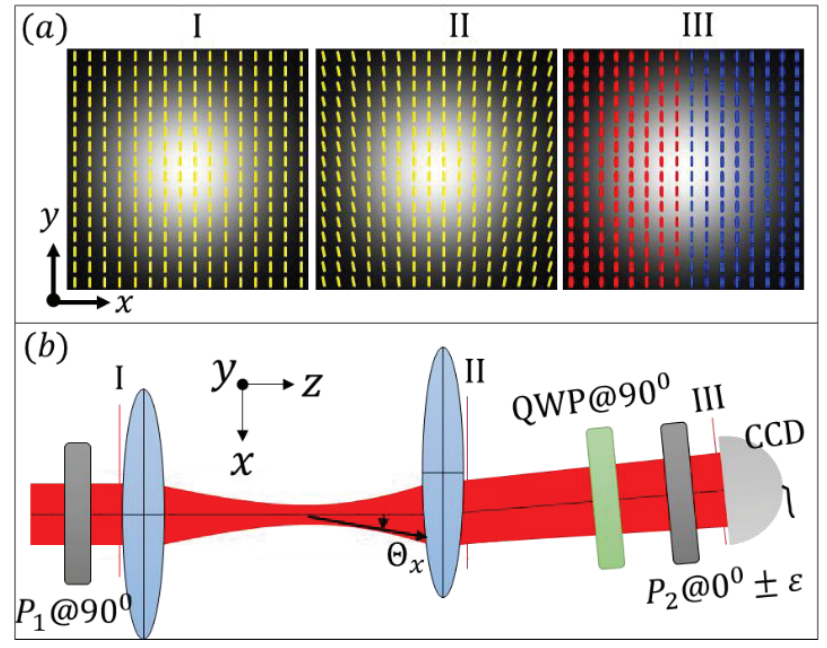

Figure 1. (a) Simulated polarization distribution of the optical beam at different planes for beam width $\omega_{0}=8$ in mesh units and aberration $\Delta=1^{\circ}$ in a $16 \times 16$ mesh. (b) The proposed experimental arrangement for the weak value amplification of the skew aberration (top view). $\mathrm{P}_{1}$ and $\mathrm{P}_{2}$ : Glan-Thompson polarizer, QWP: quarter wave plate, and CCD: charge-coupled device.

\section{Weak measurement of skew aberration}

We choose the polarization state of a Gaussian beam as the observable and the transverse momentum vector with its 
canonical conjugate transverse position as the meter for the weak measurement of the skew aberration. The Gaussian beam can be represented as a superposition of multiple plane waves with momentum vectors closely aligned to the $z$ direction:

$\mathbf{k}=k_{x} \hat{x}+k_{y} \hat{y}+k_{z} \hat{z} \sim k \Theta_{x} \hat{x}+k \Theta_{y} \hat{y}+k\left(1-\frac{\Theta^{2}}{2}\right)$.

Here $\hat{x}, \hat{y}$ and $\hat{z}$ are the unit vectors in the $x, y$, and $z$ directions; $k$ is the wave number, $\Theta_{x}$ and $\Theta_{y}$ are the angular separation of the wave vectors with respect to the $z$-axis, and $\Theta^{2}=\Theta_{x}^{2}+\Theta_{y}^{2} \ll 1$. The momentum representation of the transverse electric field of the Gaussian beam can be expressed as

$$
\tilde{E}_{\perp}(\Theta) \propto\left(\begin{array}{l}
\alpha \\
\beta
\end{array}\right) \exp \left[-k^{2} \omega_{0}^{2} \frac{\Theta^{2}}{4}\right] .
$$

Where the normalized Jones vector $\left(\begin{array}{ll}\alpha & \beta\end{array}\right)^{T}$ represents the state of polarization of the beam in $x$ and $y$ basis, and $\omega_{0}$ is the beam width. The Fourier transform of the above equation leads to the real space representation of the beam

$$
E_{\perp}(\mathrm{R}) \propto\left(\begin{array}{l}
\alpha \\
\beta
\end{array}\right) \exp \left[-\frac{R^{2}}{\omega_{0}^{2}}\right]
$$

where $R^{2}=x^{2}+y^{2}$ is the square of the transverse distance from beam axis.

Now we are in a position to employ the weak measurement on the polarization aberration. Consider a pre-selected vertically polarized Gaussian beam in momentum space [8] as the initial state

$$
\tilde{E}_{\perp}^{i}(\Theta) \propto|\psi\rangle \exp \left[-k^{2} \omega_{0}^{2} \frac{\Theta^{2}}{4}\right],
$$

where $|\psi\rangle=\left(\begin{array}{l}0 \\ 1\end{array}\right)$ represents the vertical polarization state. The linear polarization aberration arises from the accumulation of spin-redirection geometric phase associated with rays with $\Theta_{x} \neq 0$, while rays with $\Theta_{x}=0$ and $\Theta_{y} \neq 0$ exhibit zero aberration in our particular system shown in Figure 1(a). This introduces coupling between the polarization state and the momentum vector and results in a transformation of the initial beam to

$$
{\widetilde{E^{\prime}}}_{\perp}\left(\Theta_{x}\right) \propto e^{i \Delta \Theta_{x} \widehat{\sigma}_{3}}|\psi\rangle \exp \left[-k^{2} \omega_{0}^{2} \frac{\Theta^{2}}{4}\right] .
$$

The coupling strength $\Delta \ll 1$ represents the magnitude of skew aberration and $\hat{\sigma}_{3}=\left(\begin{array}{cc}0 & -i \\ i & 0\end{array}\right)$ is a Pauli matrix associated with the circular polarization Stokes parameter $S_{3}$ in the current notation. We post-select the beam desribed in equation (5) onto a polarization state $|\varphi\rangle=$ $\left(\begin{array}{l}-i \\ \pm \varepsilon\end{array}\right)$, which is nearly orthogonal to the pre-selected state with $|\varepsilon| \ll 1$. This results in

$$
\begin{aligned}
& \tilde{E}_{\perp}^{f}\left(\Theta_{x}\right) \propto\left\langle\varphi\left|e^{i \Delta \widehat{\sigma}_{3} \Theta_{x}}\right| \psi\right\rangle \exp \left[-k^{2} \omega_{0}^{2} \frac{\Theta^{2}}{4}\right] \\
& \sim\left(\langle\varphi \mid \psi\rangle+i \Delta\left\langle\varphi\left|\hat{\sigma}_{3}\right| \psi\right\rangle \Theta_{x}\right) \exp \left[-k^{2} \omega_{0}^{2} \frac{\Theta^{2}}{4}\right] \\
& =\langle\varphi \mid \psi\rangle\left(1+i \Delta\left\langle\sigma_{3}\right\rangle_{w} \Theta_{x}\right) \exp \left[-k^{2} \omega_{0}^{2} \frac{\Theta^{2}}{4}\right] \\
& \sim\langle\varphi \mid \psi\rangle e^{i \Delta\left\langle\sigma_{3}\right\rangle_{w} \Theta_{x}} \exp \left[-k^{2} \omega_{0}^{2} \frac{\Theta^{2}}{4}\right] .
\end{aligned}
$$

The quantity $\left\langle\sigma_{3}\right\rangle_{w}=\frac{\left\langle\varphi\left|\hat{\sigma}_{3}\right| \psi\right\rangle}{\langle\varphi \mid \psi\rangle}=\frac{1}{ \pm \varepsilon}$ is defined as the weak value of the observable $\hat{\sigma}_{3}$. The Fourier transform of the equation (6) results in the real space representation of the final beam

$$
E_{\perp}^{f}(x) \propto \pm \varepsilon \exp \left[-\frac{\left(x+\frac{\Delta}{2 \pi} \lambda\left\langle\sigma_{3}\right\rangle_{w}\right)^{2}}{\omega_{0}^{2}}\right] .
$$

The above equation represents a Gaussian whose centroid is shifted to $(\Delta / 2 \pi) \lambda\left\langle\sigma_{3}\right\rangle_{w}$, and thus the information on polarization aberration is transferred to the transverse position of the beam. Significantly, the sub-wavelength shift $(\Delta / 2 \pi) \lambda$, corresponding to the case where both $|\psi\rangle$ and $|\varphi\rangle$ are the same circular polarization state, is amplified to the beam width scale using weak measurement. This allows the aberration to be characterized precisely. The amplification factor $\left\langle\sigma_{3}\right\rangle_{w}=$ $1 / \pm \varepsilon$ can be regulated by the post-selection polarizer angle $\varepsilon$.

The experimental scheme to realize the weak value amplification of the beam shift is shown in Figure 1(b). A vertically oriented polarizer $\mathrm{P}_{1}$ is employed to pre-select the state of the initial beam, while a combination of a vertically oriented quarter wave plate (QWP) and a polarizer nearly oriented in horizontal direction is used to post-select the beam into its final state. The second lens in the telescopic system is slightly moved in transverse $x$ direction to enhance the linear polarization aberration over its higher order counterparts. A charge-coupled device camera is used to record the intensity profile of the final beam, from which the weak value and subsequently the polarization aberration parameter is obtained. The 
amplified shift in the beam's centroid is visualized numerically and discussed in the next section.

\section{Results and Discussion}

Weak values are complex in general and the real and imaginary parts can be switched by appropriately performing the pre- and post-selections. In general, the real part of the weak value leads to a transverse shift in real space while its imaginary part leads to a transverse shift in the corresponding momentum space. In other words, the real and imaginary parts respectively result in transverse spatial and angular shifts in real space. However, here we transformed the pure complex weak value to a real value by incorporating the QWP before the final polarizer, and thus the shift in the centroid of the final beam corresponds to the amplified spatial shift between the circular polarization components at the image plane.

Figure 2(a) shows a numerically simulated intensity profile of the initial beam and Figure 2(b), (c), and (d) correspond to the final beam for the post-selected states $\varepsilon=0^{\circ},-2^{\circ}$, and $+2^{\circ}$ respectively. The second row shows line profiles of the images at $y=0$ indicated with the weak values obtained from the shift in the centroid. (a)
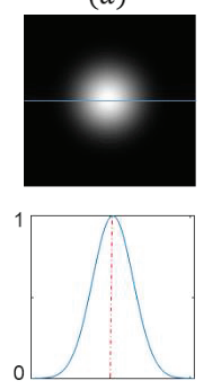

(b)
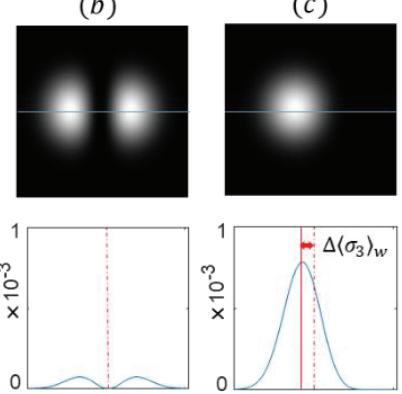

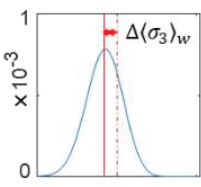

(d)
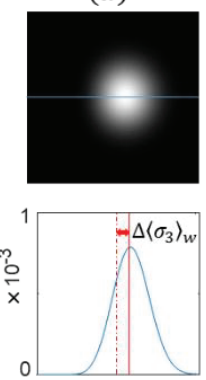

Figure 2. Simulated intensity profiles for $\omega_{0}=8$ and $\Delta=$ $0.1^{\circ}$ in a $16 \times 16$ mesh. (a) Initial beam with vertically polarized preselected state and final beam with post selected states (b) $\varepsilon=0^{\circ}$, (c) $\varepsilon=-2^{\circ}$, and (d) $\varepsilon=+2^{\circ}$.

\section{Summary}

Skew aberration is a special kind of weak polarization aberration that arises due to the geometry of the system. The actual magnitude of the skew aberration effect in paraxial systems is on the order of thousandths of the wavelength. Here we theoretically show the amplification of such skew aberration to the order of the beam width scale using the weak measurement protocol, and propose a corresponding experimental scheme. Amplification and the detailed characterization of the skew aberrations may find applications in microscopy and observational astronomy, especially when the data is polarization sensitive.

\section{References}

[1] G. Yun, C. Karlton and R. A. Chipman. "Skew aberration: a form of polarization aberration" Opt. Lett. 36, 4062-4064 (2011).

[2] R. A. Chipman, and W. S. T. Lam. "The point spread function in paraxial optics" Proc. of SPIE 9578, 957803 (2015)

[3] Y Aharonov, D. Z. Albert and L. Vaidman. "How the result of a measurement of a component of the spin of a spin-1/2 particle can turn out to be 100 " Phys. Rev. Lett. 60, 1351 (1988)

[4] I M Duck, P. M. Stevenson and E. C. G. Sudarshan. "The sense in which a "weak measurement" of a spin$1 / 2$ particle's spin component yields a value 100" Phys. Rev. D 40, 2112 (1989).

[5] N. W. M. Ritchie, J. G. Story and R. G. Hulet. "Realization of a measurement of a weak value" Phys. Rev. Lett. 66, 1107 (1991)

[6] O Hosten, and Paul Kwiat. "Observation of the spin Hall effect of light via weak measurements" Science 319, 787-790 (2008)

[7] P. B. Dixon, et al. "Ultrasensitive beam deflection measurement via interferometric weak value amplification” Phys. Rev. Lett. 102, 173601 (2009)

[8] K. Y. Bliokh, et al. "Spin-Hall effect and circular birefringence of a uniaxial crystal plate" Optica 3, 1039-1047 (2016)

[9] O S Magaña-Loaiza, et al. "Amplification of angular rotations using weak measurements" Phys. Rev. Lett. 112, 200401 (2014)

[10] B de Lima Bernardo, S. Azevedo and A. Rosas. "Ultrasmall polarization rotation measurements via weak value amplification" Phys. Lett. A 378, 2029-2033 (2014)

[11]X Qiu, et al. "Estimation of optical rotation of chiral molecules with weak measurements" Opt. Lett. 41, 4032-4035 (2016) 


\title{
Cryptanalysis of computational optical ghost imaging cryptosystems via deep learning
}

\author{
Wenqi HE*a, ${ }^{*}$, Bennian $\mathrm{HAN}^{\mathrm{a}, \mathrm{b}}$, Meihua LIAO ${ }^{\mathrm{a}, \mathrm{b}}$, Dajiang $\mathrm{LU}^{\mathrm{a}, \mathrm{b}}$, Han HAI ${ }^{\mathrm{a}, \mathrm{b}}$, Shuixin PAN ${ }^{\mathrm{a}, \mathrm{b}}$, Chenggong ZHANG ${ }^{\mathrm{a}}$ \\ b, Xiang PENG ${ }^{\mathrm{a}, \mathrm{b}}$ \\ ${ }^{a}$ Key Laboratory of Optoelectronics Devices and Systems, Education Ministry of China, Shenzhen 518060, China; \\ ${ }^{\mathrm{b}}$ College of Physics and Optoelectronic Engineering, Shenzhen University, Shenzhen 518060, China
}

\begin{abstract}
We introduce the powerful Deep Learning (DL) strategy to evaluate the security strength of computational-ghostimaging-based cryptosystems. It's well known that the Computational Ghost Imaging (CGI) technique has an excellent advantage of anti-noise ability, hence the CGI-based cryptosystems are much easier to be optically implemented, compared with previous various kinds of optical cryptosystems. However, an objective and comprehensive security analysis still must be carried out before any a cryptosystem is claimed to be safe or reliable. Unfortunately, we find out that the current CGI-based cryptosystems and even its security-enhanced ones both have security flaws. If an opponent gets a set of chosen plaintexts and their corresponding ciphertexts, an "equivalent key" or "equivalent decryption network" could be find out by training the pairs of "plaintext-ciphertext" with DL method, in which we select the Deep Neural Network (DNN) as the fundamental structure. Furthermore, we also carried out some simulations to demonstrate the performance of our proposed method under two severe conditions: down-sampling and Gaussian noise. We believe our methodology is suitable for a major part of existing optical cryptosystems including the CGI-based ones.
\end{abstract}

Keywords: Cryptanalysis, Optical encryption, Ghost imaging, Deep learning, Deep neural network

*Corresponding author: he.wenqi@qq.com

\section{Introduction}

How to ensure the privacy of messages in the process of transmission and storage is always an important issue especially in the past several decades, and as we known, various encryption techniques are the main way to guarantee that. In the past 20 years, image encryption schemes with optical means has got well development since Refregier and Javidi proposed the milestone work, Double Random Phase Encoding (DRPE) scheme based on traditional 4f optical systems in 1995 [1-5]. All of those works claimed a natural outstanding merit: the ability of parallel data processing, though most of them are hard to realize by current commercially available optical devices.

Ghost Imaging (GI), also known as correlated imaging, is a novel computational imaging technique that uses spatial correlation calculations to obtain the object information which is not captured directly by the camera. In 1995, Pittamn et al. first proposed and experimentally verified the traditional GI scheme based on entangled photon pairs [6]. Compared with most of the traditional optical imaging skills, the ghost imaging technique could improve the image quality, and most importantly it could survive in relatively harsh optical environments, that is with good anti-noise performance. In 2008, J. Shapiro presented a Computational Ghost Imaging (CGI) scheme [7]. By introducing a Spatial Light Modulator (SLM) to dynamically encode the light illuminated on object, one could retrieve the two-dimensional object information with only one Bucket Detector (BD), but without any array camera in the reference arm. This improvement greatly simplifies the experimental setup of classical GI, and thus ignite many researchers' interests [8-11]. One effort of them is to combine it with optical encryption [12-14]. In this contribution, we try to make attempts to 
crack the so-called CGI-based optical cryptosystems from the perspective view of cryptanalysis, that is Chosen Plaintext Attack (CPA) [15]. Thus, prompting further development of related encryption skills.

\section{Theoretical analysis}

\section{Brief introduction of CGI-based cryptosystems}

A schematic diagram of CGI-based optical cryptosystem is shown in Figure 1, and a brief description is provided as follows [12]: If Alice wants to send a secret message (plaintext) to Bob, suppose they both have a shared secret key in advance. Here, the key is defined as a one-dimensional random number sequence with $N$ numbers, which could control the generation of Random Phase Masks (RPMs). While encryption, $\mathrm{N}$ number of RPMs will be successively uploaded onto the SLM to modulate the light, which will then illuminate onto the two-dimensional object (usually an image, also called plaintext), finally, on the imaging plane a single-pixel BD will get a set of intensity values, which is regarded as ciphertext $\left(\left\{B_{\mathrm{i}}\right\}, i=N\right)$. In the decryption process, Bob could easily recover the plaintext by calculations in accordance with Eq. (1):

$$
P(x, y)=\frac{1}{N} \sum_{i=1}^{N}\left[B_{i}-\langle B\rangle\right] \cdot I_{i}(x, y)
$$

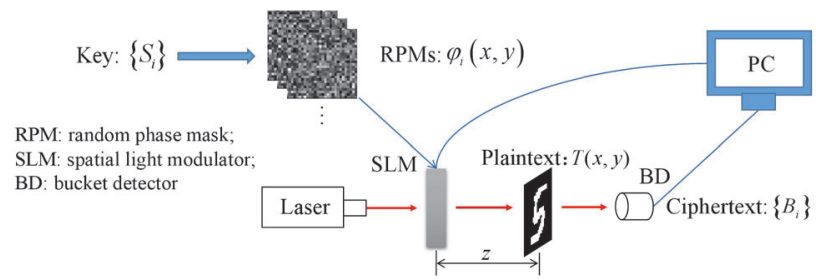

Figure 1. Schematic diagram of a CGI-based encryption

It's worthy to point out that the RPMs in Figure 1 could also be substituted by a set of orthogonal encoded fringes (structural light) [cite 2 references, Sine and Hadamard]. They are all called CGI-based cryptosystems. And our following analysis method is effective for all of them.

\section{Security analysis}

A flowchart of our crack method is illustrated by Figure 2. According to the fundamental Kerckhoffs rule, it's reasonable for us to get a set of ciphertexts while their correspond plaintexts are also known. Taking these pairs of "plaintext-ciphertext" as inputs (ciphertexts) and the constraints in the output side (plaintexts), it's possible to confirm all the crucial paraments of the designed Deep Neural Network (DNN) model by iteratively modification. The DNN model with obtained paraments is then regarded as "equivalent key" or "equivalent decryption network".

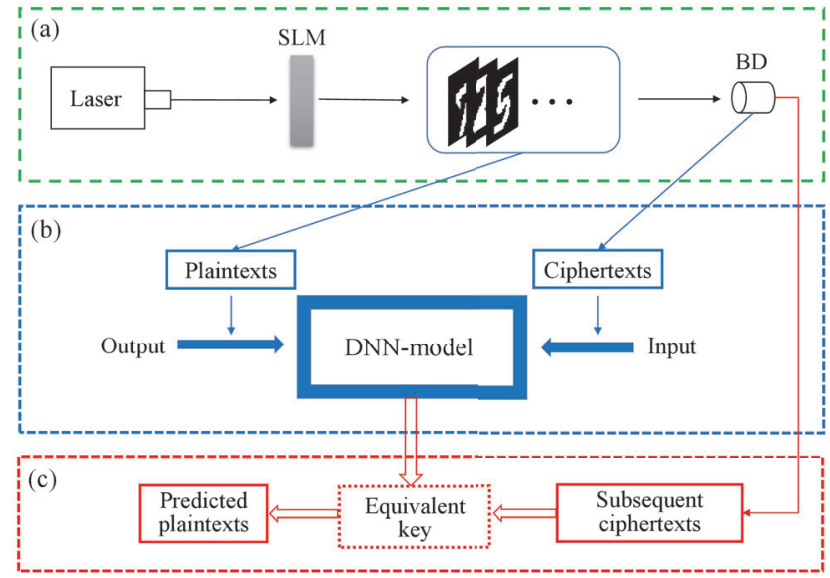

Figure 2. Flowchart of our proposed cryptanalysis

\section{Results and Discussion}

Some preliminary results are shown as Figure 3. In this test, we adopt the first CGI-based encryption scheme as the attack goal. It's easy to tell that our scheme works. More simulation and data analysis will be provided in the full-length manuscript.

(a)
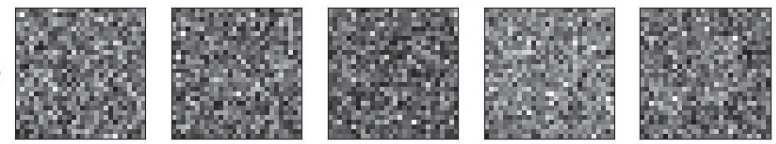

(b)
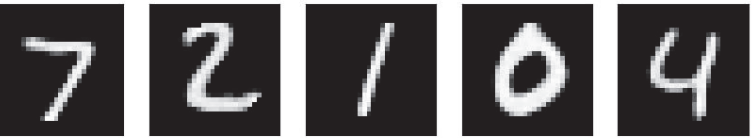

(C)
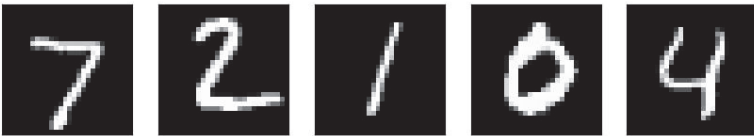

Figure 3. Simulation results, (a) five arbitrarily selected ciphertext sequences (re-arranged as two-dimensional matrixes) from the test data set; (b) predicted (cracked) plaintexts; and (c) the original plaintexts in the test data set (ground truth values) 


\section{References}

[1] Refregier, P., and B. Javidi., "Optical image encryption based on input plane and Fourier plane random encoding." Optics Letters.Papers20(7),767(1995).

[2] Matoba, O., Nomura, T., Perez-Cabre, E., Millan, M.

S., Javidi, B. "Optical techniques for information security." Proceedings of the IEEE, Papers97(6), 1128 1148(2009).

[3] Wang, X. G., D. Zhao, and L. Chen., "Image encryption based on extended fractional Fourier transform and digital holography technique." Optics Communications ,Papers260(2),449-453(2006).

[4] Deng, X. P., "Optical image encryption based on asymmetric abnormal Fourier transform." Journal of Applied Optics ,Papers28(3),262-264(2007).

[5] Jin, W. M., and C. Yan. "Optical image encryption based on multichannel fractional Fourier transform and double random phase encoding technique." Optik International Journal for Light and Electron Optics,Papers 118(1),38-41(2007).

[6] Pittman, T. B., Shih, Y. H., Strekalov, D. V., Sergienko, A. V.,"Optical imaging by means of twophoton quantum entanglement." Physical Review A, Papers52(5), R3429(1995).

[7] Shapiro, Jeffrey H., "Computational Ghost Imaging." Physical Review A,Papers78(6),1 - 2(2008).

[8] Katkovnik, V., and J. Astola., "Compressive sensing computational ghost imaging. " Journal of the Optical Society of America A Optics Image Science \& Vision ,Papers29(8),1556-67(2012).

[9] Tanha, Mehrdad, R. Kheradmand, S.

Ahmadikandjani., "Gray-scale and color optical encryption based on computational ghost imaging." Applied Physics Letters ,Papers101(10),59035907(2012).

[10]Chen, W., Chen X. D., "Object authentication in computational ghost imaging with the realizations less than $5 \%$ of Nyquist limit." Optics Letters,Papers38(4),546-8(2013).

[11]Xu, Y., and Z. Yuan., "Distance measurement by computational ghost imaging." Optik - International Journal for Light and Electron Optics,Papers124(22),5882-5884(2013).

[12] Clemente, P., Durán, V., Torrescompany, V., Tajahuerce, E., Lancis, J.,"Optical encryption based on computational ghost imaging." Optics Letters, Papers35(14), 2391-2393(2010). [13]Wu, J., Xie, Z., Liu, Z., Wei, L., Yan, Z., \& Liu, S.,"Multiple-image encryption based on computational ghost imaging." Optics Communications,Papers 359, 3843(2016).

[14] X. Li, X. Meng, X. Yang, Y. Wang, Y. Yin, X. Peng, W. He, G. Dong and H. Chen., " Multiple-image encryption via lifting wavelet transform and XOR operation based on compressive ghost imaging scheme," Optics and Lasers in Engineering., Papers102, 106-111(2018).

[15]Peng, X., H. Wei, and P. Zhang., "Chosen-plaintext attack on lensless double-random phase encoding in the Fresnel domain." Optics Letters,Papers31(22),32613(2006).

[16]Z. Zhang, S. Jiao, M. Yao, X. Li and J. Zhong., " Secured single-pixel broadcast imaging," Optics. Express ,Papers26(11), 14587-14591 (2018). [17]Le, Wang, and S. Zhao., "Fast reconstructed and high-quality ghost imaging with fast Walsh-Hadamard transform." Photonics Research,Papers4(06),31-35(2016). 


\title{
Metasurface polarizers with ultra-high extinction ratios in the telecommunication wavelengths
}

\author{
Hiroyuki kurosawa* and Shin-ichiro Inoue \\ Advanced ICT Research Institute, National Institute of Information and Communications Technology \\ (NICT), 588-2 Iwaoka, Nishi-ku, Kobe 651-2492, Japan
}

\begin{abstract}
A numerical study shows that a metasurface polarizer with an ultra-high extinction ratio greater than 3 billion is realized in the telecommunication wavelengths. The metasuface consists of stacked complementary structures which comprise of a metallic material. This kind of structure is known to serve as a polarizer. In this study, the metasurface was designed to have a double resonance of propagating and local surface plasmon polaritons (SPPs). In the presence of the double resonance, the extinction ratio of the metasurface polarizer exceeds 3 billion in the o-band of the telecommunication wavelengths. This ultra-high performance is attributed to the interference between the two SPPs. This study paves the way to an ultra-high-end polarizer with a subwavelength thickness.
\end{abstract}

Keywords: metasurface, plasmonics, polarizer, complementary structure, Babinet’s principle, interference

\section{Introduction}

Electromagnetic metamaterials exhibit an exotic optical response that cannot be available in nature. Various kinds of intriguing phenomena such as negative refraction and electromagnetic cloaking have been demonstrated in metamaterials so far [1]. Recently, extensive studies are devoted to realize the exotic response in a subwavelength region by metamaterials. This kind of metamaterial is called metasurface.

Metasurfaces are ultra-thin optical devices that have high functionality such as focusing and steering of light by a flat surface $[2,3]$. Control of polarization state of light is also possible by a metasurface [4]. The metasurface polarizer consists of three layers. The top layer comprises of a perforated metallic thin film. The middle layer comprises of transparent dielectric material and has the same geometry as the top layer. The bottom layer is complementary to the top layer. This kind of stacked complementary structure serves as a polarizer due to Babinet's principle $[5,6]$. Recently, an extinction ratio exceeding 12,000 was demonstrated experimentally in the metasurface polarizer $[7$, 8]. Even though the metasurface is very thin and has a high performance, the extinction ratio remains low compared with those of high-end prism polarizers.

In this study, we numerically show that the extinction ratio of the metasurface exceeds 3 billion, which is four orders magnitude greater than those in the previous study.

*kurosawa.hiroyuki@nict.go.jp; phone +81-78-969-2284
II Metasurface polarizer

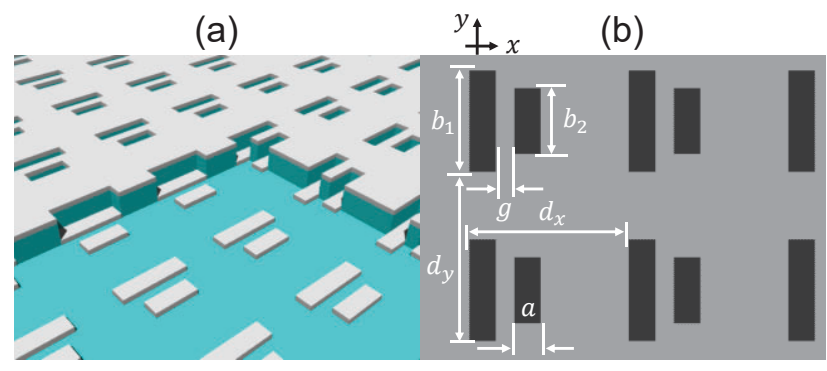

Figure 1. (a): A schematic of the metasuraface polarizer. (b): Definition of the structural parameters.

Figure 1(a) shows a schematic of the metasurface polarizer. The metasurface consists of three layers of complementary structures. The top layer is a perforated Ag (silver) thin film whose unit cell comprises of an asymmetric air holes. The second layer comprises of $\mathrm{SiO}_{2}$ and has the same air-hole-array as that of the top layer. The bottom layer comprises of $\mathrm{Ag}$ and is complementary to the top layer. This three-layered structure is on a quartz substrate. The thicknesses of the top and bottom layers are $45 \mathrm{~nm}$. The middle layer is 200 -nm-thick. The periods of the metasurface along the $x\left(d_{x}\right)$ and $y\left(d_{y}\right)$ directions are 850 and $900 \mathrm{~nm}$, respectively. The width of the air hole $(a)$ is $100 \mathrm{~nm}$. The air holes are $100 \mathrm{~nm}$ apart $(g=100 \mathrm{~nm})$. The permittivity of Ag was taken from the literature [9], while the refractive index of $\mathrm{SiO}_{2}$ and quartz was obtained from the literature [10]. We 
calculated transmittance for the $x$ and $y$ polarizations by commercial software of COMSOL Multiphysics.

\section{Results and Discussion}

\section{An ultra-high extinction ratio in the} metasurface at normal incidence

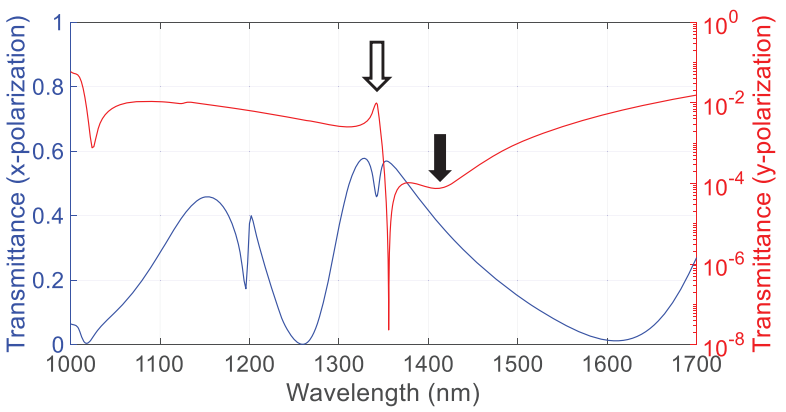

Figure 2. Transmittance spectra of the metasurface.

The left and right axes indicate the transmittance for the $x$ and $y$ polarizations, respectively.

We show the transmittance of the metasurface at normal incidence in Fig. 2. In the transmittance for the $y$ polarization $\left(T_{y}\right)$, there is an extremely sharp dip structure around $1360 \mathrm{~nm}$, while that of $x$ polarization $\left(T_{x}\right)$ has a broad peak around the wavelength. At the dip position, $T_{x}$ and $T_{y}$ are respectively 0.567 and $2.32 \times$ $10^{-8}$, resulting in an ultra-high extinction ratio about $2.45 \times 10^{7}$. This value is three orders of magnitude greater than those in the previous study. To understand the physics behind this ultra-high performance, we characterize the optical response of the metasurface.

\section{Dispersion relation}

The optical response of the metasurface is characterized by the dispersion relation. To clarify the relation, we calculated the angle resolved transmittance spectra of the metasurface. Figures 2(a) and (b) show the calculation results for the $x$ and $y$ polarizations, respectively. Figure 2(b) shows that $T_{y}$ has the extremely low transmittance responsible for the ultra-high extinction ratio only near the normal incidence. The broad dip near the sharp dip, which is indicated by a black arrow in Fig. 2, has a dispersion convex upward and has no drastic change with respect to the incident angle. In order to see the transmittance near the normal incidence clearly, we show the enlarged figure of Fig. 3(b) around $1355 \mathrm{~nm}$ near the normal incidence in Fig. 3(c). The strength of the resonance strongly depends on the incident angle. With the increase in the incident angle, the depth of the resonance increases. In particular, the transmittance becomes even lower when the incident angle is $1^{\circ}$ or $4^{\circ}$. When the incident angle is greater than $4^{\circ}$, the depth of the dip becomes shallower. The dip of the transmittance becomes minimum when the incident angle is $1^{\circ}$, where the minimum value of $T_{y}$ is about $1.9 \times 10^{-10}$. On the other hand, $T_{x}$ is about 0.56 at the minimum point. As a result, the extinction ratio is about 3 billion (Fig. 2(d)). From the analysis of the dispersion relation, we find that two modes are involved in the emergence of the extremely low transmittance responsible for the ultra-high extinction ratio. In a specific incident angle, $T_{y}$ becomes even lower. Moreover, the extinction ratio decreases when the separation between the two mods becomes greater, indicating that the interaction between the modes is crucial to enhance the extinction ratio.
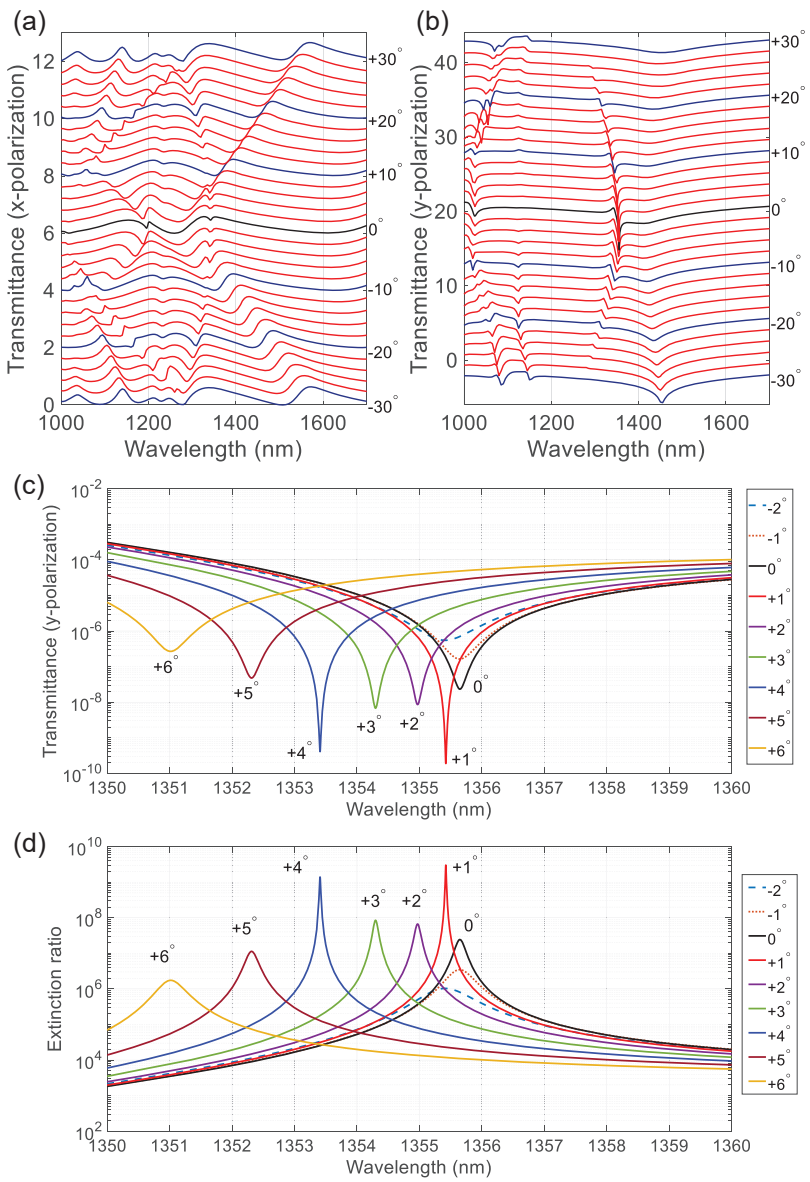

Figure 3. Angle resolved transmittance spectra for the $x$ (a) and $y$ (b) polarizations. Enlarged figure of the angle resolved transmittance of the $y$ polarization (c) and extinction ratio spectra (d) around $1355 \mathrm{~nm}$ near the normal incidence. 


\section{Eigenmodes of the metasurface}

Let us consider the origin of the modes responsible for the ultrahigh extinction ratio. To clarify the eigenmodes, we calculated the electric field distributions near the metasurface at normal incidence. Figures 3(a-f) show the calculation results. Figure 3(a) shows the field distribution at the local peak position indicated by a white arrow in Fig. 2. The electric fields are concentrated near the metal/substrate interface, indicating that propagating SPPs are excited. Figure 3(d) shows the field distribution at the broad dip indicating by a black arrow in Fig. 2. The electric fields are concentrated near the edge of the nanorods, indicating that the local SPPs are excited. Figures 3(e) and (f) show the field distribution at the extremely sharp dip in Fig. 2, where both the propagating and local SPPs are excited simultaneously. These two SPPs can interfere destructively, resulting in an extremely low transmittance. This mode analysis indicates that the ultrahigh extinction ratio is caused by the interference between the two SPPs.

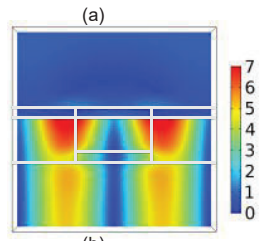

(b)
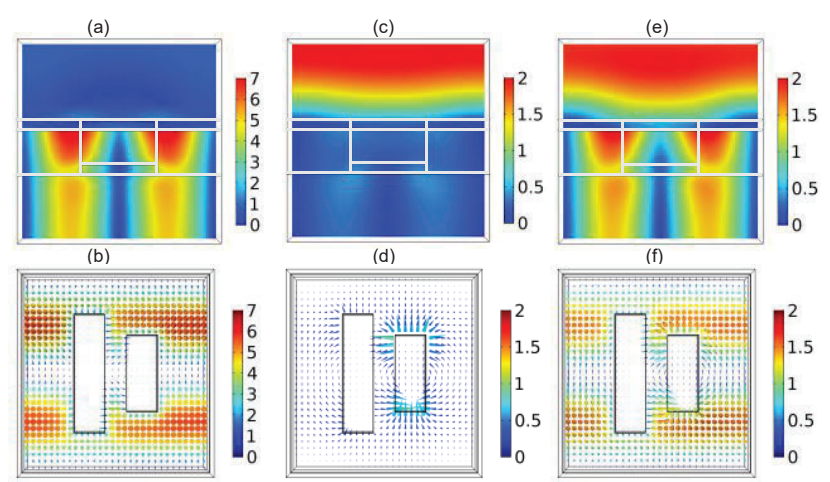

(d)
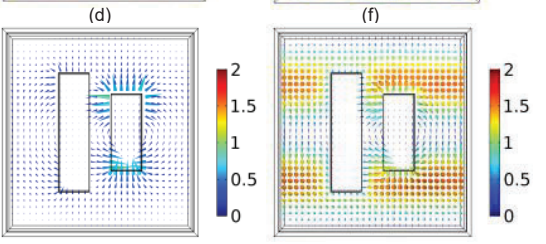

Figure 4. Electric field distribution patterns near the metasuface at $1342 \mathrm{~nm}(\mathrm{a}, \mathrm{b}), 1410 \mathrm{~nm}(\mathrm{c}, \mathrm{d})$, and $1355 \mathrm{~nm}(\mathrm{e}, \mathrm{f})$. Figures (a, c, e) show the field distributions sliced at the center of the metasurface along the $y$ direction, while Figs. (b, d, f) show those sliced at the middle of the height of the bottom structure.

\section{Conclusion}

We have numerically shown that the metasuraface has an ultrahigh extinction ratio about 3 billion in the o-band of the telecommunication wavelengths. Such an ultra-high performance is attributed to the extremely low transmittance for the $y$ polarization, which is caused by the interference between the propagating and local SPPs. Our study shows that it is possible to realize an ultra-high-end polarizer with a subwavelength thickness, opening the door for the metasurface polarizer with ultra-high performance.

\section{References}

[1] S. A. Ramakrishna and T. M. Grzegorczyk, [Physics and Applications of Negative Refractive Index Materials], CRC Press (2008).

[2] F. Aieta, M. A. Kats, P. Genevet, and F. Capasso, "Multiwavelength achromatic metasurfaces by dispersive phase compensation," Science 347, 1342-1345 (2015).

[3] N. Yu, P. Genevet, M. A. Kats, F. Aieta, J-P. Tetienne, F. Capasso, and Z. Gaburro, "Light propagation with phase discontinuities: generalized law of reflection and refraction," Science 334, 333-337 (2011).

[4] M. Iwanaga, "Polarization-selective transmission in stacked two-dimensional complementary plasmonic crystal slabs," Appl. Phys. Lett. 96:083106 (2010).

[5] M. Iwanaga, "Subwavelength electromagnetic dynamics in stacked complementary plasmonic crystal slabs," Opt. Express 18, 15389-15398 (2010).

[6] M. Iwanaga, "Photonic metamaterials: a new class of materials for manipulating light waves," Sci. Tech. Adv. Mater. 13, 053002 (2010).

[7] H. Kurosawa, B. Choi, Y. Sugimoto, and M. Iwanaga, "High performance metasurface polarizer with extinction ratio exceeding 12000," Opt. Express 25, 4446 (2017).

[8] H. Kurosawa, B. Choi, and M. Iwanaga, "Enhanced high performance of the metasuface polarizer through numerical analysis of the degradation characteristic," Nanoscale Res. Lett. 13, 225 (2018).

[9] A. D. Rakić, A. B. Djurišić, J. M. Elazar, and M. L. Majewski, "Optical properties of metallic films for verticalcavity optoelectronic devices," Appl. Opt. 37, 5271-5283 (1998).

[10] I. H. Malitson, "Interspecimen comparison of the refractive index of fused silica,” J. Opt. Soc. Am. 55, 1205-1208 (1965). 


\title{
Trapping and manipulation of microparticles using optothermal effects
}

\author{
R. Ramos-García a J. Sarabia-Alonso a, J.C. Ramirez San Juanª, T. Spezzia-Mazzocco a , G. Ortega \\ Mendoza ${ }^{\mathrm{b}}$ \\ ${ }^{a}$ Optics Department, Instituto Nacional de Astrofísica, Óptica y Electrónica, Luis Enrique Erro \#1, \\ Tonantzintla Pue, 72840 México \\ ${ }^{\mathrm{b}}$ División de Ingenierías, Universidad Politécnica de Tulancingo 43629, Hidalgo, México
}

\begin{abstract}
A review of trapping and manipulation of microparticles using optothermal effects is presented. Optothermal forces allows massive manipulation of microparticles using lower power than that needed in optical tweezers. The source of thermal effects is light absorption in highly absorbent substrates which allow the 2D massive manipulate of microparticles or nanoparticles photodeposited on the core of an optical fiber end for 3D trapping of solid particles or bubbles. Convective currents, thermophoresis and Marangoni forces interplay gives rise to different trapping configurations that will be reviewed in this work.
\end{abstract}

Keywords: agriculture, environment, remote sensing, robot arm, robust control

\section{Introduction}

Optical tweezers, which use light momentum transfer to directly microparticles at the focus of a high numerical aperture lens, were first proposed by Ashkin in 1970 [1]. Since then, optical tweezers have become an extraordinary tool in many research fields such as biology and physics. Sub picoNewton forces and nanometer spatial resolution spatial are now common in optical tweezers [2-3]. However, optical tweezers are limited to trap a few particles even if multiple traps are generated by either beam shaping or time-sharing techniques. Additionally, optical power requirements increase with the number of trapping beams, making the system more complex and expensive.

To overcome these limitations, various alternative strategies have been proposed. Plasmonic tweezers exploit the localized surface plasmon resonances of metallic nanostructures for near-field trapping [4]. The plasmon-enhanced optical forces significantly reduce the required operational power of plasmonic tweezers. Additionally, the localized plasmonic hot spots favor the trapping of nanoparticles and molecules beyond the diffraction limits [5]. However, the localized and nearfield nature of plasmonic tweezers limits its capability to dynamically manipulate a given sample [6]. An alternative approach to trapping is optodielectrophoresis which allow manipulation of particles under a nonuniform electric field [7].

Opto-thermomal manipulation creates a temperature gradient field through optical heating due to absorption in a highly absorbing material. The generation of a strong temperature gradient with low-power optical heating allows 2D collective trapping and manipulation of colloidal particles in combination with convective forces, thermophoretic and Marangoni forces

\section{Experimental description}

Two basic configurations for optothermal trapping and manipulation are studied (see Figure 1). In the first case, thermal gradient is produced by light absorption on an absorbing film [8] while in the second, light absorption occurs on photodeposited nanoparticles on the core of an optical fiber [9]. Thermal gradient may give rise to convective currents, thermophoresis and, in the case of vapor bubble, Marangoni forces. In addition, if high absorbing nanoparticles are dissolved in the solution, careful control of convective currents and optical forces may lead to particle trapping on the vicinity of the fiber core [10].

Contrary to plasmonic trapping, special control on the thickness of the film is required and no resonant absorption is needed. In fact, any light sources on the 
visible spectrum can be used since most metals or semiconductors films can be used.
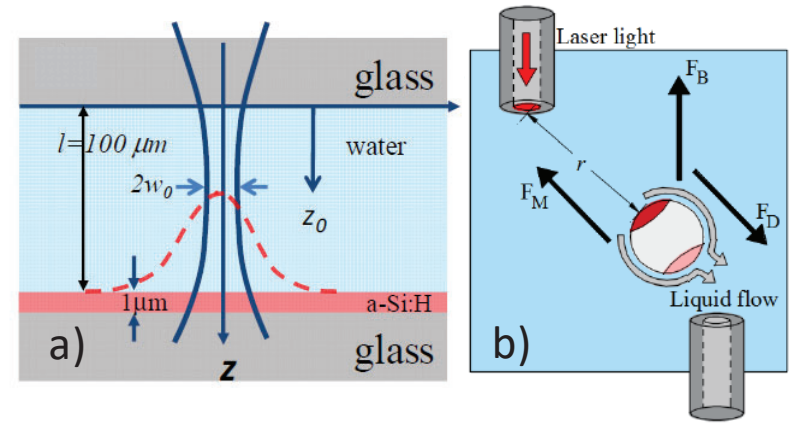

Figure 1. Different configurations for optothermal trapping: a) Thermal gradient is produced by light absorption on an absorbing film and b) absorption on photodeposited nanoparticles on the core of an optical fiber

\section{Results and Discussion}

Figure $2 \mathrm{a}$ shows $2 \mathrm{D}$ trapping of $2 \mu \mathrm{m}$ diameter colloidal particles due to convective currents using a $\mathrm{CW}$ $\mathrm{Nd}$ :YAG laser $(\lambda=1064 \mathrm{~nm})$ of $0.8 \mathrm{~mW}$ power. Even if the beam or the sample is displaced, the particles remain trapped. In Figure 2b, the power was increased to $6 \mathrm{~mW}$, in which case, the particles are expelled from the beam (indicated by the dashed circle) due to thermophoretic forces. A balance between these two forces produce trapping ring where the trapped particles moves in toroidal trajectories [8].
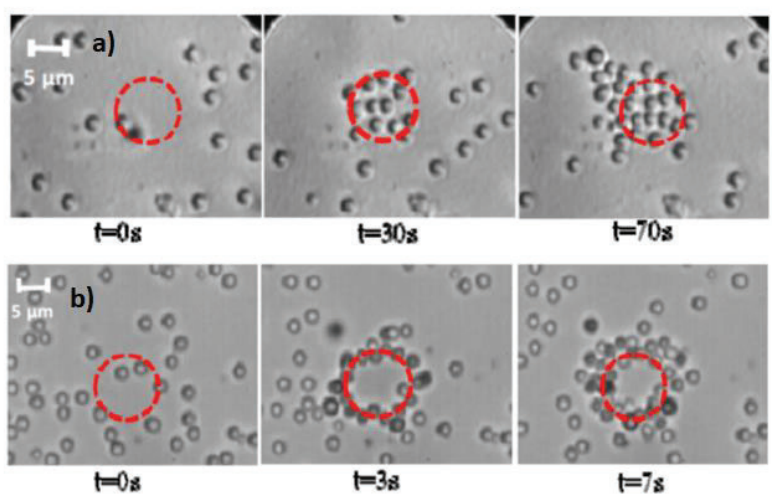

Figure 2. a) Trapping of microparticles by laser induced convective currents (power $0.8 \mathrm{~mW}$ ) and b) trapping pattern due to convective and thermophoretic forces (power $6 \mathrm{~mW}$ ).
By using a spatial light modulator, beam shaping into complex light distribution expands the capability of thermophoretic trapping. For example, in Figure 3 a ring of light produced by the Fourier transform of a Bessel beam of increasing radius allows trapping of a single particle and clearing of the area determined by the circle [8]. This circle acts as a fence that impedes the motion of particles into this area. Thus, optothermophoretic forces in combination with beam shaping may be an alternative technique to optodielectrophoresis.
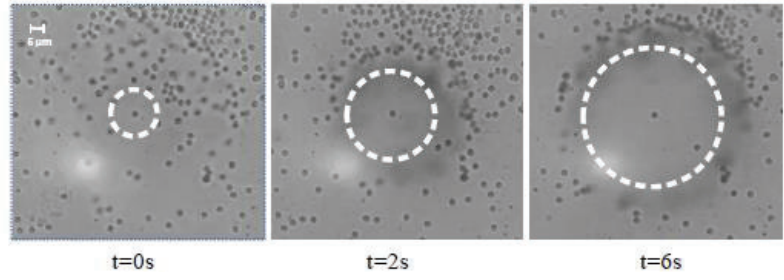

Figure 3. Optical clearing by thermal gradients produced by a circle of light.

Finally, Figure 4 shows a vapor bubble is attracted towards a multimode mode fiber on whose end, silver nanoparticles are photodeposited [9]. Light absorption at the nanoparticles produce a thermal gradient that spatially modulate the surface tension of the bubble creating a force directed towards the thermal source.
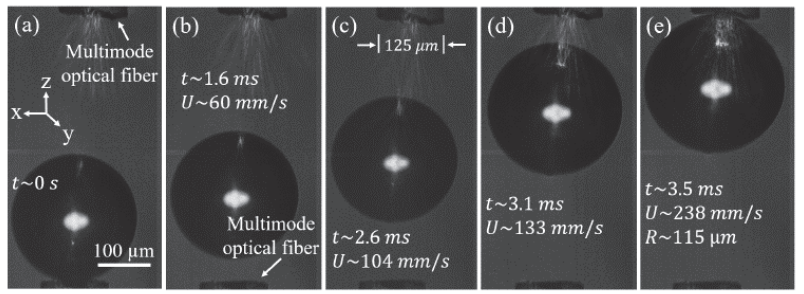

Figure 4. A bubble is attracted towards the multimode fiber due to Marangoni forces.

\section{Conclusions}

Optothermally induced forces show great promise to massively trap and manipulate colloidal particles using lower optical power than that needed in optical tweezers. The use of spatial light modulators for beam shaping may in principle be an alternative technique to optodielectrophoretic trapping. 


\section{References}

[1] Ashkin, A., "Acceleration and Trapping of Particles by Radiation Pressure," Phys. Rev. Lett., 24(4), pp. 156159 (1970).

[2] Huang, C.C., Wang, C.F., Mehta, D.S, Chiou, A., "Optical tweezers as sub-pico-newton force transducers," Optics Communications, 195, 41-48 (2001).

[3] delToro, Damian and Douglas E Smith. "Accurate measurement of force and displacement with optical tweezers using DNA molecules as metrology standards" Appl Phys Lett 104, 143701 (2014).

[4] Juan, M. L., Righini, M., and Quidant, R., "Plasmon Nano-Optical Tweezers," Nat. Photonics, 5(6), 349-356 (2011).

[5] Shoji, T., and Tsuboi, Y., "Plasmonic Optical Tweezers Toward Molecular Manipulation: Tailoring Plasmonic Nanostructure, Light Source, and Resonant

Trapping," J. Phys. Chem. Lett., 5(17), 2957-2967 (2014).

[6] Zheng, Y., Ryan, J., Hansen, P., Cheng, Y. T., Lu, T. J., and Hesselink, L., "Nano-Optical Conveyor Belt-Part II: Demonstration of Handoff Between Near-Field Optical Traps," Nano Lett., 14(6), 2971-6 (2014).

[7] Chiou, P. Y., Ohta, A. T., and Wu, M. C., "Massively Parallel Manipulation of Single Cells and Microparticles Using Optical Images," Nature, 436(7049), 370-2 (2005).

[8] Flores-Flores, E, Torres-Hurtado, S.A., Páez, R., Ruiz, U., Beltrán-Pérez, G., Neale, S. L., Ramirez-San-Juan, J.C. and Ramos-García, R. "Trapping and manipulation of microparticles using laser-induced convection currents and photophoresis," Biomed. Opt. Express 6, 4079-4087 (2015)

[9] Ortega-Mendoza, J.G., Sarabia-Alonso, J.A., ZacaMorán, P., Padilla-Vivanco, A., Toxqui-Quitl, C., RivasCambero, I., Ramirez-Ramirez, J., Torres-Hurtado, S. A. and Ramos-García, R. "Marangoni force-driven manipulation of photothermally-induced microbubbles," Opt. Express 26, 6653-6662 (2018)

[10] Ortega-Mendoza, J. G., Chávez, F., Zaca-Morán, P., Felipe, C., Pérez-Sánchez, G.F., Beltran-Pérez, G., Goiz, O., and Ramos-Garcia, R. "Selective photodeposition of zinc nanoparticles on the core of a single-mode optical fiber," Opt. Express 21, 6509-6518 (2013) 


\title{
Motion of micro-sized colloidal particles induced by optical vortex
}

\author{
Kenta Iwamoto*, Yasuyuki Kimura \\ Department of Physics, Kyushu University, 744 Motooka, Nishi-ku, Fukuoka, 819-0395, Japan
}

\begin{abstract}
Recently, the synchronization phenomena observed in living matter such as cilia has been actively studied experimentally and theoretically. In this study, we construct a simple model system composed of spheroid particles driven by optical vortex. The spheroid particles are found to exhibit self-spinning motion with tilting their long axis against the optical axis at small topological charge. We also study the cooperative motion of the particles driven by two collinearly aligned optical vortices. We also simulate hydrodynamically coupled particles on multi- circular paths and find the synchronization in the rotation.
\end{abstract}

Keywords: collective motion, colloidal particles, hydrodynamic interaction, optical vortex

\section{Introduction}

It is well known that synchronous phenomena such as cilia and flagella is important in the motion of living organisms such as bacteria and planktons. It is useful to study biomimic model systems to elucidate their physical dynamics. We have studied orbital motion of colloidal particles on an optical vortex by using holographic optical tweezers [1,2]. Various kinds of characteristic collective motion induced by the hydrodynamic interaction between the particles have been observed in multi-particles' systems. In this study, we examine the peculiar collective motion in the rotation motion of spheroid particles induced by two optical vortices. We also analyze the relevance of their hydrodynamic interaction by simulation using a simple model system.

\section{Experiment}

\section{Preparation of spheroid particles}

The spheroid particles used in the experiment were prepared following the method by Ho et. al. [3] We made a film of the mixture of polyvinyl alcohol and spherical polystyrene particles in diameter of $3.0 \mu \mathrm{m}$ and stretched it at high temperature $\left(140-170{ }^{\circ} \mathrm{C}\right)$. The particles were elongated parallel to the stretching direction and their final size are 6 $\mu \mathrm{m} \times 2 \mu \mathrm{m}$.

*iwamoto@phys.kyushu-u.ac.jp; phone 81-92-802-4109

\section{An optical vortex system}

An optical vortex is realized by using a holographic optical tweezers for various topological charge $l$ [4]. The radius of the optical vortex $r$ is proportional to $\sqrt{l}[5]$.

\section{Results and Discussion}

\section{Spheroid driven by a single vortex}

When a topological charge for vortex is large $(l=40)$, the particles are tilted from the direction of optical axis and exhibit a circular motion with keeping the tilt angle. By reducing $l$, the radius of gyration decreases, and finally it turns into the glide motion as shown in the Fig. 1.

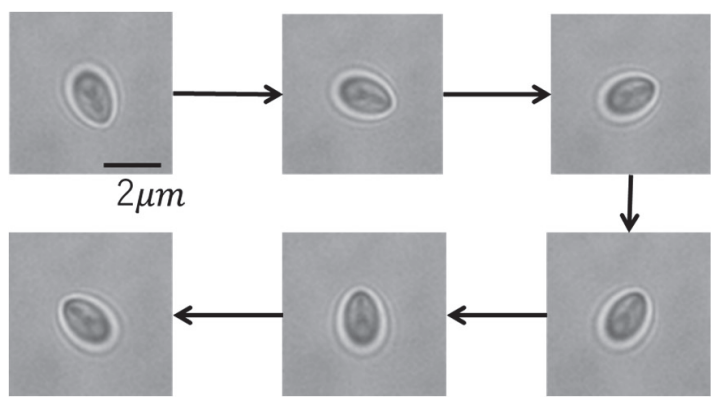

Figure 1. Glide motion of a spheroid particle in the optical vortex with $l=4$. The images are taken every 27 ms. 


\section{Spheroids driven by two optical vortices}

The collinearly aligned vortices are generated and two spheroids are trapped respective vortices of $l=4$. The centerto-center distance of the optical vortices $d$ is set to $10 \mu \mathrm{m}$. The time evolution of the rotation angle and the difference in the angles $\Delta \theta$ are respectively shown in the Figs. 2 (a) and (b). The histogram of angular difference $\Delta \theta$ exhibits the peak appears at $\Delta \theta=0.4$ and $0.6[\mathrm{rad} / 2 \pi]$. This indicates the particles temporally synchronize due to the hydrodynamic interaction.
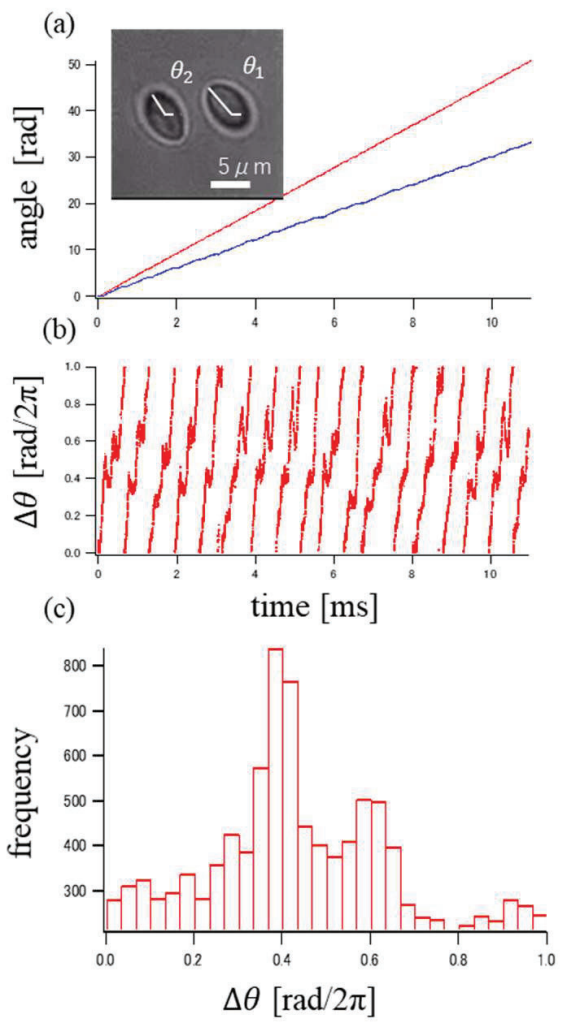

Figure 2. Two particles driven by two different vortices.

(a) Time evolution of rotation angle (blue: right, red: left). (b) Time evolution of phase difference $\Delta \theta$ ( $\Delta \theta=$ $\theta_{1}-\theta_{2}$ ). (c) Histogram of $\Delta \theta$.

\section{Simulation}

The motion of two spherical particles driven by two vortices is simulated with considering hydrodynamic interaction [5].
In the model system, it is assumed that the particles are subjected to a constant driving force in the tangential direction of the circular path and the elastic force to bind the particles to the circular path. Oseen approximation is used for their hydrodynamic interaction. When the driving forces are the same for the two vortices, the motion of the two particles finally synchronized regardless of the initial value.

\section{Conclusion}

We observe rotation motion of spheroid particles using optical vortex with small topological charge. The two particles' system, a phase locking phenomenon due to their hydrodynamic interaction is observed. Simulation reveals that hydrodynamic interaction is an important factor for synchronous phenomena in the studied system.

\section{References}

[1] Kimura Y., "Hydrodynamically induced collective motion of optically driven colloidal Particles on a circular path”, J. Phys. Soc. Jpn. 86, 101003 (2017).

[2] Sassa Y., Shibata S., Iwashita Y. and Kimura Y., "Hydrodynamically induced rhythmic motion of optically driven colloidal particles on a ring" , Phys. Rev. E 85, 061402 (2012).

[3] Ho A., Keller A., Odell J., and Ottewill R., "Preparation of monodisperse ellipsoidal polystyrene particles", Colloid Polym. Sci. 271, 469 (1993).

[4] Curtis, J., Koss, B.A., Grier, D.G., "Dynamic holographic optical tweezers," Opt. Commun. 207, 169-175 (2002).

[5] Padget M.J., Allen L., "The Poynting vector in LaguerreGaussian laser modes”, Opt. Commun., 121 (1995).

[6] Kotar J., Debono L., Bruot N., Box S., Phillips D., Simpson S., Hanna S., and Cicuta P., "Optimal Hydrodynamic Synchronization of Colloidal Rotors”, Phys. Rev. Lett. 111, 228103 (2013). 


\title{
Mass transfer and composition change during metal sphere migration
}

\section{in glass by continuous laser illumination*}

\author{
Takuya Imai ${ }^{\mathrm{a}}$, Shuko Yoshimura ${ }^{\mathrm{a}}$, Hirofumi Hidai*a, Tetsuo Kishi ${ }^{\mathrm{b}}$ \\ Souta Matsusaka ${ }^{\mathrm{a}}$, Akira Chiba $^{\mathrm{a}}$, Noboru Morita ${ }^{\mathrm{a}}$ \\ ${ }^{a}$ Department of Mechanical Engineering, Chiba University, \\ 1-33 Yayoi-cho, Inage-ku, Chiba, 263-8522, Japan \\ ${ }^{\mathrm{b}}$ Department of Materials Science \& Engineering, Tokyo Institute of Technology, \\ 2-12-1 Ookayama,Meguro-ku, Tokyo, 152-8550, Japan
}

\begin{abstract}
The authors have been proposed metal sphere manipulation in glass. Laser illumination to a metal sphere in glass heated the sphere, and surrounding glass was also heated by the thermal conduction and softened. In this paper, we report a nickel sphere migrated in a crystallized glass. After the migration, the composition of the migration trajectory and nickel sphere was measured with electron probe microanalyzer. As a result, the amount of phosphorous in the trajectory of glass decreased, and the nickel sphere contained phosphorous.
\end{abstract}

Kevwords: laser manipulation. crvstallized glass. nickel particle. dedope

\section{Introduction}

Glass is widely used in optical application because of the high transparency and chemical stability. Optical properties of glass can be controlled by changing the composition.

The authors have been proposed metal doping by metal sphere migration in the glass. A metal foil was attached on a glass, and a laser beam was illuminated to the metal foil through the glass. The metal foil was heated and melted by the laser illumination. The glass in the neighborhood of the heated metal was also heated by the thermal conduction and was softened. As a result, the metal foil became a sphere due to the surface tension and penetrated the glass [1]. The driving force of the migration was as follows: the laser illumination caused temperature gradient. The interfacial tension between the melted metal and the glass decreases with increasing the temperature. Inhomogeneous interfacial tension pushed the metal sphere to the light source, and the metal sphere migrated toward the light source $[2,3]$. Also, we have reported metal particle precipitation in the trajectory [4] and refractive index change caused by glass compositional redistribution [5].

*Corresponding author's e-mail address:

hidai@faculty.chiba-u.jp
In this paper, we report microelement in the glass condensed in the metal sphere by the migration. Specifically, a nickel sphere migrated in a crystallized glass. As a result, the amount of phosphorous in the trajectory decreased, and the nickel sphere contained phosphorous.

\section{Experimental}

Experimental set up was almost the same described in reference [4] as shown in fig. 1. Briefly, crystallized glass $\left(\mathrm{SiO}_{2} 66 \%, \mathrm{Al}_{2} \mathrm{O}_{3} 22 \%, \mathrm{Li}_{2} \mathrm{O} 4 \%, \mathrm{P}_{2} \mathrm{O}_{5} 1 \%\right.$, Neoceram N0, Nippon electric glass co., Ltd. Shiga, Japan), borosilicate glass, nickel powder (purity: $99.9 \%$, particle size: $74 \mu \mathrm{m}$, NI-314012, The Nilaco corp., Tokyo, Japan) and silica glass was stacked and fixed by a jig. A laser was illuminated to the nickel power through the crystallized glass and borosilicate glass. By the laser illumination, the nickel sphere was migrated toward the light source in borosilicate glass and then crystallized glass. Without borosilicate glass, the nickel particle could not be induced to crystallized glass directly. Therefore, the borosilicate glass was used.

The laser used was a continuous wave fiber laser. The laser power was set at $10.6 \mathrm{~W}$. The experimental procedure was as follows: Many metal spheres were induced into the borosilicate glass. Among them, two metal spheres with a diameter of $50 \mu \mathrm{m}$ were migrated 


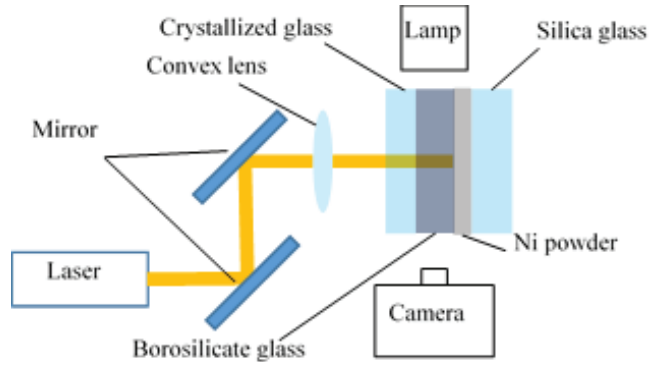

Fig. 1 Illustration of experimental equipment

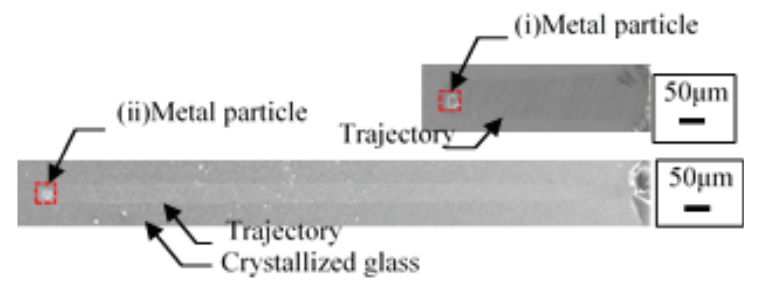

Fig.2 SEM images of the nickel spheres and modified trajectory

into the crystallized glass. They were migrated for 200 $\mu \mathrm{m}$ and $2000 \mu \mathrm{m}$ in the crystallized glass. After the migration, the glass was cut and polished to expose these two spheres and trajectory. The composition was analyzed with energy dispersive X-ray spectroscopy (JSM-6510A, EDX) and electron probe microanalyzer (EPMA; JXA8200, JEOL).

\section{Results and discussion}

Figure 2 shows the SEM images of the nickel spheres migrated for $200 \mu \mathrm{m}$ and $2000 \mu \mathrm{m}$ in the crystallized glass. The modified area with a diameter of $100 \mu \mathrm{m}$ was observed in the trajectory.

Figure 3 shows the EDX spectrum of the nickel sphere migrated in crystallized glass for $2000 \mu \mathrm{m}$. EMPA clarified that the weight percent of phosphorous in the sphere migrated for $200 \mu \mathrm{m}$ and $2000 \mu \mathrm{m}$ was $1.5 \mathrm{wt}$. \% and $5.0 \mathrm{wt}$. \%, respectively. The weight percent of nickel was 91 wt. \% from the sphere migrated for $200 \mu \mathrm{m}$ and was 86 wt. $\%$ from that for $2000 \mu \mathrm{m}$.

The weight percent of phosphorous in the glass was also measured by EPMA. The phosphorous in the trajectory was reduced to be 0.6 wt. $\%$ from 1.1 wt. $\%$ in the unmodified area. In contrast, the nickel in the trajectory was increased to be $\sim 0.5$ wt. $\%$ from $<0.1$ wt. $\%$ in the unmodified area.

The amount of phosphorous was decreased in the trajectory and was increased in the nickel sphere. Therefore, phosphorous in the crystallized glass was

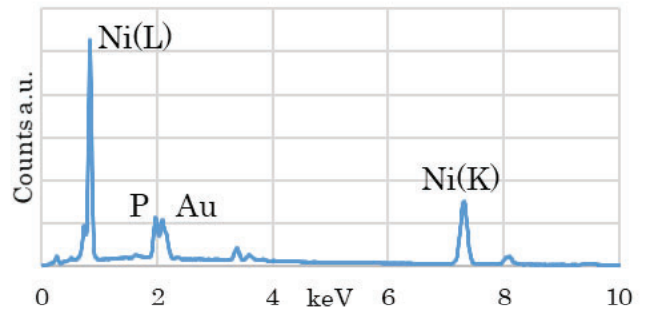

Fig. 3 EDX spectrum of the nickel sphere migrated in crystallized glass for $2000 \mu \mathrm{m}$

concentrated in the nickel sphere by the migration. In the glass, phosphorous is oxidized. However, oxygen was not detected in the nickel sphere; hence, phosphorus oxide was believed to be reduced, when phosphorous condensed in nickel sphere.

\section{Conclusion}

Amount of phosphorous in the trajectory decreased, and nickel sphere contains phosphorous by the nickel sphere migration in the crystallized glass. We demonstrated that metal sphere migration dedope minor component in a glass.

\section{References}

[1] Hidai, H., Yamazaki, T., Itoh, S., Hiromatsu, K. and Tokura, H., "Metal Particle Manipulation by Laser Irradiation in Borosilicate Glass," Opt. Exp., 18(19), 20313-20320, (2010).

[2] Hidai, H., Matsushita, M., Matsusaka, S. Chiba, A. and Morita, N., "Moving Force of Metal Particle Migration by Laser Irradiation in Borosilicate Glass," Opt. Exp. 21(16), 18955-18962 (2013).

[3] Hidai, H., Wada, J., Iwamoto, T., Matsusaka, T., Chiba, A., Kishi, T., and Morita, N., "Experimental and theoretical study on the driving force and glass flow by laser-induced metal sphere migration in glass," Sci. Rep., 6, 38545 (2016).

[4] Hidai, H., Matsushita, M., Matsusaka, S. Chiba, A. and Morita, N., "Laser-induced nickel sphere migration and nanoparticle precipitation in silica glass" Opt. Mat. Exp., 5(5), 1006-1015 (2015).

[5] Kishi, T., Kokan, T., Yoshida, Y., Iwamoto, T. Hidai, H., Noritake, F. Matsushita, N., Yano, T. "Compositional redistribution in $\mathrm{CaO}-\mathrm{Al}_{2} \mathrm{O}_{3}-\mathrm{SiO}_{2}$ glass induced by the migration of a steel microsphere due to continuous-wavelaser irradiation," Opt. Exp. 26(10), 13020-13026 (2018). 


\section{Zouheir Sekkat}

\section{Optical Manipulation by Photochemistry}

I will show that, in concept, particles should move when they are photochemically activated in a gradient of light intensity, and the motion occurs in the direction of the vector of the intensity gradient, and its efficiency depends on the respective orientations of the vectors of light polarization and intensity gradient. The theory describes well experimental observations, and it opens important perspectives for the transport of matter by light. 


\title{
Two photon photo-polymerization induced helical microfibers
}

\author{
Junhyung Lee*a, Yoshihiko Arita ${ }^{\text {b,c }}$, Reimon Matsuo ${ }^{\text {a }}$, Haruki Kawaguchi ${ }^{a}$, Katsuhiko Miyamoto ${ }^{a, b}$, \\ Kishan Dholakia ${ }^{\mathrm{a}, \mathrm{c}}$, Takashige Omatsu ${ }^{\mathrm{a}, \mathrm{b}}$ \\ ${ }^{\mathrm{a}}$ Graduate School of Science and Engineering, Chiba University, 1-33 Yayoi-cho, Inage-ku, \\ Chiba- shi 263-0022, Japan \\ ${ }^{b}$ Molecular Chirality Research Center, Chiba University, 1-33 Yayoi-cho, Inage-ku, Chiba-shi \\ 263- 0022, Japan \\ 'SUPA, School of Physics \& Astronomy, University of St Andrews, North Haugh, St Andrews, \\ KY16 9SS, United Kingdom
}

\begin{abstract}
We successfully created helical microfibers by irradiating picosecond optical vortex pulses with a wavelength of $532 \mathrm{~nm}$ to ultraviolet curing resin via a two-photon-photopolymerization process. Permanent refractive index change of resin caused by laser triggers the self-focusing of light and forms the Light-induced self-written waveguides. In this process, the orbital angular momentum of the light is transferred to make a helical microfiber. The resulting helical microfibers exhibited a length of $\sim 300 \mu \mathrm{m}$. Also, we can control the direction of twists by changing the sign of the topological charge of optical vortex.
\end{abstract}

Keywords: (060.4005) Microstructured fibers; (080.4865) Optical vortices; (130.5460) Polymer waveguides; (230.0230) Microstructure fabrication; (230.3990) Micro-optical devices.

*omatsu@faculty.chiba-u.jp; phone +81-43-290-3477

\section{Introduction}

Light-induced self-written fibers are associated with photo-polymerization owing to the self-focusing effects of an incident light [1], and they will lead to various applications for optical integrated devices.

An optical vortex [2] possessing an on-axial phase singularity and an orbital angular momentum (OAM) due to its spiral wavefront characterized by a topological charge $\ell$. The optical vortex has been widely studied in various fields, particularly, in a laser materials processing, where OAM of the irradiated optical vortex can twist or spin the materials, such as metal, silicon, and azo-polymer, so as to form chiral structures $[3,4]$.

In recent years, we have successfully demonstrated a light-induced self-written helical fiber via single photon absorption (SPA) by the irradiation of an ultraviolet light field with OAM to a curing resin [5]. The fabricated helical fiber exhibited a length of $140 \mu \mathrm{m}$, and its helicity was fully assigned with a handedness of the incident optical vortex beam. Such helical fibers can be potentially utilized as a waveguide for an optical vortex mode.
Two-photon absorption (TPA) photo-polymerization based on the irradiation of an ultrafast laser should significantly reduce the scattering and absorption losses in the photo-polymerization process, thereby enabling the extension of helical fibers.

In this paper, we report on the first demonstration of a sub-millimeter scale, self-written helical fiber created by the irradiation of pico-second laser via TPA-photopolymerization process.

\section{Experiments}

Figure 1 is a schematic diagram of an experimental setup. A homemade picosecond pulse laser with a pulse repetition frequency of $100 \mathrm{MHz}$ and a wavelength of $532 \mathrm{~nm}$ was employed, and its output was converted to be an optical vortex with the topological charge $\ell= \pm 1$ by using a spiral phase plate (SPP). The generated optical vortex beam was tightly focused by a microscope objective (Olympus, $\times 60$, $\mathrm{NA}=1.1$ water immersion) to a front glass/resin interface of the cell including cure resin. The cure resin (NOA 63) 
used has a viscosity of $2000 \mathrm{cps}$, and it exhibits a refractive index of 1.52 and 1.56 before and after curing.

The refractive index of the cure resin increases during the polymerization process. Thus, the polymerized cure resin acts as a waveguide to confine the optical field, i.e. the self-focusing effects of the irradiated beam, so as to develop into a fiber.

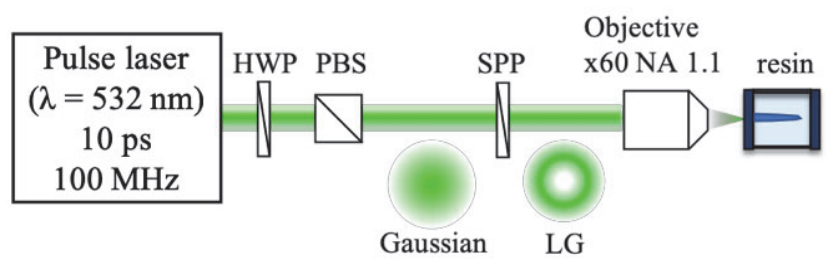

Figure 1 Experimental setup for creating helical fibers via TPP.

A clockwise or counter-clockwise helical fiber was created by the irradiation of the optical vortex with topological charge $\ell= \pm 1$. Its length was extended to double $(\sim 300 \mu \mathrm{m})$ of that of a helical fiber created via single photon photo-polymerization process. It is noteworthy that the helicity of the fiber was fully assigned by the handedness of the irradiated optical vortex, as shown in Fig. 2.
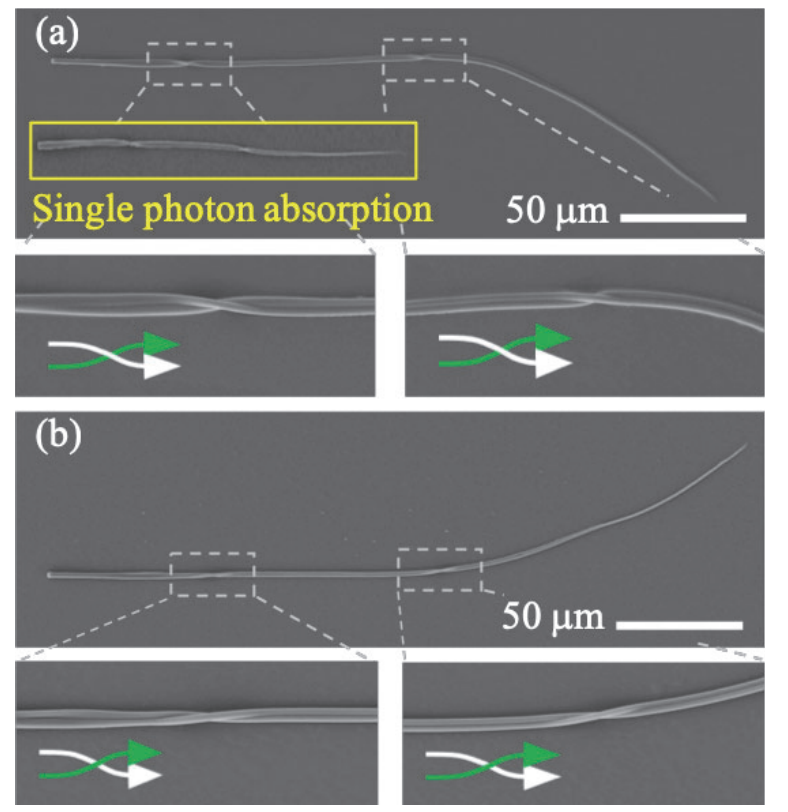

Figure 2. SEM images of helical polymeric fibers generated by the optical vortex irradiation with the topological charge $\ell=1$ (a) and $\ell=-1$ (b). Expanded views of the selected areas show twisted parts on each fiber.

\section{Conclusion}

We have successfully demonstrated the formation of a sub-millimeter scale helical fiber in a cure resin via the TPP process by the irradiation of an optical vortex picosecond laser. Also, the helical fiber length was extended to double, and it was measured to be $\sim 300 \mu \mathrm{m}$. A generated helical microfiber, manifesting that OAM of the optical vortex field can twist the photo-polymerization even through TPP, leads us to develop optical devices for space division multiplexing communications.

\section{References}

[1] M. Kagami, T. Yamashita, H. Ito, "Light-induced selfwritten three-dimensional optical waveguide," Appl.

Phys. Lett. 79, 1079 (2001)

[2] L. Allen, M. W. Beijersbergen, R. J. C. Spreeuw, J. P. Woerdman, "Orbital angular momentum of light and the transformation of Laguerre- Gaussian laser modes," Phys. Rev. A 45, 8185-8189 (1992).

[3] F. Takahashi, S. Takizawa, H. Hidai, K. Miyamoto, Ryuji Morita, T. Omatsu, “Optical vortex pulse illumination to create chiral monocrystalline silicon nanostructures," Phys. Status Solidi A, 213, 1063-1068 (2016).

[4] M. Watabe, G. Juman, K. Miyamoto, T. Omatsu, "Light induced conch-shaped relief in an azo-polymer film", Sci, Rep. 4, 4281 (2014).

[5] J. Lee, Y. Arita, S. Toyoshima, K. Miyamoto, P. Panagiotopoulos, E. M. Wright, K. Dholakia, and T. Omatsu, "Photopolymerization with Light Fields Possessing Orbital Angular Momentum: Generation of Helical Microfibers," ACS Photonics 20185 (10), 41564163 


\title{
Spectroscopy of Deoxyribonucleic Acid Film: From Ultraviolet to Terahertz *
}

\author{
Hayoung Jeong*a, Seunguk Cheon ${ }^{\mathrm{a}}$, Yong Soo Lee ${ }^{\mathrm{a}}$, Soeun Kim ${ }^{\mathrm{b}}$, Chul Kang ${ }^{\mathrm{b}}$, Inhee Maeng ${ }^{\mathrm{b}}$, and \\ Kyunghwan $\mathrm{Oh}^{\mathrm{a}}$
}

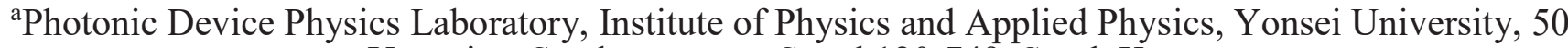 Yonsei-ro Seodaemun-gu, Seoul 120-749, South Korea
bIntegrated Optics Laboratory, Advanced Photonics Research Institute, GIST, Gwangju 61005, South Korea

\begin{abstract}
We measured the transmittance of the double-stranded deoxyribonucleic acid (DNA) film as a function of thickness in very wide frequency range, which is divided to three spectra regions: Ultraviolet/Visible/Near Infrared, Middle infrared, and Terahertz. We found that DNA film has highest transmittance in the near infrared and terahertz region among investigated frequency range.
\end{abstract}

Keywords: deoxyribonucleic acid, Ultraviolet, Infrared, Terahertz, film

\section{Introduction}

Since Deoxyribonucleic Acid (DNA) double-helix structure was discovered [1], DNA has been intensively investigated in biomedical technologies, genetic, molecular biology due to their unique properties. Recently, DNA film has drawn intense attention due to their promising optoelectronic device application such as optical amplifier as a host for photochromic dyes [2], Solar cell [3], organic light-emitting diode [4], transistor [5], and nonlinear saturable absorbers for ultrashort pulse generation [6]. In order to utilize DNA films as component of photonic devices operating in various frequency range, it is crucial to investigate transparent wavelength regions from ultraviolet to terahertz. In this study, we measured transmittance of DNA film with a various thickness ranging from a few micrometer to above a hundred micrometer in a broad spectra range including ultraviolet, visible, IR and terahertz regions. From the measured transmittance spectra, we successfully found highest transmission spectra range within investigated wavelength regions, which can provide essential information in terms of photonic device utilizing in a very wide frequency ranges and biological technologies.

\section{Sample fabrication}

Highly purified thread-like solid salmon DNA, which was widely used in DNA film fabrication for optical- electronic devices was used $[7,8]$. The solid DNA was dissolved in deionized water to prepare homogeneous DNA aqueous solution with concentration of $1 \mathrm{wt} \%$. We dropped the DNA aqueous solution onto a petri dish and dried in an oven at $25{ }^{\circ} \mathrm{C}$. Uniform freestanding DNA solid films were obtained and thickness was in the range of $2 \mu \mathrm{m} \sim 150 \mu \mathrm{m}$. The thickness of the film was controlled according to the amount of solution on the Petri dish and, surface profiler (DektakXT, BRUKER) was used to measure the thickness of films.

\section{Results and Discussion}

1. UV/Visible/NIR Measurement of DNA solid film

We measured UV/Visible/NIR transmittance of freestanding DNA solid film as a function of thickness in the wide spectra range from $200 \mathrm{~nm}$ to $3000 \mathrm{~nm}$ using a commercial spectrometer (Cary5000, Agilent), and the results are summarized in Figure 1. We observed that UV/Visible/NIR spectra of DNA solid film have two significant absorptive regions: Ultraviolet region with a wavelength range from $200 \mathrm{~nm}$ to $380 \mathrm{~nm}$ due to electronic structure of heterocyclic rings of nucleotides [9], and the stretching frequency of $\mathrm{OH}$ bond in the range of $2770 \mathrm{~nm} \sim 3330 \mathrm{~nm}$ [10]. We also found that additional absorption bands as follow: $1420 \mathrm{~nm} \sim 1520$ $\mathrm{nm}, 1900 \mathrm{~nm} \sim 2000 \mathrm{~nm}, 2250 \mathrm{~nm} \sim 2310 \mathrm{~nm}$, and 2430 $\mathrm{nm} \sim 2520 \mathrm{~nm}$, which could be attributed to a small amount of solvent $\left(\mathrm{H}_{2} \mathrm{O}\right)$ and biomolecules consisting 


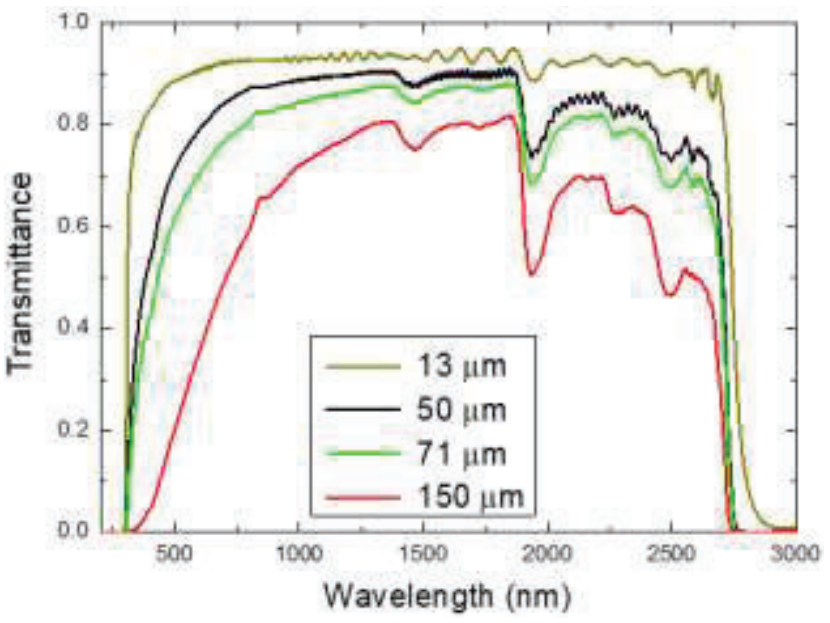

Figure 1. UV/Visible/NIR transmittance spectra of freestanding DNA solid film with a various thickness.

protein inside DNA film [11]. However, the absorption corresponding to these peak frequency is much lower in comparison with the absorption by ultraviolet and the $\mathrm{OH}$ bond stretching, and the effect is negligibly small in films with thickness below few micrometer. Note that the DNA films exhibited quite good transparency in the wide near infrared spectra range.

\section{Mid IR measurement of DNA solid film}

FTIR spectroscopy was employed in the spectra range from $3 \mu \mathrm{m}$ to $25 \mu \mathrm{m}$ to investigate transmittance of DNA film as a function of thickness. Figure 2 shows that transmittance spectra of freestanding DNA solid film with

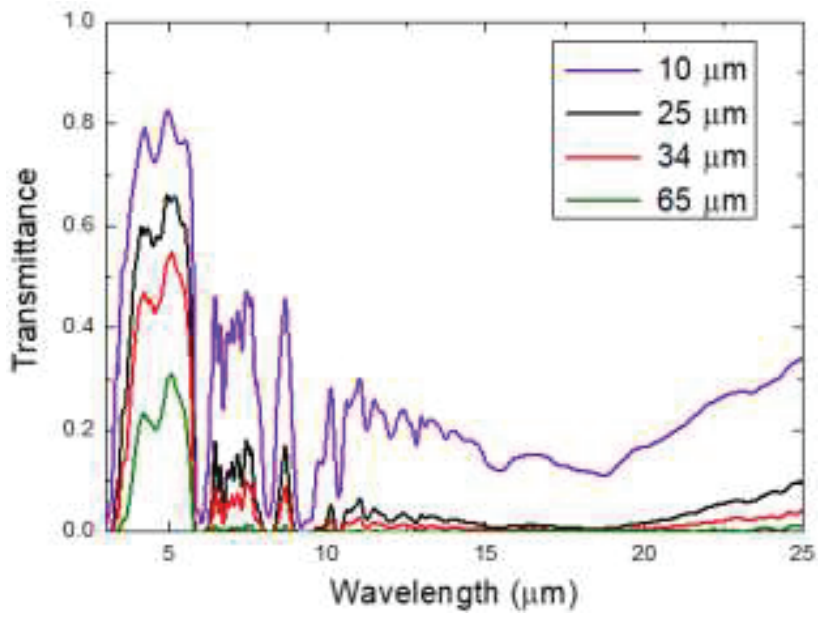

Figure 2. FTIR transmittance spectra of freestanding DNA solid films with a various thickness. a various thickness. Since FTIR absorption spectra are mainly due to resonance frequency of molecular consisting DNA structure such as $\mathrm{OH}$ stretching, four nucleobase and, sugar and phosphate backbone group [10], low transmittance in DNA solid film with thickness of few micrometer is quite predictable. We confirmed that almost all the light was blocked at a thickness of $65 \mu \mathrm{m}$ in this mid IR region as shown in the Figure 2. Note that absorption band position of DNA solid film did not change regardless of film thickness.

\section{THz-TDS spectroscopy of DNA solid film}

A standard transmission THz-TDS system was employed to measure terahertz optical properties of freestanding DNA solid film in the spectra range from $0.2 \mathrm{THz}$ to 2 THz. The electric field of terahertz pulses transmitted through reference and sample are recorded in the time domain, and the corresponding frequency domain spectra could be obtained by using numerical Fourier transforms. Figure 3 shows the amplitude transmittance of freestanding DNA solid film with thickness of $130 \mu \mathrm{m}$ and, it presents quite good transmission between $0.2 \mathrm{THz}$ and $0.7 \mathrm{THz}$. It will probably exhibits high transparency at a thickness below $100 \mu \mathrm{m}$. Refractive index was calculated using equations in terms of phase and magnitude ratio of transmitted electric field through sample and reference [12] and, its value was in the range of $1.85 \sim 2.20$ within investigated frequency range. This value was found to be higher than the refractive index of

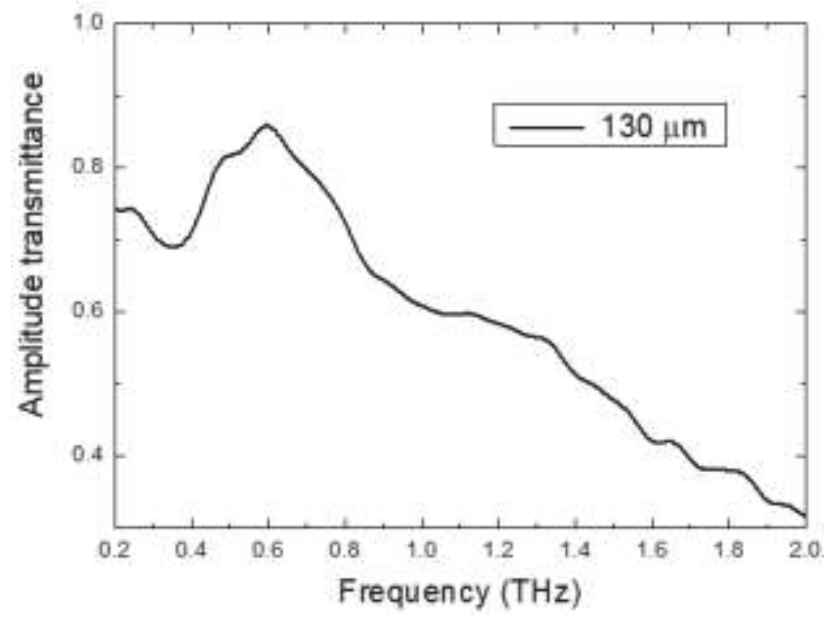

Figure 3. THz transmittance spectra of freestanding DNA solid films. 
well known transparent polymer in terahertz region [13].

\section{Conclusion}

We successfully measured transmittance of DNA film with a various thickness in the frequency range from ultraviolet to terahertz and found the wavelength range with highest transmittance of DNA film were located in the near infrared and terahertz regions. These results are expected to lead a major advance in DNA based photonics devices and DNA biology application.

\section{Reference}

[1] J. D. Watson, and F. H. Crick, "Molecular structure of nucleic acids," Nature, 171(4356), 737-738 (1953).

[2] Y. Kawabe, L. Wang, S. Horinouchi et al., "Amplified Spontaneous Emission from Fluorescent-Dye-Doped DNA-Surfactant Complex Films," Advanced Materials, 12(17), 1281-1283 (2000).

[3] W.-H. Son, M. S. P. Reddy, and S.-Y. Choi, "Hydrogenated amorphous silicon thin film solar cell with buffer layer of DNA-CTMA biopolymer," Modern Physics Letters B, 28(13), 1450107 (2014).

[4] A. Steckl, H. Spaeth, H. You et al., "DNA as an optical material," Optics and Photonics News, 22(7), 3439 (2011).

[5] N. Mohanty, and V. Berry, "Graphene-based singlebacterium resolution biodevice and DNA transistor: interfacing graphene derivatives with nanoscale and microscale biocomponents," Nano letters, 8(12), 44694476 (2008).

[6] R. Khazaeinezhad, S. H. Kassani, B. Paulson et al., "Ultrafast nonlinear optical properties of thin-solid DNA film and their application as a saturable absorber in femtosecond mode-locked fiber laser," Scientific reports, 7, 41480 (2017).

[7] A. Samoc, A. Miniewicz, M. Samoc et al., "Refractive-index anisotropy and optical dispersion in films of deoxyribonucleic acid," Journal of applied polymer science, 105(1), 236-245 (2007).

[8] B. Paulson, I. Shin, H. Jeong et al., "Optical dispersion control in surfactant-free DNA thin films by vitamin B 2 doping," Scientific Reports, 8(1), 9358 (2018).
[9] Y.-W. Kwon, C. H. Lee, D.-H. Choi et al., "Materials science of DNA," Journal of Materials Chemistry, 19(10), 1353-1380 (2009).

[10] B. Gnapareddy, S. R. Dugasani, T. Ha et al., "Chemical and physical characteristics of doxorubicin hydrochloride drug-doped salmon DNA thin films," Scientific reports, 5, 12722 (2015).

[11] O. Poch, J. Frey, I. Roditi et al., "Remote sensing of potential biosignatures from rocky, liquid, or icy (exo) planetary surfaces," Astrobiology, 17(3), 231-252 (2017).

[12] H. Yasuda, and I. Hosako, "Measurement of terahertz refractive index for plasmon waveguides." 1125 1128.

[13] Y.-S. Jin, G.-J. Kim, and S.-G. Jeon, "Terahertz dielectric properties of polymers," Journal of the Korean Physical Society, 49(2), 513-517 (2006). 


\title{
Tunable vortex parametric laser with multiple OAM states
}

\author{
Roukuya Mamuti ${ }^{\mathrm{a}}$, Shigeki Nishida ${ }^{\mathrm{a}}$, Katsuhiko Miyamoto ${ }^{\mathrm{a}, \mathrm{b}}$, Takashige Omatsu ${ }^{\mathrm{a}, \mathrm{b}}$ \\ ${ }^{a}$ Graduate School of Engineering, Chiba University, \\ 1-33, Yayoi-cho, Inage-ku, Chiba 263-8522, Japan \\ ${ }^{b}$ Molecular Chirality Research Center, Chiba University, \\ 1-33, Yayoi-cho, Inage-ku, Chiba 263-8522, Japan
}

\begin{abstract}
We demonstrate a tunable vortex laser with versatile orbital angular momentum (OAM) states based on a singly resonant optical parametric oscillator (OPO) formed of a non-critical phase-matching $\mathrm{LiB}_{3} \mathrm{O}_{5}$ (NCPM-LBO) crystal. The selective generation of a signal (idler) output with three OAMs, including an up-converted (negative) OAM, is achieved simply by appropriate shortening (or extending) of the cavity. The compact cavity configuration also allows for the generation of the signal (idler) output with various OAMs by simply tuning the signal wavelength. The vortex output is tuned within the wavelength region of $0.74 \mu \mathrm{m}$ to $1.86 \mu \mathrm{m}$ with maximum pulse energy of $1.99 \mathrm{~mJ}$ from pump energy of $8.5 \mathrm{~mJ}$.
\end{abstract}

Keywords: optical vortices, nonlinear optics, optical parametric oscillator, orbital angular momentum.

\section{Introduction}

Optical vortices are characterized by a ring-shaped spatial form and an orbital angular momentum (OAM) of

$\ell \phi[1,2]$, due to a phase singularity arising from its azimuthal phase term, $e^{i \ell \phi}$, where $\ell$ is an integer and known as a topological charge, have been widely utilized in a variety of research fields, including optical tweezing and manipulation [3], large capacity optical telecommunication [4], fluorescent microscopes with a high spatial resolution beyond the diffraction limit [5] and materials processing [6,7]. Tunable optical vortex sources with versatile OAM states are strongly desired for the above-mentioned applications to meet with the absorption bands of different materials.

In this presentation, we report on the first demonstration of selective generation of a signal and idler outputs having multiple OAM states with $\ell=-2 \sim 4$, including up-converted and negative states from a nanosecond $2^{\text {nd }}$ order vortex pumped singly resonant optical parametric oscillator (OPO) by simply shortening or extending of the cavity.

\section{Experimental setup}

Figure 1 shows a schematic diagram of the tunable optical parametric laser system. A frequency-doubled, diode-pumped, Q-switched Nd:YAG laser (a wavelength of $532 \mathrm{~nm}$; a pulse duration of $10 \mathrm{~ns}$; PRF of $100 \mathrm{~Hz}$; M $=1.1)$ was used as a pump source, and its output was converted into an optical vortex with a topological charge of +2 by using 16 segmented spiral phase plates formed of silica glass. A non-critical phase matching LBO crystal $\left(\theta=90^{\circ}, \varphi=0^{\circ}, 3 \times 3 \times 45 \mathrm{~mm}^{3}\right.$, AR-coated for $532 \mathrm{~nm}$ and $1064 \mathrm{~nm}$ ) used as a nonlinear crystal and was mounted on an oven.

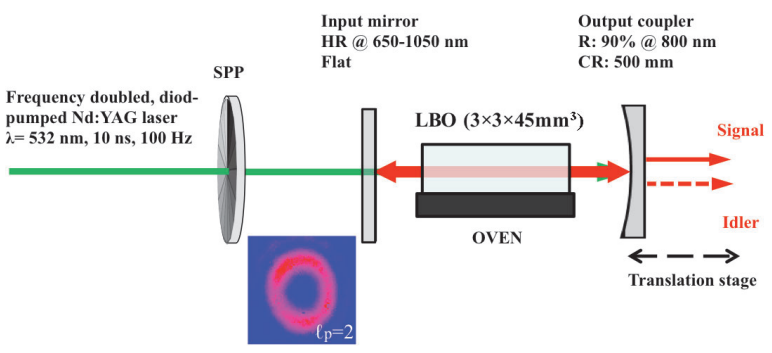

Figure 1. Schematic diagram of experimental setup.

The singly resonant cavity for the signal output was formed by a flat input mirror with high reflectivity for 0.65-1.05 $\mu \mathrm{m}$ and a concave output mirror (curvature radius, $500 \mathrm{~mm}$ ) with $90 \%$ reflectivity for $800 \mathrm{~nm}$. The signal and idler outputs were separated by a dichroic mirror, and they were observed using $\mathrm{Si}$ and $\mathrm{InGaAs}$ cameras, respectively. The cavity length was tuned within $54 \sim 250 \mathrm{~mm}$ to control the topological charge sharing 
between the signal and idler outputs. The topological charges of the signal and idler outputs were assigned by employing a self-referenced interferometer, in which the $1^{\text {st }}$ order and $-1^{\text {st }}$ order beams were diffracted with a transmission grating (10 lines/mm) and overlapped by a lens on a CCD camera to form self-interference fringes.

\section{Results and Discussion}

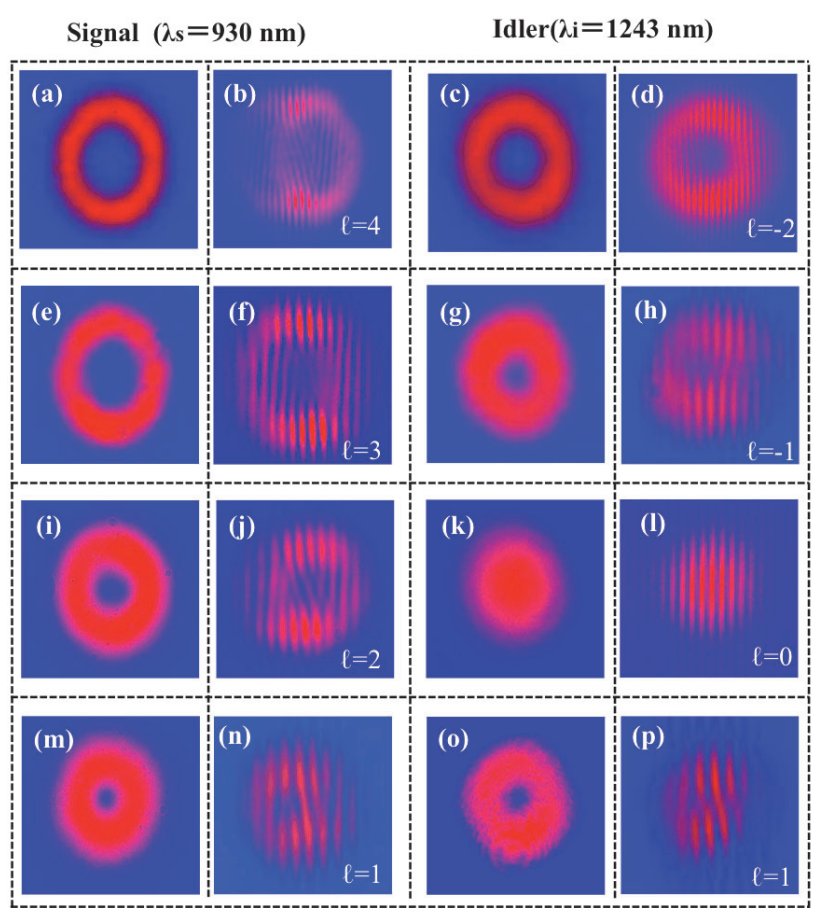

Figure 2. Spatial profiles and self-interference fringes for the $0.93 \mu \mathrm{m}$ signal and $1.24 \mu \mathrm{m}$ idler outputs from a further compact, compact, extended and further extended cavities with a length of 54, 60, 120, 230 $\mathrm{mm}$ respectively.

A cavity with a length of $54 \mathrm{~mm}$ forced the signal to lase at a vortex mode with an unexpected up-converted vortex mode with $\ell=4$, as evidenced by an annular spatial form with a pair of downward and upward five-armed self-interference fringes, as shown in Figs. 2(a,b). The resulting idler also exhibited a vortex mode with a topological charge of -2 , as evidenced by a pair of upward and downward tree-armed fringes. The compact cavity with a length of $60 \mathrm{~mm}$ forced the signal to lase at a vortex mode with $\ell=3$, thereby yielding a vortex idler with $\ell=-1$, as shown in Figs. 2(e,f) and $(\mathrm{g}, \mathrm{h})$. The extended cavity with a length of $120 \mathrm{~mm}$ forced the lasing of the signal with $\ell=+2$, as shown in Figs. 2(i,j), thereby yielding a Gaussian spatial profile without any phase singularities, as shown in Figs. 2(k,1). The further extended cavity with a length of $230 \mathrm{~mm}$ produced both signal and idler vortex modes with $\ell=+1$, as shown in Figs. $2(m, n)$ and $(o, p)$.

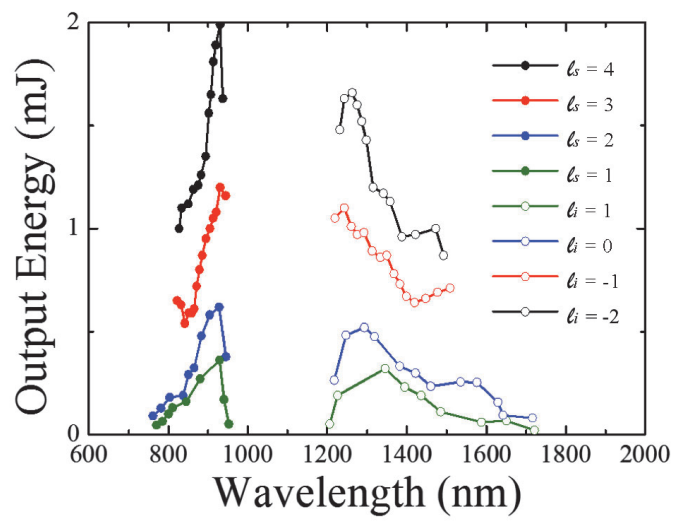

Figure 3. Tunability of the signal and idler outputs.

The signal and idler outputs with multiple OAM states were tuned in the wavelength regions of $0.74 \sim 0.96 \mu \mathrm{m}$ and 1.12 1.86 $\mu \mathrm{m}$, respectively. The maximum signal and idler output energies of $1.99 \mathrm{~mJ}$ and $1.65 \mathrm{~mJ}$ were observed at a pump energy of $8.5 \mathrm{~mJ}$, corresponding to optical efficiencies of $22.2 \%$ and $19.4 \%$.

Also, it is worth noting that the compact cavity configuration interestingly enables us to selectively operate a signal (idler) vortex output with various OAMs by simply tuning the wavelength of the signal output and without any change of the cavity length [8].

\section{Conclusion}

We have successfully demonstrated, for the first time, a selective generation of signal and idler outputs with multiple OAMs from a tunable vortex parametric laser based on a singly resonant OPO formed by a NCPM-LBO crystal. This system with a singly resonant cavity configuration allows us to produce vortex modes with seven different OAMs $(\ell=4 \sim-2)$ of the signal or idler output, simply by shortening or extending the cavity length. This system with versatile OAM states will be useful in various fields, such as materials processing, as well as tunable optical vortex generation in the midinfrared or terahertz region. Further OAM versatility in 
this system will be possible by conducting a singly resonant cavity for idler output.

\section{References}

[1] L. Allen, W. Beijersbergen, R. C. Spreeuw, and J. P. Woerdman, "Orbital angular momentum of light and the transformation of Laguerre-Gaussian laser modes," Phys. Rev. A 45, 8185 (1992).

[2] S. M. Barnett, M. Babiker, M. J. Padgett, "Optical orbital angular momentum," Phil. Trans. R. Soc. A 375: 20150444 (2017).

[3] Grier, D. G. A revolution in optical manipulation. Nature 2003, 424 (6950), 810-816.

[4] A. E. Willner, H. Huang, Y. Yan, Y. Ren, N. Ahmed, G. Xie, C. Bao, L. Li, Y. Cao, Z. Zhao, J. Wang, M. P. J. Lavery, M. Tur, S. Ramachandran, A. F. Molisch, N. Ashrafi, and S. Ashrafi, "Optical communications using orbital angular momentum beams," Adv. Opt. Photon. 7, 66-106 (2015).

[5] Stefan W. Hell and Jan Wichmann, "Breaking the diffraction resolution limit by stimulated emission: stimulated-emission-depletion fluorescence microscopy," Opt. Lett. 19, 780-782 (1994).

[6] T. Omatsu, K. Chujo, K. Miyamoto, M. Okida, K. Nakamura, N.Aoki, and R. Morita, "Metal microneedle fabrication using twisted light with spin," Opt. Express 18, 17967-17973 (2010).

[7] K. Masuda, R. Shinozaki, Y. Kinezuka, J. Lee, S. Ohno, S. Hashiyada, H. Okamoto, D. Sakai, K. Harada, K. Miyamoto, and T. Omatsu, Opt. Express 26, 2219722207 (2018).

[8] R. Mamuti, S. Araki, S. Nishida, K. Miyamoto, and T. Omatsu, Appl. Opt. 57 (34), 10004-10008 (2018). 


\title{
The PCF design for more number of OAM modes up to 101 by increasing
}

\section{the number of air-holes}

\author{
Seongjin Honga,d, Yong Soo Lee ${ }^{\mathrm{a}, \mathrm{d}}$, Hyucksu Choi ${ }^{\mathrm{a}}$, Chai Quan ${ }^{\mathrm{a}}$, Yan $\mathrm{Li}^{\mathrm{b}}$, Soeun Kim ${ }^{\mathrm{c}}$, Kyunghwan \\ $\mathrm{Oh}^{\mathrm{a}^{*}}$
}

aPhotonic Device Physics Laboratory, Institute of Physics and Applied Physics, Yonsei University, Seoul 03722, South Korea

${ }^{b}$ Department of Optoelectronics Science, Harbin Institute of Technology, Weihai 264209, China ${ }^{c}$ Integrated Optics Laboratory, Advanced Photonics Research Institute, GIST, Gwangju 61005, South Korea

${ }^{\mathrm{d}}$ These authors contributed equally to this work

\begin{abstract}
Orbital angular momentum (OAM) modes have attracted extensive attention for their wide range of applications in optical communications. OAM is considered to be promising way to increase the transmission capacity. As many as possible supported number of OAM modes in optical fiber could provide capacity increase critically. The structure design of the OAM supporting photonic crystal fiber (PCF) has recently attracted attention in optical communication research field. We proposed a new method to increase the orbital angular momentum modes up to 101 by increasing the number of air-holes at each layer from 35 to 55 in PCF, and find out that more air-holes can support more OAM modes. Additionally, higher refractive index difference, better mode quality and reduced dispersion could be achieved.
\end{abstract}

Keywords: Optical fiber, Fiber design, Communication, Orbital angular momentum, Mode division multiplexing

\section{Introduction}

Orbital angular momentum (OAM) modes have attracted extensive attention for their wide range of applications in optical communications. OAM is considered to be promising way to increase the transmission capacity [1,2]. The following factors should be considered in designing the PCF for OAM modes [35]: (1) as many as possible supported OAM modes, (2) large effective index difference $\left(>10^{-4}\right)$ in the same OAM family, (3) maintain the 'single-mode' in the radial direction, (4) Good mode quality of all supported OAM modes, (5) flat chromatic dispersion, and (6) low confinement loss.

In this paper, we proposed a new method to increase the OAM modes up to 101 by increasing the number of air-holes of each layers in PCF, for the first time to the best knowledge of the authors. Additionally, higher refractive index difference, better mode quality and reduced dispersion could be achieved for maintain the single mode with mode confinement loss lower than $10^{-}$ ${ }^{5} \mathrm{~dB} / \mathrm{m}$.

\section{Fiber design and results}

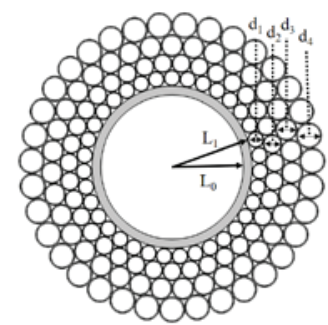

(a)

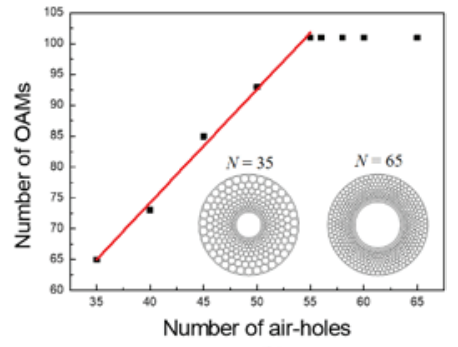

(b)
Figure 1. (a) The cross section of the designed PCF, (b) The increase number of OAM modes depending on the number of air-holes. Inset shows the cross section of designed PCF when $N=35$ and 65 .

OAM modes in fiber could be regarded as the super position of the vector eigenmodes by following equations [3].

$$
\begin{aligned}
& O A M_{ \pm l, m}^{ \pm}=H E_{l+1, m}^{\text {even }} \pm j H E_{l+1, m}^{\text {odd }} \\
& O A M_{ \pm l, m}^{\mp}=E H_{l-1, m}^{\text {even }} \pm j E H_{l-1, m}^{\text {odd }}
\end{aligned}
$$


where $m$ is the number of in radial direction, $l$ is the topological charge, \pm is the right and left of the circular polarization [3]. While the OAM modes have the same $l$ and $m$, we call them an OAM mode family. When $l=2$, the OAM family possesses 4 OAM states including $\pm \mathrm{OAM}_{21}$ from $\mathrm{HE}_{2,1}$ and $\mathrm{EH}_{1,1}$.

Figure 1(a) shows the cross-section structure of the proposed PCF for transmitting OAM modes. The background material of the designed PCF is all-silica without any doping material. The center ring was encircled by several layers of air-holes. The size of center ring was fixed with $L_{0}=8 \mu \mathrm{m}$ and $L_{1}=9 \mu \mathrm{m}$ to maintain the 'single-mode' in the radial direction. The diameter of air-holes at 1 st layer, $d_{l}$, was determined by the $L_{l}$ and the number of air-holes at 1 st layer, $N$, to encircle the center ring. The diameter of air-holes at $n$th layer, $d_{n}$, were also determined to encircle the $n$-1 th layer as shown in Figure (a) for $n>2$. For example, the parameters of the PCF are as follows when $N=35: d_{1}=1.6 \mu \mathrm{m}, d_{2}=1.86 \mu \mathrm{m}, d_{3}=$ $2.18 \mu \mathrm{m}, d_{4}=2.54 \mu \mathrm{m}$ and when $N=55: d_{1}=0.98 \mu \mathrm{m}, d_{2}=$ $1.08 \mu \mathrm{m}, d_{3}=1.20 \mu \mathrm{m}, d_{4}=1.32 \mu \mathrm{m}$. Figure $1(\mathrm{~b})$ shows the OAM mode increase depending on $N$. We could gain the increase of the number of OAM modes from 65 to 101 modes in a proposed PCF by increasing the $N$ from 35 to 55 , and the number of OAM modes was not increased more than 101 with $N=60,65$. Note that confinement loss of supported all OAM modes were kept lower than $10^{-5}$. It is clearly shown that $N$ significantly could increase the number of OAM modes in the PCF. To the best knowledge of the authors, this is the first time of the increase of the number of OAM modes by increasing the number of air-holes in PCF.
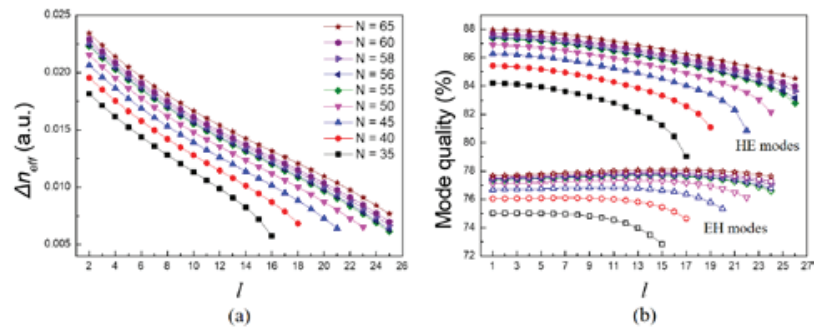

Figure 2. (a) Effective index difference of the different OAM mode family with different $N$, (b) Mode quality of supported $\mathrm{HE}$ and $\mathrm{EH}$ modes with different $N$.

It is well known that effective index difference, $\Delta n_{\text {eff, }}$, between corresponding $\mathrm{HE}_{l, 1}$ and $\mathrm{EH}_{l, 1}$ modes of the same
$\mathrm{OAM}_{l, 1}$ mode family should be larger than $10^{-4}$ to avoid modal coupling and crosstalk [3]. Figure 2(a) shows the $\Delta n_{\text {eff }}$ of the different order $\mathrm{OAM}_{l, 1}$ mode families, where $\Delta n_{\text {eff }}$ for $l=2$ denotes the effective index difference between $\mathrm{HE}_{2,1}$ and $\mathrm{EH}_{1,1}$ modes, which were composed of $\mathrm{OAM}_{2,1}$. It is clearly shown that $\Delta n_{\text {eff }}$ is significantly increased $\sim 0.005$ with $N$ increase from 35 to 65 , which confirms the number of air-holes could be solution to possess high $\Delta n_{\text {eff }}$ between the same order OAM modes in PCF.

The mode quality of OAM could ensure the stable transmission in the fiber [4]. The mode quality was evaluated by the light intensity overlap between in the ring area where the OAM modes should be confined and the whole cross-section area of the PCF.The mode quality of OAM could ensure the stable transmission in the fiber [4]. The mode quality was evaluated by the light intensity overlap between in the ring area where the OAM modes should be confined and the whole cross-section area of the PCF.

Mode quality $=\frac{\iint_{\text {core }}|\vec{E}|^{2} d x d y}{\iint_{\text {cross-section }}|\vec{E}|^{2} d x d y}$

Figure 2(b) shows the mode quality of $\mathrm{HE}_{l, 1}$ and $\mathrm{EH}_{l, 1}$ modes for all $l$ orders, which could be supported in the PCF. The filled symbol and open symbol indicate the HE and EH modes, respectively, and the order of $N$ is same with Figure 2(a). The mode quality of HE modes were higher $\sim 10 \%$ than EH modes, and also it shows that mode quality of all modes were increased at least $2.7 \%$ with increase of $N$ from 35 to 65 .

In table 1, we summarized the improved characteristics of OAM modes at $1550 \mathrm{~nm}$. With the increase of $N$ from 35 to 65 , it showed increase of the number of OAM modes (65 101), $\Delta n_{\text {eff }}(0.02038 \sim 0.02585)$, HE mode quality (75 77\%), EH mode quality (84.2 88.0\%), and the reduced dispersion of $\mathrm{OAM}_{11}$ mode (40.4 18.6ps/(nm.km)) at $l=2$. 
Table 1. Improved OAM characteristics depending on $N$

\begin{tabular}{|c|c|c|c|c|c|}
\hline $\mathbf{N}$ & $\begin{array}{c}\text { Number } \\
\text { of OAM } \\
\text { modes }\end{array}$ & $\begin{array}{c}\Delta n_{\text {eff }} \\
(\boldsymbol{l}=\mathbf{2})\end{array}$ & $\begin{array}{c}\text { HE } \\
\text { mode } \\
\text { quality } \\
(\boldsymbol{l}=\mathbf{2})\end{array}$ & $\begin{array}{c}\mathbf{E H} \\
\mathbf{m o d e} \\
\mathbf{q u a l i t y} \\
(\boldsymbol{l = 2})\end{array}$ & $\begin{array}{c}\text { OAM } \\
\text { Dispersion } \\
\text { at 1550nm } \\
{[\mathbf{p s} /(\mathbf{n m} \cdot \mathbf{k m})]}\end{array}$ \\
\hline 35 & 65 & 0.02038 & $84.2 \%$ & $75.0 \%$ & 40.4 \\
\hline 55 & 101 & 0.02469 & $87.3 \%$ & $77.4 \%$ & 24.5 \\
\hline 65 & 101 & 0.02585 & $88.0 \%$ & $77.7 \%$ & 18.6 \\
\hline
\end{tabular}

\section{Conclusion}

We proposed a new method to gain more OAM modes, higher effective index difference, better mode quality, and lower dispersion by increasing the number of air-holes in PCF. We could gain more OAM modes from 65 to 101 modes by increasing the number of air-holes at each layer from 35 to 55, and find out that more air-holes can support more OAM modes. Additionally, with increase of the number of air-holes, it showed that effective index difference increase (0.0054), HE mode quality increase (3.6\%), EH mode quality increase $(2.7 \%)$ at $1=2$, and the reduced dispersion of OAM11 mode $(21.8 \mathrm{ps} /(\mathrm{nm} \cdot \mathrm{km}))$. We believed that the proposed method could be further applied to fabricate a PCF for better OAM transmission.

\section{Acknowledgement}

This work was supported in part by ICT R\&D Program of MSIP/IITP (2014-3-00524), in part by Basic Science Research Program through the National Research Foundation of Korea (NRF) funded by the Ministry of Science, ICT \& Future Planning (No. 2016k1A3A1A09918616), and in part by Basic Science Research Program through the National Research Foundation of Korea (NRF) funded by the Ministry of Education (2018R1D1A1B07049349)

\section{References}

[1] C. Chen, G. Zhou, G. Zhou, M. Xu, Z. Hou, C. Xia, and J. Yuan, "A multi-orbital-angular-momentum multiring micro-structured fiber with ultra-high-density and low-level crosstalk," Optics Communications 368, 27-33 (2016).

[2] H. Zhang, W. Zhang, L. Xi, X. Tang, X. Zhang, and X. Zhang, "A new type circular photonic crystal fiber for orbital angular momentum mode transmission," IEEE Photonics Technology Letters 28, 1426-1429 (2016). [3] H. Zhang, X. Zhang, H. Li, Y. Deng, L. Xi, X. Tang, and W. Zhang, "The Orbital Angular Momentum Modes Supporting Fibers Based on the Photonic Crystal Fiber Structure," Crystals 7, 286 (2017).

[4] H. Zhang, X. Zhang, H. Li, Y. Deng, X. Zhang, L. Xi, $\mathrm{X}$. Tang, and W. Zhang, "A design strategy of the circular photonic crystal fiber supporting good quality orbital angular momentum mode transmission," Optics Communications 397, 59-66 (2017).

[5] Z.-A. Hu, Y.-Q. Huang, A.-P. Luo, H. Cui, Z.-C. Luo, and W.-C. Xu, "Photonic crystal fiber for supporting 26 orbital angular momentum modes," Optics express 24, 17285-17291 (2016). 


\title{
Visible vortex light source based on a diode pumped $\operatorname{Pr}^{3+}:$ YLF laser
}

\author{
Yuanyuan Ma ${ }^{\mathrm{a}}$, Jung-Chen Tung ${ }^{\mathrm{b}}$, Yung-Fu Chen ${ }^{\mathrm{b}}$, Katsuhiko Miyamoto ${ }^{\mathrm{a}, \mathrm{c}}$, Takashige Omatsu*a,c \\ ${ }^{\mathrm{a}}$ Graduate School of Advanved Integration Science, Chiba University, 1-33 Inage-ku, Chiba, 263- \\ 8522, Japan \\ ${ }^{b}$ Department of Electrophysics, National Chiao Tung University, 1001 Ta-Hsueh Rd., Hsinchu 30010, \\ Taiwan \\ 'Molecular Chirality Research Center, Chiba University, 1-33 Inage-ku, Chiba, 263-8522, Japan
}

\begin{abstract}
We successfully demonstrated the visible vortex mode generation from a diode-pumped $\operatorname{Pr}^{3+}:$ YLF laser by employing an off-axis pumping technique. A maximum output of $\sim 100 \mathrm{~mW}$ was obtained at a pump power of $1.2 \mathrm{~W}$. Such visible structured light beams will be potentially applied to super-resolution fluorescent microscope and micro-fabrications.

Keywords: optical vortices, singular optics, laser resonator, $\operatorname{Pr}^{3+}:$ YLF laser, off-axis pumping
\end{abstract}

\section{Introduction}

Vortex light fields, i.e. optical vortices (scalar vortex) and radially or azimuthally polarized (vector vortex) modes [1,2], possess unique features, such as a ringshaped spatial profile, creation of longitudinal electric field, self-healing effect, and an orbital angular momentum of $\ell \hbar$ ( $\ell$ is an integer, so-called the topological charge) per photon. In fact, they provide us a variety of applications, for instance, optical manipulations [3], space-division multiplexing optical communications [4], laser micro-fabrications [5], quantum optics, and super-resolution fluorescence microscopes [6]. Direct generation of such vortex light field from laser resonators allows us to generate very high-power vortex light beams as eigen modes with a desired wavelength and excellent beam quality without any additional phase modulating elements.

A blue InGaN laser diode pumped $\operatorname{Pr}^{3+}$ :YLF laser enables us to develop wavelength-versatile laser sources in a visible region, such as blue, green, orange, red, and deep red spectral regions [7], and it has been successfully demonstrated to achieve a continuous-wave, a Qswitching, and a mode-locking operations[8-11].

In this paper, we demonstrate, for the first time, the direct generation of a visible $(640 \mathrm{~nm})$ scalar and a vector vortex modes from a blue diode-pumped $\mathrm{Pr}^{3+}$ :YLF laser with an off-axially pumping configuration without any additional optical elements. Such visible vortex light sources will be potentially utilized in various applications.

\section{Experiments}

Figure 1 shows an experimental setup for generating the vortex light modes from a diode-pumped $\mathrm{Pr}^{3+}: \mathrm{YLF}$ laser. A 5-mm long c-cut $\operatorname{Pr}^{3+}$ :YLF crystal with 0.5 at.\% $\operatorname{Pr}^{3+}$ ions doping was used, and its input face had high reflection for a wavelength of $640 \mathrm{~nm}$ and antireflection for a wavelength of $445 \mathrm{~nm}$. The laser cavity was formed of an input face of the crystal and a highly-reflective concave output coupler ( $\mathrm{R}>98.5 \%$ ), and its length was $\sim 11 \mathrm{~mm}$. A 445-nm InGaN laser diode was used as a pump source. Its output was focused to be an elliptical

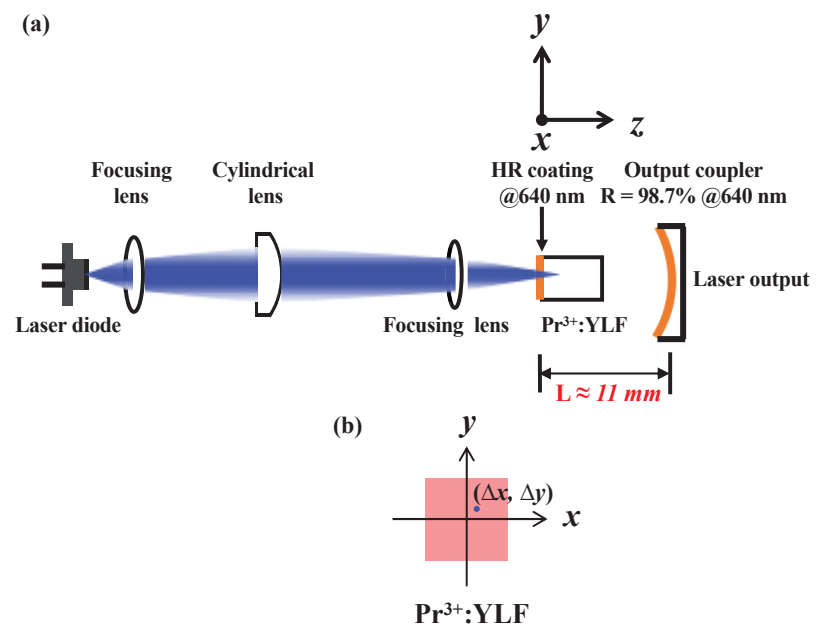

Fig 1(a). Experimental setup for $\mathrm{Pr}^{3+}$ :YLF vortex laser with the off-axis pumping. (b) Pumping position on the $\operatorname{Pr}^{3+}:$ YLF crystal. 
spot with a diameter of $100 \times 30 \mu \mathrm{m}^{2}$ onto the laser crystal, so as to off-axially pump the amplifier towards $x$ and $y$ directions with displacements of $\Delta x$ and $\Delta y$ from an onaxis of the cavity, i.e. off-axis pumping, as shown in Fig. 1(b). A maximum pump power was $1.3 \mathrm{~W}$.

\section{Results}
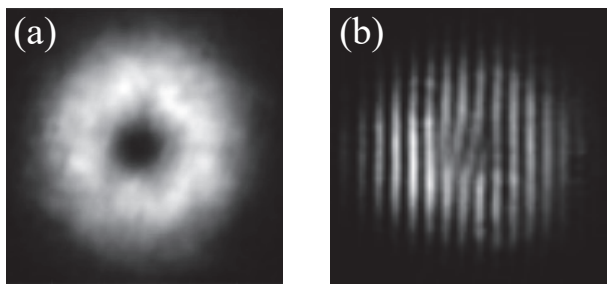

(c)

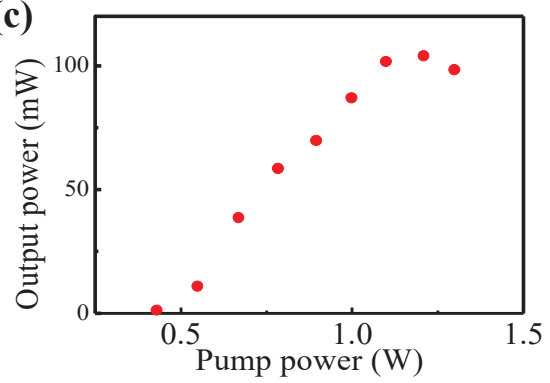

Fig. 2(a). Far-field spatial intensity profile and (b) wavefront of the generated first-order vortex beam (c) Power scaling of the vortex output.

When $\Delta x$ and $\Delta y$ were $25 \mu \mathrm{m}$ and $30 \mu \mathrm{m}$, the laser produced a scalar vortex (first-order optical vortex) mode, as evidenced by a doughnut-shaped spatial form with a central dark core and a pair of upward and downward Yshaped fringes produced by a self-referenced, laterally sheared interferometer (Figs. 2). A maximum output power was then measured to be $102 \mathrm{~mW}$ at a pump power of $1.2 \mathrm{~W}$, corresponding to a slope efficiency of $13.1 \%$.

The laser also generated a radially polarized (vector vortex) mode, as evidenced by a doughnut-shaped spatial form and a twin lobe beam along the polarization direction. A maximum power was then measured to be $90.4 \mathrm{~mW}$ (Figs. 3). The displacements $\Delta x$ and $\Delta y$ were then measured to be $20 \mu \mathrm{m}$ and $70 \mu \mathrm{m}$, respectively. Such a diode-pumped $\mathrm{Pr}^{3+}$ doped laser with an off-axially pumping configuration allows us to selectively generate a desired scalar or vector vortex mode by only controlling appropriately the location of pump spot.
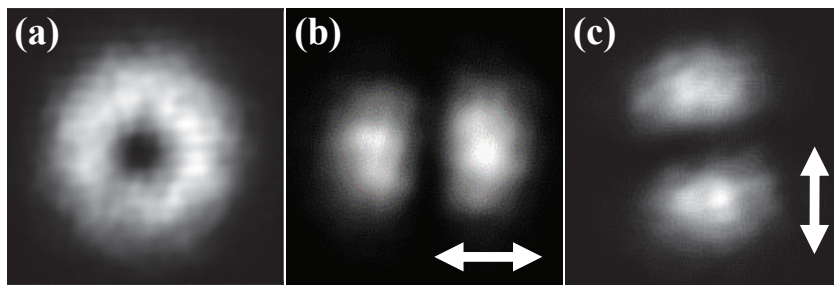

(d)

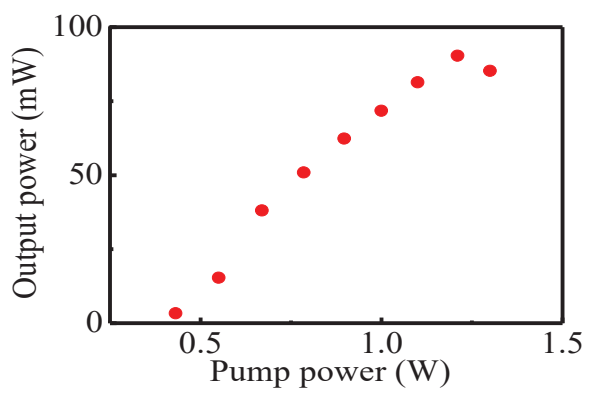

Fig 3 Intensity distributions of the obtained radially polarized vortex beam. (a) Total intensity distribution. $(b, c)$ Intensity distributions after passing through a linear polarizer with a direction of $0 \mathrm{deg}$ or $90 \mathrm{deg}$. The arrows indicate the directions of the polarizer. (d) Power scaling of the radial vector vortex output.

\section{Conclusion}

We have successfully demonstrated the direct generation of visible vortex light beams, such as scalar and vector vortex modes from a diode-pumped $\mathrm{Pr}^{3+}$ :YLF vortex laser without any additional optical elements. Such $100 \mathrm{~mW}$ level visible vortex light sources will open the door towards versatile applications, such as superresolution microscopes, and micro-fabrications.

\section{References}

[1] Allen, L., Beijersbergen, M. W., Spreeuw, R. J. C., and Woerdman, J. P., "Orbital angular momentum of light and the transformation of Laguerre-Gaussian laser modes," Phys. Rev. A 45, 8185-8189 (1992).

[2] Yao, A. M., Padgett, M. J., "Orbital angular momentum: origins, behavior and applications," Adv. Opt. Photon 3, 161-204 (2011).

[3] Grier, D. G., "A revolution in optical manipulation," Nature 424, 810 (2003).

[4] Wang, J., Yang, J. Y., Fazal, I. M., Ahmed, N., Yan, Y., Huang, H., Ren, Y. X., Yue, Y., Dolinar, S., Tur, M., and Willner, A. E., "Terabit free-space data transmission employing orbital angular momentum multiplexing," Nat. Photonics 6, 488 (2012). 
[5] Toyoda, K., Miyamoto, K., Aoki, N., Morita, R., and Omatsu, T., "Using optical vortex to control the chirality of twisted metal nanostructures," Nano Lett. 12, 3645 (2012).

[6] Watanabe, T., Iketaki, Y., Omatsu,T., Yamamoto, K., Sakai, M., and Fujii, M., "Two-point-separation in super-resolution fluorescence microscope based on up-conversion fluorescence depletion technique," Opt. Express 11, 3271-3276 (2003).

[7] Li, X.D., Yu, X., Yan, R.P., Fan, R.W., Chen, D.Y., "Optical and laser properties of $\operatorname{Pr}^{3+}$ :YLF crystal," Laser Phys. Lett. 8(11), 791794 (2011).

[8] Luo, S., Yan, X., Cui, Q., Xu, B., Xu, H., Cai, Z., "Power scaling of blue-diode-pumped Pr:YLF lasers at 523.0, 604.1, 606.9, 639.4, 697.8 and 720.9 nm," Opt. Commun. 380, 357-360 (2016).

[9] Gün, T., Metz, P., Huber, G., "Power scaling of laser diode pumped $\mathrm{Pr}^{3+}: \mathrm{LiYF}_{4} \mathrm{cw}$ lasers: efficient laser operation at $522.6 \mathrm{~nm}, 545.9 \mathrm{~nm}$, $607.2 \mathrm{~nm}$, and $639.5 \mathrm{~nm}$," Opt. Lett. 36, 1002-1004 (2011).

[10] Cheng, Y., Peng, J., Xu, B., Yang, H., Luo, Z., Xu, H., Cai, Z., and Weng, J., "Passive Q-switching of a diode-pumped Pr: $\mathrm{LiYF}_{4}$ visible laser using $\mathrm{WS}_{2}$ as saturable absorber," IEEE Photon. J. 8, 1-6 (2016).

[11] Iijima, K., Kariyama, R., Tanaka, H., Kannari, F., " $\mathrm{Pr}^{3+}$ :YLF modelocked laser at $640 \mathrm{~nm}$ directly pumped by InGaN-diode lasers," Applied Optics 55(28), 7782-7787 (2016). 


\title{
Efficient generation of intense spatio-temporally controlled light waves
}

\author{
Keisaku Yamane ${ }^{* a}$, Kohei Iwasa ${ }^{a}$, Rin Sasaki ${ }^{a}, K$ Kazuhiko Oka ${ }^{b}$, Yasunori Toda ${ }^{a}$ and Ryuji Morita ${ }^{a}$

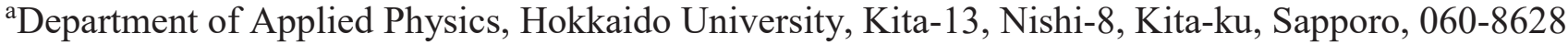 \\ Japan, \\ ${ }^{\mathrm{b}}$ Department of Mechanical Science and Engineering, Hirosaki University, 3 Bunkyo-cho, Hirosaki, \\ 036-8560, Japan
}

\begin{abstract}
We developed the high-efficiency beam converter for ultrafast spatio-temporal control of light waves based on Sagnac interferometer. The flexible control both in the azimuth and radial directions in the ultrafast regime was also realized.
\end{abstract}

Keywords: optical vortices, ring-shaped optical lattices, frequency chirping, Sagnac interferometer, spatio-temporal control

\section{Introduction}

When paired optical vortices with different topological charges interfere with each other, azimuthally periodic structure is formed [1]. The petal-like beam called a ringshaped optical lattice can be flexibly rotated by controlling the intermodal phase difference, which offers us rotational degree of freedom in applications such as optical manipulations of micro/nano particles and micro machines. The rotational control has been realized by mechanical rotation of optical components, acousto-optic devices or electro-optic modulators so far. Although their maximum rotational frequency has been limited to several $\mathrm{GHz}$ owing to their response time, we demonstrated the ultrafast rotation of optical lattices in $\mathrm{THz}$ regime by introducing a new unique technique based on frequencychirping of optical pulses [2].

In our previous work [2], the optical lattices were generated by using the axially-symmetric polarization devices, and thereafter the computer-generated holograms (CGHs) were employed for their flexible control [3]. However, owing to the low conversion efficiency (typically several \%), the output pulse energy from the beam converter has been limited to several $\mu \mathrm{J}$. In order to overcome the problem, the optical amplification

*k-yamane@eng.hokudai.ac.jp; phone +81 11 706-7883 technique was employed [4], but on the other hand, the amplification process often causes serious degradation to the amplified optical vortices. In this paper, we report the development of the high-efficiency beam converter based on a Sagnac interferometer for generation of spatiotemporally controlled light waves. The conversion efficiency theoretically reaches to $50 \%$, and therefore the additional amplification is not required in many cases. Additionally, it enables us to modulate the multiplexed two beams independently, which offers us the ultrafast control of the beam profile both in radial and azimuthal directions by introducing the radial mode index $p$ of Laguerre-Gaussian (LG) mode as well as the topological charge $m$ as control parameters. It should be also emphasized that the new our design is robust because it is the common-path interferometer.

\section{Method and Experiments}

The schematic of our Sagnac-interferometer-type beam converter is shown in Fig.1. The linearly-polarized optical pulses from a home-built regenerative amplifier (center wavelength of $\sim 800 \mathrm{~nm}$, repetition rate: $1 \mathrm{kHz}$, pulse energy: $300 \mu \mathrm{J}$, pulse duration: $130 \mathrm{ps)}$ with strong linear chirping were sent to a polarizing beam splitter and divided into two orthogonally polarized beams. The individual beams were thereafter modulated 
independently by using a reflective-type spatial light modulator. The two beams were going back and recombined at the polarizing beam splitter. In order to cause them to interfere with each other, the orthogonallypolarized two beams were sent to a polarizer. As shown Fig.2 (a), the time averaged beam profile of the output from the beam converter has no periodic structure owing to the slow response time of a CMOS camera. On the other hand, the corresponding time resolved measurements based on sum-frequency generation with ultrashort reference pulses (duration: $\sim 50 \mathrm{fs}$ ) reveals the ultrafast rotation of the petal-like beam in sub- $\mathrm{THz}$ regime (Fig.2 (b)-(d)). The conversion efficiency was typically more than $40 \%$, which was about 10 times higher than that in our previous work [3]. In addition, the ultrafast control of beam profiles in the radial directions was also demonstrated by utilizing superposition of the LG beams with mode indices $(m ; p)=(1 ; 0) ;(1 ; 2)$ (Fig.2 (f)-(h)).

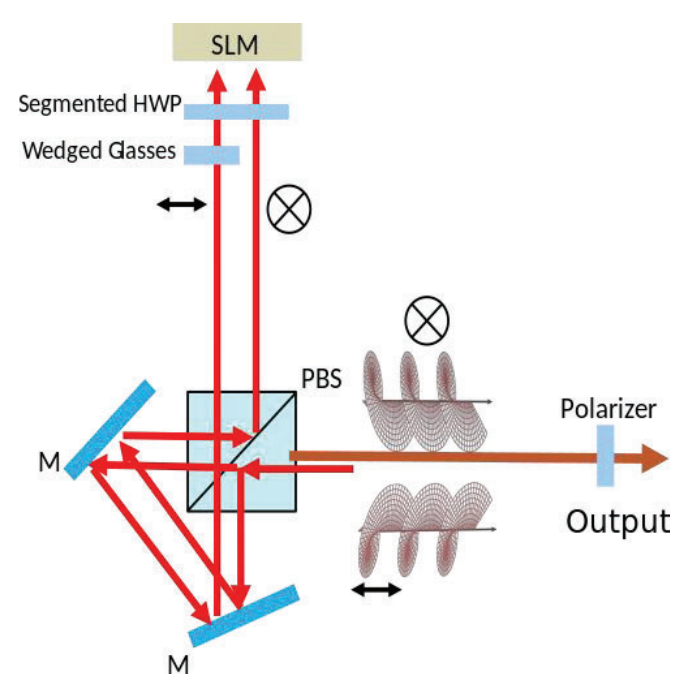

Figure 1. Schematic of yhe Sagnac-type beam converter for generation of spatio-temporally controlled light waves. PBS: polarizing beam splitter, SLM: spatial light modulator.

\section{Conclusion}

We developed the high-efficiency beam converter for generation of spatio-temporally controlled light waves. Its high conversion efficiency ( $>40 \%$ ) enables us to apply the beam to various applications without additional amplifications in many cases, and our technique will be available to flexible spatio-temporal control in lightmatter interaction in the ultrafast regime, laser processing and optical manipulations.
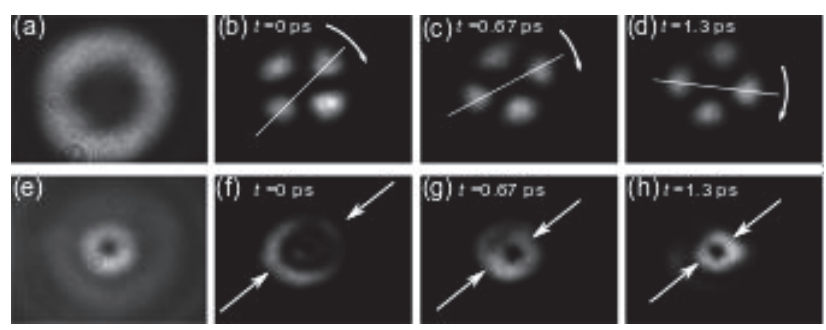

Figure 2. Experimental results. (a) The time-averaged beam profile of ring-shaped optical lattices (superposition of $(m ; p)$ $=(2 ; 0) ;(-2 ; 0))$ and $(\mathrm{b})-(\mathrm{d})$ the corresponding time-resolved images. (e) The time-averaged beam profile in the case of superposition of $(m ; p)=(1 ; 0) ;(1 ; 2)$ and the $(\mathrm{f})-(\mathrm{h})$ the corresponding time-resolved images.

\section{Acknowledgement}

This work was partially supported by JSPS KAKENHI Grant Number JP16H06506, JP17K05069) and National Institute of Information and Communications Technology (NICT).

\section{References}

[1] Paterson, L., MacDonald, M. P., Arlt, J., Sibbett, W., Bryant, P. E. and Dholakia, K., "Controlled rotation of optically trapped microscopic particles," Science 292, 912-914 (2001).

[2] Yamane, K., Sakamoto, M., Murakami, N., Morita, R. and Oka, K., "Picosecond rotation of a ring-shaped optical lattice by using a chirped vortex-pulse pair," Opt. Lett. 41, 4597-4600 (2016).

[3] Yamane, K., Kakizawa, K., Oka, K., Toda, Y. and Morita, R., "Ultrafast rotation of ring-shaped opticallattice pulses in picosecond regime," Technical digest of 7th EPS-QEOD Europhoton Conference, SSL-8 (2016).

[4] Yamane, K., Honda, A., Toda Y. and Morita, R., "Over 1-mJ Intense Ultrashort Optical-Vortex Pulse Generation with Programmable Topological-Charge Control by Chirped-Pulse Amplification," Ultrafast Phenomena XIX 809-812, (Springer International Publishing AG, Switzerland, 2015). 


\title{
Observation and temperature measurement of fast moving metal sphere in a glass with laser illumination*
}

\author{
Nobuyasu Nishioka, Hirofumi Hidai*, Souta Matsusaka, Akira Chiba, Noboru Morita \\ Department of Mechanical Engineering, Chiba University, 1-33, Yayoi-cho, Inage-ku, \\ Chiba, 263-8522, Japan
}

\begin{abstract}
We proposed a novel metal sphere manipulation method optically. Continuous-Wave (CW) laser illumination to a sample implanted a metal sphere into a glass. The sample was consisted with a glass, a metal foil, and a heat insulator stacked from the laser source direction. Low-power laser illumination to the metal sphere causes slow migration with transparent trajectory. In this presentation, we discovered that high-power laser illumination caused fast metal sphere migration with black trajectory and strong emission. The emission was analyzed with a spectrometer, and the temperature was estimated to illustrate the difference. The temperature of fast migration was $2900 \mathrm{~K}$ and the estimated viscosity of glass was lower than one tenth of slow migration.
\end{abstract}

\section{Introduction}

The authors proposed a novel glass internal processing with a laser named continuous-wave laser backside irradiation (CW-LBI). In CW-LBI, manipulation of implanted metal spheres in the glass converts optical characteristics due to changing the crystal structure of glass around the trajectory by the rapid heating and quenching and the metal diffusion from the metal sphere to the glass [1]. The movement velocity of a metal sphere in a glass was below $0.1 \mathrm{~mm} / \mathrm{s}$ (Slow movement). We found that a metal sphere moved faster than $1 \mathrm{~mm} / \mathrm{s}$ with high power density (Fast movement).

In this presentation, we observed Fast movement to compare with Slow movement and detected the emission to estimate the temperature.

\section{Experimental set up}

Figure 1 shows the schematic illustration of the experimental apparatus same as described in [2]. Borosilicate glass, a stainless steel foil (SUS304, thickness of $10 \mu \mathrm{m}$ ), and a heat insulator were clamped with a jig as a sample. Illumination of $\mathrm{CW}$ laser (RFLC020/A/2A/, Raycus corp.) to the sample imported

*Corresponding author's e-mail address:

hidai@faculty.chiba-u.jp stainless steel into the glass as a sphere.

\section{Slow movement and Fast movement}

The metal sphere moved to the laser source while illumination. The phenomenon depended on the power density. Figure 2 shows the metal sphere migration. Figure 2 (1) shows slow migration at $53 \mathrm{~kW} / \mathrm{cm}^{2}$ (Slow movement). Conversely, Fig.2 (2) shows fast migration at $62 \mathrm{~kW} / \mathrm{cm}^{2}$ (Fast movement). The trajectory of Slow and Fast movement were transparent and black, respectively.

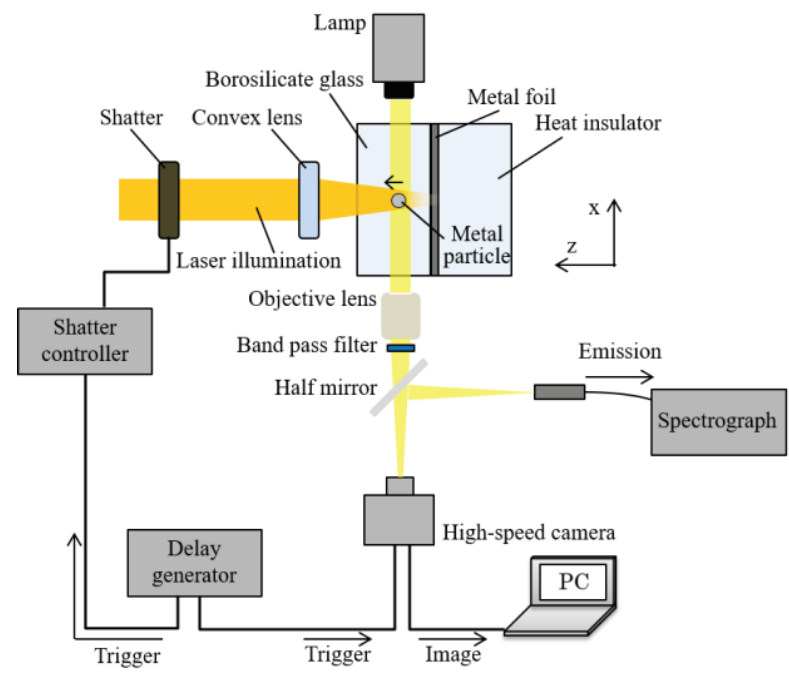

Figure. 1 Experimental setup. 


\section{Temperature calculation from emission detected}

\section{with a spectroscope}

Figure 3 shows the strong emission at laser power density of $13 \mathrm{MW} / \mathrm{cm}^{2}$. The emission moved toward the laser source at high speed $(1.5 \mathrm{~m} / \mathrm{s})$. The similar emission was reported as fiber fuse [3], when high-power laser was illuminated to a glass.

The viscosity of glass decreases with decreasing the temperature. The metal sphere moved in a glass forced by the viscous resistance from surrounding glass. So, temperature can illustrate the difference between Slow and Fast movement. Hence, the emission of Slow and Fast movement, and fiber fuse ware detected with spectrometer (SR-193i and DH334T-18FCH-03, ANDOR corp.). The temperature were calculated by the following equation, Plank's low,

$$
E_{b \lambda}=\frac{c_{1} \lambda^{-5}}{e^{c_{2} / \lambda T}-1}
$$

Figure 4 shows emission spectrum observed in Slow movement, Fast movement and fiber fuse. Fitting curve of Plank's low was also plotted. The temperature of Slow and Fast movement were 1900K and $2900 \mathrm{~K}$, respectively. The viscosity of the glass around the metal particle of Slow and Fast movement were 46.6 $\mathrm{Pa} \mathrm{s}$ and $1.37 \mathrm{~Pa} \mathrm{~s}$, respectively [4]. The similarity showed Fast movement and fiber fuse at the wavelength around $500 \mathrm{~nm}$.

\section{Conclusion}

We have shown Fast movement with black trajectory at high power density. Fast movement showed higher temperature ( $T=2900 \mathrm{~K}$ ) than Slow movement ( $T=1900 \mathrm{~K})$. The viscosity and viscous resistance of Fast movement was lower than those of Slow movement. Additionally, Fast movement may generate fiber fuse.

\section{References}

[1] H. Hidai, T. Yamazaki, S. Itoh, K. Hiromatsu, and H. Tokura, "Metal particle manipulation by laser irradiation in borosilicate glass," Opt. Express, 18(19), 20313 (2010).
[2] N. Nishioka, H. Hidai, S. Matsusaka, A. Chiba, and N. Morita, "Continuous-wave laser-induced glass fiber generation," Appl. Phys. A Mater. Sci. Process. 123(9), 600 (2017).

[3] S. I. Todoroki, "In-situ observation of fiber-fuse propagation,” Jpn. J. Appl. Phys. 44(6A), 4022-4024, (2005).

[4] L. D. Pye, I. Joseph, A. Montenero, I. [Properties of glass-forming melts], Taylor \& Francis, (2005).

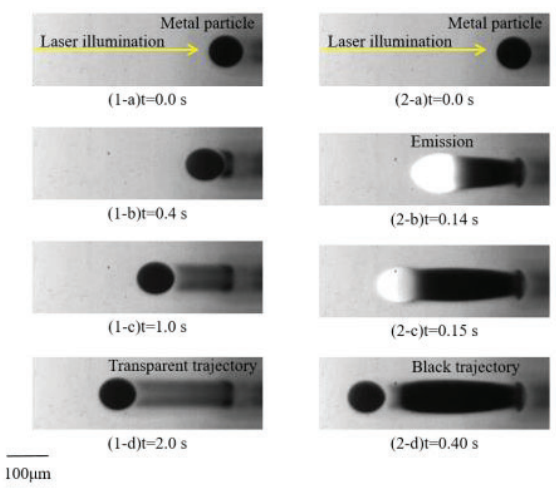

Figure. 2 Metal sphere migration.(1) Slow movement $(v=0.3 \mathrm{~mm} / \mathrm{s}),(2)$ Fast movement $(v=16 \mathrm{~mm} / \mathrm{s})$.

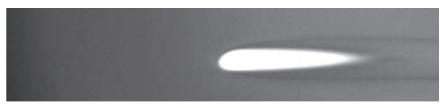

(a) $\mathrm{t}=0 \mu \mathrm{s}$

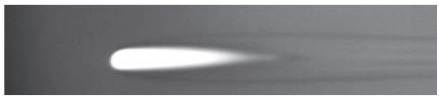

(b) $\mathrm{t}=125 \mu \mathrm{s}$

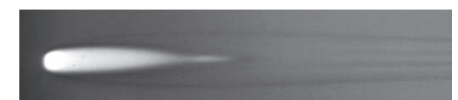

(c) $\mathrm{t}=250 \mu \mathrm{s}$

$\overline{100 \mu \mathrm{m}}$

Figure. 3 Fiber fuse in glass

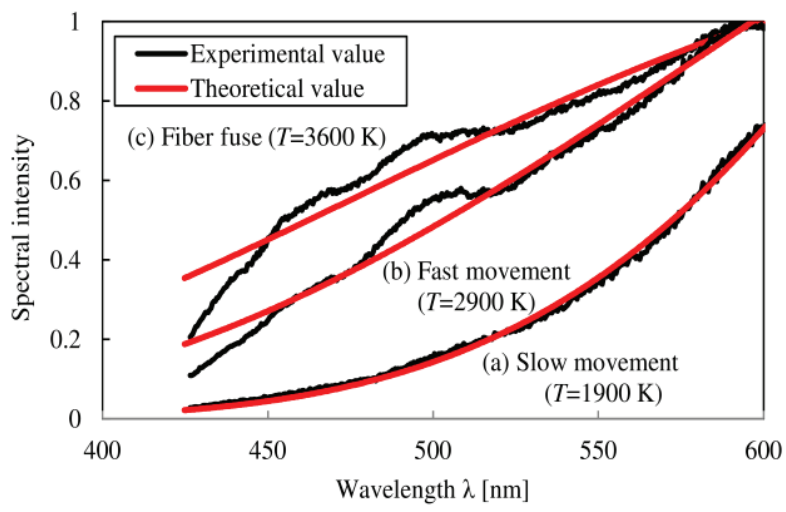

Figure. 4 Emission spectrum and Plank's low fitting of Slow movement, Fast movement and fiber fuse. 


\title{
Size- and composition-controlled synthesis of $\mathrm{Au}-\mathrm{Ag}$ nanorings for plasmonic applications
}

\author{
Tatsuya Kameyama*a,b, Kosuke Sasamoto ${ }^{\mathrm{a}}$, and Tsukasa Torimoto ${ }^{\mathrm{a}}$ \\ ${ }^{a}$ Graduate School of Engineering, Nagoya University, Chikusa-ku, Nagoya 464-8603, Japan \\ bJST-PRESTO, Kawaguchi, Saitama 332-0012, Japan
}

\begin{abstract}
Ring-shaped Au-Ag nanocrystals were successfully synthesized via galvanic replacement of Ag nanoplates as a template with $\mathrm{HAuCl}_{4}$. The average diameter of $\mathrm{Au}-\mathrm{Ag}$ nanorings could be varied from 16 to $33 \mathrm{~nm}$ with an increase in the diameter of $\mathrm{Ag}$ nanoplates from 12 to $26 \mathrm{~nm}$, while the width of rings was almost constant to ca. $4 \mathrm{~nm}$ and their composition was not significantly changed at $\mathrm{ca} . \mathrm{Au} / \mathrm{Ag}=0.7$, regardless of the diameter of rings. The Au-Ag nanorings exhibited a LSPR peak, its wavelength being red-shifted from 542 to $905 \mathrm{~nm}$ with an increase in the diameter. The wavelength of LSPR peak could be also tuned by the precise control of Au/Ag ratio of the nanorings without changing their diameter.
\end{abstract}

Keywords: localized surface plasmon resonance, nanoring, noble metal, alloy, galvanic replacement,

\section{Introduction}

Controlled synthesis of noble metal alloy nanocrystals, such as $\mathrm{Au}-\mathrm{Ag}, \mathrm{Au}-\mathrm{Cu}$, and $\mathrm{Au}-\mathrm{Pt}$, is one of the important subjects in recent years because their catalytic, electronic and optical properties are tunable by controlling the composition as well as their shape and size. ${ }^{[1]}$ Among them, Au-Ag alloy nanocrystals are attractive materials in the applications of surfaceenhanced Raman scattering, biosensing, and plasmonrelated optical manipulation, owing to both the high chemical stability and the strong electric field induced by the excitation of localized surface plasmon resonance (LSPR) peaks. ${ }^{[1]}$

Hollow metal nanostructures, such as nanocages, nanotubes, or nanospheres, have been extensively studied since they have unique properties, which were not observed in conventional solid nanocrystals, such as high specific surface area, high loading capacity in an interior space, and low packing density. A theoretical calculation predicted that nanorings, a type of hollow nanostructure, have a relatively uniform plasmon-enhanced electric field, being suitable for plasmonic applications. ${ }^{[2]}$ So far, ringshaped Au-Ag nanocrystals were successfully synthesized by Zhou and co-workers via galvanic replacement of colloidal $\mathrm{Ag}$ nanoplates with $\mathrm{HAuCl}_{4} \cdot{ }^{[3]}$ They reported that the LSPR peak of Au-Ag nanorings was dependent on the ring size which was changed by the $\mathrm{Au}$ precursors used. However, this preparation simultaneously changed not only the ring size but also the chemical composition of Au-Ag alloy nanorings, the both of which caused the shift of LSPR peak.

Here we reported a simple method to independently control the ring size and chemical composition of $\mathrm{Au}-\mathrm{Ag}$ alloy nanorings. By precisely tuning these factors individually, we successfully controlled their LSPR wavelength from visible to near-IR wavelength regions.

\section{Experimental}

\section{Preparation of colloidal Ag nanoplates}

$\mathrm{Ag}$ nanoplates as a sacrificial template for $\mathrm{Au}-\mathrm{Ag}$ nanorings were synthesized using a seed-mediated growth method. The seed solution was prepared by adding freshly prepared $\mathrm{NaBH} 4$ aqueous solution in the mixture solution containing sodium 4-polystylenesulfonate (PSS), AgNO3 and trisodium citrate under vigorously stirring for $10 \mathrm{~min}$ at $30^{\circ} \mathrm{C}$. Then, a portion of the seed solution was added into the growth solution containing $\mathrm{AgNO}$, ascorbic acid, and trisodium citrate, followed by stirring for $60 \mathrm{~min}$ at $30{ }^{\circ} \mathrm{C}$. The size of obtained $\mathrm{Ag}$ nanoplates could be controlled by changing the amount of the $\mathrm{Ag}$ seed solution added. 


\section{Synthesis of Au-Ag nanorings via galvanic replacement}

To synthesize Au-Ag nanorings, aqueous solution of $\mathrm{HAuCl} 4$ and ascorbic acid were added into the thus obtained Ag nanoplate solution with vigorous stirring for $30 \mathrm{~min}$ at $30{ }^{\circ} \mathrm{C}$. The chemical composition of Au-Ag nanorings could be controlled by varying the dosage of ascorbic acid during this procedure.

\section{Results and Discussion}

PSS and citric acid are well known as a surfactant selectively adsorbed on Ag (111) surface. ${ }^{[4]}$ Therefore, when $\mathrm{Ag}$ ions are reduced in the presence of these surfactant, the crystal growth is suppressed on Ag (111) surface, resulting in the formation of nanocrystals with a plate shape exposing (111) facet on the surface. We controlled the diameter of Ag nanoplate by changing the amount of Ag seeds used in the preparation. When a large amount of $\mathrm{Ag}$ seeds were used, the amount of $\mathrm{Ag}$ precursor for growth per particle decreased, resulting in the formation of smaller Ag nanoplates. Figures $1 \mathrm{a}$ and $\mathrm{b}$ shows typical TEM images of size-controlled Ag nanoplates. The average diameter of thus-obtained Ag nanoplates was varied from $12 \mathrm{~nm}$ (Fig. 1a) to $26 \mathrm{~nm}$ (Fig. 1b). On the other hand, the thickness of the Ag nanoplate was constant to ca. $7 \mathrm{~nm}$, regardless of the diameter of them.

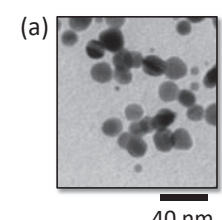

(c)

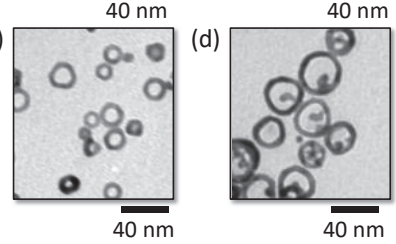

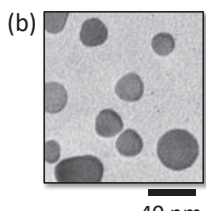

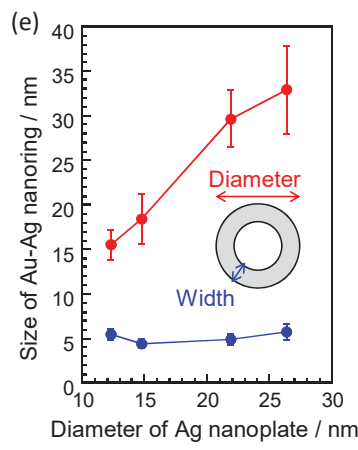

Figure 1. Typical TEM images of Ag nanoplates (a, b) and Au-Ag nanorings (c, d) with different sizes, (e) Average diameter and width of prepared $\mathrm{Au}-\mathrm{Ag}$ nanorings as a function of diameter of $\mathrm{Ag}$ nanoplates used as a precursor.
Au-Ag nanorings were prepared by galvanic replacement of $\mathrm{Ag}$ nanoplates as a sacrificial template. The size of resulting nanorings was controlled by the size of Ag nanoplates used. Figures 1c and d, show typical TEM images of $\mathrm{Au}-\mathrm{Ag}$ nanorings prepared from $\mathrm{Ag}$ nanoplates in Fig. 1a and b, respectively. The average diameter of the obtained nanorings increased with an increase in the Ag nanoplates (Fig. 1e). It should be noted that the average width (5-6 nm) of thus-obtained nanorings were unchanged even when the diameter of the rings was increased. Furthermore, the obtained $\mathrm{Au}-\mathrm{Ag}$ nanorings had almost constant chemical composition of $\mathrm{Ag} / \mathrm{Au}=0.6-0.7$, regardless of their ring size.

Based on these results, the formation mechanisms of $\mathrm{Au}-\mathrm{Ag}$ nanorings is proposed as follows. The $\mathrm{Ag}$ nanoplates act as a reducing agent and then $\mathrm{AuCl}_{4}^{-}$ions are reduced to $\mathrm{Au}$, accompanied with the oxidation of $\mathrm{Ag}$ to $\mathrm{Ag}^{+}$. Since the surfactant is adsorbed on the (111) facet of Ag nanoplates, that is, the planer surface of the plates, $\mathrm{Au}$ metal predominantly deposits at the edge of $\mathrm{Ag}$ nanoplates. A portion of thus-dissolved $\mathrm{Ag}^{+}$ions are also reduced by ascorbic acid to form $\mathrm{Au}-\mathrm{Ag}$ alloy nanorings.

Figure 2 shows the extinction spectra of the Au-Ag nanorings with different diameters. Two LSPR peaks of the Au-Ag nanorings of $33 \mathrm{~nm}$ in diameter were observed at 500 and $905 \mathrm{~nm}$, which were assigned to the plasmon resonant coupling of dipole and quadrupole, respectively. ${ }^{5}$ Furthermore these peaks were widely blue-shifted with a decrease in their diameter, in which the dipole mode LSPR peak was shifted from $905 \mathrm{~nm}$ to $520 \mathrm{~nm}$.

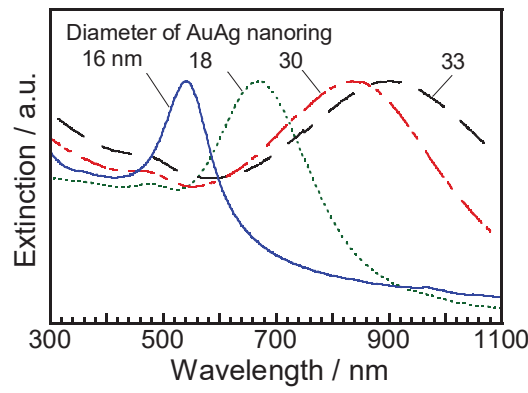

Figure 2. Extinction spectra of $\mathrm{Au}-\mathrm{Ag}$ nanorings dispersed in water. The diameter of nanorings is indicated in the figure. 
The LSPR peaks of Au-Ag nanorings were also sensitive to the alloy composition. We successfully changed the composition of Au-Ag nanorings with ca. 30 $\mathrm{nm}$ diameter and ca. $6 \mathrm{~nm}$ width, in which the ratio of $\mathrm{Au} / \mathrm{Ag}$ varied from 0.7 to 2.0 , by decreasing the dosage of ascorbic acid during the galvanic replacement. Thusobtained $\mathrm{Au}-\mathrm{Ag}$ nanorings showed the compositiondependent LSPR peak, and then the dipole LSPR peak was red-shifted from $840 \mathrm{~nm}$ to $1040 \mathrm{~nm}$ with an increase in the $\mathrm{Au} / \mathrm{Ag}$ ratio from 0.7 to 2.0.

In conclusion, we established a synthesis method to control the size and composition of Au-Ag nanorings independently and thus-obtained nanorings had a tunability of their LSPR properties in the visible and nearIR wavelength regions.

\section{References}

[1] Cortie, M. B., and McDonagh, A. M., "Synthesis and Optical Properties of Hybrid and Alloy Plasmonic Nanoparticles," Chem. Rev., 111, 3713 (2011)

[2] Aizpurua, J., Hanarp, P., Sutherland, D. S., Kall, M., Bryant, G. W., and de Abajo, F. J. G., "Optical Properties of Gold Nanorings," Phys. Rev. Lett., 90, 057401 (2003)

[3] Lia, W., Zhou, J., Jia, Z., Petti, L., and Mormile, P, "Ag@Au Hexagonal Nanorings: Synthesis, Mechanistic Analysis and Structure-dependent Optical Characteristics," J. Mater. Chem. C, 3, 9726 (2015)

[4] Aherne, D., Ledwith, D. M, Gara, M., and Kelly, J. M., "Optical Properties and Growth Aspects of Silver Nanoprisms Produced by a Highly Reproducible and Rapid Synthesis at Room Temperature," Adv. Funct. Matter., 18, 2005 (2008)

[5] Mulvaney, P., "Surface Plasmon Spectroscopy of Nanosized Metal Particles," Langmuir, 12, 788 (1996)

Proc. of SPIE Vol. 11141 1114101-138 


\title{
Three-dimensional observations of particle flows in microchannels induced by photothermal effects
}

\author{
Tetsuro Tsuji ${ }^{a}$, Takahiro Nakamoto ${ }^{\text {a }}$, Satoyuki Kawano*a \\ ${ }^{a}$ Graduate School of Engineering Science, Osaka University 1-3 Machikaneyama, \\ Toyonaka, Osaka, 560-8531, Japan
}

\begin{abstract}
Optical manipulation is expected to be a promising technique for controlling the motion of dispersed nano- and micro objects in fluids. However, the absorption of laser to a solvent and/or a target object is inevitable and can be significant, resulting in the temperature increase of the solvent and/or the targets. Such a photothermal effect induces thermal convection of the solvent or thermophoresis of the dispersed target particles and may hinder the optical trapping. Therefore, the contribution of these photothermal effects to the particle motion should be controlled carefully to isolate the optical forces. On the other hand, these thermo fluid phenomena can be useful to enhance the efficiency of optical trapping by increasing the supply of targets to the focal point. To understand the magnitude relation among the optical force, the drag force exerted by thermal convection, and thermophoretic force, we propose the three-dimensional observation of particle motion induced by the photothermal effect of near-infrared laser irradiation. By changing the channel height of a test chamber and a laser focus condition, it is found from the three-dimensional observation that there exist specific situations such that either the optical force, thermal convection, or thermophoresis is superior to other phenomena.
\end{abstract}

Keywords: Optical forces, Thermopohoresis, Thermal convection, Microchannel, Photothermal effect

\section{Introduction}

Optical manipulation of micro particles such as colloids and cells has been widely used in practical application since an innovative work on optical tweezers by Ashkin [1]. However, when the size of target shrinks to nanoscale, the optical manipulation becomes difficult because the optical force decreases as the particle diameter. To overcome such situations, higher power laser or plasmonic optical tweezers (e.g., [2]) may be used. In such situations, the absorption of the laser to the solvent, target materials, and/or the plasmonic substrate is inherent, and thus thermo fluid phenomena, i.e., thermal convection of solvents and thermophoresis [3][4] of target particles, due to photothermal effects can hinder the optical trapping. For a precise evaluation of optical forces, it is necessary to

*kawano@me.es.osaka-u.ac.jp explore a methodology to isolate the optical forces from these thermal effects. On the other hand, the thermal effects have possibilities to enhance the optical trapping by transporting the targets to the focal point [5]. Therefore, the deeper understanding on the laser-induced thermo fluid phenomena and the search for experimental settings to control them are crucial toward the development of novel optical manipulation methods. In the present paper, we carry out a three-dimensional observation of particle motion induced by laser irradiation in a microscale confinement [6] and tries to clarify the important parameter ranges for each effect to be dominant. Characterization of three-dimensional particle motion will contribute to the establishment of an experimental design such as the geometry of the confinement, which suppresses or utilizes the thermal effects. 
(a) side view

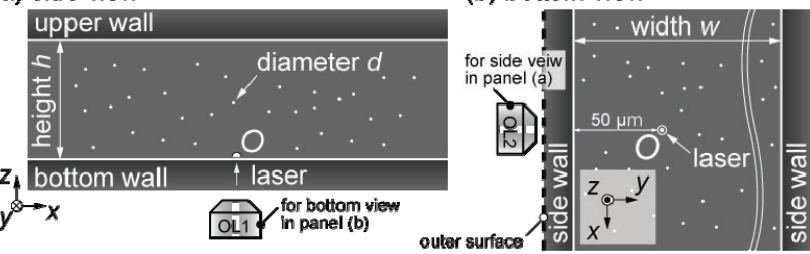

Fig. 1 Schematics of fluorescent microscopes for threedimensional observation. (a) Side and (b) bottom views.

\section{Experimental method}

An experimental setup is schematically shown in Fig. 1. We use an inverted microscope to observe the fluorescence of polystyrene (PS) particles with diameter $d$ dispersed in a Tris- $\mathrm{HCl}$ buffer solution of $10 \mathrm{mmol} / \mathrm{L}$. The solution is confined in a microchannel made of polydimethylsiloxane (PDMS) sealed with a bottom cover glass. The channel height and width are denoted by $h$ and $w=400 \mu \mathrm{m}$, respectively. A near-infrared laser with the wavelength $\lambda=$ $1,475 \mathrm{~nm}$ is irradiated from the bottom of the device through an objective lens (OL1; LCPLN100XIR, Olympus, Japan) as shown in Fig. 1(a). This objective lens has 100x magnification, numerical aperture $(\mathrm{NA})=0.85$, and $47.2 \%$ transmittance at the wavelength $\lambda=1,475 \mathrm{~nm}$. We use OL1 for the observation from the bottom, and the obtained images are schematically shown in Fig. 1(b). Another objective lens (OL2; PAL-50-L-A, Sigma Koki, Japan) with magnification $50 \mathrm{x}$ and $\mathrm{NA}=0.42$ is used to observe the channel from the side as shown in Fig. 1(b). The obtained images using OL2 are schematically illustrated in Fig. 1(a). To avoid the physical interference between OL2 and OL1, OL2 has a long working distance of $20.7 \mathrm{~mm}$. Moreover, the PDMS channel is casted so that the outer surface of the device, which is shown with the dashed line in Fig. 1(b), is flat to have high transparence.

\section{Results and Discussion}

In the following, all the figures express the origin $O$ as the laser focused position described in Fig. 1. Firstly, we show in Fig. 2 the case where the effect of optical force is dominant in the microchannel with height $h=75 \mu \mathrm{m}$. The incident laser of $200 \mathrm{~mW}$ is collimated before OL1 in Fig.

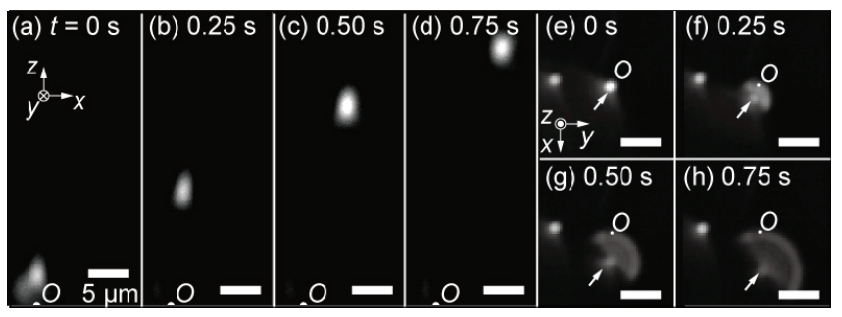

Fig. 2 (a)-(d) Side and (e)-(f) bottom views where the optical forces are prominent. Scale bar: $5 \mu \mathrm{m}$.

1(a) such that the focal spot size, $1.1 \mu \mathrm{m}$, becomes smallest in our optical setup. It is seen that the particle with $d=2 \mu \mathrm{m}$ is pushed away in the $z$ direction as shown in Figs. 2(a)-(d) with the side view. On the other hand, Figs. 2(e)-(h) show the bottom view. The particle at the origin in Fig. 2(e) is gradually defocused as time goes on, indicating the motion in the positive $z$ direction. This observation is consistent with the side view in Figs. 2(a)-(d). However, in Fig. 2(e), another particle located about $5 \mu \mathrm{m}$ away from the origin does not respond to the laser irradiation. Therefore, photothermal effects such as thermal convection or thermophoresis is negligibly weak compared with the optical force in these experimental conditions.

Next, we show in Fig. 3 the case where the thermal convection is dominant. This situation is realized by broadening the beam waist to heat the wider range of solution. In our previous study [4], the beam spot size was estimated as $7.7 \mu \mathrm{m}$. The side and bottom views of particles with $d=1 \mu \mathrm{m}$ shown in Figs. 3(a) and 3(b), respectively, are the superposition of snapshots during $5 \mathrm{sec}$ after the laser irradiation of $50 \mathrm{~mW}$. Note that the laser is irradiated at the origin and the optical forces are limited to the region close to the $z$ axis, and thus the particle motion far from the laser axis is not originated from the optical forces, but thermo fluid phenomena. It is seen from Fig. 3(a) that a vortex pair of the particle flow is produced at $z \approx 35 \mu \mathrm{m}$ and the particle speed exceeds $10 \mu \mathrm{m} / \mathrm{s}$. From the bottom view shown in Fig. 3(b), we notice that the particles are accumulated near the laser focused position. Therefore, the broader irradiation of the laser can induce a thermal convection that enhances a chance to have particles near the laser focus at the channel bottom. In the present setting, it is 


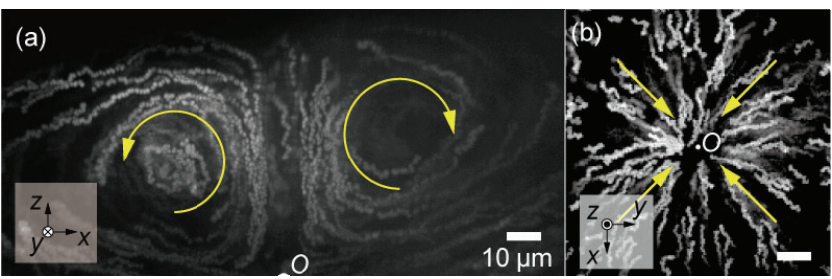

Fig. 3 (a) Side and (b) bottom views where the thermal convection is prominent. Scale bar: $10 \mu \mathrm{m}$.

difficult to separate the effects of thermal convection and thermophoresis. However, we estimated the thermophoretic velocity was $4.6 \mu \mathrm{m} / \mathrm{s}$ at most under the strong temperature gradient with the magnitude of $0.6 \mathrm{~K} / \mu \mathrm{m}$ in our previous study [7], and thus we conclude that the observed particle motion is mainly attributed to the thermal convection.

Finally, we downsize the channel height to $h=25 \mu \mathrm{m}$ to suppress thermal convection. The side and bottom views of particles with $d=1 \mu \mathrm{m}$ shown in Figs. 4(a)-(c) and 4(d)(f), respectively, are the snapshots for the laser irradiation of $300 \mathrm{~mW}$. Due to the small confinement of the channel, the thermal convection as observed in Fig. 3 cannot takes place. Instead, the temperature distribution in the $x$ direction produces thermophoretic forces acting on the particles toward the colder region. That is, the particles are repelled from the hot region created by laser irradiation. Note that it was pointed out that the PS particles in the present solution shows motion toward colder region [4][7]. Since the thermophoretic characteristics depend on the particle material and its surface condition, thermophoresis may be used for selective particle trapping by depleting the unwanted samples from the laser focus.

\section{Concluding Remarks}

In this study, particle flows induced by a laser irradiation are investigated in a microchannel. By choosing appropriate sizes of a laser spot and a channel height, we can separate the effects of optical forces, drag forces by thermal convection, and thermophoretic forces. Such an information contributes to the isolation of the optical trapping from thermo fluid phenomena or the enhancement of the efficiency of optical manipulation technique by selectively accumulating the target particle to the laser spot.

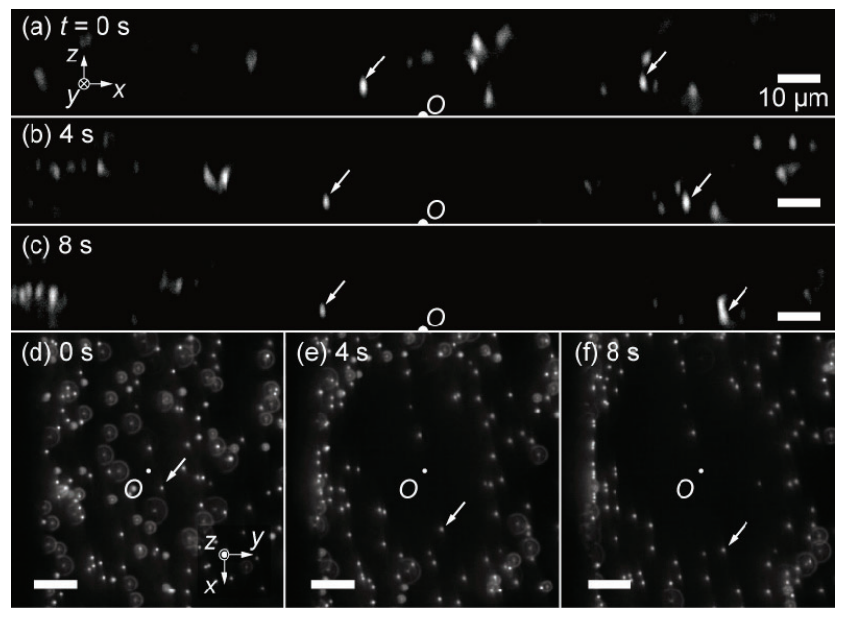

Fig. 4 (a) Side and (c) bottom views where the thermophoresis is prominent. Scale bar: $10 \mu \mathrm{m}$.

\section{References}

[1] Ashkin, A., "Acceleration and trapping of particles by radiation pressure," Phys. Rev. Lett. 24, 156-159 (1970).

[2] Shoji, T., and Tsuboi, Y., "Plasmonic optical tweezers toward molecular manipulation: Tailoring plasmonic nanostructure, light source, and resonant trapping," J. Phys. Chem. Lett. 5, 2957-2967 (2014).

[3] Piazza, R., "Thermophoresis: moving particles with thermal gradients," Soft Matter 4, 1740-1744 (2008).

[4] Tsuji, T., Sasai, Y., and Kawano, S., "Thermophoretic manipulation of micro-and nanoparticle flow through a sudden contraction in a microchannel with near-infrared laser irradiation,” Phys. Rev. Appl. 10, 044005 (2018).

[5] Braun, D., and Libchaber, A. "Trapping of DNA by thermophoretic depletion and convection," Phys. Rev. Lett. 89, 188103 (2002).

[6] Nito, F., Shiozaki, T., Nagura, R., Tsuji, T., Doi, K., Hosokawa, C., and Kawano, S., "Quantitative evaluation of optical forces by single particle tracking in slit-like microfluidic channels," J. Phys. Chem. C 122, 1796317975 (2018).

[7] Tsuji, T., Saita, S., and Kawano, S., "Dynamic pattern formation of microparticles in a uniform flow by an onchip thermophoretic separation device," Phys. Rev. Appl. 9, 024035 (2018). 


\title{
Optical control of particle oscillation through a rectangular orifice in a microchannel
}

\author{
Ryota Koyama ${ }^{\mathrm{a}}$, Fumika Nito ${ }^{\mathrm{a}}$, Tetsuro Tsuji ${ }^{\mathrm{a}}$, Kentaro Doi ${ }^{\mathrm{a}}$ and Satoyuki Kawano ${ }^{\mathrm{a} *}$ \\ ${ }^{a}$ Graduate School of Engineering Science, Osaka University \\ 1-3 Machikaneyama, Toyonaka, Osaka 560-8531, Japan
}

\begin{abstract}
In this study, to develop novel fluidic devices to measure the microparticle characteristics of diameter and electric conductivities, the oscillating flow of microparticles is generated and is controlled by optical forces. Microfluidic devices are based on the principle of Coulter counter, which is well-used for detecting and sizing microparticles such as biological cells. Using the multiple scanning by sequential translocation of single particles through an orifice, the high yield and the accurate size detection are expected. In this paper, the oscillating particle flow characteristics are investigated and a simple model based on Stokes approximation is proposed from the viewpoint of fluid mechanics. Consequently, the fluidic device proposed here is challenging development for the advanced measurement techniques in the nanobiotechnologies.
\end{abstract}

Keywords: Optical forces, Oscillating flow of microparticles, Microfluidics, High-speed observation, Biotechnologies

\section{Introduction}

Recent advances in fabrication methods enable the development of nanofluidic devices with functions of sensing nanoobjects, such as biomolecules and viruses, which have the equivalent diameter of $O(10)-O(100) \mathrm{nm}$ [1]. The devices have narrow parts, such as nanopores or nanochannels, with comparable length scale to targets. Therefore, ionic/tunneling currents in the devices show particular transient behaviors according to translocation of targets through the nanostructures. To identify single particles, it is necessary to develop novel techniques to control the translocation speed and detection frequency [25] for larger data set to be used in big data analysis. For such problems, in the present study, we propose an oscillatory manipulation technique in a narrow slit using optical trapping of targets. To be more precise, the trapped particle undergoes the sequential rectilinear translocations through the slit with a width comparable to the particle. Moreover, we discuss the control performance from the viewpoint of fluid dynamics using the Basset-BousinessqOssen equation for unsteady motion.

\section{Experimental Method}

In a previous study [6], we demonstrated the optical

*kawano@me.es.osaka-u.ac.jp manipulation of microparticles whose motion was confined in microchannels. Using microchannels that had a height comparable to the diameter of microparticles, the transport phenomena could be confined in two-dimensional spaces. In such a condition, the particle transport was discussed, focusing on the gradient force that caused the in-plane motions in the channels. As a result, the gradient force acting on the single microparticles was quantitatively evaluated as a function of distance from the focal point for various sizes of the particles. In this study, furthermore, we produce oscillating motions of microparticles in liquids by using optical trapping methods on automatically controlled stages, and investigate the effect of unsteady liquid flows

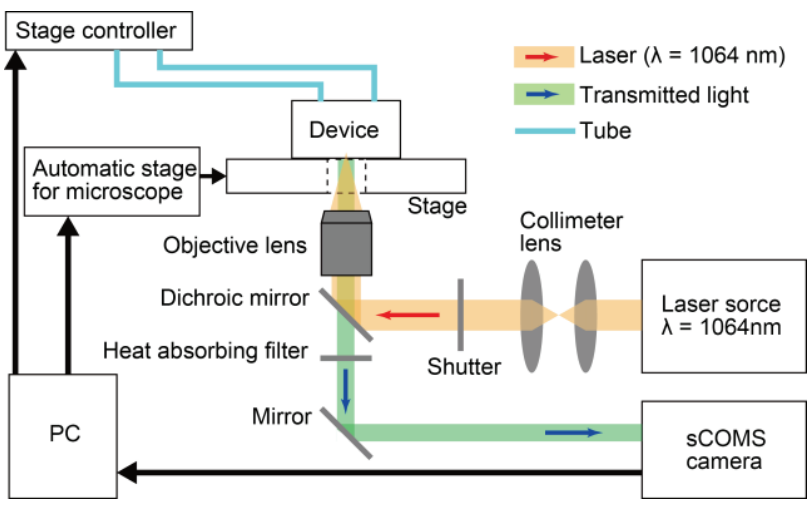

Figure 1. Schematic of experimental setup for optical manipulation of microparticles using automatic stages. 
on the viscous drag of the particles. Figure 1 shows the schematic diagram of the experimental system in which a collimated laser beam of $1064 \mathrm{~nm}$ wavelength is guided into an inverted microscope and focused at the upper surface of a microchannel to optically trap microparticles. The height of the channel is set to $3.0 \mu \mathrm{m}$ to permit the transport of microparticles whose diameter is $2.0 \mu \mathrm{m}$, avoiding stacking in the height direction. To focus on the optical effect, the background flow of the solvent is prevented by imposing the same pressure at the both ends of the channel. This is done by adjusting the water-level difference between two chambers connecting to the inlet and the outlet of the device. By bright field observation, particle motions are tracked by using a high-speed complementary metal-oxide-semiconductor (CMOS) camera. The oscillating motions of particles are produced by using automatic stages controlled by a computer.

\section{Results and Discussion}

Experimental results are presented in Fig. 2, where the laser focus is placed at the position of trapped particle and the laser power at the source is set to $0.5 \mathrm{~W}$. As shown in Fig. 2(a), the optical trapping is made at the entrance of a microscale orifice of $10 \mu \mathrm{m}$ width. The average velocities $v_{\text {stage }}$ in the $x$ direction of the automatic stage in Fig. 2(a)(e) and Fig. 2(f)-(j) are $-30 \mu \mathrm{m} / \mathrm{s}$ and $30 \mu \mathrm{m} / \mathrm{s}$, respectively. The trapped particle stays at the focal point and the orifice relatively moves with the stage. Therefore, in Fig. 2(a)-(e), the particle translocates the orifice from left to right, and, in Fig. 2(f)-(j), in the inverse direction. For the speed of the stage demonstrated in Fig. 2, an optically trapped particle stably settled at the focus. However, at the speed of $\left|v_{\text {stage }}\right|$ $=700 \mu \mathrm{m} / \mathrm{s}$, a trapped particle was released from the optical potential. In such a condition, the drag force on the particle seems to be time-dependent. From the viewpoint of theoretical fluid mechanics, the equation of unsteady motion of a single particle, which is oscillated in viscous liquids due to the optical tweezers, is not so simple.

In general, Stokes approximation for the drag force of a particle in creeping flow can be applicable only within the case of steady motion. In addition to the Stokes' drag, the consideration of the added mass force and the history force, which is so-called as the Basset term, is needed at least in the accelerated oscillating motion of a single particle. So, the following equation should be used for

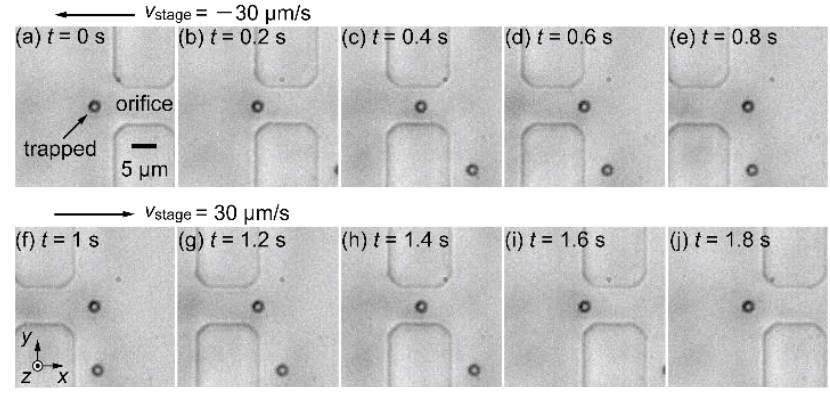

Figure 2. Oscillation through an orifice structure under optical potential: (a)-(e) $v_{\text {stage }}=-30 \mu \mathrm{m} / \mathrm{s}$ and (f)-(j) $v_{\text {stage }}$ $=30 \mu \mathrm{m} / \mathrm{s}$.

accurate estimation of the force acting on the particle as

$F_{\text {drag }}=-\frac{\pi}{12} \rho d^{3} \dot{U}-3 \pi \mu d U-\frac{3}{2} d^{2} \sqrt{\pi \rho \mu} \int_{0}^{t} \frac{\dot{U}(s)}{\sqrt{t-s}} \mathrm{~d} s$,

where $d$ denotes the diameter of particles and $U$ the relative velocity of the particle to the continuous flow velocity, which is assumed to be equal to $v_{\text {stage. }} \rho$ and $\mu$ denote the density and the viscosity of surrounding liquid, respectively. In the right-hand side of Eq. (1), the first term indicates the added mass force, second the Stokes' drag, and third the history force. Equation 1 is known as the Basset-Boussinesq-Oseen equation, which is applicable for the unsteady and creeping motion of single particles. The force on particle experimentally obtained by the highspeed observation will be compared with that by the theoretical analysis based on Eq. (1) in future.

\section{Concluding Remarks}

In the present study, using optical trapping, we have controlled the two-dimensional sequential translocation of microparticles through an orifice. We also propose the use of Basset-Boussinesq-Ossen equation for detailed analysis of such an unsteady motion. This method may be applicable for electrical identification of particle characteristics based on the Coulter counter and comparisons with the proposed model are our future works.

\section{References}

[1] Tanaka, S., et al., Sci. Rep. 6, 31670 (2016).

[2] Yano, A., et al., Jpn. J. Appl. Phys. 56, 097201 (2017).

[3] Nagura, R, et al., Micro Nano Lett. 12, 526 (2017).

[4] Doi, K., et al., J. Chem. Phys. 148, 204512 (2018).

[5] Tsuji, T., et al., Phys. Rev. Appl. 9, 024035 (2018).

[6] Nito, F., et al., J. Phys. Chem. C 122, 17936 (2018). 


\title{
Transition strength of a standing optical vortex beam in monolayer transition metal dichalcogenides
}

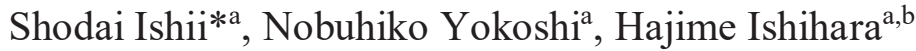 \\ ${ }^{a}$ Department of Physics and Electronics, Osaka Prefecture University, Sakai, Osaka, 599-8531, Japan \\ ${ }^{b}$ Department of Materials Engineering Science, Osaka University, Toyonaka, Osaka, 560-8531, Japan
}

\begin{abstract}
We calculated inter-band transition strength when a standing optical vortex beam is radiated to a monolayer transition metal dichalcogenides (TMD). Monolayer TMD has two valleys at the corner of the Brillouin zone. The inequivalent two valleys can be operated using circular polarized light. However, the relation between the orbital angular momentum (OAM) of light and the valleys in monolayer TMD is still elusive. We reveal an optical selection rule for the twisted lights. We also show how the transition strength is affected in changing the position of the beam waist. We expect that the result promotes the development of research fields "spin-valleytronics" and OAM of light.
\end{abstract}

Keywords: monolayer transition metal dichalcogenide, optical vortex, optical selection rule, valleytronics

\section{Introduction}

Monolayer transition metal dichalcogenides (TMD) such as $\mathrm{MoS}_{2}$ has attracted much attention because of its excellent property. Monolayer TMD has two valleys at the $\mathrm{K}$ and $-\mathrm{K}$ point in the Brillouin zone. Then, carriers have the "valley degree of freedom". The valley degree of freedom could be addressed and detected by circularly polarized light [1]. Furthermore, the valence band at $\pm K$ point has a valley-contrasting giant spin splitting, which is induced by spin-orbit coupling and broken inversion symmetry [2]. Therefore, monolayer TMD is expected as an important material of "spin-valleytronics" $[3,4]$.

On the other hand, optical vortex, light with finite orbital angular momentum (OAM), has been actively studied [5]. The optical vortices have spiral equiphase surfaces and doughnut intensity profiles. Various applications of such a light has been explored, e.g., mechanical rotation controls of micro particle [6] and nano-size chiral laser ablation [7]. Besides, the spatial structure of the optical vortex modifies the optical selection rules through transferring extra angular momentum of light to carriers in materials [8,9]. The modified optical selection rules have a large potential of new light-valley physics.

*ishii-2@pe.osakafu-u.ac.jp; phone +81 72 254-9268
Here, we aim to clarify the inter-band transition strength of a monolayer TMD by a standing wave of LaguerreGaussian beam. At first, we reveal the optical selection rules. We also show how the beam waist position affects the transition strength.

\section{Laguerre-Gaussian beam}

Light beams can be described by a vector potential A, which satisfies the Helmholtz equation. The vector potential of a beam which propagates along $\mathrm{z}$ direction describes

$$
\mathbf{A}(\mathbf{r})=A_{0} \epsilon u(\mathbf{r}) e^{i k z},
$$

where $\boldsymbol{\epsilon}$ is the polarization vector and $k$ means the wave number in the longitudinal direction. Laguerre-Gaussian beam is a solution of the Helmholtz equation in the cylindrical coordinate and paraxial approximation.

The Laguerre-Gaussian beam is described by

$$
\begin{aligned}
& u(r, \phi, z)=\frac{c_{p l}}{\omega(z)}\left[\frac{\sqrt{2} r}{\omega(z)}\right]^{|l|} L_{p}^{|l|}\left(\frac{2 r^{2}}{\omega(z)}\right) \exp (i l \phi) \\
& \times \exp \left[-\frac{r^{2}}{\omega^{2}(z)}+\frac{i k r^{2} z}{2 z_{\mathrm{R}}}-i(2 p+l+1) \arctan \left(\frac{z}{z_{\mathrm{R}}}\right)\right] .
\end{aligned}
$$

Here $L_{p}^{|l|}$ is the Laguerre function, $z_{\mathrm{R}}$ the Rayleigh range, $\omega(z)=\omega_{0}\left(1+z^{2} / z_{\mathrm{R}}^{2}\right)^{1 / 2}$ the beam width and $\omega_{0}$ is the beam waist. 
Table 1. Optical selection rules in monolayer TMD is summarized. The index $l$ means the orbital angular momentum of light. RCP (LCP) indicates right (left)handed circular polarization. Colored parts mean the inter-band transition is allowed.

\begin{tabular}{|c|c|c|c|c|c|c|c|c|c|c|}
\hline \multicolumn{2}{|c|}{$l$} & -4 & -3 & -2 & -1 & 0 & +1 & +2 & +3 & +4 \\
\hline \multirow{2}{*}{$+\mathrm{K}$} & $\mathrm{RCP}$ & & & & & & & & & \\
\hline & $\mathrm{LCP}$ & & & & & & & & & \\
\hline \multirow{2}{*}{$-\mathrm{K}$} & $\mathrm{RCP}$ & & & & & & & & & \\
\hline & $\mathrm{LCP}$ & & & & & & & & & \\
\hline
\end{tabular}

\section{Optical selection rule}

We firstly show modified optical selection rules in monolayer TMD by optical vortices. In monolayer TMD, the band edges at $\pm \mathrm{K}$ are mostly dominated by transition metal d-orbitals [10],

$$
\begin{gathered}
\left|\Psi_{\mathrm{c}}\right\rangle=\left|d_{z^{2}}\right\rangle, \\
\left|\Psi_{\mathrm{V}}^{ \pm K}\right\rangle=\frac{1}{\sqrt{2}}\left(\left|d_{x^{2}-y^{2}}\right\rangle \mp i\left|\Psi_{x y}\right\rangle\right),
\end{gathered}
$$

where the subscript c (v) means conduction (valence) band.

To reveal the optical selection rules, we calculated this transition matrix

$$
\left\langle\Psi_{\mathrm{c}}\left|\mathbf{p} \cdot \mathbf{A}^{l}+\mathbf{A}^{l} \cdot \mathbf{p}\right| \Psi_{\mathrm{v}}\right\rangle \sim \frac{2 i m}{\hbar}(\Delta E)\left\langle\Psi_{\mathrm{c}}\left|\mathbf{r} \cdot \mathbf{A}^{l}\right| \Psi_{\mathrm{v}}\right\rangle,
$$

where $\Delta E$ means the bandgap energy. The modified selection rule for the inter-band transition at $\pm \mathrm{K}$ point is summarized in Table 1. The selection rule regresses every 3 of $l$, which reflects the three-fold rotation symmetry of monolayer TMD.

\section{Beam position dependence}

Subsequently, we show the beam position dependence of the transition strength. To make the problem simple, we consider only the case $p=0$. Close to the phase singularity, the mode function can be simplified to

$$
u(r, \phi, z)=\frac{c_{p l}}{\omega(z)}\left[\frac{\sqrt{2} r}{\omega(z)}\right]^{|l|} \exp (i l \phi) .
$$

We consider the case of $l=+1, \epsilon=(1,+i, 0) / \sqrt{2}$, which excite $+\mathrm{K}$ point carriers. The vector potential for the standing vortex beam is

$$
\begin{aligned}
\mathbf{A}(\mathbf{r}) & =A_{0} \boldsymbol{\epsilon} u(\mathbf{r}) e^{i k z}+A_{0} \boldsymbol{\epsilon} u(\mathbf{r}) e^{-i k z} \\
& =2 A_{0} \frac{C_{01}}{\omega(z)}\left[\frac{\sqrt{2} r}{\omega(z)}\right]^{1} \exp (i \phi) \boldsymbol{\epsilon} \cos k z .
\end{aligned}
$$

The transition Hamiltonian $H_{\mathrm{tr}}$ in the spherical coordinate is

$$
\begin{aligned}
H_{\mathrm{tr}} & =\mathbf{r} \cdot \mathbf{A}^{l} \\
& =\frac{\alpha}{\omega_{0}^{2}} \rho^{2} \sin ^{2} \theta e^{2 i \phi}\left[1+\frac{(\rho \cos \theta)^{2}}{z_{\mathrm{R}}^{2}}\right]^{-1} \cos [k \rho \cos \theta] .
\end{aligned}
$$

As for the parameters, we assume that the wavelength $\lambda=690 \mathrm{~nm}, \omega_{0}=1 \mu \mathrm{m}, z_{\mathrm{R}}=4.55 \mu \mathrm{m}, k=9.1 \mu \mathrm{m}^{-1}$. Here, the position of TMD is $z=0$ and that of the beam waist is $z=z_{0}$ (see Fig. 1). Then, the coordinate is transformed as follows

$$
\rho^{\prime} \sin \theta^{\prime}=\rho \sin \theta, \quad \rho^{\prime} \cos \theta^{\prime}=\rho \cos \theta-z_{0} .
$$

Using this approximation $\left(\rho \cos \theta-z_{0} \approx-z_{0}\right)$, the final form of transition matrix is

$$
\left\langle\Psi_{\mathrm{c}}\left|\mathbf{r} \cdot \mathbf{A}^{l}\right| \Psi_{\mathrm{v}}\right\rangle \propto\left(1+\frac{z_{0}^{2}}{z_{\mathrm{R}}^{2}}\right)^{-1} \cos \left[-k z_{0}\right] .
$$

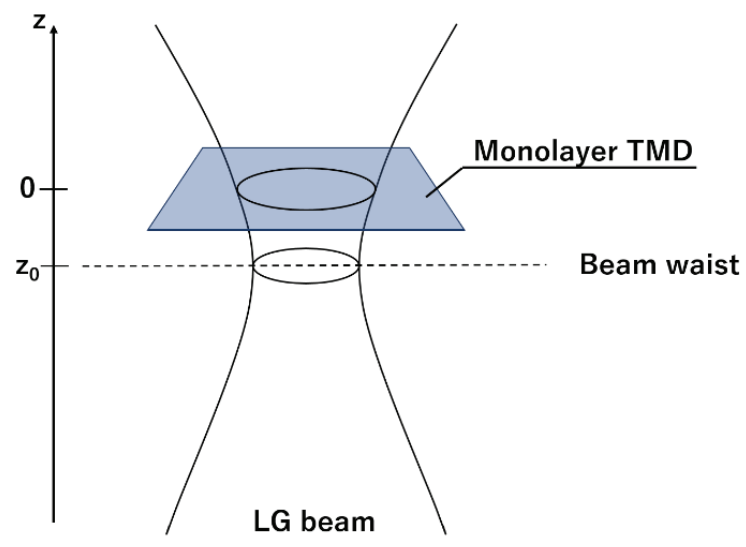

Figure 1. Image of position relationship between monolayer TMD and Laguerre-Gaussian beam waist.

In Fig. 2, we show the result of the position dependence of the transition strength. One can see that the standing Laguerre-Gaussian beam can transfer its OAM of light to carriers in monolayer TMD. Furthermore, it is also found that the transition strength oscillates with the beam waist position even though the thickness of monolayer TMD can be neglected.

\section{Ш Summary}

In this work, we have shown the optical selection rules of optical vortex in a monolayer TMD and evaluated the 


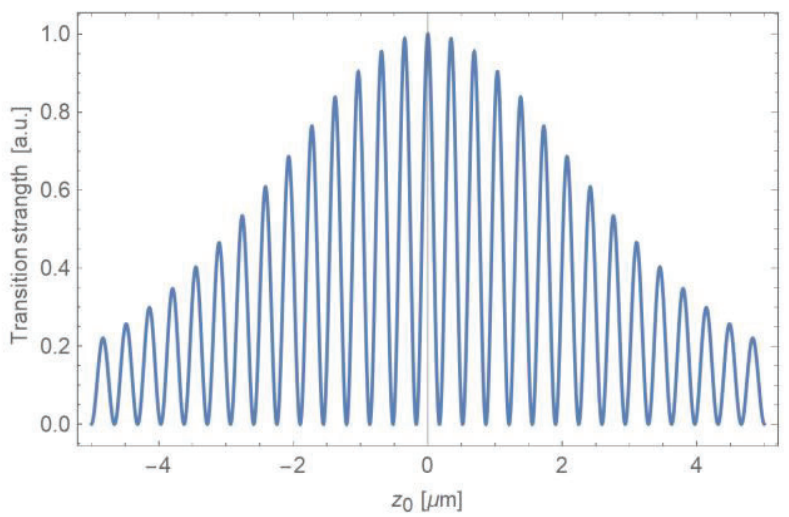

Figure 2. $z$-dependence of transition strength using the standing wave of Laguerre-Gaussian beam in monolayer TMD. $z_{0}$ indicates the distance between the beam waist and center of monolayer TMD.

inter-band transition strength for the case of the standing vortex beam. In future, we will calculate the absolute value of the transition strength and formulate the interaction between OAM of light and the carrier spins in TMD. We expect that the new interaction enables various new operations of spins and the valley degree of freedom. We want to extend the field of "spin-valleytronics" and "twodimensional materials" by optical vortices.

\section{References}

[1] Cao, T., Wang, G., Han, W., Ye, H., Zhu, C., Shi, J., Niu, Q., Tan, P., Wang, E., Liu, B. and Feng, J. "Valley-selective circular dichroism of monolayer molybdenum disulphide" Nature Communication 3887 (2012).

[2] Zhu, Z. Y., Cheng, C. Y., and Schwingenschlog̈l, U. "Giant spin-orbitinduced spin splitting in two-dimensional transition-metal dichalcogenide semiconductors" Physical Review B 84, 153402 (2011).

[3] Xiao, D., Gui-Bin, L., Wanxiang, F., Xiaodong, X., and Wang, Y. "Coupled Spin and Valley Physics in Monolayers of $\mathrm{MoS}_{2}$ and Other Group-VI Dichalcogenides” Physical Review Letters 108, 196802 (2012).

[4] Xiaodong, X., Wang, Y., Xiao, D and Heinz, T. F. "Spin and pseudospins in layered transition metal dichalcogenides" Nature Physics 10, 343-350 (2014).

[5] Allen, L., Beijersbergen, M. W., Spreeuw, R. J. C. C. and Woerdman, J. P., "Orbital angular momentum of light and the transformation of Laguerre-Gaussian laser modes," Physical Review A 45, 8185-8189 (1992).
[6] Friese, M. E. J., Nieminen, T. A., Heckenberg, N. R. and RubinszteinDunlop, H., "Optical alignment and spinning of laser-trapped microscopic particles," Nature 394, 348-35025 (1998).

[7] Toyoda, K., Takahashi, F., Takizawa, S., Tokizane, Y., Miyamoto, K., Moriya, R., Omatsu, T., "Transfer of Light Helicity to Nanostructures," Physical Review Letters 110, 143603 (2013).

[8] Schmiegelow, C. T., Schulz, J., Kaufmann, H., Ruster, T., Poschinger, U. G. and Schmidt-Kaler, F. "Transfer of optical orbital angular momentum to a bound electron" Nature Communication 712998 (2016).

[9] Peshkov, A. A., Seipt, D. Surzhykov, A. and Fritzsche, S. "Photoexcitation of atoms by Laguerre-Gaussian beams" Physical Review A 96, 023407 (2017).

[10] Coohoorn, R., Haas C. and de Groot R. A. "Electronic structure of MoSe2, MoS2, and WSe2. II. The nature of the optical band gaps" Physical Review B 35, 6203 (1987). 


\title{
Generation of three-dimensional dark hole by hybrid phase plate in super- resolution microscopy
}

\author{
Koumei Nagai *a, Akira Kodaira a , Takashi Maruyama a, Satoshi Oku a, \\ Hiroshi Kumagai ${ }^{b}$, Bokor Nándor ${ }^{c}$, Yoshinori Iketaki ${ }^{b, d}$ \\ ${ }^{a}$ NTT Advanced Technology Corporation, 3-1 Morinosato Wakamiya, Atsugi, 243-0124, Japan \\ ${ }^{\mathrm{b}}$ School of Allied Health Sciences Physics, Kitasato University, 1-15-1 Kitasato, Minami, \\ Sagamihara, 252-0373, Japan \\ ${ }^{\mathrm{c}}$ Department of Physics, Budapest University of Technology and Economics, Budafoki u. 8., \\ Budapest 1111, Hungary \\ ${ }^{\mathrm{d}}$ Olympus Corporation, 2-3 Kuboyama-cho, Hachioji, 192-8512, Japan
}

\begin{abstract}
The two-color phase plates (TPP) can achieve super resolution microscopy (SRM) based on the two-color fluorescence depletion. TPP modulates the erase beam to a donut shape having a dark spot at the center while maintaining the pump beam in the Gaussian shape near the focal region. The hybrid type phase-plate (HPP) with TPP function is designed so as to have both structure of annular phase plate and spiral phase plate and is expected to realize the improvement of depth and breadth resolution simultaneously. The precise HPP is fabricated by photolithography and etching process technology. We confirmed the formation of a dark hole which suppresses fluorescence in three-dimensions by mounting the HPP in the SRM.
\end{abstract}

Keywords: Super-resolution microscopy, two color phase plate, fluorescence depletion, lateral and depth resolution.

\section{Introduction}

Super-resolution microscopy based on the two-color fluorescence depletion is expected to be a powerful measurement tool in life science, since it can provide high

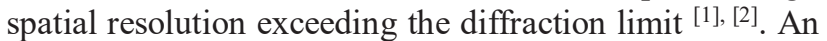
erase beam with $\lambda_{e}$ quenches a molecule in the $S_{1}$ state generated by a pump beam with $\lambda_{\mathrm{p}}$, and prevents fluorescence from the $\mathrm{S}_{1}$ state. The TPP modulates the erase beam to a donut shape having a dark spot at the center while maintaining the pump beam in the initial Gaussian shape near the focal region. When the pump and erase beam passing the TPP is focused onto a dyed sample, the fluorescence spot becomes smaller than the diffraction limit. To achieve high three dimensional spatial resolution, The HPP with TPP function is designed so as to have both structure of an annular phase plate and a spiral phase plate and is expected to realize the improvement of depth and lateral resolution simultaneously. The precise HPP is fabricated by photolithography and etching process technology, where their processing accuracy is improved compared with the previous one. The plate can be applied to a commercial microscope without modification. We confirmed the formation of a dark hole which suppresses fluorescence in three-dimensions by mounting the HPP in the SRM.

*koumei.nagai@ntt-at.co.jp

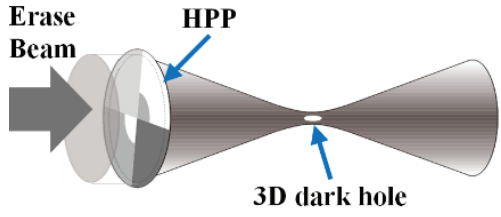

(a)

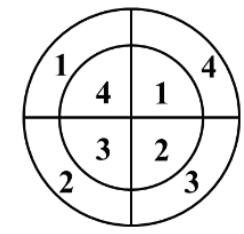

(b)
Figure 1. Hybrid type phase-plate (HPP)

(a) Schematic of HPP and 3D dark hole.

(b) Diagram of phase plates consisting of multiple.

\section{Design and fabrication}

\section{Design}

In our design, we assumed the following physical parameters (corresponding to our experimental conditions for erase and pump lasers, and a Synthetic quartz substrate): $\lambda_{\mathrm{e}}=647 \mathrm{~nm}, \lambda_{\mathrm{p}}=532 \mathrm{~nm}, \mathrm{n}_{\mathrm{e}}=1.475$, and $\mathrm{n}_{\mathrm{p}}=1.479$. In a manner similar to that Bokor and Iketaki did ${ }^{[3]}$, we designed the etching depths of regions for HPP (Table 1). 
Table 1. Design thicknesses of the segments of the HPP.

\begin{tabular}{|c|c|c|c|}
\hline $\begin{array}{c}\text { Number of } \\
\text { segment }\end{array}$ & $\begin{array}{c}\text { Etching } \\
\text { Depth } \\
\text { (nm) }\end{array}$ & $\begin{array}{c}\text { Phase } \\
\text { delay at } \lambda_{\mathbf{e}} \\
\text { (deg.) }\end{array}$ & $\begin{array}{c}\text { Phase } \\
\text { delay at } \lambda_{\mathbf{p}} \\
\text { (deg.) }\end{array}$ \\
\hline 1 & 0 & 0 & 0 \\
\hline 2 & 4605 & 90 & -45.7 \\
\hline 3 & 2125 & 180 & 34.29 \\
\hline
\end{tabular}

\section{Fabrication}

We fabricated HPP by etching used in the semiconductor manufacturing process. By using the etching method, unlike the vapor deposition method, each HPP having a constant density and dense can be obtained.

For photolithography, a reduction projection type exposure apparatus (stepper) was used. Since the positioning accuracy of the stepper is extremely high as a submicron level, it is possible to manufacture a phase plate with high alignment accuracy as compared with the conventional method. For quartz substrate processing, reactive ion etching (RIE) with CF gas was used.

\section{Results and Discussion}

To confirm the optical characteristics of the HPP, we inserted these into the scan unit of the LSM system (Olympus Fluoview 1000) and measured the 3D intensity distribution of the erase beam by scanning fluorescence microbeads smaller than the diffraction limit size (Figure 2 ), which can emit fluorescence by irradiating the pump or erase beam.

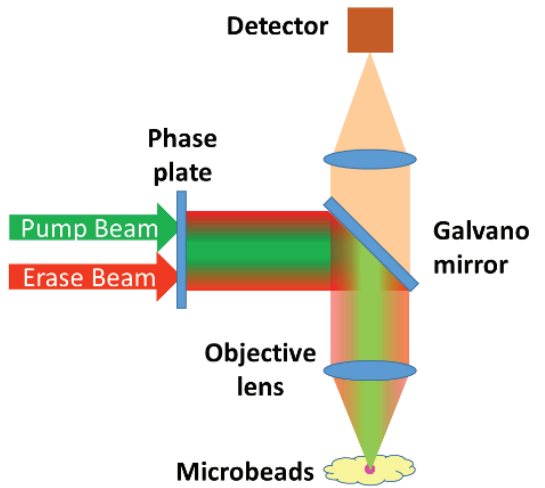

Figure 2. Principle of a super resolution microscopy.
Figure 3 shows the theoretical and observed intensity profiles of the 3D dark hole generated by HPP near the focal point of SRM. From comparing these results, it is confirmed that the HPP produced using etching process are accurately manufactured according to the design values, and it was found that the HPP has sufficient specifications for the SRM. In this conference, we will report details of fabrication processing of HPP and its performance for SRM.
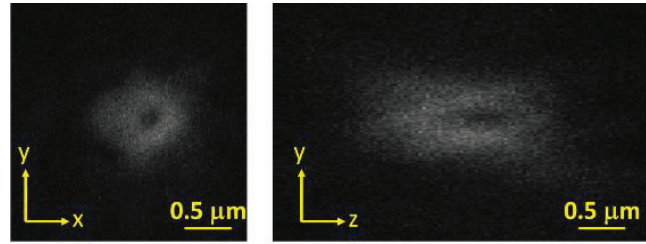

(a) Measured
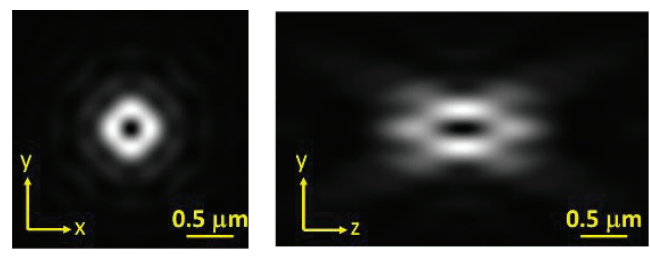

(b) Calculated

Figure 4. Principle of a super resolution microscopy

\section{Acknowledgements}

This study is entirely funded by the project of "Department of Advanced Measuring Technology" in Japan Science and Technology Agency.

\section{Reference}

[1] Hell, S. W. and Wichmann, J., " Breaking the diffraction resolution limit by stimulated emission: stimulated-emission-depletion fluorescence microscopy," Optics Letter, 19(11), 780-782 (1994).

[2] Y. Ikelaki, T. Omatsu, O. Satio, T. Suzuki and M. Fujii, "Super-resolution fluorescence microscopy by upconversion-depletion using two color lasers," Conference on Lasers and Electro-Optics (CLEO 2000). Technical Digest. Postconference Edition. TOPS Vol.39, San Francisco, CA, USA, 2000, pp. 448-449 (2000). [3] Bokor, N. and Iketaki, Y., "New design method for a phase plate in super-resolution fluorescence microscopy," Applied spectroscopy, 68(3), 353-361 (2014). 


\title{
Manipulation of DNA using Nano-structured Semiconductor-assisted (NASSCA) Optical Tweezers
}

\author{
Ryota Takao ${ }^{1}$, Tatsuya Shoji ${ }^{1}$, Denver Linklater ${ }^{2}$, Saulius Juodkazis ${ }^{2}$, Yasuyuki Tsuboi ${ }^{1 *}$ \\ 1 Graduate School of Science, Osaka City University, 3-3-138 Sugimoto, \\ Sumiyoshi, Osaka 558-8585, Japan \\ 2Centre for Micro-Photonics, Swinburne University of Technology, \\ PO Box 218, Hawthorn, 3122 Australia
}

\begin{abstract}
Recently, we have developed non-plasmonic nano-structured semiconductor-assisted (NASSCA) optical tweezers using a silicon with nanostructured surface $(\mathrm{B}-\mathrm{Si})$ to trap nanoparticles efficiently. In the present study, we demonstrate optical trapping of DNA using NASSCA optical tweezers. Fluorescence microspectroscopy showed that irradiation of a near-infrared laser beam led to micro-assembly formation of a DNA with random coil structure, which has hardly been trapped with conventional optical tweezers. Turning off the irradiation, the DNA micro-assembly on B-Si was rapidly dissipated in solution. These behaviors clearly indicated that NASSCA optical tweezers was able to perform the efficient trap-and-release behavior of DNA. NASSCA optical tweezers will open new horizons in nanoscience for biomolecular manipulation.
\end{abstract}

Keywords: Optical tweezers, Black silicon, NASSCA, Enhanced optical force, DNA

*twoboys@sci.osaka-cu.ac.jp; phone +81-6-6605-3693

\section{Introduction}

Direct optical manipulation of biomolecules such as DNA and proteins without attaching a microbead to them is currently a challenging task. Although optical manipulation requires high polarizability of such biomolecules, their polarizabilities are too small to be stably trapped with conventional optical tweezers. An alternative approach for biomolecular manipulation is to enhance an electric field of light using localized surface plasmon ${ }^{[1]}$. Recently, we have developed non-plasmonic nano-structure semiconductor-assisted optical tweezers (NASSCA-OT) to trap nanoparticles efficiently ${ }^{[2]}$. This technique uses a nanostructured silicon (B-Si) to enhance optical forces due to multi-scatterings of incident light among the nano-needles on the surface. It should be noted that NASSCA-OT is free from any photothermal effect.

In the present study, we explored optical trapping of DNA molecules using NASSCA-OT. We demonstrated that NASSCAOT can perform trapping of DNA in a trap-and-release mode of a DNA taking random coil structures. This result indicates that NASSCA-OT will be a promising method for novel manipulation of biomolecules.

\section{Experiments}

We used $\lambda$-DNA (48.5 kbp) stained with fluorescence molecular probe YOYO-1 in a Tris-borate-EDTA buffer solution. B-Si was fabricated by reactive ion etching of a single crystal silicon wafer. In this process, nano-needles with $400 \mathrm{~nm}$ height were formed on the surface. We used near-infrared laser light $(\lambda$ $=1064 \mathrm{~nm}$ ) for NASSCA optical trapping and visible laser light $(\lambda=473 \mathrm{~nm})$ for fluorescence excitation. These laser beams were coaxially introduced into an inverted microscope, and focused on a surface of B-Si. All the experiments were performed under ambient conditions at room temperature.

\section{Results and Discussion}

We carried out NASSCA optical trapping of DNA at $0.15 \mathrm{~W}$ of laser power (corresponding to $0.52 \mathrm{MW} / \mathrm{cm}^{2}$ at the focal point), at which we never observed any sign of trapping events with conventional optical tweezers or a flat silicon substrate. Figure 1 shows fluorescence micrographs during irradiation on the B-Si. Turning on trapping laser irradiation, fluorescence of DNA was observed from the irradiation area. Upon further irradiation, the fluorescence intensity gradually increased. 
$0 \mathrm{~s}$

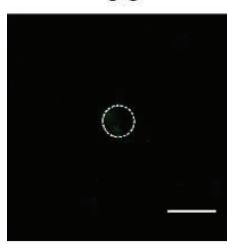

$5 \mathrm{~s}$

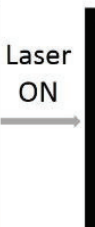

Laser

ON
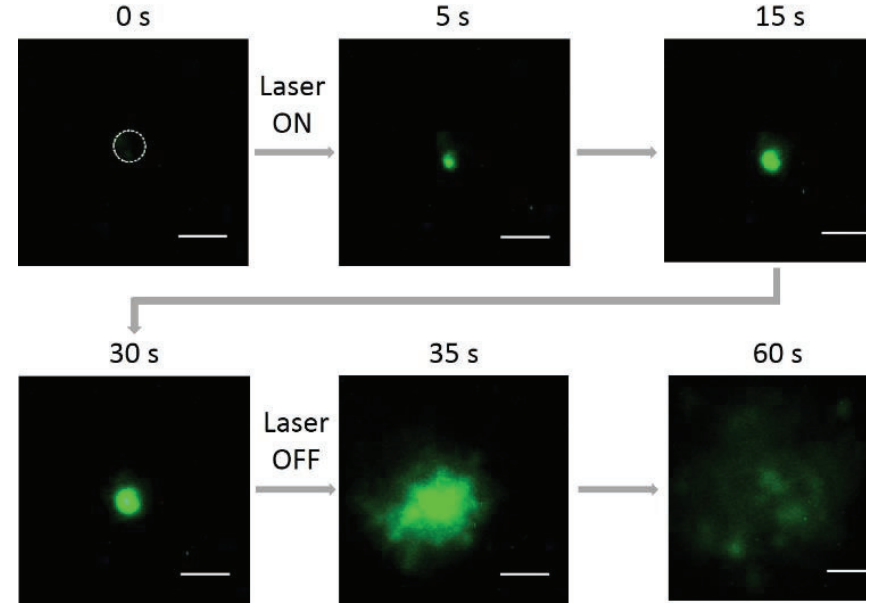

Figure 1. Fluorescence micrographs during NASSCA optical trapping of DNA on B-Si. $0 \mathrm{~s}$ means the time just starting laser irradiation. Laser irradiation area is expressed as a white circle. Scale bar is $5 \mu \mathrm{m}$.

At 30 seconds, the size of the DNA assembly reached about $2 \mu \mathrm{m}$, indicating that numerous DNA molecules were efficiently trapped on B-Si. Turning off the irradiation, the trapped DNA molecules were immediately released, and dissipated from the area by Brownian motion. The smooth trap-and release behavior was also observed by means of fluorescence microspectroscopy.

Here, we discuss the trapping mechanism of DNA using NASSCA-OT. We have experimentally determined the temperature elevation during NIR laser irradiation on a B-Si surface, indicating that the DNA molecules were stably trapped without any photothermal damages. This is supported by fluorescence microspectroscopy.

\section{Conclusion}

What is important here is that NASSCA-OT can stably trap $\lambda$ DNA taking random coil structures. Our NASSCA-OT will be a promising candidate for a biomolecular manipulation technique.

\section{Reference}

[1] T. Shoji, Y. Tsuboi, et al, "Permanent Fixing or Reversible Trapping and Release of DNA Micropatterns on a Gold Nanostructure Using Continuous-Wave or Femtosecond-Pulsed Near-Infrared Laser Light" J. Am. Chem. Soc. 135 (17), 6643$6648(2013)$
[2] T. Shoji, A. Mototsuji, Y. Tsuboi, et al., "Optical tweezing and binding at high irradiation powers on black-Si" Scientific Reports 7, 12298 (2017) 


\title{
Laguerre-Gaussian self-trapped beams in optical lattices
}

\author{
Miguel Angel Cruz-Gomez ${ }^{\mathrm{a}}$, Daniel Lopez-Aguayo ${ }^{\mathrm{b}}$, Servando Lopez-Aguayo*a \\ ${ }^{a}$ Tecnologico de Monterrey, Escuela de Ingeniería y Ciencias, Ave. Eugenio Garza Sada 2501, \\ Monterrey, N.L., México, 64849 \\ ${ }^{\mathrm{b}}$ Tecnologico de Monterrey, Escuela de Ingeniería y Ciencias, Hidalgo 42080, México
}

\begin{abstract}
We study families of self-trapped optical beams generated by Laguerre-Gaussian modes that constitute the stationary solutions of a pure local nonlinear Schrödinger equation with a potential described by a combination of also LaguerreGaussian functions and a harmonic term. The pure local nonlinear medium here studied resembles, up to a certain point, a strongly nonlocal medium allowing thus to make several connections between linear and nonlinear systems. We obtain the corresponding analytical expressions for some conserved quantities and we find that these modes can be stabilized provided they remain below of a level of power threshold.
\end{abstract}

Keywords: laser beam shaping, optical solitons, nondiffracting beams, optical lattices

*servando@tec.mx; phone 8358-2000 ext. 4631

\section{Introduction}

Transverse light modulation in media where optical fields can be propagated, offers several interesting phenomena to study and observe by routing and steering light, where a particular technique of interest is by using optical lattices ${ }^{1}$. On the other hand, self-trapped nonlinear modes, or spatial solitons have been a topic of intense study over the last decade due to their potential to become a fundamental part for the future of all-optical technologies ${ }^{2}$. Thus, the control of solitons by optical lattices deserves several studies from different conditions of structured-light. Of the special interest, Laguerre-Gaussian modes are two dimensional modes that have rotational symmetry along their propagation axis and besides, these modes have a rotational orbital angular momentum that allows to transmit a torque to an object located in the propagation axis $^{3}$. As a concrete example, this property of the Laguerre Gaussian modes has been used to move particles, and these beams, together with the nondiffracting Bessel beams, constitute a very special case of beam shaping examples that can be useful for the study of transferring angular momentum from light to matter ${ }^{4}$.

In this work, we study soliton solutions that can be generated by Laguerre-Gaussian beams supported by optical lattices for the $(2+1) \mathrm{D}$ case, where both, the soliton and the potential are completely described by pure analytical functions. Therefore, this model offers a possibility to gain a deeper understanding of their physical properties. Remarkably, we find that the LaguerreGaussian solitons can be stable in propagation if their power remains below a critical threshold value, as similar as in the case of Hermite-Gaussian solitons for the unidimensional scenario ${ }^{5}$.

\section{Physical Model}

We start our analysis by considering the evolution of a scalar paraxial optical complex field $\psi$, described by a dimensionless $(2+1) \mathrm{D}$ nonlinear Schrödinger equation (NLSE)

$$
i \frac{\partial \psi}{\partial z}+\frac{1}{2} \nabla_{T}^{2} \psi+|\psi|^{2} \psi+V \psi=0,
$$

where $\mathrm{z}$ is the longitudinal coordinate of evolution, while the transverse Laplacian operator is given by $\nabla_{T}^{2} \psi=\frac{\partial \psi^{2}}{\partial x^{2}}+$ $\frac{\partial \psi^{2}}{\partial y^{2}}$ and $\mathrm{V}=\mathrm{V}(\mathrm{x}, \mathrm{y})$ is the corresponding potential of the system generated by an optical lattice. Eq.(1) admits as the following conserved quantities the power or total energy flow:

$$
P=\int_{-\infty}^{\infty} \int_{-\infty}^{\infty}|\psi|^{2} d x d y
$$


and the Hamiltonian

$H=\frac{1}{2} \int_{-\infty}^{\infty} \int_{-\infty}^{\infty}\left(\nabla \psi \cdot \nabla \psi^{*}-2 V|\psi|^{2}+|\psi|^{4}\right) d x d y$

Besides, even that it is not properly a conserved quantity, another important parameter to study due to the particular geometry of the generated modes, it is the corresponding projection of the angular momentum in the z-axis:

$$
L_{z}=\int_{-\infty}^{\infty} \int_{-\infty}^{\infty} \psi^{*}(\vec{r} \times \nabla \psi) \cdot e_{z} d x d y
$$

where $\overrightarrow{\boldsymbol{r}}=(\mathrm{x}, \mathrm{y})$ is a two dimensional vector position and $\boldsymbol{e}_{\mathbf{z}}$ is unit vector along the $\mathrm{z}$ axis. Note that the integration of these quantities is done over the entire $x^{-} y$ plane.

\section{Results}

Next, we start by looking for soliton solutions of the form

$$
\psi(r, \theta, z)=\varphi(r) e^{i \lambda z} e^{i m \theta},
$$

where the parameter $\lambda$ is a real propagation constant and $\varphi$ is a real-valued function. As boundary conditions, we set $\varphi(r) \rightarrow 0$ as $|\mathrm{r}| \rightarrow \infty$, in order to have well-localized wave solutions. Substituting this kind of function in Eq.(1) and solving for the potential we get

$$
V=\lambda-|\varphi(r)|^{2}-\frac{1}{2} \frac{\left[\partial_{r}^{2} \varphi(r)+\frac{1}{r} \partial_{r} \varphi(r)-\frac{m^{2}}{r^{2}} \varphi(r)\right]}{\varphi(r)},
$$

As a next step, we study soliton solutions in the form

$$
\varphi(r)=r^{m} \mathrm{f}\left(r^{2}\right) \exp \left(-r^{2} / \omega_{0}^{2}\right),
$$

where the real parameter $\omega_{0}$ stands for the corresponding width of the soliton obtained. After some mathematical work, we obtain the following soliton solution:

$$
\begin{gathered}
\psi(r, \theta, z)=A\left(\frac{r}{\omega_{0}}\right)^{m} L_{n}^{m}\left(2\left(\frac{r}{\omega_{0}}\right)^{2}\right) \exp \left[-\left(r / \omega_{0}\right)^{2}+\right. \\
i(\lambda z+m \theta)],
\end{gathered}
$$

Where $L_{n}^{m}$ is the associated Laguerre Polynomial with parameters $\mathrm{m}$ and $\mathrm{n}$ and $\mathrm{A}$ is a real parameter. This selftrapped solution corresponds to the potential function

$V(r, \theta, z)=\lambda-|\psi(r, \theta, z)|^{2}+4 n+2 m+2-2 r^{2}$, and with the power

$$
P=\frac{A^{2} \pi \omega_{0}^{2}}{2^{m+1}} \cdot \frac{(n+m) !}{n !},
$$

and Hamiltonian

$$
H=\frac{1}{2}\left[3 \zeta\left(\omega_{0}\right)-\frac{A^{2} \omega_{0}^{2} \pi \lambda}{2^{m} n !} \cdot(n+m) !\right],
$$

Where $\zeta\left(\omega_{0}\right)=c \int_{-\infty}^{\infty} \int_{-\infty}^{\infty}|\psi|^{4} d x d y$, can be obtained analytically, in spite of being a cumbersome function. In a similar way,

$$
L_{z}=m \frac{A^{2} \pi \omega_{0}^{2}}{2^{m+1}} \cdot \frac{(n+m) !}{n !}=m P,
$$

Thus, Eqs. (8)-(12) constitute the main analytical results from our work and they constitute a full analytical soliton solution to a particular (2+1) D NLSE.

\section{Numerical Simulations}

We validate our results by using pseudo-spectral methods, propagation of these stable solutions was performed. The following figures show the results of these numerical simulations.
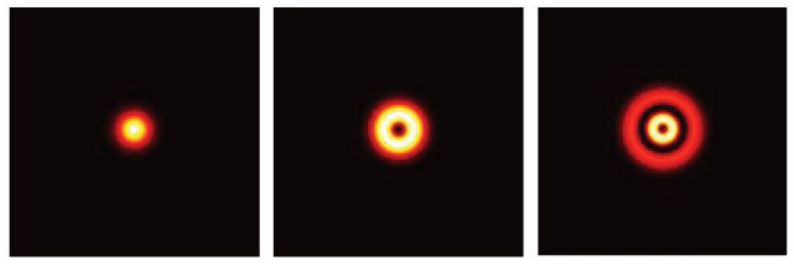

Figure 1. Transversal mode images of stable solutions $\psi(m, n)$. From left to right: $\psi(0,0), \psi(0,1), \psi(1,1)$. Modes $\psi(0,1)$ and $\psi(1,1)$ show a dislocation (i.e. a region where the optical field's phase is not defined). Due to this as well as their radial symmetry, these modes are typically used to transfer angular momentum.

It's important to recall that $\psi$ by no means is a solution to Nonlinear Schrödinger equation, in fact, it's a Helmholtz equation solution in cylindrical coordinates, but it's stabilized on NLSE by V(r). In order to study stability of these solutions, we used standard perturbation theory and found that there are regions of power where these Laguerre-Gaussian solitons are unstable, but also regions where these beams can be stabilized, provided they power remain under certain threshold value.
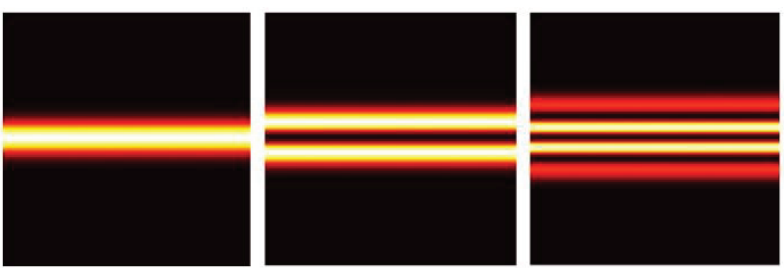

Figure 2. Longitudinal propagation of stable solutions. It's clearly observed invariant properties along propagation coordinates. From left to right: $\psi(0,0)$ $, \psi(0,1), \psi(1,1)$. 


\section{Conclusions}

In summary, we have obtained a family of analytical twodimensional nonlinear modes that are solutions to a nonlinear Schrödinger equation supported by local potential described by a harmonic term and LaguerreGaussian functions. We derive the corresponding expressions for two quantities of conservation: the power and the projection of angular momentum along the $\mathrm{z}$ coordinate, and we find that both quantities are related through the topological charge. We find that the corresponding solitons solutions of the nonlinear system can be completely described by also Laguerre-Gaussian functions. Finally, we found a correspondence between our pure local system and nonlinear models with high nonlocality.

\section{References}

[1] Kartashov, Y. V., Vysloukh, V. A., and Torner, L., "Soliton shape and mobility control in optical lattices," Progress in Optics 52, 63-148 (2009).

[2] Kivshar, Y. S. and Agrawal, G., [Optical solitons: from fibers to photonic crystals ], Academic press (2003).

[3] Allen, L., Beijersbergen, M. W., Spreeuw, R., and Woerdman, J., "Orbital angular momentum of light and the transformation of Laguerre-Gaussian laser modes," Physical Review A 45(11), 8185 (1992).

[4] Dholakia, K. and Cizmar, T., "Shaping the future of manipulation," Nature Photonics 5(6), 335 (2011).

[5] Trejo-Garcia, D., Gonzalez-Hernandez, D., LopezAguayo, D., and Lopez-Aguayo, S., "Stable HermiteGaussian solitons in optical lattices," Journal of Optics 20(12), 125501 (2018). 


\section{High Q-factor planar toroidal metamaterial with the ability of strong magnetic field localization Maria V. Cojocari ${ }^{\mathrm{a}, \mathrm{b}}$, Vitaly I. Chuguevskya, ${ }^{\mathrm{a} b}$, Nikita A. Volsky ${ }^{\mathrm{a}, \mathrm{b}}$ Alexey A. Basharinn ${ }^{\mathrm{a}, \mathrm{b}, \mathrm{c}}$

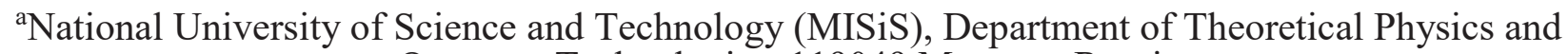 Quantum Technologies, 119049 Moscow, Russia \\ bNational University of Science and Technology (MISiS), The Laboratory of Superconducting metamaterials, 119049 Moscow, Russia \\ 'Politecnico di Torino, Department of Electronic and Telecommunications, Torino 10129, Italy \\ Abstract}

In this paper we propose toroidal planar metamaterial with advantage of magnetic field control. This structure supports toroidal dipole moment excitation, which has major contribution to metamaterial properties, i. e. high Q-factor and extremely strong magnetic field localization. The electromagnetic properties of the proposed design were studied both theoretically and experimentally. The resulting transmission spectra represents a sharp Fano-resonance. Due to its properties, the proposed metamaterial can be applied novel devices in field of light-matter interactions of magnetic, wireless transmission, and magnetic resonance imaging methods..

Keywords: metamaterial, toroidal dipole moment, magnetic field, high Q-factor

\section{Introduction}

The fast development of artificial magnetism makes it possible to control magnetic field on the same level as it is possible with the electric field, which is strongly required both for the development of fundamental solid state physics and many technological applications. The idea is to simulate the natural diamagnetic properties of matter by specific currents distribution in engineered structures such as metamaterials. The idea of "magnetic" metamaterial is to enhance magnetic response of a medium by constructing a particular periodic structure. Such a structure should comprise unit cells with dimensions smaller than the wavelength.

Still, the main purpose of magnetic moment enhancement in metamolecules is to localize magnetic field in sub-wavelength area. Thereafter, here we suggest an alternative approach. For the first time we demonstrate how magnetic field can be strongly captured by metamaterial with dominating toroidal mode. Toroidal moment appears in an imaginary torus due to the magnetic field being rotated by poloidal currents. It has been proved recently [1-3] that it is crucial to include toroidal moment in multipole expansion for structures of toroidal topology. Starting with 2010-s, when the existence of toroidal moment was demonstrated experimentally for the first time, [2] a great number of three-dimensional structures with toroidal topology was proposed [1, 4, 5-12].

\section{Body}

In this work we have designed and fabricated a high Qfactor planar toroidal metamaterial (Figure 1) with the ability of strong magnetic field localization. It represents a metallic surface with periodically arranged voids of the epsilon-letters form. Incident plain electromagnetic wave, polarized perpendicularly to the central strips of cut-outs, excites two counter-directed currents flowing along epsilon voids. As a result, magnetic field vectors are organized in a head-to-tail way around the central wire. The latter causes toroidal moment excitation. 


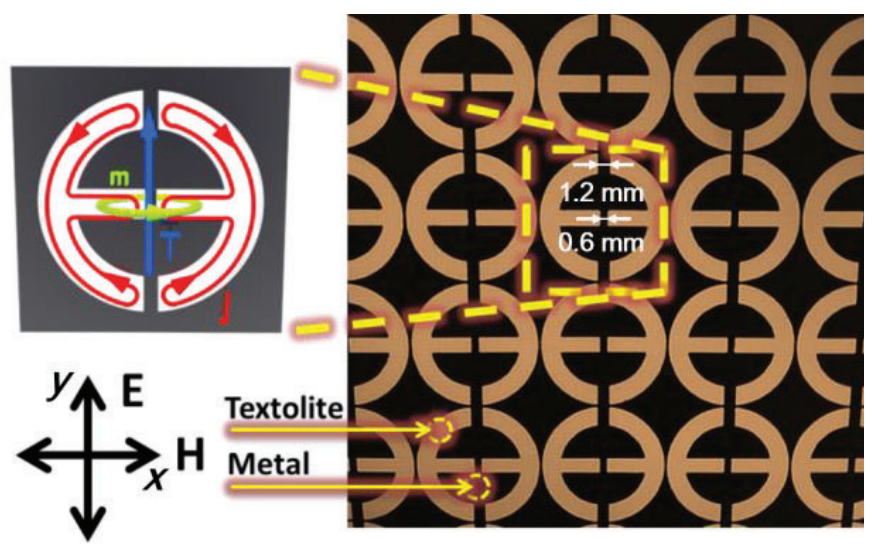

Figure 1. The proposed metamaterial, fabricated using the method of chemical etching (a metal foil on a textolite substrate), and the currents distribution on the surface of a separate metamolecule. $\mathrm{T}$ is toroidal moment, $\mathrm{m}$ is magnetic field, $\mathrm{j}$ are electric currents.

As a result, there can be seen a sharp Fano-resonance (Figure 2a) in the transmission spectra of proposed metamaterial. It demonstrates lack of radiation losses. This can be explained if we consider the interaction between two strongest multipoles, excited in the metamaterial at the resonance frequency (Figure 2b): toroidal dipole and magnetic quadrupole moments in the terms of their radiation patterns overlap. Toroidal moment, characterized by the same radiation patterns as electric dipole moment, influences four-lobe patterns of magnetic quadrupole moment through the elongation of one lobes in the direction of reflection, so that zero forward scattering is attained. Another property ensuing from described processes is as was mentioned above extremely strong magnetic field localization in the subwavelength area.

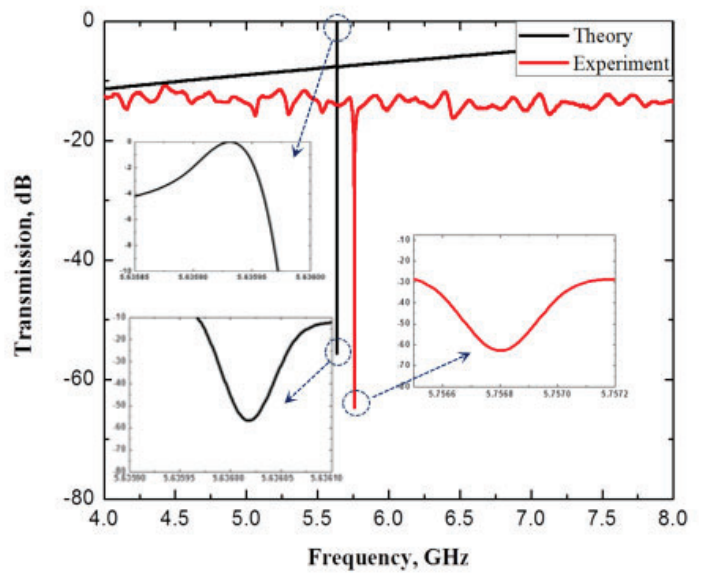

(a)

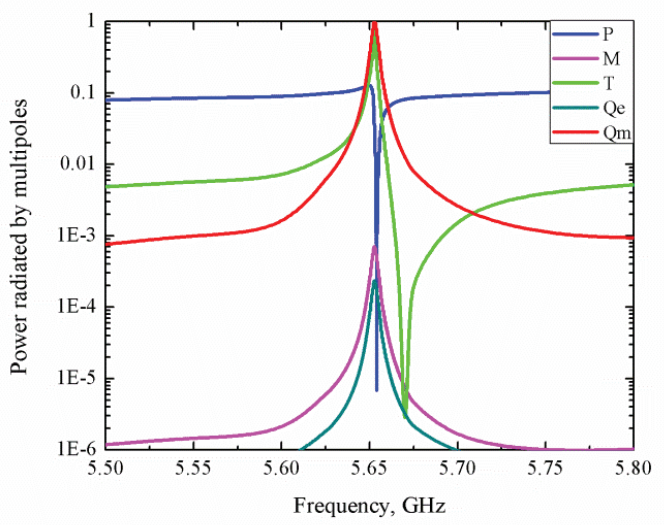

(b)

Figure 2. (a) The transmission spectra for theoretical (black line) and experimental (red line) studies of the metamaterial presented in Fig. 1. The insets show both dips and peaks within smaller frequencies ranges;

(b) the relative electromagnetic power of five strongest multipoles $(\mathrm{P}-$ is an electric dipole, $\mathrm{M}-$ is a magnetic dipole, $\mathrm{T}-$ is a toroidal dipole, $\mathrm{Qe}-$ is an electric quadrupole, Qm - is a magnetic quadrupole) in the metamolecule far-field zone at frequencies around resonance frequency.

\section{Results and Discussion}

Proposed structure benefits compared to other approaches of magnetic field enhancement through achieved values 
of Q-factor and magnetic field localization. Besides, it has several other advantages. For example, in comparison with magnetic dipole excitation in all-dielectric metamaterials, in our metamaterial magnetic field is concentrated in vacuum (air), while in dielectric particles - inside the particle. The advantage of field localization in air is the ability to manipulate it directly. Also, the fabrication of proposed metamaterial will be easier in $\mu \mathrm{m}$ dimensions as long as it is a planar structure. Finally, metal is cheaper than dielectric materials. Such high Qfactor value in planar structures provides a variety of possibilities in the magnetic light-matter interaction, especially at higher frequencies.

\section{References}

1. N. Papasimakis, V. A. Fedotov, V. Savinov, T. A. Raybould \& N. I. Zheludev, Nature Materials 15, 263 (2016)

2. Kaelberer T., Fedotov V.A, Papasimakis N., Tsai D.P., Zheludev N.I., Science 330, 1510 (2010)

3. V. Savinov, V. A. Fedotov, and N. I. Zheludev, Phys. Rev. B 89, 205112 (2014)

4. A. M. Zagoskin, A. Chipouline, E. Il'ichev, J. R. Johansson, F. Nori, Sci Rep. 5, 16934 (2015)

5. Juan S. Totero Gongora, Andrey E. Miroshnichenko, Yuri S. Kivshar \& Andrea Fratalocchi, Nature Communications 8, 15535 (2017)

6. Zheng-Gao Dong, J. Zhu, Junsuk Rho, Jia-Qi Li, Changgui Lu, Xiaobo Yin, and X. Zhang, Appl. Phys. Lett. 101, 144105 (2012)

7. Burcu Ögüt, Nahid Talebi, Ralf Vogelgesang, Wilfried Sigle and Peter A. van Aken, Nano Lett. 12, 5239 (2012)

8. Fan YC, Wei ZY, Li HQ, Chen H, Soukoulis C.M, Phys Rev. B 87, 115417 (2013)

9. Song Han, Manoj Gupta, Longqing Cong, Yogesh Kumar Srivastava, and Ranjan Singh Journal of Applied Physics 122, 113105 (2017)

10. Longqing Cong, Yogesh Kumar Srivastava, and Ranjan Singh, Appl. Phys. Lett. 111, 081108 (2017)

11. Gupta Manoj, Singh Ranjan, Advanced Optical Materials, 4, 2195 (2016)

12. Alexey A. Basharin, Vitaly Chuguevsky, Nikita Volsky, Maria Kafesaki , Eleftherios N. Economou, Phys Rev B 95, 035104 (2017) 


\title{
Nano-structured Semiconductor-assisted (NASSCA) Optical Tweezers for Size sorting of polystyrene nanospheres
}

\author{
Sayaka Hashimoto ${ }^{\mathrm{a}}$,Tatsuya Shoji ${ }^{\mathrm{a}}$, Saulius Juodkazis ${ }^{\mathrm{b}}$, Yasuyuki Tsuboi*a \\ ${ }^{a}$ Graduate School of Science, Osaka City University, 3-3-138 Sugimoto, \\ Sumiyoshi, Osaka 558-8585, Japan \\ ${ }^{\mathrm{b}}$ Centre for Micro-Photonics, Swinburne University of Technology, \\ PO Box 218, Hawthorn, 3122 Australia
}

\begin{abstract}
Recently, we have developed a novel optical manipulation technique with a silicon substrate with a nanostructured (black silicon): NASSCA optical tweezers. This method allows us to trap nanoparticles over a wide area $(10 \mu \mathrm{m} \times 10 \mu \mathrm{m})$, being capable of optically separating and aligning them on the basis of chemical composition, shape and size. Here, we demonstrate such optical sorting of polystyrene nanospheres according to their size on a black silicon. Upon near-infrared laser irradiation, larger nanospheres were preferentially trapped than smaller nanospheres on a black silicon. The larger sphere was trapped at the center of the focal spot, while smaller spheres were trapped with surrounding the larger sphere. On this way, we succeeded in size sorting of polystyrene nanospheres.
\end{abstract}

Keywords: optical force, silicon nanostructures, Black silicon, Fluorescence microspectroscopy

\section{Introduction}

Optical tweezers is a non-contact and non-destructive method to trap and manipulate micro- and submicro-metersized objects with a tightly focused laser beam. Since an optical force exerted on nanospheres strongly depends on their polarizability, optical tweezers have great potential for separating and trapping them according to their sizes and shapes. Such optical sorting has been recently investigated using holographic optical tweezers and optical waveguides. Nowadays, for developments of the optical sorting, an optical manipulation technique to trap a large amount of nanospheres with a large-scale area is highly demanded.

Recently, we have developed a novel manipulation technique using a silicon crystal with a nanostructured surface (Black silicon; B-Si). We named it nanostructured semiconductor assisted optical tweezers (NASSCA-OT). The NASSCA-OT can trap numerous polystyrene nanospheres in a large-scale area with weak laser irradiation, because multiple light scattering on $\mathrm{B}-\mathrm{Si}$ results in an enhancement of optical force. Furthermore, due to the low absorption coefficient in the near-infrared region and the high thermal conductivity of silicon, any photothermal effects are negligible in NASSCA-OT. These advantages make it possible to demonstrate the novel optical sorting. In the present study, we show optical separation of different sized polystyrene nanospheres using NASSCA-OT.

\section{Experiments}

A B-Si substrate was fabricated using dry etching in $\mathrm{SF}_{6} / \mathrm{O}_{2}$, resulting in nanoneedles with sub-micrometer height on the surface. The fabricated needles had $400 \mathrm{~nm}$ in height (Figure 1). As a trapping target, we used two kinds of fluorescent polystyrene nanospheres (PSs). One was yellow-green fluorescence emitting (YG) PSs with $500 \mathrm{~nm}$ in diameter, while the other was blue (BL) emitting PSs with $1000 \mathrm{~nm}$ in diameter (Figure 2). These PSs were dispersed in water.

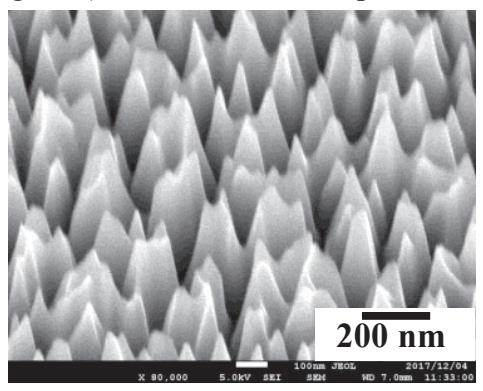

Figure 1. A SEM image of B-Si 


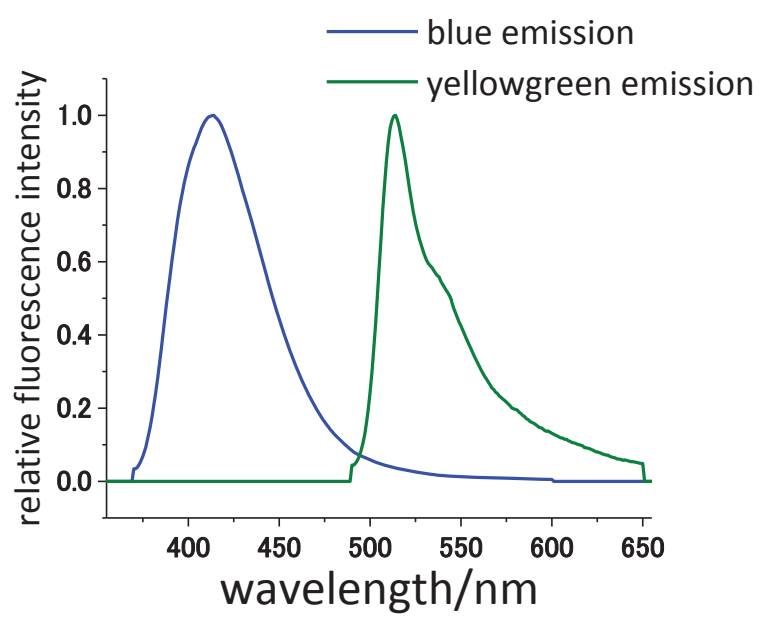

Figure 2. Normalized emission spectra of PSs (blue and yellowgreen)

A continuous wave (cw) near-infrared (NIR) laser beam $(\lambda=808 \mathrm{~nm})$ for NASSCA-OT was coaxially introduced into an inverted microscope together with a cw ultraviolet (UV) laser beam $(\lambda=375 \mathrm{~nm})$ for fluorescence excitation. These laser beams were focused on a B-Si surface with an oil-immersion objective lens $(\times 100$, N.A. $=1.4)$. Since the laser power of UV laser was very weak in comparison with that of NIR laser, we observed no sign of optical trapping and any photo bleaching under the UV laser irradiation.

\section{Results and Discussion}

We examined NASSCA optical trapping for a mixture solution of YG PSs and BL PSs at $700 \mathrm{~kW} / \mathrm{cm}^{2}$ of NIR laser intensity. Turning on NIR laser irradiation, a BL PS was stably trapped at the center of the irradiation area. YG PSs were trapped around the trapped BL PS during the irradiation, leading to formation of an ordered assembly like a flower (Figure 3). Also, we often observed a billiard-like-stacking behavior: BL PSs pushed pre-trapped YG PSs away from the center position of the irradiation area. The pushed YG PSs were trapped again around the trapped BL PSs. These results indicate that optical force sorted different sized nanospheres.
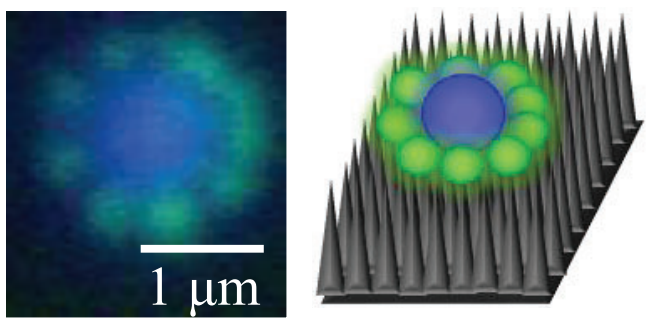

Figure 3. A .fluorescence micrograph and a schematic illustration of NASSCA optical trapping of BL PSs (1000 nm) and YG PSs (500 nm) on a B-Si (light intensity: $700 \mathrm{~kW} / \mathrm{cm}^{2}$ ).

\section{Conclusion}

We succeeded in optical size sorting of PSs by NASSCA-OT. We found that larger PSs were preferentially trapped at the center of the irradiation area and smaller PSs were trapped around the trapped larger PSs. The origin of this trapping behavior is sorting of different sized PSs by optical force. Optical sorting with NASSCA-OT has potential application of optical manipulation technique to separate and trap according to size and shape.

\section{References}

[1]M. Toshimitsu, T. Shoji, Y. Tsuboi, et al, " MetallicNanostructure-Enhanced Optical Trapping of Flexible Polymer Chains in Aqueous Solution As Revealed by Confocal Fluorescence Microspectroscopy" J. Phys. Chem. C 116, 14610-14618 (2012)

[2]T. Shoji, A. Mototsuji, Y. Tsuboi, et al., "Optical tweezing and binding at high irradiation powers on black-Si" Scientific Reports 7, 12298 (2017)

[4] Gediminas, G., Saulius, J., et al., "Surface-enhanced Raman scattering sensing on black silicon" Ann. Phys. 525 (12), 907 914 (2013) 


\title{
Structured nanofiber-based optical cavity for quantum electrodynamics
}

\author{
Maki Maeda, Priscila Romagnoli*, Viet Giang Truong, Wenfang Li, Jinjin Du, Jonathan M. Ward \\ and Síle Nic Chormaic
}

\section{Light-Matter Interactions Unit, Okinawa Institute of Science and Technology Graduate University, 1919-1 Tancha, Onna-son, Okinawa 904-0495, Japan}

\begin{abstract}
Nanofiber-based optical cavities enable the creation of devices with strong atom-photon coupling; the cavity can be obtained by milling Bragg mirrors on a nanofiber. Here, we present experimental progress to optimize the quality of a triplex air-nanocube structured nanofiber cavity. The structures are produced by focused $\mathrm{Ga}^{+}$ion beam milling. The quality factor obtained was $\sim 784$ in a periodic structure of only 20 mirrors and structures with up to 50 mirrors were fabricated to increase the quality factor. Subsequently, single quantum emitters, such as quantum dots, deposited onto the cavity should achieve strong coupling. The proposed nanofiber-based cavity creates a small, integrated device for the study of single quantum emitters for quantum networks.
\end{abstract}

Keywords: optical nanofiber, optical cavities, Bragg mirrors, single quantum emitters, focused ion beam

\section{Introduction}

Optical nanofibers (ONFs) have proven to be very useful devices for studying light-matter interactions, in particular for quantum optics applications. This is largely due to the strong transverse confinement of the guided mode and high evanescent field at subwavelength diameters, and the ease with which they can be integrated into experimental setups. ONFs are produced by a tapering technique [1], which consists of heating and stretching the fiber in a controlled manner.

In our work, we increase the functionality of ONFs by structuring them so that they consist of optical cavities. Optical cavities enhance the strength of light-matter interactions by confining photons to a small volume and are used for studying strong-coupling in cavity quantum electrodynamics (cQED) systems. Strong coupling has already been reported in the literature, and extensive studies show that if one combines cavities with an ONF it is possible to increase the photon coupling for single quantum emitters [2-6].

Here, we present our experimental progress to optimize the fabrication of high-quality optical cavities by milling out Bragg mirror patterns on an ONF. The Bragg mirrors consist of a triplex air-nanocube structure milled on a nanofiber with $\sim 800 \mathrm{~nm}$ diameter. Our group first demonstrated this cavity structure in [6]. The structures are produced by a focused ion beam (FIB) milling technique, using $\mathrm{Ga}^{+}$ions. The experimental quality factor (Q-factor) obtained was $\sim 784$ in a periodic structure of only 20 mirrors. However, it should be possible to improve the structure by changing the number of mirrors and/or the length of the cavity. This nanofiber-based cavity creates a small, integrated device for the study of single quantum emitters for quantum networks.

\section{Structured Nanofiber Cavity Fabrication,}

\section{Results and Discussion}

A fiber pulling rig was used to fabricate ONFs by using the flame brushing method [1]. In this setup, the fiber is fixed on oscillating stages that pull on the fiber ends, while a stationary hydrogen/oxygen flame heats a small portion of the fiber. Light transmission was monitored during the pulling process. Exponentially shaped tapers with waist diameters of $\sim 800 \mathrm{~nm}$ were produced with optical transmissions up to $98 \%$.

After fabrication, the ONF was coated with a conductive and transparent layer of indium tin oxide (ITO). The layer is $\sim 20 \mathrm{~nm}$ thick and does not affect the optical characteristics of the cavity. Finally, the structure was milled by the FIB. The beam diameter was $\sim 9 \mathrm{~nm}$ with $30 \mathrm{kV}$ of voltage and $7 \mathrm{pA}$ of current. Figure 1 shows a scanning electron microscope (SEM) image of the final structure. The milled structure consists of nanoholes of 
$100 \mathrm{~nm} \times 100 \mathrm{~nm}$, pitch of $310 \mathrm{~nm}, 20$ mirrors, and a cavity length of $2.2 \mu \mathrm{m}$.

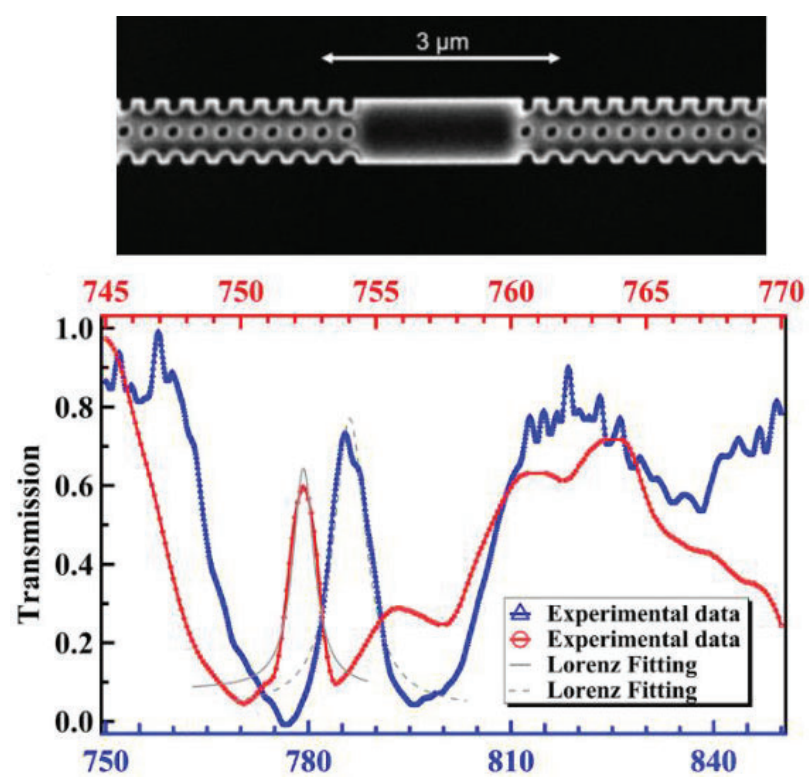

Figure 1. SEM image of the milled structured nanofiber cavity with 20 mirrors (top). Transmission characterization of the cavity (bottom).

The resulting ONF cavity, shown in Fig. 1, was optically characterized by coupling the ONF to a supercontinuum light source and recording the transmitted spectra. It is possible to observe the cavity's resonant peak ( $752 \mathrm{~nm}$ for $\mathrm{x}$ - and $\sim 786 \mathrm{~nm}$ for $\mathrm{y}$ - polarization). The estimated Q-factor of the cavity was $\sim 784$.

It is important to note that the structure provides a promising platform to study light-matter interactions. However, the cavity still can be further improved, but this is experimentally challenging due to vibrations and alignment of the ion beam. Figure 2 shows an SEM image of a structured ONF produced with 50 mirrors.

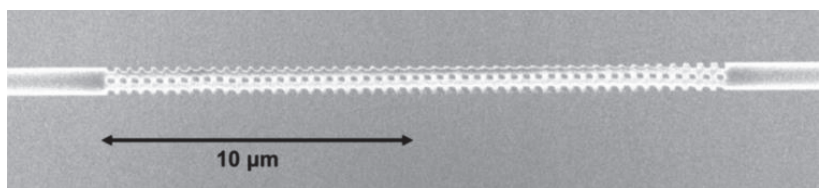

Figure 2. SEM image of the milled structured nanofiber cavity with 50 mirrors.
We have shown that, by ion milling directly on an ONF, high-quality cavities can be obtained even with a low number of Bragg mirrors. The low number of mirror greatly simplifies the milling process. Parameters of the cavity's structure, like the length and number of mirrors, can be optimized in order to improve the quality factor. A high Q-factor indicates low losses of energy and higher confinement of photons in the cavity. In future work, quantum dots will be placed on the structured ONF cavity for the purpose of acting as single quantum emitters to study cavity enhanced coupling.

\section{References}

[1] Ward, J. M., Maimaiti, A., Le, Vu H. and Nic Chormaic, S., "Contributed review: optical micro- and nanofiber pulling rig," Rev. Sci. Instrum. 85. 111501(2014).

[2] Nayak, K. P., Sadgrove, M., Yalla, R., Le Kien, F. and Hakuta, K., "Nanofiber quantum photonics," J. Opt. 20, 073001(2018).

[3] Wuttke, C., Becker, M., Bruckner, S., Rothhardt and M., Rauschenbeutel, "Nanofiber Fabry-Perot microresonator for nonlinear optics and cavity quantum electrodynamics," A. Opt. Lett. 37, 1949(2012).

[4] Kato, S. and Aoki, T., "Strong coupling between a trapped single atom and an all-fiber cavity," Phys. Rev. Lett. 115. 093603(2015).

[5] Keloth, J., Nayak, K. P. and Hakuta, K., "Fabrication of a centimeter-long cavity on a nanofiber for cavity quantum electrodynamics," Opt. Lett. 42, 1003(2017).

[6] Li, W., Du, J., Truong, V. G. and Nic Chormaic, S., "Optical nanofiber-based cavity induced by periodic airnanohole arrays," Appl. Phys. Lett. 110, 253102(2017).

\section{Conclusion and Outlook}




\title{
Non-destructive dispersion of quantum dots into buffer gases
}

\section{toward their optical manipulation}

\author{
Mitsutaka Kumakura*a, Yuta Baba ${ }^{\text {a }}$, Takayuki Shimomura ${ }^{\text {a }}$, Takayuki Takiyama ${ }^{\text {, }}$ \\ Tatsuya Kameyama $^{\mathrm{b}}$, Tsukasa Torimoto ${ }^{\mathrm{b}}$, and Takeshi Moriyasu ${ }^{\mathrm{a}}$ \\ a Department of Applied Physics, Graduate School of Engineering, University of Fukui, \\ 3-9-1 Bunkyo, Fukui, 910-8507, Japan \\ ${ }^{b}$ Department of Crystalline Materials Science, Graduate School of Engineering, Nagoya University, \\ Chikusa, Nagoya, 464-8601, Japan
}

\begin{abstract}
To carry out optical manipulation and spectral measurement of isolated quantum dots (QDs) in buffer gases, we are constructing experimental setups for dispersing QDs by using droplets of their organic solutions. The droplet was generated with a mesh-type nebulizer with a piezoelectric oscillator and was monitored by observing the scattered light. The QD density was also monitored by observing the fluorescence from the QDs. In this paper, we present our recent experimental results on the dispersion of $\mathrm{ZnS}-\mathrm{AgInS} \mathrm{S}_{2}$ solid solution QDs and $\mathrm{AgInS} / \mathrm{ZnS}$ nano-pyramid QDs into a dry nitrogen buffer gas, and discuss their isolation and optical lifetimes in comparison with our previous results on CdSe/ZnS QDs. We also discuss the experimental results of miniaturizing micro-droplets into nano-clusters.
\end{abstract}

Keywords: quantum dot, spectroscopy, fluorescence, droplet, cluster, evaporation, buffer gas

\section{Introduction}

Semiconductor quantum dots (QDs) are attracting great interest in many fields [1], since their electronic structures and spectral properties vary significantly with their size and environment [2]. For their future applications to efficient opt-electronic devices, more precise size control is considered to be one of key factors. The aim of our study is to develop a size-separation method for nm size QDs by their manipulation. In liquid helium, the optical size-separation has been successfully demonstrated for $\mathrm{CuCl}$ QDs created by laser ablation [3]. For realizing similar optical separation for a variety of chemically synthesized QDs at normal temperature, we are developing a new experimental approach by using buffer gases, which have low viscosity and high thermal diffusivity. So far, we constructed an experimental setup by using a mesh-type nebulizer with a piezoelectric oscillator, and successfully confirmed the dispersion of $\mathrm{CdSe} / \mathrm{ZnS}$ coreshell QDs [4] in a dry nitrogen buffer gas [5]. However, the quenching of the QD fluorescence was observed along with their isolation. Since the exciton in the CdSe QD is weakly binding, one of possible causes for this quenching is consider to be the relaxation of the exciton on the bare surface of the QD. In this study, to suppress such relaxation, we carried out a similar dispersion experiment on (Ag In) $\mathrm{Zn}_{2(1-x)} \mathrm{S}_{2}$ solid solution (ZAIS) QDs [6] and $\mathrm{AgInS}_{2} / \mathrm{ZnS}$ nano-pyramid (NP) QDs. Since the emission of these new QDs is considered to be due to the lattice defect, the excitation relaxation is expected to be insensitive to the surface. Moreover, to avoid the attachment of impurities on the QD surface, we miniaturized the micron-size droplet into nano-size clusters with a cluster generator by using a metal mesh rotor. Based on these experimental results, the cause of the fluorescence quenching is discussed.

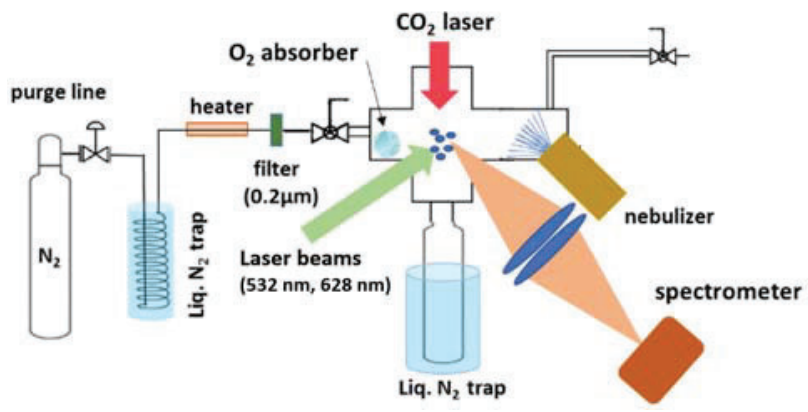

Figure 1. Experimental apparatus 


\section{Experiment}

Figure 1 shows our experimental setup for dispersing QDs in a buffer gas. The droplets of the dilute hexane solution of ZAIS and NP QDs were generated with a commercial meshtype nebulizer (Omron NE-22). The nominal value of the most probable diameter of the droplet is around $5 \mu \mathrm{m}$. They were dispersed in a sealed gas cell filled with a dry nitrogen. Residual contamination, oxygen, and water vapor were removed with a mesh filter, oxygen absorber, and a liquidnitrogen trap. To enhance the evaporation of the solvent, the gas cell was heated to the temperature of nearly $320 \mathrm{~K}$ (the boiling point of the hexane is $341 \mathrm{~K}$ ). Moreover, the $\mathrm{CO}_{2}$ laser was irradiated on the droplets for the further enhancement of the evaporation. The solvent evaporated from the droplet was collected in the cold trap.

To monitor the evaporation of the droplet, we introduced the $628 \mathrm{~nm}$ laser beam into the gas cell and observed the scattered light intensity. In addition, to monitor the number density of the QD, we introduced the $532 \mathrm{~nm}$ laser beam $(\sim 100$ $\mathrm{mW} / \mathrm{mm}^{2}$ ) into the gas cell and observed the fluorescence from the QDs. To distinguish these two signals, we used the CCD spectrometer.

For generating miniaturized droplets, we used a remodeled commercial cluster generator (Corona CNR-400B) instead of the mesh-type nebulizer. The diameter of the created clusters nominally ranges from $10 \mathrm{~nm}$ to $100 \mathrm{~nm}$. In this case, the evaporation was monitored without the $\mathrm{CO} 2$ laser heating.

In this experiment, we used ZAIS QDs with a diameter of about $5 \mathrm{~nm}$ and the stoichiometric ratio $x=0.3$ and 0.7 . The absorption edge wavelength is approximately $500 \mathrm{~nm}$ and 600 $\mathrm{nm}$ for the former and the latter, respectively. Therefore, in the case of the former QD, the excitation wavelength is around the absorption edge.

The length of one side of the NP QD is about $11.5 \mathrm{~nm}$ and the volume is 2.7 times that of the ZAIS QD. Since the NP QD has a $\mathrm{ZnS}$ shell much thicker than the ZAIS QD, the influence of the surface on the excitation relaxation is expected to be much smaller. The absorption edge wavelength of this QD is around $600 \mathrm{~nm}$, which is similar to the one for the $\operatorname{ZAIS}(x=0.7)$ QD.

The typical particle number density was approximately $7 \times 10^{13} \mathrm{dots} / \mathrm{ml}$. So that, the number of the QDs in the single droplet is estimated to be nearly $5 \times 10^{3}$ and 0.04 , respectively, for the droplets with diameters of $5 \mu \mathrm{m}$ and $100 \mathrm{~nm}$.

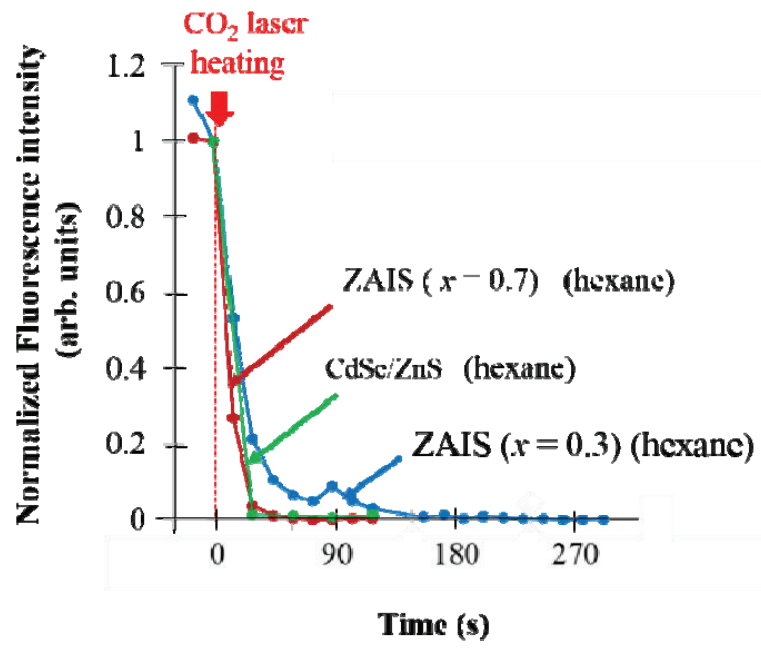

Figure 2. Fluorescence intensity from the quantum dots

\section{Results and Discussion}

Figure 2 shows the time variation of the fluorescence intensity of the ZAIS QDs after the dispersion and the $\mathrm{CO}_{2}$ laser heating. Our previous experimental result for the $\mathrm{CdSe} / \mathrm{ZnS}$ QD (Sigma-Aldrich Lumidot640, the diameter is around $6.3 \mathrm{~nm}$.) is indicated simultaneously. In the case of the ZAIS $(x=0.7)$ QD and the CdSe/ZnS QD, the fluorescence from the QDs is quenched within $30 \mathrm{~s}$. In contrast, in the case of the ZAIS ( $x=0.3$ ) QD, the fluorescence can be observed for a longer period of nearly $120 \mathrm{~s}$. From these results, it is suggested that the fluorescence quenching might be mainly caused by the thermal relaxation after the optical excitation .

In the case of the NP QD, the fluorescence was observed for a period similar to the ZAIS $(x=0.3)$ QD in spite of its absorption edge wavelength similar to the ZAIS $(x=0.7)$ QD. One of possible causes for such a long lifetime could be its larger heat capacity resulted from the larger volume.

Even in the case of the miniaturized clusters, the observed fluorescence lifetimes of the ZAIS QDs did not change considerably. This result indicates that impurities in the droplet is not be a major cause for the fluorescence quenching.

\section{Summary}

In the nitrogen buffer gas, we dispersed the droplets and clusters of the hexane solution of the ZAIS and NP QDs, and observed the time variation for the scattering from the droplet 
and the fluorescence from the QD. The observed fluorescence intensity indicated the quenching along with the isolation of the QD by the evaporation of the solvent. From the comparison between several QDs with different characteristics, one of major causes for this fluorescence quenching is suggested to be the thermal relaxation after the optical excitation.

This work was supported by JSPS KAKENHI Grant Numbers JP16H06505 in Scientific Research on Innovative Areas "Nano-Material Optical-Manipulation".

\section{References}

[1] Smith, A. M. and Nie, S., "Semiconductor nanocrystals: structure, properties, and band gap engineering," Acc. Chem. Res. 43(2), 190-200 (2010).

[2] Kumakura, M., Kinan, A. and Moriyasu, T., "Influence of dilution with organic solvents on emission spectra of CdSe/ZnS quantum dots," Proc. SPIE 10252, 1025219/1-4 (2017).

[3] Inaba, K., Imaizumi, K., Katayama, K., Ichimiya, M., Ashida, M., Iida, T., Ishihara, H. and Itoh, T.," Optical manipulation of $\mathrm{CuCl}$ nanoparticles under an excitonic resonance condition in superfluid helium," Phys. Status Solidi B 243(14) 3829-3833 (2006).

[4] Dabbousi, B. O., Rodriguez-Viejo, J., Mikulec, F. V., Heine, J. R., Mattoussi ,H., Ober, R., Jensen, K. F. and Bawendi, M. G., "(CdSe)ZnS core-shell quantum dots: synthesis and characterization of a size series of highly luminescent nanocrystallites,” J. Phys. Chem. B 101(46) 9463-9475 (1997). [5] Kumakura, M., Kinan, A. and Moriyasu, T., "Dispersion of quantum dots into gases toward their optical manipulation," Proc. SPIE 10712, 107121I/1-3 (2018). 


\title{
Wavefront restoration of high-intensity pulsed laser radiation
}

\section{by acousto-optics}

\author{
Vladimir Ya. Molchanov*a ${ }^{\text {a }}$ Konstantin B. Yushkova \\ ${ }^{a}$ Acousto-Optical Research Center, National University of Science and Technology MISIS, 4 \\ Leninsky Prospekt, Moscow, 119049, Russia
}

\begin{abstract}
A new technical principle for the correction of the wavefront of high-intensity pulsed laser radiation is proposed. The method is based on the creation of a multichannel matrix two-dimensional dispersion structure in an acousto-optic crystal. The temporal response of the proposed device is more than an order of magnitude higher than the temporal response of the known wavefront correction devices. Preliminary experiments confirm the proposed principle.
\end{abstract}

Keywords: acousto-optics, chirp, dispersive method, piezo transducer

\section{Introduction}

Among different techniques and methods for controlling the phase fronts of optical radiation various systems of adaptive mirrors and spatial modulators based on liquid crystals are widely used. It is well known that with insufficient speed of control to compensate for thermal and turbulent distortions of laser radiation, the compensation quality decreases. The control speed of mechanical adaptive systems and systems based on liquid crystals usually do not exceed $10 \mathrm{kHz}$, but the dynamics, for example, of atmospheric turbulence, scattering media in biomedicine, are usually even lower.

The situation is changing if it is necessary to observe a fast process in dynamics, for example, a moving object, when the number of iterations during mathematical processing increases sharply. The challenge is to develop fast adaptive wavefront correction devices with a control speed that is an order of magnitude higher than the existing adaptive optical systems. Acoustic-optical (AO) methods allows to make a new step in this direction.

* v.ya.molchanov@gmail.com

\section{Conception}

The possibility of one-dimensional recording of the laser field phase structure by the AO devices was apparently first expressed in (Balakshy,1981)

In this study, we solve the inverse problem: control of the wave front by an acousto-optic matrix device. The matrix ultrasonic structure in the crystal can be formed in an AO geometry, similar to the geometry of a multichannel optical switch (Aubin,2004). In the claimed design, each acoustic channel (column of the array) is emitted by a separate piezoelectric transducer. The column is filled with an ultrasonic field with a programmable amplitude-frequency structure, as it is done in the dispersive AO delay lines (Verluise,2000; Molchanov,2009) or in AO filter with synthesized transmission functuion (Yushkov,2015). A spatial matrix structure is formed, which controls the angular position and intensity of the wavefront along one coordinate in the plane of diffraction. For two-coordinate control, after the first AO matrix, an orthogonally oriented second matrix is located (Figure 1). One of the features of the proposed method is that for control MxN elements matrix only $\mathrm{N}$ drivers are required. 


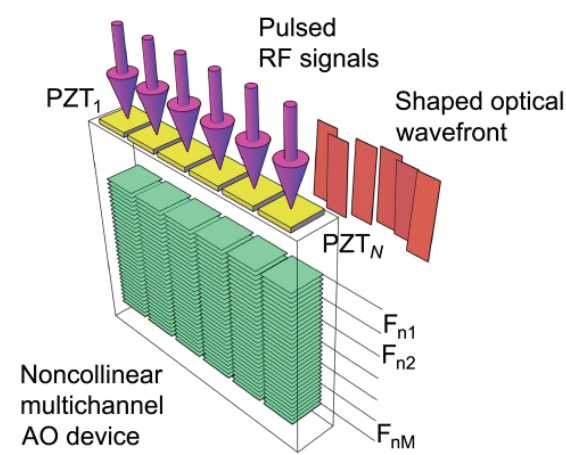

Figure 1. Diagram of an acousto-optic corrector with the architecture of $6 \times 6$ matrix elements.

\section{Experiment}

One-coordinate prototype of $\mathrm{AO}$ corrector was custom designed and fabricated. The $\mathrm{AO}$ cell is based on $\mathrm{TeO}_{2}$ crystal. Slow shear BAW is propagated along [110] axes, optical radiation - along [001] axes. An array of 4 independent transducers was placed on (1-10) plane. To verify the principle of wavefront restoration, we used two adjacent channels: one reference channel and one control channel.

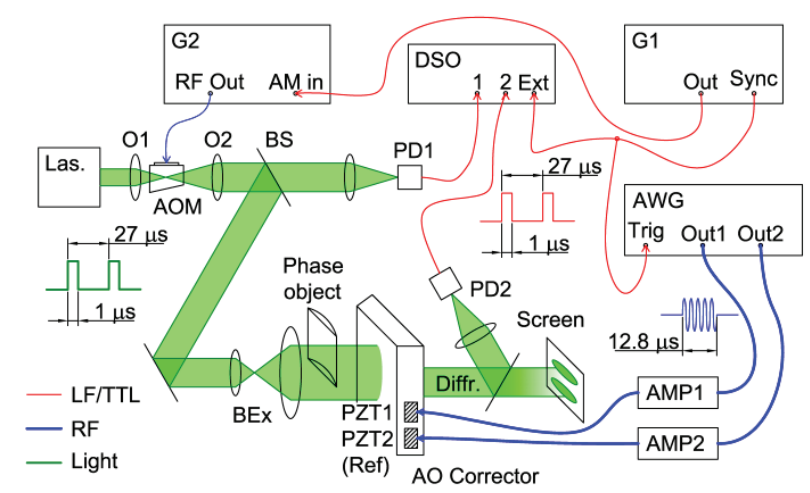

Figure 2. Experimental setup for verifying the principle of AO correction of laser radiation.

The setup is shown in Figure 2. The Laser Quantum Torus CW laser wavelength was $532 \mathrm{~nm}$. The emission was converted to pulsed mode with an acousto-optical modulator synchronized with the phase corrector. The collimated laser beam after the beam expander (BEx) had an almost flat phase front. The additional phase object could be installed in front of the working channel. The reference channel was not affected. The phase object was a cylindrical lens with a focal length of $1 \mathrm{~m}$, the focusing plane is perpendicular to the diffraction plane. Global synchronization is distributed by the RF generator Agilent 33220A (G1); the AOM is controlled by the driver G\&H 1150 (G2). Two-channel arbitrary waveform generator (AWG) Agilent N8241A and two amplifier modules AMP1, AMP2 (Empower RF) feed two transducers of the AO corrector: the working one PZT1 and the reference PZT2 one.

\section{Results and Discussion}

The distribution of the diffracted field after $\mathrm{AO}$ corrector was observed on the screen (Figure 3). On the left - the zero order, on the right - the diffracted field.

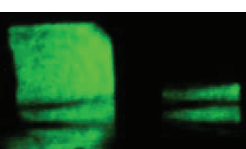

(a)

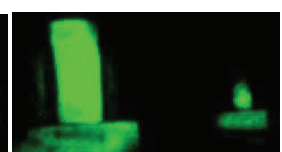

(b)

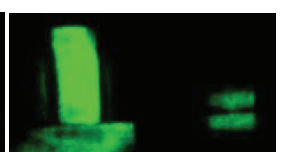

(c)
Figure 3. AO restoration of the incoming field.

In Figure 3(a), identical RF signals with a frequency of $63 \mathrm{MHz}$ are fed to both channels of the $\mathrm{AO}$ corrector. The upper channel is working and the lower one is reference. In Figure 3(b), an external phase object is installed in front of the working channel, the control RF mode is the same for both channels. The focusing of laser radiation in the working channel is observed. In Figure $3(\mathrm{c})$, the dispersion control of ultrasound in the working channel is implemented to compensate for the wavefront distortion. The central diffraction frequency in the working channel is $63 \mathrm{MHz}$, the RF chirp is $57-69 \mathrm{MHz}$. The distribution of the diffracted field in the working channel is restored to the original one. The prototype switching time does not exceed $25 \mu$ s. In all experiments, the AWG generated high-frequency pulses with a duration of $12,8 \mu$ s resulting in the maximum waveform update rate of $\sim 80 \mathrm{kHz}$.

\section{Conclusion}

We proposed and experimentally demonstrated a new dispersive matrix acousto-optic method for phase front control of the laser high intensity pulsed radiation. The temporal response of the proposed matrix $\mathrm{AO}$ system is 
an order of magnitude higher than the speed of existing wave front correction systems. One of the features of the method is that $\mathrm{MxN}$ matrix can be controlled by either $\mathrm{M}$ or $\mathrm{N}$ drivers.

The research was supported by the Russian Foundation for Basic Research (Project 18-29-20019) and the Ministry of Science and Education of the Russian Federation (Project 02.A03.21.0004 / Grant K2-2017079).

\section{References}

Aubin G., Sapriel J., Molchanov V., Gabet R., Grosso P., Gosselin S., and Jaouen Y. "Multichannel acoustooptic cells for fast optical crossconnect," Electronics Letters 40, 448-449 (2004).

Balakshy V.I., Parygin V.N., Upasena H.A. "On possibility of light field phase structure registration by an acousto-optic method", Kvantovaya Electronica 8, 865-872 (1981).

Molchanov V.Ya., Chizhikov S.I., Makarov O.Yu., Solodovnikov N.P., Ginzburg V.N., Katin E.V., Khazanov E.A., Lozhkarev V.V., and Yakovlev I.V. "Adaptive acousto-optic technique for femtosecond laser pulses shaping," Appl. Opt. 48(7), 118-124 (2009).

Verluise F., Laude V., Huignard J.-P., Tournois P. "Arbitrary dispersion control of ultrashort optical pulses with acoustic waves," Opt. Soc. Am. B. 17(1), 138-145 (2000).

Yushkov K.B., Molchanov V.Ya. "Acousto-optic filters with arbitrary spectral transmission" Optics Communications 355,177-180 (2015). 


\title{
Two techniques for experimental generation of spiral light beams
}

\author{
K.V. Efimova*a,b, S.A. Kishkin ${ }^{a}$, S.P. Kotova ${ }^{a}$, N.N. Losevsky ${ }^{a}$, D.V. Prokopova ${ }^{a, b}$, S.A. Samagin ${ }^{a}$ \\ ${ }^{a}$ Lebedev Physical institute, 221 Novo-Sadovaya, Samara, 443011, Russia, \\ ${ }^{\mathrm{b}}$ Samara University, 34A Moskovskoe Shosse, Samara, 443086, Russia
}

\begin{abstract}
Various methods of generating spiral beams have been analyzed. And the two methods, amplitude-phase and holographic, were subjected to experimental research. They were compared by their effectiveness and the quality of the beams being formed. Besides, a model sample of the system designed for the formation of spiral beams of light was presented, and a specialized software able to calculate complex amplitudes of structurally stable light fields with a given intensity distribution was described.
\end{abstract}

Keywords: spiral beams, spatial light modulator, amplitude mask, phase mask, holograms

\section{Introduction}

Spiral beams form the class of fields with phase singularities that retain their intensity distribution shape (except for scaling and rotation) during propagation and focusing. These beams were first discovered and described by E. Abramochkin and V. Volostnikov [1,2] as a class of solutions of the parabolic equation for the light field. The spiral beam optics provides a new fundamental possibility for forming structured vortex light fields. Their use extends the functional resource of laser tweezers, makes achievable a super resolution in optical microscopes, and increases the noise immunity of the quantum transmission systems. In this regard, a practical task often arises of producing the spiral beams with predetermined desired characteristics. This work also contributes to the formation of spiral beams of light and to improvement of their efficiency.

\section{Formation of spiral beams of light}

A spiral beam can be experimentally formed by several methods, such as intracavity, astigmatic transformation, amplitude-phase mask method and holographic one. On the basis of the analysis of the available literature on this issue, two ways for experimental testing of the generation of spiral beams were selected: holographic method and amplitude-phase masks. These two methods are relatively easily implementable, including the optical alignment. In our experiments the specially made stationary phase masks based on dichromate gelatin and liquid crystal spatial light modulator (LC SLM) HOLOEYE HEO-1080p (active area dimensions: 15,36 x 8,64 mm; display resolution: $1920(\mathrm{H}) \times 1080(\mathrm{~V}))$ were used for specifying the required phase distributions. As a radiation source, a solid-state laser was used $\left(\lambda=532 \mathrm{~nm}, P_{\max }=50 \mathrm{~mW}\right)$. The resulting images were recorded using a CCD Matrix of Canon EOS 350D digital camera.

For the amplitude-phase masks method, the two separate transparencies were used, an amplitude photomask and stationary phase mask, and their amplitude and phase transmittance corresponded to the amplitude and phase of the spiral beam. In addition, the phase profile of the converging lens was recorded on the phase masks.

In the holographic method, the phase profile of the transparencies was described by the expression that is the interference term for the case of a helical beam and a plane wave superposition:

$\varphi_{H}=A_{S} A_{F} \cos \left(\varphi_{S}-\varphi_{F}\right) \frac{\pi}{2}$,

where $A_{S}, A_{F}$ and $\varphi_{S}, \varphi_{F}$, are the spatial distribution of the amplitude and phase of the spiral beam and the plane wave, respectively.

The efficiency and quality of the spiral beams formation were estimated. The efficiency was determined as the ratio of the power attributable only to the obtained spiral beam to the total power in the detection plane. For the amplitude-phase masks method the efficiency was $3 \%$, while for the holographic one it achieved $15 \%$. The quality of the generated fields was estimated by the following parameters: heterogeneity of the curve width 
(RMSw) and deviation of the experimentally obtained width from the calculated curve (nw).

For the amplitude-phase masks method: $\mathrm{RMSw}=5.5 \%$, nw $=48 \%$. For the holographic method: $\mathrm{RMSw}=5.5 \%, \mathrm{nw}=21 \%$.

Thus it can be concluded that the holographic method is obviously preferred for the spiral beams formation. It is easier to align, energetically more efficient and allows generating of beams of a better quality.

\section{Hardware and software complex for the con- struction of spiral beams of light.}

In order to work with the mathematical model of spiral beams, in general, and to calculate the complex amplitudes, in particular, a specialized software was developed.

Analytical expression [3] given below, affords us to construct a complex amplitude of a spiral beam with the intensity in the form of an arbitrary plane curve $\mathrm{z}(\mathrm{t})$.

$$
\begin{aligned}
& S(z, \bar{z} \mid \zeta(t), t \in[0, T])=\exp \left\{\frac{-z \bar{z}}{\rho^{2}}\right\} f(z)= \\
& =\exp \left\{\frac{-z \bar{z}}{\rho^{2}}\right\} \times \int_{0}^{\tau} \exp \left\{-\frac{\zeta(t) \overline{\zeta(t)}}{\rho^{2}}+\frac{2 z \overline{\zeta(t)}}{\rho^{2}}+\right. \\
& \left.+\frac{1}{\rho^{2}} \int_{0}^{t}\left[\overline{\zeta(\tau)} \zeta^{\prime}(\tau)-\zeta(\tau) \overline{\zeta^{\prime}(\tau)}\right] d \tau\right\}\left|\zeta^{\prime}(t)\right| d t,
\end{aligned}
$$

where $\rho$ is the Gaussian beam parameter, the bar means derivative in the variable $t$, dash denotes complex conjugation .

Thus, figure 1 shows the results of numerical taking the integral (2) for the curve in the form of the Christmas tree contour and oak leaf.
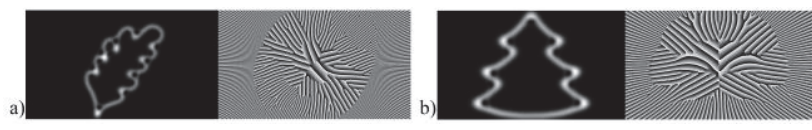

Figure 1. Intensity and phase of the spiral beam in the form of various curves.

Among other things, the software allows to calculate digital holograms by the method described in detail by the authors in [2]. It also allows to introduce the lens, choose its focal length, specify the angle of the hologram recording, change the modulation depth from 0 to $2 \pi$, and choose a sinusoidal or triangular dash profile.

The algorithm of the software use is as follows: we draw a curve and save it on a sheet of the size of
$1920 \times 1080$ pixels (correlation with the LCD PMS) in the format of 24-bit bmp. Then we upload the file into our software. Afterwards, the complex amplitude of the spiral beam in the form of the given curve is constructed using numerical methods. Here we can build a hologram of the spiral beam, introduce the lens, change the bands frequency.

The digital holograms were further used in the hardware of our system. With the aid of the LC SLM, a phase hologram was formed. To restore the helical beam hologram, a solid-state laser beam expanded by a collimator was applied.

Figure 2 shows experimental results of the reconstruction of a spiral beam hologram in the form of a Christmas tree and oak leaf. It is seen that the spiral beam image is scaled and is rotating. The effectiveness of the fields formation by this method was $11 \%$.
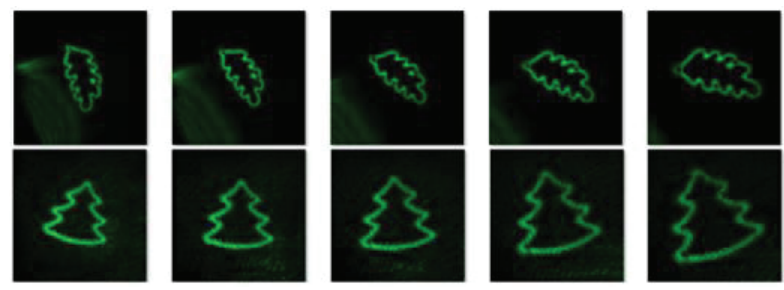

Figure 2. Experimentally obtained intensity distributions of the spiral beam with a step of $4 \mathrm{~cm}$ from the focusing plane $\mathrm{F}=42 \mathrm{~cm}$.

\section{Conclusion}

Of the two methods considered, the holographic one allows the formation of spiral beams of a better quality and with a higher efficiency. To implement this method, a hardware-software system has been developed that allowed the in-real-time formation of the corresponding spiral beams along a given curve.

\section{References}

[1] Abramochkin, E.G., Volostnikov, V.G., [Modern optics of Gaussian beams], Phyzmathlit, Moscow (2010) (In Russian).

[2] Abramochkin, E.G., Volostnikov, V.G., "Spiral-type beams: Optical and quantum aspects," Optics Communications 125(4-6), 302-323 (1996).

[3] Afanasiev, K.N., Kishkin, S.A. "Formation of spiral beams by using phase holograms," Proceedings of the Samara scientific center, Russian Academy of Sciences 14(4), 184-188 (2012) (In Russian). 


\title{
What will be done with the magnetically trapped superconducting micro particle?
}

\author{
M. Takanune ${ }^{1}$, J. Naoi ${ }^{1}$, S. Sasaki ${ }^{1}$, M. Kumakura ${ }^{2}$, M. Ashida ${ }^{3}$, F. Matsushima ${ }^{1}$, and Y. Moriwaki ${ }^{1}$ \\ ${ }^{1}$ University of Toyama, 3190 Gofuku, Toyama, 930-8555, Japan \\ ${ }^{2}$ University of Fukui, 3-9-1 Bunkyo, Fukui, 910-8507, Japan \\ ${ }^{3}$ Osaka University, 1-10 Machikaneyama, Toyonaka, Osaka, 560-0043, Japan
}

\begin{abstract}
A spherical micro superconducting particle fabricated by laser ablation of the base material in superfluid helium is trapped in a magnetic quadrupole field. The trapped particle is free from contact with materials other than superfluid helium, and because of the superfluid helium physical property such as optical transparency, low temperature, small viscosity, high thermal conductivity and so on allow us to carry out several interesting experiments with the superconducting micro particle.
\end{abstract}

Keywords: micro particle, magnetic trap, superconductivity, optical manipulation, laser ablation,

\section{Introduction}

We have fabricated superconducting micro particles by laser ablation of their base material in superfluid helium and then trapped them in a space using the quadrupole magnetic field [1]. This trap is based on the Meissner effect and is robust for the liquid helium temperature below the critical value of the superconductivity. We have measured the critical temperature for micro particles produced from indium and rhenium. As the result, the critical temperature of indium has been lowered from that of the bulk value as the size of the particle decrease, on the contrary, as for rhenium the critical temperature has been heightened much larger than the bulk value. The size of the trapped particles is hard to measure opticalmicroscopically on the spot, but some of them could be successfully dropped into a hollow produced on the copper plate which was placed below the trap, and then after the cryogenic experiment they could be observed by the scanning electron microscope and the size could be measured. The present and future work of this research will be discussed in this paper.

\section{Experiments}

Scheme of fabrication and trapping of a superconducting micro particle is described in the previous paper [1]. Flat surface of indium metal as a base metal placed in superfluid helium is irradiated by a focused beam of a pulsed Nd:YAG laser with a pulse width of $5 \mathrm{~ns}$ and a pulse energy of $1 \mathrm{~mJ}$, which causes laser ablation plume. A micro particle produced by the plume and then cooled down below its critical temperature of superconductivity by surrounding liquid helium is trapped by a quadrupole magnetic field, which is produced by a pair of neodymium magnets placed in front of the base metal as shown in Fig. 1. By the irradiation of a low power laser, the scattering light from the trapped particle can be observed using a microscope and a CMOS camera. At the end of the experiment, placing the copper plate below the trapped particle and raising the liquid helium temperature gradually, then the particle drops onto the plate, which is picked out from the cryostat after the cryogenic experiment and the collected particles can be detected by a scanning electron microscope.

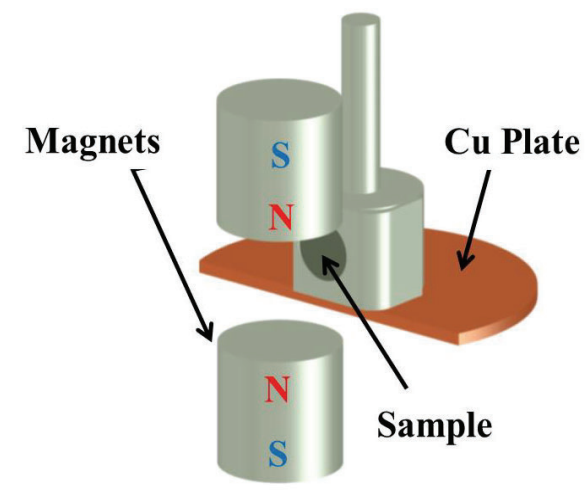

Figure 1. Experimental set up for magnetic trap 


\section{Discussion}

The present and future of our study is listed as

follows:

(1) A trapped particle in the superfluid helium can be pushed away from its original trapped position by a laser irradiation. The displacement increases with the laser power and it depends on the wavelength of the laser. This displacement cannot be observed for the particle in the normal fluid helium. The displacing force is much stronger than the optical pressure of the laser. Displacement analysis manifests some optical feature of the superconducting particle and the thermal properties of superfluid helium.

(2) A particle released from the displaced position moves along the force of the trapping potential and the viscous force of the superfluid helium against this. By tracking the particle motion we can deduce physical property of liquid helium and trapped particle.

(3) Angular distribution of the scattering light from the spherical trapped particle will be the subject for the Mie scattering analysis, which gives us optical properties of the superconducting particle.

In the talk we will present and discuss the progress of our research.

\section{References}

[1] Y. Takahashi et al., Appl. Phys. Express, 10, 022701 (2017). 
..«Diagnosis of semiconductor materials such as cadmium chalcogenides by the method of exiton-polariton luminescence»

\author{
B.Z. Polvonov, Ferghana polytechnic institute, 150107 Ferghana, \\ Uzbekistan.E-mail:bakhtiyorp@mail.ru
}

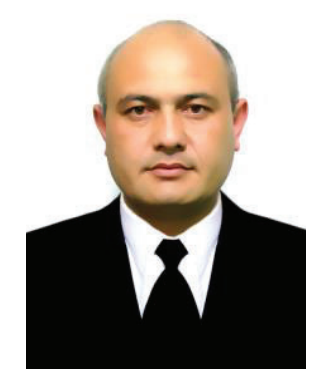

\title{
Introduction
}

Low-temperature photoluminescence (LTPL) spectroscopy is one of the most sensitive and informative optical methods for studying semiconductors and film structures. LTPL spectra of CdTe crystals have been investigated in detail, and new methods for predicting and controlling electrical properties of semiconductor structures have been proposed on their basis. In particular, based on the study of the dynamics of changes in photoluminescence spectra $[1,2]$, a method for deep sample purification was developed, and polycrystalline CdTe of stoichiometric composition with a photoluminescence spectrum containing only the exciton component and no impurity contributions was obtained. The role of grain boundaries in the formation of properties of coarse-grained cadmium telluride was investigated by photoluminescence microprobe methods; it was shown that the impurity-defect compositions of the boundary and internal regions of singlecrystal grains with sizes of 1-2 $\mathrm{mm}$ are significantly different [3, 4]. However, the influence of structural and point defects on the formation of the photoluminescence (PL) spectrum of fine-grained semiconductor samples has not been considered to date.In this paper, we report the results of studying the mechanisms of formation of LTPL spectrum and their relationship with the anomalous photovoltaic (APV) properties of obliquely deposited CdTe and CdTe:In films in dependence of the degree of their structural imperfection. The LTPL spectra of fine-grained polycrystalline CdTe and CdTe:In films with APV properties, in contrast to the spectra of single crystals and coarse-grained polycrystals, were found to contain no contributions from excitons and donor-acceptor pairs (DAPs), which are due to the generation of photo-emf in the boundary regions of crystallites (that leads to stimulation of intrinsic $(e-h)$ luminescence and build-up of longitudinal optical (LO-) phonon repetitions in undoped samples). The half-width of this band correlates with the peak value of the anomalously large photovoltage generated in the film.

\section{Technology. Experimental results}

The objects of study, pure and In-doped obliquely deposited polycrystalline films with a thickness of and an area of $5 \times 20 \mathrm{~mm}^{2}$, were obtained from CdTe powder by thermal evaporation in vacuum (residual gas pressure $(1-4) \times 10^{-2} \mathrm{~Pa}$ ) onto a glass substrate at a temperature $T_{s}=500-550 \mathrm{~K}[7,8]$. The sizes of individual crystallites were $0.7-1.0 \mu \mathrm{m}$. CdTe films at room temperature, illuminated by an 
incandescent lamp with intensity $L \approx 10^{4} 1 \mathrm{x}$, generated photovoltage $V_{A P V} \approx 600 \mathrm{~V}$ and short-circuit current $I_{s c} \approx 10^{-10} \mathrm{~A}$. As-prepared CdTe:In samples with a thickness $d=0.5-0.8 \mu \mathrm{m}$ turned out to have a lower resistivity and rather weakly pronounced APV properties $\left(V_{A P V}=50-100 \mathrm{~V}\right)$. However, after a TT at a temperature $450-550 \mathrm{~K}$ for $3-5 \mathrm{~min}$ in air in the presence of coactivator $\mathrm{CdCl}_{2}$ vapor, they generated a photovoltage up to $(2-4) \cdot 10^{3} \mathrm{~V}$, and the short-circuit photocurrent was as high as $I_{s c} \approx 10^{-8}$ A. The photovoltaic parameters of the films practically did not degrade during a year.

\section{Conclusions}

1.The LTPL spectra of fine-grained CdTe films excited by a $\mathrm{cw}$ gas-discharge $\mathrm{Ar}^{+}$laser contain a fundamental emission band with a half-width $\Delta_{\mathrm{A}} \approx 10-20 \mathrm{meV}$ ( $A$ line) and its LO- and 2LO-phonon repetitions.

2. A correlation was found between the LTPL spectrum and the APV properties of CdTe and CdTe:In films. The intrinsic luminescence band is due to the presence of potential barriers at grain boundaries, which generate surface photoemfs; the asymmetry of the latter results in APV properties. The method of joint analysis of the LTPL spectra and photoelectric properties of fine-grained CdTe polycrystals, proposed in this study,can successfully be used to investigate properties of other semiconductor film structures.

\section{References}

[1]. A.V. Kvit and other... Semiconductors 34(1), 19 (2000).

[2]. V.S. Bagaev, YU.V. and other... Fiz. Tverd. Tela 52(1), 37 (2010).

[3]. V.P. Veleschuk, and other..., Fiz. Tverd. Tela 52(3), 439 (2010).

[4]. S.A. Permogorov, and other... Fiz. Tverd. Tela 40(5), 897 (1998). 


\title{
Second-harmonic generation in swift $\mathrm{O}^{5+}$ ion irradiated $\mathrm{KTiOPO}_{4}$ ridge waveguide*
}

\author{
Yazhou Cheng*a, Shengqiang Zhou ${ }^{\mathrm{b}}$
}

\author{
a School of Physics, State Key Laboratory of Crystal Materials and Key Laboratory of Particle \\ Physics and Particle Irradiation (MOE), Shandong University, Jinan 250100, China \\ ${ }^{b}$ Helmholtz-Zentrum Dresden-Rossendorf, Institute of Ion Beam Physics and Materials Research, \\ Dresden 01314, Germany
}

\begin{abstract}
We report on the fabrication of ridge waveguide in potassium titanyl phosphate (KTP) crystal by using swift $\mathrm{O}^{5+}$ ion irradiation and precise diamond blade dicing. An end-face coupling system was arranged to realize second harmonic generation (SHG) through KTP ridge waveguide: 1064-nm TE-polarization wave $\rightarrow 532-\mathrm{nm}$ TM-polarization wave, resulting in a conversion efficiency of $25.4 \%$. This result implies potential applications for $\mathrm{O}^{5+}$ ion irradiated ridge KTP waveguide to be an efficient frequency-doubling device.
\end{abstract}

Keywords: Optical waveguide, KTP crystal, Ion irradiation, Second harmonic generation

\section{Introduction}

Potassium titanyl phosphate (KTP) is a widely used frequency-doubling crystal for realizing high-efficiency laser sources from near infrared to visible wavelengths through phase matching (PM) and quasi-phase matching (QPM) configurations ${ }^{[1]}$. Optical waveguides are basic photonic components which confine the light propagation in very small volumes with dimensions of micron or submicrometer scales. Swift heavy-ion irradiation (with energies not less than $1 \mathrm{MeV} / \mathrm{amu}$ ) has emerged to be a very powerful technique for waveguide fabrication in various materials ${ }^{[2]}$.

\section{Body}

In this work, the KTP crystal with dimensions of $6.0 \times 3.1 \times 1.7 \mathrm{~mm}^{3}$ was cut along the direction optimal for SHG at $532 \mathrm{~nm}$ from the fundamental 1064-nm wavelength $\left(\theta=90^{\circ}, \varphi=23.5^{\circ}\right)$ under the Type II phase matching configuration, i.e., $\mathrm{e}^{\omega}+\mathrm{o}^{\omega} \rightarrow \mathrm{e}^{2 \omega}$. Figure 1 depicts the schematic plot of the ridge waveguides fabrication process. In the first step, the sample was irradiated by $17 \mathrm{MeV} \mathrm{O}^{5+}$ ions at fluence of $1.5 \times 10^{15}$ ions $/ \mathrm{cm}^{2}$ on one surface $\left(6.0 \times 1.7 \mathrm{~mm}^{2}\right)$ through the $3 \mathrm{MV}$ tandem accelerator, forming a planar waveguide layer with thickness of $\sim 8 \mu \mathrm{m}$ beneath the sample surface. In the second step, a diamond rotating blade on top of the irradiated planar waveguide surface moving in the direction parallel to the blade was used to construct air grooves with depth of $12 \mu \mathrm{m}$. The rotating speed and cutting velocity were set to $20.000 \mathrm{rpm}$ and $0.2 \mathrm{~mm} / \mathrm{s}$, respectively. As a result, a ridge waveguide with $40-\mu \mathrm{m}$ width and 8 - $\mu \mathrm{m}$ depth was formed. Figure 2 exhibits the microscope image of the cross section of the KTP ridge waveguide.

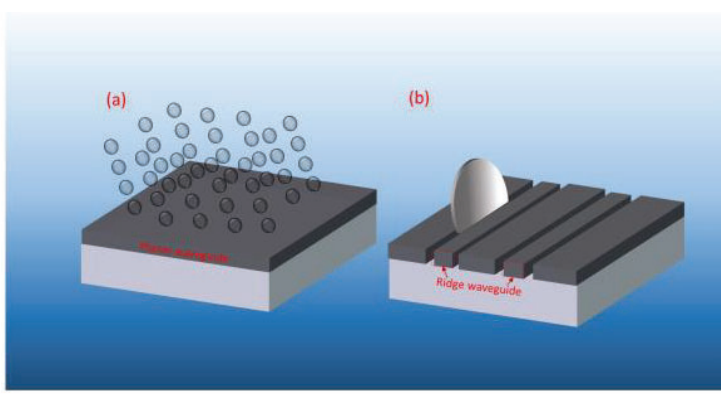

Figure 1. The schematic plot of the KTP ridge waveguide fabrication process.

*chengyazhou@s,sdu.edu.cn 


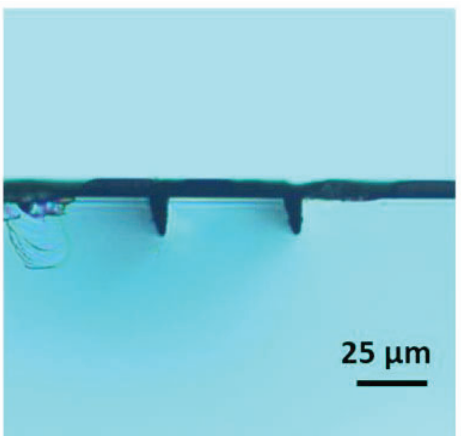

Figure 2. The microscope image of the cross section of the KTP ridge waveguide.

An end-face coupling system, as shown in Fig. 3, was used to realize SHG through KTP ridge waveguide. We utilized $1064 \mathrm{~nm}$ pulsed laser $(80 \mu \mathrm{J}$ pulses with duration of $11.05 \mathrm{~ns}$ at a repetition of $5 \mathrm{kHz}$ ) as the fundamental laser source. The incident laser beam at 1064 $\mathrm{nm}$ was coupled into the KTP ridge waveguide using a convex lens $(\mathrm{NA}=0.4)$, and the generated $\mathrm{SH}$ laser beam at $532 \mathrm{~nm}$ from the waveguide exit facet was captured by using a $20 \times$ microscope objective and characterized by a CCD camera, a spectrometer, or a power meter.

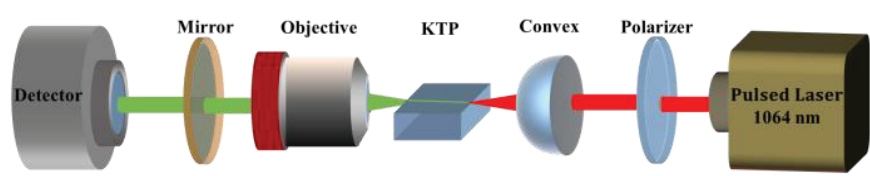

Figure 3. End-face coupling system used to realize SHG through $\mathrm{KTP}$ ridge waveguide.

\section{Results and Discussion}

Figure 4 depicts the typical laser spectra of fundamental wave at $1064 \mathrm{~nm}$ and $\mathrm{SH}$ wave at $532 \mathrm{~nm}$ under pulsed laser pump configuration. It was noted that the fundamental wave at $1064 \mathrm{~nm}$ was with TE polarization and the generated wave at $532 \mathrm{~nm}$ exhibited TM polarization, which was in agreement with the bulk type II $\left(\mathrm{e}^{\omega}+\mathrm{o}^{\omega} \rightarrow \mathrm{e}^{2 \omega}\right) 1064 \mathrm{~nm} \rightarrow 532 \mathrm{~nm}$ of $\mathrm{KTiOPO}_{4}$ crystal.

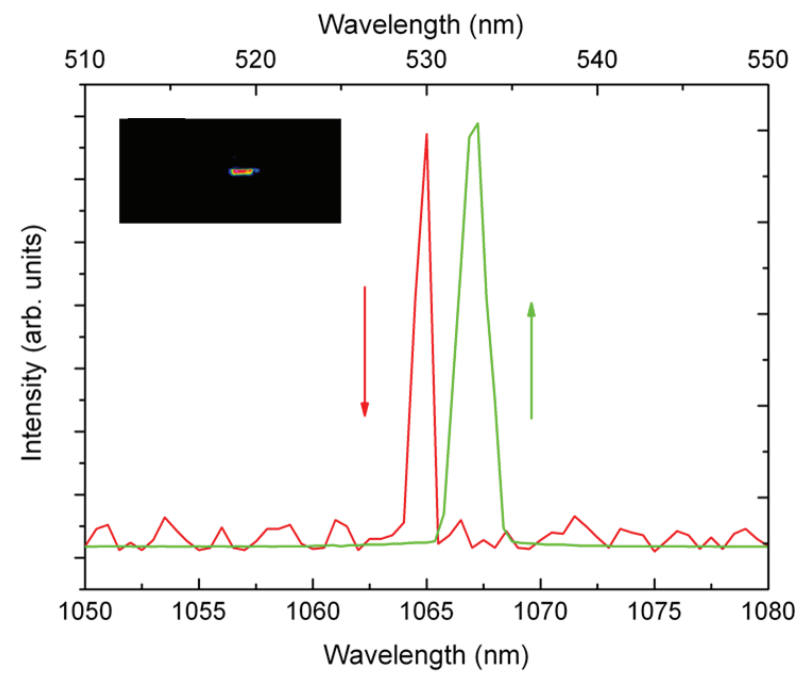

Figure 4. The typical laser spectra of fundamental wave at 1064 $\mathrm{nm}$ and $\mathrm{SH}$ wave at $532 \mathrm{~nm}$ under pulsed laser pump configuration.

Figure 5 depicts the power of the generated $\mathrm{SH}$ wave at $532 \mathrm{~nm}$ and SH conversion efficiency as a function of the $1064 \mathrm{~nm}$ fundamental wave for the ridge waveguide in $\mathrm{KTiOPO}_{4}$. The solid circles and the lines are the experimental data and the linear fit, respectively. As we can see, the maximum output power of the $\mathrm{SH}$ light was $19.05 \mathrm{~W}$ when the pump power was $75 \mathrm{~W}$, resulting in an SH conversion efficiency of $\eta=25.4 \%$. Compared with the reported value of $\mathrm{Nd}$ :GdCOB ridge waveguide ${ }^{[3]}$, the conversion efficiency of KTP ridge waveguide is significantly higher. This result implies potential applications for $\mathrm{O}^{5+}$ ion irradiated ridge KTP waveguide to be an efficient frequency-doubling device.

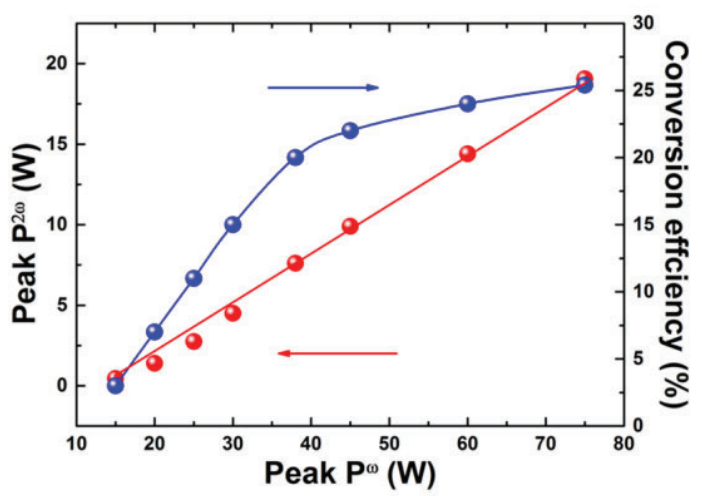

Figure 5. The power of the generated $\mathrm{SH}$ wave $\left(\mathrm{P}^{2 \omega}\right)$ at $532 \mathrm{~nm}$ and $\mathrm{SH}$ conversion efficiency as a function of the $1064 \mathrm{~nm}$ fundamental wave $\left(\mathrm{P}^{\omega}\right)$ for the ridge waveguide in KTP. 


\section{References}

[1]X. Deng et al., "Generation of blue light at $426 \mathrm{~nm}$ by frequency doubling with a monolithic periodically poled $\mathrm{KTiOPO}_{4}$," Opt. Express 21(22), 25907-25911 (2013).

[2]F. Chen, "Micro-and submicrometric waveguiding structures in optical crystals produced by ion beams for photonic applications," Laser Photon. Rev. 6(5), 622-640 (2012).

[3] Y. Jia, et al, "Femtosecond laser micromachining of $\mathrm{Nd}$ :GdCOB ridge waveguides for second harmonic generation,” Opt. Mater. 34(11), 1913-1916 (2012). 


\title{
Advanced nanoantenna
}

\author{
Dmitrii Poletaev, Bogdan Sokolenko, Alexander Nudga, Alexander Starosek, Andrei Prisyazhniuk \\ V.I. Vernadsky crimean federal university, Vernadsky av. 4, Simferopol, 295007, \\ Russia
}

\begin{abstract}
In this paper an advanced nanoantenna was proposed. The aim of the work is a theoretical analysis of the construction of the proposed nanoantenna. It was shown that proposed nanoantenna has more than 2 times less reactive resistance than the monopole antenna. The area occupied by the proposed nanoantenna compared with monopoly antenna increases only twice.
\end{abstract}

Keywords: skin-layer, capacitance, inductance, resonant circuit

\section{Introduction}

Nanoantennas are widely used in modern science and technology $[1,2]$. Some of those devices are used for directry convert light into electrical energy [2]. However, the range of wavelengths in which a nanoantenna operates effectively is rather narrow [2]. To extend it the sets of nanoantenas were used [1]. The disadvantage of such system is a large surface area occupied by a single element. In scientific works $[1,2]$ tie-bow nanoantennas are described. However, they can not operate over a wide range of wavelengths. It is advisable to propose a construction of a nanoantenna that capable efficiently operate on a wide range of wavelengths. The aim of the work is a theoretical analysis of new advanced nanoantenna's constructionight of the symbol to reduce white space.

\section{Theoretical part}

The proposed advanced nanoantenna consists of two connected monopole antennas formed by square copper prisms. The height of the first prism is $d_{l}$, the second - is $d_{2}$. The sides of the square of the base of the prisms are the same and equal to $b$. Each square copper prism of this nanoantenna has its own capacitance and inductance. Their values depend on the geometric and electrophysical parameters. The capacitance and inductance of each square copper prism form an oscillatory circuit with its own resonant wavelength. The degree of connection of these resonant circuits varies depending on the wavelength, because of the presence of the skin layer in real conductors.

Values of square copper prism's inductance and capacitance are calculated with sufficient accuracy, for example in [3]. The magnitude of the resistances $R_{1}$ and $\mathrm{R}_{2}$ for the first and second prisms, respectively, are nonlinearly dependent on the length of the electromagnetic wave, due to the presence of the skin effect [4].

The electromagnetic wave interacts simultaneously with both prisms (upper and lower). The amplitude of an electromagnetic wave propagating along the upper prism decreases at least 7 times, due to its size, passing from its upper surface to the surface of the lower prism. An electromagnetic wave interacting with the upper surface of the upper prism is transmitted to the radiation receiver. Therefore, the highest part of the energy of the electromagnetic wave will take the upper prism.

\section{Results and Discussion}

Antenna operates efficiently in a range of wavelengths where its impedance is purely active [3]. The graph of the dependence of the imaginary part of the impedance of a copper nanoantenna (1) with optimized sizes $d_{1}=d_{2}=35$ 
$\mathrm{nm}, \mathrm{b}=48 \mathrm{~nm}$ from the wavelength is shown in figure 1 . In this graph other curve is shown for comparison the dependence of the imaginary part of a single monopole antenna formed by a square copper prism with height $\mathrm{d}_{1}=35 \mathrm{~nm}$ with the side of the square's side $\mathrm{b}=48 \mathrm{~nm}$.

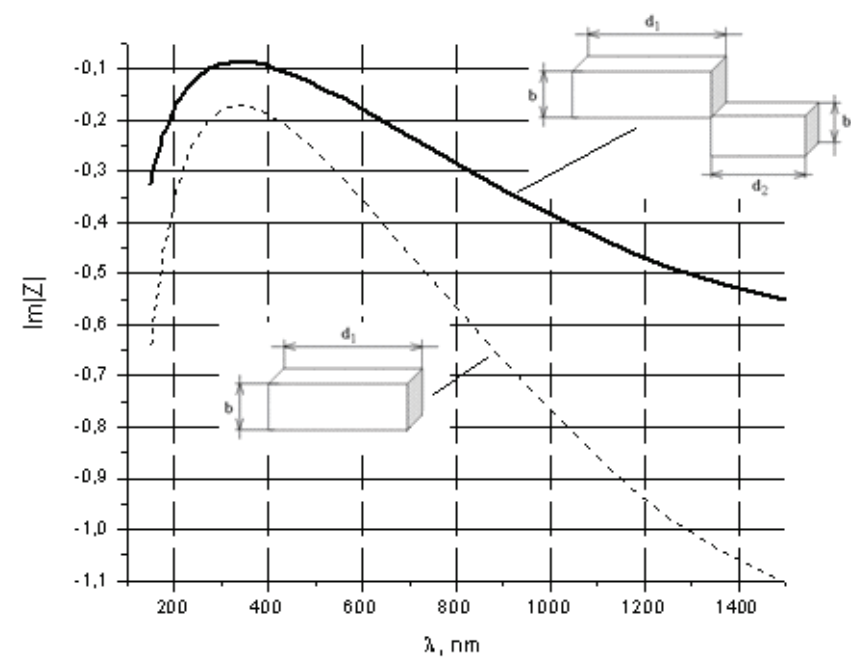

Figure 1. Dependence of the imaginary part of the nanoantenna's impedance from the wavelength

As can be seen from the graph in the solar radiation wavelength range, the proposed nanoantenna has more than 2 times less reactive resistance than the monopole antenna. However, the effectiveness of this nanoantenna is much greater than the efficiency of the monopole antenna, due to a wider wavelength range. The minimum of the reactance is in the near ultraviolet region. This makes it possible to compensate the absorption of the ultraviolet part of the spectrum in the atmosphere. The proposed nanoantenna can be used in photovoltaics.

A patent for this construction of nanoantenna had been applied. A positive decision regarding the patent in the Russian Federation was received.

\section{Acknowledgments}

This work was partially supported by the V.I. Vernadsky Crimean federal university development program for 2015 - 2024 within the framework of grant support for young scientists.

\section{References}

[1] Spinelli, P., Ferry, V., Groep, J., Lare, M., Verschuuren, M., Schropp, R., Atwater, H., Polman, A. "Plasmonic light trapping in thin-film si solar cells," journal of optics 2(14) (2012).

[2] Olmon, R., Raschke, M. "Antenna-load interactions at optical frequencies: impedance matching to quantum systems" nanotechnology 9(23). (2012).

[3] Schelkunoff, S., Friis, H. 1952 antennas: theory and practice. New York:Wiley.

[4] Balanis, C. 1982 antenna theory: analysis and design. New York: Harper \& Row. 


\title{
The resemblance of polarization spectra of polymers between photo- and mechanically- induced microstrains
}

\author{
Irakli Chaganava ${ }^{\mathrm{a}, \mathrm{b}}$, Barbara Kilosanidze*a and Irine Kobilashvili ${ }^{\mathrm{a}}$ \\ a - Laboratory of Holographic Recording and Processing of Information. Institute of Cybernetics of \\ Georgian Technical University. 5 Sandro Euli str., 0186 Tbilisi. Georgia; \\ b - Georgian State Teaching University of Physical Education and Sport, 49 Chavchavadze ave., \\ 0179 Tbilisi, Georgia
}

\begin{abstract}
The study of the mechanism of the phenomenon of vector polyphotochromism led to the opinion that it has an interference nature. This paper presents the first experimental data on the correspondence of this effect with the manifestation of the photoelasticity of the polymer component of the material. We verified the accordance of the transmission spectra of polarized light on the one hand of a photoactivated polarization-sensitive material and on the other hand under the influence of a mechanical stress. The profiles of the obtained polarization spectra match each other.
\end{abstract}

Keywords: optical manipulation, polarization spectroscopy, vector optical signal, photoelasticity, frequency selection

\section{Introduction}

Earlier, a light-induced effect on the material was detected in our laboratory, which was later named as vector polyphotochromism [1]. It is noteworthy that both the induction of this effect and its further registration is possible only with polarized light and it manifests itself in the form of the possibility of tuning the polarization spectral characteristics over the entire visible range. The authors continue to investigate this phenomenon and are studying the mechanism of its formation. In previous works, the interference nature of the occurrence of polyphochromism has been found out in organic materials obtained on the basis of azo dyes and polymers [2-3].

\section{The phenomenon under investigation}

The phenomenon of vector photochromism was found in some highly efficient polarization-sensitive polymeric materials with azocomponents as vectorial selective receivers of the recording light. The results depend on the illumination dose of the linearly polarized actinic light being exposed on that materials. When radiant exposure exceed certain value specific to each material, a sharp change in the transmission spectrum of the probing linearly polarized nonactinic beam is observed after analyzer. While probing with unpolarized light the transmission spectrum is practically unchanged and does not depend on the magnitude of the radiant exposure. The effect is an interference nature and depends on the path difference between the ordinary and extraordinary rays in material with photo-induced anisotropy. It is found that in this case birefringence has made the main contribution in photoanisotropy [3].

\section{Experimental results and discussion}

During the study of this phenomenon, we noticed that when material is exposed to the actinic light, its spectral properties change in the same sequence as observed in the Michel-Lévy interference color chart [4]. Due to interference between of the ordinary and extraordinary rays within optically anisotpopic medium a fringe pattern develops along with the irradiation of polarizationsensitive material. It is important to note that at the beginning of the sequence of changing color of photochromic area is the same one that the Michel-Lévy interference color chart starts (In both cases, there is transmission of the probing light in the yellow region of spectrum). Further development of polyphotochromism in the polarization-sensitive material is in a precise sequence in accordance with this chart. For instance in the process of increasing exposure to the actinic light after yellow the spectral properties of the irradiated medium changes for 
transmission of orange, then switches to red range, magenta, blue, cyan and ends the series ends with transmission in the green region of the spectrum. Thus, it became necessary to check the accordance of the transmission spectra of polarized light in one case of a photoactivated material and on the other hand under the influence of a mechanical stress. The profiles of the obtained polarization spectra match each other. Figure 1 and Figure 2.

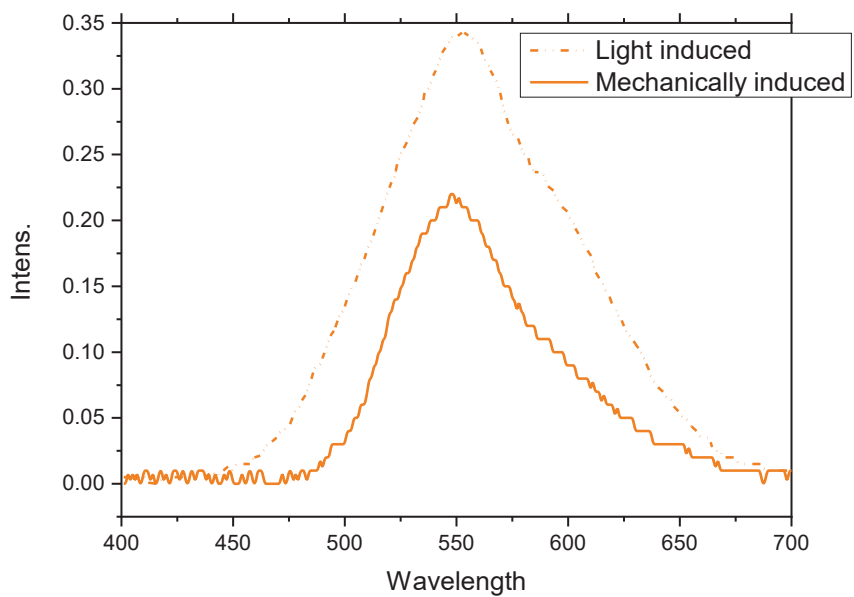

Figure 1. The resemblance of polarization spectra of the photoanisotropic material between the photo- and mechanicaly-induced strained area for the max transmission on range of 545-555 $\mathrm{nm}$.

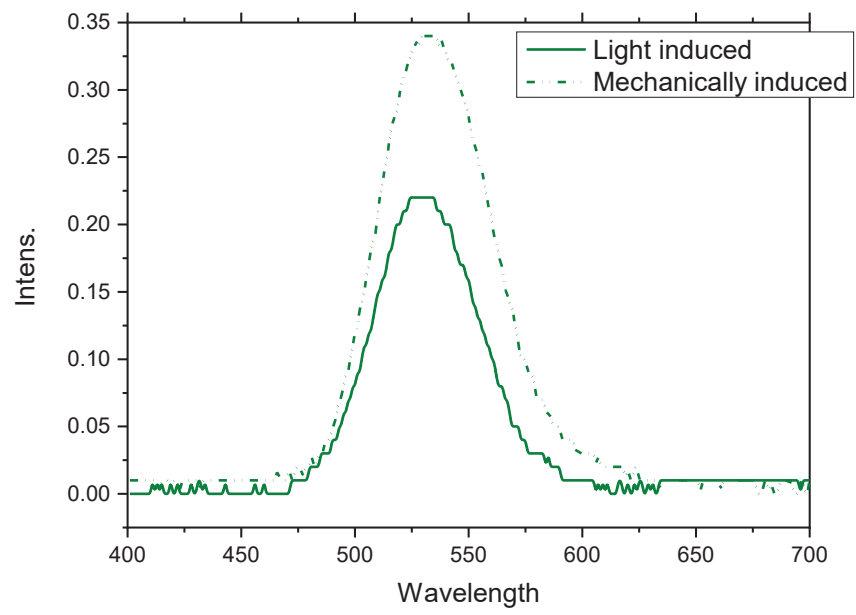

Figure 2. The resemblance of polarization spectra of the photoanisotropic material between the photo- and mechanicaly-induced strained area for the max transmission on range of 525-535 nm.

\section{Conclusion}

The observed behavior of the material in the same way, both under the influence of light and under mechanical action, suggests that the phenomenon of the vector polyphotochromism is based on the photoelasticity of the polymer component of the material.

\section{References}

[1] Irakli Chaganava, George Kakauridze, Barbara Kilosanidze, and Yuri Mshvenieradze, "Vector photochromism in polarization-sensitive materials," Opt. Lett. 39, 3841-3844 (2014)

[2] Irakli Chaganava; George Kakauridze; Barbara Kilosanidze and Yuri Mshvenieradze. "Lightcontrolled vector polyphotochromism", Proc. SPIE 9137, Organic Photonics VI, 913712 (May 1, 2014); http://dx.doi.org/10.1117/12.2051756

[3] Irakli Chaganava, Barbara Kilosanidze, George Kakauridze, Luis Oriol, Milagros Piñol, Alfonso Martinez-Felipe, Induction of the vector polyphotochromism in side-chain azopolymers, Journal of Photochemistry and Photobiology A: Chemistry, Volume 354, 2018, Pages 70-77, ISSN 1010-6030;

[4] John Gustav Delly., "The Michel-Lévy Interference Color Chart," 22 September 2016, https://www.mccrone.com/mm/the-michel-levyinterference-color-chart-microscopys-magical-colorkey 


\title{
Controlling the electrical size of a conducting cylinder by eccentric coating of Matched Impedance Zero Index Metamaterial
}

\author{
Tayyab H. Malik \\ Department of Electronics, Quaid-i-Azam University, Islamabad - Pakistan
}

\begin{abstract}
Metamaterials that exhibit zero index of refraction have been experimentally realized by many research groups and the existence of such metamaterials inspired the present study. In this article, the scattering properties of a conducting cylinder coated eccentrically with Matched Impedance Zero Index Metamaterial (MIZIM) are presented and it is shown that the MIZIM layer can be used to control the electrical size of the object. The coordinates transformation techniques along with superposition of cylindrical wave functions are elegantly employed to obtain a simple form of solution for a better insight of the eccentric problem. The effects of eccentricity and thickness of coating layer on radar cross section (RCS) of the conducting cylinder are explored and discussed. Our study suggests that, the MIZIM coating layer can be used to scale the RCS of the conducting cylinders, as desired in many applications. Moreover, the same scattering properties can be obtained without employing expensive techniques for depositing a precise and uniform coating layer.
\end{abstract}

Index Terms-EM Scattering, Metamaterials, Zero-Index, MIZIM .

\section{INTRODUCTION}

The scattering and propagation properties of electromagnetic (EM) waves mainly depend upon the constitutive parameters of the obstacle material and/or the medium of propagation. Therefore, naturally occurring materials could only provide a limited control on these properties [1]. Recent advances in interdisciplinary sciences and engineering enabled the researchers to realize and develop variety of artificial materials to control the flow of EM waves in unprecedented ways [2]. By employing the modern engineering techniques, one can create artificial materials with such characteristics that are not usually found in naturally occurring materials, i.e. classical materials.

Electromagnetic materials are often characterized by two constituent parameters i.e. permittivity $(\epsilon)$ and permeability $(\mu)$. In almost all classical materials (with some exceptions e.g. metals, plasmas) these two quantities takes positive values or at least one of the two remains positive over a wide range of frequencies. However, such metamaterials can be engineered with (real part of) permittivity $(\epsilon)$ negative, permeability $(\mu)$ negative or both $(\epsilon)$ and $(\mu)$ negative known as ENG, MNG and DNG materials respectively [3], [4].

There is another set of metamaterials having neither positive nor negative values of permittivity and permeability over a narrow frequency range. They are recognized as 'epsilon near zero' $\operatorname{ENZ}(\epsilon \rightarrow 0)$, mu near zero $\operatorname{MNZ}(\mu \rightarrow 0)$ metamaterials. As the refractive index of a medium is defined by $n=\sqrt{\epsilon_{r} \mu_{r}}$ (assuming $\epsilon_{r}, \mu_{r}$ relative to free space), if one of the parameter takes a zero value, the index of refraction will become zero. Thus, it is also known as zero index medium (ZIM). Similarly, if both $\left(\epsilon_{r}, \mu_{r}\right)$ becomes (zero in this case) the impedance of medium $n=\sqrt{\mu_{r} / \epsilon_{r}}$ is matched with that of free space impedance and hence termed as 'matched impedance zero index medium' (MIZIM) [5], [6] .

A fascinating feature of ZIM (Zero Index Metamaterials) that has been reported recently is the propagation of electromagnetic (EM) waves through it with zero phase delay. It has been observed that a radiating source placed inside a bounded ZIM region behaves similar to if it is placed on the boundaries.

In recent years, several studies have been carried out to investigate the EM properties of structures involving ZIM and MIZIM both theoretically and experimentally [6]-[14]. For instance, the $n=0$ structures have been experimentally verified for visible range [15]. It has been reported that ZIM can be used to enhance the directive emission of an embedded source [16]. The propagation properties through ZIM and MIZIM structures were reported in [17]. It was suggested that the ENZ medium can squeeze the EM waves in a narrow waveguide [18]-[20] and the similar tunneling effect was later demonstrated in microwave experiments [21]. The possibility of using ENZ materials to tailor the phase pattern of the output radiation from an arbitrary source was studied in [22]. The enhancement of light transmission through dielectric cylinders embedded in an ENZ host due to Mie resonances was demonstrated in [23]. It has been shown in [24] that perfect transmission of EM energy can be achieved by using ENZ material junctions. Reshaping schemes based on ZIM were proposed [25]-[27] and phase matching-free devices and spatial power combination for omnidirectional radiation via anisotropic metamaterials demonstrated recently [28], [29].

In this article, the study of scattering properties of a conducting cylinder coated eccentrically with Matched Impedance Zero Index Metamaterial (MIZIM) is presented. Generally, the expressions for eccentric coating problems appears to be quite complicated, however, here we applied the coordinates transformation techniques along with superposition of cylindrical wave functions to obtain a simple form of solution for a better insight of the eccentric problem. The effects of eccentricity and thickness of coating layer on radar cross section (RCS) of the conducting cylinder are explored and discussed. 


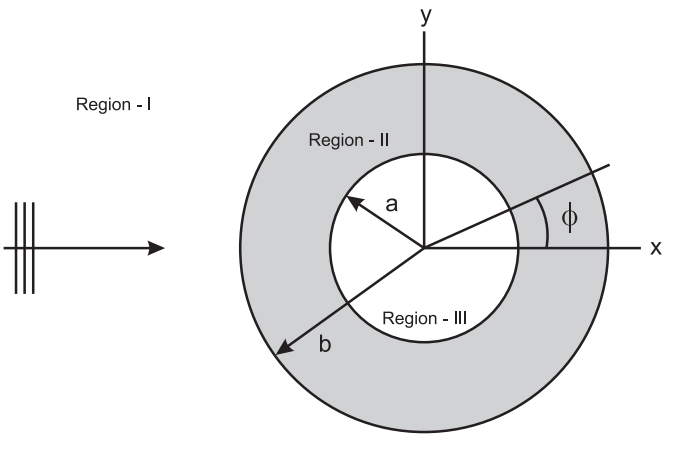

(a)

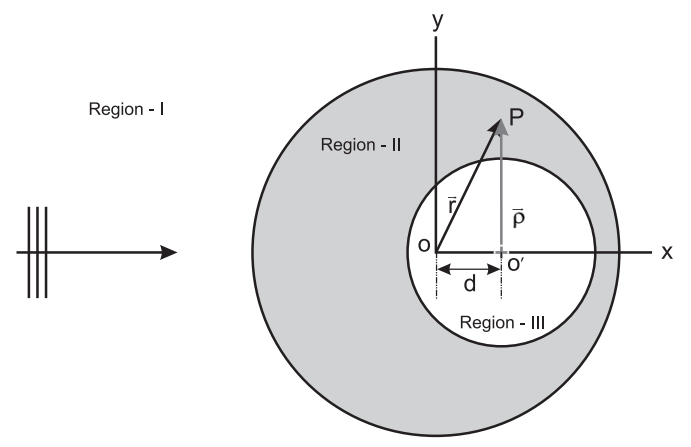

(b)

Fig. 1. Geometry of the problem.(a) Concentric case. (b) Eccentric case

\section{Problem Formulation}

\section{A. Concentric Case}

Let a PEC circular cylinder coated uniformly with a layer of MIZIM metamaterial. The radii of the core and coated cylinders are denoted by $a$ and $b$ respectively. The problem can be divided into three regions, region-I $(\rho>b)$ is considered to be the free space while the region-II $(a<\rho<b)$ is a layer of MIZIM characterised by $\epsilon_{2}$ and $\mu_{2}$ and the region-III $(\rho<a)$ is a PEC cylinder.

Assume a $T M^{z}$ polarized plane wave traveling in $x$ direction is normally incident on the structure as shown in 1(a). The time dependency $e^{j \omega t}$ is considered and suppressed throughout the text.

Hence, the total electric field (which is only z-component in this case) in region - I can be written as;

$$
\mathbf{E}_{I_{z}}^{t o t}=E_{0} \sum_{n=-\infty}^{n=+\infty} j^{-n}\left[J_{n}\left(k_{1} \rho\right)+a_{n} H_{n}^{(2)}\left(k_{1} \rho\right)\right] e^{j n \phi}
$$

where, $E_{0}$ is the amplitude of the incident field, $J_{n}($.$) and$ $H_{n}^{(k)}($.$) are the Bessel and Hankel functions of first and k^{t h}$ kind respectively, $k_{1}$ is the wave number in region-I defined as $k_{1}=\sqrt{\epsilon_{0} \mu_{0}}$, and $a_{n}$ is the unknown scattering coefficient. The electric and magnetic fields in each region is then described in terms of unknowns coefficients by making use of incident field and Maxwell equations. After applying the appropriate boundary conditions, the matrix form of the mathematical model of the problem is given below;

$$
\begin{gathered}
A=\left(\begin{array}{ccc}
H_{n}^{(2)}\left(k_{1} b\right) & -H_{n}^{(1)}\left(k_{2} b\right) & -H_{n}^{(2)}\left(k_{2} b\right) \\
H_{n}^{(2)^{\prime}}\left(k_{1} b\right) & -\frac{k_{2} \mu_{1}}{k_{1} \mu_{2}} H_{n}^{(1)^{\prime}}\left(k_{2} b\right) & -\frac{k_{2} \mu_{1}}{k_{1} \mu_{2}} H_{n}^{(2)^{\prime}}\left(k_{2} b\right) \\
0 & H_{n}^{(1)}\left(k_{2} a\right) & H_{n}^{(2)}\left(k_{2} a\right)
\end{array}\right) \\
B=\left(\begin{array}{c}
-J_{n}\left(k_{1} b\right) \\
-J_{n}^{\prime}\left(k_{1} b\right) \\
0
\end{array}\right) \\
X=\left(\begin{array}{c}
a_{n} \\
b_{n} \\
c_{n}
\end{array}\right)
\end{gathered}
$$

Where $b_{n}$ and $c_{n}$ are the transmission and reflection coefficients in region - II respectively. The prime denotes the derivative with respect to whole argument.

\section{B. Eccentric Case}

Let us now consider a non-uniform coating over the MIZIM cylinder as shown in 1(b). The core is displaced from the original center $O$ by a distance $d$ to a new center $O^{\prime}$ as shown in 1 (b). We will refer $d$ as the eccentricity parameter. It can be seen that the transformation between the two cylindrical coordinate systems $O$ and $O^{\prime}$ may be given by:

$$
\vec{r}=\vec{\rho}+\vec{d}
$$

It should be noted that the Helmholtz equations in region - II will not be changed, while the cylindrical (Bessel and Neumann) functions in this region would be modified as prescribed by the Graf's addition theorem [30] i.e.,

$$
\begin{aligned}
& \left.\mathbf{Z}_{n}(k \rho) e^{j n \phi}=\sum_{p=-\infty}^{p=+\infty} Z_{p}(k d) Z_{n+p}(k r)\right) e^{j(n+p) \phi} \\
& \left.\mathbf{Z}_{n}^{\prime}(k \rho) e^{j n \phi}=\sum_{p=-\infty}^{p=+\infty} Z_{p}(k d) Z_{n+p}^{\prime}(k r)\right) e^{j(n+p) \phi}
\end{aligned}
$$

The $a_{n}$ scattering coefficient in region- $\mathrm{I}$ is then determined by using the modified system of equations.

In general, the total scattering cross-section is given by the ratio of scattered power to the incident power per unit area [31], i.e.,

$$
\sigma=\frac{4}{k_{1}}\left|\sum_{n=-\infty}^{n=+\infty} a_{n} e^{j n \phi}\right|^{2}
$$

where $k_{1}$ is the free space wave number.

The backscattering cross-section is indispensable for many practical applications, which can be given by setting the $\phi=\pi$ in equation (5) as;

$$
\sigma_{b}=\frac{4}{k_{1}}\left|\sum_{n=-\infty}^{n=+\infty}(-1)^{n} a_{n}\right|^{2}
$$




\section{NumericAl Results AND Discussion}

In this section, the selected numerical results are presented and discussed. The frequency of the incident wave is considered to be $10 \mathrm{GHz}$ throughout the analysis or otherwise specified. In fig-2, the normalized bistatic RCS of a perfectly conducting cylinder coated with MIZIM in comparison with uncoated and coated with ordinary dielectric is presented. It is evident that the RCS of the core cylinder is reduced by dielectric coating, which is obvious because of the passive nature of coating material. However, an increment in the RCS of core cylinder is observed when coated with MIZIM and the enhanced RCS seemed to be a scaled version of the RCS of an uncoated PEC cylinder. It was also observed that the RCS of the core cylinder have a linear relationship with the thickness of MIZIM coating layer as shown in fig-3a. Further investigations revealed that the RCS of the MIZIM coated PEC cylinder is exactly overlapped the RCS of an uncoated PEC cylinder with radius $b$ i.e. equal to the outer radius of the coated configuration. It was then confirmed by varying the coating thickness and comparing the results with PEC cylinder of corresponding radius as given in fig $-3 \mathrm{~b}$. The RCS of a target object is usually proportional to its electrical size and to obtain a larger RCS, we generally use a higher frequency EM source for a fixed physical size. We found that the MIZIM layer can be used to enhance the RCS of a conducting cylinders without changing its physical size or the frequency of the incident wave. This is worth noting that the profile of the scattered fields does not change (but scaled) in case of MIZIM coating on PEC cylinder.

Another, interesting feature observed in the present study is depicted in fig-4a, where it is shown that the scattering properties of the core cylinder are independent from eccentricity between the core and Lossless MIZIM coating centers. The justification behind this interesting phenomenon could be quite simpler as; the phase velocity of the wave propagating inside the MIZIM region is infinite, hence the small differences in distances from core to the outer interface should not matter. However, for a Lossy MIZIM the backscattering can be controlled with appropriate position (eccentricity) of the core and coating centers as shown in fig- $4 \mathrm{~b}$.

It has been reported in [17] that inside the MIZIM medium, spatial components of the total electromagnetic fields behave like a combination of static electric and magnetic fields while the temporal components remains dynamic. It has also been shown in the above cited paper that the elementary sources can radiate when placed inside the MIZIM medium and the phase remains unchanged throughout the MIZIM region after steady state conditions are obtained. In the light of above mentioned observations, we can demonstrate the phenomenon of scattering from MIZIM coated PEC cylinder as; The incident wave does not see any impedance (mismatch) when arrive at the MIZIM boundary of the coated cylinder and hence no reflection should be occurred at that interface. As the wave number ' $k$ ' is zero inside the MIZIM medium, no spatial variation is expected and the static fields coupled the surface charges of the core cylinder to the outer interface. These fields excite time harmonic surface currents on the surface of the

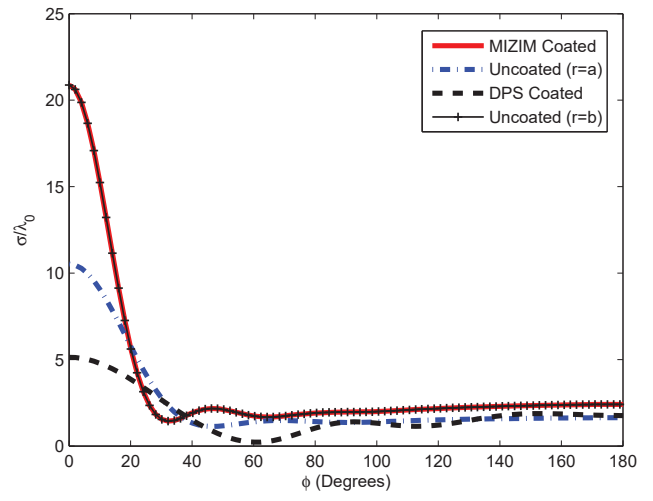

Fig. 2. Comparison MIZIM coating with PEC (uncoated) and ordinary dielectric coating. Core and coating radii: $a=0.5 \lambda_{0}, b=0.75 \lambda_{0}$, Dielectric parameters: $\epsilon_{r}=9.8, \mu_{r}=1$,

PEC cylinder. The PEC cylinder behaves like a collection of infinite line sources distributed along the circumference with certain phase difference. These line sources then reradiates and the EM fields propagates with infinite phase velocity through MIZIM region and reaches the outer interface.

\section{CONCLUSION}

The scattering properties of a conducting cylinder coated eccentrically with MIZIM are presented. The effects of eccentricity and thickness of coating layer on RCS of the conducting cylinder are explored and discussed.

The two key findings in the present work are:

1- The electrical size of the conducting cylinder varies systematically with the thickness of the MIZIM coating layer and is independent of its physical size.

2- The scattering properties of the core cylinder do not depend upon the non-uniformity of Lossless MIZIM coating layer while the backscattering cross section depends linearly on eccentricity.

Hence, the MIZIM coating layer can be used to scale the RCS of the conducting cylinders, as desired in many applications. Moreover,the same scattering properties can be obtained without employing expensive techniques for depositing a precise and uniform coating layer.

\section{REFERENCES}

[1] A Sihvola. Metamaterials, 1(1):2-11, 2007.

[2] isbn 978-92-79-07563-6, KI-NA-24409-EN-C, 2010

[3] V.G Veselago. Physics-Uspekhi, 10(4):509-514, 1968.

[4] H.K Yuan, U.K Chettiar, W Cai, A.V Kildishev, A Boltasseva, V.P Drachev, and V.M Shalaev. Optics Express, 15(3):1076-1083, 2007.

[5] A Alù, M.G Silveirinha, A Salandrino, and N Engheta. Physical Review $B, 75(15): 155410,2007$.

[6] M Silveirinha and N Engheta. Physical Review B, 75(7):075119, 2007.

[7] R.J Pollard, A Murphy, W.R Hendren, P.R Evans, R Atkinson, G.A Wurtz, A.V Zayats, and Viktor A Podolskiy. Physical review letters, 102(12):127405, 2009.

[8] M Silveirinha and N Engheta. Physical review letters, 97(15):157403, 2006.

[9] R Liu, Q Cheng, T Hand, J.J Mock, T.J Cui, S.A Cummer, and D.R Smith. Physical review letters, 100(2):23903, 2008.

[10] B Edwards, A Alù, M.E Young, M Silveirinha, and N Engheta. Physical Review Letters, 100(3):033903, 2008. 


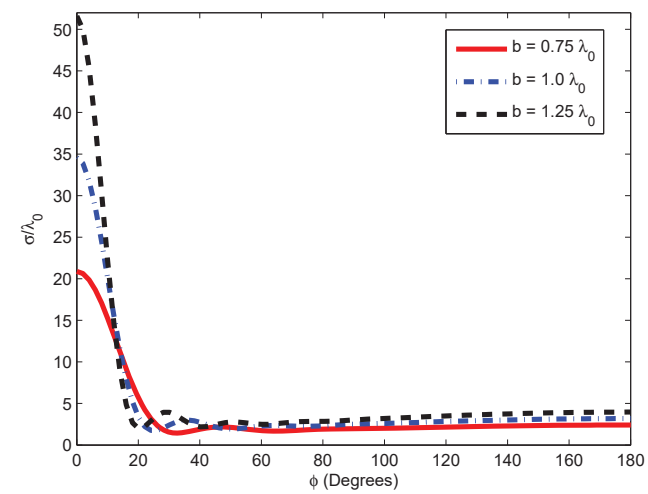

(a)

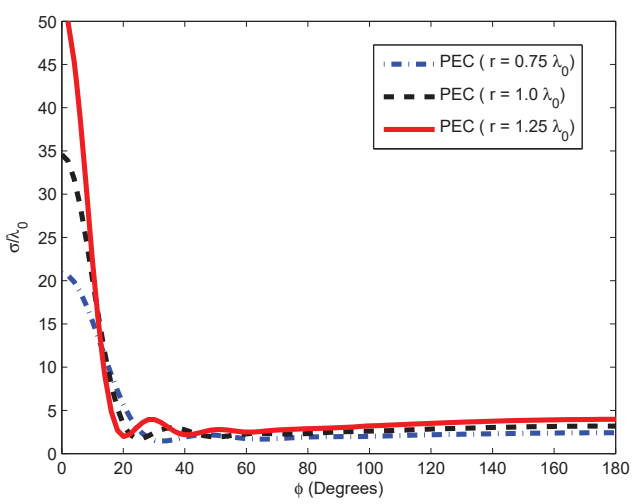

(b)

Fig. 3. Effects of MIZIM layer thickness.

(a). $a=0.5 \lambda_{0}$ (b). The RCS of the Uncoated PEC Cylinder (for reference)

[11] B Edwards, A Alù, M.G Silveirinha, and N Engheta. Journal of Applied Physics, 105(4):044905, 2009.

[12] K Halterman and S Feng. Physical Review A, 78(2):021805, 2008

[13] W.G Spitzer, D Kleinman, and D Walsh. Physical Review, 113(1):127, 1959.

[14] R.W Ziolkowski. Physical Review E, 70(4):046608, 2004

[15] E.J.R Vesseur, T Coenen, H Caglayan, N Engheta, and A Polman. Physical review letters, 110(1):013902, 2013.

[16] S Enoch, G Tayeb, P Sabouroux, N Guérin, and P Vincent. Physical Review Letters, 89(21):213902, 2002.

[17] R.W Ziolkowski. Physical Review E, 70(4):046608, 2004.

[18] M Silveirinha and N Engheta. Physical review letters, 97(15):157403, 2006.

[19] M Silveirinha and N Engheta. Physical Review B, 75(7):075119, 2007.

[20] M.G Silveirinha and N Engheta. Physical Review B, 76(24):245109, 2007.

[21] R Liu, Q Cheng, T Hand, J.J Mock, T.J Cui, S.A Cummer, and D.R Smith. Physical review letters, 100(2):23903, 2008.

[22] A Alù, M.G Silveirinha, A Salandrino, and N Engheta. Physical Review $B, 75(15): 155410,2007$.

[23] A.A Basharin, C Mavidis, M Kafesaki, E.N Economou, and C.M Soukoulis. Physical Review B, 87(15):155130, 2013.

[24] A Ourir, A Maurel, and V Pagneux. Optics letters, 38(12):2092-2094, 2013.

[25] J.J Yang, M Huang, and J.H Peng. Radioengineering Journal, 18, 2009

[26] B Wang and K.M Huang. Progress In Electromagnetics Research, 106:107-119, 2010.

[27] Q Cheng, W.X Jiang, and T.J Cui. Applied Physics Letters, 99(13):131913, 2011.

[28] H Suchowski, K O'Brien, Z.J Wong, A Salandrino, X Yin, and X Zhang. Science, 342(6163):1223-1226, 2013.

[29] Q Cheng, W.X Jiang, and T.J Cui. Physical review letters, 108(21):213903, 2012.

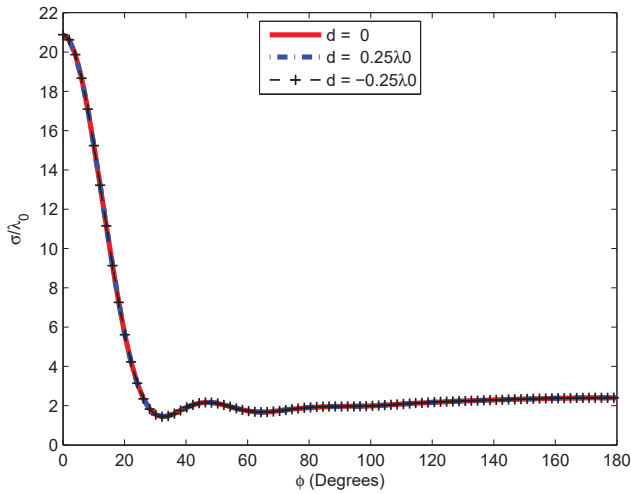

(a)

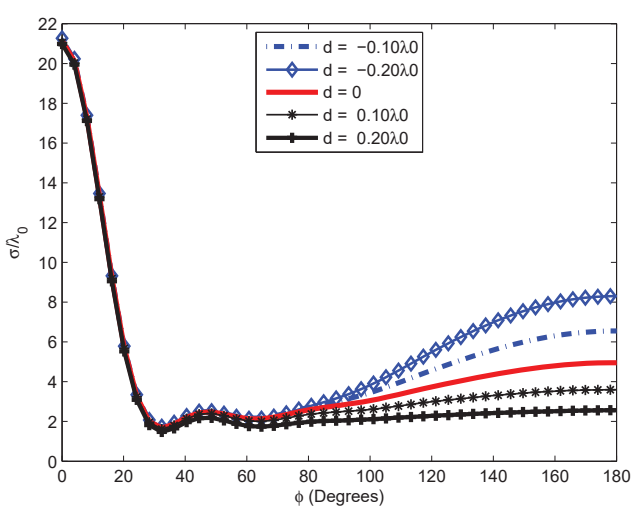

(b)

Fig. 4. Effects of Eccentricity $a=0.5 \lambda_{0}, b=0.75 \lambda_{0}$ (a) Lossless MIZIM Coating (b) Lossy MIZIM Coating

[30] M Abramowitz, I.A Stegun, et al. volume 1. Dover New York, 1972.

[31] C.A Balanis. volume 20. Wiley New York, 1989. 


\title{
Spin momentum locking in a tightly focused Gaussian beam
}

\author{
Debapriya Pal, Nirmalya Ghosh, ${ }^{*}$ and Ayan Banerjee ${ }^{\dagger}$ \\ Department of Physical Sciences, IISER Kolkata, Mohanpur, Nadia 741246, India \\ Subhasish Dutta Gupta \\ School of Physical Sciences, Hyderabad Central University, Hyderabad, India
}

(Dated: December 14, 2018)

\begin{abstract}
We demonstrate by a simple calculation that tight focusing of a Gaus- sian beam in optical tweezers leads to spin-momentum locking, where the spin momentum density is rendered independent of helicity while the Poynting vec- tor becomes helicity dependent. We hypothesize that the presence of a stratified medium in the path of the trapping beam would enhance the transverse spin momentum leading to exotic controlled rotation of optically trapped single mesoscopic particles for input circularly polarized light.
\end{abstract}

\section{INTRODUCTION}

Light carries both orbital and spin angular momentum, and the Poynting vector - which is considered to be the vector representative of the flow of energy - has contribution from both the orbital and spin part of the momentum. The spin contribution in Poynting vector, introduced by Belinfante through the equation $\vec{p}=\vec{p}^{o}+\vec{p}^{s}$ [1], where $\vec{p}^{s}$ represents the contribution of spin in poynting vector, is rather enigmatic. This is because the term $\vec{p}^{s}$, though being responsible for spin angular momentum, does not contribute to the energy flow, so that it may be considered to be a virtual quantity. On the other hand, $\vec{p}^{o}$ represents canonical momentum that is responsible for generating orbital angular momentum (OAM) $\left(\vec{l}=\vec{r} \times \vec{p}^{o}\right)$.

It has recently been discovered that a phase shifted longitudinal component of field plays a major role in the appearance of spin (polarization) dependent transverse momentum and spin (polarization) independent transverse spin angular momentum $(\mathrm{SAM})\left(\vec{p}^{s}\right)[1-4]$. This particular feature is well known as spin momentum locking in condensed matter physics in the context of topological insulators $[5,6]$. On the surface of a topological insulator, special states exist which fall within the bulk energy gap and allow surface metallic conduction [7]. The carriers in these surface states have their spin locked at a right-angle to their momentum (spin-momentum locking) [8]. In optics, this feature is manifested as the transverse component of the Poynting vector - which represents the flow of momentum - being independent of helicity (spin) of the beam [9]. In case of evanescent fields, such non-trivial structures of spin and momentum density have been observed and reported [9]. In fact, such a transversely spinning electric field arising in the case of transverse SAM of light, and resembling the spinning

\footnotetext{
* nghosh@iiserkol.ac.in

† ayan@iiserkol.ac.in
}

movement of the spokes of a rolling bicycle wheel, has recently been experimentally achieved [10]. It has also been shown that the general solution of Mie scattering from a spherical particle, which has phase-shifted longitudinal component indeed has the helicity dependent transverse component of poynting vector (generally addressed as 'transverse (spin) momentum') and helicity independent transverse spin angular momentum density $[11,12]$. Thus, keeping in mind that a tightly focused Gaussian beam has a longitudinal field component which is phase shifted from the transverse components, the obvious question that arises is whether a tightly focused Gaussian beam also contains these interesting and exotic properties.

In this paper, we show that a tightly focused Gaussian beam indeed possesses the very same properties of spinmomentum locking as has been observed for evanescent fields and Mie scattering.

\section{THEORY}

\section{A. Spin momentum locking for tight focusing of a Gaussian beam}

Tight focusing is well understood with the help of Debye-Wolf diffraction integral method and angular spectrum method. The generation of longitudinal component which is phase shifted from the transverse components is well known. The expression of the tightly focused beam can be written in a matrix formation as

$$
\left(\begin{array}{c}
E_{x} \\
E_{y} \\
E_{z}
\end{array}\right)=C\left(\begin{array}{ccc}
I_{0}+I_{2} \cos 2 \psi & I_{2} \sin 2 \psi & 2 i I_{1} \cos \psi \\
I_{2} \sin 2 \psi & I_{0}-I_{2} \cos 2 \psi & 2 i I_{1} \sin \psi \\
-2 i I_{1} \cos \psi & -2 i I_{1} \sin \psi & I_{0}+I_{2}
\end{array}\right) \vec{X}
$$

where $\vec{X}$ is Jones polarization vector. The $I_{0}, I_{1}$ and $I_{2}$ are well known diffraction integrals. $\mathrm{C}$ is a constant. In case of left circular and right circular polarization input (with $\vec{X}=\left[\begin{array}{lll}1 & \pm i\end{array}\right]^{T}$ for L.C.P. and R.C.P. 
respectively) the output electric field becomes:

$$
\left(\begin{array}{c}
E_{x} \\
E_{y} \\
E_{z}
\end{array}\right)=\left(\begin{array}{c}
\left(I_{0}+I_{2} \cos (2 \psi) \pm i I_{2} \sin (2 \psi)\right) \\
I_{2} \sin (2 \psi) \pm i\left(I_{0}-I_{2} \cos (2 \psi)\right) \\
-2 i I_{1} \cos (\psi) \pm 2 I_{1} \sin (\psi)
\end{array}\right) \vec{X}
$$

To calculated magnetic field we exploit the symmetry that tightly focused electric and magnetic field enjoy[14].

$$
\begin{aligned}
\mathbf{H}_{x} & =-\mathbf{E}_{y}\left(\frac{\pi}{2}-\psi\right) \\
\mathbf{H}_{y} & =\mathbf{E}_{x}\left(\frac{\pi}{2}-\psi\right) \\
\mathbf{H}_{x} & =-\mathbf{E}_{z}
\end{aligned}
$$

The spin momentum density is given in SI units as $[2,10,15]$

$$
\mathbf{S}=\frac{\epsilon \mathbf{E} \times \mathbf{E}^{*}+\mu \mathbf{H} \times \mathbf{H}^{*}}{\omega\left[\epsilon|\mathbf{E}|^{2}+\mu|\mathbf{H}|^{2}\right]}
$$

The Poynting Vector in SI unit is given as:

$$
\mathbf{P}=\frac{1}{2} \operatorname{Re}\left(\mathbf{E} \times \mathbf{H}^{*}\right)
$$

Here we calculate the Spin momentum density and Poynting vector.For Right Circularly Polarized light the expressions are:

$S_{x}=4 I_{1}(\cos (\psi)+\sin (\psi))\left(I_{0}-I_{2} \cos (2 \psi)+I_{2} \sin (2 \psi)\right) ;$

$S_{y}=4 I_{1}\left(-\left(I_{0}+I_{2}\right) \cos (\psi)+I_{0} \sin (\psi)+I_{2} \sin (3 \psi)\right)$;

$S_{z}=4\left(I_{0}^{2}-I_{2}^{2}\right)$
$P_{x}=2(\cos (\psi)+\sin (\psi))\left(I_{0} I_{1}-I_{1} I_{2}(\cos (2 \psi)+\sin (2 \psi)) ;\right.$

$P_{y}=-2(\cos (\psi)-\sin (\psi))\left(I_{0} I_{1}+I_{1} I_{2}(\cos (2 \psi)+\sin (2 \psi))\right.$;

$P_{z}=2\left(I_{0}^{2}-I_{2}^{2} \cos (4 \psi)\right)$

For Left Circularly polarized light the expressions are:

$$
\begin{aligned}
& S_{x}=4 I_{1}(\cos (\psi)+\sin (\psi))\left(I_{0}-I_{2} \cos (2 \psi)+I_{2} \sin (2 \psi)\right) \\
& S_{y}=4 I_{1}\left(-\left(I_{0}+I_{2}\right) \cos (\psi)+I_{0} \sin (\psi)+I_{2} \sin (3 \psi)\right) ; \\
& S_{z}=-4\left(I_{0}^{2}-I_{2}^{2}\right)
\end{aligned}
$$

$P_{x}=-2(\cos (\psi)+\sin (\psi))\left(I_{0} I_{1}-I_{1} I_{2}(\cos (2 \psi)+\sin (2 \psi)) ;\right.$

$P_{y}=2(\cos (\psi)-\sin (\psi))\left(I_{0} I_{1}+I_{1} I_{2}(\cos (2 \psi)+\sin (2 \psi))\right.$;

$P_{z}=2\left(I_{0}^{2}-I_{2}^{2} \cos (4 \psi)\right)$

Clearly we can see that under the change of helicity from +1 to -1 , the transverse component of spin angular momentum remains same, but the transverse components of Poynting Vector flip direction. This confirms that helicity dependence of traverse Poynting vector and helicity-independence of transverse spin angular momentum are properties of a tightly focused Gaussian beam. Acknowledgements: The authors acknowledge the financial support by Indian Institute of Science Education and Research, Kolkata, an autonomous research and teaching institute funded by the Ministry of Human Resource Development, Govt. of India. The authors also acknowledge DST-SERB grant for this project.DP acknowledge 2018 SPIE Optics and Photonics Scholarship for support.
[1] K. Y. Bliokh and A. Y. Bekshaev and F. Nori, Nat. Communications 5, 330 (2014).

[2] A. Aiello and P. Banzer and M. Neugebauer G. Leuchs , Nat.Photonics 9, 789 (2015).

[3] K. Y. Bliokh and D. Smirnova F. Nori , Science 348, 1448 (2015).

[4] A. Y. Bekshaev and K. Y. Bliokh F. Nori , Phys. Rev. X 5, 011039 (2015).

[5] M. Z. Hasan et al., Rev. Mod. Phys. 82, 3045 (2010).

[6] F. Ortmann and S. Roche and S. Valenzuela, Topological Insulators: Fundamentals and Perspectives (John Wiley and Sons, 2015).

[7] F. Zhang and C. L. Kane E. J. Mele, Physical Review B 86(8), 081303 (2012).

[8] D. Hsieh and Y. Xia et al., arXiv preprint arXiv:1001.1590 82, 3045 (2010).
[9] T. Van Mechelen and Z. Jacob, Optica 3(2), 118-126 (2016).

[10] T. Bauer and M. Neugebauer G. Leuchs P. Banzer , Phys. Rev. Lett. 117, 013601 (2016).

[11] S. Saha et al., Opt. Lett. 41, 4499 (2016).

[12] C. F. Bohren and D. R. Huffman, 2004 Absorption and Scattering of Light by Small Particles (Weinheim: Wiley-VCH).

[13] S.D. Gupta and N. Ghosh and A. Banerjee, Wave Optics: Basic Concepts and Contemporary Trends (CRC Press, 2015).

[14] B. Richards and E. Wolf , Proc. R. Soc. A 253, 358 (1959).

[15] M. V. Berry and M. R. Dennis , Proc. R. Soc. A 457, 141 (2001).

[16] Basudev Roy et al., Phys. Rev. A 87, 043823 (2013).

[17] Basudev Roy et al., New Journal of Physics, 16, 083037 (2014). 


\title{
Nano-post arrays for optical interconnects*
}

\author{
Shulang Lin*a, Huarong $\mathrm{Gu}^{\mathrm{a}}$ \\ State Key Laboratory of Precision Measurement Technology and Instruments, Department of \\ Precision Instrument, Tsinghua University, Beijing 100084, China
}

\begin{abstract}
In this paper, we present a compact solution using high-contrast all-dielectric nano-post arrays for optical interconnects in optoelectronic integrated neural networks. The nano-post arrays are made of amorphous silicon which has a high refractive index and high transmittance in the near infrared. The radius of each post is changed to generate different phase delay. The simulated arrays approximated by phase geometry can focus and deflect the plane wave of $1550 \mathrm{~nm}$ simultaneously. To obtain a higher efficiency at designed area of detector, we assume the intensity distribution on focal plane as Gaussian-shaped. We also use the Rayleigh-Sommerfeld diffraction formula and Gerchberg Saxton iteration to calculate the phase profile of optical interconnects. The connection efficiencies of post arrays are calculated by numerical simulations and compare to zone plates. Simulation results show that the proposed nano-post arrays have superior performances.
\end{abstract}

Keywords: optical interconnects, neural networks, diffraction, zone plates, all-dielectric nano-post

\section{Introduction}

Recently, artificial neural networks (ANNs) have received extensive attention because of their excellent performance in image processing, pattern recognition, medical diagnosis and so on. These applications are mainly implemented in software which work on CPU. Due to CPU's low efficiency with processing big data, and increasing importance of real-time processing, much work of improving ANNs' computing speed and efficiency has a great development such as GPU, FPGA, ASIC. For example, NVIDIA's Xavier processor is designed for unpiloted driving. Electronic neural hardware are demonstrated that their efficiencies have great improvement while it is still limited by electronic clock rates and ohmic losses. Similarly, ANNs can be implemented in optoelectronic or all-optical ways. The optoelectronic implementations have more superiority thanks to flexibility of electronic components and parallelism of optic connects. Figure 1 shows a basic structure of the optoeletronic neural network that one neuron is connected to nine neurons in next layer by nine optical interconnects. The detector receive light signal emitted by the neurons on the upper layer. Hence, an optoeletronic neuron requires at least three different optical interconnects which are called center, edge and diagonal for short. However, current optical interconnects, including diffractive optical elements such as zone plates and holographic devices, are typically above 100 microns in size. Typically, such a focal length of the diffractive optical element are more than 100 microns. If they are required to $r$ a large deflection angle, overlay in the depth direction of the current process is difficult to achieve. In other words, the diffractive optical element is not easy to achieve high numerical aperture (NA). Considering integration of an electro-optical neural processor, the size of the optical interconnects must be small enough, the focal length is short, and the efficiency of focal area is high enough. Recent work on subwavelength flat optics shows that metasurface-based metalens can meet the need of size, focal length and efficiency.

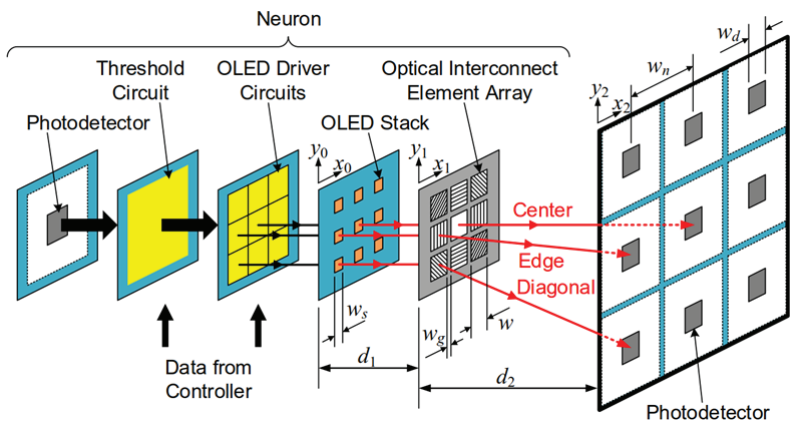

Fig. 1. A basic structure of an optoelectronic neural network. The function of optic interconnects consist of nine light source and nine connect devices. 
Metasurface can control light by subwavelength-scale structures such as V-shaped antennas, which are capable of modulating the phase, amplitude, or polarization of transmitted light. Through gradual phase accumulation or local phase shifts, we can construct ultrathin optical components with converging function which are called metalens. In this paper, we proposed three kinds of nanopost arrays to realize requirements of the optical interconnects. The structure consist of $\alpha$-silicon post arrays and silica substrate. Through changing the radius of post, depending on high contrast grating theory, the generated phase shift can realize $2 \pi$. The arrays can focus light and deflect big angle simultaneously with high efficiency.

\section{Design of nano-post arrays}

The design of transmit arrays start with simulations of post with in a square lattice. The arrays is normally incident by plane wave with wavelength of $1550 \mathrm{~nm}$. The post arrays are $700 \mathrm{~nm}$ tall in order to ease fabrication difficulties, which is different with previous simulation. The index of amorphous silicon is 3.37 according to actual processing parameters of film growth and the index of silica is 1.44 . The period of square lattice are $720 \mathrm{~nm}$ to increase the sampling rate so that the phase shift of arrays can be closer to the calculated continuous phase distribution. The unit with lattice period less than 720 could not cover $2 \pi$ phase range with suitable radius composition. The phase and amplitude response of the unit as shown in Fig 2. Because the lattice is square and the unit is post, there is no difference between the polarization direction and the $\mathrm{x}$ direction and the $\mathrm{y}$ direction.

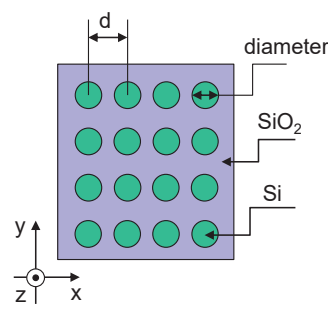

a

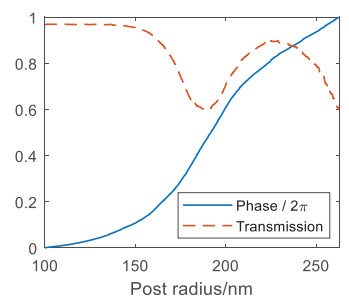

$\mathrm{b}$
Figure 2(a) Post arrays with same radius. Every unit is amorphous silicon post set on square lattice of fused periodic post array with radius varying from $100 \mathrm{~nm}$ to $261 \mathrm{~nm}$.
We have calculated 600 points of radius from $100 \mathrm{~nm}$ to $300 \mathrm{~nm}$ and used linear interpolation to obtain fitting curve between phase, transmission and corresponding radius. We have trimmed the region from $100 \mathrm{~nm}$ to $261 \mathrm{~nm}$ in the curve because they can meet the need of $2 \pi$ phase shift, otherwise, the transmission are more than $60 \%$ for each case. The diameter of $200 \mathrm{~nm}$ to $522 \mathrm{~nm}$ can ease the difficulty of fabrication for such deep silicon etching. In our previous simulation whose period is $800 \mathrm{~nm}$, the transmission is more than $85 \%$ with same radii range. When the lattice constant decrease from $800 \mathrm{~nm}$ to $720 \mathrm{~nm}$, the transmission falls. Since the phase delay of different unit are relative value, we delimit the phase of $100 \mathrm{~nm}$ as zero and at $261 \mathrm{~nm}$ the phase change to $2 \pi$ relatively. With the curve of phase and radius, any phase distribution can be realized.

We use center, edge and diagonal to refer to each element that the center only focus the plane wave on the distance of $65 \mu \mathrm{m}$ at $\mathrm{z}$ direction, the edge should focus on the same distance and deflect $\mathrm{w}=30 \mu \mathrm{m}$ at $\mathrm{y}$ direction, and the diagonal should deflect $\mathrm{w}=30 * \sqrt{2} \mu \mathrm{m}$ at diagonal direction. Take the edge case as an example, the deflection angle for central part is about $24.8^{\circ}$ which corresponds to about 5 units in a $2 \pi$ period under the condition of wavelength of $1550 \mathrm{~nm}$ and periodicity of $720 \mathrm{~nm}$. The structure away from the central part need to deflect a longer distance, causing some of them become useless. When less than two posts in a period, there does not exist phase gradient. With plane wave of $\lambda=1550 \mathrm{~nm}$ normally incident in our simulation, every element has size of $50.4 \mu \mathrm{m} \times 50.4 \mu \mathrm{m}$ which consist of $70 \times 70$ posts with lattice's periodicity of $720 \mathrm{~nm}$. In most work about metalens, the desired output wavefront is considered as spherical waves for incidence of plane wave like traditional lens. To improve the efficiency of the designed spot, we assume the intensity distribution on focal plane as Gaussian-shape and used the Rayleigh-Sommerfeld diffraction formula (RS) and Gerchberg Saxton (GS) algorithms for 20000 iterations of forward and reverse propagation. According to the size of the detector we designed, we found that the efficiency calculated by RS is highest when the waist is set as $2 \mu \mathrm{m}$. Fig3 is the post arrays for center, edge, and diagonal connections instead by corresponding post. 


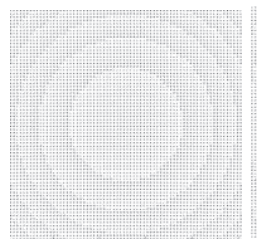

a

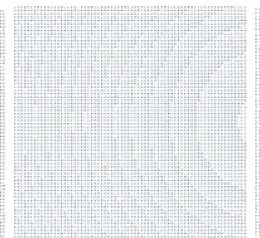

b

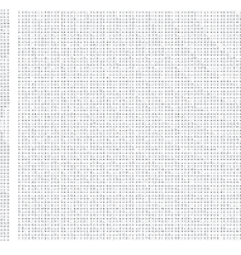

Figure 3, (a), (b) and (c) are posts array which consist $\alpha$-silicon post set on $\mathrm{SiO} 2$ substance for center, edge, and diagonal connections

\section{Results and Discussion}

\section{Figures and captions}

The normalized intensity distributions of three connections show as Fig4. The focusing efficiency is defined as the transmission within a $16 \mu \mathrm{m} \times 16 \mu \mathrm{m}$ detector around the focal point.

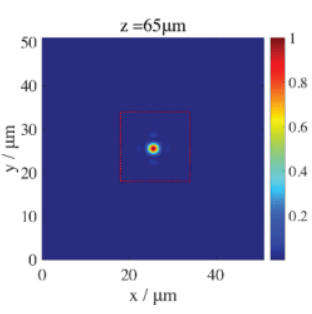

a

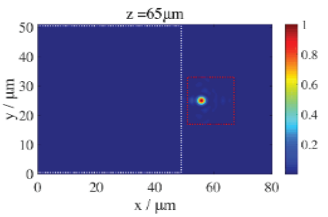

$\mathrm{c}$

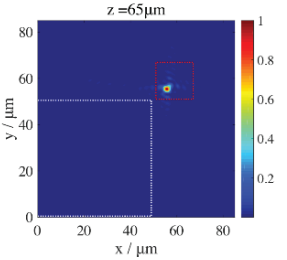

e

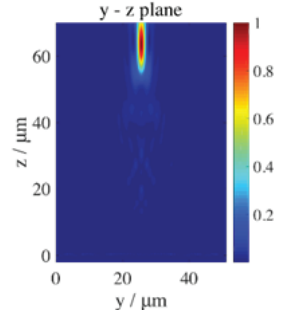

b

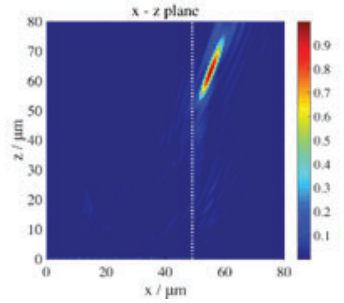

d

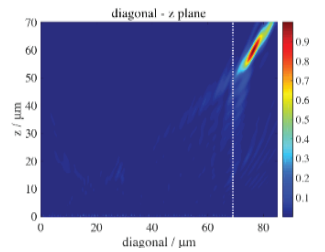

f
Figure 4, The absolute value of scatter electric field of $x-y$ plane at $z=65 \mu \mathrm{m}$. (a) center, (c) edge and (e) diagonal, the electric field of propagate plane (b) center, (d) edge and (f) diagonal. The white dotted line is the projection surface of the optical interconnection element and the red dotted line is the monitor.

For Gaussian-shape design, the normalized intensity distributions of edge and diagonal connections show as Fig5. The spot sizes are bigger than spherical one.

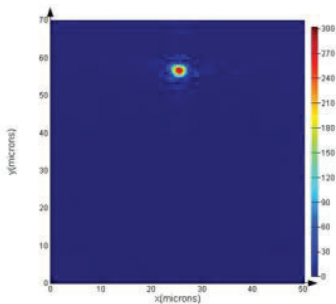

a

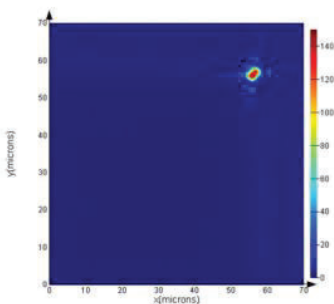

b
Figure 5, The absolute value of scatter electric field of $x-y$ plane at $\mathrm{z}=65 \mu \mathrm{m}$. (a) edge and (b) diagonal.

In order to compare under the same conditions, we also use FDTD to simulate the zone plate with a focal length of $65 \mu \mathrm{m}$. Fig5 shows the two steps zone plate phase profile and simulation results. The phase profile calculate with sampling of $100 \mathrm{~nm}$ considering the smallest line width is about $1 \mu \mathrm{m}$ at practice. The windows chose for monitor the efficiency are same to nano-post arrays.

To have a fair comparison, the sampling period of the phase profile for the zone plates is set as $0.1 \mu \mathrm{m}$, the phase profiles of the zone plates are quantized into two steps as shown in Fig. 9, the numerical simulations are carried out using the same version of Lumerical FDTD Solutions, and the same set of detectors are employed to calculate the focusing efficiencies. The performance of nano-post arrays and zone plates are listed in the Table 1. For each connections, the focal efficiency of nano-post arrays are always higher than zone plate owing to two step phase gradient can't well approximate real continuous phase distribution. The nano-post arrays solution can mostly imitate real geometry phase with infinite steps with enough sampling. 
Table 1. Comparison of nano-post arrays and zone plates

\begin{tabular}{|c|c|c|}
\hline items & & efficiency \\
\hline \multirow[t]{3}{*}{ center } & $\begin{array}{c}\text { Nano-post } \\
\text { (sphere) }\end{array}$ & $42 \%$ \\
\hline & $\begin{array}{l}\text { Nano-post } \\
\text { (Gaussian) }\end{array}$ & $52 \%$ \\
\hline & Zone plate & $18 \%$ \\
\hline \multirow[t]{3}{*}{ edge } & $\begin{array}{l}\text { Nano-post } \\
\text { (sphere) }\end{array}$ & $26 \%$ \\
\hline & $\begin{array}{l}\text { Nano-post } \\
\text { (Gaussian) }\end{array}$ & $40 \%$ \\
\hline & Zone plate & $23 \%$ \\
\hline \multirow[t]{3}{*}{ diagonal } & $\begin{array}{l}\text { Nano-post } \\
\text { (sphere) }\end{array}$ & $21 \%$ \\
\hline & $\begin{array}{l}\text { Nano-post } \\
\text { (Gaussian) }\end{array}$ & $24 \%$ \\
\hline & Zone plate & $21 \%$ \\
\hline
\end{tabular}

\section{Conclution}

This article present nano-post arrays solution for optic interconnects based on high contrast refractive index. The center connection can concentrate the light and the edge and diagonal can focus and deflect it to the detector out of the element. The Gaussian-shape design have higher efficiencies. The focal efficiency still need more research, for example, trying to enlarge the sampling or considering of multi-level diffraction. The nano-post arrays solution have higher efficiency than two-step zone plate with mature CMOS processing technology. With these binary optic optimization methods we can obtain better beam shaping and multifocal element for special interconnects.

\section{References}

Guo, X. G. Wang, and M. Pietikainen, "Local binary features for texture classification: Taxonomy and experimental study," Pattern Recognit. 62, 135-160 (2017).

K. Kamnitsas, C. Ledig, V. F. J. Newcombe, J. P. Sirnpson, A. D. Kane, D. K. Menon, D. Rueckert, and B. Glocker, "Efficient multi-scale 3D CNN with fully connected CRF for accurate brain lesion segmentation," Medical Image Analysis 36, 61-78 (2017).

S. Z. Zhang, J. J. Wang, X. Y. Tao, Y. H. Gong, and N. N. Zheng, "Constructing Deep Sparse Coding Network for image classification," Pattern Recognit. 64, 130140 (2017).
S. Han, X. Y. Liu, H. Z. Mao, J. Pu, A. Pedram, M. A. Horowitz, W. J. Dally, and Ieee, "EIE: Efficient Inference Engine on Compressed Deep Neural Network," in 2016 Acm/Ieee 43rd Annual International Symposium on Computer Architecture(Ieee, 2016), pp. 243-254.

Y. Shen, N. C. Harris, S. Skirlo, M. Prabhu, T. Baehrjones, M. Hochberg, X. Sun, S. Zhao, H. Larochelle, and D. Englund, "Deep learning with coherent nanophotonic circuits," Nature Photonics 11, 189-190 (2016).

D. Psaltis, D. Brady, X. G. Gu, and S. Lin, "HOLOGRAPHY IN ARTIFICIAL NEURAL NETWORKS," Nature 343, 325-330 (1990).

W. Herrington, H. Gu, S. Lin, and C. Warde, "Design, fabrication, and testing of zone-plate interconnects for a compact optoelectronic integrated neural coprocessor," Applied Optics 56, 4606-4612 (2017).

V. P. Kozemiako, O. K. Kolesnytskyj, T. S. Lischenko, W. Wojcik, and A. Sulemenov, "Optoelectronic Spiking Neural Network," in Optical Fibers and Their Applications 2012, R. S. Romaniuk, and W. Wojcik, eds. (2013).

C. Sun, M. T. Wade, Y. Lee, J. S. Orcutt, L. Alloatti, M. S. Georgas, A. S. Waterman, J. M. Shainline, R. R. Avizienis, S. Lin, B. R. Moss, R. Kumar, F. Pavanello, A. H. Atabaki, H. M. Cook, A. J. Ou, J. C. Leu, Y.-H. Chen, K. Asanovic, R. J. Ram, M. A. Popovic, and V. M. Stojanovic, "Single-chip microprocessor that communicates directly using light," Nature 528, 534538 (2015).

F. Duport, B. Schneider, A. Smerieri, M. Haelterman, and S. Massar, "All-optical reservoir computing," Optics Express 20, 22783-22795 (2012).

A. G. Rudi, and S. Jalili, "A parallel optical implementation of arithmetic operations," Optics \& Laser Technology 49, 173-182 (2013).

$\mathrm{Xu}$, Ning, et al. "Multiring pure-phase binary optical elements to extend depth of focus." Applied optics 57.32: 9643-9648(2018).

$\mathrm{H}$. $\mathrm{Gu}$, and $\mathrm{C}$. Warde, "Evaluation of organic lightemitting diodes as light sources for a compact optoelectronic integrated neural coprocessor," Applied Optics 55, 8576-8581 (2016).

K. H. Wagner, and Ieee, "Deep Optical Learning Devices and Architectures,"Photonics Society Summer Topical 
Meeting Series (SUM), IEEE, 179-180(2016).

N. Yu, P. Genevet, F. Aieta, M. A. Kats, R. Blanchard, G. Aoust, J.-P. Tetienne, Z. Gaburro, and F. Capasso, "Flat Optics: Controlling Wavefronts With Optical Antenna Metasurfaces," Ieee Journal of Selected Topics in Quantum Electronics 19, 4700423 -4700423 (2013).

N. Yu, and F. Capasso, "Optical Metasurfaces and Prospect of Their Applications Including Fiber Optics," Journal of Lightwave Technology 33, 23442358 (2015).

M. A. Kats, P. Genevet, G. Aoust, N. Yu, R. Blanchard, F. Aieta, Z. Gaburro, and F. Capasso, "Giant birefringence in optical antenna arrays with widely tailorable optical anisotropy," Proceedings of the National Academy of Sciences of the United States of America 109, 12364-12368 (2012).

H.-T. Chen, A. J. Taylor, and N. Yu, "A review of metasurfaces: physics and applications," Reports on Progress in Physics 79,076401-076442 (2016).

Z.-L. Deng, and G. Li, "Metasurface optical holography," Materials Today Physics 3, 16-32 (2017). 


\title{
Multiring pure-phase binary optical elements to tunable axial multi-focus \\ beam intensity
}

\author{
Ning $\mathrm{Xu}^{*}$, Qiaofeng Tan

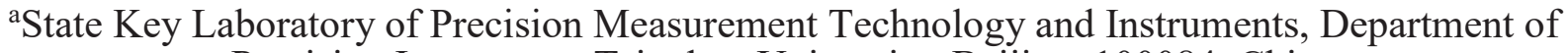 \\ Precision Instrument, Tsinghua University, Beijing, 100084, China
}

\begin{abstract}
Tunable multi-focus spots (TMS) as a technique potentially offers extremely convenient in scientific and industrial applications. However, the general approach used for forming axial multi-focal spots based on Fresnel zone plate limits the conceivable applications. In this paper, we introduce a method to generate TMS by phase-only results and a novel modified Gerchberg-Saxton algorithm. The main advantage of the approach introduced here is that it is intuitive, effective, and straightforward. As an example, we applied it to design the axial tunable spots intensity, which can be controlled easily. Theoretical and numerical simulations demonstrate how TMS can offer the possibility of versatile applications.
\end{abstract}

Keywords: Laser beam shaping; Diffractive optics; Binary optics; Multi-focus spots

\section{Introduction}

Multi-focus beam offers a broad range of applications of spots mode, and is being applied in numerous scientific and industrial fields. Particularly interesting applications for this kind of beams are reading in multi-layered peptide array system [1], intraocular lenses, long depth of beam [2-3] and multi-layer optical disc storage [4] in general. Such beams are attractive not only because of their multifocus spots properties but also we can modulate the spots mode.

Many approaches have been put forward to design multi-focus beam, such as micro-lens arrays, beam splitters, freeform surface, Fresnel zone plate, and diffractive optical elements. Although different approaches have been carried out for designing multifocus lenses, our goal is to present an approach based on pure phase binary optical elements (BOEs) by optimization algorithm. However, to the best of knowledge, no thorough analysis of axial intensity distribution of the generated multi-focus spots modes have been reported so far. Here, we apply it to design axial multi-focus points with different modes distribution. The approach allows us a straightforward method to control normalized intensity distributions. Meantime,

*xn18@,mails.tsinghua.edu.cn; various algorithms about such a light field have been bring forward in literature [5]. Calculating an accurately phase distribution to generate a high-quality demandoriented light field is a challenge problem, because phase distribution generally cannot be solved analytical results. We report the realization of iterative algorithm for multifocus spots, which can obtain intensity profile accurately. Since such beam can be generated by modified methods, the accurate problems are overcome and to reach in many applications, i.e. direct laser writing, demanding in a variety of modes spots.

In this paper, we propose a practical method to show how to engineer different kinds useful forms beam of multi-focus spots. Firstly, we describe theoretical considerations by $\mathbf{k}$ space Fourier transformation, and the expression of axial light field is discussed. Based on the theory, the axial light field controlled by phase-only expression is derived, which can be used for tunable multi-focus spots control in the hereabouts focal region. Then, a novel modified Gerchberg-Saxton (GS) algorithm is presented to optimize the BOEs. Through this algorithm, the axial light field can be tailoring accurately. Eventually, numerical simulation and experiment are carried out to confirm the feasibility of the proposed design and demonstrate the tunable tailoring mode of different foci spots intensity by pure phase 
spatial light modulator (SLM). We also provide a brief summary of the method, the most significant results, and the potential applications.

\section{Design Methods}

With a scalar and paraxial description, our system is limited in the small numerical aperture, which is justified for the diffraction from the $\mathrm{BOE}$ and lens can be neglected and the feature size of BOEs $>>\lambda$. For an $\mathrm{N}-$ ring pure phase BOE, when an ideal plane wave incidents commonly, the two-dimensional Fourier transform of a field $E\left(r_{2}, z\right)$ defined in axial position at distance $\mathrm{z}$ can be expressed as [6]

$$
E\left(r_{2}, z\right)=\left(\frac{2 \pi}{\lambda z}\right) \int_{0}^{\mathrm{R}} t\left(r_{1}\right) \exp \left[\frac{\mathrm{i} \pi r_{1}^{2}}{\lambda}\left(\frac{1}{z}-\frac{1}{f}\right)\right] \mathrm{J}_{0}\left(\frac{2 \pi r_{1} r_{2}}{\lambda f}\right) r_{1} d r_{1},
$$

where $r_{1}$ and $r_{2}$ represent the radial coordinates on the input and output planes, respectively. $\lambda$ is the wavelength, $f$ is the focal length of lens, $\mathbf{J}_{0}$ is the zero-order Bessel function. It worthwhile to note that $t\left(r_{1}\right)$ denotes the complex amplitude function in the radial direction on the input plane, which can be written as

$$
t\left(r_{1}\right)=\sum_{j=1}^{N} \exp \left(i \varphi_{1 j}\right) \operatorname{rect}\left(\frac{r_{1}-(2 j-1) R / N / 2}{R / N}\right),
$$

where $\varphi_{1 j}, j=1, \ldots, N$ defines the phase of zone $j$ on the input plane, $R$ is the radii of the $\mathrm{BOE}$, and $\operatorname{rect}(\cdot)$ denotes a rectangle function defined as

$$
\operatorname{rect}(x)=\left\{\begin{array}{ll}
1, & |x| \leq \frac{1}{2} \\
0, & \text { else }
\end{array} .\right.
$$

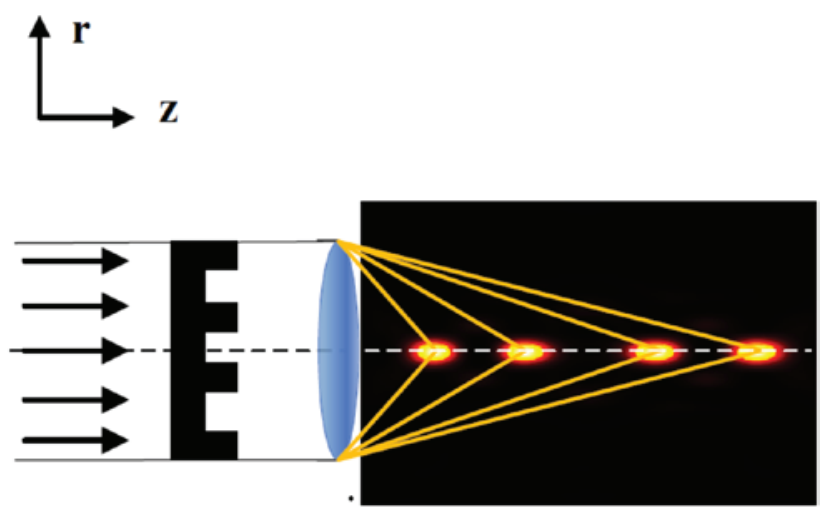

Figure 1. Multi-focus spots generated by a BOE and a lens.

Furthermore, we adopt a wave-vector $\mathbf{k}$ space to numerical analyze the propagation of the beam behind the lens by Hankel transformation. If we assign a zero value to the on-axis position, the light field just behind the lens $(\mathrm{z}=0)$ can be written as

$$
S(\xi, z=0)=\iint E\left(r_{2}, z=0\right) \exp (-\mathrm{i} \xi k r) d r,
$$

Considering an azimuthally independent light field distribution with distance $\mathrm{z}=0$, the Fourier transform can be expressed in the form of a zero-order Hankel transform in the cylindrical coordinate system

$$
S(\xi, z=0)=k \int_{0}^{R} E\left(r_{2}, z=0\right) J_{0}(\xi k r) r d r .
$$

We describe such a beam propagate the spectrum in the $\mathbf{k}$ space with a distance $\mathrm{z}$ based on angular-spectrum method [6], then given by

$$
S(\xi, z)=S(\xi, 0) \exp \left(\mathrm{i} k_{z} z\right),
$$

where $k_{z}=k \sqrt{1-\xi^{2}}$ is the wave vector in the propagation direction usually termed as the propagation constant. Using the inverse Hankel transform, the light field distribution on-axis can be obtained

$$
E\left(r_{2}, z\right)=k \int_{0}^{1} S(\xi, z) J_{0}(\xi k r) \xi d \xi \text {. }
$$

Based on inverse transform to Eq. (12), we may express the corresponding axial light field as,

$$
E\left(r_{2}=0, z\right)=\int_{0}^{k} S(\xi, z=0) k_{z} \exp \left(\mathrm{i} k_{z} z\right) d k_{z} .
$$

It is clearly to note that the function Eq. (13) describes the spatial spectrum field distribution lined with the axial propagation generated by one-dimensional Fourier transform.

\section{Simulation Results}

In the previous sections, we show how to design a BOE to modulate the light field by phase-only results and a novel modified GS algorithm. In this section, we will work within the framework of below sections, and we show how to achieve tailored TMS along the optical axis. Furthermore, spots intensity can be easily tunable. The design parameters are shown in Table 1.

Table 1. Design parameters in the simulation.

\begin{tabular}{l|c}
\hline Focal length of lens & $\mathrm{f}=100 \mathrm{~mm}$ \\
\hline $\begin{array}{l}\text { Number of sampling points } \\
\text { (Axial direction) }\end{array}$ & $\begin{array}{c}\mathrm{M}=600 \text { or } 800 \text { (For four-focus } \\
\text { spot) }\end{array}$ \\
\hline $\begin{array}{l}\text { Number of sampling points } \\
\text { (Radial direction) }\end{array}$ & $\mathrm{N}=100$ \\
\hline Wavelength & $\lambda=632.8 \mathrm{~nm}$ \\
\hline The diameter of BOEs & $\mathrm{D}=7 \mathrm{~mm}$ \\
\hline $\begin{array}{l}\text { Number of modified algorithm } \\
\text { iterations }\end{array}$ & 120 times/plane \\
\hline
\end{tabular}


In Fig. 9, we show the light intensity distribution with the propagation distance, and it consists of tunable spots energy distribution. The axial light intensity distribution can be designed as the tendency of " $\Lambda$ or $v$ ", which is shown in Fig. 2 (a1-a3). The axial intensity of the submaximal value is set to $50 \%$ of the maximal value. It can be shown the method process good regulation ability to realize the target field.
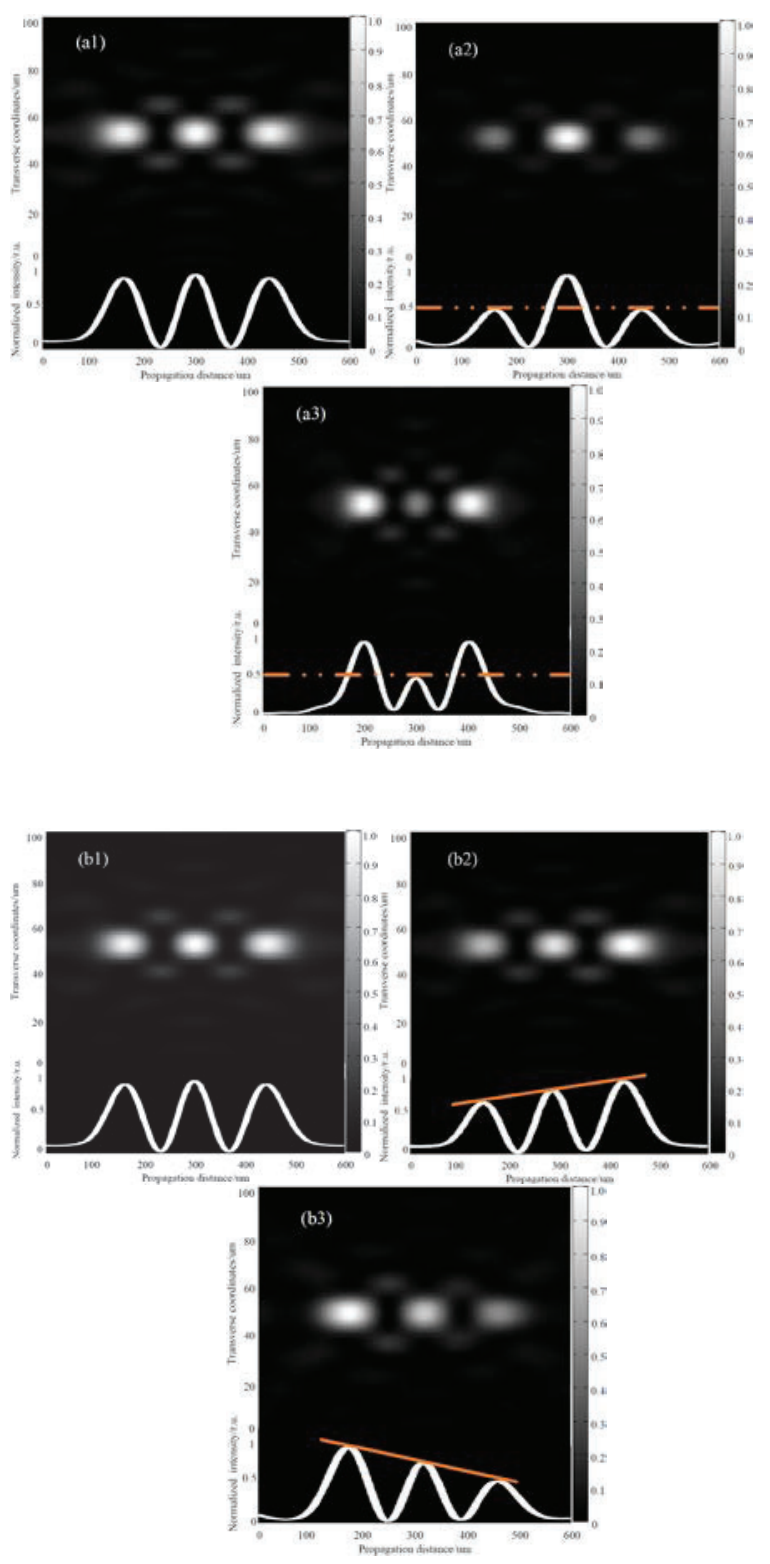

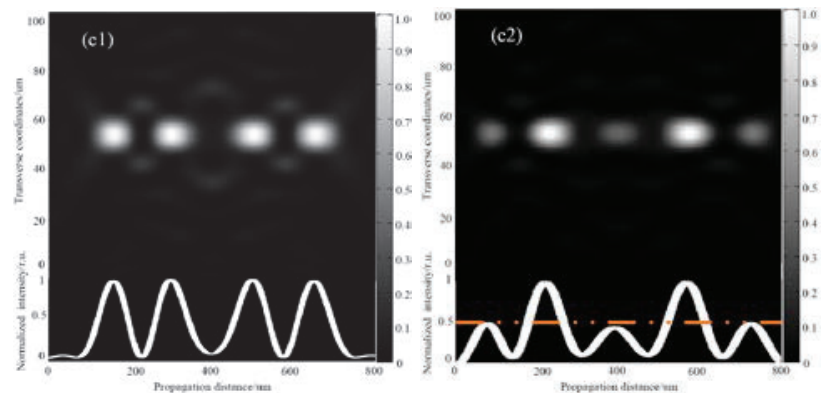

Figure 2. Numerical simulation results with tunable spots energy distribution.

Moreover, the sidelobes intensity also be constrained. We set the peak intensity of the sidelobe should be lower than $25 \%$ of the maximum intensity on-axis.

\section{Conclusions}

In summary, we have shown a useful method for designing TMS based on phase-only results and a novel modified GS algorithm. Compared to the frequently general method, the phase-only pattern targeted to control the TMS can be more efficiently to obtain using our proposed method. We have shown that TMS can be generated different various modes of beam. This pure phase designing method has potential applications in interdisciplinary fields. Point-based method is used to obtain the straightforward and effective initial phase, and a modified GS algorithm is put forward to design BOEs, and the sidelobe are also constrained in the design. Specifically, the experiment TMS light field distribution is naturally consistent with numerical simulations. We believe that the ability to generate TMS beams will be fully used for potential laser beam applications.

\section{References}

R. Cohen, G. Gannot, and M. Ben David, et al, "Three dimension optical imaging in a high throughput layered expression system," Opt. Express 19(21), 19822 -19835 (2011).

N. Xu, Z. Kong, and Q. Tan, et al, "Multiring pure-phase binary optical elements to extend depth of focus," Appl. Opt. 57 (32), 9643-9648 (2018).

N. Xu, Q. Tan, and Y. Fu, "Design of diffractive optical elements for controllable axial long depth of focus," 
Proc. SPIE 10744, Laser Beam Shaping XVIII, 107440K (14 September 2018);

T. Ide, S. Kimura, and E. Tatsu, "Interlayer crosstalk reduction of a multilayer Blu-ray Disc using a grating in a three beam optical system," Appl. Opt. 49 (12), 2309-2315 (2010).

S. Lin, H. Gu, and C. Warde, "Optical interconnects based on high-contrast all-dielectric nano-post arrays," Appl. Opt. 57(29), 8664-8672 (2018).

J. W. Goodman, Introduction to Fourier Optics, Roberts \& Co. Publishers, Englewood, Colo, 3. ed., (2005).

N. Xu, H. Xiao, and Z. Kong, et al, "Axial multi-focus beams formed by binary optical elements," IEEE Photonics J. accept (2019). 


\title{
Simultaneously achieving a large negative dispersion and a high birefringence over Er and Tm dual gain bands in a square lattice photonic crystal fiber*
}

\author{
Yong Soo Lee, ${ }^{\mathrm{a}}$ Chung Ghiu Lee, ${ }^{\mathrm{b}}$ Faouzi Bahloul, ${ }^{\mathrm{c}}$ Soeun Kim, ${ }^{\mathrm{d}}$ and Kyunghwan $\mathrm{Oh}^{\mathrm{a}}$ \\ aPhotonic Device Physics Laboratory, Institute of Physics and Applied Physics, Yonsei University, Seoul 03722, South \\ Korea \\ ${ }^{b}$ Department of Electronic Engineering, Chosun University, Gwangju 501-759, South Korea \\ ${ }^{\mathrm{c} U n i v e r s i t y ~ o f ~ T u n i s ~ E l ~ M a n a r, ~ N a t i o n a l ~ E n g i n e e r i n g ~ S c h o o l ~ o f ~ T u n i s, ~ U n i v e r s i t y ~ o f ~ C a r t h a g e, ~ T u n i s i a ~ P o l y t e c h n i c ~}$ \\ School, SERCOM laboratory, 2078, Marsa, Tunisia \\ ${ }^{\mathrm{d} I n t e g r a t e d ~ O p t i c s ~ L a b o r a t o r y, ~ A d v a n c e d ~ P h o t o n i c s ~ R e s e a r c h ~ I n s t i t u t e, ~ G I S T, ~ G w a n g j u ~ 61005, ~ S o u t h ~ K o r e a ~}$
}

\begin{abstract}
We proposed a novel photonic crystal fiber composed of a double-cladding square lattice that could be used in dualband, Er and Tm optical gain bands, simultaneously supporting a large negative dispersion and a high birefringence. We theoretically investigated the light guiding property through the proposed PCF by using a vectorial finite element method (FEM) with a perfectly matched layer (PML). By optimizing the structural parameters, we obtained an ultra-large negative dispersion of $-20,186 \mathrm{ps} /(\mathrm{nm} \cdot \mathrm{km})$ and a very high birefringence of $9.27 \times 10^{-3}$ at the wavelength of $1.55 \mu \mathrm{m}$ in the Er gain band and a very large negative dispersion of $-8,067 \mathrm{ps} /(\mathrm{nm} \cdot \mathrm{km})$ and a high birefringence of $1.0 \times 10^{-3}$ at the wavelength of $1.87 \mu \mathrm{m}$ in the Tm band.
\end{abstract}

Keywords: photonic crystal fiber, birefringence, negative dispersion

\section{Introduction}

Photonic crystal fibers (PCFs) have optical properties distinctively unique in comparison to conventional fibers due to its cladding composed of regularly arrayed air holes. By controlling the air-hole cladding structure, PCFs have demonstrated novel optical properties that were not attainable in conventional fibers, such as flexibly controllable chromatic dispersion, high birefringence, tailored nonlinearity, and endless single mode guidance, to name a few [1-4]. Among them, the ability to adjust the chromatic dispersion has drawn intense attention due to its fundamental roles to enable high capacity optical communication systems by effective dispersion compensation $[5,6]$, and to overcome the trade-off between the optical nonlinearity and the negative dispersion in conventional step-index fibers [7].

In this study, we proposed a new double-clad squarelattice silica PCF design that can overcome the prior trade-off between $D$ and B so that we were able to obtain both a large negative $D$ and a high B not only in the Er gain band but also in the Tm-gain band for the first time to the best knowledge of the authors. By parametric analyses, the impacts of our double-clad square-lattice PCF design were thoroughly investigated to understand impacts of the waveguide parameters on $D$ and $\mathrm{B}$ in the dual band.

\section{Fiber design and numerical analysis}

Figure 1 shows the cross-section of the proposed square-lattice PCF with a double-cladding square lattice (DC-SL) structure. The square lattice cladding consists of two regions, the inner cladding, and the outer cladding, which have their own air hole diameter $\left(d_{\text {in }}, d_{\text {out }}\right)$ and air hole lattice parameter $\left(\Lambda_{\text {in }}, \Lambda_{\text {out }}\right)$. To implement the double-cladding structure, we removed 6 air holes with the diameter of $d_{\text {out }}, 2$ rows and 3 columns, from the center and then replace it with smaller air holes with the diameter $d_{\text {in }}$ arranged with the spacing $\Lambda_{\text {in }}\left(=\Lambda_{\text {out }} / 3\right)$, which serves as the inner cladding. Then, we further removed 6 smaller air holes with the diameter of $d_{\text {in, }}, 2$ 


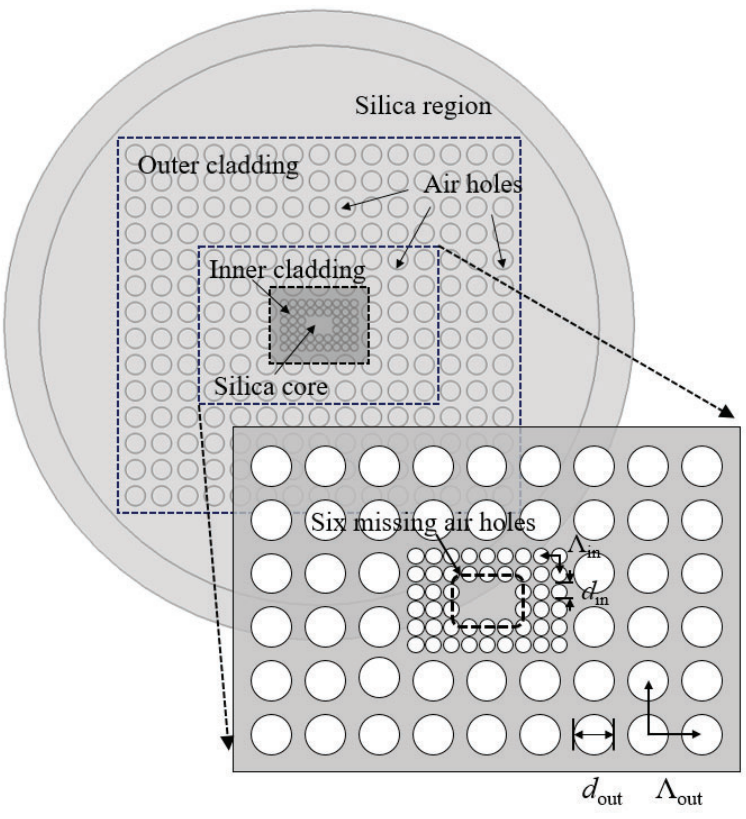

Figure 1. Transverse cross section of the proposed PCF

rows and 3 columns, in order to make a rectangular core with a high spatial asymmetry as shown in Figure 1.

The proposed PCF simultaneously realized a large negative $D$ and a high B in both Er and Tm gain bands. In an optimized structure, the air filling fraction of the inner cladding was set higher than that of the outer cladding so that the proposed PCF induced a large negative dispersion. A high-birefringence was obtained independently by controlling the size and the shape of the rectangle core [8]. Using the proposed DC-SL PCF structure, we obtained an ultra-large negative dispersion of $-20,186 \mathrm{ps} /(\mathrm{nm} \cdot \mathrm{km})$ and a very high birefringence of $9.27 \times 10^{-3}$ at the wavelength of $1.55 \mu \mathrm{m}$ in the Er gain band and a very large negative dispersion of $-8,067 \mathrm{ps} /(\mathrm{nm} \cdot \mathrm{km})$ and high birefringence of $1.0 \times 10^{-3}$ at the wavelength $1.87 \mu \mathrm{m}$ in the Tm band as shown in Figure 2.

In the mode analyses, we used a full vectorial finite element method (FEM) package (COMSOL Multiphysics) and applied the perfectly matched boundary layer (PML) boundary condition to understand the light guiding properties of the proposed PCF. In order to fully include the material dispersion in the chromatic dispersion, we used the refractive index of fused silica given by Sellmeier equation.

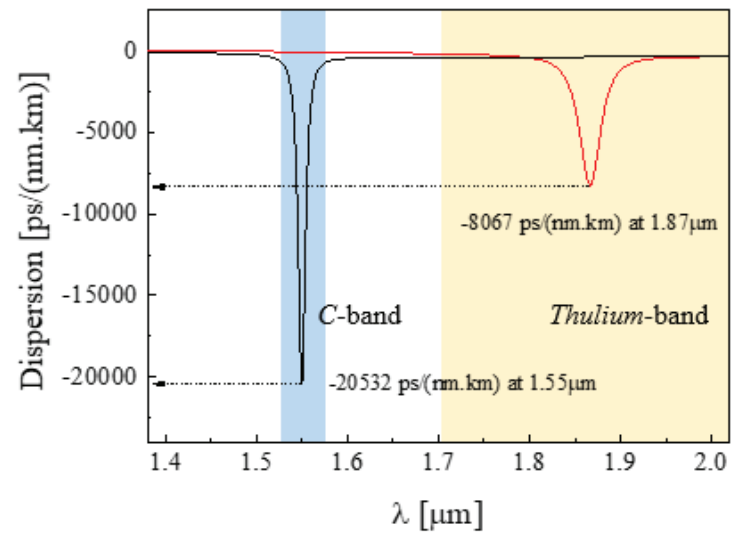

(a)

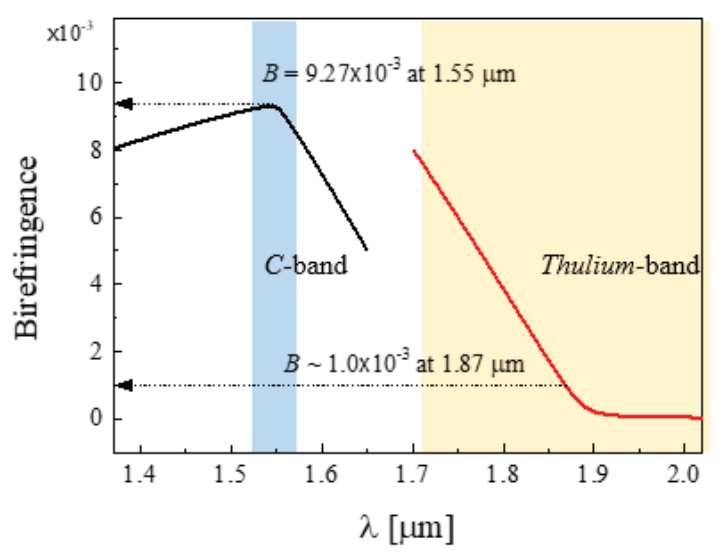

(b)

Figure 2. Light guiding properties of the proposed PCF.

(a) chromatic dispersion of the polarized fundamental modes in of the $y$-polarized fundamental mode at $\lambda=1.55$ $\mu \mathrm{m}$ in Er gain band and $x$-polarized fundamental mode $\lambda$ $=1.87 \mu \mathrm{m}$ in Tm gain band, and (b) birefringence. The black line indicates optical properties of $y$-polarized fundamental mode with $\Lambda_{\text {out }}=1.5 \mu \mathrm{m}, d_{\text {out }} / \Lambda_{\text {out }}=0.75, \Lambda_{\text {in }}$ $=\Lambda_{\text {out }} / 3, d_{\text {in }} / \Lambda_{\text {in }}=0.878$. The red line indicates optical properties of $x$-polarized fundamental mode with $\Lambda_{\text {out }}=$ $1.6 \mu \mathrm{m}, d_{\text {out }} / \Lambda_{\text {out }}=0.75, \Lambda_{\text {in }}=\Lambda_{\text {out }} / 3, d_{\text {in }} / \Lambda_{\text {in }}=0.878$.

\section{Conclusion}

A square lattice PCF with a double cladding structure was proposed to break the technical trade-off between the large negative dispersion and the high birefringence for the first time. The asymmetric rectangular core surrounded by square-lattice double-cladding enabled very flexible tuning of the negative dispersion and the birefringence independently not only in the Er gain band 
but also in the Tm gain band. For optimized waveguide parameters, the proposed PCF can simultaneously provide an extremely large negative dispersion of $-20,186$ $\mathrm{ps} /(\mathrm{nm} \cdot \mathrm{km})$ and a high birefringence of $9.27 \times 10^{-3}$ and at $\lambda=1.55 \mu \mathrm{m}$ in the Er gain band. The proposed PCF can also provide a very large negative dispersion of $8,067 \mathrm{ps} /(\mathrm{nm} \cdot \mathrm{km})$ and a high birefringence of $\sim 1.0 \times 10^{-3}$ at $\lambda=1.87 \mu \mathrm{m}$ in the Tm band. The PCF can be used for dispersion compensation in both optical communication fiber links and pulsed fiber laser cavities in the dual band, maintaining a high polarization selectivity, which can open a new avenue of wavelength division of multiplexing in the dual band and ultrafast mid-infrared fiber laser source developments.

Acknowledgement: This work was supported by National Research Foundation of Korea Grant funded by the Korean Government (2015R1D1A1A01058057) and Basic Science Research Program through the National Research Foundation of Korea(NRF) funded by the Ministry of Science, ICT \& Future Planning (No. 2016k1A3A1A09918616) and the Ministry of Education(2018R1D1A1B07049349)

\section{References}

[1] Lu, S., Li, W., and Guo, H., "Analysis of birefringent and dispersive properties of photonic crystal fibers," Appl. Opt. 50, 5798-5802 (2011).

[2] Lee, J. H., Teh, P. C., and Yusoff, Z., "A holey fiber based nonlinear thresholding device for optical CDMA receiver performance enhancement," IEEE Photon. Technol. Lett. 14, 876-878 (2002).

[3] Knight, J. C., Birks, T. A., and Cregan, R. F., "Large mode area photonic crystal fibre," Electron. Lett. 34, 1347-1348 (1998).

[4] Birks, T. A., Knight, J. C., and Russell, P., "Endlessly single-mode photonic crystal fiber," Opt. Lett. 22, 961-963 (1997).

[5] Nielsen, M. D., Jacobson, C., Mortensen, N. A., Folkenberg, J. R., and Simonsen, H. R., "Low-loss photonic crystal fibers for transmission system and their dispersion properties," Opt. Expr. 12, 1372-1376 (2004).

[6] Shen, L. P., Huang, W. P., and Jian, S. S., "Design and optimization of photonic crystal fibers for broad-band dispersion compensation," IEEE Photon. Technol. Lett. 15, 540-542 (2003).

[7] Finazzi, V., Monro, T. M., and Richardson, D. J., "Small-core silica holey fibers: nonlinearity and confinement loss trade-offs," J. Opt. Soc. Amer. B 20, 1427-1436 (2003).

[8] Lee, Y. S., Lee, C. G., Jung, Y., Oh, M, and Kim, S., "Highly Birefringent and Dispersion Compensating Photonic Crystal Fiber Based on Double Line Defect Core,” J. Opt. Soc. Korea 20, 567-574 (2016). 


\title{
Diffraction-free mapping of arbitrary modes from pump to probe beam via coherent population oscillation in two-level system
}

\author{
Onkar N. Verma*, Koustav Dey and Sourabh Roy \\ Department of Physics, National Institute of Technology Warangal, \\ Warangal-506004, Telangana, India
}

\begin{abstract}
We study an efficient way for diffraction-free mapping of multimode transverse profiles from one laser beam to another laser beam. Both beams are mutually interacting with a system of two-level atoms moving in a hot vapor cell. The two optical beams, namely, a weak probe and a strong control beam are counter-copropagating and driving same transition of two-level atom. We show that two or three diffraction-limited Gaussian modes carried by pump beam can be efficiently transferred to probe beam within a Rayleigh length. Such spatial transfer is attributed to pump field intensitydependence of both absorption and refractive index of probe beam. This method of information transfer may find potential applications in all-optical imaging and lithography technologies.
\end{abstract}

Keywords: Diffraction, saturated absorption, all-optical imaging, lithography technologies.

\section{Introduction}

Paraxial diffraction of laser beams poses a great challenge in all-optical imaging, wave-guiding and lithography techniques. ${ }^{1}$ Diffraction, causing the divergence of a laser beam, determines its size and shape during its propagation in free space or in a medium. A laser beam incident on an optical system such a circular lens is also diffracted and thus, can not be focused into desired spot. ${ }^{2}$ This sets a fundamental limit to the resolution of optical systems to resolve or create smaller details. ${ }^{3}$ The smaller is waist of output beam from a laser, the quicker it diverges. It is possible to reduce or even reverse the divergence of a laser beam by using a nonlinear optical effect known as Kerr effect. ${ }^{4}$ In a Kerr effect, the refractive index increases with the light intensity and thus, creating a waveguide-like structure similar to a graded-index fiber. ${ }^{5}$ The light field remains confined within the waveguide it has created and propagates without changing its shape and size. This is referred as self-focusing in which the nonlinear effect induced by a laser beam balances its paraxial diffraction. Spatial solitons of distinct shape are generated on the same principle of self-focusing due to a strong Kerr effect. ${ }^{6}$ However, the Kerr effect is limited to high intensity light beams propagating through conventional nonlinear media. ${ }^{7}$ Therefore, it is very important to search a new class of optical medium that can produce optical solitons at very low light intensities. ${ }^{8}$

Here, we propose a method to control the paraxial diffraction of transverse multimode light fields in a saturated absorption medium composed of gas atoms. ${ }^{9-11}$ In a such medium, two counter-propagating laser beams (namely, a weak probe and a strong pump) originated from a single laser beam are driving the same transition of two-level atoms in a vapor cell.

\section{Atomic Model and Dynamical Equations}

We consider a inhomogeneously broadened gas of ${ }^{87} \mathrm{Rb}$ atoms. The atoms in a vapor cell are modelled as two level system and driven by two counter-copropagating optical fields, namely, a weak probe and a strong control field. The dynamics of atom-field interaction is governed by the standard master equation under the rotating-wave approximation. ${ }^{12}$ The probe's atomic coherence for positive probe frequency is obtained from density matrix equation under steady state condition and in weak probe field limit. This coherence gives rise to complex susceptibility $(\chi)$. The real and imaginary part of susceptibility is responsible for the dispersion and absorption of probe beam, respectively. The effect of medium response on probe beam propagation in $\mathrm{z}-$ direction can be observed by numerically solving the wave equation in paraxial domain. ${ }^{13}$ 


\section{Results and Discussion}

\section{Probe's susceptibility}

We use multimode Gaussian-shaped pump beam profile to spatially manipulate the probe's susceptibility in transverse direction.
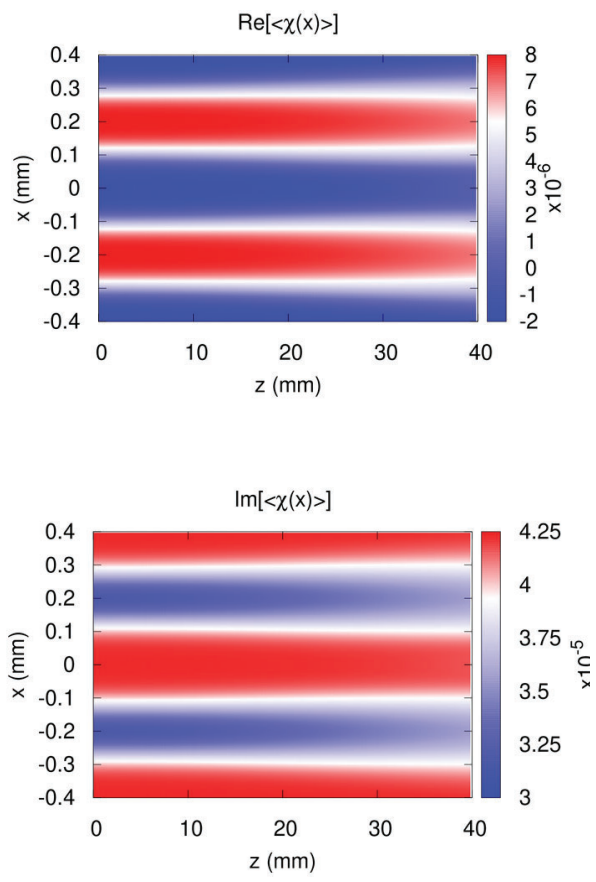

Figure 1. Transverse variation of real part (a) and imaginary part (b) of the averaged susceptibility in the presence of the two focused Gaussian modes of pump beam with input amplitude $\mathrm{G}_{0}=1$, waist radius $\mathrm{w}_{0}=$ $100 \mu \mathrm{m}$ and separation of $4 \mathrm{w}_{0}$.

In the presence of these two modes, Fig.1 depicts spatial variation of the averaged susceptibility against radial coordinate $\mathrm{x}$ at $\mathrm{y}=0$ plane. The pump field is assumed to be at resonance, $\Delta=0$. The common parameters used in all curves for ${ }^{87} \mathrm{Rb}$ D1-line transition are chosen as: coherence decay rate $\Gamma=0.5 \gamma$, collisional dephasing rate $\gamma_{c}=0.001$, Doppler width $W_{D}=90 \gamma$, and atomic density $\mathrm{N}=1 \times 10^{12}$ atoms $/ \mathrm{cm}^{3}$. Here, $\gamma$ is the spontaneous decay rate of excited state. The real and imaginary components that correspond to refractive index and absorption of the medium, are shown in Fig.1(a) and Fig.1(b), respectively. From Fig. 1(a), it is clear that the refractive index is maximized locally at higher intensity regions of pump beam and resembles two parallel waveguide-like structures with cores $(0.3 \mathrm{~mm} \geq 1$ $\mathrm{x} \mid \geq 0.1 \mathrm{~mm}$ ) and claddings ( $0.1 \mathrm{~mm} \geq|\mathrm{x}| \geq 0.3 \mathrm{~mm}$ ). This gradient in refractive index is responsible for guiding and focusing of probe beam and results in a smaller feature size of probe beam. Similarly, Fig.1(b) shows that the probe field is essentially transmitted at higher intensity regions of pump beam due to formation of two transparency windows centered in core regions of waveguide. In the following, we use this inhomogeneous susceptibility to illustrate the Diffraction-free imaging of different transverse modes carried by pump beam onto a plane wave probe beam.

\section{Probe Beam propagation}

Fig.2 shows the peak normalized intensity of both input and output pump beam along with transmitted probe beam after a propagation of 4-cm-long medium. The size of individual peaks of imaged probe beam profile is measured to be equal to $25 \mu \mathrm{m}$. We define a new parameter called finesse as ratio of the spacing between peaks to the width of peaks. Thus, the finesse of the transmitted probe intensity profile is 4 times smaller than the input pump intensity profile. We also find that the spacing between two peaks of the transmitted probe intensity profile can be controlled by changing focusing position of intense pump beam inside the vapor cell.

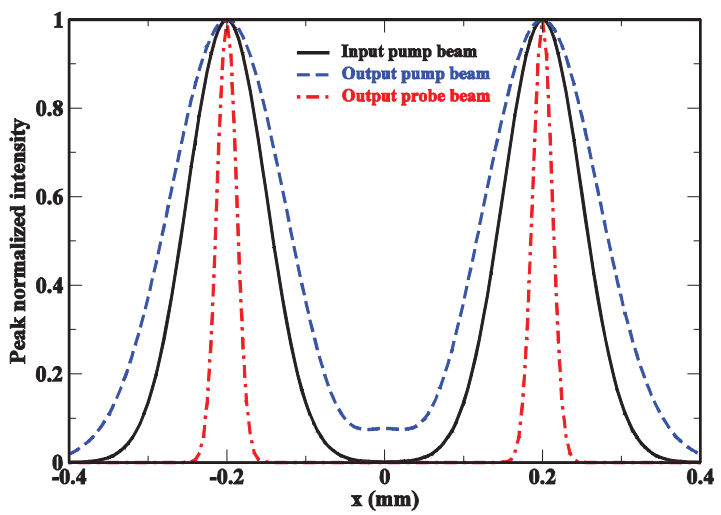

Figure 2. Peak normalized intensity profile of the output imaged probe beam is compared with input and output pump beam. The initial amplitude of probe beam is set as $g_{0}=0.1$. All other parameters are same as in Fig 1. 


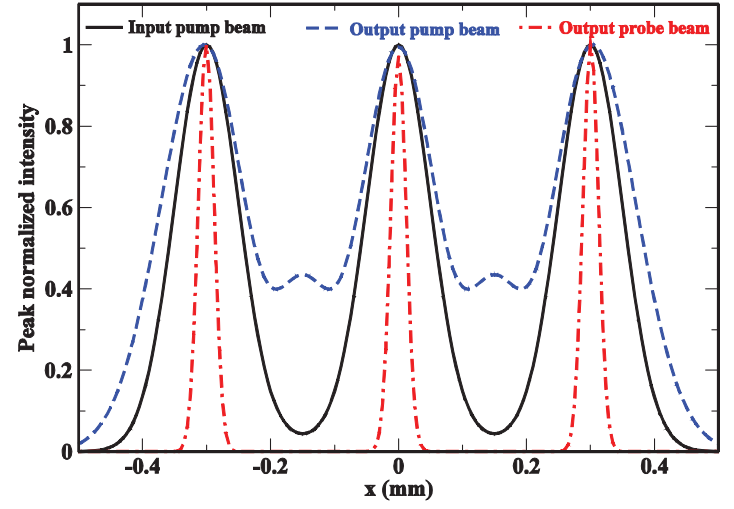

Figure 3. Peak normalized intensity profile cross sections of three Gaussian-modes of pump and imaged probe beam at the output of 4-cm-long vapor cell. All other parameters are the same as in Fig 2.

Next, we proceed to show the transfer of three Gaussian-modes of pump beam onto probe beam. The locations of the three peaks are chosen at $x_{1}=0$, $\mathrm{x}_{2}=0.15 \mathrm{~mm}$ and $\mathrm{x}_{3}=-0.15 \mathrm{~mm}$, as depicted by a solid black line in Fig. 3. In this case, the individual peaks are separated by a distance equal to $3 \mathrm{w}_{0}$ which is less a unit as compared to previous two modes. For convenience, we consider that the individual peaks have equal widths as before. The transverse variation of the probe field susceptibility for three peaks is similar to earlier case of two Gaussian-modes. Fig. 3 shows a comparison between input or output pump and transmitted probe intensity profiles at the exit of 4-cm-long vapor cell. The width of transmitted probe beam is measured to be $25 \mu \mathrm{m}$. This shows that the finesse of the imaged probe is again 4 times smaller than that of pump.

\section{Conclusion}

We have predicted diffraction-free transferring of arbitrary modes carried by a pump beam onto a probe beam. Our method is based on saturated absorption technique arising in a two-level Doppler-broadened atomic vapor system. On numerical simulation shows that two or three Gauusian-modes profiles of the pump beam are efficiently mapped onto the probe beam. Even though the pump image is severely distorted after propagating several Rayleigh length, the probe beam acquires the multimode structure nicely. The feature size of the transmitted probe images is reduced by 4 times compared to that of the original pump beam structure. The present work opens the possibility in all-optical imaging techniques and creating small structures in optical lithography.

\section{Acknowledgement}

Authors gratefully acknowledge funding from SERBDST, Government of India (File No. PDF/2016/002679).

\section{References}

[1] Born, M. and Wolf , E., [Principles of Optics], Cambridge University Press, Cambridge England, (1999). [2] Saleh, B. A. A. and Teich, M. C., [Fundamentals of Photonics], John Wiley \& Sons, New York, (1991).

[3] Lord Rayleigh, "Investigations in optics, with special reference to the spectroscope”,Philos. Mag. 8, 261 (1879). [4] R. Y. Chiao, E. Garmire, and C. H. Townes, "SelfTrapping of Optical Beams” Phys. Rev. Lett. 13, 479 (1964).

[5] Y. S. Kivshar, B. Luther-Davies, "Dark optical solitons: physics and applications” Phys. Rep. 81 298, (1998).

[6] J. E. Bjorkholm and A. A. Ashkin, "cw Self-Focusing and Self-Trapping of Light in Sodium Vapor”, Phys. Rev. Lett. 32, 129 (1974).

[7] T. Hong, "Spatial Weak-Light Solitons in an Electromagnetically Induced Nonlinear Waveguide” Phys. Rev. Lett. 90, 183901 (2003).

[8] H. Kang and Y. Zhu, "Observation of Large Kerr Nonlinearity at Low Light Intensities”, Phys. Rev. Lett. 91, 093601 (2003).

[9] S. Haroche and F. Hartmann, "Theory of SaturatedAbsorption Line Shapes”, Phys. Rev. A 6, 1280 (1972).

[10] B. Couillaud and A. Ducasse, "Refractive Index Saturation Effects in Saturated Absorption Experiments", Phys. Rev. Lett. 35, 1276 (1975).

[11] M. Sargent III, "Spectroscopic techniques based on Lamb's laser theory”, Phys. Rep. C 43, 223 (1978).

[12] O. N. Verma, L. Zhang, J. Evers, and T. N. Dey, "Optical cloning of arbitrary images beyond the diffraction limits,” Phys. Rev. A 88, 013810 (2013).

[13] O. N. Verma and T. N. Dey, "Enhancement of image resolution beyond the diffraction limit by double dark resonances,” Phys. Rev. A 89, 033830 (2014). 


\title{
Optical and Thermal Time-Dependent Analysis for Simulating Thermal Lens Effect by High Power Lasers
}

\author{
Shinji Kameda \\ ${ }^{a}$ Laser Optics Department in Sumitomo Electric Hardmetal Corporation, \\ 1-1-1 Koyakita, Itami-shi, Hyogo, 664-0016, Japan
}

\begin{abstract}
In the conference, it will be discussed what kind of simulating method is needed to calculate thermal lens effects, especially for transmitting optical components in laser manufacturing machines, such as beam shapers. A finite element method (FEM) is used for the thermal analysis, and a ray-trace based optical simulation is used for the optical analysis. We developed a time-dependent simulating tool as a co-working system between these analyses. Furthermore, we compared simulated results and experimental results, and good correlation is obtained. Finally, distortion with aberration is calculated for a beam shaper.
\end{abstract}

Keywords: thermal lens effect, high power, beam shaping, analytical model.

\section{Introduction}

There are lots of laser manufacturing systems using high power lasers for cutting, welding, or drilling. During these processes, optical elements such as focusing lenses, beam shapers and protective windows in these systems are heated by laser resources [1-2]. In a bad case, usual glasses might be broken as soaring temperature, so pure fused silica are often used as a material of these optical elements.

Because silica has a low value of its heat transfer coefficient, the temperature distribution in a protective window made of silica is slowly modified depending on the irradiating time. On the other hand, for good productivity, high power lasers are turned on in a few ten seconds as a process. Therefore, the temperature distribution in optical elements is not saturated, before the laser is turned off. So, a time-dependent simulation is needed in order to simulate focal shifts, especially using glass material for an optical wavelength band from $\sim 1.0 \mu \mathrm{m}$ to $\sim 0.2 \mu \mathrm{m}$.

In this paper, a time-dependent simulation is adopted for calculating temperature distributions. Temperatures are converted into refractive indices, so that the focal shift is simulated in optical analysis based on ray tracings. Analytical and Experimental results have a good correlation.

\section{Analytical Model and Experimental Setup 1. Thermal lens effect}

A schematic of the thermal lens effect is shown in figure 1. Optical lenses or protective windows used with high power lasers $(>\sim 100 \mathrm{~W}$ of the average output) are heated even if they have little absorption $(<\sim 100 \mathrm{ppm}$ of the absorption coefficient). Heated lenses or windows have a temperature distribution. Refractive indices are able to be calculated as a product of $\mathrm{dn} / \mathrm{dT}$ and $\Delta \mathrm{T}$, where $\mathrm{dn} / \mathrm{dT}$ is a coefficient to convert temperatures into refractive indices and $\Delta \mathrm{T}$ is temperature shift from a reference. Therefore, lenses or windows become to have index distributions, as apparent lenses.

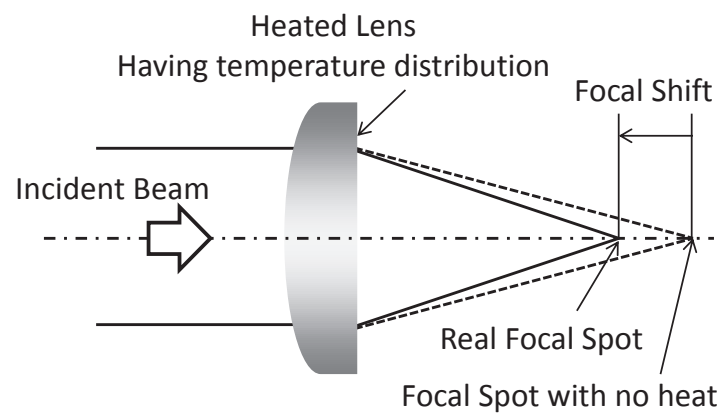

Figure 1. A schematic of thermal lens effect 


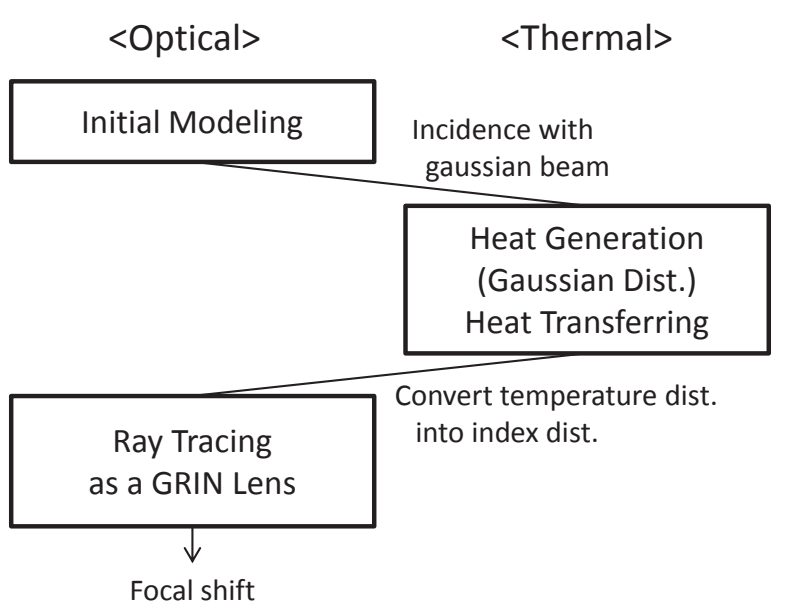

Figure 2. A flow chart of the analytical model

\section{Analytical Model}

The flow chart of thermal and optical simulations is shown in figure 2. First of all, an initial model is made optically. The Gaussian beam is usually used as an incident beam, so the heat generation in thermal analysis has a Gaussian distribution. Simulated temperature distribution is converted into refractive-index distribution. Finally, ray tracing with a graded index lens derives focal shift amount.

\section{Experimental Setup}

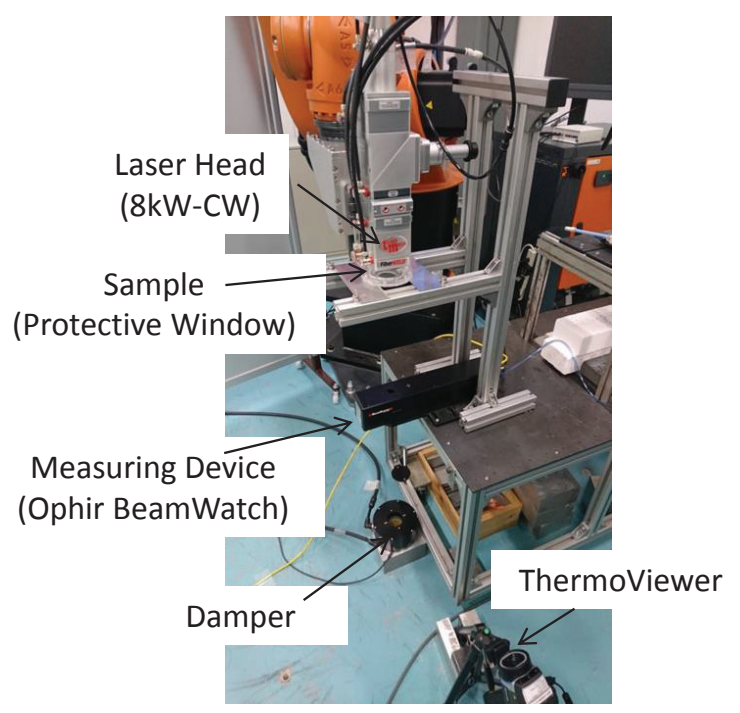

Figure 3. Experimental setup

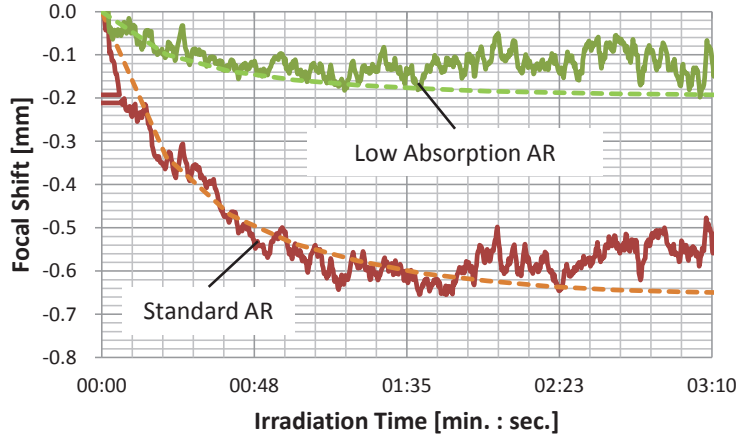

Figure 4. Results from analysis(dotted line) and from experiment(solid line)

Figure 3 shows an experimental setup to measure focal shifts. The Incident beam from the $8 \mathrm{~kW}-\mathrm{CW}$ fiber laser head is focused with a focal length of $500 \mathrm{~mm}$. Samples are $(\phi 50-5 \mathrm{t})$ various AR(Anti-Reflective) coated protective windows whose material is a fused silica, and are placed on an acrylic plate with a hole at $250 \mathrm{~mm}$ from the head.

The focal shift is measured with the BeamWatch ${ }^{\mathrm{TM}}$ which is a product of Ophir Optronics Solutions Ltd.[3]. A thermo-viewer is used to measure temperature distributions. All experiments are prepared and conducted at the Laser R\&D Center in NADEX PRODUCTS CO., LTD. [4].

\section{Accuracy of the Analytical Model}

Results are shown in figure 4. Focal shift amount against irradiation time is calculated as the dotted line and measured as the solid line. Upper and lower lines are for each sample with AR coatings which have lower (60 66ppm) and standard (200 240ppm) absorption coefficients, respectively.

Both lines have good correlation with each other. It is reproduced how two kinds of AR coating effect to the focal shift amount. Time-dependency is also met with measured results. The accuracy of this simulation is sufficiently confirmed. 

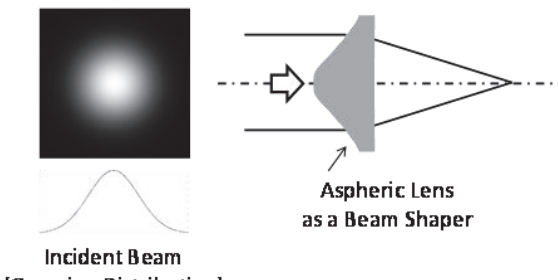

(Gaussian Distribution)

Figure 5. A conceptual diagram of a beam shaper.

Aspheric coefficients are designed for realizing a spot

with a flattop distribution from Gaussian beam.

\section{Results and Discussion}

In the conference, simulated result of an aspheric lens which is used as a beam shaper will be shown. Not only focal shift but also aberrations and intensity profiles are to be explained.

Figure 5 shows a conceptual diagram for a kind of beam shapers. The incident beam has an intensity with Gaussian distribution. The aspheric lens is designed here for realizing flattop distribution as a focal spot. By the way, beam shapers are for various spots such as ring or turned-T shapes in order to realize welding with beautiful beads or less sputtering.

With the thermal lens effect, simulated result from the time-dependent analysis is shown as figure 6. The wavefront is distorted at the exit pupil of the beam shaper. Thus, flattop distribution is also distorted. With standard
AR coating, the homogenity gets worse from $2[\%]$ to $10[\%]$. With low absorption AR coating, the homogenity of $4[\%]$ is to be. It is confirmed that adopting the low absorption AR is valid for suppressing the thermal lens effect as distortions of intensity distributions.

\section{References}

[1]J. Paulo Davim, [Lasers in Manufacturing], John Wiley and Sons Inc., Chapter 2 (2012).

[2]Narendra B. Dahotre, Sandip Harimkar, [Laser Fabrication and Machining of Materials], Springer Science and Business Media (2008).

[3]https://www.ophiropt.com/laser--measurement/beamprofilers/products/High-Power-Beam-

Profiling/BeamWatch

[4]http://www.nadex-p.jp/laser_rd/index.html

\section{Acknowledgement}

The auther especially thanks to NADEX PRODUCTS CO., LTD. who prepared and conducted the experiment. (a)

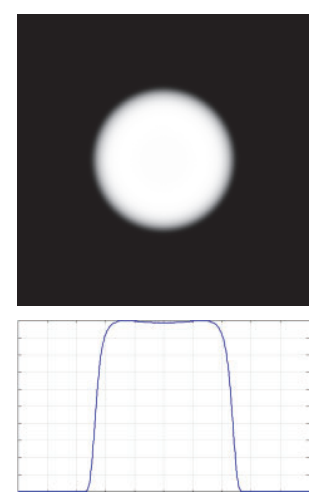

(b)

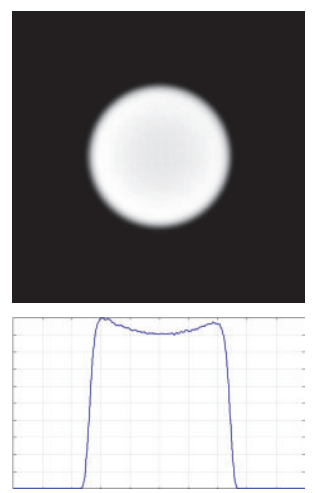

(c)

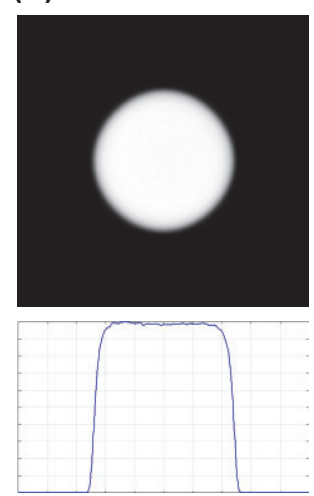

Figure 6. Intensity distributions and their cross-sections of the output spots at 40sec. after turning on the laser.

(a) ideal with no heating, (b) standard AR, and (c) low-absorption AR. 


\title{
Emission lifetime measurement of optically trapped single particles by using stimulated emission
}

\author{
Syoji Ito*a $^{*_{a}}$,Shunsuke Okamoto ${ }^{a}$, Kenji Setoura $^{a}$, Hikaru Sotome $^{a}$, Hiroshi Miyasaka $^{a}$ \\ Division of Frontier Materials Science and Center for Promotion of Advanced Interdisciplinary \\ Research, Graduate School of Engineering Science, Osaka University, Toyonaka, Osaka 560-8531, \\ Japan
}

\begin{abstract}
To detect excited-state dynamics in small area, fluorescence detection is a powerful approach because of its high sensitivity even at the single-molecule level. Though TCSPC is a typical method for emission dynamics measurement under optical microscope the temporal resolution is limited by the instrumental response time (ca. $50 \mathrm{ps}$ ). To attain higher temporal resolution, we propose an approach not limited by the instrumental response time in the present study. We have demonstrated emission lifetime measurement on the basis of pump-dump process of the excited state of a fluorescent dye whose time resolution is in principle determined by the temporal duration of light pulse. The approach was successfully applied to the emission lifetime measurement of dyes in single optically trapped droplets in water.
\end{abstract}

Keywords: optical tweezer, single droplet, fluorescence lifetime, stimulated emission

\section{Introduction}

Photofunctional mesoscopic materials have been attracting considerable attention and intensively studied in the last two decades. There have been a wide variety of demonstrations such as, organic nano/microcrystals exhibiting photomechanical responses [1], semiconductor nanoparticles with photocatalytic activities [2], plasmonic particles as light-field enhancers for spectroscopy [3] and efficient photothermal converters [4], etc.

For the rational design of photofunctional mesoscopic materials, it is crucial to investigate ultrafast dynamics in the electronically excited state by means of time-resolved spectroscopic techniques with high temporal resolution. Such mesoscopic photofunctional systems intrinsically include microheterogeneity, the investigation at the single particle level is therefore a powerful approach for elucidation of their excited-state dynamics depending on size, shape, and microscopic environment.

A common specimen for single-particle spectroscopic measurement is individual particles immobilized on solid surface or in transparent solid matrix. Meanwhile the measurement of ultrafast excited-state dynamics of single particles suspended in solution phase is rather difficult owing to their Brownian motion. Optical tweezer is a promising candidate to trap single particle at a certain position in solution for single-particle spectroscopy.

To detect excited-state dynamics in such small area, fluorescence detection is a powerful approach compared with absorption spectroscopy. Fluorescence measurement is a background-free detection leading to the high sensitivity enabling the detection even at the singlemolecule level. Time-correlated single-photon counting (TCSPC) is a typical method for time-resolved emission detection under optical microscope. The temporal resolution of TCSPC is limited by the instrumental response time of a detection system including photonmultiplier and other electronic systems. To attain higher temporal resolution, a different approach not limited by the instrumental response time is required. In the present study, we have developed a method based on the pump and dump process whose time resolution is in principle determined by the temporal width of the light pulse so as to attain higher temporal resolution of microscopic fluorescence detection.

*E-mail: $\underline{\text { sito@chem.es.osaka-u.ac.jp }}$ 


\section{Experiment}

\section{Experimental setup}

Two light pulses with different wavelengths were used for the measurement. The first light pulse (pump pulse; wavelength $400 \mathrm{~nm}$, pulse duration $100 \mathrm{fs}$ ) was used for photoexcitation of samples. The second pulse (wavelength $800 \mathrm{~nm}$, pulse duration $12 \mathrm{ps}$ ) was introduced after a timedelay, $\Delta \mathrm{T}$, with respect to the first pulse. The second pulse is used for deactivation of the excited state of sample through stimulated emission. The $800-\mathrm{nm}$ pulse was also used for optical trapping of single particle. The emission intensity of sample was measured as a function of the time interval $\Delta \mathrm{T}$, providing the time profile of emission that was reversely proportional to the emission decay curve of the sample.

The temporal resolution of this measurement was determined by the pulse duration of the dump pulse. The detection efficiency is limited by the dumping efficiency and the two-photon absorption cross section of sample.

\section{Sample}

As a sample for fluorescence lifetime measurement on the basis of the pump-dump scheme under optical microscope, we prepared ethanol solution of a fluorescent dye, 4-(2-(6-(Dibutylamino)-2-naphtalenyl)-ethenyl)-1-(3sulfopropyl)-pyridinium hydroxide inner salt (Di-4ANEPPS). To demonstrate emission lifetime measurement of single optically trapped particles, we also prepared microdroplets of chloroform containing LDS722 suspended in water.

\section{Results and Discussion}

Figure 1 shows the time profile of the fluorescence signal of di-4-ANEPPS in solution measured with the present method. The time constant of the decay is determined to be $311 \mathrm{ps}$; this value is in very good agreement with the fluorescence lifetime obtained by the conventional TCSPC method. This agreement demonstrates the validity of the present approach.

The plot in figure 1 shows that when the delay time is slightly negative, the integrated emission intensity decays at approximately $30 \mathrm{ps}$, which was obtained by analyzing the time when the intensity decreases from $90 \%$ to $10 \%$. Since the temporal resolution of this method is approximately $10 \mathrm{ps}$, the decay time is sufficiently longer than the pulse width of the dump pulse.

Furthermore, we applied this technique to measure emission lifetime of a dye, LDS722, in a single microdroplet of chloroform optically trapped in water. The emission lifetime of the dye in the trapped single droplet showed very good agreement with that in bulk solution. This demonstrated the validity of the present approach for track the excited-state dynamics in single particle suspended in solution.

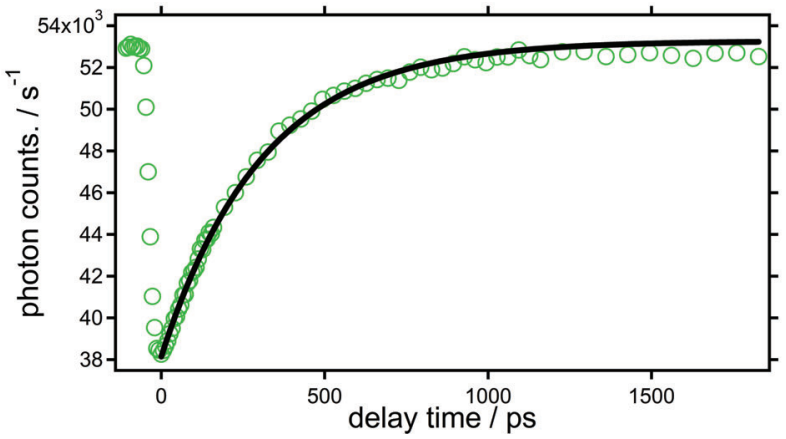

Figure 1. The time profile of integrated emission intensity of Di-4-ANEPPS in chloroform measured by the present pump-dump method (open circles). The solid curve in the plot is the analytical result.

\section{References}

[1] Irie, M., Fukaminato, T., Matsuda, K. and Kobatake, S., "Photochromism of Diarylethene Molecules and Crystals: Memories, Switches, and Actuators," Chem. Rev. 114, 12174-12277 (2014).

[2] Pal, B., Torimoto, T., Okazaki, K. and Ohtani B., "Photocatalytic syntheses of azoxybenzene by visible light irradiation of silica-coated cadmium sulfide nanocomposites," Chem. Commun. 2007, 483-485.

[3] Mulvaney, P., "Surface Plasmon Spectroscopy of Nanosized Metal Particles," Langmuir, 12, 788-800 (1996).

[4] Setoura, K., Ito, S. and Miyasaka, H., "Stationary bubble formation and Marangoni convection induced by CW laser heating of a single gold nanoparticle," Nanoscale 9, 719-730 (2017). 


\title{
Metalens array generated structured light for distance sensing
}

\author{
Mu Ku Chen*a,b, Cheng Hung Chu ${ }^{\mathrm{b}}$, Hsin Yu Kuo ${ }^{\mathrm{a}, \mathrm{b}}$, Ren Jie Lin ${ }^{\mathrm{b}}$, , and Din Ping Tsai ${ }^{\mathrm{a}, \mathrm{b}}$ \\ ${ }^{a}$ Research Center for Applied Sciences, Academia Sinica, Taipei 11529, Taiwan \\ ${ }^{b}$ Department of Physics, National Taiwan University, Taipei 10617, Taiwan \\ Email: d04245001@,ntu.edu.tw
}

\begin{abstract}
Metalens are composed of designed subwavelength nanostructures at an interface which can control the properties of incident light. Here we demonstrated a GaN metalens array to project a light spots array which can be a light shape generator in the structured light applications. The distance between two light spots is a function with the distance of target. A $10 \times 10$ achromatic metalens array which arranged by the single metalens diameter is $20 \mathrm{um}$. The advantages of this metadevice is light weight, small, ultrathin, durable and easy to compact with other device. Our design provides a new avenue for the structure light application such as distance sensing and 3D environmental construction.
\end{abstract}

Keywords: Metalens, Structured light, distance sensing.

\section{Introduction}

Metalenses consist of a large number of optical nanoantennas capable of focusing the incoming light wavefront [1-7]. Metalens have great ability in light focusing and can be tailored to exhibit varied functionalities in ultrathin optical applications. A metalens is realized by using integrated-resonant unit elements whose geometric phase are combined with phase compensation from integrated-resonant unit elements. Metalens are composed of designed subwavelength nanostructures at an interface which can control the properties of incident light. Here we demonstrated a GaN metalens array to project a light spots array which can be a light shape generator in the structured light applications. The distance between two light spots is a function with the distance of target. A $10 \mathrm{x}$ 10 achromatic metalens array which arranged by the single metalens diameter is $20 \mathrm{um}$. The advantages of this metadevice is light weight, small, ultrathin, durable and easy to compact with other device. The working wavelength is cover all visible and can be extent in to the near infrared region. Our design provides a new avenue for the structure light application such as distance sensing and $3 \mathrm{D}$ environmental construction which can make us live better.

\section{Results and Discussion}

For the experiment setup, we use a 532 laser irradiated on the $60 \times 60$ achromatic metalens array and a 20x objective was used to collected the focused spot from the achromatic metalens array in transmission. A Screen was placed behind the objective and set several distance from the metalens array. Figure 1 shows the photographs of light focused spots array on the screen with distance from $30 \mathrm{~cm}$ to $80 \mathrm{~cm}$. The size of $10 \times 10$ light focused spots array was increased with the distance was increased. To quantify the experimental results, we measured the separation between two spots on each photograph as shown in Figure 2. When the real distance was set from $30 \mathrm{~cm}$ to $90 \mathrm{~cm}$, the separation between two spots was increased from 47 pixels to 103 pixels.
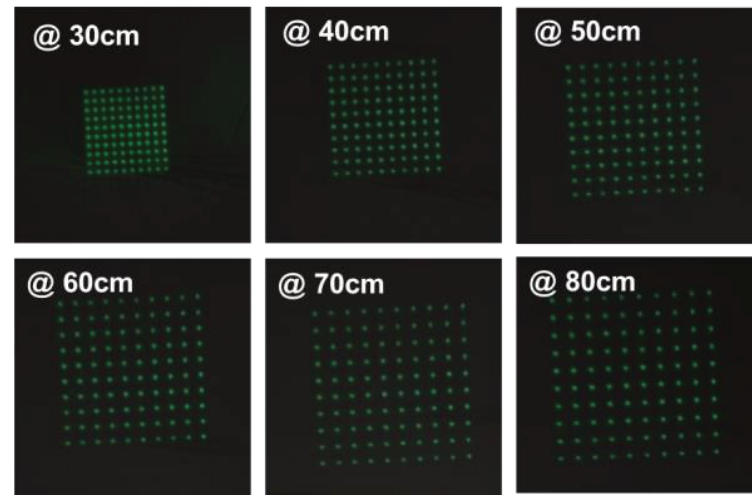
Figure 1. Photograph of light focused spots array on the screen with distance from $30 \mathrm{~cm}$ to $80 \mathrm{~cm}$.

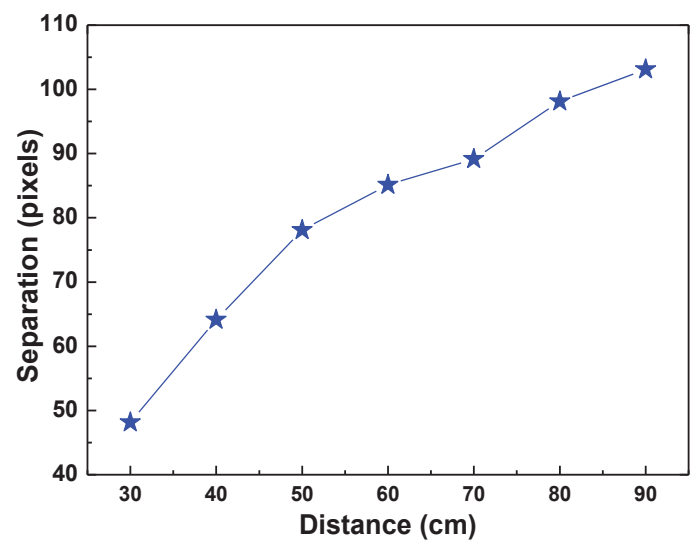

Figure 2. Measurement results of separation of light focused spots array corresponding with real distance between metalens array and target.

\section{Acknowledgment.}

The authors acknowledge financial support from the Ministry of Science and Technology, Taiwan (Grant No. MOST-107-2112-M-001-042-MY3, MOST-107-2911-I001-508, MOST-107-2911-I-001-510, MOST-107-2923M-001-010-MY3) and Academia Sinica (Grant No. AS103-TP-A06, AS-TP-108-M12, AS-iMATE-108-41), They are also grateful to the National Center for Theoretical Sciences, the NEMS Research Center of National Taiwan University, the National Center for High-Performance Computing, Taiwan, and the Research Center for Applied Sciences, Academia Sinica, Taiwan for their support.

\section{References}

1. S. M. Wang, P. C. Wu, V.-C. Su, Y.-C. Lai, C. H. Chu, J.-W. Chen, S.-H. Lu, J. Chen, B. B. Xu, C.-H. Kuan, T. Li, S. N. Zhu and D. P. Tsai, Nature Comm. 8, 187 (2017). 2. B. H. Chen, P. C. Wu, V.-C. Su, Y.-C. Lai, C. H. Chu, I. C. Lee, J.-W. Chen, Y. H. Chen,Y.-C. Lan, C.-H. Kuan and D. P. Tsai, Nano Lett. 17, 6345 (2017).

3. S. M. Wang, P. C. Wu, V.-C. Su, Y.-C. Lai, M.-K. Chen, H. Y. Kuo, B. H. Chen, Y. H. Chen, T.-T. Huang, J.-
H. Wang, R.-M. Lin, C.-H. Kuan, T. Li, Z. Wang, S. Zhu and D. P. Tsai, Nature Nanotechnology 13, 227 (2018).

4. V.-C. Su, C. H. Chu, G. Sun and D. P. Tsai, Optics Express 26, 13148 (2018).

5. M. L. Tseng, H. - H. Hsiao, C. H. Chu, M. K. Chen, G. Sun, A. - Q. Liu and D. P. Tsai, Adv. Optical Mater. 6, 1800554 (2018).

6. H. - H. Hsiao, Y. H. Chen, R. J. Lin, P. C. Wu, S. Wang, B. H. Chen and D. P. Tsai, Adv. Optical Mater. 6, 1800031 (2018).

7. R. J. Lin, V. -C. Su, S. M. Wang, M. K. Chen, T. L. Chung, Y. H. Chen, H. Y. Kuo, J. W. Chen, J. Chen, Y. T. Huang, J.H. Wang, C. H. Chu, P. C. Wu, T. Li, Z. Wang S. Zhu and D. P. Tsai, Nature Nanotechnology, doi:10.1038/s41565-018-0347-0 (2019). 


\title{
Emergence of optical extreme events from a modified Fresnel zone plate
}

\author{
Amanda K. Fritsch ${ }^{\mathrm{a}}$, Ricardo R. B. Correia ${ }^{\mathrm{a}}$, Cristian Bonatto ${ }^{\mathrm{a}}$ \\ ${ }^{a}$ Instituto de Física, Universidade Federal do Rio Grande do Sul, 91501-970 Porto Alegre, Brazil
}

\begin{abstract}
This work discusses theoretical results of the investigation of light beams that have their intensity structure during propagation defined by non markovian distribution of phases on the Fresnel zone plate (FZP) framework. Through the probability density function (PDF) of intensity events on the FZP focal plane, a remarkable deviation from the Rayleigh distribution of a markovian distribution of phases is established for these other stochastic cases. Experimentally all computed cases are reproduced using the wavefront shaping displayed on a spatial light modulator (SLM) considering the resolutions of this device and from the acquisition camera. Such extreme events observed for a linear superposition of waves are of general interest for optical systems, since further amplification of the observed structured intensity profiles within gain media have to be carefully considered.
\end{abstract}

Keywords: wavefront shaping, diffraction and scattering, image forming, beam characteristics, extreme events.

\section{Introduction}

For centuries, sailors and fishermen have told tales of "freak", rogue waves (RW) that can peak more than $30 \mathrm{~m}$ above the surface appearing from nowhere and disappearing without a trace. These gigantic oceanic rogue waves are the most known example of rare high-amplitude events called extreme events. Such events are not restricted to the ocean, but are also observed in many other physical systems. In the past decades, an increasing number of studies have been dedicated to extreme events in various systems $[1,2]$.

Systems featuring extreme events can be identified by their characteristic long-tailed distribution of the wave height. Conventional statistical models accounting for describing the height of ocean waves (for example, Gaussian or Rayleigh) suggest that ocean RW's should only be observed one time in centuries, which contradicts the observations. In fact the height of ocean waves follow a "L-shaped" statistic, i.e., most waves have small amplitudes and high-amplitude events far from the median are also observed with low probability, but more frequently than previous models predicted [3, 1]. Extreme events have been quantitatively identified in a number of different ways. A common way to define a rogue wave by the abnormality index, which is the ratio between the height of the wave and the average wave height among one-third of the highest waves. Every event whose abnormality index is larger than 2 is considered a rogue wave $\left(I_{R W} / I_{1 / 3}>2\right)$. RW can also be defined based on the standard deviation $(\sigma)$ of the distribution for wave heights, i.e., how its height deviates from the average. These definitions have the advantage of being precise and the drawback of being quite arbitrary, although it has been applied in several studies [1,2].

In optics, the more accessible information is not the field amplitude, but rather the light intensity. A statistical analysis can be made computing a histogram of the intensity peaks and RW are identified according to a chosen definition. The mechanisms that form a extreme event are still actively studied, it has been showed that they can be associated with different effects: from linear effects such as directional focusing to nonlinear effects associated with the growth of surface noise forming localized wave structures [1].

It is possible to control the emergence of extreme events in a light beam through wavefront modulation, a powerful and flexible tool to generate light with specific profiles and apply to study new systems. The modulation form employed here is based on the linear superposition of a Fresnel zone plate (FZP) construction, which is ordinarily used to focus light. A FZP consists in several radially symmetric zones of equal area, a binary phase alternating between 0 and $\pi$ being is assigned to each zone. The zones are spaced such that all the point sources at the plate constructively interfere at the focal plane. Conventional FZP as the one described above is completely coherent case. By drawing the phases randomly through the plate following a markovian process a completely incoherent pattern can be produced. 
a)

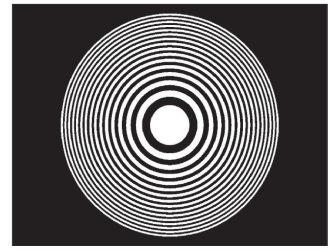

b)

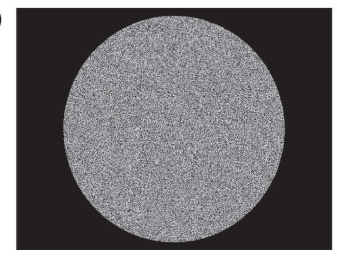

Figure 1. a) Conventional FZP. b) Completely incoherent phase pattern, with markovian draw of phases.

\section{Modified Fresnel zone plate}

In this work we introduce a phase pattern based on the FZP, designed to produce a light beam with a null central intensity along the propagation axis, while carrying non-markovian properties in two dimensions. To generate the null intensity center we rely on the principle of the FZP, except that in this case we produce a destructive interference. This can be achieved dividing each zone in angular regions of equal area and of different phases, ensuring that once a region receives a phase $\varphi$, another region in the same zone receives a phase $\varphi+\pi$. Therefore we guarantee the same amount of point sources with opposite phases and the geometry of the phase pattern makes certain that the destructive interference occurs at the center, along all the propagation axis. The phase of each region are drawn following a non-markovian criteria such that we have the same portion of each phase in both radial and angular directions. An example of phase pattern is shown in Figure 2, the pattern presents 8 phase levels distributed in 32 regions and 32 zones.

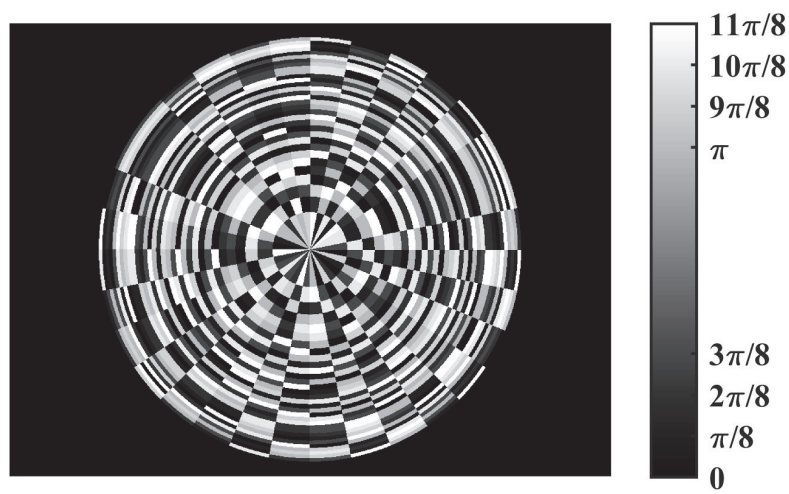

Figure 2. Phase pattern of a modified FZP with a non-markovian draw of phases. This phase pattern has 8 phase levels assigned in 32 zones and 32 regions.

We also investigate some variations of this pattern where we introduce more correlation to the drawn, separate the group of phases in two sub-groups (the first and the third quadrant) and proceed to draw without mixing them. Since the two dimensions are independent, we can compare cases without this separation with cases presenting one dimension divided (testing one dimension at time) and cases with the two dimensions divided. We also analyze the case of modified FZP presenting a markovian drawn that does not requires the destructive pairs. a)

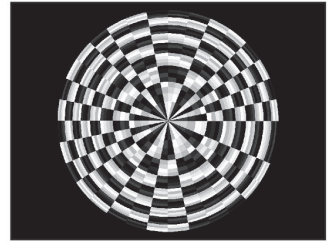

c)

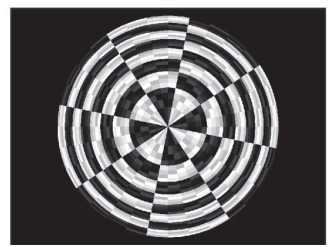

b)

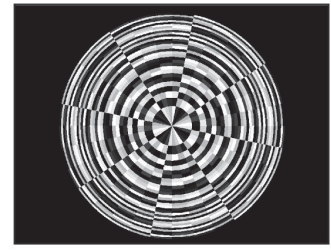

d)

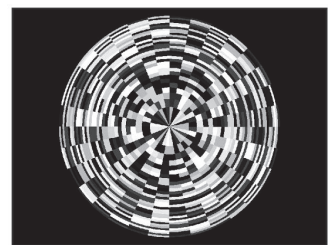

Figure 3. Variations of modified FZP with a) only radial sub-division, b) only angular sub-division, c) both dimensions sub-divided and d) markovian draw of phases in both dimensions. These phase patterns have the same 8 phase levels assigned in 32 zones and 32 regions.

\section{Intensity profile}

We numerically simulate the propagation of a light beam modulated by the phase pattern described above. Here we analyse the intensity at a distance from the mask corresponding to the focal plane of the equivalent FZP, which is a well known plane for the coherent case. Figure 4 presents the average intensity distribution after 100 realizations with differents draws for each case of correlation. A dark spot can be observed at the center of the beam for cases with non markovian draw of phases, as well as intense events close to this spot. This is a characteristic of extreme events, it has been showed that RW often appears accompanied by "holes", i.e. deep vales occurring close to the largest crest [4].

All numerical parameters were chosen to reproduce the experimental conditions present in our setup. Briefly, we illuminate a SLM (Spatial Light Modulator, 1024x768) with the collimated beam of an expanded beam of a $405 \mathrm{~nm}$ laser. The propagated profile is registered with a CMOS (1280 x 960) camera. Phase 
values associated to grey image levels are directly sent to the SLM, displaying the modified FZP's. So far we reproduce all simulated images with an exceptional quality, limited to the resolution of these devices. a)

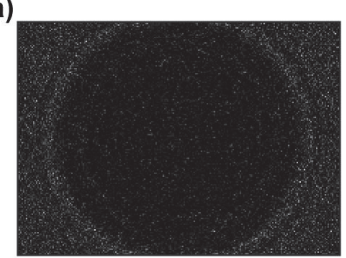

c)

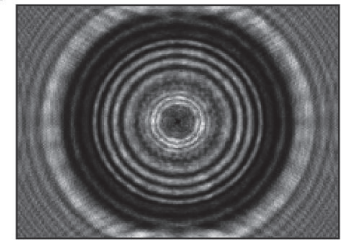

e)

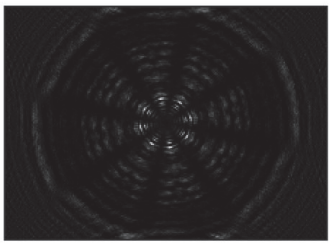

b)

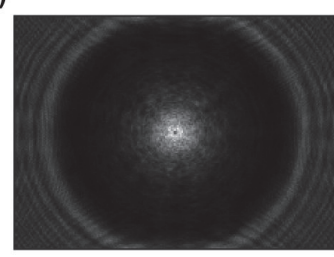

d)

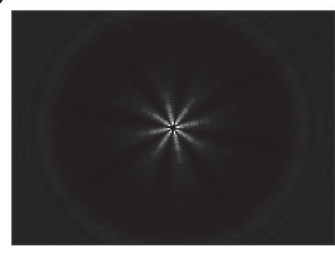

f)

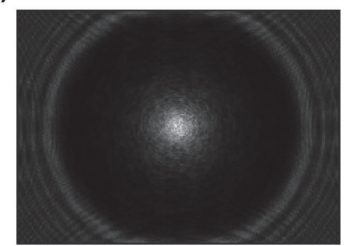

Figure 4. Average intensity profile of beam modulated by a) completely incoherent phase pattern, by modified FZP's with non-markovian draw b) without sub-division, c) only radial sub-division, d) only angular sub-division, e) both dimensions sub-divided and f) markovian draw of phases in both dimensions.

\section{Results and Discussion}

The intensity profile of light beams modulated by these phase pattern obeys a "L-shaped" statistic, as expected for systems presenting extreme events. When comparing its probability density functions (PDF) to a negative exponential, characteristic of the Rayleigh distribution, one can establish how much each case deviate from a completely markovian case, allowing to identify which case of phase distribution favours the emergence extreme events.
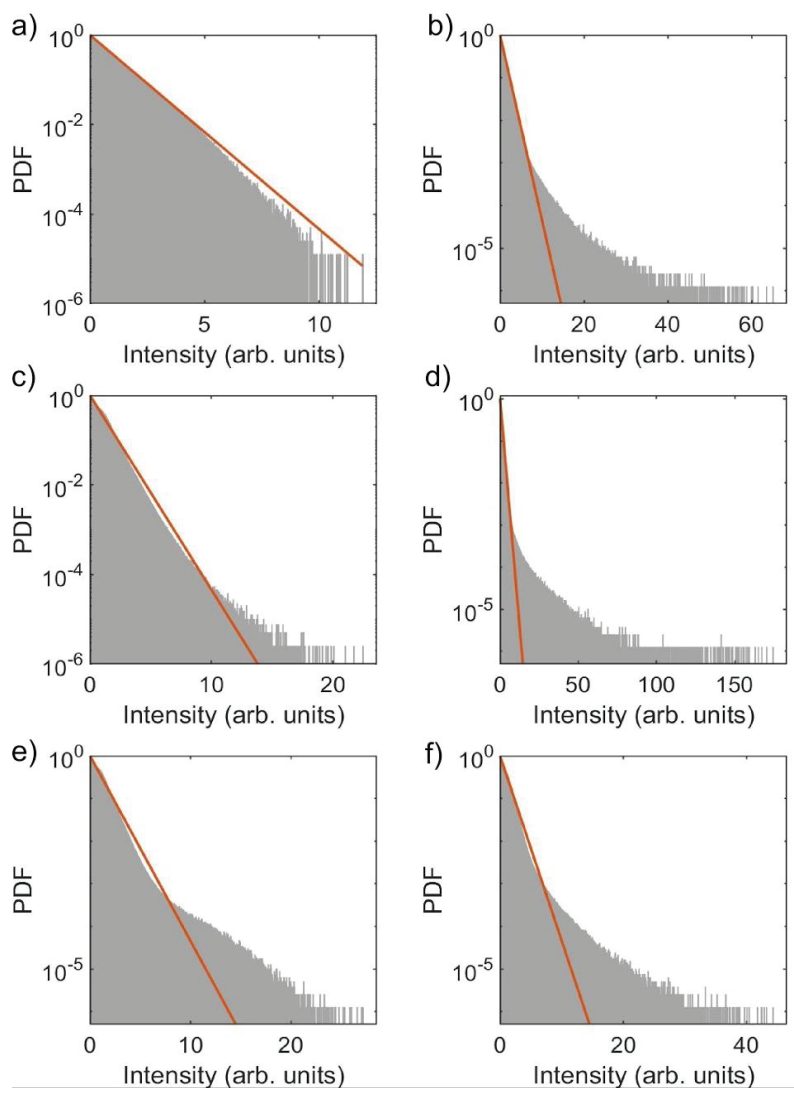

Figure 5. Probability density function (PDFs) for intensity profile of the described cases in figure 4 , in the presented order. The red line denotes a negative exponential fitting, which is a signature of Rayleigh statistics.

The completely incoherent (markovian) phase pattern leads to a intensity distribution following a negative exponential, which is a signature of Rayleigh statistics [5]. Deviations from the Rayleigh statistics can be observed in all tested cases of modified FZP. Such deviations can be attributed to the structural organization of the pattern, even when the phase distributions presents a low correlation, suggesting that the FZP phase relation is not completely lost by the modifications and some constructive interferences still occur close to the center, as illustrated in Figure 4.

We observe that the correlation level of the phase distribution is not the only characteristic that affects the deviation from Rayleigh's statistics, and therefore the probability of emergence of extreme events, the dimension which presents the correlation is also important. Increasing angular correlation results in greater probability and events with higher intensities, on the other hand the deviation is reduced when radial correlation is increased. Such dependence can be 
understood by recalling the conventional FZP, where two successive zones have a phase difference of $\pi$. When the phases were divided in first and third quadrant and organized with a radial correlation, implying that four successive zones will have a phase from the same quadrant the constructive interference does not occur as in the FZP, leading to lower intensities. On the other hand, a less correlated case has more chances to presents two successive zones with a phase difference of approximately $\pi$, presenting more constructive interferences, and hence higher intensities close to the center. A similar argument can be used to explain the case with high angular correlation and low radial correlation. In this case four successive regions of the modified FZP have phase in the same quadrant, e.g., 0 to $\pi / 2$. Consequently the next four zones of the FZP have phases from the opposite quadrant, here from $\pi$ to $3 \pi / 2$. This composition resembles slices of FZP's, which produces intense spots organized angularly and close to the center.

\section{Conclusion}

Non markovian processes and related byproducts, e.g., phase profiles on optical elements, are ubiquitous in nature and in manufactured products. Therefore the structured phase waveforms and the corresponding intensity profiles analyzed in this work provides the comprehensive portrait of how a linear stochastic process may generate optical extreme events. Through statistical analysis we also provided some insight of how the relation between phases affects the emergence of extreme events.

\section{References}

[1]. Dudley, J. M., Dias, F., Erkintalo, M. and Genty, G., "Instabilities, breathers and rogue waves in optics", Nature Photonics, volume 8, pages 755-764 (2014).

[2]. Bonatto, C. et al. Deterministic optical rogue waves. Phys. Rev. Lett. 107, 053901 (2011).

[3]. Solli, D. R., Ropers, C., Koonath, P. and Jalali, B. Optical rogue waves. Nature 450, 1054-1057 (2007).

[4]. Osborne, A. R., Onorato, M. and Serio, M., "The nonlinear dynamics of rogue waves and holes in deep-water gravity wave trains". Phys. Lett. A 275, 386-393 (2000).

[5]. Rayleigh, Lord. On the resultant of a large number of vibrations of the same pitch and of arbitrary phase. Phil. Mag. 10, 73-78 (1880).

\section{Acknowledgement}

This research was supported by Coordenação de Aperfeiçoamento de Pessoal de Nível Superior (CAPES) and Universidade Federal do Rio Grande do Sul (UFRGS). 


\title{
Giant enhancement of cooperative effect in superfluorescence of arranged molecules by nanoscale metallic structures
}

\author{
Hirofumi Shiraki*a, Masayuki Hoshina ${ }^{\mathrm{a}}$, Nobuhiko Yokoshia ${ }^{\mathrm{a}}$, and Hajime Ishihara ${ }^{\mathrm{a}, \mathrm{b}}$ \\ aDepartment of Physics and Electronics, Osaka Prefecture University, 1-1 Gakuen-cho, Naka-ku, \\ Sakai-shi, Osaka, 599-8531, Japan \\ ${ }^{b}$ Department of Materials Engineering Science, Osaka University, 1-3 Machikaneyama-cho, \\ Toyonaka-shi, Osaka, 560-8531, Japan
}

\begin{abstract}
We investigate a superfluorescence of emitters, which are coupled with metallic optical antennas. Superfluorescence is a phenomenon in which high-density emitters in a population inversion state spontaneously form a macroscopic dipole moment through a radiation field and spontaneously emit a pulsed light. We find that the correlation between the emitters is drastically enhanced by a localized surface plasmon of the optical antenna. In addition, when the conditions such as the metal structure and the emitter position are changed, the behavior of the superfluorescence is affected.
\end{abstract}

Keywords: superfluorescence, plasmon, metamaterial, luminescence, nanoparticle

\section{Introduction}

When high-density quantum emitters are in population inversion state, their polarizations come to be correlated with each other through radiation fields. By this process, a large number of the quantum emitters spontaneously form macroscopic dipole moment, and a synchronized photoemission (superfluorescence) occurs. Superfluorescence has three main features. The first is that the peak of the emission intensity is proportional to the square of the number $N$ of the quantum emitters. The second is that the pulse width of the emission is proportional to $1 / N$. Third, the coherence and the directivity exist in emission [1].

Conventional theories of superfluorescence consider all the emitters to exist within the same phase of the radiated light. On the other hand, we have recently developed a theory to analyze the emission time profile of arbitrarilypositioned emitters in arbitrary environment [2].

As is mentioned above, in order for superfluorescence to occur, it is necessary for the quantum emitters to grow the correlation through the radiation field. Meanwhile, when the metallic optical antenna is irradiated, the localized surface plasmons are excited, and the effective electric field is enhanced. By arranging the quantum

*shiraki-2@pe.osakafu-u.ac.jp; phone 072-254-9268 emitter nearby the antenna, the correlation between the quantum emitters may be enhanced due to the correlation between the quantum emitters and the metal optical antennas. On the other hand, because the correlation between the quantum emitters grows due to Purcell effect, it may be attenuated before superfluorescence occurs. Therefore, it is considered that these two effects compete.

Here, we consider a system combining the quantum emitters and the metal optical antenna, and calculate the time profile of the fluorescent intensity. In particular, we examine the change of enhancement by calculating the emission intensity for various metal structures and emitters' position.

\section{Theory}

For calculation of superfluorescence in the dispersed particle system, the following Hamiltonian was used from [2].

$\widehat{H}=\sum_{i}^{N} \hbar \omega_{i} \sigma_{10}^{i} \sigma_{01}^{i}+\sum_{\lambda} \int \mathrm{d} \mathbf{k} \hbar \omega_{\mathbf{k}} b_{\mathbf{k} \lambda}^{\dagger} b_{\mathbf{k} \lambda}$

$-\int \mathrm{d} \mathbf{r} \sum_{i}^{N} \hat{\mathbf{d}}_{i} \cdot \hat{\mathbf{E}}(\mathbf{r})$

The operator $\sigma_{10}^{i}, \sigma_{01}^{i}$ mean the ladder operators, $b_{\mathbf{k} \lambda}^{\dagger}, b_{\mathbf{k} \lambda}$ mean the creation and annihilation operator. $\lambda$ means the polarization direction, $\boldsymbol{k}$ means wavenumber. $\hbar \omega_{i}, \hbar \omega_{\mathbf{k}}$ 
mean the energy of the two level system and photon. $\hat{\mathbf{d}}_{i}$ means the dipole moment, $\hat{\mathbf{E}}(\mathbf{r})$ means the electric field.

The intensity of superfluorescence describes by

$$
\begin{aligned}
n_{p}(t) & =\sum_{\lambda} \int_{d \mathbf{k}} \frac{\partial}{\partial t}\left\langle b_{\boldsymbol{k} \lambda}^{\dagger} b_{\boldsymbol{k} \lambda}\right\rangle(t) \\
& =\frac{2}{\hbar \epsilon_{0}} \sum_{i}^{N} \operatorname{Im}\left[\mathbf{d}_{i} \cdot \mathbf{G}\left(\mathbf{r}_{i}, \mathbf{r}_{i}, \omega_{i}\right) \cdot \mathbf{d}_{i}\left\langle\sigma_{10}^{i} \sigma_{01}^{i}\right\rangle\right] \\
& +\frac{2}{\hbar \epsilon_{0}} \sum_{i \neq j}^{N} \sum_{j \neq i}^{N} \operatorname{Im}\left[\mathbf{d}_{j} \cdot \mathbf{G}\left(\mathbf{r}_{j}, \mathbf{r}_{i}, \omega_{i}\right) \cdot \mathbf{d}_{i}\left\langle\sigma_{10}^{i} \sigma_{01}^{j}\right\rangle\right],
\end{aligned}
$$

where the operator $\mathbf{G}$ is the dyadic green function.

This function contains the position of the particle and the spatial information. Then, for convolving the spatial structure information, we used the numerical calculation method, which is described by the equation as,

$$
\begin{aligned}
\mathbf{G}\left(\mathbf{r}, \mathbf{r}^{\prime}, \omega\right) & =\mathbf{G}_{0}\left(\mathbf{r}, \mathbf{r}^{\prime}, \omega\right) \\
& +\int d \mathbf{r}^{\prime \prime} \mathbf{G}_{0}\left(\mathbf{r}, \mathbf{r}^{\prime \prime}, \omega\right) \chi_{\text {metal }}\left(\mathbf{r}^{\prime \prime}, \omega\right) \mathbf{G}\left(\mathbf{r}^{\prime \prime}, \mathbf{r}^{\prime}, \omega\right) .
\end{aligned}
$$

In order to numerically calculate $\mathbf{G}$, the discrete dipole approximation (DDA) was applied by deforming as follows [3], [4].

$$
\begin{aligned}
\mathbf{G}_{i j}^{0} & =\mathbf{G}_{i j}-\sum_{j^{\prime}} V \mathbf{G}_{i j^{\prime}}^{0} \chi_{j^{\prime}} \mathbf{G}_{j^{\prime} j} \\
& =\sum_{j^{\prime}}\left(\delta_{i j^{\prime}} \mathbf{I}-V \chi_{j^{\prime}} \mathbf{G}_{i j^{\prime}}\right) \mathbf{G}_{j^{\prime} j} \\
& =\sum_{j^{\prime}} \boldsymbol{A}_{i j^{\prime}} \mathbf{G}_{j^{\prime} j} .
\end{aligned}
$$

This makes it possible to calculate $\mathbf{G}$ for arbitrary shapes and conditions. We solve the problem as a simultaneous linear equation of $\mathbf{G}$ in each points.

\section{Results and Discussion}

We calculate the time profile of the fluorescent intensity in the condition that, when a metal plate $(\mathrm{Au}, 30 * 30 * 5$ $\mathrm{nm}^{3}$ ) and 15 emitters are arranged as shown in Fig.1. Here, the emitter radius is $1 \mathrm{~nm}$, and the resonance energy is 1.4 $\mathrm{eV}$. The susceptibility of Au is given by the Drude model. The spacing between the metals is $12.7 \mathrm{~nm}$, whereas the spacing between the emitters is $2.82 \mathrm{~nm}$. For comparison, the calculations are also done when 15 emitters are not evenly spaced.

The calculated result is shown in Fig. 2. We can see that, when the emitters are evenly spaced in the gap region, the fluorescent intensity dramatically increases. Its time profile has the feature of superfluorescence. On the other hand,

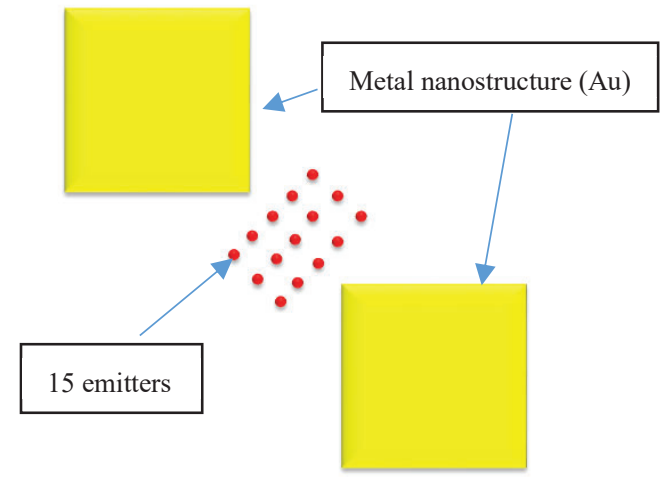

Figure 1: The metal nanostructure and 15 emitters.

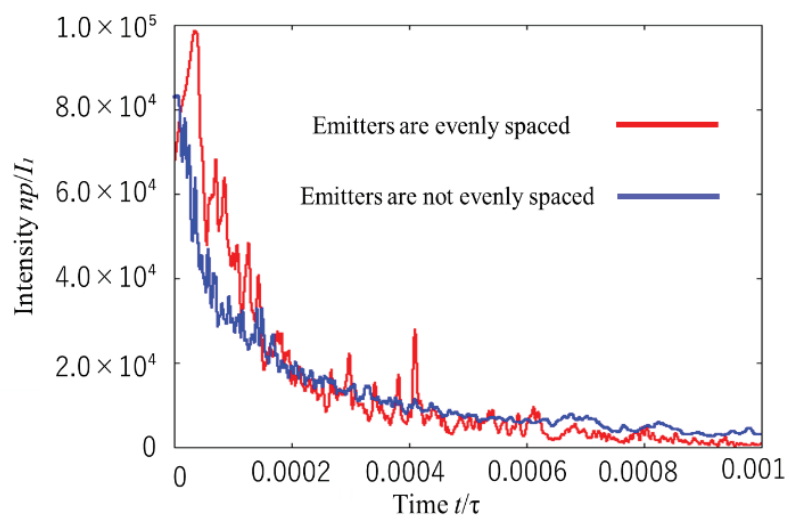

Figure 2: Fluorescent time profiles of the quantum emitters in one gap. $\tau$ is the radiative lifetime of the emitter in vacuum. $I_{1}$ is the initial value of intensity of one emitter in vacuum.

when emitters are not evenly spaced, the feature of superfluorescence does not appear though the strong Purcell effect occurs. Therefore, these results imply that the enhancement the inter-emitter correlation nearby the optical antenna strongly depends on the emitter spacing even though all the emitters lie within a few ten nanometer radius.

\section{Summary and Outlook}

We have shown that a giant enhancement of superfluorescence occurs when quantum emitters are coupled with optical antennas. The spatial configuration of the emitters strongly affects the enhancement of the cooperative effect in the superfluorescence. The present result will open new possibility and potential of quantum emitter ensemble coupled with optical antennas as highefficient luminous materials. 


\section{References}

[1] Dicke, R. H., "Coherence in spontaneous radiation processes." Physical Review 93, 99, (1954).

[2] Yokoshi, N., Odagiri K., Ishikawa, A., and Ishihara, H., "Synchronization dynamics in a designed open system." Physical Review Letter 118, 203601, (2017).

[3] Purcell, E. M. and Pennypacker, C. R., "Scattering and absorption of light by nonspherical dielectric grains."

Astrophysical Journal 186, 705, (1973).

[4] Hoshina, M., Yokoshi, N., Okamoto, H., and Ishihara, H., "Super-resolution trapping: A nanoparticle manipulation using nonlinear optical response." ACS Photonics 5, 318-323, (2018). 


\title{
Generation of pure vector field from the interference of two ellipse fields embedded with C-points and V-points
}

\author{
Sushanta Kumar Pal*a, and P. Senthilkumaran ${ }^{\mathrm{a}}$ \\ *sushanta1985@gmail.com \\ ${ }^{a}$ Department of Physics, Indian Institute of Technology Delhi, New Delhi-110016, India
}

\begin{abstract}
In this article we show that the interference of two three beam pairs can lead to generation of lattice of V-points. Interestingly each three beam pair is embedded with C-points and V-points but their resultant field is embedded with only V-points. A careful observation reveals that interference of a right handed C-point (star) with a left handed C-point (star), both oriented in the same direction, results in a linear polarization. But the interaction between two similar V-points leads to same V-point. The resultant field is embedded with the lowest order generic V-point singularities.
\end{abstract}

Keywords: Singular optics, polarization singularity, interference, optical vortices, stokes vortices

\section{Introduction}

In recent times spatially varying polarization distributions embedded with polarization singularities (C-points and Vpoints) are widely studied among the scientific communities. These are isolated points where some of the parameters related to polarization ellipse is indeterminate ${ }^{1-5}$. C-points and $\mathrm{V}$-points are the singularities in the ellipse and vector fields respectively. Unlike V-points the $\mathrm{C}$-points can occur at any value in the intensity distribution. The neighborhood polarization distributions around the singularity helps in distinguishing the types of polarization singularities. For example the intensity is zero at the singularity for both V-point and dark C-point but the neighborhood polarization distribution is linear for V-point and elliptical for dark Cpoint. A C-point can be left or right handedness. Recently it is shown that interference of three or more vector beams can be used to generate array of these singularities ${ }^{6-10}$. We extend this idea to interference of two three vector beam interference pairs to generate pure vector field distributions embedded with lowest order generic V-point singularities.

\section{Three beam Interference}

We have taken two pairs of three axially equidistant noncoplanar linearly polarized plane beams whose electric field vectors are oriented in a desired fashion for the formation of C-point-V-point lattice structure. The wave vectors and electric field vectors corresponding to these interfering beams are taken as given in $\operatorname{ref}^{10}$. The propagation vectors of all the interfering beams have same $z$-component as shown in Fig. 1(a).

(a)
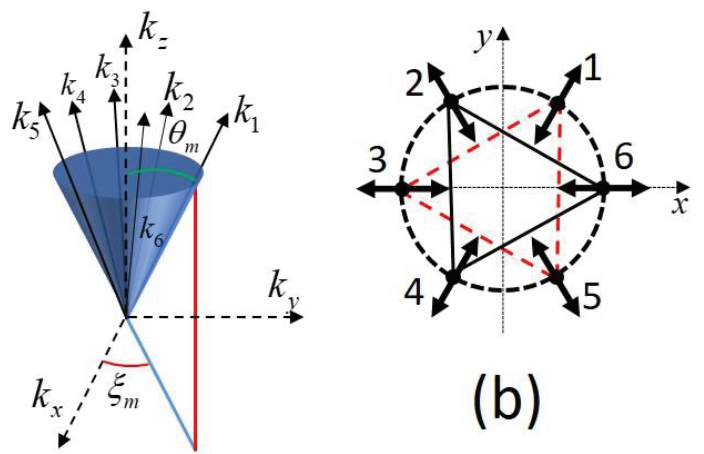

(b)

Fig. 1: (a) Schematic representation of wave vectors of the interfering plane waves; (b) radial orientation of the electric field vectors in the transverse plane.

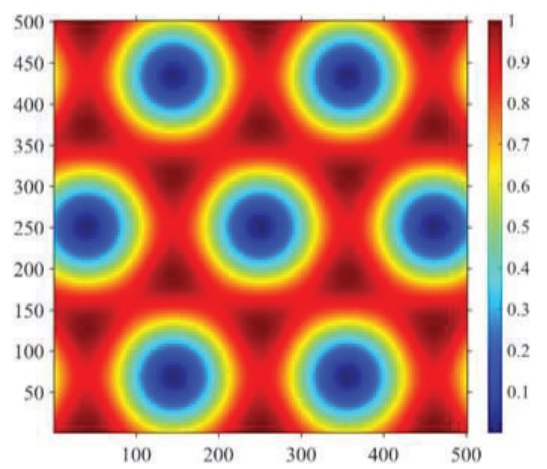

Fig. 2: Simulated transverse intensity distribution. 
The radial orientation of electric field vectors are shown in Fig. 1(b). Beams (1, 3, 5) and beams (2, 4, 6) correspond to two three beam interference pairs. The total transverse intensity for the resultant field corresponding to beams $(1,3,5)$ and beams $(2,4,6)$ is shown in Fig. 2. Here both the three beam pairs correspond to same total intensity distribution.

Normalized Stokes parameters $\left(\mathrm{S}_{0}, \mathrm{~S}_{1}, \mathrm{~S}_{2}, \mathrm{~S}_{3}\right)$ are widely used to describe the spatial distribution of states of polarization in an optical field ${ }^{11-12}$. Spatially varying polarization distributions embedded with singular points can be understood from the complex Stokes fields, which are constructed from the normalized Stokes parameters. In the complex Stokes field $S_{12}=S_{1}+i S_{2}$, constructed from $S_{1}$ and $S_{2}$, the lowest order C-points and V-points appear as phase vortices of topological charge \pm 1 and \pm 2 respectively.

\section{Results and Discussion}

The Stokes phase distributions and Stokes intensity distributions corresponding to two three beam pairs are shown in Fig. 3(a) and Fig. 3(b) respectively. Interestingly both the three beam pairs gives same Stokes phase as well as Stokes intensity distributions. In the Stokes phase distributions the C-points and V-points are appear as phase vortices of charge -1 and +2 respectively.
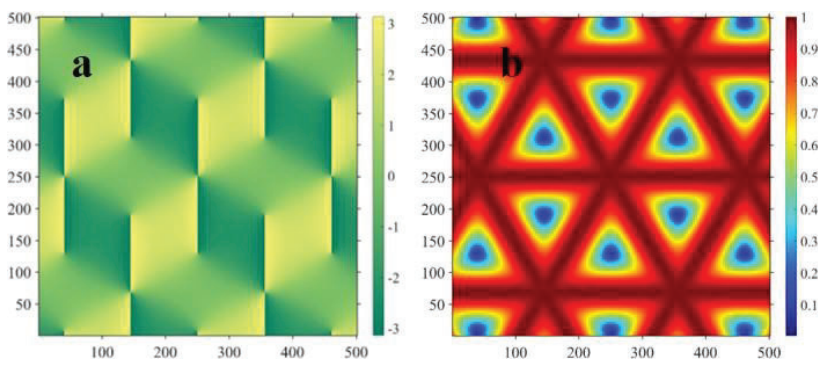

Fig. 3: Simulated a) Stokes phase distribution and b)

Stokes intensity distribution.

Surprisingly the locations of C-points and V-points remains same in both the three beam interference pairs. The polarization distribution corresponding to the resultant field of the interference of beams 1, 3 and 5 is shown in Fig. 4. Polarization distribution corresponding to the resultant field of beams 2, 4 and 6 is shown in Fig. 5. In both the polarization distributions (Fig. 4 and Fig. 5) the right handed and left handed C-points are marked by red and blue colors respectively.

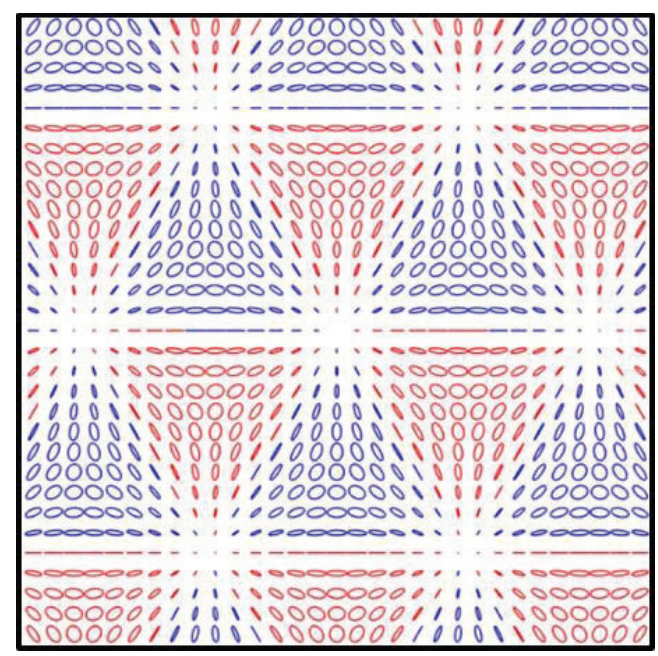

Fig. 4: Simulated polarization distribution corresponding to the interference of beams 1,3 and 5 .

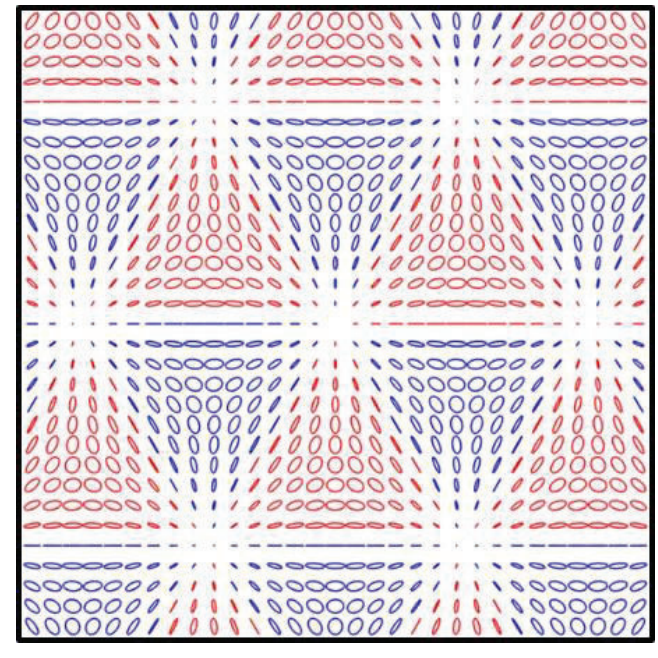

Fig. 5: Simulated polarization distribution corresponding to the interference of beams 2, 4 and 6 .

By comparing Fig. 4 with Fig. 5 one can see that the handedness of $\mathrm{C}$-points, located at the same positions in both the distributions, opposite to each other. When we interfere all these six beams (interfering these two three beam pairs) the resultant polarization distribution turns out to be purely vector field (only spatially varying linear polarizations). The resultant of two opposite handed Cpoints, oriented in the same direction, leads to linear 
polarization, whereas the interference of a V-point with similar V-point remain as the V-point. So in case of six beam interference the resultant field is embedded with only V-points as shown in Fig. 6. The resultant intensity distribution and Stokes phase distribution corresponding to the six beam interference are shown in Fig. 7(a) and (b) respectively. Here the resultant field is infested with Vpoints of opposite polarity but same Poincare Hopf indices.

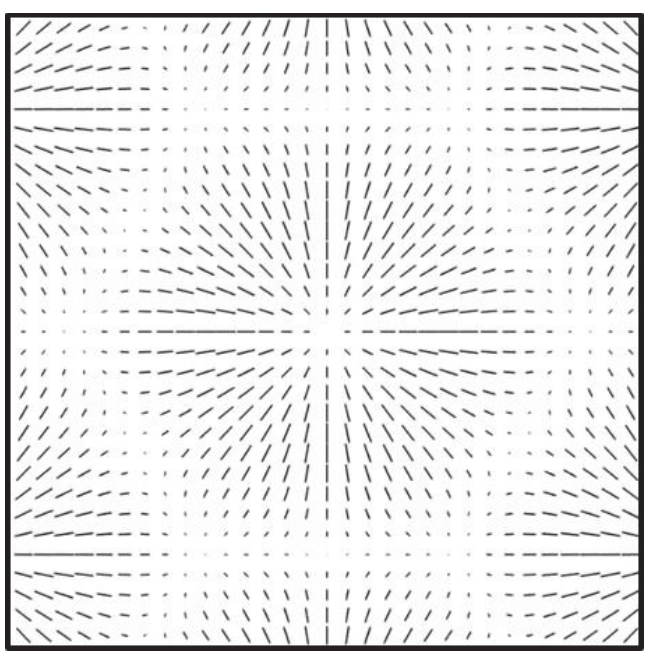

Fig. 6: Simulated polarization distribution corresponding to six beam interference.
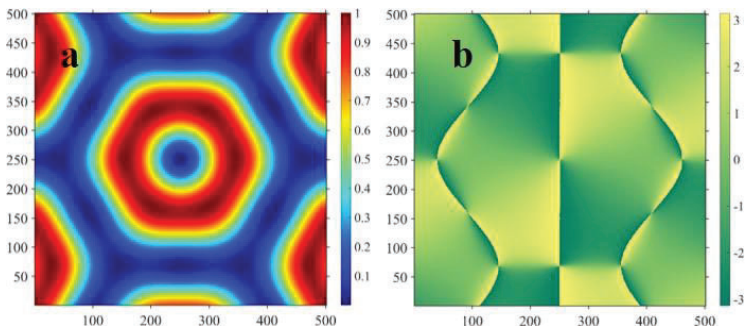

Fig. 7: Simulated a) intensity b) Stokes phase distributions of six beam interference.

\section{Conclusion}

In conclusion we have shown that the interference of two three beam pairs can lead to generation of lattice of Vpoints. Interestingly both the three beam pairs are embedded with C-points and V-points but their resultant field is embedded with only generic V-points. The interference of two opposite handed C-points, both oriented in the same direction, results in a linear polarization. We expect that such polarization lattice structure may lead to novel concept of structured polarization illumination methods in super resolution microscopy.

\section{References}

[1] I. Freund, "Polarization singularity indices in Gaussian laser beams," Opt. Commun. 201, 251-270 (2002).

[2] M. R. Dennis, "Polarization singularities in paraxial vector fields: morphology and statistics," Opt. Commun. 213, 201-221 (2002).

[3] I. Freund, M. S. Soskin, and A. I. Mokhun, "Elliptic critical points in paraxial optical fields", Opt. Commun. 208, 223-253 (2002).

[4] I. Freund, "Mobius strips and twisted ribbons in intersecting Gauss-Laguerre beams", Opt. Commun. 284, 3816-3845 (2011).

[5] A. I. Mokhun, M. S. Soskin, and I. Freund, "Elliptic critical points: C-points, $a$-lines, and the sign rule," Opt. Lett. 27, 995-997 (2002).

[6] S. K. Pal, Ruchi, and P. Senthilkumaran, "C-point and $\mathrm{V}$-point singularity lattice formation and index sign conversion methods," Opt. Commun. 393, 156-168, (2017).

[7] R. Yu, Y. Xin, Q. Zhao, Y. Chen, and Q. Song, “Array of polarization singularities in interference of three waves," J. Opt. Soc. Am. A 30, 2556-2560 (2013).

[8] P. Kurzynowski, W. A. Wozniak, M. Zdunek, and M. Borwinska, "Singularities in interference of three waves with different polarization states," Opt. Express 20, 26755-26765 (2012).

[9] R. W. Schoonover, and T. D. Visser, "Creating polarization singularities with N-pinhole interferometer," Phys. Rev. A 79, 043809 (1-7) (2009).

[10] Ruchi, S. K. Pal, and P. Senthilkumaran, "Generation of V-point polarization singularity lattices," Opt. Express 25, 19326-19331 (2017).

[11] D. Goldstein, Polarized Light (CRC Press, Florida, USA, 2011).

[12] M. Born and E. Wolf, Principle of Optics

(Cambridge University Press, Cambridge, 1999). 


\section{SPIE. DIGITAL}

CONFERENCE PROCEEDINGS

$\begin{array}{lll}\text { PAPERS PRESENTATIONS JOURNALS EBOOKS } & \end{array}$

\section{Search the world's largest collection of optics and photonics applied research}

\section{SPIE. PHOTONICS GO> SPIE. MEDICAL SPIE. ADVANCED}

Newly Published Proceedings Proceedings of SPIE

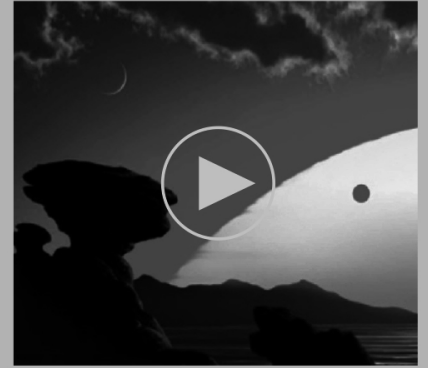

Featured Presentation

Watch the video

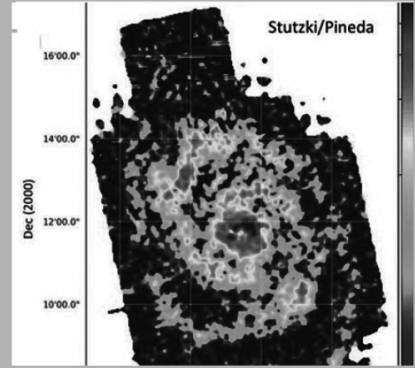

Featured Journal Article

Featuring 490,000 papers from SPIE Conferences, Journals and eBooks.

\section{wWw.spiedl.org}

